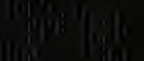


eases. The extent of the growth of the subject of ritamins to which Funk has given such a tremendous impetus can be judged not only by exces. sive popularity, which it unfortunately accutured, but by the unusual volume of work it had inspired in laboratories all over the world. In the valuable monograph of Sherman and Smith, the second and revised edition of which we gladly welcome, the bibliography alone occupies 175 printed pages, which is half the space occupierl by the text of the monograph (350 pages.) The bibliography contains approximately 3500 titles and, assuming that this list is exhaustive of the studies on vitamins which have been published for the last quarter of a century, this represents an output of a new paper perhajs every two or three day's over that entire period. Undoubtedly, the quality of the work produced at such a prolific rate clid not keep pace with the aunantity; nevertheless, it signifies both an intense interest in as well as a deep importance of the sulpject of vitamins. Sherman and Snith trace the growth of the subject in all its cletails and ramifications. From an original number of three, our knowledge has expanded within a very few years to a definite recognition of six different vitamins. And if one reads the signs correctly, the "vitamin $P$ " which has only recently been split into two separate entities may, in the near future, unclergo still further fractionation. Tn the case of a sul)- 
Reviewed in the "Collecting Not" August 22, 1931 , by Dr. S. Morgulis, and presented to the Marine Biological Laboratory Iibrary 



\section{THE VITAMINS}

BY

H. C. SHERMAN

MITCHILL PROFESSOR OF CHEMISTRY

COLUMBIA UNIVERSITY

and

S. L. SMITH

SENIOR CHEMIST, OFFICE OF EXPERIMENT STATIONS,

UNITED STATES DEPARTMENT OF AGRICULTURE

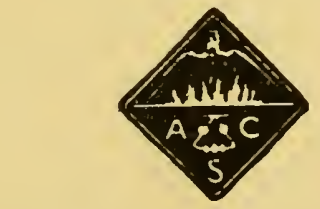

American Chemical Society

Monograph Series

SECOND EDITION

BOOK DEPARTMENT

The CHEMICAL CATALOG COMPANY, Inc. 419 FOURTH AVENUE, AT 29TH STREET, NEW YORK, U. S. A. 
COPYRIGHT, 1931, BY

The CHEMICAL CATALOG COMPANY, Inc.

All rights reserved 


\section{GENERAL INTRODUCTION}

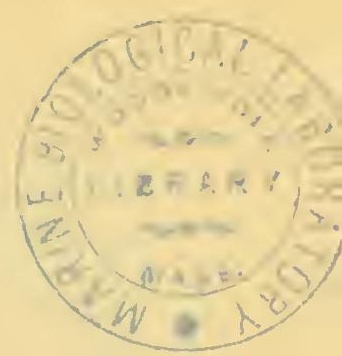

\section{American Chemical Society Series of Scientific and Technologic Monographs}

By arrangement with the Interallied Conference of Pure and Applied Chemistry, which met in London and Brussels in July, 1919, the American Chemical Society was to undertake the production and publication of Scientific and Technologic Monographs on chemical subjects. At the same time it was agreed that the National Research Council, in coöperation with the American Chemical Society and the American Physical Society, should undertake the production and publication of Critical Tables of Chemical and Physical Constants. The American Chemical Society and the National Research Council mutually agreed to care for these two fields of chemical development. The American Chemical Society named as Trustees, to make the necessary arrangements for the publication of the monographs, Charles L. Parsons, Secretary of the American Chemical Society, Washington, D. C.; John E. Teeple, Treasurer of the American Chemical Society, New York City; and Professor Gellert Alleman of Swarthmore College. The Trustees have arranged for the publication of the American Chemical Society series of (a) Scientific and (b) Technologic Monographs by the Chemical Catalog Company of New York City.

The Council, acting through the Committee on National Policy of the American Chemical Society, appointed the editors, named at the close of this introduction, to have charge of securing authors, and of considering critically the manuscripts prepared. The editors of each series will endeavor to select topics which are of current interest and authors who are recognized as authorities in their respective fields. The list of monographs thus far secured appears in the publisher's own announcement elsewhere in this volume.

The development of knowledge in all branches of science, and especially in chemistry, has been so rapid during the last fifty years and the fields covered by this development have been so varied that it is difficult for any individual to keep in touch with 
the progress in branches of science outside his own specialty. In spite of the facilities for the examination of the literature given by Chemical Abstracts and such compendia as Beilstein's Handbuch der Organischen Chemie, Richter's Lexikon, Ostwald's Lehrbuch der Allgemeinen Chemie, Abegy's and Gmelin-Kraut's Handbuch der Anorganischen Chemie and the English and French Dictionaries of Chemistry, it often takes a great deal of time to coördinate the knowledge available upon a single topic. Consequently when men who have spent years in the study of important subjects are willing to coördinate their knowledge and present it in concise, readable form, they perform a service of the highest value to their fellow chemists.

It was with a clear recognition of the usefulness of reviews of this character that a Committee of the American Chemical Society recommended the publication of the two series of monographs under the auspices of the Society.

Two rather distinct purposes are to be served by these monographs. The first purpose, whose fulfilment will probably render to chemists in general the most important service, is to present the knowledge available upon the chosen topic in a readable form, intelligible to those whose activities may be along a wholly different line. Many chemists fail to realize how closely their investigations may be connected with other work which on the surface appears far afield from their own. These monographs will enable such men to form closer contact with the work of chemists in other lines of research. The second purpose is to promote research in the branch of science covered by the monograph, by furnishing a well digested survey of the progress already made in that field and by pointing out directions in which investigation needs to be extended. To facilitate the attainment of this purpose, it is intended to include extended references to the literature, which will enable anyone interested to follow up the subject in more detail. If the literature is so voluminous that a complete bibliography is impracticable, a critical selection will be made of those papers which are most important.

The publication of these books marks a distinct departure in the policy of the American Chemical Society inasmuch as it is a serious attempt to found an American chemical literature without primary regard to commercial considerations. The success of the venture will depend in large part upon the measure of coöperation which can be secured in the preparation of books 
dealing adequately with topics of general interest; it is earnestly hoped, therefore, that every member of the various organizations in the chemical and allied industries will recognize the importance of the enterprise and take sufficient interest to justify it.

\section{A MERICAN CHEMICAL SOCIETY}

\section{BOARD OF EDITORS}

Scientific Series:-

William A. Noyes, Editor, S. C. LIND, Lafayette B. Mendel, Arthur A. Noyes, Julius STIEgLiTz.
Technologic Series:-

Harrison E. Howe, Editor, Walter A. Schmidt, F. A. Lidbury, Arthur D. Litthe, Fred C. Zeisberg, JoHn JoHnston,

R. E. WiLson, E. R. WeIdLEIN, C. E. K. MEES, F. W. WiLLARD. 



\section{Preface to First Edition}

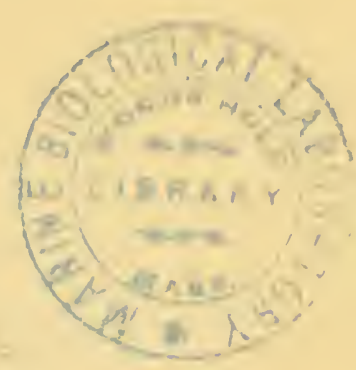

Although the chemical nature of vitamins is still unknown much of both scientific and practical importance has been learned regarding them, and the present work is designed to summarize this knowledge in as judicial a manner as possible. To those who "don't believe in vitamins because we have never seen one" or who hold that we know nothing about them chemically because no structural formula can yet be assigned, we would commend the view of Hopkins that it is easier to sympathize with the farmer who will believe in vitamins only when their price per hundredweight can be quoted in the market than with the scientific man who refrains from an endeavor to appraise their importance until they have been separated in pure condition. "To be logical he should then avert his eyes from such agents as toxins and antitoxins, not to speak of enzymes; powerful realities all of them, which though of unknown constitution by no means elude objective and quantitative study." It is hoped that the present work may do something to stimulate such quantitative research as distinguished from the merely qualitative tests which have now largely served their purpose, and at the same time may serve to present the chief facts now known in such a way as to show their true significance and avoid exaggerated impressions. ...

The greater part of the text was written near the middle of the year 1921 but on a few points we have been able to bring our summary down to about the end of that year, thus covering the work of the decade since the term was introduced and the conception of the vitamins began to be current. A carefully selected alphabetical bibliography of about one thousand titles is included. Names and dates are so used in the text that the reader may readily locate original places of publication by turning to the bibliography, the need of footnote references being thus avoided. It is hoped that this bibliography will suffice to put the reader in touch with practically all of the more significant literature of the vitamins to the end of 1921 . The writers will be glad to be notified of any serious omissions in case a second edition should be called for. 



\section{Preface to Second Edition}

Few groups of substances can rival the vitamins in the extent of the development of knowledge regarding their scientific significance in advance of the working out of their structural formulas.

In the pages which follow, an attempt has been made to summarize the very considerable amount of information which has been gained regarding the chemical natures of the individual vitamins, and the more voluminous knowledge regarding their rôles in life processes, their formation and distribution in nature, their relative abundance in different types of food materials and stability under the conditions to which these are likely to be subjected.

It is doubtless only because the history of the discovery of the vitamins and of the recognition of their great scientific and practical importance has been made so rapidly as to outrun the investigation of their chemical natures, that the chemically very different substances here considered are grouped together under one collective designation. With increasing knowledge it becomes more and more evident that several (at least) of the vitamins are of very different chemical nature from each other. The trend of the findings of recent research is decidedly toward diversification rather than unification of the chemistry of the vitamins. The time is already past for any such unified style of treatment as would be suggested by the literal interpretation of the term monograph: to attempt it would not be consistent with present knowledge of the wide chemical differences between individual vitamins.

Hence we have attempted rather to summarize the more important of our knowledge of each vitamin in a separate chapter, letting each chapter develop into the form best suited to the presentation of its own subject-matter, and to append a collective bibliography sufficiently complete to guide the reader to practically all the important literature of the vitamins.

In general both the text and the bibliography have been brought down to about the middle of the year I930; but our endeavor has been to serve the reader as effectively as circumstances would permit rather than to maintain a meticulously uniform date-limit, and it will doubtless be found that contributions near at hand have been included down to a somewhat more recent date than those published at a great distance or for other reasons less readily accessible. 
To have mentioned every worthy contribution to vitamin research in the text would have made this volume so large and expensive as to have limited its usefulness. In accordance with the policy adopted by the Editors for this series of monographs, we have sought conciseness for the sake of the reader rather than completeness of citation of the work of every author who has contributed to the rapidly growing literature of the vitamins.

Recognizing that knowledge of vitamins touches closely the intellectual and professional interests of many other scientifically trained persons as well as professional chemists, we have sought to treat the subject with the interests of all probable readers in view, and have thus included more of physiological, pathological, nutritional and health aspects than would otherwise be expected in a chemical monograph.

The authors are greatly indebted to Dr. M. L. Caldwell, Dr. F. L. MacLeod and Dr. H. K. Stiebeling for collaboration in the present revision. 


\section{Table of Contents}

Chapter I. General Introduction to the Vitamin Theory . . . . . . . . . . . 13

Chapter II. Vitamin B $\left(\mathrm{B}_{1}\right)$. . . . . . . . . 24

Antineuritic and Growth-promoting Functions. Chemical Nature. Physiological Properties and Relation to Nutrition and Health. Distribution in Foods. Quantitative Determination. Physico-chemical Properties.

Chapter III. Vitamin G $\left(\mathrm{B}_{2}\right)$. . . . . . . . .

Differentiation from Vitamin B. Concentration. Quantitative Determination. Physico-chemical Properties. Distribution in Foods. Relation to Nutrition.

Chapter IV. Vitamin C . . . . . . . . .

Vitamin C Requirements of Different Species. Relation to Nutrition and Health. Quantitative Determination. Occurrence and Stability in Foods. Chemical Nature.

Chapter V. Vitamin A . . . . . . . . . tion in Nature. Measurement of Vitamin A Values. Occurrence in Foods. Relation to Nutrition and Health.

Chapter VI. Vitamin D

Antirachitic Effects and Relations to Nutrition and Health. Formation by Irradiation. Chemical Nature. Quantitative Measurement. Food Sources of Vitamin D.

Chapter Vil. Vitanin E

Distribution in Foods. Relation to Nutrition. Physical and Chemical

Properties. Attempted Isolation.

Bibliography

INDEX OF AuthoRS

INDEX OF SubJects 



\section{THE VITAMINS}

CHAPTER I

\section{GENERAL INTRODUCTION TO THE VITAMIN THEORY}

Until recent years the discussion of food values and of nutrition and other life processes from the chemical point of view was hampered by the embarrassing fact that all attempts at prolonged feeding upon artificial mixtures containing the substances known to be necessary in nutrition had ended in failure. Nor were such feeding experiments any more successful when great care was devoted to the chemical purity of the substances fed. Whether nutritive failure resulted from the need of other substances than those known as essential, or from faulty selection or quantitative combination of the nutrients entering into the artificial food mixture remained obscure until the work of Hopkins in England, and of Osborne and Mendel and McCollum and Davis in this country, made it clear that a natural food supply furnishes, and normal nutrition requires, other substances in addition to proteins, fats, carbohydrates, water, and salts. With this fact now convincingly established, it is easier to see that it was foreshadowed by many earlier observations than it is to say definitely when or by whom the existence of the substances now known as vitamins was discovered. Our present "vitamin theory" or point of view in regard to this branch of chemistry is rather the product of development than of any isolated discovery, and much of this development antedates the introduction of the word vitamin.

\section{Early Evidence from Observations upon Disease}

As early as 1720 , Kramer wrote in his Medicina Castrensis that neither medicine nor surgery could give relief in scurvy, "But if you can get green vegetables; if you can prepare a sufficient quantity of fresh antiscorbutic juices, if you have oranges, lemons, citrons, or their pulp and juice preserved with whey in cask, so that you can make a lemonade, or rather give to the quantity of 3 or 4 ounces of their juice in whey, you will, without other assistance, cure this dreadful evil." And Lind confirmed this doctrine by well-controlled experiments upon human subjects. As surgeon of the Salisbury he had, during one voyage, twelve scurvy patients, without sufficient supplies of oranges 
and lemons for them all. He therefore gave all the patients the best care and housing that the circumstances permitted, and to two of them he gave oranges and lemons while others received cider, cream of tartar, elixir of vitriol, or other medicaments which had been recommended by different medical writers of that day. The men receiving oranges and lemons made rapid recovery, those having cider showed improvement, while the different medicines tried were found to be without effect upon the scurvy. With these and other experiences in mind, Lind, writing in 1757 , pointed out that while lemon juice retained its antiscorbutic property sufficiently well to make it useful for long voyages, there was no hope of preventing scurvy by means of dried spinach since this had lost during its preparation "something contained in the natural juices of the plant" which "no moisture whatever could replace." The idea that the antiscorbutic properties of certain foods is due to some definite (though not yet identified) thing, was again clearly stated by Budd who in 1841 ascribed the action of antiscorbutic foods "to an essential element, which, it is hardly too sanguine to state, will be discovered by organic chemistry or the experiments of physiologists in a not far distant future" (Hess, 1920).

In 1804 the regular issue of a ration of lemon juice was made compulsory in the British navy and thereafter scurvy was a comparatively rare disease among British sailors, whereas a few years earlier thousands of cases were reported every year. (British Committee Report, 2 nd ed. p. 126.) The relative ease with which scurvy can thus be prevented under ordinary conditions doubtless contributed to delay the rigorous search for its cause.

In the Japanese navy the disease beriberi was for a long time exceedingly prevalent. The number of cases sufficiently severe to be officially reported ranged annually from 25 to 40 per cent of the entire navy force during the years 1878 to 1882 . At about this time Takaki (1885) became convinced that the diet had some relation to the disease and succeeded in having the ration modified in several respects, the most important change being the substitution of barley for a considerable part of the polished rice which had previously been the chief article of food. Immediately following this change in the ration, beriberi practically disappeared from the Japanese navy.

Eijkman, in 1897, summarized the results of a large number of observations which he had made during 1890-1896 upon "an illness of fowls similar to beriberi" which he was able to produce experimentally by feeding the fowls upon polished rice, and to prevent or cure by feeding an extract of the rice polishings. By means of systematic experi- 
ments he refuted the theories that beriberi might be due to the presence of pathogenic organisms in the rice, to lack of mechanical stimulation of the intestine, or to insufficiency of total food value, protein, or salts. The explanation first offered by Eijkman was to the effect that the condition is a state of intoxication brought about by the metabolism of excessive quantities of starch and that in the silver-skin of the rice (and in some other foods) there is to be found a substance or substances which counteract the toxic products of the disturbed metabolism. As has been pointed out in the British Committee Report, the pharmacological bias thus given by Eijkman to the interpretation of his observations resulted in obscuring the fact that he had here adduced experimental evidence of the presence in rice polishings, and some other foods, of a substance or substances playing an important part in nutrition and whose existence was previously unknown.

Later Eijkman (1906) withdrew the hypothesis of a nerve poison and stated clearly that there is present in rice polishings a substance of a different nature than protein, fat or salts which is indispensable to health and the lack of which causes "nutritional polyneuritis,"-this designation being used by Eijkman as the title of his second paper of this date. Thus the existence of an antineuritic substance as something different from the known foodstuffs but essential to normal nutrition was clearly discovered by Eijkman long before the term vitamin was proposed. Eijkman, therefore, seems to have been the first to produce experimentally a nutritional-deficiency disease. Eijkman's excellent pioneer work in this field does not seem to have received the attention that it deserves, either from the chemical or the physiological point of view. Even as regards the cause and cure of beriberi, the significance of his work was not generally recognized until after several years during which much new evidence relating to the dietary origin of the disease beriberi and the substantial duplication of beriberi in the experimental polyneuritis of fowls was brought forward. Only a few of the investigations of this period can be mentioned here.

Grijns, in 1901, showed that the polyneuritis of fowls, produced by feeding polished rice, could be prevented by adding the native bean, katjang idjo (Phaseolus radiatus), to the polished rice diet. HulshoffPol (1902) then tested this bean and found it effective both as a preventive and curative agent in human beriberi. He emphasized: (1) that a diet consisting too largely of highly milled rice will produce beriberi, (2) that the disease can be prevented by the addition of beans to the diet, (3) that beriberi must be due to some deficiency in the diet since if the rice were toxic the disease could hardly be prevented simply 
by the addition of the beans. He then made a decoction of the beans which, by the successful treatment of 18 patients, he showed to contain the substance needed for the cure of beriberi. As a further step he purified the decoction by precipitation with basic lead acetate and subsequent removal of the lead. With this purified preparation he cured four cases of human beriberi, and both cured and prevented the polyneuritis of fowls. Plainly these experiments afforded very strong evidence that the polyneuritis of fowls induced by feeding polished rice is essentially the same condition as beriberi and that both are due to the lack of a definite substance, which occurs in rice polishings, beans, and some other foods.

Chamberlain and Vedder of the United States Army medical commission for the study of tropical diseases in the Philippines made considerable progress in the study of the chemical nature of the substance which prevents and cures beriberi in man and experimental polyneuritis in fowls and concluded that it was an organic base but not an alkaloid.

The work looking toward chemical identification of the active substance thus begun by Hulshoff-Pol and by Chamberlain and Vedder was then taken up by Funk, who in a paper published in December 1911, first claimed to have isolated the active substance in an approximately pure state. He gave to it the name beriberi vitamine. The name vitamine thus introduced by Funk was obviously designed to indicate that the substance in question was an amine essential to life or bearing special relationship to vitality. He believed this substance to be a combination of nicotinic acid with a pyrimidine base, and predicted that other substances would be found to bear the same relation to other "deficiency diseases" which this "beriberi vitamine" bears to beriberi and experimental polyneuritis.

Even in 1907, Holst had described experimental studies of ship beriberi and scurvy, and now at practically the same time that Funk put forward this suggestion, Holst and Frölich of Christiania published (1912) the results of several years of systematic work upon experimental scurvy. Because of its importance to the Norwegian fisherman and merchant marine, they had been interested in the study of ship beriberi and had felt that the application of laboratory experiments to human therapeutics and prophylaxis would rest upon a more satisfactory basis if they could produce the disease experimentally in mammals rather than fowls or pigeons. They found, however, that the guinea pig when kept upon a diet of polished rice developed the symptoms of scurvy instead of beriberi, and that scurvy also developed when whole 
grains, such as would ensure protection against beriberi, were fed. From this they proceeded to a thorough study of experimental scurvy in the guinea pig, the results of which (to be described in Chapter IV) indicated clearly that scurvy is due to the deficiency in the diet of a substance soluble in water and rather easily destroyed by heating under ordinary conditions, but more stable in acid than in neutral or alkaline medium.

Shortly after this, Osborne and Mendel (1913a) found that their experimental animals became subject to a characteristic eye disease when kept upon diets devoid of fat, while the simple addition of butter fat to the diet sufficed either to prevent or to cure the disease. Further experiments showed that cod-liver oil shared this property with butter fat, and that beef fat showed it in lesser degree, while it was not shown under like conditions by certain other fats such as lard or cottonseed oil. As this eye disease involves an infection it is not so purely a "deficiency disease" as scurvy and beriberi now appear to be, yet this distinction does not seem to us to be particularly significant since the susceptibility to the eye trouble induced by a deficiency in the diet is the chief determining cause of the disease. (It may also be noted that many of the physicians who have had to do with beriberi and scurvy have emphasized the view that the clinical forms of these diseases usually show infection superimposed upon the nutritional deficiency.)

Thus observations upon disease led to the conception of the existence of several substances of the vitamin type, needed for the prevention of the "deficiency" diseases and for the maintenance of that condition of healthy resistance which safeguards against susceptibility to infections in other ways than through the ordinary immunological mechanism.

While in this way the conception of the vitamins has arisen largely in connection, and is intimately associated, with that of the deficiency diseases, yet from our present standpoint an even greater interest attaches to vitamins as chemical substances occurring in natural food materials and having important functions in the normal processes of nutrition.

\section{Early Evidence from Experiments upon Normal Nutrition}

In 1881, Lunin, in the course of a report upon investigations of the significance of certain inorganic substances in animal nutrition, remarked that since mice can be successfully nourished under laboratory conditions upon milk but not upon purified proteins, fats, carbohydrates, salts and water, it follows that milk must contain other 
substances indispensable for nutrition. This statement was given wide circulation in Bunge's Textbook of Physiological and Pathological Chemistry, but never became influential, probably because the evidence upon which it was based was not sufficiently convincing. Limitations of space make it impracticable for us to attempt to review and discuss here the hints which might be gathered from the writings of other authors ${ }^{1}$ and which in the light of present knowledge can be construed as foreshadowing, rather than as constituting, an effective discovery of vitamins as factors in normal nutrition.

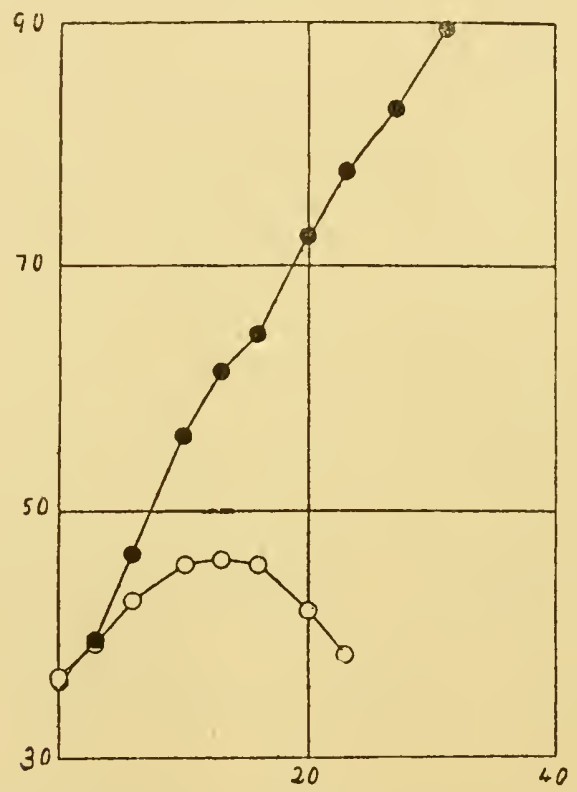

FIG. 1.-Growth curves of rats with and without the vitamins furnished by small amounts of milk. The lower curve shows the average weight of six rats receiving a diet of purified foodstuffs; the upper curve that of six similar rats receiving the same diet with the addition of small amounts of milk. Abscissætime in days; ordinates-average weight in grams. (Courtesy of Professor Hopkins and the Medical Research Committee of Great Britain.)

To Hopkins is due, in our opinion, the honor of having first made clear that natural food contains and normal nutrition requires some other substance or substances beside proteins, fats, carbohydrates, and mineral matters. As early as 1906, he had determined and reported that, "no animal can live upon a mixture of pure protein, fat, and carbohydrate and even when the necessary inorganic material is carefully

\footnotetext{
1 Notable among such writings is that of Pekhelharing (1505) recently recalled by van
} Leersum, Science, October 8, 1926. 
supplied, the animal still cannot flourish. The animal body is adjusted to live either upon plant tissues or other animals and these contain countless substances other than the proteins, carbohydrates, and fats." In his experiments, using rats as subjects, Hopkins found that the addition of small amounts of milk to diets otherwise composed of purified foodstuffs resulted in growth (Figs. 1 and 2), and that this was due to an alcohol-soluble organic substance or substances in the

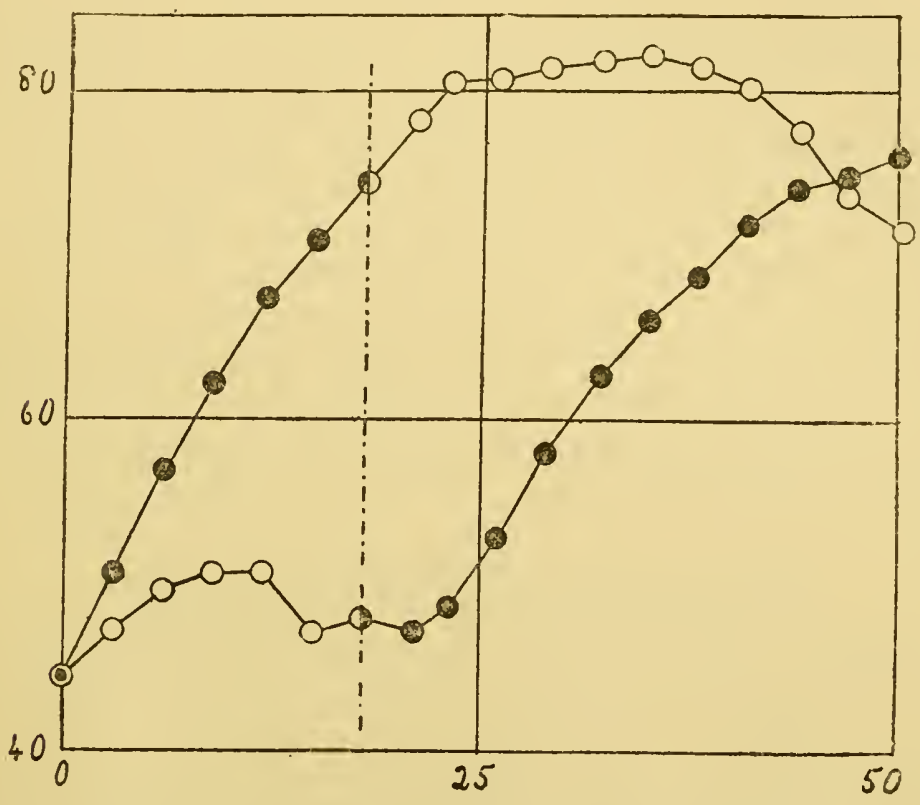

FIG. 2.-Growth curves of rats with and without small amounts of milk as source of vitamins. The lower curve up to the eighteenth day represents rats on purified food; the upper curve similar rats having milk each day in addition to this food. On the eighteenth day marked by the vertical dotted line the milk was transferred from one set to the other. Abscissæ-time in days; ordinates-average weight in grams. (Courtesy of Professor Hopkins and the Medical Research Committee of Great Britain.)

milk and not to any of its previously known constituents. Certain vegetables had the same property in lesser degree than milk. As described in the following chapter, Osborne and Mendel demonstrated that a similar growth-promoting property was possessed by their "proteinfree milk," a powder prepared by removing the fat, casein, and albumin from cows' milk and evaporating the filtrate to dryness. A little later it was found both by McCollum and Davis (1913) and by Osborne and Mendel (1913a) that the fat of milk also possesses a 
growth-promoting property which is not an attribute of the triglycerides themselves but rather of a fat-soluble substance. Both the water-soluble and the fat-soluble growth-promoting substances are fairly soluble in alcohol which accounts for the fact that Hopkins' alcoholic extract of dry milk contained both (or all) of these essentials and supplied all that was needed for the growth of rats when added to his mixture of previously recognized foodstuffs. The theory soon gained currency that the water-soluble and fat-soluble substances essential to growth were the same as the water-soluble and fat-soluble "vitamins" which prevent beriberi and ophthalmia, respectively. The addition of these vitamins to a diet otherwise consisting of properly selected isolated foodstuffs appeared to provide all that was needed for the growth of rats. The normal growth of babies and of young monkeys and guinea pigs (and doubtless of many other species also) required, however, the feeding of sufficient amounts of the antiscorbutic vitamin as well.

Thus by 1920 it was well established that the normal growth of the young, at least in the human and some other species, demands adequate supplies of at least three substances believed to be identical with the substances which are essential to the prevention of the three "deficiency diseases" of scurvy, beriberi, and the characteristic ophthalmia.

\section{Terminology and Recent Developments}

The names which best expressed the distinctive properties of these three newly discovered nutritional essentials were perhaps "antineuritic," "antiscorbutic," "antiophthalmic" substance, respectively. From the standpoint of food chemistry and normal nutrition, however, it seems an unnecessary and generally undesirable circumlocution that a substance having an important rôle in normal processes should be named according to the abnormal condition which arises when it is absent. Moreover, Funk's term vitamine was criticized both because it implies that these substances are amines, which is not proven in any case and certainly not probable in all, and because the choice of "vita" as a designation was thought by some to carry an exaggerated implication of unique responsibility for life and vitality whereas other substances such as tryptophane are no less essential. But if "vita" seems to claim too much, the designation "accessory" suggested by Hopkins is certainly too modest, since an accessory substance would ordinarily be judged to be dispensable whereas the indispensability of these substances is one of their most marked characteristics.

In order to avoid these difficulties McCollum suggested that, until 
such time as chemical names can properly be assigned to them, these substances be known by alphabetical designations qualified only by such statement of their solubilities as may seem helpful. In his earlier rations of supposedly purified foodstuffs McCollum unwittingly furnished water-soluble vitamin in the lactose fed. Hence he at first concluded that the fat-soluble substance of butter, egg-fat, etc., was the only unidentified substance essential to normal nutrition. Thus this became, in his alphabetical terminology, "fat-soluble A," and the water-soluble substance which he next recognized as essential was designated as "water-soluble B." A little later the antiscorbutic substance was included in the alphabetical terminology as "water-soluble C."

In 1920, Drummond suggested that the designations then most common, those of Funk and of McCollum, be combined and simplified both for convenience and to free them from questionable implications as follows: That the familiar alphabetical designations be retained but without the antecedent statements of solubility: and that the original designation of Funk be retained in part but the final " $\mathrm{e}$ " be dropped, so that the resulting word vitamin shall carry no implication as to the chemical constitution of the substance. The three substances then recognized as belonging to this group thus became vitamin $A$, vitamin $\mathrm{B}$, and vitamin $\mathrm{C}$, respectively, and the way was left open for any others, whose existence might be demonstrated before they are chemically identified, to be added in chronological order and in alphabetical sequence in one series regardless of solubilities. This suggestion has now been so generally adopted that it will here be regarded as established usage, though the adjective indicating the originally observed solubility or the physiological significance of a vitamin may still be used in any case in which it seems helpful to the discussion in hand. Especially important is it in our judgment to avoid habits of thought or expression which might prejudice the interpretation of future experiments; and, on the other hand, terminology should be recognized for merely what it is and should not be blamed for "misdirected research."

Since 1920, all qualified students of the subject have recognized the existence of at least the three vitamins, A, B, and C; though perhaps not all have recognized with equal clearness that any one of these terms as originally introduced might, with the growth of knowledge through further investigation, be found to have covered more than one substance. As a matter of fact, the subsequent additions to the vitamin alphabet have arisen not so much by wholly new discovery as by differentiation.

Whereas formerly the terms vitamin A and fat-soluble vitamin were 
employed interchangeably the existence has now been established of at least two other fat-soluble factors not identical with vitamin A. These are the antirachitic substance now usually called vitamin $\mathrm{D},{ }^{2}$ and a substance essential to reproduction (though no more so than is vitamin A) which was discovered by Evans and by him at first designated as $\mathrm{X}$, but which is now commonly known as vitamin $\mathrm{E}$. Vitamins $\mathrm{D}$ and E were differentiated from vitamin A by observed differences in occurrence, certain physical and chemical characteristics, and definite physiological properties without unduly altering the original significance of vitamin A.

What was formerly known as vitamin $\mathrm{B}$, as explained in Chapters II and III, has ceased to exist as hitherto inderstood; and to explain the functions previously attributed to it, at least two independent factors must be postulated, neither of which alone fulfills the conception for which the term vitamin B had stood. One of these is the antineuritic vitamin which is also essential to growth, while the other is (or others are) essential to growth as well. This has resulted in considerable confusion in terminology which was not the case in naming the fat-soluble vitamins.

At first it seemed best, in the interest of clarity, to use two new designations for the two factors into which the old "vitamin B complex" had been resolved- "vitamin F" for the antineuritic substance, and "vitamin G" the more heat-stable "Goldberger factor" (described in Chapter III beyond); and these designations have been used by some of the scientific bureaus of the United States Government as well as individual American writers, especially during the years 1927 and 1928. Meanwhile, however, the term vitamin $F$ had been suggested in connection with a postulated new fat-soluble vitamin which conception, however, was subsequently dropped. Thus the usage regarding the letter $\mathrm{F}$ became somewhat confused, and the recent tendency of American writers has been to employ the designation "vitamin B" (new style) for the antineuritic substance and continue the use of the term "vitamin G" in the sense indicated above and developed in Chapter III; while the British have suggested the tentative use of the terms $B_{1}, B_{2}$, etc., to designate the different nutritionally essential substances of the original vitamin $\mathrm{B}$ complex. As will appear in Chapters II and III there is now abundant evidence of the existence of more than two such substances.

As the vitamin theory of the present day has so largely grown out

\footnotetext{
2 The term vitamin $\mathrm{D}$ has also been applied by some writers to the substance generally known as bios, which is characterized by its accelerating influence upon the growth of yeast. Bios has been quite fully discussed by Tanner (1925).
} 
of, and developed around, the study of the antineuritic factor, we shall, in the chapters which follow, discuss first the antineuritic vitamin $B$ $\left(B_{1}\right)$, then vitamin $G\left(B_{2}\right)$ as the factor (substance or substances) most directly differentiated from, and usually regarded as most closely related to, vitamin $B$; and then will follow, in what seems to be the most logical sequence, the chapters deroted to the water-soluble vitamin $\mathrm{C}$ and to the fat-soluble vitamins $\mathrm{A}, \mathrm{D}$, and $\mathrm{E}$, respectively. 


\section{CHAPTER II}

\section{VITAMIN B $\left(B_{1}\right)$}

\section{Development of Knowledge of Vitamin B as an Antineuritic Substance}

We have seen that Eijkman, in his papers published from 1897 to 1906 , clearly set forth his discovery that a diet of polished rice produces in fowls a condition of nutritional polyneuritis which appears to be essentially identical with typical beriberi in man, and that this disease is due to the lack of a substance essential to normal nutrition, which substance exists in the rice polishings (i.e., in the outer layers and embryo of the rice kernel) as well as in other natural foods. After the publication of the first of these papers, Grijns (1901) took up the subject, confirmed Eijkman's earlier work and extended it to show that the antineuritic or protective substance which Eijkman had demonstrated to occur in rice, occurs also in legumes and in this case not wholly in the outer layers of the seed.

Failure to isolate the antineuritic substance from rice polishings led Grijns to determine whether the heating of unpolished rice (or of the phaseolus seeds) destroyed the protective power. Of four fowls fed unpolished rice which had been heated at $120^{\circ} \mathrm{C}$., two died of polyneuritis after five or six months, while two remained well at the end of eleven months. On polished rice fowls may be expected to develop polyneuritis in about three weeks, while on unpolished and unheated rice there should be entire freedom from the disease. Hence Grijns concluded that heating at $120^{\circ} \mathrm{C}$. destroyed much but not all of the antineuritic substance of rice. Similarly heating the beans (phaseolus) for one hour at $120^{\circ} \mathrm{C}$. destroyed their antineuritic property to such an extent that four fowls, fed such quantities of the beans as would ordinarily protect them, all died of polyneuritis in from 33 to 37 days. Grijns also observed that fowls fed raw meat were protected, while those fed sterilized meat were not.

Eijkman in 1906 described experiments designed to throw light upon the chemical nature of the antineuritic substance. He found that it was soluble in water, was dialyzable, and was not readily precipitated from water solution by alcohol. In Eijkman's experiments the antineuritic substance appeared to be entirely destroyed when unpol- 
ished rice was heated in an autoclave for two hours at $125^{\circ} \mathrm{C}$, , but only partially destroyed when the temperature of heating was $115^{\circ} \mathrm{C}$. Similar results were obtained in experiments with other grains. Eijkman also showed that fowls rendered polyneuritic by feeding with grain which had been heated in the autoclave could be cured by giving water extracts of raw grain but not by phosphorus compounds prepared from such extracts - a result of considerable importance at that date since under the teaching of Schaumann there was a tendency to ascribe the antineuritic property to some organic phosphorus compound. As further evidence on this point Eijkman showed in 1911 that an extract of rice polishings containing only the slightest traces of phosphorus yet had marked curative effects in polyneuritis. He also showed that the curative substances could be administered either by mouth or by injection.

Simultaneous with much of this work by Eijkman were the experiments of Hopkins $(1906,1912)$ and those of Osborne and Mendel (1911). The former showed that milk and some at least of the vegetables contained an organic substance or substances soluble in water and alcohol which induced growth in young animals fed upon mixtures of purified foodstuffs whereas when these foodstuffs were well purified and fed without accessory substances growth always failed. Osborne and Mendel further showed that the water solution remaining after removal of fat, casein and lactalbumin from milk (the so-called proteinfree milk) was much more efficient in inducing growth than was a corresponding mixture of lactose and pure salts or milk ash, thus implying the presence of some water-soluble organic growth-promoting substance.

It was, however, principally in connection with the study of beriberi and experimental polyneuritis in birds that the vitamin conception was actively developed at the beginning of the second decade of this century. Takaki's conquest of beriberi in the Japanese navy by a change of ration did not carry conviction to many at the time because the only explanation which he had to offer, namely, that the new ration was richer in protein than the old, was seen to be inadequate; and the work of Eijkman and of Grijns did not at first command much attention because many were slow to accept the view that the experimental polyneuritis of fowls was the same disease as human beriberi. Between 1900 and 1910, however, there was accumulated indubitable evidence in support of the view that beriberi is essentially a nutritional disease which may be prevented by the consumption of adequate amounts of any of the foods containing the unknown antineuritic substance. 
Chamberlain, Vedder, and their associates (1911) of the United States army medical commission for the study of tropical diseases in the Philippines were able to report in 1910 the rapid eradication of beriberi from the native troops known as Philippine Scouts, and promptly began a systematic investigation looking toward the chemical identification of the antineuritic substance. As has been mentioned briefly in the preceding chapter, they found the neuritis-preventing substance to be soluble in water and alcohol but insoluble in ether, readily dialyzable through an ordinary parchment membrane, rather readily adsorbed by bone black, and gradually decomposed by heating at temperatures in the region of $115^{\circ} \mathrm{C}$. to $125^{\circ} \mathrm{C}$. Attempts to identify it with any one of a number of salts and organic phosphorus compounds, with choline or lipoids of the lecithin group, and with arginine, histidine, asparagine or other amino acids were unsuccessful. They suggested that, though not identical with any of the nitrogen compounds tested, the antineuritic substance might prove to be a nitrogenous base but not an alkaloid.

Simultaneously with this work, Fraser and Stanton (1911) had shown that the antineuritic substance is soluble in both alcohol and water, stable to heat in acid solution, and much more readily destroyed in alkaline solution.

Cooper and Funk (1911) reported that dried, pressed yeast hydrolyzed for 24 hours with 20 per cent sulfuric acid still retained its curative properties. They also confirmed the results of Fraser and Stanton concerning the antineuritic action of the alcoholic extract of rice polishings and carried the process further by finding that the active substance was completely precipitated from a water solution of the extract by means of phosphotungstic acid and that on decomposing the precipitate with barium hydroxide an active substance free from phosphorus, carbohydrate, and protein was obtained. Following this, Funk (1911) announced the isolation from rice polishings of a crystalline nitrogenous compound which he held to be the curative substance. Shortly afterward (1912a) he corrected some of his previous statements regarding this substance, which he provisionally named beriberi vitamine (1912c). At this time he offered evidence indicating that it was a free base probably belonging to the pyrimidine group analogous to uracil and thymine and possibly a constituent of nucleic acid.

While later work has hardly supported such definite conclusions as to the chemical nature of the antineuritic substance, yet considerable interest attaches to the experimental evidence which led to the intro- 
duction of the term vitamin and helped to crystallize the conception for which the term stands.

The method of extraction first adopted by Funk (1911) was a modification of that used by Fraser and Stanton, based on the customary procedure for isolating simple natural bases.

On account of the small amount of curative substance present, 54 kilograms of rice polishings was used. This was extracted in separate portions of 1500 grams each with 4 liters of alcohol containing gaseous hydrochloric acid to the extent of from two to five per cent, the extraction being conducted in a shaking machine and the liquid afterwards filtered on a Büchner funnel, the residue pressed out in an hydraulic press and the liquid added to the original filtrate. On evaporation in vacuo at $38^{\circ} \mathrm{C}$. a fat-like residue was obtained which melted at $50^{\circ} \mathrm{C}$. This was heated with water on a water bath, and filtered at $38^{\circ}-40^{\circ}$ C. The filtrate formed two layers which were separated and the aqueous layer extracted three times with ether to remove fatty substances. The resulting aqueous solution, which is described as containing no proteins and showing no reaction for amino acids when subjected to the Millon, glyoxylic acid, bromine, xanthoproteic, diazo and diacetyl tests, was found to be effective in curing polyneuritic pigeons in doses corresponding to 20 grams of the original rice. On adding to such an aqueous extract sulfuric acid to five per cent concentration, followed by phosphotungstic acid, the active substance came down in the precipitate while the filtrate, although containing nitrogen, proved inactive. The precipitate was washed with 5 per cent sulfuric acid, ground with barium hydroxide in a mortar, water added, and the mixture shaken for three hours, then filtered and the precipitated barium phosphotungstate washed with water. The filtrate, after removal of ammonia by aeration and of excess barium by precipitation with sulfuric acid and filtration, was neutralized with hydrochloric acid and evaporated in vacuo at room temperature. The alcoholic extract of this residue in doses equivalent to 40 grams of the rice polishings was an effective cure for polyneuritic pigeons.

On treating this extract with alcoholic mercuric chloride and allowing the solution to stand in the cold for some time a crystalline precipitate (I) was obtained which was soluble in water and recrystallized from the water solution in needle-like crystals consisting largely of choline but containing some of the active substance. At this point the active substance was also found in the alcoholic filtrate from the mercuric chloride precipitate (III), and in the aqueous filtrate after recrystallization (II). These filtrates were treated separately as follows: The mercury was removed from the aqueous filtrate (II) by hydrogen sulfide, the filtrate evaporated, the residue taken up by alcohol, the choline removed by precipitation with platinic chloride and filtration, and the filtrate finally treated with phosphotungstic acid. A crystalline precipitate was obtained which showed activity on decomposition of the phosphotungstate with barium hydroxide and carbon dioxide. The alcoholic filtrate (III) was evaporated, the residue taken up in water, the mercury removed by hydrogen sulfide and the filtrate freed from chloride by silver sulfate, the silver by hydrogen sulfide, and the sulfuric acid by barium hydroxide. The filtrate thus purified was then acidified with nitric acid and treated with silver nitrate and barium hydroxide according to the methods used in isolating histidine and like bases as described, e.g., by Barger (1914), the silver nitrate being added until a drop of the solution no longer gave a white precipitate with ammoniacal silver nitrate. The precipitate thus obtained was washed thoroughly with nitric acid, decomposed with hydrogen sulfide, freed from the last traces of barium hydroxide with very dilute sulfuric acid and concentrated in vacuo. On evaporating with alcohol in a desiccator the active extract thus prepared, a small yield ( 0.4 gram from 50 kilograms of the rice polishings) of microscopic needle-like crystals was obtained which in doses of about $0.02 \mathrm{gram}$ effected a rapid cure in polyneuritic pigeons. These crystals melted at $233^{\circ} \mathrm{C}$., were difficultly soluble in cold water and alcohol, more soluble in hot water, and were free from chlorine, ash. and sulfuric acid. A single analysis of the substance gave the percentage composition: $\mathrm{C}, 55.63 ; \mathrm{H}, 5.29 ; \mathrm{N}, 7.68$ per cent, figures which were thought to correspond best to the formula $\mathrm{C}_{17} \mathrm{H}_{20} \mathrm{O}_{7} \mathrm{~N}_{2}$. 
The following year Funk (1912a) reported the isolation of the same or similar substances from yeast, milk, ox brains and lime juice. The yeast was treated in two different ways. In the first, the alcoholic extract was evaporated and the residue hydrolyzed with sulfuric acid, after which the solution was treated as in the case of the rice polishings by precipitation first with phosphotungstic acid and then with silver nitrate and barium hydroxide. In the second method the alcoholic extract after evaporation was extracted with water and then precipitated with silver nitrate and barium hydroxide. This method proved unsatisfactory as the bulk of the vitamin remained in the filtrate and could not be precipitated except after hydrolysis. This was thought to indicate that the vitamin is present in yeast in a combined form.

Edie, Evans, Moore, Simpson and Webster (1912) after considerable experimentation obtained from commercial fresh-pressed yeast an active crystalline substance which they named toruline.

In their method methyl alcohol slightly acidulated with hydrochloric acid was used for the first extraction. This extract, after evaporation to remove the alcohol, was set with plaster of Paris and the resulting matrix ground to a fine powder and extracted on a shaking machine with successive portions of the alcohol. Basic lead acetate and absolute alcohol were used as reagents to separate the inactive constituents of the extract, and silver nitrate and barium hydroxide to precipitate the active substance. On decomposition of this precipitate by the usual means, a small quantity of a brown sticky hygroscopic mass was obtained, 3 milligrams of which is said to have been equivalent to 15 grams of the original yeast. "A dose of 0.006 gram administered to a bird with severe convulsions and lameness relieved the convulsions in four hours; the bird was flying strongly in 20 hours, and the lameness disappeared in 48 hours. Two further doses of 0.003 gram were given on the third and eighth days; the bird appeared normal, and gained weight on polished rice diet, but died on the fifteenth day without return of lameness or convulsions."

The crude material was insoluble in ether or acetone, very soluble in water and soluble in alcohol, from which it could be crystallized in feathery crystals. On analysis these crystals, to which the name toruline was given, gave results corresponding to a formula $\mathrm{C}_{7} \mathrm{H}_{17} \mathrm{~N}_{2} \mathrm{O}_{5}$ or $\mathrm{C}_{7} \mathrm{H}_{16} \mathrm{NO}_{2}\left(\mathrm{HNO}_{3}\right)$. Assuming the presence of the trimethylamine group the formula was further written as $\left(\mathrm{CH}_{3}\right)_{3} \mathrm{~N} \cdot \mathrm{C}_{4} \mathrm{H}_{7} \mathrm{O}_{2} \cdot \mathrm{HNO}_{3}$.

In June 1912, Schaumann (1912a) described the preparation of a crystallizable active base similar to that of Funk and proposed the theory that this substance functioned as an activator in the body, 
helping to restore to normal the degenerated nerve tissues the actual rebuilding of which, he emphasized, must of necessity call for other materials also. His work contributed little so far as methods of isolation are concerned as he used the mercuric chloride precipitation which, as in the case of Funk's early work, left the active substance scattered in three or four fractions.

In July 1912, Suzuki, Shimamura and Odake published a paper representing the results of four years of chemical investigation of the antineuritic substance of rice bran.

The method of these Japanese investigators resembled Funk's original procedure through the precipitation of the active substance by phosphotungstic acid. This precipitate was decomposed by trituration with an excess of a solution of barium lydroxide until the thick paste reacted strongly alkaline, or was dissolved in acetone and water and enough of the barium hydroxide added to give a strongly alkaline reaction. After freeing the filtrate from barium by sulfuric acid and evaporating under reduced pressure there remained a slightly acid light brown sirup which, on drying over sulfuric acid, formed a resinous mass to which the name Crude Oryzanine I was given. The yield at this point was 0.4 per cent of the original bran (1.2 grams from 300 grams of bran).

Crude Oryzanine I proved to be ten times as active as the alcoholic extract. From 0.03 to 0.04 gram, dissolved in a little water and administered either by mouth or subcutaneously, cured polyneuritic pigeons in a day, while half the dose prolonged the life of the pigeon but did not effect a complete cure. It was readily soluble in water and dilute alcohol, forming a slightly acid solution which gave no biuret reaction, a deep red color with Millon's reagent, and a flocculent precipitate with phosphotungstic or phosphomolybdic acid in acid solution, and in concentrated solution was precipitated by lead acetate and ammonia and partially precipitated by mercuric chloride, acetate, or nitrate or by tannic acid. On hydrolysis by dilute acid two acids described as $\alpha$-and $\beta$-acids were obtained as well as nicotinic acid, and glucose. To the $\alpha$ - acid was ascribed the formula $\mathrm{C}_{10} \mathrm{H}_{8} \mathrm{NO}_{4}$ and to the $\beta$-acid $\mathrm{C}_{18} \mathrm{H}_{6} \mathrm{~N}_{2} \mathrm{O}_{9}$.

Precipitation with tannic acid was made the basis of further concentration of the active material. Four grams of Crude Oryzanine I was dissolved in 100 cubic centimeters of water and treated with 20 per cent aqueous tannic acid until a whitish brown flocculent precipitate appeared. This on decomposition and concentration, as in the case of the phosphotungstate, yielded a small amount of a light brown sirup "Crude Oryzanine II." The yield was only about 6 per cent of the weight of the Crude Oryzanine I taken for purification, but the antineuritic potency was about three times as high, 0.01 gram proving sufficient to cure a polyneuritic pigeon. Several modifications of this method are described as yielding similar products.

Picric acid was used in the next and final step of the concentration. After repeated trials to prevent the simultaneous precipitation of nicotinic acid, traces of which still remained in Oryzanine II, it was found that by adding small amounts of the picric acid to Oryzanine II and triturating in the cold, the oryzanine picrate appeared as a yellowish-brown, flocculent precipitate becoming crystalline on standing in the cold, while the nicotinic acid remained in solution. On recrystallization from acetone solution the picrate was obtained as yellowish brown, small microscopic needles generally in clusters. These were insoluble in ether or petroleum ether, rather difficultly soluble in cold water, and readily soluble in hot water or alcohol. Unfortunately the yield of picrate was so small that attempts to obtain free oryzanine were unsuccessful.

For protection from polyneuritis, pigeons required 3 grams of bran daily, or 0.3 gram of alcoholic extract, or 0.03 gram of Crude Oryzanine I, or $0.01 \mathrm{gram}$ of Crude Oryzanine II, or about 0.005 gram of "Pure Oryzanine." This would imply that the purification process had concentrated the active substance at least 
500 -fold. If the final substance were in fact pure, then the concentration of this substance in the original rice bran was presumably about 0.2 per cent, or in the whole rice grain perhaps about 0.05 per cent. If, however, the final product were still a mixture, then the percentage of the actual antineuritic substance in the rice kernel must be smaller than this estimate would indicate.

Suzuki and his coworkers also attempted by feeding experiments to determine whether oryzanine plays as important a rôle with other species as with pigeons and reported it to be indispensable for fowls, mice, and dogs. In view of the later work of Cowgill, their experiments with dogs are of particular interest. It was reported that dogs when fed cooked rice and the boiled-out residue of horse flesh began to lose appetite in about three weeks and after from five to seven weeks showed marked losses in weight. If, however, 3 or 4 grams of the alcoholic extract or 0.3 to 0.4 gram of Crude Oryzanine I were administered daily to a dog which appeared about to die as a result of the deficient diet, the appetite was quickly restored and the body weight increased until the doses of oryzanine were omitted, when the same symptoms recurred. In a full-grown dog these changes were brought about four times in seven months.

Vedder and Williams (1913) reported observations which raised the question whether rice polishings contain antineuritic substances other than the vitamin as apparently isolated by Funk or whether the one substance is only partially separated by his method.

They stated that before Funk's papers had reached them in the Philippines they had attempted to concentrate the antineuritic constituent of rice polishings by extraction with alcohol, precipitation with phosphotungstic acid, and decomposition of the phosphotungstate with barium hydroxide, but had not succeeded in obtaining any protective action with the resulting material. In comparing methods, they found that Funk had added 2.5 per cent of hydrochloric acid to the alcohol and had used curative tests. On modifying their procedure by hydrolyzing the alcoholic extract with 5 per cent hydrochloric or sulfuric acid they were able to confirm his results in curative experiments. A comparison of the unhydrolyzed and hydrolyzed extracts showed the former to be non-poisonous and only slowly curative and the latter poisonous in larger doses and very promptly curative in smaller doses. This difference was ascribed to the hydrolysis of the active material into a more active and probably simpler form. On the theory that the marked losses in activity of the phosphotungstate on decomposition with barium hydroxide might be due to the destructive action of the barium hydroxide, the method was modified by extracting the phosphotungstate precipitate by repeated prolonged shaking with 50 per cent alcohol and adding barium hydroxide to the extract, less being required than when added to the original precipitate. After removal of the barium phosphotungstate the extract was treated with silver nitrate and barium hydroxide as in Funk's method.

On testing the various fractions they found that polyneuritis in fowls was prevented not only by the fraction containing Funk's base, but also by the filtrate from it and even by the "purine fraction" precipitated by silver nitrate from neutral solution, if this fraction were fed in sufficient quantity. Of these three groups of substances conferring protection, only one, the fraction containing Funk's base, was promptly curative. 
In their attempts to discover a better method for isolating the base, Vedder and Williams also tried the method of Suzuki, Shimamura and Odake but do not seem to have regarded it as constituting any marked improvement over the method of Funk. The method noted above was also abandoned as a quantitative procedure as the greater part of the curative base was lost during the final precipitation with silver nitrate in the presence of barium hydroxide. A method which proved more successful consisted in neutralizing a quantity of unhydrolyzed extract of rice polishings with barium hydroxide, adding an excess of barium acetate, treating the precipitate thus obtained with 5 per cent sulfuric acid for three hours and filtering. The filtrate after the removal of the excess sulfuric acid with barium carbonate proved to have retained practically all of the curative power of the original extract as shown by curative experiments with fowls suffering from advanced polyneuritis.

In the same year, Cooper (1913a) described the preparation from animal tissue (horse flesh) of a substance capable of curing polyneuritis in birds.

In Cooper's method, absolute alcohol followed by ordinary alcohol was used to extract the active substance from the dried powdered flesh, ether to remove lipoidal material from the extract, partial crystallization of an aqueous solution of the alcoholic extract to remove carnosine, and lead acetate to remove other impurities. The precipitation of the active material was effected first by silver nitrate, and then by silver nitrate with barium hydroxide. It was estimated that at least three-fifths of the total amount of antineuritic substance present in the filtrate from the lead acetate precipitation was precipitated by the addition of silver nitrate only, and about one-fourth of the remainder was carried out of solution by the subsequent addition of baryta. The remaining three-twentieths had probably decomposed under the influence of the alkali. The residue obtained by evaporation of the curative solution resulting from dissolving the first silver nitrate precipitate in hydrochloric acid was next extracted with chloroform. Only a small amount of inactive substance was extracted. The active substance was thus insoluble in chloroform and also insoluble in benzene and in ethyl acetate. It was destroyed by standing in ammoniacal solution, and was not destroyed by hydrogen sulfide but appeared to be largely lost by adsorption on precipitates of metallic sulfides.

Cooper also (1914b) made use of acetone precipitation in the preparation of an antineuritic concentrate from cardiac muscle; and in that year made the first use of autolyzed yeast as a starting point for the isolation of the antineuritic vitamin (1914).

In a paper published in June 1913, Funk (1913b) gave the results of further studies upon the chemistry of the vitamin fraction of yeast and rice polishings. By recrystallizing from alcohol the active fraction of yeast prepared by his first method he obtained three substances, one of which had the composition $\mathrm{C}_{24} \mathrm{H}_{19} \mathrm{O}_{9} \mathrm{~N}_{5}$ and one $\mathrm{C}_{29} \mathrm{H}_{23} \mathrm{O}_{9} \mathrm{~N}_{5}$, 
while the third appeared to be nicotinic acid. The vitamin fraction from rice polishings yielded nicotinic acid and a substance to which the formula $\mathrm{C}_{26} \mathrm{H}_{20} \mathrm{O}_{9} \mathrm{~N}_{4}$ was assigned. The work of Suzuki ct al. was discussed in this paper and in greater detail in one by Drummond and Funk (1914) in which were reported the results of an extended study of the phosphotungstate precipitate from rice polishings obtained according to Funk's earlier method. They were not only unable to obtain the active products mentioned by the Japanese investigators but failed also in isolating the curative substance of rice polishings by an extension of their previous method which had pointed to success. From an exhaustive fractionation of the phosphotungstic acid precipitate they succeeded in isolating comparatively large amounts of choline, nicotinic acid and betaine, together with small amounts of the purines, guanine and adenine, and traces of a substance which they considered might possibly be guanidine.

The substance to which Funk had previously assigned the formula $\mathrm{C}_{26} \mathrm{H}_{20} \mathrm{O}_{9} \mathrm{~N}_{4}$ and which, with nicotinic acid, had been obtained from the portion containing practically the whole of the curative substance was definitely proved to be also nicotinic acid. "All trace of the curative substance had disappeared and a fraction which originally had shown very marked curative properties now consisted of nothing other than nicotinic acid which possesses very slight action. All attempts which we have made to isolate the elusive curative substance from this fraction, in which it originally occurs, have failed." This throws doubt upon Funk's earlier isolation of a vitamin of melting point $233^{\circ} \mathrm{C}$. and gives point to Barger's suggestion that this may have been only nicotinic acid contaminated with the active substance.

Seidell (1916), adopting a method used by Lloyd in working with alkaloids, found that Lloyd's reagent, a special form of fuller's earth (hydrous aluminum silicate), effectively adsorbed the vitamin from autolyzed yeast solution. When 50 grams of the fuller's earth per liter of the autolyzed yeast solution was used the vitamin was practically all adsorbed and the solid could then be washed with successive portions of very dilute acid, water, and small amounts of alcohol, and finally dried in a vacuum desiccator, thus affording a stable form of the vitamin. Both preventive and curative experiments were made with this material. Prompt and effective cures of completely paralyzed pigeons resulted from 0.05 gram doses of the activated solid, corresponding to 1 cubic centimeter of the original yeast filtrate. The same amount fed alternate days to pigeons on a polished rice diet proved sufficient to keep the birds in normal health and weight. 
Williams (1916) published the results of unsuccessful attempts at the isolation of the vitamin from rice polishings by various modifications of Funk's original method. He obtained a very slight yield of crystals melting at $233^{\circ} \mathrm{C}$. which were curative in doses of 10 to 20 milligrams. An amorphous material also gradually separated which was curative in doses of 20 to 30 milligrams and proved effective in treating cases of human beriberi. After exhausting every resource to avoid mixed deposits from the final liquor and to increase the yield of needles melting at $233^{\circ} \mathrm{C}$. he abandoned the hope of isolating large quantities of an individual vitamin from rice polishings and decided to attempt the synthesis of curative substances, which might throw light on the nature of the vitamin. The facts adduced by Funk and others seemed to Williams to point to the pyridine ring as the most promising nucleus. The most important facts in favor of this choice were the occurrence of nicotinic acid with the vitamin in several natural substances and the stability of the vitamin on heating with sulfuric acid. Nicotinic acid, trigonelline, and p-oxy-nicotinic acid given by mouth to polyneuritic fowls caused little improvement, but some prolongation of life. The hydrochloride of the methyl ester of nicotinic acid brought about marked, but temporary, improvement.

Later in the same year Williams (1916a) published the results of experiments upon the antineuritic properties of some synthetic hydroxypyridines. These were selected on account of the fact that antineuritic concentrates when treated with phosphotungstic acid and alkali had given the blue color produced by substances containing hydroxy groups in the benzene ring.

Polyneuritic pigeons were given doses of from 10 to 100 milligrams by intramuscular injections. In some cases similar doses were administered by mouth as a supplementary test. The substances tested included nicotinic, cinchomeric, quinolinic, 6-hydroxynicotinic and citrazinic acids, $\alpha$-hydroxypyridine, glutazine, 2,4,6-trihydroxypyridine and its anhydride, and finally 2,3,4-trihydroxypyridine and the socalled tetrahydroxypyridine.

Only $a$-hydroxypyridine, 2,4,6-trihydroxypyridine, and 2,3,4-trihydroxypyridine were found to have curative power. Moreover, these compounds were curative only when freshly prepared and invariably lost their curative properties on standing. As there was no evidence of decomposition it seemed probable that the change was due to isomerization. A study of the isomeric forms and curative properties of $\alpha$-hydroxypyridine showed that of the two crystalline forms one exhibited activity and the other did not. Similar results were obtained 
with $\beta$ - and $\gamma$-hydroxypyridine, leading Williams to state: "The antineuritic properties of these substances suggest that an isomerism is at least partially responsible for the instability of the vitamin in foodstuffs and that the antineuritic property may be inherent in the potentiality of this type of isomerism. We may not conclude that vitamins are necessarily hydroxypyridines since a similar isomerism may exist in substances containing other heterocyclic nitrogenous nuclei which are known to occur widely as constituents of animal tissues."

With this conception in mind attention was directed to the natural antineuritic substances of yeast. Starting with activated fuller's earth, Williams and Seidell (1916) attempted to separate the vitamin from it by extraction with acidified aqueous alcohol but failed in this attempt on account of simultaneous extraction of aluminum compounds. On shaking the activated fuller's earth with a five per cent solution of sodium hydroxide in dilute alcohol and evaporating the extract, they obtained a crystalline antineuritic substance the physiological action of which was apparently not due to adhering mother liquor. In attempts to purify this substance further by recrystallization they found the resulting product to be identical with adenine and to have no antineuritic properties. When, however, 10 milligrams of this inactive substance was heated with one cubic centimeter of absolute alcohol in a sealed tube at $180^{\circ} \mathrm{C}$. for three hours it acquired antineuritic properties and the power to give a blue color with the FolinMacallum phosphotungstic sodium carbonate reagent. These results were thought to be in harmony with the conclusions of the previous paper and to suggest the probability than an isomer of adenine is "the chemical entity responsible for the characteristic physiological properties of the vitamin under investigation."

Voegtlin and White on the other hand, reported (1916) that they were unable to cause adenine to acquire antineuritic properties and that there probably exists no relation between adenine and the antineuritic vitamin.

In a third paper Williams (1917) described further work with synthetic substances from which he concluded that "the curative form of $\alpha$-hydroxypyridine is a pseudo base and that a structure conforming more or less closely to the type of a betaine ring is probably an essential characteristic of antineuritic vitamins." Attention was called to the theoretical possibility of the existence of such a substance in some of the simpler nitrogenous constituents of animal tissues, especially the nuclein bases. It was also suggested that nicotinic acid may exist in a betaine form and that the curative properties of the 
vitamin fractions of yeast and rice polishings may have been due in part to this isomeric form of nicotinic acid.

Harden and Zilva (1917), repeating Williams' experiments with $\alpha$-hydroxypyridine, were able to confirm the chemical properties of that substance as reported by Williams but were unable by its use to effect a cure or even an improvement in the condition of the polyneuritic birds. Pure adenine, as well as adenine treated with sodium ethylate in a sealed tube for five hours at $100^{\circ} \mathrm{C}$., also yielded negative results in agreement with Voegtlin and White but in disagreement with Williams and Seidell.

Meantime the water-soluble, growth-promoting factor for rats considered by many to be identical with the antineuritic vitamin was receiving an increasing share of the attention of investigators, and no further report on the attempted isolation of the antineuritic vitamin as such was noted until May 1920, when Myers and Voegtlin reported the results of an investigation covering a period of several years in which they had used autolyzed yeast as a starting material, and mastic, Lloyd's reagent and ferric phosphate as adsorbing agents, but with little success.

The method adopted by Myers and Voegtlin consisted in the extraction of dried brewers' yeast by 95 per cent methyl alcohol containing 2 cubic centimeters of concentrated hydrochloric acid per liter and the precipitation of the purine fractions by hot silver acetate and of the active substance by barium hydroxide and silver acetate. A further concentration was effected by decomposing this precipitate with hydrogen sulfide, and precipitating most of the histidine fraction with mercuric sulfate, and the active material with absolute alcohol. This precipitate, on decomposition as before, yielded a filtrate which, after concentration in vacuo at low temperatures, gave a purple solution with ninhydrin, a negative biuret test, and no precipitate with picric acid, but gave a heavy precipitate with phosphotungstic acid, and was highly curative for polyneuritic pigeons. The solution on further concentration in vacuo over soda-lime yielded spindle-shaped crystals which were active as long as the mother liquor was present. On drying, the crystals changed from spindles to prisms and became inactive. When the prisms were dissolved in a relatively large volume of water and again allowed to crystallize, spindle-shaped crystals were again formed. While realizing that the activity might be in the non-crystallizable mother liquor as well as in the crystals, Myers and Voegtlin were of the opinion that there were at least two substances in the final solution, both of a distinctly basic character. One of the impurities was a histamine-like substance as shown by the positive Pauly reaction.

Hofmeister (1920) in an attempt to separate the antineuritic substance from commercial rice meal made use of bismuth potassium iodide as precipitant.

The material was first shaken with 80 per cent alcohol, and the extract distilled in vacuo to a thick sirup, acidified with 3 per cent hydrochloric acid and shaken with ether. The aqueous fraction, after removal of the ether, was again evaporated to a thick sirup and the extraction with 80 per cent alcohol repeated. After the alcohol had been expelled, the extract was made slightly alkaline with 
sodium carbonate and precipitated with bismuth potassium iodide. After about five hours the grayish yellow precipitate, which at this point consisted chiefly of choline, was filtered off, the filtrate treated with one-tenth its volume of 20 per cent hydrochloric acid and again precipitated with bismuth potassium iodide, with long and thorough shaking. At this point the active material was in the precipitate, a brick red to purple powder to which the name oridin fraction was given. This was immediately filtered with strong suction, triturated with silver carbonate and again filtered. The slightly alkaline, silver-containing, active filtrate was at once made slightly acid with hydrochloric acid, and, after removal of the precipitated silver chloride, evaporated to dryness in vacuo at low temperature. A slightly colored, deliquescent mass of crystals formed which, in doses of 5 to 10 milligrams daily, was capable of curing polyneuritis in pigeons. On treatment with gold chloride a yellow double salt, corresponding to the formula $\mathrm{C}_{5} \mathrm{H}_{11} \mathrm{NO}_{2} . \mathrm{HCl}$. AuCl was obtained which on decomposition yielded a free base isomeric with betaine and valine and probably containing the pyridine or piperidine groups, but which possessed no antineuritic properties.

In discussing this loss in activity two possible explanations were suggested, (1) that the antineuritic vitamin (or antineuritin as it was called) was present in the oridin hydrochloride only as an impurity and was set free from it and lost on further purification and (2) that the oridin hydrochloride was actually the salt of antineuritin, but that in the process of purification it became an inactive isomer. The latter view, in harmony with the theory of Williams, was apparently favored by Hofmeister at this time. Later, Hofmeister (1923) attributed the losses of vitamin, in attempts to purify it, to adsorption by precipitates of other substances, as had Drummond in 1917.

Seidell (1921a) recorded the preparation of a stable silver vitamin compound from activated fuller's earth.

Saturated aqueous barium hydroxide solution in the proportion of one liter per 100 grams of the activated fuller's earth was used for the extraction of the active material, the mixture being shaken vigorously for three minutes and the solid removed as rapidly as possible, preferably by means of a De Laval cream separator. The nearly clear liquid was immediately acidified with a slight excess of concentrated sulfuric acid, a moderate excess of powdered barium carbonate was added to remove the excess of sulfuric acid, and the mixture filtered after about thirty minutes. After the removal of non-active material from the filtrate by precipitation with a saturated lead acetate solution, and of the excess lead by hydrogen sulfide, the resulting filtrate was concentrated to a small volume by rapid vacuum distillation, the white amorphous material, separating out toward the end, removed by filtration, and the final concentrate reduced to a thick, pasty, semisolid condition by allowing it to stand several days in a vacuum desiccator.

On diluting to a volume of 25 cubic centimeters the extract thus prepared from 300 grams of activated fuller's earth and adding gradually an almost saturated aqueous silver nitrate solution, a voluminous silver precipitate devoid of antineuritic properties was obtained. When the liquid resulting from centrifuging and washing this precipitate was treated with an excess of fairly concentrated aqueous ammoniacal silver nitrate solution another voluminous precipitate formed. This second precipitate, which proved to be highly antineuritic as tested by feeding to pigeons on a polished rice diet, was further purified of an easily crystallizable inactive compound by adding a slight excess of hydrochloric acid to a suspension in water, removing the silver chloride formed and slowly evaporating the clear aqueous solution in a vacuum desiccator until crystals ceased to form. On remov- 
ing these crystals and repeating the precipitation with silver nitrate followed by ammoniacal silver nitrate, a voluminous silver precipitate was again formed, but free from the crystallizable inactive fraction. This substance, the yield of which in the dry state was 0.7 gram from 300 grams of activated fuller's earth, when administered in doses of 0.004 gram on alternate days to pigeons on a polished rice diet prevented the development of polyneuritic symptoms. In view of recent work, it may be of significance that this substance did not prevent a slight loss in weight as did the activated solid or crude extract.

Williams (1921) reaffirmed much of his earlier work upon the antineuritic properties of substances of known structure and continued the development of his theory that antineuritic activity may be connected with isomerism of the type above described. He reported that $\beta$-hydroxypyridine and $\beta$-methylpyridine gave some evidence of antineuritic property in curative tests on polyneuritic pigeons and trimethyluracil and 4-phenylisocytosine in protective tests. In his opinion protective experiments are much more convincing than curative.

The following year Seidell (1922) announced that when a concentrated extract prepared from yeast by the method of Osborne and Wakeman (described beyond) was used in place of autolyzed yeast in the preparation of activated fuller's earth the resulting product contained less nitrogen and about twice as much of the active antineuritic material as the product made in the original way. This new method of preparing the activated solid was used by Seidell (1922b) in a further study of the various silver fractions obtained by his previous method. In this and later work the test for activity of the various fractions consisted (Seidell, 1922a) in preparing a solution of definite concentration, adsorbing it on such an amount of fuller's earth that each 0.1 gram corresponded to a definite number of milligrams of the solids present, and using this preparation as the sole source of antineuritic vitamin for pigeons of known weight on an exclusive polished rice diet. The unit of activity was defined as "that quantity of vitamin, given to a normal pigeon on each alternate day, which is just sufficient to replace the vitamin deficiency of an exclusive diet of polished rice."

The activity of the silver nitrate precipitate was much higher than previously found. This was attributed to the presence of adenine (also precipitable by silver nitrate) in the extracts prepared from autolyzed yeast and its absence in the extracts obtained by the new method. Only a little over half of the total active vitamin, however, was present in the two silver precipitates. The loss of active material by this method was thought to be due to the rather high solubility of the silver-vitamin complex.

The fractions obtained by silver precipitation were quite stable 
both in solution and in the dried state, if, before drying, the silver was removed by hydrochloric acid or hydrogen sulfide. A comparison of the nitrogen content of the various fractions with their activity showed that in the ammoniacal silver nitrate fraction a smaller amount of nitrogen was required for protection than in the other fractions, although this was almost four times as much as in an ammoniacal silver nitrate fraction obtained after silver acetate precipitation. "Reasoning in this manner, it may be concluded that the extracts prepared from activated solid are mixtures of vitamin with one or more other nitrogenous bases. By silver fractionation the proportion of vitamin may be increased to a certain extent, but a considerable degree of purification can not be effected by the method."

Abandoning the silver precipitation, Seidell (1924) next used picric acid as a precipitant of the active material from the extract obtained from activated solid as previously. The new method, an important preliminary step of which was thought to be the removal of potassium sulfate from the extract before the addition of the picric acid, was as follows :

The extract was dissolved in water and diluted to a definite volume. An aliquot of this was evaporated to dryness, the residue ignited, and the resulting ash titrated with tenth-normal sulfuric acid, with methyl orange as indicator. From the amount of acid required the calculated amount of normal acid equivalent to the bases present in the entire solution was added to the main solution and the mixture distilled under reduced pressure almost to dryness. The residue was treated with 66 per cent ethyl alcohol and the insoluble potassium sulfate removed by filtration. The protective dose of the alcoholic filtrate thus obtained was found to be 4 milligrams fed every other day.

The picrate was prepared by adding to this alcoholic extract an amount of picric acid dissolved in methyl alcohol, approximately equal in weight to the solids present in the extract. The alcohol was gradually removed by evaporation under reduced pressure, water being added at intervals to replace it. The precipitate was finally separated by centrifugation and washed with small portions of water. The crude picrate was purified by adding to 5 grams of the material 8 cubic centimeters of 95 per cent acetone, stirring for 3 minutes, centrifuging, and decanting the clear reddish yellow solution. The process was repeated successively with 4,2 , and finally 1 cubic centimeter of the acetone. Ten cubic centimeters of water was added to the combined extracts and the mixture evaporated in a partially evacuated desiccator containing sulfuric acid until the volume had been reduced about one-third, when 2 cubic centimeters of water was added and the process continued until the further addition of water caused no further precipitation. The solid was then filtered on a paper filter resting on a small perforated porcelain disc, and washed with a little water, and the entire process repeated. The twicecrystallized picrate was reported to consist of irregularly-shaped transparent crystalline flakes capable of protecting fully grown young pigeons in doses as low as 2 milligrams daily.

The portion of the crude picrate insoluble in 95 per cent acetone proved to be soluble in dilute acetone, yielding on recrystallization pale yellow rods or prismatic crystals possessing very little activity, although 
combustion analyses of the two picrates gave results which agreed closely and corresponded to the formula $\mathrm{C}_{6} \mathrm{H}_{18} \mathrm{O}_{2} \mathrm{~N}_{3} \cdot \mathrm{OH} \cdot \mathrm{C}_{6} \mathrm{H}_{2}\left(\mathrm{NO}_{2}\right)_{3}$. In commenting upon the difference in activity of the two picrates, Seidell expressed the opinion that the crystalline picrate obtained by Suzuki et al. was the inactive form of the present investigation, but containing a small amount of the active form as an impurity.

Peters (1924) used the vegetable charcoal "norite" as an adsorbent of the antineuritic vitamin from an extract prepared from the Osborne-Wakeman yeast concentrate. He first freed this extract of much inactive material by precipitating with neutral lead acetate, acid mercuric sulfate, and barium sulfide, clearing the extract of sulfides, neutralizing it exactly, and making it just acid with acetic acid. To the amount of extract thus obtained from 7 pounds of fresh yeast, 30 grams of norite was added. After thorough stirring and standing for 30 minutes the charcoal was separated in a Büchner funnel and washed thoroughly with distilled water. The washings, if colored, were treated with 20 grams of norite, which was then combined with that previously used. The active material was set free from the norite by heating for half an hour on the water bath with 300 cubic centimeters of 50 per cent alcohol containing 1 cubic centimeter of concentrated hydrochloric acid per 100 cubic centimeters and repeating the process twice with 150 cubic centimeters of the alcohol. The extract, after concentration to about 20 cubic centimeters by evaporation at a temperature not exceeding $60^{\circ} \mathrm{C}$., was still very active and the activity was not destroyed by drying at $100^{\circ} \mathrm{C}$. If the hydrochloric acid was not removed the dried material retained its activity for several months at room temperature.

In testing the activity of his preparations, Peters adopted the earlier curative tests with polyneuritic pigeons, giving as his reason for not using the weight or growth test as employed by Seidell the objection that such tests assume the identity of the curative and growth-promoting substances; and, for not using tests involving the protection of pigeons from symptoms of polyneuritis while upon a polished rice diet, the difficulties raised by the variability of the times of onset of convulsions of different pigeons placed upon the same diet. The conditions considered by him to be essential in the curative test are as follows :

"Only those pigeons should be used for the test which show wellmarked head retraction, in which the symptoms have appeared within 30 to 35 days, and which appear strong enough to withstand treatment. If the symptoms clear up completely upon administration of the 
extract within a period of 6 to 12 hours, recovery must be a persistent one for two or better three days, in order to establish the activity of an extract. If the symptoms do not clear up, it is not proof of the non-activity of an extract unless (a) the bird is strong, and (b) the extract is administered within 12 hours from the start of the convulsions if it is the first attack, and within three hours if it is the second."

As thus tested, the yeast concentrate before the final evaporation to dryness was of such strength that 0.05 cubic centimeter cured the usual pigeon of symptoms within six hours and kept it from convulsions upon a diet of polished rice for about three and one-half days. After evaporation to dryness 3 milligrams was required. The extract did not lose its activity on treatment with an acid solution of sodium nitrite, thus apparently excluding primary amines and many secondary amines as responsible for antineuritic action. Negative, or only temporary, results were obtained in curative tests with various substances of basic character, including hexylamine, guanidine, and dimethyl urea. The administration of histamine, however, produced cures in a small percentage of cases. Peters was at a loss to explain the occasionally curative action of histamine except by attributing it to some temporary vascular action.

In a later paper Kinnersley and Peters (1925) reported further attempts at concentrating the active material obtained by the method described in 1924 with the exception that treatment with barium sulfide was omitted and the charcoal adsorption was applied to the acid fluid after removal of the mercury precipitate.

In testing the activities of their various preparations they adopted as the standard of comparison:

$$
\text { Torulin activity (T.A.) }=\frac{\text { Number of days' protection after cure }}{\text { Weight in mg. of the dry preparation }} \times 100 \text {. }
$$

Torulin, the term introduced by Edie ct al. (1912), was defined as "the principle in yeast which cures symptoms of head retraction in pigeons induced by feeding upon polished rice."

By successive treatments of the charcoal concentrate with ethyl alcohol to a concentration of 60 per cent, 25 per cent lead acetate, methyl alcohol to a concentration of not less than 90 per cent, 10 per cent "dialyzed iron," followed by the rapid addition at room temperature or below of normal sodium hydroxide until a precipitate ceased to form, and finally ethyl alcohol in increasing concentrations followed by ether, an active fraction was obtained which was soluble in absolute alcohol, and the activity of which varied from 50 to 100 T.A. The purpose of this "rather empirical" fractionation method, the final stages of which required much care, was to free the extract from gum and ash. The final treatment of the fraction is described as follows: 
"By fractionation of a product of 63 T.A. in mixtures of ether and alcohol in which the amount of water was reduced to a minimum, three fractions were obtained: (a) a gummy fraction thrown out of the alcohol by the addition of mere traces of ether, (b) an intermediate fraction thrown out by ether up to four volumes of ether to one of alcohol, and (c) a fraction soluble in etheralcohol. The last contained an oil, comparatively insoluble in water. Fractions (a) and (c) were relatively inactive. The intermediate fraction when treated with silver sulfate in acid solution threw down an inactive precipitate. From the centrifugate after treatment with silver nitrite in the presence of hydrochloric acid, ammoniacal silver hydroxide threw down a precipitate, which was extracted with hydrochloric acid and alcohol, and yielded a preparation of 1190 T.A. . . . This contained ammonium chloride as an impurity, and some evidence has already been obtained that the activity may be pushed to 3,000 T.A. This being so, it does not seem unlikely that the activity of torulin itself may prove to lie between 10,000 and 100,000 T.A. or in the region of 0.001 milligram per day for the normal pigeon. This would only bring it into line with substances like pituitrin."

Two feeding experiments were reported with growth curves showing that doses of this concentrate which protected against polyneuritis for many days did not cause an increase in weight of the pigeons after cure of the polyneuritic symptoms. This was thought to support the view that vitamin $\mathrm{B}$ consists of two factors. (Compare Williams and Waterman, 1927.)

Influenced by the work of Levene and van der Hoeven (described later), Seidell (1926) modified his general procedure for obtaining an antineuritic vitamin concentrate from yeast in two essentials-first, by using sodium hydroxide in place of barium hydroxide to liberate the vitamin from its combination with fuller's earth, and glacial acetic acid in place of sulfuric to acidify the resulting extract, (in 1929 the use of sulfuric acid was again recommended,) and second, by using strong alcohol to precipitate the active material from this extract after removal of the sodium salts and other inactive material. The addition of successive portions of 98 to 100 per cent alcohol to the thick viscous paste resulting from the evaporation under reduced pressure of the purified extract from the fuller's earth converted it into a nonhygroscopic powder. From pigeon tests (1926) it was estimated that approximately one-third of the total vitamin contained in 5 kilograms of activated solid is found in about 150 grams of the non-hygroscopic powder. The losses of active material were attributed to the impossibility of liberating all of the vitamin from its combination with fuller's earth, the destruction of the vitamin by the various treatments to which the solutions are subjected during the process, and the impossibility of separating the vitamin completely from the inactive fraction by the method employed. Numerous tests for activity of the powder have shown that it prevents loss in weight in pigeons in doses of about 0.010 gram given on alternate days. In 1929, Seidell pointed out that 
the methods of separating the antineuritic vitamin from soluble inactive material in aqueous solution may, in general, be classified as those which depend upon differential solubility of the constituents of the mixture in various concentrations of alcohol or similar solvents, those based on precipitation by suitable reagents, and those based on fractional adsorption. He inclined to favor the last as permitting the most complete separation. He found that about 28 per cent of the original vitamin activity of brewers' yeast remained in the coagulated protein which separates out upon heating a suspension of the yeast in water. Three successive adsorptions of the aqueous yeast extract on fuller's earth (30 grams per liter) resulted in preparations of "activated solids" containing respectively 55 per cent, 5 per cent, and practically none of the activity of the original yeast. About $S S$ per cent of the vitamin originally in the yeast was thus accounted for.

Later (1929a) Seidell described a method for converting the dry non-hygroscopic antineuritic concentrate (prepared by essentially the method described in 1926) into a still more active fraction. To an aqueous solution of the concentrate made alkaline with sodium carbonate an excess of benzoyl chloride was added, and the mixture was extracted with chloroform. The aqueous layer was then acidified and repeatedly extracted with chloroform. About 90 per cent of the vitamin activity and 25 per cent of the nitrogen remained in the aqueous solution. When this solution was poured into 10 to 12 volumes of acetone, salts were precipitated accompanied by nitrogenous material which protects pigeons from loss in weight on a diet of polished rice in doses containing 0.15 milligram of nitrogen. Judged on the basis of nitrogen content, this final product represents a concentration of antineuritic substance of 100 times that of dried brewers' yeast.

Jansen and Donath (1926, 1926a, 1927), working in the same laboratory in which Eijkman carried on his pioneer studies on the antineuritic vitamin, prepared from rice polishings a crystalline substance which they believe to be the hydrochloride of the antineuritic vitamin.

The method consists essentially in the extraction of the rice polishings with water acidified with sulfuric acid to an acidity of $\mathrm{pH} 4.5$, and containing 20 cubic centimeters of alcohol per liter; adsorption on acid clay (a kind of fuller's earth, hydrated aluminum silicate); extraction of the activated clay with barium hydroxide at $\mathrm{pH} 12$ or 13 , followed by sulfuric acid; fractionation of the acid extract with silver nitrate and barium hydroxide to $\mathrm{pH} 4.5,6.5$, and 8, followed, after decomposition with hydrochloric acid, by a second treatment of the combined silver fractions which concentrates the vitamin in the second 
fraction; decomposition of the vitamin-containing fraction with hydrochloric acid and reprecipitation with phosphotungstic acid and 5 per cent sulfuric acid; purification of the phosphotungstate by solution in acetone and water and reprecipitation with 5 per cent sulfuric acid; decomposition of the purified phosphotungstate with barium hydroxide, removal of the barium phosphotungstate and excess sulfuric acid, and acidification of the filtrate with hydrochloric acid; evaporation to dryness, and solution in absolute alcohol, then precipitation with platinic chloride. The platinum chloride precipitate contains about 25 per cent of the original vitamin. Then followed decomposition of the platinum precipitate suspended in dilute hydrochloric acid with hydrogen sulfide and repeated fractional crystallization of the chloride by dissolving it in absolute alcohol, adding a small amount of acetone, decanting the solution from the small amount of deposit which forms on the side of the flask and repeating the acetone treatment. These elaborate fractional recrystallizations often require months to complete. Another method of treating the decomposed platinum salt was by precipitation with picrolonic acid and treatment of the picrolonate with ether or ethyl acetate and hydrochloric acid. A portion of this substance was transformed into a crystalline double salt with chloride of gold.

By either method there is finally obtained from 300 kilograms of rice polishings about 100 milligrams of "vitamin-hydrochloride," a crystalline substance which after recrystallization has a melting point of $250^{\circ} \mathrm{C}$. (corrected). As judged by protective experiments chiefly on small rice birds known as "bondols" and later occasionally on pigeons, the crystalline substance is an exceedingly potent antineuritic. Complete protection was afforded by from 1 to 2 parts of the material to one million parts of rice. In commenting on this, attention is called to the fact that the dosage is of the same order of magnitude as that of thyroxine or adrenaline.

The vitamin hydrochloride before final purification crystallizes into bundles of needles and after purification in small bars joined into rosettes. It is not hygroscopic but dissolves readily in water. It is also readily soluble in methyl alcohol and less readily in ethyl alcohol. A 2 per cent aqueous solution gives a scanty precipitate with mercuric chloride, increasing with the addition of sodium acetate, a heavy precipitate with mercuric sulfate in dilute sulfuric acid, a fine black precipitate with iodine-potassium iodide, a thick red precipitate with a solution of bismuth iodide in potassium iodide, and opacity with picric acid. No precipitate is formed with zinc chloride, cadmium chloride, lead acetate, cupric acetate, potassium chromate, potassium thiocyanate, 
and perchloric acid. With picrolonic acid and with gold chloride needleshaped crystals are formed. Pauly's diazo reaction is given with sulfanilic acid and nitrous acid, followed by sodium hydroxide. Elementary analyses of the hydrochloride and gold chloride gave results conforming closely to the empirical formulas $\mathrm{C}_{6} \mathrm{H}_{10} \mathrm{ON}_{2} . \mathrm{HCl}$ and $\mathrm{C}_{6} \mathrm{H}_{10} \mathrm{ON}_{2} \cdot \mathrm{HCl} . \mathrm{AuCl}_{3}$. From this work and the previous findings of other investigators indicating that the antineuritic vitamin does not react with nitrous acid and consequently contains neither primary nor secondary amino groups, it is considered probable that the vitamin contains either an imidazole or pyrimidine ring.

In 1929, Jansen modified the above method by substitution of silicotungstic acid for phosphotungstic to increase the yield of purified vitamin; and cadmium chloride for platinic chloride for the sake of economy.

Kinnersley and Peters (1927) described further improvements in their technique of preparing torulin (see above) which simplified the procedure, doubled the yield of active material and increased the activity to such an extent that only 0.5 to $1 \mathrm{mg}$. daily was required in the curative tests. The principal change from the technique previously employed consisted in treating the filtrate from the neutral lead acetate precipitate with cold saturated baryta before adding mercuric sulfate. This served to flocculate the gums present in the autolysate and thus facilitate subsequent filtrations. The optimum reaction for adsorption of the active material by norite was found to be at $\mathrm{pH} 7$ for material treated with mercuric sulfate. Starting with 14 pounds of bakers' yeast the adsorption was found to be most complete when carried out in two steps using 60 grams of norite in the first and 20 in the second step. The active material was set free from each charcoal fraction by cubic centimeters of tenth-normal hydrochloric acid, followed in some two treatments on the hot water bath for an hour with about 200 cases by two extractions with about 150 cubic centimeters of 50 per cent alcohol (by volume) containing 1 per cent strong hydrochloric acid. The combined extracts, freed from the last traces of sulfuric acid by dilute barium chloride, were concentrated in vacuo at a temperature not exceeding $60^{\circ} \mathrm{C}$.

If it is considered desirable to avoid the use of mercuric sulfate, as in the preparation of a concentrate to be used in long-continued feeding tests, the bulk of the substances precipitable by this reagent can be removed by adsorption on norite at $\mathrm{pH} 2.5$ previous to the adsorption of the active material at $\mathrm{pH} 7$. The yield, however, is not as high. 
Their attempts to concentrate torulin still further by the method of Jansen and Donath proved unsuccessful. In commenting upon this, Kinnersley and Peters say, "We lay no stress upon our failure to concentrate torulin in our fractions by a method applicable to the protective (? antiberiberi) factor in rice polishings. Doubtless it lies in some peculiarity of the yeast extracts. It is, however, possible that the antiberiberi factor is distinct from torulin. The question at issue seems to be whether torulin is distinct from the antiberiberi factor, and what relation these properties of extracts bear to the protective factor in the sense of Jansen and Donath. It is indeed possible that the train of symptoms cured by torulin can only arise when absence of some other factor has accentuated a special phase of metabolism. This would explain certain abnormal responses to test doses, and would also justify the feeling that curative tests upon pigeons are not a reliable guide where human beriberi is concerned."

This uncertainty concerning the identity of the "curative" and "protective" substance for pigeons was accentuated by the evidence which by this time had become convincing through the various studies reviewed in detail in the following chapter that yeast and other sources of vitamin $B$ contain at least two separate factors for rat growth differentiated in part by their unlike stability to heat and that (see Chick and Roscoe, 1927; Roscoe, 1927) the thermolabile factor is present in concentrates of the curative substances for pigeons. With the provisional designation of the thermolabile and thermostable factors as vitamin $B_{1}$ and $B_{2}$, Kinnersley and Peters (1928) in their next paper adopted the term yeast vitamin $B_{1}$ (curative) in place of torulin to designate their yeast concentrate. In this paper they reported attempts to purify and concentrate further the fraction obtained by extracting activated charcoal with tenth-normal hydrochloric acid. Although they were able in one case to concentrate ten-fold about 30 per cent of the vitamin of a preparation of activity of 0.9 milligram by further adsorption at $\mathrm{pH} 7$ with norite, yet in general the relations of the highly active fractions to charcoal could not be defined with certainty, inasmuch as further treatment with norite removed no more vitamin. They concluded that adsorption upon norite in the earlier stages is due to the presence of a co-adsorbent, and considered it preferable to depend upon alcohol fractionation rather than upon successive adsorptions to give reproducible results to an activity of 0.15 to 0.3 milligram per day. In order to eliminate the large losses during the subsequent alcohol fractionations the tenth-normal hydrochloric acid extracts must first be freed of all traces of metals, and of sulfuric acid 
with barium chloride, sodium hydroxide, and hydrogen sulfide. The final alcohol fractions were about as active as the preparation which Jansen and Donath obtained at the phosphotungstic acid stage. Although Kinnersley and Peters obtained very active fractions by the use of the silver nitrate method (Seidell, 1922) they found that this method does not always prove reliable. The use of phosphotungstic acid with a preliminary charcoal adsorption step as suggested by Jansen and Donath gave fractions of 0.07 to 0.08 milligram per day activity, but with marked losses, particularly when acetone was used as a solvent. The authors think that precipitation with phosphotungstic acid is a "genuine property of the active principle." With chloroplatinic acid they have obtained very active preparations but with large losses and variable yields.

The active preparations give a slight Pauly test, contain 13 to 20 per cent nitrogen, give qualitative tests for sulfur, negative Benedict test, and positive Molisch reaction.

In discussing the properties of this yeast vitamin $B_{1}$ Kinnersley and Peters emphasized that the properties of preparations change during the process of concentration. Thus while in the early stages of fractionation from yeast, vitamin $B_{1}$ is thrown out upon alcohol precipitation, at a later stage after the removal of certain substances relatively insoluble in alcohol, vitamin $B_{1}$ is soluble in alcohol of high concentration; in their experience vitamin $B_{1}$ (activity 1.0-0.03 milligram per day) is soluble in alcohol or water, but insoluble in chloroform, carbon tetrachloride, ether, acetone, or ethyl acetate. When fractionated from mixtures of benzene and alcohol or ether and alcohol in which water is reduced to a minimum, active preparations are thrown out of solution, but much seems to depend on the acidity.

The stability to alkali appeared to depend upon various factors. Alcohol and hydrogen peroxide appeared to accelerate the destruction by alkali. It is considered advisable to carry out any alkaline stage in the process of concentration and purification at a low temperature and in the absence of alcohol. In general the active material proved stable to oxidizing agents and fairly resistant to reducing agents.

On account of criticisms from various sources on the reliability of short curative tests upon pigeons in vitamin B studies, Kinnersley, Peters, and Reader (1928) subjected the test as originally described by Peters (1924) to rigid examination as to its quantitative value with the conclusion that if certain factors are taken into account the test can be used with confidence. Two possible cures other than that due to the curative substance were recognized. One is the so-called "heat" 
cure and the other the "glucose" cure. The former can be avoided by leaving the birds in a warm place for at least two hours before treatment and the latter by giving a small dose of glucose in water at the same time that the bird is being tested for the heat cure. This technique was followed by Guha and Drummond (1929) in testing various concentrates obtained in an effort to isolate vitamin $B\left(B_{1}\right)$ from wheat embryo. Since they were primarily interested in this vitamin as required for normal nutrition of rats and other animals, their investigation will be noted in greater detail in the following section, but it is of interest that in following the procedure of Jansen and Donath full confirmation of their results were obtained at various stages of fractionation through that of precipitation with platinic chloride. According to Drummond (1930) the concentrate at various stages had the following activity in curative day doses for pigeons (the material being injected subcutaneously): Material separated from "activated" fuller's earth, 2.6 milligrams; silver fraction at $\mathrm{pH} 6.5,0.18$ milligrams; phosphotungstic acid fraction, 0.014 milligram; alcohol-soluble fraction 0.014 milligram, and platinum chloride fraction 0.005 milligram.

A further examination of the Jansen-Donath method has been reported by Williams and Eddy (1927-28, 1928-29) and Williams, Waterman, and Gurin (1930), using both yeast and rice polishings as the original material, and both rats and pigeons as test subjects. Various deviations from the original Jansen-Donath procedure and the use of protective rather than curative tests make impossible a quantitative comparison of their findings with those of Jansen and Donath, but they report a good qualitative but poor quantitative relationship between their concentrates and those of Jansen and Donath at various stages. Vitamin hydrochloride crystals supplied by Jansen and Donath were used in protective tests for pigeons on a polished rice diet. With doses of 0.04 milligram daily polyneuritis and death were much retarded.

deCugnac (1929) applied the method of fractional precipitation to extracts prepared by the Seidell (1926) method, using as solvents water, acetic acid, and formic acid and as precipitants alcohol, acetone, ether, and picric acid. With successive precipitations with 70,80 and 87 per cent alcohol and acetone, fractions were obtained similar in their vitamin B content, as determined by the Seidell (1924) method, but at each step freer from inorganic material. This was interpreted as indicating that the apparent precipitation of vitamin $B\left(B_{1}\right)$ is really a phenomenon of adsorption rather than precipitation. The active 
preparations were never in definite crystalline form, although some of them showed under the microscope a mixture of crystalline fragments and amorphous material.

deCugnac used acetone as a precipitant of vitamin B. Smith (1930) on the contrary prepared a concentrate of vitamin B from the supernatant fluid decanted from the precipitate obtained when 1 volume of vitamin-rich solution was poured into 9 volumes of acetone. His method was as follows: After triturating dried brewers' yeast in a mortar with 5 volumes of twenty-fifth normal acetic acid, and allowing it to stand overnight, the extract was centrifuged, decanted, and poured with stirring into 2 volumes of 95 per cent alcohol. The supernatant liquid was concentrated to one-twentieth its volume, poured into 9 volumes of acetone, and again the supernatant solution decanted and concentrated to one-twentieth its volume. After diluting 10 times with water $(\mathrm{pH} \mathrm{4})$ it was stirred with purified norite 3 times, the norite filtered and washed with water. The combined norites were treated 3 times successively on a water bath with 5 volumes of twentieth-normal hydrochloric acid in 50 per cent alcolol. The extract thus obtained was concentrated to one-twentieth of its volume under reduced pressure, and was found curative to polyneuritic rats in single doses of 0.5 to 1.0 milligram solids.

A comparison of the relative potencies of various vitamin $\mathrm{B}$ concentrates by this method and by the Seidell method showed a decided lack of agreement. Referring to the confusion arising from using different tests, Smith emphasizes a point which cannot fail to impress any one who attempts to review the long and as yet unsuccessful attempts to isolate the antineuritic vitamin and determine its chemical nature. "Apart from the question as to which of the two methods represents a more accurate index of the antineuritic potency of the above or any other concentrate, these results at least emphasize one point clearly, and that is the impossibility of comparing the merits of the various chemical procedures used by different men in the concentration and purification of the antineuritic vitamin unless one biologic method is adopted in common for testing of the potency of such concentrates."

\section{Suggestions as to the Chemical Nature of the Antineuritic Vitamin}

As has been noted, attempts have been made from time to time to assign empirical formulas to the antineuritic vitamin on the basis of analyses and reactions of the concentrates prepared, and also to determine the chemical nature of the vitamin by testing definite com- 
pounds for antineuritic properties (Williams, 1916, 1916a, 1917, 1921 ; Harden and Zilva, 1917).

Sahashi $(1925,1926,1926 a)$ prepared 300 grams of the $\beta$-acid of oryzanine (Suzuki) and attempted to determine its chemical nature. On heating crystals of the acid to $150^{\circ} \mathrm{C}$. water of crystallization was driven off, leaving an anhydrous salt conforming to the empirical formula $\mathrm{C}_{10} \mathrm{H}_{7} \mathrm{NO}_{4}$ instead of $\mathrm{C}_{10} \mathrm{H}_{8} \mathrm{NO}_{4}$ ascribed to it by Suzuki. From various tests the constitution of the compound was considered to be 2,6-dioxy-quinoline-4-carboxylic acid.

The $\alpha$-acid was thought to be the $\beta$-acid containing one molecule of water of crystallization. The resemblance of this compound to $\alpha$-hydroxypyridine and its derivatives found by Williams (1916a) to have temporary antineuritic properties, led Sahashi (1927) to determine whether the 2,6-dioxyquinoline group in the $\beta$-acid has any relation to the curative property of the compound for polyneuritic pigeons. Pure crystalline 2,6-dioxyquinoline hydrochloride obtained from the $\beta$-acid of oryzanine showed marked curative properties, suggesting that the nucleus of the antineuritic factor may consist either of the 2,6-dioxyquinoline group or its tautomeric form.
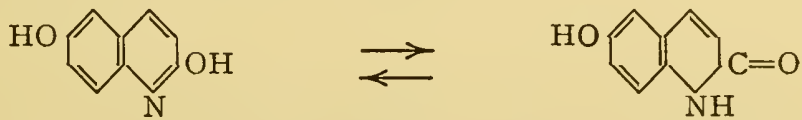

Later, in an attempt to synthesize a glyoxaline derivative of the same empirical formula as that of Jansen and Donath's (1927) crystalline compound, Sahashi (1928) prepared 4-(or 5)-glyoxalinemethylethyl carbinol. Injections of this he found to be temporarily curative for polyneuritic pigeons.

Resuming earlier attempts by Williams to determine the nature of the antineuritic vitamin by testing various synthetic substances for antineuritic activity, Williams and Eddy (1927-28) tested various di-keto piperazines as sources of the antineuritic vitamin for rats. The enol forms of certain of these compounds were found to have a definite effect on growth and the keto forms to be entirely without effect, thus conforming to the earlier theory of isomeric changes as a factor in the lability of antineuritic substances. "The chemical features associated with the apparent curative activity of the earlier synthetic preparations are also present in the di-keto piperazines. Feeding results to date with pigeons are not convincingly positive, and those with rats, while apparently beyond the limits of experimental error, still lack the excellence obtainable with natural antineuritics. We seem to be 
dealing with substances kindred to but not identical with the natural product."

The various claims as to the curative properties of certain quinoline and glyoxaline derivatives to pigeons suffering from polyneuritis led Gulland and Peters (1929) to repeat some of these experiments under very carefully standardized conditions. They give a standard dose of a torulin preparation which is curative for about 3 days, before giving the substance under test. If, after this, the test substance is not effective, but it is subsequently possible to relieve the symptoms by the use of a second dose of torulin, the negative answer is considered decisive. Neither 4-(nor 5) glyoxalinemethylethyl carbinol hydrochloride, nor 2,6-dihydroxyquinoline showed curative properties similar to torulin. The following compounds containing glyoxaline or pyrimidine rings had no curative effects: 2,6-dihydroxyquinoline-4-carboxylic acid; 4-hydroxy-2,6-dimethyl pyrimidine; 2-hydroxy-4,6-methyl pyrimidine; 2-thiol-4 (or 5)-aminomethyl glyoxaline; ergothioneine hydrochloride; 2-methyl glyoxaline; glyoxaline-4-(or 5)-formaldehyde; 4-(or 5)-[glyoxaline-4(or 5)-methyl] glyoxaline-5-(or 4)-methyl alcohol; glyoxaline-4-(or 5 )-acetic acid; $d l$ - $\alpha$-hydroxy- $\beta$-glyoxaline-4-(or 5 )-propionic acid.

The question is still open whether Sahashi's temporarily positive results are attributable, as Peters suggests, to "pseudo-cures" or whether the negative results obtained with some substances are to be ascribed to the duality of the nature of vitamin $B_{1}$ postulated by Guha and Drummond (1929).

In spite of the uncertainty which still exists concerning the nature of vitamin B after nearly twenty years of intensive research in many laboratories, Drummond (1930) is of the opinion that "there are grounds for some optimism regarding the future of research into the nature of the antineuritic vitamin $B$. It appears to be a basic substance of relatively simple composition and it should, therefore, be only a matter of carrying out the fractionation on a sufficiently large scale to obtain enough of the active substance, or substances, to establish their nature and constitution."

\section{Development of Knowledge of the Growth-Promoting Function of Vitamin B}

Osborne and Mendel in 1911 and again in 1912 had pointed out the superiority of their experimental rations containing "protein-free milk" over mixtures of purified proteins, fats, carbohydrates and salts, 
as food for growing animals, even though the purified food substances were themselves derived from milk.

In 1913 they developed the idea more fully, giving experimental evidence which pointed plainly to the presence in milk of a watersoluble substance important for growth and different from any of the known constituents of the diet. Their chief evidence on these points at that time was as follows: Young rats fed solely upon milk food (a paste consisting of milk powder, 60 per cent, starch, 12 per cent, lard, 28 per cent) not only grew from infancy to full maturity but gave birth to litters of normal young which in turn throve on a diet precisely like that furnished to their parents. Therefore this food was considered to contain all that is essential for both growth and maintenance.

Mixtures of starch, lard, purified protein from milk, and a salt mixture made in imitation of milk ash never supported growth. But on mixtures of purified protein, lard, starch, and "protein-free milk," young rats grew well for 60 days to 100 days or more; when finally there was a sudden decline, and death followed unless a change was made in the diet.

The observation that the nutritive decline which eventually sets in on such a diet can be averted and growth renewed by the addition of butter fat to the diet led to the conclusion that a substance exerting a marked influence upon growth is present in butter and tended at the time to concentrate attention upon this factor rather than to emphasize the presence of another growth-promoting substance in the protein-free milk. In December, 1914, Mendel (1915) in a lecture on Nutrition and Growth delivered before the Harvey Society of New York stated: "It is not unlikely, to speak conservatively, that there are at least two 'determinants' in the nutrition of growth. One of these is furnished in our 'protein-free milk' which insures proper maintenance even in the absence of growth. When this was fed we have maintained rats without growth for very long periods. Without this 'determinant' (as, for example, in diets of isolated food substances containing artificial substitutes for 'natural' protein-free milk) the special components of butter fat or cod-liver oil or egg fat induce only limited gains at best. Another 'determinant' is furnished by these natural fats." Meantime Hopkins (1912) had published the results of his discovery dating back to 1906, of the remarkable effect of the addition of small amounts of milk to a diet of purified foodstuffs and had drawn the conclusion that certain natural foods contain an alcohol-soluble, growth-promoting substance or substances. Since both of the substances 
discussed by Mendel in the paragraph quoted above are soluble in alcohol, the work of Osborne and Mendel was in accord with that of Hopkins and carried the subject further in showing the existence of two factors in the problem.

A paper published by McCollum and Davis (1915) did much to advance the view that "there are necessary for normal nutrition during growth two classes of unknown accessory substances, one soluble in fats and accompanying these in the process of isolation of fats from certain foodstuffs and the other soluble in water, but apparently not in fats." By means of feeding experiments with young rats, it was found that polished rice could not be supplemented so as to produce rations capable of inducing growth by the addition of purified protein, fats which possess the growth-promoting property, and salt mixtures, but that the addition of quantities of wheat embryo or of milk powder as small as two per cent of a food mixture consisting of polished rice, casein, salts and butterfat, furnished enough of an essential accessory to induce growth. This substance essential for growth was shown to be present in the water and alcohol extracts of wheat embryo and of egg yolk and to be apparently stable to heat. That it was not identical with the growth-promoting constituent of butterfat was shown by the fact that the addition of 20 per cent of butterfat to the basal ration did not induce growth unless the other accessory was supplied nor did the addition of the water-soluble accessory suffice to support growth in the absence of the fat-soluble accessory. That the amounts of the water-soluble factor necessary to induce normal growth are very minute was shown by the fact that amounts of an alcohol extract of wheat embryo carrying as little as 0.6 gram of solids and 0.0095 gram of nitrogen, equal to 0.33 per cent of the total nitrogen of the ration, sufficed to induce normal growth.

Attention was also called to the probability that lactose of the ordinary purity may be contaminated with sufficient of the watersoluble, growth-promoting accessory to cause pronounced increase in the body weight of animals whose ration was otherwise adequate. Later in the same year McCollum and Davis (1915a) reported that supposedly pure casein as well as lactose may contain appreciable amounts of the water-soluble, growth-promoting substances. Casein was therefore purified by twice repeated precipitation, followed by washing, drying and grinding. With casein prepared in this way, together with dextrin, butterfat and salts, they obtained no appreciable growth even during the first month. Nor was growth obtained in the presence of excess of the water-soluble factor with absence of the fat-soluble factor. 
Thus an explanation was afforded for the observations previously noted by Hopkins and Neville (1913) and others, that when purification of the various ingredients of an artificial dietary is carried to a further degree than usual the animals decline at a much more rapid rate than when fed upon a similar diet which is less pure.

Shortly after these papers Drummond (1916) published more detailed evidence leading to the same conclusion, that lactose refined to a degree ordinarily regarded as pure is likely to carry considerable amounts of a growth-promoting substance soluble in water and alcohol and not destroyed by exposure to $100^{\circ} \mathrm{C}$. for six hours. This helped to explain the discordant results obtained by various investigators in growth experiments in which lactose had been a part of the experimental dietary and to make more convincing the proof of the indispensability for normal nutrition of the water-soluble and fat-soluble vitamins.

Similarity in occurrence and properties of the water-soluble growthpromoting and the antineuritic vitamins had led to the suggestion, and belief on the part of most workers, that these two were identical.

McCollum and Kennedy (1916) held that the water-soluble B essential to growth is identical with the substance which prevents and cures polyneuritis and that it may be extracted from fat-free wheat embryo not only, by water and alcohol but also by acetone, benzene and ethyl acetate. They found evidence of the presence of this substance in the juice of potato and cabbage as well as in extracts of wheat and oats.

Funk and Macallum (1916a), in attempts to concentrate the watersoluble growth-promoting vitamin in yeast by methods such as Funk had used in his studies on the antineuritic vitamin. found that the growth-promoting substance was almost entirely precipitated by phosphotungstic acid, but that practically all of its activity was lost on subsequent fractionation with silver salts. While their results did not entirely parallel those of Funk, they concluded that the water-soluble, growth-promoting substance is analogous to, if not the same as, the antineuritic vitamin. At about the same time Eddy (1916) obtained from sheep's pancreas an alcoholic extract containing a water-soluble, growth-promoting vitamin capable of adsorption with Lloyd's reagent, and of precipitation from the resulting extract by phosphotungstic acid. In the treatment of the phosphotungstate both Funk and Macallum and Eddy used the amyl alcohol method of Jacobs which does not seem to have been used in the various methods of concentrating the antineuritic vitamin.

In both of the foregoing studies the activity of the various fractions was tested by using them as supplements to a basal diet of casein, 
sugar, starch, lard, agar, and salts in feeding experiments conducted on young rats. The necessity for providing the fat-soluble vitamin, as pointed out by Osborne and Mendel and by McCollum, had apparently not been recognized by these workers. Osborne and Mendel (1917a) called attention to the absence of butterfat in the diet used by Funk and Macallum and reiterated their belief that "unless all the other necessary factors of the diet are adequately supplied the presence or absence of the water-soluble vitamin can not be demonstrated." In the light of later developments this paper contains several significant statements. For the first time emphasis was given to what is now considered to be one of the most important properties of vitamin $\mathrm{B}$, its stimulation of the appetite. In attempting to explain this, the opinion was expressed that the water-soluble vitamin exerts a favorable influence upon metabolism, improving the general condition of the animal and thus increasing its appetite, rather than merely rendering the food mixture more palatable and thus inducing the animal to eat more. Subsequently it was demonstrated experimentally that an animal's appetite for vitamin B-free food can be improved by feeding the vitamin separately in the form of a small amount of dried yeast or dried spinach. Another significant observation made at this time was that the rapidity of growth was related to the quantity of yeast fed. To promote normal growth in white rats with dried yeast as the sole source of watersoluble vitamin in their experiments required the presence of about 2 per cent of yeast solids in the food mixture. The question as to whether or not the water-soluble vitamin with which they were dealing was identical with the antineuritic vitamin was considered unanswered, although attention was called to the opinion of Funk and Macallum that "the growth-promoting substance is analogous to and possibly identical with the beriberi vitamin, but that considerably larger quantities of vitamins are necessary for stimulating growth than for curing beriberi." The necessity for carrying feeding experiments through more than one generation, the recognition of which led later to the discovery of vitamin $\mathrm{E}$, was brought out in the concluding statement in this paper in which attention was called to the observation that yeast as a source of water-soluble vitamin had not given as good results as were obtained from the use of protein-free milk. "Although some of the animals brought up on the yeast-containing foods have given birth to young, thus far none of the latter have been reared." The work of Osborne and Mendel made it plain that this water-soluble vitamin so often referred to as "growth-promoting" is certainly essential to normal nutrition at all ages. 
In another paper published later in the same year Osborne and Mendel (1917b), in calling attention to the fact that up to this time milk and pancreas were the only products of animal origin that had been tested for the presence of the water-soluble vitamin, stated that "indirect evidence that animal tissues may contain this hormone is afforded by studies of the protective, curative or antineuritic properties of some of these in relation to beriberi. That the substance which induces the remarkable recoveries which have been described in these cases is identical with the water-soluble hormone which is so essential for growth and maintenance is as yet merely a matter of conjecture." In the experimental work reported in this paper it was shown that muscle tissue and meat extract contain but little of the water-soluble, growth-promoting vitamin but that liver contains a larger proportion of this substance. It was pointed out that these results parallel the findings of Cooper in respect to the relative amounts of antineuritic vitamin in muscle and liver. Attention was also called to the fact that both liver and pancreas, in contrast to muscle tissue, are exceptionally rich in glandular cells.

The question as to whether the antineuritic and growth-promoting properties of yeast are different properties of the same vitamin or indicate two different vitamins in the yeast was complicated in all of the work reviewed by the fact that pigeons or fowls were used for testing the antineuritic and rats or mice the growth-promoting properties. Recognizing this, Emmett and McKim (1917) attempted to determine whether a yeast preparation known to have been effective in curing polyneuritis in pigeons would completely supplement polished rice to the extent of bringing about normal gains in weight in the cured pigeons on continued feeding. They found that activated fuller's earth prepared from autolyzed yeast by the original method of Seidell, when added as a supplement to polished rice not only prevented the recurrence of polyneuritis but brought about some gain in weight for from 75 to 90 days, after which growth came to a standstill. Barley, shelled corn, and brown rice fed as the sole diet brought about better gains, the brown rice giving the best results. Brown rice plus the vitamin extract stimulated growth beyond normal values. They concluded that, "(a) the activated fuller's earth when given as a rational supplement to a polished or brown rice diet, acts as a partial stimulant to increase the weight of the treated polyneuritic pigeons; (b) it does not, however, in the case of the polished rice, accelerate the increase in weight to anything like that which is produced under similar conditions with brown rice alone, corn, barley, or hulled oats; and 
(c) comparatively speaking, this activated yeast vitamin is not a complete supplement to a polished rice diet. There are apparently two so-called vitamins associated with rice polishing, one which cures polyneuritis and one which produces weight, and of these two, the Seidell yeast vitamin preparation contains chiefly the curative fraction, along with a small per cent of the other." This is of interest in connection with the later work of Williams and Waterman (1927) and of Randoin and Lecoq (1927d, e).

Drummond (1917) attributed losses in purification not so much to instability of the vitamin as to its ready adsorption by colloidal precipitates. "Further proof that this is probably the explanation of the great loss which is so often encountered in the isolation of the substance is to be found in the fact that whenever in the course of the fractionation recourse to precipitation is made, the precipitate, particularly if bulky and flocculent in nature, will usually carry down the majority, if not all, of the active substance from solution." The greater difficulty in tracing the growth-promoting than the antineuritic vitamin during fractionation was attributed by Drummond to the fact that much smaller quantities of the extracts were required to improve the condition of polyneuritic pigeons than to cause an appreciable increase in the body weight of rats.

McCollum and Simmonds (1918) studied the solubility and stability of vitamin $\mathrm{B}$, using white beans or wheat embryo as sources of the vitamin. Young rats were fed a diet of purified food substances (casein, salt mixture, agar-agar and dextrin) together with 5 per cent of butterfat to supply an abundance of vitamin A. The rats were confined to this diet for about five weeks until they had either become stationary in weight or were declining with evidences of paralysis. The material to be tested was then added to the diet, the animals continuing to decline or responding with growth according as the material was deficient or rich in vitamin B. This method was considered to show within two weeks whether the preparation under investigation contained vitamin B in significant amounts. As tested by these criteria, it was shown that vitamin $B$ is not extracted directly from beans, wheat germ or pig kidney by ether, benzene or acetone, but is readily extracted in great part by alcohol (95 per cent). McCollum and Simmonds concluded that: "The probability that there should be two or more physiologically indispensable substances in what we term watersoluble $\mathrm{B}$, both or all of which should show the same solution relations with three solvents, is relatively small and lends support to our view that the substance which protects animals against polyneuritis is the 
only essential complex in the extracts described." (These experiments probably deal with $B_{1}$, in the present sense of the term.) A few experiments on the stability toward acids and alkalies of water-soluble $B$ in the form of an alcohol solution of wheat embryo were also reported. These tended to confirm similar tests of the antineuritic vitamin in its rapid destruction by alkalies and relatively marked stability toward hydrochloric acid. Nitrous acid had slight if any action, this fact constituting a strong indication that the active substance is neither a primary nor a secondary amine.

Osborne and Wakeman (1919) in an attempt to obtain from yeast a concentrate of vitamin $B$ relatively free from proteins and related substances first coagulated the protein by boiling water, thus removing all but a comparatively small proportion of the total solids of the yeast, and then subjected the water extract to fractional precipitation with alcohol. The preliminary treatment of the yeast, which was later adopted by Seidell and others in their attempts to isolate the antineuritic vitamin, was described as follows: "Several liters of fresh bottom yeast were obtained directly from a brewery, and immediately diluted with ice water. After centrifuging, the sediment was washed twice more in the same way. The moist, washed yeast weighed 264 grams, equal to 48 grams dried at $107^{\circ} \mathrm{C}$. This was stirred gradually into 1 liter of boiling distilled water containing 10 cubic centimeters of 1 per cent acetic acid. After boiling for about 2 minutes the solids were separated from the extract with the centrifuge. The residue was washed once by boiling with 0.01 per cent acetic acid and, after centrifuging, the extracts were united and concentrated to 500 cubic centimeters. This concentrated extract contained 8.14 grams of solids, equal to 17.1 per cent of dry yeast, and 0.666 gram of nitrogen, equal to 14.4 per cent of the original yeast nitrogen or to 8.18 per cent of the solids of the extract."

This concentrate although containing less than one-fifth of the solids and only one-seventh of the nitrogen of the yeast contained nearly all of the vitamin B as then understood. Daily doses of 17 milligrams of the solids of the extract, equivalent to 0.1 gram of the original dried yeast, sufficed to bring about the recovery and rapid growth of young rats declining on a diet free from the water-soluble vitamin. By treating the extract successively with alcohol to 52,79 , and 90 per cent concentration by weight, three flocculent precipitates were obtained, all of which contained some of the vitamins. Fraction II, however, contained by far the greater part. This fraction, after washing with 79 per cent alcohol, twice dissolving in 100 cubic centimeters of 
water (in which it was very soluble), reprecipitating by alcohol to 90 per cent concentration, digesting under absolute alcohol and drying over sulfuric acid yielded a light-colored friable product equivalent to 37 per cent of the solids of the extract or 6.2 per cent of the dried yeast. It contained 7.5 per cent of nitrogen, equal to 4.5 per cent of the nitrogen of the original yeast, and 10.65 per cent of ash. Numerous feeding experiments showed it to be highly efficient in promoting the growth of young rats when fed as the sole source of water-soluble vitamin and in restoring animals which were declining on a diet lacking in these vitamins.

Osborne and Wakeman determined the distribution of nitrogen between humus, ammonia, amino, basic, and purine forms in this yeast fraction before and after hydrolysis with results indicating the presence of relatively large proportions of nucleic acid, amino acids, and peptides. An aqueous solution of the fraction was distinctly acid to litmus, requiring considerable alkali for neutralization and still more to produce an alkaline reaction. The unneutralized solution gave a heavy precipitate with lead acetate, but only slight turbidity with barium chloride or with silver nitrate unless previously neutralized with sodium hydroxide, when an abundant precipitate formed. Barium hydroxide gave a voluminous precipitate containing about 25 per cent of the solids of the fraction and very little nitrogen. On adding silver nitrate to the filtrate from the barium hydroxide precipitate about 25 per cent more of the fraction was precipitated. This, when thoroughly washed, contained nearly one-half of the nitrogen of the fraction. The aqueous solution of Fraction II on acidification with sulfuric acid gave an abundant precipitate with phosphotungstic acid, a precipitate with mercuric chloride, but none with copper sulfate, and a precipitate with an aqueous or alcoholic solution of picric acid if added in considerable quantity.

In a further study of this yeast fraction Osborne and Leavenworth (1921) found that its efficiency as a source of vitamin B, as indicated by growth experiments with rats, was only slightly, if appreciably, affected when the material was dissolved in tenth-normal sodium hydroxide and allowed to stand at a temperature of $20^{\circ} \mathrm{C}$. from one-half hour to 18 hours, whereas when such a solution stood for 90 hours at $20^{\circ} \mathrm{C}$. its efficiency was very greatly reduced, indicating the destruction of a large proportion of the vitamin present. A still larger proportion, if not the whole, of the vitamin present was destroyed when a solution of the same alkalinity after standing for 18 hours at $20^{\circ} \mathrm{C}$. was heated for one hour at $90^{\circ} \mathrm{C}$. The authors concluded that, in at- 
tempting to concentrate or isolate vitamin $\mathrm{B}$, dilute alkaline solutions can be used without materially affecting its activity provided that a low temperature and a short time of exposure to the alkali be employed.

Levene and van der Hoeven (1924) in a brief note in Science announced that they had succeeded in preparing from the Osborne and Wakeman fraction by precipitation with barium hydroxide a material which was from five to ten times more active as a source of vitamin $B$ than the original fraction. An extension of this method, together with others, was described later in the same year. In this paper Levene and Van der Hoeven (1924a) called attention to the fact that work hitherto reported on the purification of the crude vitamin had followed two distinctive principles, one aiming to find a specific reagent for precipitation or for extraction (as in the early studies on the antineuritic vitamin) and the other to remove gradually the inert material and thus increase progressively the potency of the material (as in the work of Osborne and Wakeman). Seidell's use of Lloyd's reagent to adsorb the active material from the Osborne-IVakeman fraction was considered to represent further progress in the choice of methods, and was the first method used in the present study.

Acting on the theory that the adsorbing power is dependent upon the degree of ionization of the active surface of the adsorbent and the degree of dissociation of the substance to be adsorbed, and that there must exist for each substance a certain hydrogen-ion activity at which the adsorption is optimal, the adsorbing power of Lloyd's reagent was tested at different hydrogen-ion activities. The optimal adsorption of the vitamin was found to take place at $\mathrm{pH} 4$, while the optimal extraction from the adsorbed material was at $\mathrm{pH}$ 9. By this means a fraction was obtained which was from two to three times as active as the original material. The use of kaolin as adsorbent gave unsatisfactory results. With silica gel better results were obtained than with any other adsorbent. The optimal hydrogen-ion activity for adsorption with silica gel was at $\mathrm{pH} \mathrm{5,} \mathrm{and} \mathrm{for} \mathrm{subsequent} \mathrm{extraction} \mathrm{was} \mathrm{at} \mathrm{pH} \mathrm{9,} \mathrm{although}$ a considerable portion was extracted by acids at $\mathrm{pH} 3$.

By treating with silica gel the solution containing the active material obtained by the barium process noted in the preliminary report, and subsequently extracting at $\mathrm{pH} \mathrm{9,} \mathrm{a} \mathrm{material} \mathrm{was} \mathrm{obtained} \mathrm{which} \mathrm{was}$ from 200 to 400 times as potent as dried yeast, but at the expense of considerable loss of the original material. A better method consisted in suspending the original barium precipitate in water and passing a stream of carbon dioxide through it. Under these conditions most of the potent material remained in the precipitate, while the inert material 
passed into solution. The precipitate, freed from barium, was active in amounts furnishing about 0.00017 gram of nitrogen per day.

In testing the activity of the various fractions, rats about six weeks old which had been on a vitamin B-free diet (McCollum) for three weeks and were losing weight were given definite quantities, as determined by nitrogen content, of the solution to be tested, carefully neutralized and preserved in 30 per cent alcohol. After 3 or 4 days the rats were again weighed and the strength of the solution being tested was calculated from the weight gains by a method which is described as follows: "From 'normal growth' curves of several rats the average increase in weight during 3 days for a body weight of 40 to 70 grams is found ( 5 to 7 grams). Considering this increase normal, the amount of the substance (measured by the quantity of nitrogen) was determined which would be required to give a normal increase in weight in the test animals during the feeding period. It is realized that the adopted unit may be insufficient to bring about a normal course of the entire period of growth up to maturity. However, it offers a rapid and reliable method for standardizing the relative strength of various products."

Levene and van der Hoeven called attention to Seidell's crystalline picrate (described in the preceding section), with the comment that: "The standards of testing employed by Seidell and by us are so different that it is difficult to compare the results of the two investigations. It seems unlikely that the picrate of Seidell and our concentrate have potencies of different orders of magnitude. Yet we are certain that our material is not an individual substance, but a complex mixture. The pure vitamin, if ever isolated, will prove many times more potent than the picrate of Seidell or than our present concentrate."

In their next paper Levene and van der Hoeven (1925, 1925a) reported a further concentration of the Osborne-Wakeman fraction involving a preliminary precipitation with basic lead acetate, with removal of the lead by sulfuric acid instead of hydrogen sulfide as had generally been the custom. By this step alone the potency of the product was increased three or four times. Precipitation of this material by barium hydroxide as in the original method yielded a product approximately as active as the one previously prepared by the silica gel adsorption process. Quantities of from 2 to 4 milligrams, containing from 0.1 to 0.2 milligram of nitrogen ( 5.2 per cent on an ash-free basis) sufficed in the usual feeding tests. Adsorption of the material by silica gel and subsequent extraction twice with alcohol at $\mathrm{pH} 3$ yielded products of only moderate potency. A third extraction with alcohol at $\mathrm{pH} 9$ gave the most potent material thus far prepared. This was active 
in amounts of about 0.1 milligram, containing 0.015 milligram of nitrogen. The yield, however, was very small, amounting to from 3 to 5 per cent of the original Osborne-Wakeman fraction. Attempts were made to increase the yield by removing the salts of phosphoric acid from the lead-barium product before the silica gel adsorption, either by precipitating the active material with absolute alcohol from a sulfuric acid ( $\mathrm{pH}$ 2) extract of the precipitate or by dissolving it in a minimal amount of hydrochloric acid of specific gravity 1.19 and adding to the solution enough 98 per cent alcohol to make the alcohol concentration 70 per cent. The materials thus prepared were active in daily amounts of 2.5 and 1.25 milligrams respectively, but subsequent adsorption on silica gel increased the activity to a much less extent than was the case with the phosphorus-containing fraction, the minimum effective dose of the purified fraction after adsorption being 0.6 milligram and of the phosphorus-containing fraction 0.1 milligram daily. This was thought to suggest the possibility that the phosphorus-containing impurity is essential for selective adsorption by silica.

A further modification of their method was described the following year by Levene and van der Hoeven (1926) essentially as follows:

The Osborne-Wakeman fraction was extracted repeatedly with absolute alcohol until a dry nonhygroscopic powder was obtained. This was dissolved in water and adjusted to $\mathrm{pH} 4$, at which reaction some inactive material precipitated out on standing. The remaining filtrate was active in amounts furnishing 0.5 milligram nitrogen per day, as compared with 2 milligrams for the original Osborne and Wakeman fraction. This was deaminized with barium nitrite and sulfuric acid, the strongly acid solution after concentration under diminished pressure was precipitated by adding alcohol to 80 per cent concentration, and the process repeated with the filtrate from the first precipitation. This material was active in amounts containing about 0.15 milligram nitrogen per day. The final stage consisted in the adsorption of the active material from the preceding step by silica gel at $\mathrm{pH} 5$, followed by two extractions at $\mathrm{pH} 3$, and one at $\mathrm{pH} 9.5$, neutralization and concentration of the extraction product, filtration through collodion membrane, further concentration, and precipitation with dry acetone. The activity of the final fraction was reported to be such that in one case normal growth for a 14-day period was secured with 0.15 milligram substance per day. When observations were extended to longer periods, the material was usually found ineffective after the first week.

"The final product obtained is still a complex mixture, containing complex carbohydrates and nitrogenous substances and esters of phos- 
phoric acid. It contains no amino nitrogen, but does contain sulfur, which is given off on treatment with alkali in the form of hydrogen sulfide. The sulfur content increases with purification, but it is premature to pass an opinion as to whether the sulfur is a part of the active substance." (Cf. Guha and Drummond, 1929.)

Another method for the extraction of vitamin $B$ was suggested by McCollum and Kruse (1926). On the assumption that vitamin B might be a tertiary base capable of combining with acids, and that the resulting salts may be more soluble in 95 per cent alcohol than the vitamin itself, extracts were prepared from wheat germ with hot 95 per cent alcohol containing 1 per cent of various organic and inorganic acids. After evaporation of the solvent, these extracts were tested for their content of vitamin B by feeding experiments with young rats according to the technique of McCollum and Simmonds (1918).

An extract thus prepared from wheat embryo with 95 per cent of alcohol alone showed at the most only slight traces of vitamin $B$. This was also true of extracts containing acetic, citric, sulfuric, hydrochloric, or phosphoric acid, but those containing nitric, benzoic, or lactic acid showed the presence of appreciable amounts of the vitamin and excellent results were obtained with extracts containing 1 per cent of either gallic or tannic acid. The active extracts were thought to offer promise in further attempts at isolating the vitamin.

The possibility of forming soluble double salts of the vitamin with a metallic salt was also tested. An extract prepared from wheat embryo with 95 per cent alcohol containing 1 per cent of either zinc chloride or cadmium chloride was said to show some activity, but several other salts tested gave negative results. (Cf. Jansen and Donath, 1929.)

As will be developed more fully in Chapter III, much experimental evidence has been adduced since 1919 indicating that a number of individual substances contribute to the effects formerly ascribed to "watersoluble B." Peters in a memorandum to Nature (September 14, 1929) indicates that there are at least five factors concerned, and it is by no means certain that the list is complete.

Peters recognizes ( 1 ) the heat-labile antineuritic vitamin, necessary for both pigeons and rats; (2) a second heat-labile factor needed for pigeons (Williams and Waterman); (3) another heat-labile factor necessary to rats (Reader); 4 ) a relatively heat-stable factor needed by the rat (Smith and Hendrick); and (5) a heat-stable waterinsoluble (?) yeast factor necessary for rats (Hunt).

It is not possible to evaluate all of the earlier data regarding vitamin $\mathrm{B}\left(\mathrm{B}_{1}\right)$, the growth promoting and polyneuritis-preventive vitamin for 
rats, because the basal diets and the bodily store of the rats may have provided the other essential factors in variable amounts and proportions. Tests for the presence of vitamin $B\left(B_{1}\right)$ may have failed or its true concentration may have been underestimated because other nutritive essentials were furnished by the food or bodily store in concentrations too low to support the rate of growth permitted by vitamin $B$ $\left(B_{1}\right)$. It is of interest that Guha and Drummond (1929) in the fractionation of vitamin $B_{1}$ concentrates, have observed no fixed ratio between the rat-dose and pigeon-dose of the various preparations investigated. Thus the rat-doses of their picrolonic filtrate, of the phosphotungstic fraction and of the platinic chloride fraction were respectively, 10 times, 7 times, and 3 times the pigeon-dose. These authors are inclined to attribute this difference to the errors in the biological methods of assay rather than to a difference between the rat-factor and pigeonfactor. But the fact that both their rat experiments and pigeon experiments appear to corroborate a duality of the nature of the antineuritic $B_{1}$ raises the question as to whether various investigators even when using curative tests in pigeons were all dealing with the same factor. Certainly we have not adequate reason to assume that preventive or curative tests measure the factor necessary for weight-maintenance in pigeons, or that the first two are necessarily identical.

With the definite acceptance of at least two factors in growthpromoting vitamin $B$ (the relatively heat-labile $B\left(B_{1}\right)$ and heat-stable $G\left(B_{2}\right)$ ), attention was paid to their distribution in the various steps in the fractionation process for concentrating vitamin $B$.

In 1928, Salmon, Guerrant and Hays noted that fuller's earth, particularly when used in low concentrations, adsorbs vitamin B preferentially from a solution containing both vitamins $B$ and $G\left(B_{2}\right)$ even though vitamin $G$ is preponderant in the original solution. Fractionation of the vitamin G-rich filtrate left after three successive adsorptions, with alcohol to a concentration of 51 per cent by weight yielded a precipitate which was rejected; concentration of the resulting filtrate and washings and precipitation at an alcohol concentration of 82.7 per cent gave a fraction comparable to the Osborne-Wakeman fraction II, which carried a large part of the vitamin G originally contained in the crude extract, and which on the basis of total net solids appeared to have 3 to 4 times the original concentration of vitamin G. This precipitate was, however, less rich in vitamin $B$ than the filtrate left after the fractionation.

The effect of hydrogen-ion concentration upon the adsorption of vitamins $B$ and $G$ by fuller's earth from extracts of maize and yeasts 
has been studied extensively by Salmon, Guerrant and Hays (1928a). In experiments in which they kept the volumes and the amounts of fuller's earth proportional to the weight of the original material, they found the effect of $\mathrm{pH}$ upon the antineuritic potency of the "activated solids" by comparing the number of protective doses for pigeons per 100 grams adsorbate prepared over a range of $\mathrm{pH}$ 's from 0.5 to 11.9 . Maximum adsorption of vitamin $\mathrm{B}$ occurred between $\mathrm{pH} 3.0$ and $\mathrm{pH}$ 5.5 (equilibrium reaction), with inactivation beginning at $\mathrm{pH} 6.8$ and increasing significantly throughout the alkaline range. Poor adsorption in the alkaline range could not be attributed entirely to inactivation of the vitamin, for a second adsorption of the same solutions adjusted to a favorable $\mathrm{pH}$, removed 10 times as much antineuritic vitamin as had the initial adsorption process. This inactivation emphasizes, however, the importance of using weakly alkaline solutions and a short exposure to alkali when using alkali to remove the adsorbed substance from fuller's earth.

The effect of $\mathrm{pH}$ upon adsorption of the antineuritic factor by fuller's earth from maize and yeast extracts seemed to be similar to its effect upon the adsorption of quinine; whereas the effect of $\mathrm{pH}$ upon adsorption of the more heat stable factor seemed to be similar to its effect upon the adsorption of glucose. Fuller's earth seemed to be a more efficient adsorbent for vitamin $B$ than for vitamin $G$.

From brewers' yeast, 30 milligrams daily of which contained enough vitamin $B\left(B_{1}\right)$ for normal growth, Levene (1928) made a concentrate similar to Osborne and Wakeman's. The wet brewers' yeast was treated with 95 per cent alcohol for 4 hours at $60^{\circ} \mathrm{C}$., filtered, and the filtrate concentrated under reduced pressure. The addition of an equal volume of 95 per cent alcohol precipitated foreign material which was removed by centrifuging. To the resulting clear supernatant liquid, sufficient 98.5 per cent alcohol was added to bring the final concentration to 80 per cent, whereupon most of the vitamin was precipitated. This precipitate was washed with absolute alcohol and extracted with acetone, and served as a starting material for further concentration.

Fifty grams of this fraction in a 4 liter volume, with the acidity adjusted to $\mathrm{pH} 3$ by hydriodic acid, was treated 6 times successively with silica gel. The filtrate after being neutralized with lithium hydroxide, concentrated, and centrifuged to remove foreign material, was brought to an 80 per cent alcohol concentration, upon which treatment a vitamin $G\left(B_{2}\right)$-containing material precipitated out, apparently essentially free from vitamin $B_{1}$, and 30 milligrams of which supported growth when vitamin B was supplied. 
The silica gel-vitamin complex was washed at $\mathrm{pH} \mathrm{3}$, suspended in 6 liters of water, and the vitamins were extracted at $\mathrm{pH}$ 9.8. This vitamin-rich solution was neutralized and concentrated, inactive material was precipitated by adding an equal volume of 95 per cent alcohol, and the supernatant fluid was poured into acetone. The resulting precipitate contained a high concentration of vitamin $B\left(B_{1}\right)$ and some vitamin $G\left(B_{2}\right)$. This preparation, of which 2.2 milligram daily, or 0.07 milligram when supplemented with the heat-stable factor, supported normal growth (18 to 24 days) was apparently about 30 times as rich in vitamin $B\left(B_{1}\right)$ as in vitamin $G\left(B_{2}\right)$. It was soluble in water and partly soluble in alcohol. It gave negative biuret and Millon tests, and reduced Fehling's solution only after hydrolysis. Upon heating with strong alkali, hydrogen sulfide was evolved, as demonstrated by means of lead acetate, and a strong odor of indole or skatole was developed (Cf. Guha and Drummond, 1929). It showed the following composition: $\mathrm{C}, 31.54 ; \mathrm{H}, 4.99 ; \mathrm{N}, 8.92 ; \mathrm{NH}_{2}, 1.9 ; \mathrm{P}, 8.92$ per cent.

Levene also reported at this time that by deaminizing the starting material with nitrous acid before adsorption on silica gel it was possible to obtain a concentrate of $B_{1}$ containing no detectable quantities of the heat-stable material $\left(\mathrm{B}_{2}\right)$.

Williams and Eddy (1927-28), in their investigation already referred to in the previous section, compared the activity of various yeast concentrates on pigeons and rats by conducting 60-day feeding experiments on young rats with the materials being tested as the source of vitamin $\mathrm{B}$ and determining the minimum protective dose against polyneuritis in adult pigeons on a polished rice diet in a 30- to 40-day test, and concluded that per unit weight young rats used about 10 times as much vitamin $B_{1}$ as adult pigeons.

As has also been noted in the previous section, Guha and Drummond (1929) followed the various steps in the concentrations of vitamin $\mathrm{B}_{1}$ by tests on both rats and pigeons.

The rat tests were carried out by studying the influence of the supplement on the growth of rats whose development had been inhibited by a diet deficient in vitamin $B_{1}$. Young rats averaging 50 grams in weight, kept in separate cages with screened bottoms, were fed a basal diet consisting of 75 per cent rice starch, 21 per cent commercial casein and 4 per cent salt mixture (McCollum), with a daily supplement of 1 drop of cod-liver oil, and 1 cubic centimeter of a 50 per cent solution of marmite (a commercial yeast preparation) autoclaved for 3 hours at 14 to 15 pounds pressure in an alkaline medium. Compari- 
sons of vitamin activity were made by finding the minimum amount of supplementary food which would permit a weekly gain of 10 to 12 grams for 2 to 4 weeks. Longer experiments were avoided as the authors believed that in too long an experiment the result might be vitiated by some of the other factors necessary for growth. In a number of fractions the curative property was also investigated by subcutaneous injection into rats exhibiting typical beriberi convulsions as a result of prolonged deprivation of vitamin $B\left(B_{1}\right)$.

As starting material for their fractionations, wheat embryo was extracted twice with alcohol (50 per cent by volume) acidified with hydrochloric acid, each extraction being conducted for 3 to 4 hours between $60^{\circ}$ to $70^{\circ} \mathrm{C}$. The filtrate was concentrated in vacuo to a yellow viscous mass which retained its potency (active in doses corresponding to 0.5 gram embryo daily) for at least 10 months when stored in the dark at $0^{\circ} \mathrm{C}$. in well-closed vessels.

This concentrate was subjected to two fractionation processes. In the first, from a solution of the concentrate inert materials were removed by precipitation with lead acetate; to the filtered supernatant liquid was added enough hydrochloric acid to make it just acid to Congo red, and the resulting filtrate was freed from lead by hydrogen sulfide. Upon subsequent removal of the hydrogen sulfide this solution was active in rat experiments in doses corresponding to 0.5 gram to 0.75 gram embryo daily, and contained 2.3 per cent nitrogen in the organic matter.

The active material was found to be preferentially adsorbed under their conditions on norite at $\mathrm{pH} 4$ and 5. (Vitamin $\mathrm{B}$ from yeast extracts was found to be adsorbed by norite at $\mathrm{pH} \mathrm{7,} \mathrm{as} \mathrm{stated} \mathrm{by} \mathrm{Kin-}$ nersley and Peters, 1928.) After two successive adsorptions at each of these hydrogen-ion activities the active material was extracted from the charcoal-vitamin complex by refluxing twice successively with 50 per cent alcohol containing 1 per cent hydrochloric acid, each extraction lasting 30 minutes at $60^{\circ} \mathrm{C}$. The extracts corresponding to $\mathrm{pH} 4$ and 5 were combined, and the acidity of the mixture brought to $\mathrm{pH} 5$ by adding $2 \mathrm{M}$ sodium hydroxide, which process darkened the solution and precipitated some inert material. The active material was then precipitated by an excess of phosphotungstic acid in 5 per cent sulfuric acid. After freeing the potent material by treatment with concentrated hydrochloric acid and with amyl alcohol and ether, most of the active material could be adsorbed on freshly precipitated silver oxide, from which it could again be released by extraction with acidified alcohol. After removal of the alcohol in vacuo the resulting red solution 
proved active in doses equivalent to 2 grams wheat embryo, containing 1.3 milligrams organic solids of which 17.8 per cent was nitrogen. Subsequent fractionation with 50 per cent alcohol, and acidified absolute alcohol yielded a product active in doses containing 0.6 milligram organic matter, and corresponding to 3.7 grams embryo.

The filtrates from the alcoholic fractionation were evaporated to dryness in vacuo at $40^{\circ}$ to $50^{\circ} \mathrm{C}$., taken up in water, and adjusted to $\mathrm{pH}$ 6.5, with sodium hydroxide, whereupon an inactive precipitate could be removed. Treatment of the filtrate with an absolute alcohol solution of picrolonic acid produced a semi-crystalline yellow precipitate, which when freed from the acid by hydrochloric acid and ether, was inactive in doses corresponding to 12 grams embryo, while the supernatant fluid was active in doses corresponding to 4.5 to 6 grams embryo and carrying 0.4 to 0.5 milligram organic matter. Nearly 11 per cent of the activity of the original extract of wheat germ was present in this solution.

Attempts to concentrate further the active material of this preparation were unsuccessful. These included: (a) a second precipitation with phosphotungstic acid followed by fractional crystallization from hot water and from mixtures of acetone and water; (b) adsorption on silica gel at $\mathrm{pH} 3,4$ or 5 followed by subsequent extraction at $\mathrm{pH} 9.5$ (Levene and van der Hoeven, 1925, 1925a ; Levene, 1928) ; and (c) fractionation with platinic chloride.

The second method followed by Guha and Drummond in fractionating their concentrate made from wheat embryo approximated that of Jansen and Donath (previously described). Upon decomposing the aqueous suspension of the platinum precipitate with hydrogen sulfide, a colorless solution was obtained which was evaporated to dryness over soda lime. The residue was found to be active in doses equivalent to 4.6 grams embryo and containing 0.015 milligram total solids. "About 11 per cent of the activity of the original extract was thus retained in this material. The product was colourless and for the greater part microcrystalline but obviously impure." The average day-dose in curative tests on pigeons was 0.0051 milligram.

Upon extracting the active preparation from the platinum compound with absolute alcohol and treating the filtrate with an alcoholic solution of gold chloride a slight turbidity resulted which cleared up on warming. When the preparation was slowly evaporated in a desiccator over calcium chloride a small amount of microcrystalline deposit was formed, which did not appear homogeneous and did not show the 
characteristic appearance of the gold double salt obtained by Jansen and Donath. When the solution had been concentrated to about 1.5 cubic centimeters the precipitate was washed with absolute alcohol, and both the aqueous suspension of the precipitate and the filtrate were decomposed with hydrogen sulfide. The resulting solutions were evaporated to dryness in vacuo over soda lime. In doses corresponding to 36 grams of embryo, the insoluble gold salt fraction produced bur subnormal growth, while the soluble gold fraction was inactive. Administering these two fractions in combination, however, revealed a supplementary action both in rat-growth and pigeon-curative tests. This would suggest that the action of vitamin $\mathrm{B}_{1}$ is probably to be ascribed to more than one factor. The gold precipitate would appear to contain most of one factor and a little of the other, whereas the soluble fraction would appear to contain the second factor almost exclusively.

The active picrolonic filtrate gave a very strong Pauly reaction comparable with that of histidine. Heated with 40 per cent sodium hydroxide this fraction emitted a strong smell suggestive of alkylamines, but no smell of skatole or indole (Cf. Levene, 1928). No sulfur reaction with lead acetate was obtained. Millon's, xanthoproteic, and purine reactions were negative, which would appear to exclude the possibility of the vitamin being a hydroxyphenyl or purine derivative. The platinum fraction and the preparation from the gold precipitate also produced a very strong Pauly reaction, whereas the gold filtrate gave a very faint one. The gold precipitate produced a very faint coloration with Folin and Denis' reagent. Using the preparation obtained by Jansen and Donath, Eijkman (1927) had observed a blue coloration with this reagent, which, however, was stated to be weaker than that produced by uric acid of the same concentration. These authors indicate the importance of this observation in view of statements in the literature claiming Folin and Denis' reagent a valuable guide in the detection of vitamin $\mathrm{B}$.

In a discussion of their observations, Guha and Drummond say: "As regards the properties of vitamin $B_{1}$, its behavior appears to be determined to an extraordinary extent by the presence of other substances and by the previous treatment of a given preparation, a fact pointed out by Kinnersley and Peters (1928). These investigators observed that the solubility of their curative preparations from yeast in alcohol varied with their activity and with the hydrogen-ion concentration of the solutions treated with alcohol. Similar variability has been observed with fractionation by silver nitrate. In our first method of 
fractionation it will be observed that the active material was not precipitated by platinic chloride, whilst in the second it was. In the first method the precipitation of the active substance appeared to be dependent on the presence of an excess of phosphotungstic acid, whereas in the second the amount of this reagent required was much less. Moreover, charcoal adsorbed most of the active material at $\mathrm{pH} 4$ and 5 in our first method, whereas the curative substance in yeast extracts has been found by Kinnersley and Peters to be adsorbed selectively by the same reagent in the zone of neutrality, an observation which we have been able to confirm. Jansen and Donath (1926) described a procedure in which the activity was found to be associated with the precipitate obtained by treatment of their concentrate from rice-polishings with picrolonic acid, whereas in the first procedure which we adopted for the concentration of wheat-embryo extract we have been able to recover most of the activity in the picrolonic acid filtrate. Similar apparently conflicting statements regarding the properties of vitamin $B_{1}$ have appeared repeatedly in the literature. It has been suggested that the factors which various workers were attempting to isolate from different sources were probably not identical. In this paper, however, it has been shown that even when the same biological techniques are adopted, and the same substance chosen as the raw material, the properties of the active factor are profoundly modified according to the treatment undergone. It does not, therefore, appear strange that the substance should exhibit even greater differences in behaviour in the hands of investigators, starting from different raw materials and adopting different biological methods."

Williams and Eddy (1929) stated that the Jansen-Donath method had been applied to the fractionation of rice polishings with greater success than had been the case with yeast, but without confirmation of all of the claims of Jansen and Donath. They were also inclined to attribute some of the discrepancies in estimates of recoveries to differences in the method of physiological testing. Continued protection of pigeons against polyneuritis, but at the same time loss in weight as compared with growth in rats, was thought to confirm their previously expressed theory of a third factor in rice polishings necessary for the growth of pigeons but not of rats. In a more detailed report of these studies Williams, Waterman, and Gurin (1930) state that in their opinion the most important result of their work was the evidence that the pure antineuritic vitamin, in small doses at least, has scarcely any appreciable effect upon the weight curves of pigeons on a polished rice diet. 


\section{Physiological Properties and Relation to Nutrition and Health}

The nervous symptoms induced by a shortage of the (antineuritic) vitamin $B$ are so striking and respond so readily to treatment that they have tended to obscure the other effects resulting from a partial or total deficiency of this vitamin in the diet. Attention was called to this by McCarrison (1919). Working first with pigeons, he was able to demonstrate a certain parallelism between the effects of simple starvation and lack of vitamins. In cases uncomplicated by bacterial invasion, both simple starvation and a diet of polished and autoclaved rice brought about loss in weight, a progressive fall in body temperature, and slowing of respiration. The weights of the various organs of the body immediately after death showed atrophy of the thymus, testicle, spleen, ovary, pancreas, heart, liver, kidneys, stomach and thyroid in decreasing order; and marked hypertrophy of the adrenals. The pituitary gland and the brain showed no change in the starved pigeons, while on the vitamin-deficient diet a slight tendency to enlargement of the pituitary was noted in the male birds and slight atrophy of the brain in both male and female. The central nervous system as a whole underwent little atrophy, the paralytic symptoms being mainly due to impaired functional activity of the nerve cells rather than to their degeneration.

Recently (1928) Marrian confirmed the earlier observations on the hypertrophy of the adrenals in starving pigeons receiving vitamin $\mathrm{B}$ and in pigeons forcibly fed on an artificial vitamin B-free diet, and in addition obtained evidence indicating that while a deficiency of both vitamin $B$ and $G$ is involved in the hypertrophy occurring in the vitamin $\mathrm{B}$-deficient pigeons, the deficiency of $\mathrm{B}$ is of greater importance than of $\mathrm{G}$. He suggested that in starvation the changes occur mainly in the medulla and in vitamin B deficiency in the cortex.

In a more detailed study of the histological changes in the intestines of pigeons on vitamin-deficient diets, McCarrison (1919c) reported the chief microscopical changes observed at autopsy to be atrophy and congestion, the latter more largely confined to the upper part of the alimentary tract, and tending to open the way to systemic infection from the diseased intestine. Interpreted in terms of bowel function the derangements to which these pathological changes may ultimately lead were classified as impairment of the neuromuscular control of the bowel; impaired transport of the intestinal contents along the alimentary canal; impairment of assimilative power ; impairment of secretory function; impaired protective resources leading to infection of the mucous membrane of the bowel by pathogenic saprophites or 
ingested bacteria and to systemic infection therefrom. That these gastro-intestinal lesions are much more deep seated than those of the nervous system is indicated from the observation that the nervous symptoms present in avian polyneuritis may be rapidly ameliorated and recovered from, and yet the bird dies in consequence of gastro-intestinal lesions. In the nervous system the symptoms were considered to be mainly the result of functional disorder, while in the intestines a greater destruction of tissue cells takes place.

Findlay (1921a) in a very carefully controlled comparative study of starvation and beriberi in pigeons and fowls confirmed the work of $\mathrm{McCarrison}$ to the extent that the histological changes in beriberi were found to be similar in gross manifestations to those of starvation, including hypertrophy of the adrenals, little or no change in the pituitary gland, and atrophy of the other organs. Microscopical examination of the tissues was considered to reveal evidence of nuclear degeneration in line with McCarrison's theory of the relationship of vitamin B to nuclear metabolism.

These investigations indicate the profound effect which a qualitatively insufficient diet lacking in vitamin B may have upon the bodily functions. It should be emphasized, however, that in experiments in which polished rice serves as the sole nutrient aside from the vitamin containing substances, other nutritive deficiencies are involved which may complicate the issue in so far as the physiological properties of vitamin B are concerned. Some investigators, particularly Simonnet (1920, 1921) and Randoin and Simonnet (1924), have used more complete basal rations for pigeon experiments, but investigations conducted with mammals, particularly those at Yale with dogs, on controlled rations adequate except for vitamin $B$ seem of greater import. It is of interest that in many respects the more quantitative work upon other species confirms the qualitative studies of McCarrison on pigeons.

Relation to Appetite.-A conspicuous effect of lack of vitamin B is the abrupt failure of appetite after a preliminary period, varying in length with the age and species of animal. This loss of appetite had been noted in the classical studies of Hopkins and others, leading up to the discovery of the vitanins, but probably the earliest allusion to it as a specific effect of lack of vitamin B was in the 1917 paper of Osborne and Mendel in which, as noted previously, it was pointed out that the stimulating effect of vitamin $B$ on the appetite is not simply a matter of improved palatability of the food mixture through its presence. Improvement in appetite naturally leads to increase in food consumption and in the young to growth, and the attribute "growth- 
promoting" has always been associated with vitamin B. In this same paper Osborne and Mendel showed for the first time that there is a quantitative relationship between the appetite-stimulating vitamin $\mathrm{B}$ and the amount of growth, an observation confirmed the following year by Drummond (1918). The outward effect on rats of deprivation of vitamin B was described by Drummond as follows: "For some time after the water-soluble factor has been withheld from the diet the general appearance of the animals is apparently normal. They are lively, sleek-coated, and bright-eyed. As the period of maintenance at constant body weight draws to a close, the rats gradually lose this appearance. The fur loses its gloss and becomes ruffled, whilst the rats assume a hunched attitude, and become lethargic. During the final decline the rats appear pale and emaciated, feeling cold and thin to the touch, and they usually remain huddled in corners away from the light." Occasionally symptoms resembling those of avian polyneuritis were noted by Drummond, but usually the rats died before the appearance of such symptoms.

Karr (1920), working in Mendel's laboratory, demonstrated with dogs failure of appetite and decreased food consumption on diets deficient in vitamin $B$, followed by a revival of appetite and increased food consumption when vitamin B preparation was fed. As the vitamin preparation was given separately from the food the effect could not be attributed to increased palatability. The quantity of the vitamin preparation necessary to sustain the appetite, while varying slightly with different animals, appeared to be fairly constant for the same animal. It was noted in this paper that in certain of the animals which continued to eat their food for a longer time without the addition of vitamin $\mathrm{B}$, characteristic polyneuritic symptoms appeared which were promptly alleviated by administration of the vitamin extract.

This suggestion that stimulation of the appetite is a function of the antineuritic factor B is confirmed by Sandels' (1928) study of the food consumption of rats receiving respectively, (a) diets free from vitamin B (Sherman-Spohn basal diet supplemented by autoclaved yeast) and (b) diets practically free from vitamin G (Sherman-Spohn basal diet supplemented by a strong-alcohol extract of ground whole wheat). Chick and Roscoe (1928) also state that on a basal diet adequate except for vitamin $G$, young rats can be maintained for three months or more at practically constant weight, with fair appetites. Metabolism studies reported by Karr (1920a) suggested that the nitrogen utilization in the digestive tract was unaffected by the absence of vitamin $\mathrm{B}$, although it was pointed out that "metabolism experiments 

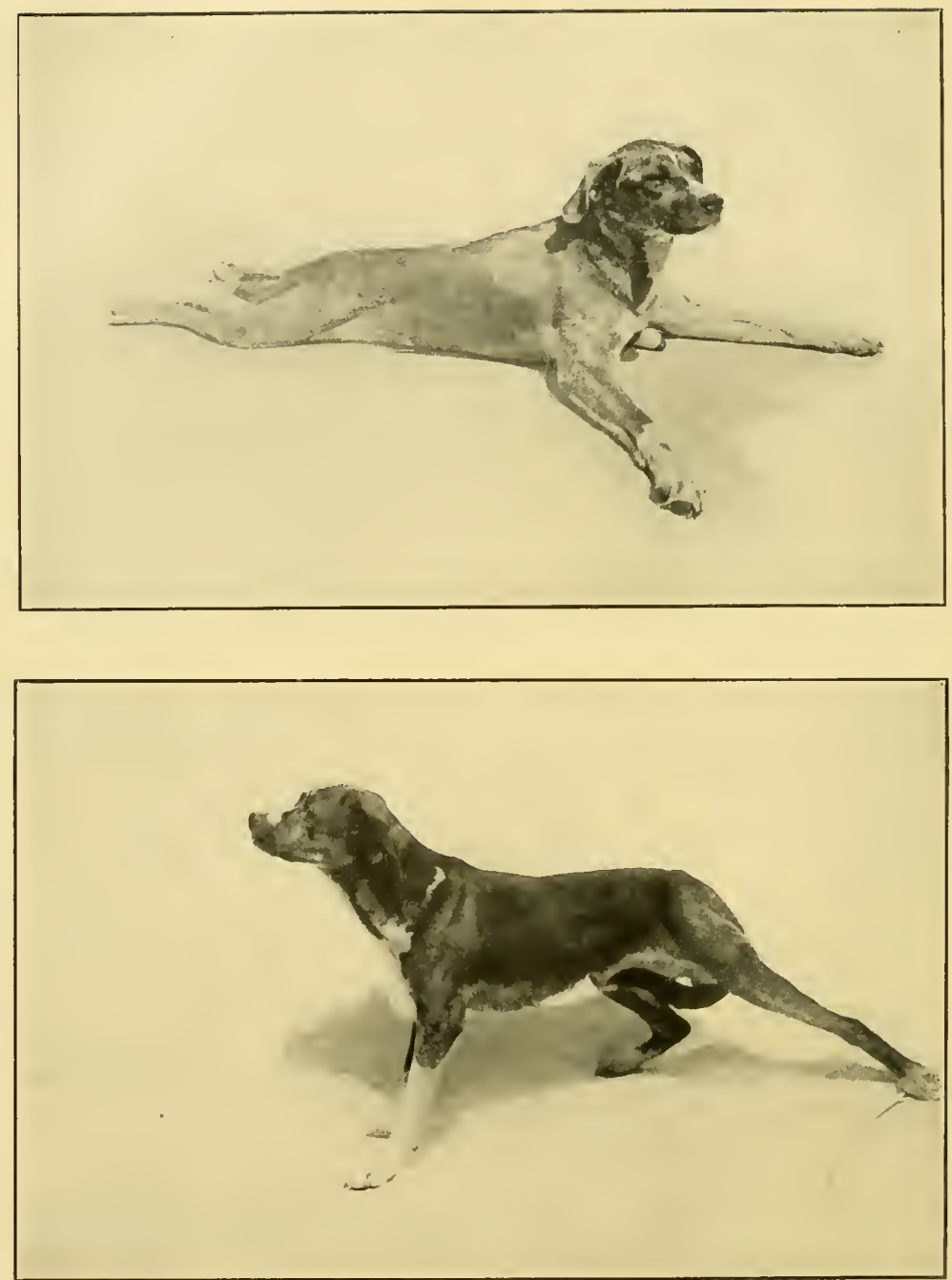

FIG. 3.-(Upper photograph.) Dog showing polyneuritis with marked paralysis of hind legs as result of diet lacking vitamin B. (Courtesy of G. R. Cowgill and Professor Dendel and of the American Journal of Physiology.)

FIG. 4.- (Lower photograph.) Dog shown in Fig. 3 cured by vitamin B given in form of tomato juice. (Courtesy of G. R. Cowgill and Professor Mendel and of the Anerican Journal of Physiology.) 
on vitamin-free diets are complicated by many factors, especially the intimate relation of water-soluble $B$ vitamin to food intake, and also the possible supplementing action of the protein in the crude vitamin substance added."

A further study along the same lines was reported by Cowgill (1921b). Dried brewery yeast, neutralized tomato juice, and alcoholic extracts of wheat embryo, of rice polishings, and of navy beans were employed as sources of vitamin B. All these materials show pronounced curative effects when fed to polyneuritic pigeons and all were found capable of restoring the desire for food in a dog which had lost appetite through being confined to a diet lacking vitamin B. Dogs on diets adequate in other respects but lacking vitamin $B$ usually show loss of appetite in from five to fifteen days and thereafter eat very irregularly if at all. If the dog continued to eat some food it eventually showed symptoms of polyneuritis differing somewhat in appearance from those exhibited by pigeons and fowls. Paralysis of the hind legs, resulting first in a peculiar dragging of the feet and then in complete loss of control of the hind limbs, was the most characteristic feature in the dog. Often the first signs of paralysis were accompanied by vomiting and a noticeably foul breath. Convulsions usually began not long after the appearance of the paralysis. If, however, vitamin $B$ in any of the forms above mentioned was given after the paralysis had appeared but before it was too late, the polyneuritis was cured by the vitamin in these mammalian experiments, as in the earlier experiments with pigeons and fowls. Figures 3 and 4 from photographs published by Cowgill (1921b) show the same dog before and after treatment with tomato juice.

Cowgill agreed with Karr that the changes in body weight of the animals follow the food intake, so that the loss of weight is not a symptom characteristic of vitamin deficiency as against loss of appetite from other causes, or simple starvation.

Relation to Secretory Function.-The stimulating effect of vitamin $\mathrm{B}$ on the appetite has led to many attempts to establish a relationship between this vitamin and the secretory function of various glands. Voegtlin and Myers (1919a) suggested the possible identity of vitamin $B$ with secretin, but the validity of the evidence upon which they based their conclusions was questioned by Anrep and Drummond (1921), Cowgill (1921), and Downs and Eddy (1921), who were unable to substantiate their findings. Cowgill and Mendel (1921) reviewed critically much of the literature of the subject in introducing their own investigation which consisted in testing the effects of preparations 
known to contain vitamin $\mathrm{B}$ on the secretory activity of the pancreas, liver, and salivary glands of dogs. Not only were these preparations without effect, but the intestinal mucosae from polyneuritic dogs were shown to contain secretin. Moreover, Cowgill (1923a), by demonstrating that convulsions in dogs on vitamin $\mathrm{B}$-free rations could be relieved by intravenous and intraperitoneal injections of a vitamin $\mathrm{B}$ concentrate free from protein, showed that substances containing vitamin $\mathrm{B}$ do not require action upon them by the enzymes contained in the various digestive juices before the vitamin can be effective.

Relation to Motor Functions.-A possible clue to the apparent discrepancy in findings concerning the relationship between vitamin B and digestive secretions may be found in the gastric and intestinal atony noted in vitamin $\mathrm{B}$ deficiency by various workers. McCarrison mentioned impairment of the neuromuscular control of the bowels, impaired transport of the intestinal contents along the alimentary canal, and impairment of excretory functions as among the important effects of lack of vitamin $\mathrm{B}$ in pigeons and monkeys.

Gross (1923, 1924), repeating and extending some of McCarrison's observations, but with rats on diets designed to be deficient in only one vitamin at a time, confirmed his suggestion of impairment of the motor function of the intestinal tract in vitamin B deficiency, and expressed the opinion that vitamin $\mathrm{B}$ deficiency probably plays an important part in the intestinal stasis often encountered in everyday life.

A demonstration of gastric atony in extreme vitamin B deficiency was reported by Cowgill, Deuel, Plummer, and Messer (1926). Four dogs having permanent gastric fistulas were fed basal diets free from, or extremely low in, vitamin B but adequate in other respects. From time to time the motility of the empty stomach was recorded by means of an inflated rubber balloon placed in the stomach and connected with a chloroform manometer and recording device. When the animals had lost their desire to eat, beef juice was administered but with no effect upon the appetite. A vitamin B preparation was then administered with prompt restoration of the appetite. The tracings of the hunger contractions showed no remarkable changes in character in the early stages of the vitamin B deficiency but almost complete atony in later periods. The administration of vitamin $\mathrm{B}$ was followed by rapid improvement in the stomach musculature as shown by the regularity of muscle contraction.

In discussing the possible relationship between failure of appetite and gastric atony, Cowgill and his coworkers expressed the opinion that "it is difficult, when dealing with such a parallelism as has been 
described, to decide whether or not vitamin B maintains the desire to eat simply by aiding in the preservation of the normal gastric tone. The marked systemic manifestations characteristic of advanced cases of vitamin B deficiency suggest that the loss of the desire to eat in such cases is due as much to a generalized systemic disturbance as it is to an abnormal condition localized in the alimentary canal."

An editorial comment in the Journal of the American Medical Association (1926) on this paper was: "The demonstration of gastric atony as a part of the syndrome characteristic of an advanced stage of deficiency disease in which lack of vitamin B is concerned is in harmony with clinical observations on patients with beriberi. The list of disorders attended with gastric atony is a long one, including high fever, parathyroid tetany, acute gastritis, pneumonia, and general peritonitis. It is also now demonstrated to characterize prolonged subsistence on a diet poor in vitamin $B$. The suggestion has been made, therefore, that the dietary factor should never be overlooked where gastric atony is known to occur."

Relations to Metabolism.-McCollum, Simmonds and Pitz (1916c) concluded from the results of their rat-feeding experiments, as had Andrews (1912) in his study of human beriberi, that vitamin $B$ passes into the milk only about in proportion as it is supplied by the food of the mother.

Drummond (1918) found the food consumption of rats fed upon a diet deficient in vitamin $B$ to be low. The addition to the diet of commercial meat extract, which is deficient in vitamin $B$, or of extracts containing vitamin $\mathrm{B}$ brought about increased food consumption, but growth took place only when the extract contained vitamin $B$ and the extent of growth was, within certain limits, proportional to the amount of vitamin $B$ added. The length of time that an animal is able to maintain itself on a diet deficient in vitamin B without suffering serious loss in body weight appeared to be directly proportional to the age at which the restriction is imposed. A restriction in the supply of vitamin $B$ in the diet of a pregnant rat was shown to be followed by serious consequences both to herself and her offspring. "This was particularly marked when the restriction was imposed before parturition. When imposed at or after delivery, the young usually lived and in some cases even showed a subnormal rate of growth for a short period but the fatal decline was not long in appearing. By returning the mother to a normal diet the young could be saved, although the improvement in them was not immediately appreciable." Actively growing tissues (embryos, tumors, etc.) did not contain appreciable amounts of vitamin 
$\mathrm{B}$, and desiccated pituitary gland (whole gland and anterior lobe), thyroid, thymus, testicles and ovarian tissues were also found to be deficient in this vitamin.

The only apparent deviation from the normal noted in the nitrogen metabolism of rats on diets deficient in vitamin $B$ was the appearance of creatinuria accompanied by a slow wasting of the skeletal muscles. Drummond was unable to determine the cause of the fatal decline which invariably follows such deficiency. Symptoms of nerve disorder were found in only three of the many cases studied.

Osborne and Mendel had suggested, in their earlier studies of vitamin $B$, a quantitative relationship between vitamin $B$ requirements and growth. Definite proof that the requirement is somewhat proportional to the amount of active tissue was afforded by the results obtained in a long continued study (Osborne and Mendel, 1922b) of the growth of rats of different ages on varying amounts of yeast as the sole source of vitamin B. Groups of rats weighing 40, 70, 150 and 240 grams, not less than five rats in each group, were fed the basal diet with the addition of $25,50,100$ or 200 milligrams daily of yeast, each amount of yeast being fed to a group of each initial weight.

In the four groups receiving 200 milligrams of dried yeast daily, the animals, with few exceptions, reached the average adult size within the average time. With 100 milligrams, the results were less satisfactory, particularly after the animals reached a weight of 200 grams. With the smaller doses, 50 and 25 milligrams, the rate of growth was more or less retarded from the very beginning of the experiment, and in the case of the larger animals 25 milligrams failed to secure even maintenance of body weight.

On recalculating the various dosages to the amount in milligrams per 100 grams of body weight, the daily requirement appeared to have a quantitative relationship to the mass of active tissue and to approximate from 50 to 60 milligrams of the dried yeast daily. The observation that a number of the larger animals appeared to require a smaller amount of the vitamin per unit of body weight was explained on the ground that these animals were comparatively rich in fat and the actual amount of active tissue was smaller than the weights would indicate. Another illustration of the dependence of the rate of growth upon the vitamin dosage was afforded by the growth curves of six rats from the same litter, each of which was put, when it reached a weight of 100 grams, on the standard diet, but with differing amounts of yeast. With decreasing amounts of yeast the growth was about the same for 
a short time, after which there was a decrease in rate, more marked the smaller the amount of yeast fed.

The correlation between the size of the animal and its requirement of vitamin $B$ was further shown in this and in a later paper (Osborne and Mendel, 1925) by the growth curves of rats fed varying dosages of the vitamin $\mathrm{B}$ concentrate of Osborne and Wakeman. Similar relationships between the vitamin B requirement of an animal and its size and metabolic rate were demonstrated by Pilcher and Sollmann (1925) for pigeons, by Beard (1926) for mice, and by Cowgill and coworkers for dogs, mice, rats and pigeons.

The effect of vitamin $\mathrm{B}$ upon oxygen consumption and the metabolism of various food constituents has been studied with variable results. The lowered temperature and difficulties in respiration noted in the final stages of vitamin B deficiency led some investigators to the conclusion that vitamin $\mathrm{B}$ is concerned in maintaining the activity of the oxidizing mechanism of the body, and many unsuccessful attempts have been made to discover the cause of the alleged lowered consumption of oxygen. Papers from Drummond's laboratory on the physiological rôle of vitamin $B$ have done much to clear up the confusion in the literature and to relate the various phenomena observed in vitamin $B$ deficiency to inanition following failure in appetite. Since these papers contain a critical discussion of the conflicting literature, together with a repetition under carefully controlled conditions of much of the earlier work, they are reviewed somewhat fully here.

Drummond and Marrian (1926) first attempted to verify the assumption that deprivation of vitamin $B$ leads to a reduction of the oxygen utilization of the animal, by testing the washed and unwashed tissues of normal pigeons and rats, with those of others killed in the final stages of vitamin B deficiency, for their ability to reduce methylene blue according to the method of Thunberg (1918). Contrary to the results reported by W. R. Hess (1921, 1922, 1923), Hess and Messerle (1922), and Rohr (1923) in similar studies, but with the use of $m$-dinitrobenzene as indicator, the normal and "beriberi" muscles showed no difference in their rate of decoloration of the dyestuff, and hence in their oxidizing mechanism; nor was the reduction in the beriberi muscle hastened by the addition of small quantities of yeast as claimed by Abderhalden and his colleagues. The results obtained by this indirect method were confirmed by the direct method of determining the oxygen uptake of isolated tissue preparations in a respiration apparatus. The uniformity of results with normal and beriberi tissues was in complete disagreement with the findings of Abderhalden and Schmidt (1920) and Shinoda (1924) but in agreement with those of Roche (1925):

Concluding that the tissues themselves show no appreciable impairment of oxidative activity in vitamin B deficiency, Drummond and Marrian next made a prolonged study of the gaseous metabolism of rats in various states of nutrition, as determined in a closed circuit system with constant temperature control and provided with a recording spirometer. The data obtained on rats during progressive vitamin $\mathrm{B}$ deprivation indicated that the oxygen consumption remains at a normal level until the final phase of the disturbance which, as has been noted previously, is characterized by a marked loss in weight, rapid fall in body tem- 
perature, and difficulties in respiration. In this phase only were low values obtained for oxygen consumption. In most cases after the body temperature was raised to normal by placing the animal in a warm place for about two hours, the oxygen consumption rose to a nearly normal level and there was a marked although temporary improvement in the condition of the animal-such as noted by Peters (1924), and Kinnersley and Peters (1925) in the case of pigeons ("heat cure"). This is thought to demonstrate conclusively that the oxidative mechanism is not damaged in vitamin B deficiency.

It was next shown that the figures for rectal temperature, body weight, and oxygen consumption of rats fed a complete artificial diet plus 5 per cent yeast extract, in amounts corresponding to the estimate of what would have been voluntarily consumed of the vitamin B deficient ration, were strikingly similar to those obtained with rats of the same initial weight receiving a vitamin B-deficient ration ad libitum, and that in the smaller rats of the first group, there was eventually the same sharp decline in weight, rapid fall of temperature and oxygen consumption, followed by death, invariably resulting on the vitamin B deficient diet. Starving rats and rats receiving only yeast were likewise shown to maintain their body temperature and oxygen consumption until shortly before death. "To our minds it seemed clear from these results that the lowered metabolism and body temperature observable during the last phase of the condition resulting from deficiency of vitamin $B$ were merely indications that the animal was dying and that the immediate cause was general starvation."

This conclusion was confirmed by the striking similarity found by Woollard (1927) in the histological appearance of the nerve tissues of the rats deprived of all food and of vitamin B alone, although the lesions were more marked in the latter case. The only changes noted were in the intermuscular medullated motor and sensory nerves and their endings, these changes subsiding rapidly at a little distance from the muscles. The sympathetic and central nervous systems showed no abnormal anatomical changes.

That many of the phenomena of polyneuritis in pigeons may also be traced to starvation, as suggested earlier by McCarrison from qualitative studies, was demonstrated by Kon and Drummond (1927) in a later paper of the same series. In order to separate the effects of inanition from those which might be due more directly to the vitamin deficiency itself, each of a group of eight pigeons was matched as closely as possible in weight and age with another pigeon to serve as control. The first group received a modified artificial vitamin B-free diet of the type used by Randoin and Simmonet. Each of the pigeons of the second group of matched controls was forcibly fed the same quantity of food that the corresponding pigeon had received the previous day plus 1 gram of yeast extract (marmite).

The records of body weight, food consumption, and, in the later stages of the experiment, body temperature were strikingly similar for corresponding pigeons in the two groups. An initial loss in body weight, attributed to lack of appetite during adjustment to confinement in separate cages, followed by slight gain and then a gradual loss, was characteristic of the pigeons in both groups. At about the beginning of the fourth week there was sometimes a partial but transient recovery of appetite. A progressive fall in body temperature occurred in both groups up to the onset of acute symptoms, when there was a rapid drop in temperature in the five pigeons in the first group which developed typical polyneuritic symptoms. Four of the five pigeons developing acute symptoms recovered from the first convulsive seizures without being treated, and with recovery there was a rise in temperature from the low level during the attack. "It seems fair to assume that the thermal changes occurring in pigeons during vitamin $\mathrm{B}$ deficiency are two-fold: firstly, a progressive decrease which is most probably due to insufficient food intake, and secondly, a profound and rapid fall manifesting itself in the course of a few hours and associated with the onset and gravity of acute nervous symptoms. There seems a possibility that this phenomenon is more directly related to vitamin B."

Histological examination by Woollard of pigeons from the two groups showed similar changes in both groups attributable to the wasting resulting from starvation. No relation could be traced between the occurrence of acute nervous symptoms and degenerative changes in the nerves. "It can not be sufficiently emphasized 
that no nerve regeneration is possible in the course of a few hours, and that the nervous symptoms, vanishing so rapidly under the action of vitamin B or even disappearing spontaneously, must be due to a quite different cause. There is no logical impediment, however, in assuming that the paralytic form of pigeon's beriberi which is not curable or very hardly curable by vitamin administration may be associated with nerve degeneration."

As noted by Kon and Drummond, a connection between vitamin B and carbohydrate metabolism has been suggested by various investigators including Funk and von Schönborn (1914), Funk (1919), Randoin and Lelesz (1925) largely because of hyperglycemia observed in pigeons on vitamin B-deficient diets. Collazo (1922), who also observed this phenomenon, found the same hyperglycemia to be present in starving pigeons. Collazo's observations were confirmed in the present study where no appreciable difference could be noted in the blood sugar level of the two groups of pigeons. The average in both groups was somewhat higher than normal, a difference attributed to inanition. Drummond and Marrian in their study with rats had confirmed the observations of Eggleton and Gross (1925) that in vitamin B deficiency in rats the blood sugar level is above normal until the final stage of the disorder when a definite hypoglycemia takes place. A few experiments with growing rats indicated the same hyperglycemia followed by hypoglycemia.

A repetition of the work of Farmer and Redenbaugh (1925a) failed to confirm their reported findings of a complete loss of amylolytic and decrease of proteolytic enzymes in pigeons on a B-deficient diet, but showed clearly that the digestion of starch is normal.

In reviewing their experimental observations, Kon and Drummond state that: "The actual food intake of a pigeon from which vitamin B is withheld, be it forcibly fed or partaking freely of the diet, is greatly diminished, but the ingested quantum is metabolized just as completely as in a pigeon receiving liberal amounts of vitamin $B$ in the form of yeast extract; and this is true even in the stage of the deficiency disease where the characteristic convulsions appear. The similarity of the curves for food intake and body weight, the similar rates of passage of a meal through the intestines, the blood-sugar levels and composition of the feces all support this view. Furthermore, it is believed that the diminished food intake and subsequent partial starvation are suffcient to explain any hyperglycemia encountered in vitamin B-deficient pigeons."

A suggested explanation of the spontaneous cures, which have been noted in this and other investigations as of frequent occurrence, is a liberation of the reserves of vitamin $\mathrm{B}$ in the tissues during periods 
when there is little or none in the diet and when there is considerable tissue wasting. "The question immediately arises whether what is perhaps the most characteristic symptom of the deficiency (the loss of the desire to partake of the deficient food), is to be looked upon merely as a morbid phenomenon or as an attempt of the organism to liberate by starvation and subsequent tissue wasting the body stores of the lacking vitamin, bound in the tissues so as to be inaccessible to the animal under normal conditions, and thus make available a substance essential for life. The occurrence of spontaneous cures on diets such as we employed suggests that there may be a liberation following a convulsive seizure which gives temporary relief and sometimes leads to a transient return of appetite. Further investigation must follow before an opinion on this question can be formed. It seems evident that almost all the pathological changes observed in beriberi pigeons are directly attributable to inanition. There are, however, certain differences between a vitamin B-deficient pigeon and one partially starving but receiving a liberal supply of the vitamin, namely, the occurrence in the former of two well-defined symptoms which in the order of their appearance are: (1) a diminished desire to partake of the deficient food, (2) symptoms known as opisthotonos, emprosthotonos, convulsive seizures, 'cart-wheel' turning. Leg weakness is also frequently present, but is not characteristic as a symptom. It yields only with difficulty to vitamin $\mathrm{B}$ treatment and is often observed in the premortal stage in control birds."

Reader and Drummond (1926) reviewed the various claims which have been made as to the quantitative relationship between vitamin $B$ and carbohydrate metabolism as previously noted, protein (Hartwell, 1924a, 1925c), protein and carbohydrate (Tscherkes, 1926), and total food or energy metabolism (Osborne and Mendel, 1922b, 1925; and Cowgill et al., 1925, 1926) and reported growth data on rats fed diets containing varying ratios of protein to vitamin $B$ (yeast). In their experiments, satisfactory growth was secured only when the ratio of protein to the yeast extract used had a value of 5 or under. This was thought to confirm the views of Hartwell and of Tscherkes that vitamin $B$ is related quantitatively to the amount of protein in the diet and not to lend support to the opinion that it is related to carbohydrate or total energy metabolism, at least as far as growth is concerned. In the course of an attempt to throw further light on the actual relationship between protein and vitamin B, Hassan and Drummond (1927) discovered that it is apparently not vitamin B which is concerned in the supplementary effect of yeast in the high protein diets 
but the more alkali-stable vitamin G. This was confirmed by Hartwell (1928), and indirectly by Kon (1929).

Sherman and Gloy (1927) found no differences in the outcome of vitamin $B$ experiments for the age period covered in the usual tests for vitamin $B$ in foods when the protein of the standard basal ration was varied from 12 to 54 per cent.

Stucky and Rose (1929) reported that the blood sugar of vitamin B deficient dogs remained normal, while the blood chloride fell sometimes to a marked extent; anhydremia developed accompanied by an increase in the hemoglobin content of the blood. Sure and Smith (1929a) reported a rise in the reducing non-sugars of the blood of the rat.

Peters (1929) suggests that vitamin B may be concerned with the removal of lactic acid. The symptom of opisthotonus in pigeons appears to be due to excess of lactic acid in the brain and is cured most quickly by injecting concentrated preparations of torulin locally. Absence of another vitamin $\mathrm{B}$ fraction apparently leads to heart block and a remarkably slow pulse, and the puzzling question of edema (wet and dry beriberi) may be due to another fraction which in conjunction with torulin regulates distribution of water in the body.

Kinnersley and Peters (1929) found that increased lactic acid in the brain is a concomitant of the symptoms in avitaminosis $B_{1}$ and disappears within a short time after the administration of torulin.

"A forcibly fed bird suffering from lack of vitamin $B_{1}$ has a permanent hyperglycaemia, so that the tissue cells are being continually bathed with an abnormal concentration of blood sugar. The animal which feeds normally doubtless does not eat so much in the later stages and is often found to have practically normal blood-sugar. It is not improbable that the lack of appetite is correlated with an adjustment of the blood-sugar."

Continuing this investigation, Kinnersley and Peters (1930, 1930a) have reported very recently that the abnormal amounts of lactic acid found in the brains of vitamin $\mathrm{B}_{1}$-deficient pigeons are unevenly distributed, the increase being especially marked in the lower parts of the brain. These findings are thought to furnish convincing proof of the theory so often advanced that vitamin $B_{1}$ is associated with intermediary metabolism of carbohydrate. Kinnersley and Peters go so far as to suggest that vitamin $B_{1}$ is concerned with the oxidative removal of lactic acid, though admitting that a substantive proof of this is at present lacking.

A new approach to the question of the relation of vitamin $B_{1}$ to 
carbohydrate metabolism was made by Lepkovsky, Wood and Evans (1930) through glucose tolerance tests conducted on carefully paired rats on diets designed to differ only in their content of vitamin $B_{1}$. In animals suffering from only slight deficiency in vitamin $B_{1}$ the glucose tolerance curves did not vary greatly from the controls, but in others showing typical signs of beriberi there was evidence of definite interference in carbohydrate metabolism.

Evans and Lepkovsky (1929) have presented evidence of the sparing action of fat on antineuritic vitamin $B$, but not on the heat-stable vitamin $G$, which function they show to be due to fat itself and not to the presence of the vitamin as an impurity.

Relation to Reproduction and Lactation.-One of the findings emphasized by McCarrison was the constant and pronounced atrophy of the testes and similar but less marked atrophy of the ovaries among animals which had been confined to diets lacking in vitamin $B$. The atrophy of the male gonads was said to be much more pronounced in pigeons than in monkeys. Funk and Douglas (1914) had previously reported atrophy of the testicles, with shrunken tubules and absence of spermatozoa in polyneuritic pigeons. Allen (1919) examined histologically the testes of rats which had been fed on Osborne and Mendel's vitamin $\mathrm{B}$-deficient diet and reported considerable but irregular degeneration, not all of the tubules being equally affected. Drummond (1918) reported that male rats deprived of vitamin B for more than 14 days cease to breed with normally-fed females. For a more detailed discussion of the effect of vitamin B deficiency on reproduction in the male rat, see Parkes and Drummond (1925). While admitting that degenerative changes may be initiated in the testes of pigeons by a wide range of unfavorable conditions, Marrian and Parkes (1928) were of the opinion that the testicular degeneration resulting from a deficiency in vitamin $B$ is not necessarily the result of inanition. Vitamin $G$, however, is considered by them to be less necessary than vitamin $B$ for the maintenance of nutrition of the testis.

Findlay (1928) pointed out that the testicles of some rats deprived of vitamin $G$ showed spermatozoa while in all of those deprived of vitamin $\mathrm{B}$ there was complete absence of spermatozoa. On the other hand, Mattill (1927) and also Evans (1928b) hold that if vitamin E is adequately provided, neither acute nor chronic deficiency of vitamin $B$ impairs the male germinal elements, although a decrease in sex interest is observed. Evans and Bishop (1922b) found that ovulation is affected more readily by lack of vitamin $B$ than by the general condition of the body. 
Many observations in the literature indicate that for successful lactation as well as reproduction much more vitamin $B$ is needed even than for rapid growth.

In the opinion of Sure (1927a, b) even a fairly abundant intake of vitamin $\mathrm{B}$ by the nursing mother does not always insure an adequate supply for the young. He found that a smaller amount of vitamin $B$ concentrate sufficed to carry female rats through the lactation period if a part of the concentrate were administered directly to the young instead of the entire amount being given to the mother, and concluded that it may be advisable in infant feeding to supply some source of vitamin $B$ as a routine measure, as orange juice is given for vitamin $C$ and egg yolk or cod-liver oil for vitamins A and D. Later Sure and Schilling (1928) reported that 10 per cent wheat embryo furnished sufficient vitamin B for good growth, but even 30 per cent was inadequate for successful lactation. In the young of mothers on the deficient diets partial to complete loss of control of the rear limbs was observed. The stomachs of the young were well distended with curd, thus indicating that the failure of lactation was due to poor quality rather than deficient quantity of milk. Hemorrhagic conditions were present in osteogenetic tissues, and sometimes in internal organs. Sure, Kik and Walker (1929) found that an insufficiency of antineuritic vitamin in nursing young caused loss of weight, posterior paralysis, labored respiration, cyanosis, and death.

Daniels, Jordan and Hutton (1929) reported a failure in the nursing young of mothers receiving high protein-low fat diets with insufficient vitamin $\mathrm{B}$. These young failed to gain or lost weight, showed muscular incoördination, convulsions, and unusual outcries with subsequent death, usually between the thirteenth and sixteenth days. Cerebral hemorrhages particularly at the junction of the occipital and parietal bones, were the most frequent pathological findings.

It is believed that animals have little or no ability to synthesize vitamin $\mathrm{B}$, and that the requirement of the body must be met by the vitamin preformed in the food. It is necessary then to conceive of all material in the digestive tract as food not yet, technically speaking, in the body. An investigation at the Pennsylvania Experiment Station of the "vitamin B" requirements of dairy calves suggested in the earlier stage of the work (Bechdel, 1925) that unlike other species calves seemed to have a very low requirement for vitamin $\mathrm{B}$. The finding that milk of certain cows which had never received more than small amounts of vitamin $B$ in their rations was not deficient in this vitamin led to the hypothesis that cows and other ruminants are able to synthesize 
vitamin $\mathrm{B}$ through a bacterial symbiosis in the digestive tract. To test this an artificial opening was made into the rumen of a cow which had always received the vitamin B-poor food and the contents of the rumen were tested for vitamin B by the usual feeding experiments on rats (Bechdel, Honeywell et al., 1928). Weight for weight, the dried residue of the bacteria isolated from the rumen appeared as rich in vitamin $B$ as dried yeast. That synthesis of vitamin $B$ in the digestive tract is a common occurrence is suggested by the finding of vitamin B in the excreta. Salmon (1925) showed that the feces of rats may be rich in vitamin $B$ even when the diet is low in this factor, particularly if through the presence of undigested food residues in the intestinal tract the conditions are favorable for bacterial growth. It would thus seem that cows and other ruminants are not unlike other species in their vitamin $B$ requirement, but that, through their peculiar digestive apparatus, conditions are particularly favorable for the synthesis of vitamin $\mathrm{B}$ by bacteria and for the utilization of the vitamin thus synthesized.

Fridericia and coworkers (1927) reported that young rats occasionally recover spontaneously from the effect of vitamin $\mathrm{B}$ deficiency and are able to live for months with no vitamin $B$ in their diet and even to transmit this immunity to their young. This phenomenon, which has been named "refection," is said to be accompanied by a marked change in the character of the feces which become white from unchanged starch granules. The refection may be transmitted artificially by feeding the white feces for a few days provided the diet contains starch. The phenomenon has been confirmed by Roscoe (1927a) who noted its spontaneous occurrence in some rats on a vitamin $\mathrm{B}$-free diet and was later able to transmit it to other rats by feeding the feces of refected rats. Refection was also induced in rats on diets containing either vitamin $\mathrm{B}$ or vitamin $\mathrm{G}$ but not on diets containing dextrin or cooked instead of raw starch. These findings point to the synthesis of vitamins $B$ and $G$ by bacterial processes in the intestinal tract under conditions involving defective digestion of starch.

Mendel and Vickery (1929) report that they have never encountered "refection" in their rats receiving a variety of uncooked starches.

The extent of elaboration of vitamin B by bacteria depends on the medium upon which the organisms are grown, however, as was shown by the variations in the vitamin content of yeasts grown on beet molasses, cane molasses and malt extract (Randoin and Lecoq, 1928). So far as we know, the synthesis of vitamin B appears to be associated only with the metabolism of plant life. 
Qualitative Reactions to Different Degrees of Deficiency of Vita$\min B$. - A pronounced deficiency of vitamin B is followed by failure of appetite, decreased food consumption, and finally starvation, resulting in gastric and intestinal atony, fall of body temperature and death. Both Sandels (1928) and Chase (1928) found that on a diet adequate in other respects but free from vitamin $B$, young albino rats usually die before showing definite symptoms of polyneuritis. When measurable but insufficient amounts of vitamin B are fed, the animals make larger gains, but finally suffer a sharp loss in weight, develop the classical symptoms of polyneuritis and die, usually within a few days thereafter. When sufficient vitamin $B$ is fed to allow net maintenance of weight during the experimental period many of the animals develop a chronic type of polyneuritis, in which condition they may survive for several weeks. This state is characterized by a moderate head retraction, a spastic gait, a highly excitable nervous condition, and upon handling, a temporary paralysis in the animal. McCarrison (1928a) pointed out that whereas typical avian polyneuritis is produced by entire absence of the antineuritic vitamin, a condition resembling human beriberi is produced in pigeons by an unfavorable proportion but not absolute deficiency of the vitamin.

In young rats receiving a basal diet adequate so far as known except for vitamin $B$, growth is proportional to the added vitamin B between levels large enough to prevent polyneuritis and small enough to prevent normal growth. The deficiency of vitamin B tending to retard gain in weight, produces a lesser retardation in length than in weight, and smaller chests in proportion to body weight than is found in normal rats (Quinn, King and Dimit, 1929).

A serious deficiency of vitamin $B$ in human nutrition will lead to beriberi, or under less drastic conditions, to failure of appetite, possibly minor digestive disturbance, and loss of stamina. Many Japanese physicians hold that in actual clinical beriberi an infection is usually involved.

\section{Distribution of Vitamin B in the Body and in Food Materials}

Until recently the reported occurrence of vitamin $B$ was based on either pigeon- or rat-feeding experiments. Some of the earlier work can now be reinterpreted in terms of vitamins $B$ and $G$, but since it is both and not either one alone which is essential to mammalian nutrition, too elaborate an attempt at such reinterpretation might be premature.

Distribution in the Body.-Cooper (1914b) recorded in terms of the natural food material and dry weight, respectively, the following 
amounts of animal products as necessary to prevent polyneuritis: $\mathrm{Ox}$ voluntary muscle (beef) 20 grams and 5 grams, ox cardiac muscle (beef heart) 5 and 1.7, ox cerebrum 6 and 1.2, ox cerebellum 12 and 2.4 , ox liver 3 and 0.9 , cow's milk 35 and 3.5 , and egg yolk 3 grams and 1.5 grams.

By feeding animal tissues as the sole source of vitamin B for the support of growth in rats, Osborne and Mendel (1918a) showed that skeletal muscle (ordinary meat) is poor in this vitamin, while heart muscle, liver, kidney, and brain contain it in larger proportion.

Eddy (1916) had also shown the presence of notable amounts of water-soluble vitamin in pancreas tissue.

McCollum (1918) speaks of a relatively high vitamin B content in glandular organs. Conversely Funk and Douglas (1914) had reported that with few exceptions the organs of internal secretion are atrophied in pigeons rendered polyneuritic through lack of vitamin B.

Brodie (unpublished data) has studied in as strictly quantitative a manner as possible the distribution of vitamin $B\left(B_{1}\right)$ in the principal tissues of adult rats which had received an adequate ration. From her work, liver and heart muscle appear to be about ten times as rich as skeletal muscle; kidney about five times, and brain tissue about three times. Blood is even poorer in vitamin B than muscle; and lung and spleen appear to contain very little of it. The corresponding tissues are relatively more potent in meeting the nutritive requirements of the growing rat for vitamin G (Carlsson, 1929) than vitamin B; neither vitamin $B$ nor vitamin $G$ appears to be stored in the liver in any such marked degree as is vitamin $\mathrm{A}$.

Meat.-From the distribution of vitamin $\mathrm{B}$ in the animal body, as indicated above, it is clear that ordinary meats are poor in vitamin B, while heart, liver and kidney contain it in somewhat larger proportion. The experiments of Osborne and Mendel (1918a) made it plain that most ordinary meat is not to be depended upon to furnish the vitamin $B$ needed for growth. With 20 per cent of dry meat (beef) in the food mixture given to growing rats there was early and marked failure for lack of vitamin $\mathrm{B}$. On the other hand, rations containing the dried tissue of heart, liver, or brain in the proportions of 19,22 , and 32.5 per cent respectively seemed to furnish enough vitamin $B$ for the support of normal growth. The contrast between ordinary meat on the one hand and heart, liver or brain on the other appears even more strikingly here than in Cooper's comparison of the amounts required to prevent polyneuritis (cited above). By comparison with experiments reported by Osborne and Mendel elsewhere it would appear 
that heart, liver and brain contain vitamin B in proportions somewhat similar to those in which it is found in milk, eggs and whole cereals (all being compared on the basis of solid matter), whereas ordinary muscle meat showed too small an amount to admit of any quantitative comparison.

Hoagland (1923) in a study of the content of antineuritic vitamin (as determined by preventive tests with young pigcons) in the voluntary muscles and edible organs of the ox, sheep, and hog, obtained data indicating that beef and sheep muscle are of little value as a source of antineuritic vitamin, but that hog muscle is much richer in this vitamin. This observation was confirmed and extended by Hoagland (1924) in a further study of the antineuritic value of hog muscle and has also been confirmed incidentally by Wright (1923) in a study of the presence of vitamin B in frozen meat. Although no difference could be noted in the vitamin $B$ content (as tested on chickens on a mixed vitamin $B$-free diet) of fresh and frozen meat, pork which had been kept in cold storage for nine years, showed a much higher content of vitamin $B$ than lamb, mutton, or beef.

The data reported by Hoagland on the edible organs did not lend themselves to quantitative interpretation, but Osborne and Mendel (1923a) reported that the livers of rats which had been kept on a vitamin $\mathrm{B}$-free ration contained much less vitamin $\mathrm{B}$ than those from rats which had been on a normal diet. In the latter case the dried liver proved to be almost as good a source of vitamin B as dried yeast. "From the clean-cut outcome of this study the conclusion seems inevitable that when an adequate supply of vitamin B is lacking in the diet the store of this factor in the liver tissue where it is ordinarily found in abundance becomes largely depleted."

Eggs.-Osborne and Mendel (1923) have stated that "judged by the comparative trials on rats, the average sized hen's egg is equivalent in vitamin $\mathrm{B}$ potency to about 150 cubic centimeters of cow's milk, or a quart of milk and six or seven whole eggs on the average have an approximately equivalent vitamin B value." This estimate was made on the basis of the amount of dried egg yolk or a water extract of egg yolk required as the sole source of vitamin $\mathrm{B}$ for rats. For a 100 gram rat at least 0.8 gram of dried egg yolk per day (equivalent to 10 per cent of the food mixture) was required even to approximate normal growth.

Since even slight deterioration of an egg is readily perceptible to the senses, it is probable that eggs preserved by any method sufficiently efficient to keep them salable will retain their original vitamins 
in practically undiminished proportion. Tso (1926) has reported that Chinese preserved duck's eggs, "pidan," are apparently as rich in vitamins $A$ and D as fresh duck's eggs, but are practically devoid of vitamin $B$, showing that the process used in preservation has destroyed vitamin $B$, doubtless through its strong alkalinity. Hoagland and Lee (1924) have reported a possible slight destruction in the antineuritic vitamin of eggs as the result of 7 minutes' boiling.

Milk.-The mammary gland is believed not to synthesize the vitamin but to take it from the blood, or through the blood from the tissues, of the mother and convey it to the young through the milk. Since the total amount of vitamin $B$ available in the tissues is relatively small the concentration in which it appears in the milk is largely dependent upon the vitamin content of the mother's food; or upon the bacterial synthesis of this vitamin in the rumen of the cow (Bechdel et al., 1928). This doubtless is one reason that different investigators have received different impressions as regards the richness of milk in this vitamin. Another reason is to be found in the observation of Osborne and Mendel (1918c) that individual animals vary in their requirements for vitamin $\mathrm{B}$; and still another in the differing lengths of experiments and criteria of satisfactory growth. In some cases the experiment has been considered successful and the food satisfactory when growth at a nearly average rate was maintained for a few weeks, while other investigators, such as Osborne and Mendel, have discussed the question in terms of the requirement for optimal growth. If, to a basal ration, milk is added in increasing proportions, it is probable that the diet, even though adequate before, will be improved by the increased proportion of milk in other respects as well as in its content of vitamin $\mathrm{B}$, and so the experimenter may be led to the use of more milk than is really necessary to supply the needed amount of vitamin B. From a study of the work of various investigators it appears that milk solids contain about as much vitamin $\mathrm{B}$ as the solids of eggs and much more than the solids of ordinary meat; and that, on the average, an amount of milk sufficient to furnish from 10 to 20 per cent of the total solids or total calories of the diet will furnish as much vitamin $B$ as is needed to make the food mixture adequate for growth.

Commercially dried milks appear to be practically as rich in vitamin $\mathrm{B}$ as the solids of the original milk. Dutcher and Francis (1926), from a study of the vitamin B content of evaporated milk made by the vacuum and aeration processes from milk, the original vitamin $B$ content of which was determined, concluded "that vitamin B is not readily destroyed by the evaporation method described and only under unusual 
conditions would we expect the vitamin B deficiency of commercial evaporated milks to be due to methods of manufacture." Daniels $e t$ al. (1929) found evidence that both heat and subjection to aeration lower the antineuritic value of evaporated and pasteurized milks. Sherman and Spohn (1923), using powdered milk and reconstituted milk as a vehicle for vitamin $B$ in the dry and liquid state, respectively, found that prolonged heating at $100^{\circ} \mathrm{C}$. was more destructive of the vitamin $\mathrm{B}$ in the liquid milk than in the dry. While these experiments were conducted before the differentiation of vitamins $B$ and $G$, it is certain that vitamin $B$ was being measured, both because it is the limiting factor in cow's milk for growth, and because it is the more thermolabile of the two.

Human milk may perhaps contain vitamins $\mathrm{B}$ and $\mathrm{G}$ in different proportions from cow's milk. Osborne and Mendel (1922a) found 5 cubic centimeters daily of mixed human milk to furnish insufficient vitamin $B$ for normal growth in young rats, but when dried and fed in the form of tablets, the equivalent of 10 cubic centimeters sufficed for normal growth. Macy, Outhouse, Graham, and Long (1927a) reported that from 14 to 15 cubic centimeters daily of mixed human milk were required to secure even subnormal growth in young rats. From 25 to 30 cubic centimeters were required for normal growth and development in the younger animals and 35 cubic centimeters for continued growth in larger animals weighing 200 to 240 grams. The young of female rats receiving as much as 35 cubic centimeters of the milk were subnormal and few survived the nursing period. The addition of 0.4 gram daily of autoclaved yeast brought about renewed growth in a few rats which had ceased to grow. In a comparative study of cow's milk, using identical methods, Outhouse, Macy, Brekke, and Graham (1927) found that from 20 to 25 cubic centimeters of milk daily were required for normal growth in young rats for a period of 12 weeks. A daily supplement of 0.4 gram of autoclaved yeast was without effect, but the same amount of fresh dried yeast brought about an immediate response in growth in rats which had ceased to grow on the 25 cubic centimeters allowance of milk. These results would suggest that of these two water-soluble, growth-promoting vitamins. in human milk the limiting factor is the heat-stable vitamin $G$ and in cow's milk the heat-labile vitamin $B$. This difference if confirmed might be attributed to the food; the human diet may be poorer in vitamin $G$ than that of a cow on a ration consisting more largely of leaves.

Cereals and Breadstuffs.-The whole grains of the cereals are fairly rich in vitamin $B$ and a diet consisting largely of grain products would 
probably always contain an ample amount of this vitamin were the entire grain used.

Relative to the vitamin B content of dried yeast, Plimmer and his coworkers (1927) have assigned the following values to cereal foods: (Based on maintenance tests with pigeons)

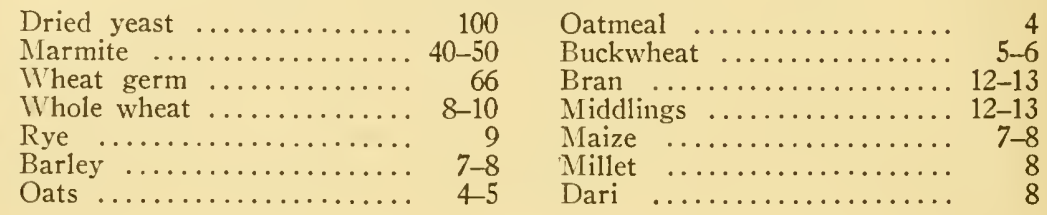

In the Japanese navy, these authors point out, beriberi was prevented by a ration of barley amounting to approximately one-third of the dry weight of the food consumed.

Although the different cereals apparently vary in the distribution of vitamin $\mathrm{B}$ in their structural parts, the interior of the endosperm in general contains less of the vitamin than the other parts of the grain and consequently the more highly refined the mill product the greater the danger that the vitamin content may be too greatly reduced.

In wheat, vitamin B has been shown by Bell and Mendel (1922) to be widely dispersed throughout the entire kernel. Their calculation of the percentage distribution in the true structural parts of the grain is: embryo from 8 to 16 per cent, bran 24 , and endosperm from 60 to 68 per cent. The percentage distribution of the vitamin in the different milling products calculated on the basis of the fraction of the entire kernel represented by each of these milling products is patent flour from 0 to 5 , first clear from 10 to 15 , second clear 5 , low grade 16 , middlings 40 , and bran 24 per cent. The explanation advanced by Bell and Mendel for the poverty of patent flour in this vitamin as compared with pure endosperm is that "in the process of milling the softer, more friable parts of the endosperm which are rich in this vitamin adhere to the embryo and bran as they are flaked off from the grain. Harder lumps of endosperm, poor in vitamin, are left behind and when sufficiently freed from germ and bran are themselves ground into fine white flour." Bell and Mendel emphasize the fact that different varieties of wheat may vary widely in their content of vita$\min \mathrm{B}$.

Wheat embryo has been found by Chick and Roscoe (1927) and ground whole wheat by Sherman and Axtmayer (1927) to be relatively richer in vitamin $B$ than in vitamin $G$.

Nearly all of the vitamin B of maize or corn has been found by 
Croll and Mendel (1925) to be located in the embryo. The endosperm contains hardly a measurable amount of vitamin B and the bran very little. Two varieties, one yellow and one white, contained about the same amount of vitamin $B$, from 20 to 30 per cent of the ration being necessary for normal growth. The content of vitamin B in commercial milling products of corn used for human and animal food depends largely on the relative amounts of the embryo left in the fraction by the milling process. Whole corn, corn germs, corn germ meal, gluten feed, hominy feed, and whole ground corn meal were found to contain considerable amounts of vitamin B. Corn products consisting largely of endosperm or hulls, such as degerminated corn meal, corn flour, crude and refined corn oils, cornstarch, grits, gluten hulls, steep water, and corn flakes were deficient in this vitamin.

Whole unpolished "brown" rice and both the endosperm and embryo ends of the unpolished rice kernel (separated by hand dissection) were found by Croll (Illinois Agricultural Experiment Station, Annual Report, 1927) to contain vitamin B, the embryo end being richer than the endosperm end. Since the outer bran coats are retained in the unpolished rice kernel, the presence of vitamin B in the endosperm end may be due to its presence in bran rather than in the endosperm itself. Pure white polished rice is very deficient in vitamin B. Rice polishings, long known to be a very rich source of the antineuritic factor (vitamin B) have been shown by Munsell (1929) to be relatively poor as a source of vitamin $G$.

With all three of these cereals (wheat, maize, rice) the use of the highly milled products in preference to the whole grain will markedly decrease the vitamin B content of the diet. In commenting on this, particularly as applied to whole wheat vs. patent flour, Osborne and Mendel (1919a) state: "So long as the diet of man is sufficiently varied to include enough vitamin from sources other than the cereals the milling question need not awaken much concern from the vitamin standpoint; but when the diet is restricted the danger of a shortage of vitamin may become real if a large proportion of sugar, fat, or other products, low in their content of water-soluble vitamin, are included in the diet." Since this is often the case and since we now have reason to believe that an intake of vitamins much more abundant than is absolutely necessary is often advantageous, we believe that the use of whole grain in preference to highly milled products should be encouraged, except in those cases in which there is reason to fear that the "roughage" of the whole-grain product is unduly irritating to the individual digestive tract. 
Since yeast is rich in vitamin $\mathrm{B}$, the introduction of yeast in the making of bread must raise its vitamin content; but the increase is only very slight because the amount of yeast used is so small. The use of a "lower" grade of flour (less purely the interior of the endosperm) may be expected to increase the vitamin content of the bread much more than does the yeast used for leavening. Successful attempts have been made to raise the vitamin $B$ content of bread through incorporating in the dough a concentrated extract of vitamin $B$ and to increase both this and other factors by the use of considerable amounts of dried milk.

As has been noted, cow's milk is relatively rich in vitamin $G$ and its use in bread making is thus of special value in supplementing wheat and other cereal products, in this respect as well as in respect to vitamin A and calcium, and in somewhat lesser degree vitamin B.

Beans, peas, and seeds in general, when eaten entire or approximately so, are apparently all rather similar as sources of vitamin B. McCollum states that with 25 per cent of white beans in the diet as the sole source of vitamin B the supply was fully adequate for the growth of rats. The requirement of barley was reported by Steenbock to be about 20 per cent, whole wheat by Bell and Mendel (1922) from 20 to 30 per cent, and lentils by Jones and Murphy (1924) at least 25 per cent of the ration. Osborne and Mendel (1919) speak of whole wheat, soy beans, dried eggs, and milk solids as each being required in proportions twice as large as of dried spinach, when serving as sole sources of vitamin B. Green peas are considered by Eddy, Kohman, and Carlsson (1926) to be richer in vitamin B, weight for weight, than either milk or tomato juice. As noted previously, considerable evidence has accumulated recently to the effect that seeds contain relatively more of vitamin B than of vitamin G. Hauge and Carrick (1926), Goldberger et al. (1926), and Hunt (1928a) have shown this to be true for corn; Smith and Hendrick (1926) for rolled oats; Salmon (1927) for soy beans and velvet beans; Sherman and Axtmayer (1927) and Hunt (1928a) for wheat; and Munsell (1929) for rice polishings.

Vegetables.-The potato was found by McCollum, Simmonds and Parsons (1918a) to be capable of supplying all the vitamin B needed by rats, but since large proportions of potatoes were used in all of the experiments which they describe, their data do not show how the potato compares with other foods quantitatively in this respect. Osborne and Mendel (1920a) found it to compare favorably with roots as a source of vitamin B. Lyman and Blystone (1926), questioning 
the earlier data on account of failure to prevent the animals from having access to their excreta, reinvestigated the vitamin $B$ content of two varieties of white potatoes and found them to be considerably less rich in this vitamin than previously reported. Good growth was secured only when potatoes constituted at least 80 per cent of the diet. They concluded that the white potato can not be depended on safely to make good any considerable deficiencies of vitamin $B$ in other articles of the diet. Possibly a repetition of much of the earlier work on the vitamin $\mathrm{B}$ content of other foods would tend to lower the values assigned. This, however, would not necessarily invalidate comparisons of the relative values of different foods when tested by the same methods. Steenbock and Gross (1919) reported the sweet potato as containing less vitamin B than carrots, rutabagas, or dasheens of which 15 per cent sufficed to make the food mixture adequate. Osborne and Mendel (1919c, 1920a) found spinach, cabbage, carrot, turnip, onion, beet leaves or beet stems to contain about twice as much vitamin $\mathrm{B}$ as beet roots, about half as much as tomato and one-fourth as much as yeast when calculated on the dry basis. In this comparison the amount of tomato was 0.5 grams daily. Osborne and Mendel (1922) found green asparagus to be very rich, the minimum daily requirement approximating 0.2 grams of the dried material equivalent to 2.2 grams of fresh. Corresponding values for other vegetable materials on the dry basis were blanched celery 0.5 to 0.8 grams, and lettuce and parsley 0.4 grams. These comparisons have all been on the dry basis, with growth of rats as the evidence of relative amounts of vitamin $\mathrm{B}$ in the foods tested. As determined on the fresh basis and with maintenance for a given period instead of growth as the criterion, the vitamin $B$ values of fresh vegetables other than greens are not strikingly different. The values reported by Sherman (1926) in terms of the older units per gram are string beans 0.35 , cabbage $0.35-0.5$, carrot 0.3 , onion 0.2 , potato 0.3 , tomato $0.3-0.6$, and turnip 0.3. Compared with these figures spinach has a value of $0.6-0.8$ and according to Burton (1928) collards and turnip greens have even higher values. Preliminary studies at the Kentucky Experiment Station have shown kale and mustard greens to be rich in vitamin $B$. Quinn, Burtis and Milner (1927) have recently shown string beans and green peppers to be equal to lettuce or cabbage but not as rich as spinach in vitamin $B$.

Fruits.-Compared in the fresh fluid form, orange, lemon, and grapefruit juice were found by Osborne and Mendel (1920d) to contain about the same concentration of vitamin B as they had reported 
for milk (about 10 cubic centimeters daily being required for satisfactory growth in rats). Fresh prunes were comparatively rich ( 5 grams daily promoting rapid growth), apples and pears less rich, and grape juice markedly deficient in vitamin B. Spohn (personal communication) has found the vitamin $B$ content of two varieties of apples to vary by about 15 per cent. Miller, working in Hawaii, has found pineapples, fresh and canned (1924), to compare favorably with citrus fruits and the native papaya (1926) to be only about half as rich in its vitamin $\mathrm{B}$ content. The avocado was reported by Santos (1922) to be about as rich in vitamin B as togi or okra, 0.5 grams of dried material daily being sufficient in curative tests for rats. In preliminary studies Tilt (personal communication) reports 1.4 grams daily of fresh Florida-grown avocado sufficient to maintain the weight of rats for an 8 weeks experimental period. According to Eddy and Kellogg (1927) the banana has about the same vitamin $B$ value as tomato juice. From the limited data available apples, peaches, and grapes appear to be similar to bananas as sources of vitamin $B$.

So far few attempts have been made to determine the relative richness of vitamins $B$ and $G$ in fruits. Eddy (1928a) has shown that the banana is relatively richer in the heat-stable vitamin $G$ than in the antineuritic vitamin $\mathrm{B}$.

Nuts.-Cajori (1920), working with rats which consumed 6 to 8 grams of total food per day, found that if almond, English walnut, black walnut, chestnut, Brazil nut, or pecan were included in the ration to the extent of 1 to 2 grams per day, the nut sufficed as sole source of the $B$ vitamin. This would imply that nuts are nearly but not quite as rich in vitamin $\mathrm{B}$ as are the whole cereals, the dry legumes, or the solids of milk or eggs. Salmon and Livingston (1925) tested pecans for vitamin $B$ with pigeons and rats. The minimum protective dose against polyneuritis was from 3 to 3.4 grams daily, representing from 17 to 20 per cent of the total ration. Rats declining on a ration lacking in vitamin $B$ were able to maintain their weight when 10 per cent of pecans was added to the basal ration, but concentrations of 20,40 or even 60 per cent failed to produce normal growth. The rats on the 60 per cent ration were consuming about 2 grams of pecans a day. This would seem to indicate that the values reported by Cajori were perhaps too high, as were most of the data obtained before the necessity was realized of preventing access of the experimental rats to their excreta. ${ }^{1}$

\footnotetext{
1 It should be noted, however, that some laboratories used cages with raised screen floors to prevent access to excreta in vitamin work, on the general principle of permitting the test animal to eat nothing but the food, before the necessity for this had been explicitly demonstrated by Steenbock in his work upon vitamin $\mathbf{B}$.
} 
Using pigeon tests, Plimmer, Raymond and Lowndes (1929), have found pulses and nuts to have the following vitamin B potencies, taking the value of dried yeast as 100: Split peas, whole dried green peas, lentils, and soya beans, 13 ; haricot beans, ground almond, whole almonds, and dried chestnuts, 10; hazelnuts and peanuts, 20; cocoanut and roasted coffee, 0 .

\section{Quantitative Determination}

As explained earlier in this chapter, two methods have been chiefly employed in work with vitamin $B$, namely (1) the prevention or cure of polyneuritis in fowls, pigeons, or other birds, and (2) the promotion of growth in young experimental animals, most frequently rats.

For a time, attempts were also made to utilize the rate of growth, or rate of reproduction, of yeast as a simpler and more rapid method of testing foods for vitamin B values; but such stimulation of yeast is now considered as a measure of "bios" rather than of vitamin B value. Bios has been shown to be relatively stable both to alkali and to heat (Guha and Drummond, 1929), which properties differentiate it from vitamin $\mathrm{B}\left(\mathrm{B}_{1}\right)$. In view of this fact and the pressure for space to review here the present knowledge of the substances universally regarded as vitamins, we are regretfully obliged to omit discussion of bios and of the yeast method, and to confine attention here to a very brief mention of the pigeon method of testing for antineuritic values of foods and a fuller account of the rat growth method as the one chiefly used in attempts to measure quantitatively the relative amounts of vitamin B in different materials or in the same material before and after different treatments, as in the studies of stability summarized further on in this section.

Quantitatively, experiments with rats have usually seemed more satisfactory than those with pigeons. Cure of the polyneuritis induced by a polished rice diet is qualitatively a striking demonstration; but there is no satisfactory way of determining the degree of polyneuritis from the symptoms or of judging the exact time at which the curative experiment should be performed in order to justify quantitative comparisons of the amounts of foods (or other materials) required to effect a cure. It may readily happen that a vitamin-containing food which shows good antineuritic effects upon one bird may fail to cure a second because in the second case the disease had progresser so far as to preclude recovery. Still greater difficulty arises from the fact that birds which have developed typical polyneuritis on vitamin-free or vitamin-poor diet sometimes recover spontaneously without any 
change in their food or the administration of vitamin in any form (Kon and Drummond, 1927; Kon, 1927), and temporary cures have been reported effected by a variety of substances which may bear no relation (in chemical nature or normal nutritional function) to the antineuritic vitamin. This being the case it necessarily follows that neither the positive nor the negative results obtained in "curative" experiments can be entirely conclusive in any individual case. Safe conclusions can be drawn only from a preponderance of the evidence resulting from numerous experiments conducted under rigidly controlled conditions. Peters and coworkers (Peters, 1924; Kinnersley and Peters, 1925, 1927, 1928; Kinnersley, Peters and Reader, 1928) describe their technique for the use of the pigeon as a reagent. These papers will repay careful study by those interested in this method. Under wellcontrolled conditions there is usually a quantitative relationship between the time of protection after cure and the amount of curative dose given.

With a well-chosen basal dietary fed to an animal of suitable species and of standard age and weight, the amount of the food under investigation which will be needed for protection from deficiency disease or for support of a standard rate of growth will be inversely proportional to the concentration of vitamin in the food, provided no disturbing factor is allowed to enter into the test. This minimum allowance of the food in question may be determined either in terms of the weight required per day when the test food is fed separately from the rest of the diet, or of the proportion in which the test food must enter into the food mixture in order to make the ration adequate. In either case the total food intake should be determined and the adequacy of the diet in respect to all other nutritive requirements should be assured. Obviously in either case the determination of the "minimum protective dose" of the food or of the minimum allowance for support of a standard rate of growth is likely to require a series of experiments in which the quantity or proportion of the test food is systematically varied while all other conditions are held constant. Such a series of quantitative experiments is naturally much more exacting than the few tests which would suffice to demonstrate the presence of the vitamin qualitatively, and it is not surprising that in the years which have been so largely spent in demonstrating the actual existence of vitamins and learning something of their occurrence in plant and animal organisms, the majority of investigators should have felt it more interesting to survey the general field qualitatively than to spend the large amount of time and labor necessary to make quanti- 
tative comparisons. Now, however, the qualitative survey has (in our opinion) served its purpose and henceforth no investigator should be satisfied with anything less than at least approximately quantitative work.

The importance of accurate and quantitative measurements in experimental work upon nutrition with special reference to vitamin problems was well emphasized by Chick and Hume who said (1919): "Since the general conception of accessory food factors (or vitamins) was brought forward, a constant succession of researches has been made to assign values to various foodstuffs as antineuritic, as antiscorbutic, or as growth-promoting materials respectively, and further to determine whether these values are lessened or destroyed by heating, drying, or other methods of preservation. In most of this work one fundamental consideration-namely that of quantity, has been to a large extent overlooked in the methods adopted for experiment, and the conclusions drawn have been untrustworthy to a corresponding degree.

"In comparing the values of a series of foodstuffs as regards their value in content of some accessory food factors, it is obvious that the first step necessary is to determine in each case the minimum daily dose which will maintain health in the experimental animal and to institute comparison between these amounts. . . .

"Similarly, in investigating the effect of heat, drying, or any other method of preservation upon the accessory factors contained in any foodstuff, it is necessary to determine the minimum daily dose (1) of the original untreated material and (2) of the same material after heating, drying, etc., which will protect the same experimental animal under similar conditions of experiment. Then only is it possible to state to what extent, if any, the foodstuff has suffered deterioration as the result of the treatment.

"Neglect of this simple and fundamental, but admittedly tedious procedure is evident in many papers recently published upon the influence of various treatments upon the antineuritic and antiscorbutic and other properties of certain foodstuffs and the results obtained are in consequence vague, and may even be erroneous. It is clear, for example, that if a foodstuff is rich in any particular accessory factor and is fed to an animal in large excess of the amount actually required, any destruction of the factor that may take place during heating cannot be assessed and may even remain undetected if the heated material is fed in similar large amount. It is absolutely necessary to determine the minimum required in both cases, and to institute a comparison between 
these two results before any satisfactory conclusion on this point can be drawn."

In experiments designed to determine the antineuritic values of foods by finding the amounts required to protect pigeons or fowls from polyneuritis, it has been common to use polished rice as the basal diet, although this plainly does not meet the desideratum of being nutritionally adequate in all respects other than the vitamin under investigation.

In order that all other nutritional needs should be adequately met, Simonnet (1920) proposed the use of the following ration for the study of polyneuritis in pigeons:

Meat residue prepared by double extraction with boiling alcohol and ether 11 grams, Osborne and Mendel's salt mixture 4, pulverized agar 5, peanut oil heated for 3 hours at $130^{\circ}$ C. 5 , cellulose 5 , butter melted, decanted and filtered at a temperature below $40^{\circ} \mathrm{C} .10$, and potato starch 60 grams. These ingredients are intimately mixed with 80 per cent of their weight of water and made into pellets, which are introduced into the crops of the experimental pigeons in two daily doses and in amounts equal to one-fifth of the weight of the bird. The pigeons are kept in very large cages to allow considerable freedom.

On the above diet the pigeons are said to maintain their weight but to develop a form of avian polyneuritis unaccompanied by paralytic and atrophic symptoms, in the development of which inanition and multiple deficiencies are considered to play the important rôle. The disease appears generally in about 30 days, and is usually fatal in 4 days if no treatment is given. The administration of dry or autolyzed yeast brings about prompt recovery. By supplementing the deficient ration by the administration of 0.5 gram of dried yeast daily, the pigeons can be maintained in excellent health for more than 7 months.

Experiments with pigeons using weight-maintenance as a criterion for the antineuritic potency of a product must now be questioned, for recent work of Williams and Waterman (1928) and Randoin and Lecoq (1928) indicate that at least two factors are here involved (vitamins $B_{1}$ and $B_{3}$ ).

Slight modifications of the Simonnet ration (Lopez-Lomba and Randoin, 1923a; Randoin and Simonnet, 1923), and others of the same type (Gulick, 1922; Hoet, 1923), have since been described and have been used particularly in certain studies of the physiological rôle of vitamin $B$.

Most investigators have, however, inclined to the use of the growing rat as a means of testing foodstuffs for their vitamin $B$ values. 
In the earlier studies on the distribution of vitamin B, notably those of Osborne and Mendel, of Steenbock, and of McCollum, comparisons were made on the basis of the amounts of foods required for approximately normal growth in young rats on an artificial basal ration free from vitamin $\mathrm{B}$ and adequate in all other respects: and it is largely through such experiments that estimates such as those quoted in the preceding section were obtained. Difficulty in securing sufficient consumption of the food being tested without displacing too much of the basal diet, together with uncertainty as to what constitutes normal growth, has led some investigators to adopt maintenance of weight over a given period rather than gain in weight as the criterion. Dunham (1921) described such a method as follows: "Albino rats from 4 to 5 weeks old were fed ad libitum a basal ration of casein 20, starch 61, butter fat 15 , and salt mixture 4 per cent until growth had ceased and in some cases a slight decrease in weight had occurred. The vegetable to be tested, air-dried and pulverized, was then fed in decreasing amounts apart from the daily ration until the weight became constant at some point between 75 and 100 grams, when the rat was 100 to 130 days old. The feeding of the basal ration plus this amount of vitamin was continued for 30 days, during which time the weight should remain constant with an allowable variation of \pm 4 per cent. At the end of this period 500 milligrams daily of dried yeast was added to the diet in place of the substance tested. Immediate restoration of growth would indicate that the lack of growth was due to insufficiency of vitamin B."

An important point overlooked in much of the earlier work was that the feces of rats on the experimental diets may contain appreciable amounts of vitamin $\mathrm{B}$ and that unless care is taken to prevent the rats from consuming their feces the results of feeding experiments may be of little quantitative value. Steenbock, Sell and Nelson (1923) called attention to this and their conclusions were confirmed by Dutcher and Francis (1924), Smith, Cowgill and Croll (1925) and others. Salmon (1925) showed that when conditions are favorable for bacterial growth in the intestines the feces of rats may be rich in vitamin $B$ even when the diet is deficient in this vitamin. Cages with raised screen floors of sufficiently large mesh to allow the feces to drop through are now used in practically all vitamin B studies.

Feeding Method.-Healthy young rats, 25 to 29 days of age, and weighing usually 40 to 55 grams are placed in individual metal cages with raised wire screen floors and given ad libitum distilled water and a basal vitamin-B-free diet now known to be practically free from 
both vitamins $\mathrm{B}$ and $\mathrm{G}$, and designed to be not only adequate but approximately optimal (for growth of rats) in other respects. The diet (Sherman and Spohn, 1923, as modified by Chase, 1928) consists of casein (purified by extraction with 60 per cent alcohol) 18, Osborne and Mendel (1919b) salt mixture 4, butterfat 8, cod-liver oil 2, autoclaved bakers' yeast ${ }^{2} 15$, and starch 53 per cent.

The casein is freed from vitamin B by cold extraction with 60 per cent (by weight) alcohol. Four hundred grams of casein are treated with two liters of 60 per cent alcohol and the whole stirred for onehalf hour, then allowed to stand $5 \mathrm{I} / 2$ hours, filtered with suction and thoroughly washed with one liter of 60 per cent alcohol. It is again treated with two liters of 60 per cent alcohol, and stirred for another half hour. After standing 18 hours it is filtered, washed with one liter of 60 per cent alcohol and finally with one liter of 90 per cent (by weight) alcohol to facilitate air drying. Each time, the casein is carefully freed from the alcoholic solution and washings. To prepare the autoclaved yeast, powdered bakers' yeast is heated for 6 hours after addition of 0.1 molar sodium hydroxide at 15 pounds pressure $\left(120^{\circ}\right.$ C.), enough alkali being added to make a smooth paste (125 cubic centimeters per 100 grams yeast), and neutralized after autoclaving by adding equivalent amounts of standard hydrochloric acid. The product is dried at room temperature before an electric fan, and finally ground to an impalpable powder.

On this diet, practically devoid of vitamin $B$ while containing an abundance of vitamin $G$ and all other known factors required for the nutrition of rats, the experimental animals usually continue to gain in weight for from one to two weeks by virtue of the vitamin $B$ stored in their bodies before they were placed on the diet. This is therefore considered a "depletion period" preliminary to the test period proper. At the end of this depletion or fore period, when all gain in weight has ceased, those of the experimental animals which are to serve as "negative controls" are left on this basal diet only.

Other rats of the same initial age, and so far as possible from the same litters as the negative controls, are given, in addition to the basal ration, graduated daily doses of the food to be tested as their sole source of vitamin B. Any advantage which they show over the negative controls should be attributable to the vitamin $B$ which they obtain from the weighed portions of the food under examination. If the amount of vitamin $B$ thus obtained is very small it results simply

The particular yeast used must be investigated as to completeness of destruction of vitamin $\mathrm{B}\left(\mathrm{B}_{1}\right)$ on autoclaving. 
in a less rapid loss of weight and a prolongation of the survival period; but as the allowance is increased, growth at increasing rate is obtained.

Different workers have made quantitative comparisons at different levels of vitamin intake and maintenance or gain of weight. To facilitate comprehensive comparisons it may therefore be desirable to feed at several different levels.

The average results of feeding the same vitamin-B-containing food at nine different levels is shown, along with the results of the ("negative") controls in Fig. 5 from the work of Chase (1928).

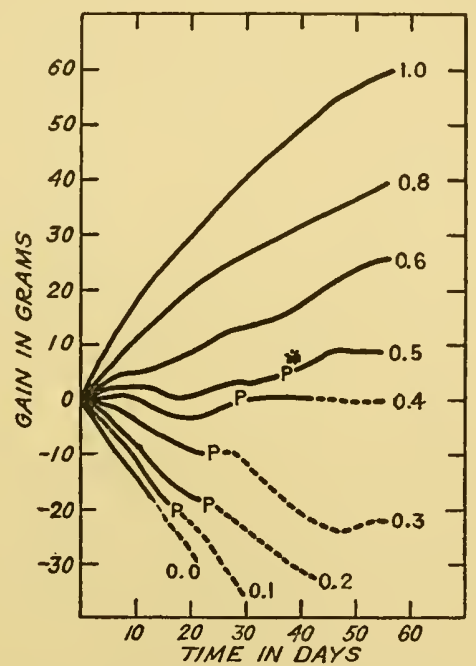

FIG. 5.-Average gain curves of rats on vitamin B-free diet (No. 513) plus daily supplements of ground whole wheat of $0.1,0.2,0.3,0.4,0.5,0.6,0.8$, and 1.0 grams. Negative controls (marked 0.0 ) received basal diet only.

$* \mathrm{P}=$ Point where, on the average, chronic symptoms of polyneuritis appeared.

Lines broken from the points at which some individuals died.

(Courtesy of Dr. E. L. Chase.)

In all cases in which the results are to be compared directly, the feeding experiments are conducted in parallel between groups matched as to sex and weight after depletion, and containing representative animals of the same litters. The response of the rats has been observed to vary with graded increments of vitamin-B-containing food material, from death with or without severe polyneuritis in from 20 to 30 days; through maintenance of body weight for 8 weeks with moderate polyneuritis during the latter half of that time; and through growth with apparent protection from polyneuritis; up to a normal rate of growth throughout. 
The doubt introduced by the work of Williams, Waterman and Gurin (1929) as to the adequacy of destruction of vitamin B and conservation of vitamin $\mathrm{G}$ in the autoclaving of yeast, has apparently been cleared up by Bisbey (1930) who shows that the treatment used and recommended by Chase is entirely satisfactory as applied to the type of yeast ("bakers'") which she (Chase) used, whereas other types of yeast may require a different treatment or may be less suitable for this purpose. Each investigator should, therefore, assure himself by experimentation upon the yeast he proposes to use, that it is, after autoclaving, sufficiently freed fron 1 vitamin $B$ and a sufficiently good source of vitamin $G$ to be satisfactory for this purpose. Or the general method here described may be used with something else than autoclaved yeast as the source of vitamin G. (See also the work of Chick and Roscoe, 1929.)

Under the conditions described, the rate of growth appears to be satisfactorily uniform (when averages of sufficiently large numbers are considered), and proportional to the vitamin B intake, but not necessarily arithmetically proportional. Quantitative comparisons are, therefore, most accurate when made at the same levels of gain in weight.

It is suggested that quantitative comparisons of vitamin $B$ values be based on the determination of the amount of test material which when fed daily under the conditions just described permits a gain of 3 grams per week during an experimental period of from 4 to 8 weeks. This amount of vitamin B may be taken as a "unit" for use in those comparisons or discussions in which such a unit (necessarily somewhat arbitrarily chosen) is considered a convenience.

The unit here suggested is no more arbitrary than any such unit must necessarily be in the present state of knowledge, and has the advantage of direct relationship to the experimental operation upon which it is based. It is also believed that a gain of weight of about 3 grams per week is favorable to a combination of delicacy and reliability in quantitative comparisons, in that it ensures sufficiently healthy test animals for reasonable constancy of results, yet is so far below normal growth that there is ample room for response to an increased intake of the vitamin.

Tentatively, then, a unit of vitamin $B$ may be defined as that amount which, when fed as a daily allowance, results in a rate of gain of 3 grams per week in a properly standardized and controlled rat during an experimental period of 4 to 8 weeks. The direct results of the feeding test show what weight of the food under test contains one 
unit of the vitamin; and from this the number of such units of vitamin B contained in a gram, a kilogram, a pound, an once, or a 100-calorie portion of the food can readily be calculated.

In much of the work of the past in which special attention was being given to the quantitative aspect, a test period of 8 weeks has been used in the belief that this was safer, and apt to be more accurate, than any shorter period; but in view of recent evidence that after several weeks on the basal diet other vitamins, not yet well defined, may begin to be limiting factors also, and thus complicate the measurement of vitamin $B$, it now seems perhaps better to base the quantitative comparison upon the rate of gain during a period of four weeks beginning at the end of the "depletion period"; or, if the body weight should decline for a few days after this, then the measured test period may be counted as beginning at the minimum point of the weight curve during the first week of the feeding of the test portions of the material under investigation. (Attention should, perhaps, be called to the fact that the "unit" here suggested is not identical with that formerly used by one of us: Sherman, 1926.)

Many of the precautions advised to insure accuracy in vitamin A determinations (see Ch. V) are probably equally applicable to this method for determining vitamin $B$. In this connection attention may be called to work which, while published before the clear differentiation of vitamin $G\left(B_{2}\right)$, is yet applicable to the control of the present method for the measurement of vitamin $B\left(B_{1}\right)$ values: Sherman and McArthur, 1927; Sherman and Gloy, 1927.

Chick and Roscoe (1929), in the method which they have adopted for the assay of vitamin $B_{1}$, use washed brewery yeast autoclaved at $120^{\circ} \mathrm{C}$. for 5 hours as their source of vitamin $B_{2}$, although admitting that this occasionally carries traces of vitamin $B_{1}$ and also has a tendency to cause diarrhea. The basal diet for the assay of either vitamin $B_{1}$ or $B_{2}$ consists of purified casein 10 , rice starch 30 , cottonseed oil 7.5, McCollum salt mixture (185) 2.5, and distilled water 50 parts, the whole being cooked in a double boiler for 3 hours at $100^{\circ} \mathrm{C}$. to prevent refection. Cod-liver oil is administered separately in daily doses of 3 to 5 drops ( 0.05 to $1 \mathrm{gm}$.) depending upon the size of the animal. In the assay of vitamin $B_{1}$ the young rats are placed at weaning on the basal diet plus the cod-liver oil and when growth has ceased are given the material to be tested as a supplement. The unit of vitamin $B_{1}$ which they have selected is the amount which will restore growth and induce a weekly increase in weight of about 10-14 grams under these conditions. 
The question has occasionally been raised as to whether or not the rat is a suitable animal for use in studies of the antineuritic vitamin. The symptoms of the specific deficiency (polyneuritis) had not in the past been produced with regularity in rats, and because of this, doubt has arisen as to the value of the rat growth technique. As recently as 1928 Kinnersley, Peters and Reader make the statement that "it is not yet absolutely certain that rat tests can differentiate the curative factor in the vitamin B complex." Chick and Roscoe (1927) rarely observed symptoms of polyneuritis in their animals on their basal diet $\mathrm{K}$ alone.

Drummond (1917) noted paralytic symptoms in three only out of a large number of rats on diets deprived of water-soluble vitamins. Opposed to these negative results, Schaumann (1911) found that rats confined to a diet of denatured horse flesh developed symptoms of paralysis. He states that three rats on this diet became lame after 25 to 28 days on this diet. All three animals survived in this condition for the eighty days of the experimental period. McCollum and Simmonds (1918) had observed paralysis in their rats as a sign of depletion of the bodily store of vitamin B. Hofmeister (1922) was able to produce symptoms of polyneuritis in rats with considerable regularity.

When the diet is lacking or is very low in vitamin $B$, rats may die before the classical nervous symptoms of polyneuritis appear; whereas a small amount of vitamin B will allow them to live longer, though they may exhibit severe nerve symptoms for as long as three weeks to a month.

Beyond a certain minimum which does not prolong life sufficiently to permit the development of polyneuritis, gradually increasing the allowance of vitamin B first permits the full development of the disease, while further increasing the allowance postpones the time of onset and reduces the proportion of animals afflicted with observable symptoms. Head retraction is usually one of the earliest symptoms. In its mildest form it appears as a nervous jerking of the head when the animal becomes excited. Animals are apt to hold their heads on one side and walk in circles. In more aggravated cases the opisthotonic position of the head is quite marked. There is sometimes complete paralysis of the hind legs, but more frequently the nervous involvement manifests itself in a spastic gait, or in loss of muscular control, especially when handled. In many cases this loss of control results in animals becoming prostrated, sometimes rolling over completely many times when attempting to move forward, and is probably comparable to 
the "cart-wheel turnings" of pigeons described by Kon and Drummond (1927).

An amount of the vitamin which allows approximately net maintenance of the weight of the experimental animal over an eight week period, is frequently insufficient for protection from the deficiency, and, as pointed out by Sandels (1930) may permit the development of a subacute or chronic type of polyneuritis. Animals in this condition usually maintain life for several weeks, and the majority of these show definite symptoms of polyneuritis continuously after the deficiency has once become manifest. Vedder and Clark (1912), Weill and Mouriquand (1919), and Randoin and Lecoq (1927i) reported a similar condition in fowls receiving an amount of vitamin insufficient for full protection. Hofmeister (1922) also described this subacute type of polyneuritis, and associated the condition with shortage rather than complete absence of the antineuritic vitamin. For a fuller and recent discussion of this point, see the paper of Sandels (1930).

Proposed Injection Method.-Smith (1930) suggests that the relative antineuritic potency of preparations be determined by finding the minimum dose which when injected intravenously will cure polyneuritic rats for 3 days. He finds that the period of recovery is roughly proportional to the size of the dose administered, and that when paralysis intervenes again it can be alleviated a second or third time, thus making it possible to use the same animal for several tests with but little change in its response to the influence of the vitamin. In his investigation the autoclaved brewers' yeast used as a source of vitamin G apparently contains enough vitamin $B$ to prolong the life of the rat sufficiently to permit quite regular development of polyneuritis.

\section{Stability of Vitamin B}

Sherman and Spohn (1923) found no measurable diminution of vitamin $\mathrm{B}$ in milk powder when heated dry with free access to air at $100^{\circ} \mathrm{C}$., when this heating was continued even for 48 hours. When the milk was heated in the fluid state for 6 hours at $100^{\circ} \mathrm{C}$. about onefourth of the vitamin $B$ content was destroyed. Although these experiments were conducted before vitamin $G$ was recognized, it is quite certain that the results were not vitiated by lack of vitamin $G$ both because it has been amply demonstrated that cow's milk is relatively richer in vitamin $G$ than vitamin $B$ (at least, in terms of the nutritional needs of the albino rat) and because vitamin $B$ is the more thermolabile of the two.

Daniels, Giddings and Jordan (1929) report that the degree of 
aeration as well as temperature may be a factor in the destruction of vitamin $B$ in fluid milk.

The effect of heating vitamin $B$ in tomato juice at its natural acidity, approximately $\mathrm{pH} 4.3$, at $100^{\circ}, 110^{\circ}, 120^{\circ}$, and $130^{\circ} \mathrm{C}$. for 4 hours was studied by Sherman and Grose (1923), by finding how much of the juice heated at each temperature as compared with untreated juice was necessary to maintain weight in carefully matched groups of rats during a similar eight-week experimental period. From their results the rate of destruction of vitamin $\mathrm{B}$ was calculated to be as follows : at $100^{\circ}$ C., 20 per cent; at $110^{\circ} \mathrm{C}$., 33 per cent; at $120^{\circ} \mathrm{C}$., 45 per cent; at $130^{\circ}$ C., 58 per cent. These figures establish a low temperature coefficient of destruction for vitamin $\mathrm{B}$, under such conditions. For an increase of $10^{\circ} \mathrm{C}$. in the range $100^{\circ}$ to $130^{\circ} \mathrm{C}$, the rate of destruction under the conditions described was increased about 1.4-fold as compared with the about 2 -fold increase in most chemical reactions.

The successive increases in the rate of destruction at $100^{\circ} \mathrm{C}$. of vitamin $\mathrm{B}$ in tomato juice resulting from definite changes in hydrogenion concentration, shifting the natural reaction of $\mathrm{pH} 4.28$ to $\mathrm{pH} 5.2$, $\mathrm{pH}$ 7.9, $\mathrm{pH} 9.2$ and $\mathrm{pH}$ 10.9, was investigated by Sherman and Burton (1926). Comparisons were made in solutions (filtered tomato juice) heated at the natural acidity, and in parallel portions unheated; and between portions heated at natural acidity ( $\mathrm{pH} 4.28)$ and portions heated after shifting the $\mathrm{pH}$ by graded additions of alkali $(\mathrm{pH}$ determined electrometrically). Hence their results may be stated both in terms of the approximate percentage of vitamin $B$ destroyed at each $\mathrm{pH}$ studied, and in terms of the increased destruction due to lowering the concentration of hydrogen ions during the process of heating.

The increased destruction of vitamin $\mathrm{B}$ over that at $\mathrm{pH} 4.28$ (natural acidity of clear filtered tomato juice), on heating for 1 hour at

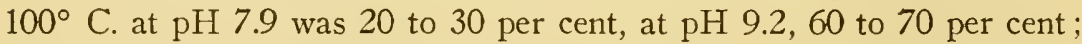
at $\mathrm{pH} 10.9,90$ to 100 per cent. On heating for 4 hours at the same temperature, the increased destruction over that occurring at $\mathrm{pH} 4.28$ was 10 to 20 per cent at $\mathrm{pH} 5.2 ; 60$ to 70 per cent at $\mathrm{pH} 7.9$.

Of the total initial amount of vitamin $\mathrm{B}$ the percentages destroyed by 1 hour of heating at $100^{\circ} \mathrm{C}$. were as follows : at $\mathrm{pH} 5.2$, about 10

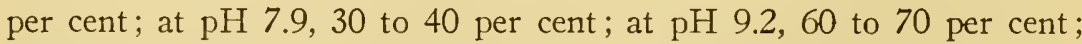
at $\mathrm{pH} 10.9,90$ to 100 per cent; while the corresponding percentages destroyed by 4 hours of heating at $100^{\circ} \mathrm{C}$. were: at $\mathrm{pH} 4.28$, about 20 per cent; at $\mathrm{pH} 5.2$, about 30 per cent; at $\mathrm{pH} 7.9$ about 70 per cent. 
The decrease in acidity from $\mathrm{pH} 4.28$ to $\mathrm{pH} 5.2$ increased the rate of destruction of vitamin $\mathrm{B}$ to about the same extent as did the increase in temperature from $100^{\circ}$ to $110^{\circ} \mathrm{C}$. in the experiments of Sherman and Grose, and a change from $\mathrm{pH} 4.28$ to $\mathrm{pH} 7.9$ accelerated the destruction to a greater degree than did a change in temperature from $100^{\circ}$ to $130^{\circ} \mathrm{C}$.

Sherman and Burton also found that this destruction of vitamin B appears not to be due in any appreciable degree to oxidation, for substantially identical results were obtained whether the heating was done in loosely covered flasks or under very strictly anaerobic conditions. This was true both when experiments were conducted on tomato juice at its natural acidity or at an alkalinity such that about one-half of the vitamin $\mathrm{B}$ was destroyed in the experimental heating of 4 hours. They suggested that the destruction of vitamin $B$ in these cases may have been due to an hydrolysis or intramolecular rearrangement, and that in either case the destruction reaction was catalyzed by hydroxyl ions. On both sides of neutrality, the rate of destruction was a function of the $\mathrm{pH}$ of the medium in which the vitamin $\mathrm{B}$ was dissolved.

Kinnersley and Peters (1928) reported that while alkali is destructive to the vitamin certain other factors appear to accelerate the inactivation. Whereas a less pure preparation (activity 1.0 milligram) was kept in 99 per cent alcohol made more acid than $\mathrm{pH} 2$ for 3 years at room temperature with no apparent alteration in activity, the purest preparations have been found to lose activity at room temperature in a few weeks under these conditions. In alkaline alcoholic solutions the vitamin was completely inactivated. They confirmed the stability of vitamin $\mathrm{B}$ to oxidizing agents in acid solution. Heating with 5 per cent nitric acid, or with potassium permanganate in acid solution or hydrogen peroxide in acid solution did not appreciably inactivate the vitamin, whereas hydrogen peroxide in alkaline solution at room temperature produced rapid destruction. Guha and Drummond (1929) reported upon the stability of a comparatively concentrated preparation of vita$\min B_{1}$ (charcoal concentrate) upon heating at different hydrogen-ion concentrations for different periods of time. Boiling for 24 hours at $\mathrm{pH} 1 \mathrm{did}$ not cause any appreciable inactivation, while at $\mathrm{pH} 5$ it destroyed about half; boiling for 1 hour at $\mathrm{pH} 9$ destroyed about half, while shorter periods of boiling resulted in correspondingly less destruction. They confirmed the finding of McCollum and Simmonds (1918), Peters (1924), and Levene (1928) that nitrous acid does not destroy vitamin $B_{1}$ (antineuritic). Neither their picrolonic acid filtrate 
fraction nor the charcoal concentrate obtained by the method of Kinnersley and Peters (1928) was appreciably inactivated by treating with hydrochloric acid and sodium nitrite, then warming for half an hour and finally boiling a few minutes.

Whitsitt (unpublished experiments) found that a more drastic treatment with nitrous acid than heretofore used seemed to result in a considerable destruction of vitamin B in "protein-free milk."

That vitamin B may be not wholly resistant to oxidation even at $\mathrm{pH} 3$ or $\mathrm{pH} 4$ is suggested by the fact that Halliday (1929) observed less inactivation of vitamin B when "protein-free milk" was shaken with Lloyd's reagent under an atmosphere of nitrogen than when this process was conducted in air.

\section{Solubility (Extractability)}

Sandels (1928) studied the solubility of vitamin B from yeast and ground whole wheat in 80 per cent (by weight) alcohol. Four hundred grams of the dry material were treated with 1,500 cubic centimeters of the alcohol, thoroughly stirred, and allowed to stand at room temperature $\left(20^{\circ}\right.$ to $25^{\circ} \mathrm{C}$.) for 24 hours, then filtered through a Büchner funnel and washed with 750 cubic centimeters alcohol, extracted a second time with 1,500 cubic centimeters alcohol, stirred, and allowed to stand again for 24 hours, after which it was filtered and washed as before. The residue was dried at room temperature. The extract was concentrated to approximately one-fourth of its original volume on a steam bath in 2 hours, and dried on cornstarch at room temperature before a fan.

The 80 per cent alcoholic extract of yeast appeared to contain much the larger portion (about three-fourths) of the antineuritic vitamin originally present in the yeast. The extracted residue, fed in equivalent amounts, was correspondingly inactive.

On the other hand, the 80 per cent alcohol extract of ground whole wheat appeared to contain about half as much vitamin $B$ as the untreated wheat, similar growth resulting from supplementing the basal diet 3 times per week with 1.6 grams whole wheat or the alcoholic extract of 3.2 grams whole wheat.

Alleman (unpublished experiments) finds no difference in the solubility (extractability from skimmed milk powder) of vitamin $B$, in 80 per cent alcohol and in alcohol of that strength containing 0.1 molar hydrochloric, benzoic or gallic acid. Bisbey (1930) finds that acidification of alcohol with acetic acid does not increase the solubility of vitamin $B$ either in 80 per cent or absolute alcohol. 


\section{Adsorption}

For many years it has been known that vitamin B (as the term has been heretofore understood) was readily adsorbed on charcoal, fuller's earth and other adsorbing agents as evidenced by the frequent use of this step in the processes of concentration. In the work of Salmon and Guerrant (1928) adsorption of vitamin B from extracts of yeast or maize with fuller's earth or Lloyd's reagent, was found to occur best at about $\mathrm{pH} 4$ (measured after the adsorption process). They considered that vitamin $\mathrm{B}$ behaved in adsorption like a basic substance, such as an alkaloid.

According to Kinnersley and Peters (1928), the other substances present, and perhaps the concentration of the vitamin, determine the $\mathrm{pH}$ at which adsorption most effectively occurs. They found that the relations of a highly purified concentrate to charcoal could not be defined with certainty, and that successive adsorptions removed no more of the vitamin. They believe that adsorption in the earlier stages is due to the presence of a coadsorbent. When yeast extract had not been treated with mercuric sulfate, adsorption of the vitamin took place at $\mathrm{pH} 5.0$ to 6.0 ; whereas when so treated it was best adsorbed at $\mathrm{pH}$ 7.0. Guha and Drummond (1929) confirmed these investigators' findings that vitamin $B_{1}$ is best adsorbed by norite from yeast extract at $\mathrm{pH} \mathrm{7,} \mathrm{but} \mathrm{pointed} \mathrm{out} \mathrm{that} \mathrm{under} \mathrm{their} \mathrm{conditions} \mathrm{it} \mathrm{is} \mathrm{best} \mathrm{adsorbed}$ by norite from an extract from wheat embryo at $\mathrm{pH} 4$ to $\mathrm{pH} 5$. Peters emphasized that the character of an adsorption is exceedingly variable, and that it is dangerous to draw conclusions from one class of mixtures and apply them to another.

Halliday (1929) made a quantitative comparison of the adsorption of vitamins $B$ and $G$ from "protein-free milk," using varying amounts of Lloyd's reagent ( 80 grams to 5 grams per liter of solution) with the hydrogen-ion activity of the solution adjusted to $\mathrm{pH} 4.0$ and to $\mathrm{pH} 3.0$. The activated solids were dried at room temperature and fed incorporated in the basal diet. The filtrates were evaporated to about onefourth of their volume under reduced pressure, at about $30^{\circ}$ to $40^{\circ} \mathrm{C}$., neutralized to about $\mathrm{pH} 5.7$, and fed as daily supplements. All portions were fed in quantities corresponding to definite amounts of milk powder or protein-free milk, the initial vitamin content of which had been determined. The results as a whole seem to indicate that equally good adsorption of vitamin $\mathrm{B}$ may be expected at hydrogen-ion activities of about $\mathrm{pH} 3$ to $\mathrm{pH} 5$ (measured at equilibrium). The amount of adsorbent may be varied within rather wide limits. The upper limit is governed by the amount which the animal can or will eat in addition 
to its basal ration. With the solutions used in this work it was found that, with 5 grams of adsorbent to a liter of solution, a demonstrable amount of vitamin $B$ was left in the filtrate.

\section{Other Factors}

(See the section on quantitative determination and the concluding paragraph of Chapter III.) 


\section{CHAPTER III}

\section{VITAMIN G $\left(\mathrm{B}_{2}\right)$}

\section{Differentiation of the Water-soluble Growth-promoting Vitamins}

Mitchell, in 1919, after referring to the then common belief that the antineuritic and water-soluble growth-promoting vitamins are identical, pointed out that "This belief ranges from positive conviction through tacit acceptance, to a frank weighing of the probabilities." His paper constituted an excellent review of the literature on the subject up to that time with a critical evaluation of the evidence then in hand. According to Mitchell, "The conclusion that the two vitamins are identical seems to be based upon the following grounds. (1) The distribution of the two substances in natural food products is very similar and the correlation between the actual amounts (in as far as these have been measured by biological tests) found in different products appears to be close. (2) The lack of known sources of water-soluble B in the diet of various species of experimental animals seems generally, if not always, to result in symptoms of nerve degeneration and paralysis. (3) Extracts of natural food products possessing growth-promoting properties are said to contain very probably only one indispensable vitamin, though supplementing satisfactorily a ration containing no other possible source of antineuritic vitamin. (4) The solubilities of the two vitamins in the common solvents are said to be identical. (5) Attempts at isolating the two vitamins have shown that they possess identical precipitants and adsorbents. (6) The stabilities of the two substances, especially to acids, alkalies, and elevated temperatures, seem to be similar if not identical." (It is to be kept in mind that this was published by Mitchell in 1919.)

In discussing the first point, attention was called to instances in the literature in which the correlation between the distribution of the antineuritic and the growth-promoting factors was not close. This seemed to be particularly the case in green vegetables, roots and tubers, which appeared to contain rich or moderate amounts of the growthpromoting water-soluble vitamin (or vitamins) (Osborne and Mendel, 1919, 1919c; McCollum, Simmonds and Parsons, 1918a; Sugiura and 
Benedict, 1918), and little if any of the antineuritic vitamin (McCollum and Kennedy, 1916; Chick and Hume, 1916-17; Vedder and Clark, 1912; Chamberlain, Vedder and Williams, 1912). While Mitchell pointed out that if this relation should be abundantly confirmed by future research "it may of itself effectively dispose of any contention of the identity of the two vitamins," he showed that the evidence as it stood at the time (1919) did not amount to finality on account of the variability of the vitamin content of vegetables, roots and tubers in different stages of freshness and maturity of the material, the lack of sufficient experiments on the antineuritic and growth-promoting properties of the same vegetable samples and the unreliability of experimental results in investigations of the comparative antineuritic properties of foods when polished rice, in itself defective in several factors other than the antineuritic vitamin, is used as the basal diet.

Experiments with unpolished rice were considered to afford some evidence of the lack of identity of water-soluble $B$ and the antineuritic vitamin, as shown by the observation of McCollum and Davis (1915b) that unpolished rice is adequately supplemented by casein, salt and butterfat, thus implying a sufficiency of water-soluble B; and of Gibson and Concepcion (1914) that unpolished rice does not furnish complete protection against polyneuritis in pigeons, which, if confirmed, would show an insufficiency of the antineuritic vitamin. The experience of other investigators, however, does not bear out that of Gibson and Concepcion.

The second point, that lack of vitamin $B$ in the diet of various species of experimental animals results in symptoms of nerve degeneration and paralysis, was dismissed with references to the observation of Osborne, Wakeman and Ferry (1919) that such symptoms do not always develop on a diet lacking in water-soluble B, and of Hart, Miller and McCollum (1916) that "malnutrition histologically characterized by nerve degeneration may result from the presence of toxic materials in apparently normal food products and in the presence of all known factors essential for continued growth and well-being." (It is now known that nerve symptoms usually develop in rats only in the presence of small but insufficient allowances of vitamin B. With complete deficiency, experimental animals may die before the neuritis develops.)

As an illustration of the third point, that similarity in extraction points to the identity of the two vitamins, the statement of McCollum and Simmonds (1918) was quoted; their argument of the improbability of two or more physiologically indispensable substances possessing the 
same solubility relations toward several solvents was criticized. "In fact it is quite conceivable that the treatment of natural foods with boiling alcohol breaks up combinations of vitamins with other substances so that the removal of the water-soluble B by this solvent may have been a combination of chemical and purely solvent action."

In reviewing the literature on the solubilities of the water-soluble growth-promoting and the antineuritic vitamin, both were shown to be soluble in water and dilute alcohol and, generally speaking, insoluble in fat solvents. Water-soluble B was said by Osborne and Mendel (1917a) and by Drummond (1917) to be insoluble in absolute alcohol, and by McCollum and Simmonds (1918), only incompletely soluble in 95 per cent alcohol. On the other hand, the antineuritic vitamin had been said to be soluble in absolute alcohol by Eijkman (1911), Funk (1911), Fraser and Stanton (1910) and others who had worked on its extraction and concentration. The conclusion that the growth-promoting factor is of somewhat lower solubility than the antineuritic was thought to be even more clearly shown in the case of acetone and benzene. From McCollum's laboratory, it had been reported that acetone and benzene extract the antineuritic vitamin from fat-free wheat embryo (McCollum and Kennedy, 1916) but that the same solvents do not extract the water-soluble growth-promoting substance to any appreciable extent (McCollum and Davis, 1915b; McCollum and Simmonds, 1918). After extraction with alcohol, however, the latter appears to be only slightly soluble in acetone but more readily soluble in benzene.

(Recently, however, Williams and Waterman (1926) repeated the experiments of McCollum and Simmonds, using rice polishings as the raw material and pigeons as the experimental animals in preventive tests, and found the active material to be soluble in benzene only in the presence of small amounts of water such as might readily be present in an alcohol extract. "It seems safe to assume," they concluded, "that the effective solvent is the low boiling ternary mixture of alcohol 18.5 per cent, water 7.4 per cent, and benzene 74.1 per cent, which distils off at $64.85^{\circ} \mathrm{C}$. from a mixture of any proportions.")

In discussing the attempts at isolating the two vitamins, Mitchell criticized the work of Funk and Macallum (1916a) and of Eddy (1916) on the ground that the basal rations used in making the feeding tests, besides their deficiency of water-soluble B, "could not have contained more than a trace of fat-soluble A." The results of Drummond (1917) while supporting "the view that water-soluble B and the antineuritic vitamin are identical do not constitute a demonstration. The 
lack of food intake records complicates their interpretation while the large losses of vitamin as soon as precipitation was resorted to find no parallel in analogous work with the antineuritic substance."

"The ready adsorption of water-soluble $B$ by precipitates of all descriptions postulated by Drummond, in explaining the large losses during chemical manipulation, does not seem to be a property of the antineuritic vitamin. Thus, Enmett and McKim (1917) show that while this vitamin is adsorbed by fuller's earth and Lloyd's reagent it is not adsorbed by the kieselguhrs or infusorial earths, indicating a selective adsorption by the former. Being readily dialyzable, there seems to be no compelling reason for believing it to be indiscriminately adsorbed by precipitates of all kinds."

With regard to the stability of the two vitamins, evidence was cited that both are stable to acids and to even concentrated alkalies at room temperature. At the boiling temperature the antineuritic vitamin appeared to be rapidly destroyed by alkali (Steenbock, 1917), while the growth-promoting vitamin was more stable although the results with alkalies were somewhat confusing (Drummond, 1917; Osborne, Wakeman and Ferry, 1919; McCollum and Simmonds, 1918; and Daniels and McClurg, 1919).

As to stability toward heat, the evidence was also conflicting for temperatures above $120^{\circ} \mathrm{C}$., although a greater stability of the growthpromoting vitamin was again indicated. (Chick and Hume, 1917b; McCollum and Davis, 1915c; McCollum, Simmonds and Pitz, 1917; Daniels and McClurg, 1915; and Drummond, 1917.)

Mitchell concluded that there seemed to be very good reason for doubting the identity of the two vitamins but that in settling the question definitely there was need of quantitative experiments in which the same materials are tested for their growth-promoting and antineuritic properties.

The following year Emmett and Luros (1920) reported an investigation of this kind, the results of which cast further doubt upon the then usual assumption that the antineuritic and water-soluble growthpromoting vitamin were identical. The particular phase of the problem considered was the stability of the water-soluble vitamin to heat. A compilation of the available data on this point, with some consideration of the possibility that the amount of ration consumed might carry an excess of the vitamin beyond minimum requirements and so mask partial destruction, showed that the antineuritic and the growth-promoting vitamins, as measured by polyneuritic pigeons and young rats respectively, were fairly stable at temperatures around $100^{\circ}$ to $105^{\circ} \mathrm{C}$., 
while at higher temperatures the antineuritic vitamin seemed to be less stable to heat and alkali than the rat growth-promoting vitamin.

Comparable results were obtained in a series of experiments in which the same source of water-soluble vitamins, unmilled rice, was used for studies on polyneuritis in pigeons, and on the rate of growth in young rats. In the case of pigeons, the unmilled unground rice constituted the sole food while with rats it was supplemented with lactalbumin, salt mixture, butterfat and lard to form a balanced ration for growth. The results obtained in feeding experiments in which the rice had been subjected to different heat treatments showed that "while the antineuritic vitamin is stable to heat at $120^{\circ} \mathrm{C}$. and 15 pounds pressure for 1 hour, partially altered by heating in the air oven at $120^{\circ} \mathrm{C}$. for 2 hours and totally destroyed at $120^{\circ} \mathrm{C}$. and 15 pounds pressure in 2 and 3 hours, the water-soluble B vitamin (rats) appears to be stable to heat at these same temperatures, that is, it is not distinctly or totally broken down.

"These findings suggest, tentatively, at least, that the antineuritic (pigeons) and the water-soluble $B$ (rats) vitamins are not the same, and that it would be better to consider them as being different until there is further proof to the contrary." The question could not then be definitely settled because of lack of quantitative methods and of accurate knowledge of the nutritional requirements of the experimental animals.

Funk and Dubin (1921) reported a separation by fractional adsorption of autolyzed yeast with norite of two vitamins of the B type, both of which fractions appeared to be needed by rats. On either alone, rats grew only for a few weeks at a slow rate, and after two months death ensued. Increasing the amount of either did not have any effect; feeding both produced rats normal in regard to growth and appearance. These findings were so briefly reported that they did not influence greatly the thinking on the subject.

Further evidence of the nonidentity of the two vitamins (or the interference of unknown factors) was furnished by Levene and Muhlfeld (1923) in a comparison of the behavior of three samples of bakers' and one of brewers' yeast in protective experiments with pigeons and growth experiments with young rats. The basal diet for the rats consisted of casein 18 , salt mixture 4 , starch 54 , butterfat 9 , lard 13, and cod-liver oil 2 parts. Young rats were placed in individual cages and fed this diet until they had definitely lost in weight, when the yeast was given in tablet form. Four or more animals were used for each sample of yeast tested. For the pigeon experiments, birds weighing over 300 
grams were used, three for each of the yeasts. The birds were kept in individual cages and were forcibly fed 20 grams of ground white rice, 18 cubic centimeters of distilled water, and the yeast, the experiment being continued for at least 63 days.

Extremely variable results were obtained with the different yeasts in the pigeon tests but fairly concordant ones in the rat tests. This was also true with the Osborne-Wakeman fraction II of the various yeasts. Moreover, although the concentration of the protective factor was higher in one of the samples of bakers' yeast than in the brewers' yeast, the condition was reversed in the respective fractions. It was doubtless the irregularity of results in the pigeon tests that led Levene to use the rat in his later attempts to determine the chemical nature of vitamin $\mathrm{B}$ as noted previously.

Hauge and Carrick (1926) criticized these studies of Emmett and Luros and of Levene and Muhlfeld in that the diets for the pigeons were deficient in substances other than water-soluble vitamins and that different species and different stages of life were involved in testing for the two factors. To avoid both of these sources of error, they used baby chicks as their experimental animals for testing both factors. Preliminary experiments had shown that with dried brewers' yeast as the sole source of vitamin B, young chicks grew very rapidly but developed polyneuritis in a short time; while with corn as the sole source of the vitamin, polyneuritis was prevented but growth was very slow. This suggested the probability that the corn was rich in the antineuritic factor but relatively poor in a growth-promoting factor, while the opposite was true of the yeast. To test this further, corn and yeast were fed at different levels, separately and combined, in rations otherwise identical and furnishing adequate protein, salts and fat-soluble vitamins. With 30 per cent of corn as the sole source of vitamin B, the chicks grew slowly and showed no symptoms of polyneuritis during the 18 weeks of the test; whereas, when the corn was replaced by yeast, the chicks grew rapidly but developed polyneuritis during the tenth week and died within two weeks thereafter. With 20 per cent of corn and 10 per cent of yeast, growth was rapid and only a few cases of polyneuritis developed. With lower levels of corn and yeast, some polyneuritis developed in all of the lots. Growth was rapid on as small an amount as 10 per cent of yeast and slow even with large amounts of corn.

In discussing these results Hauge and Carrick emphasized the nonidentity of the antineuritic vitamin and the so-called water-soluble, growth-promoting vitamin and the simultaneous occurrence of the two 
factors in varying proportions in different food materials, as shown by the supplementing action of one material for another in meeting the combined nutritional need of the prevention of polyneuritis and the promotion of growth.

That vitamin $\mathrm{B}$ as the term was then used might be a composite of two or more factors occurring in varying proportions in different materials had been suggested incidentally by McCollum, Simmonds and Becker (1925) in an attempt to explain the apparent inferiority of yeast as contrasted with wheat germ (as the sole source of vitamin B) in protecting rats against faulty salt mixtures. Suggesting as an explanation that vitamin B is more than one substance, they said, "It may be possible that yeast contains both of these principles but in different proportions than are contained in wheat germ, and that on this basis one may explain the inferiority of yeast as contrasted with germ in protecting the rats against our faulty salt mixtures. For example, one might speculate that there are two nutritive essentials composing vitamin $\mathrm{B}$, which may be designated as $y$ and $z$. In yeast, $y$ may be present in lesser amount than $z$, and so when the minimum amount of yeast is fed which can support growth in the rat when the rest of the diet is well constituted, $y$ is the limiting factor, and $z$ is fed more liberally than $y$. In wheat germ $y$ and $z$ might be present in more favorable proportions (in certain samples) than in yeast, so that when fed to the amount of 4 per cent of the diet, likewise well constituted, neither $y$ nor $z$ would be provided in minimal amount, hence the physiological stability would be greater in this case than when the minimum amount of yeast is fed. When the diet is appropriately constituted, little or no difference might be demonstrable between the values of yeast and germ as sources of vitamin B $(y$ and $z)$, but the superiority of one over the other might be demonstrable when the diet is made unfavorable through the addition of inappropriate salt mixtures."

In the course of an investigation of the influence of dietary deficiencies on experimental tuberculosis in the albino rat, Smith (1926) found that a diet containing 40 per cent of rolled oats (considered to be rich in vitamin B), together with an adequate amount of casein, salts, and vitamin A, failed to promote normal growth in young rats. An attempt to determine the nature of the deficiency in this diet led to the important discovery (Smith and Hendrick, 1926) that the diet could be made adequate for growth by the addition of 5 or 6 per cent of dried brewery yeast even after the yeast had been autoclaved for 6 hours at 15 pounds pressure, a procedure believed to destroy vitamin 
B. This demonstration that yeast contains a heat-stable factor essential for growth was confirmed by a further series of feeding experiments in which it was shown that young rats failed to grow on a diet of purified casein 18, salt mixture 4, 2 per cent Seidell vitamin B picrate in milk sugar 1, cod-liver oil 2, olive oil 8, and starch 67 parts, but began to gain weight promptly upon the substitution of 5 per cent of autoclaved yeast for an equivalent amount of starch. The same diet including the autoclaved yeast but not the vitamin $\mathrm{B}$ picrate resulted in a gradual loss in weight, followed by death within 3 or 4 weeks.

In another series of experiments, rats which were declining in weight on a vitamin-B-free diet were given graded amounts of a vitamin B fraction, with 500 milligrams daily of autoclaved yeast. The growth response to 2.5 and 5 milligrams daily of the vitamin B fraction in combination with the autoclaved yeast was approximately the same as that obtained with 200 and 500 milligrams, respectively, of dried brewery yeast under the same experimental conditions. Since fair growth resulted when the fraction was fed in doses of 15 milligrams or more without being supplemented with autoclaved yeast, it was concluded that some of the heat-stable factor was present in the yeast fraction.

Seidell (1926a) repeated the work of Smith and Hendrick with rats and also tested the supplementary effect of autoclaved yeast for one of his antineuritic concentrates in pigeon experiments. When young rats were fed a diet in which the antineuritic vitamin concentrate served as the sole source of vitamin $B$, there was no growth response until the autoclaved yeast was added, when there was a rapid response as had been noted by Smith and Hendrick. On the other hand, pigeons on a diet of polished rice supplemented by just enough of the vitamin concentrate to keep them at constant weight showed no growth response following the addition of autoclaved yeast. On the assumption that vitamin $B$ is composed of the antineuritic vitamin and another constituent, in itself active or inactive as regards growth, Seidell suggested the possibility that the rat (and probably other mammals) needs both of these constituents and the pigeon only the antineuritic vitamin. (Cf. Williams and Waterman, 1927-8.)

The paper of Smith and Hendrick was followed closely by one from Goldberger, Wheeler, Lillie, and Rogers (1926) in which it was suggested that the heat-stable substance in yeast is concerned with the prevention and cure of pellagra. A long-continued investigation of the relation of diet to pellagra, begun in 1914 by Goldberger, Waring and Willets of the U. S. Public Health Service, had led to the announce- 
ment by Goldberger and Tanner (1925) that, "in the prevention (and presumably causation) of pellagra there is concerned a heretofore unrecognized or unappreciated dietary factor, which we designate as factor P-P. This may be effective with but little, possibly without any, coöperation from the protein factor. Factor P-P may possibly play the sole essential rôle in the prevention (and causation) of pellagra. Factor P-P is present in brewers' yeast, in milk, and (on the basis of our experience with fresh meat) in lean beef; it is very low or lacking in dry soy beans, dry cowpeas, butter, cod-liver oil, and canned tomatoes."

The use of yeast in the treatment of pellagra was suggested by the favorable results which Goldberger and Wheeler had obtained with it in experimental black tongue of dogs, a disease which Chittenden and Underhill (1917) had shown to be very similar to, if not identical with, human pellagra and which Goldberger and Wheeler had produced in dogs by diets similar to those associated with the occurrence of pellagra. In their study of black tongue, white and yellow corn meal, casein, cod-liver oil, and butter had appeared to be very deficient or lacking in the black tongue preventive factor, milk to have slight but inferior preventive properties, and fresh lean beef and yeast to be very active, as was also Seidell's activated fuller's earth and autoclaved yeast. From the similarity in behavior of these substances in pellagra and black tongue, Goldberger and his associates were of the opinion that one and the same factor is responsible for the prevention of both these diseases.

After confirming the pellagra-preventing properties of beef and of fresh and autoclaved yeast in experiments upon human pellagra, Goldberger, Wheeler, Lillie and Rogers (1926) conducted a series of feeding experiments on young rats on a vitamin-B-free diet with various supplements with the following results: When 30 or 40 per cent of autoclaved yeast, believed to contain the pellagra-preventing vitamin but not the antineuritic, was fed to young rats on a diet free from water-soluble, growth-promoting vitamins, there was a short initial gain in weight, followed by rapid decline and death, with or without symptoms of polyneuritis. With a supplement of an alcoholic extract of corn meal (equivalent to 40 per cent of corn in the diet), capable of curing polyneuritis, there was again a brief gain in weight, followed by a rapid decline. When, however, a combination of as small an amount as 8 or 10 per cent of the autoclaved yeast and alcoholic extract of corn meal (equivalent to 5 per cent of corn in the diet) was fed, the rats grew normally. With 20 per cent of dried fresh lean beef, 
similar results were obtained as with the autoclaved yeast alone until the beef was supplemented with alcoholic extract of corn (equivalent to as little as 5 per cent of corn in the diet), when prompt recovery and growth followed.

These results are in harmony with those reported by other workers, especially Hauge and Carrick and Smith and Hendrick, but in the interpretation of them Goldberger and his associates went farther than these investigators through suggesting a correlation of the pellagrapreventing vitamin with the other factors present in yeast as follows:

"Thus, autoclaved yeast and beef muscle contain a factor distinct from the polyneuritis-preventing vitamin which in combination with the antineutric is essential for the growth of the rat. From the facts presented, it seems probable that this is the same as factor P-P, and some of the work in the very confusing literature relating to the identity of the 'growth-promoting' complex of 'water-soluble B' with bios appears to us to be in harmony with this interpretation. Further investigation will, however, be required to determine this.

"In any event investigators using the rat-growth test must hereafter recognize and take due account of at least two essentials (B sensu stricto and P-P) where heretofore only one was considered. This is, perhaps, of special importance to those heretofore occupied in the chemical isolation of the beriberi vitamin. It may well be suspected that the highly 'active' concentrates supposedly of vitamin B (sensu stricto) that some of these workers have succeeded in preparing, in proportion as they enable the rat to grow in the absence of any other source of the 'water soluble B' in the diet, are concentrates of at least two factors. The rat-growth test may continue to be used as a test of the purity of a concentrate, but must be interpreted in a sense opposite to that heretofore current. The pure concentrate will be seemingly inert. The complete test of such a concentrate (or a food substance) will necessitate combining it alternately with an adequate proportion of a proved preparation of the antineuritic and of the P-P factor, respectively, and, perhaps, of both, and this or some equivalent test will have to be made before an apparently inactive preparation (or 'food) can be adjudged as really inert. It is, at least, possible that in the past, workers in discarding 'inactive' fractions have unwittingly been throwing away the very thing they were laboriously seeking. This may perhaps explain, at least in part, the somewhat unaccountable losses of vitamin in the process of fractionation of 'active' preparations."

In a more detailed report of their study of the effect of the pellagrapreventing vitamin upon rats, Goldberger and Lillie (1926) illustrated 
the principle outlined in the preceding paragraph by tests in which the Seidell activated solid (activated fuller's earth) was used as the sole source of vitamin B for rats. When this was fed at a 5 or 6 per cent level, good growth to approximately adult size resulted. When, however, the amount was reduced to between 0.5 and 1 per cent, growth was quickly arrested and the rat declined without evidence of polyneuritis, but in some cases with the development of symptoms more or less suggestive of pellagra. On adding to the diet 9 per cent of autoclaved yeast or 6 per cent of a fuller's earth preparation activated with autoclaved yeast, growth was resumed with improvement and disappearance of the "pellagra-like" condition. This was considered to indicate that in Seidell's activated fuller's earth the limiting factor for growth was the thermostable substance.

The diet selected by Goldberger and Lillie for their study of the effect of lack of this factor alone upon young rats was the familiar vitamin $\mathrm{B}$-f ree diet of casein, salt mixture, cod-liver oil, hydrogenated cottonseed oil, and corn starch. To this was added an 85 per cent alcoholic extract of corn meal dried on cornstarch. Such an extract had been shown in their previous work to be rich in the antineuritic vitamin (B) but to contain little of the heat-stable antipellagric factor (now called vitamin G). On the basal diet supplemented by 6 per cent or more of this extract, healthy young rats after some initial growth invariably lost weight rapidly. An increase in the amount of extract even to 71 per cent of the ration failed to check this decline. In no case were symptoms of polyneuritis noted, but in many of the rats a condition showing considerable similarity to pellagra developed after varying lengths of time (never less than 7 weeks). The symptoms as described included a form of ophthalmia followed closely by a dermatitis, manifesting itself by a falling out of the fur and dry incrustations of the skin. Other symptoms characteristic of pellagra were occasionally noted, including ulceration at the angles of the mouth, inflammation of the anterior part of the floor of the mouth, and diarrhea. All of these symptoms if taken in time disappeared promptly upon the addition of 6 per cent of fuller's earth activated by autoclaved yeast. Goldberger and Lillie, although convinced of the similarity of this condition with human pellagra, were of the opinion that additional evidence is required to establish it beyond reasonable doubt.

Meanwhile Laird (1926), in McCollum's laboratory, found in the testing of numerous extracts obtained from natural foods by means of different (usually acidulated) solvents, many instances in which the antineuritic and growth-promoting potencies did not run parallel, and 
some cases in which growth was not promoted by either of two extracts but was induced by a mixture of them.

Further evidence that the term vitamin $B$ had formerly included at least two active factors, both of which are necessary for growth, was furnished by Salmon (1927) in the report of an investigation which had as its object (1) a comparison of the antineuritic and growthpromoting values of the same samples of plant materials and (2) a separation of extracts from these materials into fractions which might possess either the antineuritic or the growth-promoting action alone. The materials tested were seeds of soy beans and of velvet beans, and leaves of velvet beans and of rape. The seeds proved to be more potent than the leaves in their antineuritic value for pigeons and their protective value against polyneuritic symptoms and loss in weight in rats. The leaves had a much greater growth-promoting action for rats than the seeds. The observation that some of the rats on small allowances of the seeds showed a tendency to lose their fur in patches and occasionally to develop an inflammation around the eye was in harmony with the results reported by Goldberger and Lillie for similar diets.

The extracts used in the second part of the investigation consisted of fuller's earth fractions (activated solid) of alcoholic extracts of the seeds, and of acidulated extracts of the leaves prepared according to the customary method of concentrating the antineuritic vitamin; and the residue left after the fuller's earth adsorption of the leaf extract. All of the activated solids prevented polyneuritis in pigeons and rats. Relatively larger amounts were required when prepared from the extracts of the leaves than of the seeds. The residue had extremely weak antineuritic and growth-promoting action when fed alone but when added in small amounts with the antineuritic fractions showed marked growthpromoting properties.

Hassan and Drummond (1927) in the course of an investigation of the possible relation between vitamin $\mathrm{B}$ and protein metabolism obtained evidence of the multiple nature of vitamin $\mathrm{B}$ through the discovery that an extract of yeast autoclaved at $120^{\circ} \mathrm{C}$. for one hour at a strongly alkaline reaction and subsequently adjusted to the original hydrogen-ion concentration of the extract by the addition of acid, was capable of supplementing to a marked degree the growth-promoting effect of a yeast concentrate prepared by the Seidell (1926) method, although possessing no growth-promoting property when fed as the sole source of vitamin $B$.

At about the same time, Chick and Roscoe (1927) added to the 
rapidly accumulating evidence of at least two water-soluble growthpromoting factors by a series of experiments in which various extracts and preparations of yeast and of wheat embryo were used singly and combined as the source of vitamin $B$ in preventive and curative tests on rats. A concentrate of the antineuritic vitamin prepared by the Peters' method (vitamin extracted from charcoal concentrate by 50 per cent acidified alcohol) was found to be ineffective for growth until supplemented in this case by yeast heated at $120^{\circ} \mathrm{C}$. for 5 hours, when recovery and normal growth ensued. The autoclaved yeast by itself had no effect in curing the paralytic symptoms sometimes developing in rats on a vitamin B-deficient diet, but promptly relieved a pellagra-like condition similar to that described by Goldberger and by Salmon and observed to occur frequently when the Peters' concentrate constituted the source of vitamin B. Brewers' yeast was shown to be rich in both these factors and pure wheat embryo to be rich in the antineuritic vitamin, but deficient in the heat-stable factor; the Peters' concentrate was considered to be a good source of antineuritic vitamin uncontaminated by the heatstable factor, and autoclaved yeast to be devoid of the antineuritic vitamin.

Evidence of a more quantitative nature as to the supplementing growth-promoting action of one material for another and therefore of the existence of more than one water-soluble growth-promoting vitamin was obtained by Sherman and Axtmayer (1927) working with ground whole wheat, autoclaved yeast and dried skimmed milk as sources of vitamin $\mathrm{B}$ in quantitative feeding experiments. They fed comparable groups of rats the basal diet alone, or the basal diet supplemented by 0.8 gram daily of ground whole wheat, 0.8 gram of autoclaved yeast or 0.4 gram each of the wheat and yeast. The autoclaved yeast was incapable of sustaining growth as the sole source of water-soluble vitamins but was effectively supplemented by the wheat. Since autoclaving tends to destroy the heat-labile vitamin B the limiting factor for growth in wheat must be vitamin G. Using skim milk as a source of both vitamins, and supplementing it with autoclaved yeast and ground whole wheat respectively, it was found that vitamin $\mathrm{B}$ is the limiting factor in cow's milk, or that milk is relatively richer in vitamin $G$ than in vitamin $B$ while the reverse is true of wheat.

Salmon, Guerrant and Hays (1928), in fractionating an acidulated aqueous extract of velvet bean leaves, by treating the extract with fuller's earth, obtained a preparation which prevented beriberi in pigeons in daily doses of 0.01 gram but did not promote growth in rats in doses as high as 0.1 gram daily. They estimated that more than one- 
third of the antineuritic fraction (vitamin B) was adsorbed by the fuller's earth, while only a negligible amount of the P-P factor (vitamin $G$ ) was thus adsorbed. To obtain a concentrate of the latter free from the former, a combination of treatment with small amounts of fuller's earth to remove vitamin $B$, and fractionation of the resulting filtrate with alcohol was attempted. The filtrate left after the first adsorption with fuller's earth was concentrated by vacuum distillation and then treated with successive portions of fuller's earth. The resulting filtrate and washings were again concentrated, and 2 liters poured into 3 liters of 93 per cent alcohol according to the method of Osborne and Wakeman. The precipitate (Fraction I), after washing with 51 per cent alcohol, was rejected; the filtrate and washings were concentrated to 300 cubic centimeters and poured into 3 liters of 93 per cent alcohol. This second precipitate was mixed with cornstarch, macerated in absolute alcohol, filtered, washed with absolute alcohol, and dried at from $50^{\circ}$ to $60^{\circ} \mathrm{C}$., making Fraction II. The remaining filtrate and washings were reduced to small volume by vacuum distillation, taken up on cornstarch, and dried (Fraction III).

Fraction II, by itself, had no growth-promoting properties, but prevented pellagra-like symptoms in rats. When combined with a small amount of the antineuritic factor, growth was promoted. Fraction III was found to retain more of the antineuritic factor (vitamin B) than Fraction II, and to have a more laxative action.

In discussing their experimental work, the authors advanced the opinion that in the preparation of a vitamin G concentrate, it is preferable to remove vitamin $\mathrm{B}$ as completely as possible by the method outlined.

They found that the maximum adsorption of the vitamin $G$ by fuller's earth occurred at $\mathrm{pH} 0.08$, the most acid solution tested. This adsorption decreased gradually to a minimum at $\mathrm{pH} 6.3$, and remained approximately constant to $\mathrm{pH} 12.07$, the most alkaline solution tested. Fuller's earth seemed to be a more efficient adsorbent for vitamin $B$ than for vitamin G. They pointed out certain discrepancies which they interpret as suggesting that vitamin $\mathrm{G}$ may be a composite rather than one substance. (Salmon, Guerrant and Hays, 1928; Salmon, Hays and Guerrant, 1928.)

Hogan and Hunter (1928) reported that exposure of materials containing the vitamin $\mathrm{B}$ complex to ultra-violet light apparently does not affect the antineuritic properties (vitamin B), but does destroy the activity usually described as growth-promoting (vitamin G). However, Kennedy and Palmer (1929) do not confirm these authors that ultra- 
violet irradiation can be relied upon to destroy vitamin $G\left(B_{2}\right)$. See also Chick and Roscoe (1929).

Willians and Waterman $(1927,1928)$ postulated the tripartite nature of "vitamin B" in reporting the results of a quantitative comparison of the effects upon adult pigeons and young rats of various yeast preparations used as supplements to the Sherman-Spohn watersoluble-vitamin-free diet. The yeast preparations included (1) an aqueous extract of brewers' yeast, (2) fuller's earth activated with the aqueous extract of brewers' yeast with the interposition of a collodion membrane, and (3) brewers' yeast autoclaved for 6 hours at $121^{\circ} \mathrm{C}$.

Pigeons on this test diet without supplement declined in weight to from 60 to 70 per cent of normal and died of polyneuritis in 30 to 40 days. When the diet was supplemented by 0.2 to 0.4 gram of the yeast extract, by 0.01 gram of activated fuller's earth, or by 0.01 gram of activated fuller's earth plus 1 gram of autoclaved yeast, the pigeons showed slight loss in weight and a slight dejection but were otherwise healthy. The addition of minute quantities of whole wheat or untreated yeast prevented this loss, and promoted growth. A repetition of these experiments substituting polished rice for the Sherman-Spohn diet gave essentially the same results, except that the birds receiving no supplement died sooner.

Young rats receiving no supplement lost weight rapidly and died of polyneuritis. On the basal diet supplemented with 0.03 gram of yeast extract plus 1 gram of autoclaved yeast or with 0.01 gram of activated fuller's earth plus 1 gram of autoclaved yeast, growth was normal. With 0.01 gram of activated fuller's earth as the supplement, there was slow growth at first, followed by a decline of several weeks' duration. With 1 gram of autoclaved yeast as the supplement there was slight growth, followed by decline, polyneuritis, and death.

The authors interpreted these findings to confirm the conclusion of previous workers that at least two factors formerly included in the concept "vitamin B," a heat-labile vitamin (B) and a more heat-stable vitamin (G) are necessary for the growth and well being of young rats. Pigeons appear not to require the heat-stable factor, but to need in addition a third factor which is found in untreated whole wheat or yeast, but not in autoclaved yeast nor in some purified antineuritic preparations.

Experimenting with pigeons on a "synthetic diet," adequate except for the "vitamin B complex," Lecoq (1927) and Randoin and Lecoq (1927d) found very different values for different yeasts and their extracts as sources of the substance necessary to prevent polyneuritis and 
of that required to maintain weight and promote growth. The requirement of this latter factor seemed to be dependent upon the type and proportion of carbohydrate furnished in the basal diet.

Hunt $(1928,1928 \mathrm{a}, 1928 \mathrm{~b})$ has reported the existence in yeast of three vitamins necessary for the growth of rats.

\section{Concentration of Vitamin G}

The finding that Peters' antineuritic concentrate made from yeast extract is practically free from vitamin $G\left(B_{2}\right)$ led Chick and Roscoe (1929a) to study at what point the latter is left behind in the process of fractionation, and to attempt a separation of vitamin $G\left(B_{2}\right)$ free from vitamin $B\left(B_{1}\right)$. They found that the vitamin $G\left(B_{2}\right)$ of the acidulated aqueous extract of washed brewers' yeast is precipitated largely by lead acetate, the amount depending upon the acidity, and that most of any remainder was precipitated with the barium hydroxide. At $\mathrm{pH} 2.6$, the lead acetate precipitated about one-half of the vitamin $\mathrm{G}\left(\mathrm{B}_{2}\right)$; at $\mathrm{pH} 4.7$, about three-fourths; and in neutral or alkaline medium, practically all. Under these conditions they were unable to separate vitamin $G\left(B_{2}\right)$ from vitamin $B\left(B_{1}\right)$; about one-half of the original vitamin $\mathrm{B}$ being found in the vitamin $\mathrm{G}$ fraction, the amount apparently depending both on the acidity and the relative amounts of the vitamins in the source material.

These authors found that after an acidified ( $\mathrm{pH} 3$ ) aqueous extract of yeast was dialyzed for 4 days in a refrigerator in a "cellophone" bag against distilled water, the concentration of vitamin $G\left(B_{2}\right)$ was the same on both sides of the membrane. In their experience, ultraviolet irradition inactivates both vitamins $B\left(B_{1}\right)$ and $G\left(B_{2}\right)$, the latter to a greater degree. They confirmed earlier workers in the finding that vitamin $\mathrm{G}\left(\mathrm{B}_{2}\right)$ is insoluble in 83 to 95 per cent alcohol. The precipitate formed when an aqueous (acid) concentrate of this vitamin was slowly added with vigorous stirring to a large volume of 94 per cent alcohol, was much reduced in vitamin potency after repeated washings and shaking with 94 per cent alcohol. This loss appeared not to be due either to mere prolonged contact with air at room temperature in the dry condition, nor could it be explained only on the basis of contact with alcohol of that strength. They suggested that the destructive effect of alcohol on vitamin $G\left(B_{2}\right)$ may be related to the reaction of the solution.

Chick (1929) found no diminution of the vitamin $B_{2}$ (G) potency when the amino nitrogen of the preparation was reduced to one-sixth of the original by the action of nitrous acid. The treatment consisted 
of adding 50 cubic centimeters water, 5 cubic centimeters of a 30 per cent solution of sodium nitrite and 10 cubic centimeters of 10 per cent sulfuric acid to 50 cubic centimeters of a concentrate of vitamin $B_{2}(G)$. The mixture was shaken 5 minutes, allowed to stand overnight, heated on a water bath until foaming ceased, and then boiled for 10 minutes.

In 1930 Narayanan and Drummond reported success in separating bios from vitamin $\mathrm{B}_{2}(\mathrm{G})$. This they did by concentrating in vacuo a yeast extract prepared from brewers' yeast at $60^{\circ}$ to $70^{\circ} \mathrm{C}$. with alcohol of 50 per cent concentration, hydrolyzing the product at 15 pounds pressure with 20 per cent barium hydroxide, freeing the filtrate from barium with dilute sulfuric acid, and treating with an excess of lead acetate solution (110 grams lead acetate to the extract from 7 pounds yeast). The resulting filtrate and precipitate were both treated with hydrogen sulfide, and the lead-free liquids were brought to $\mathrm{pH} 6.8$. Vitamin $G\left(B_{2}\right)$ was found to be concentrated in the fraction prepared from the lead acetate precipitate whereas bios remained in the fraction made from the filtrate.

These investigators attempted to concentrate vitamin $B_{2}(G)$ from the lead acetate precipitate obtained as above with or without the alkaline hydrolysis step. The precipitate was decomposed by suspending in warm water and slowly adding 10 per cent sulfuric acid with constant stirring until acid to Congo red, whereupon the lead sulfate was removed by filtration and the filtrate neutralized with sodium hydroxide. The filtrate and subsequent fractions were tested for the vitamin by finding the minimal daily doses which, when fed to rats depleted of their bodily reserves of vitamin $B_{2}(G)$ (vitamin $B$, furnished by the Kinnersley and Peters concentrate), permitted a gain of at least 10 grams weekly during a three weeks test period.

The filtrate, active in daily doses of 10 to 15 milligrams organic matter, was subjected to adsorption at various hydrogen-ion activities by fuller's earth and by norite, and to fractional precipitation with alcohol. Between $\mathrm{pH} 6.8$ and $\mathrm{pH} 0.1$ adsorption of vitamin $\mathrm{B}_{2}(\mathrm{G})$ on fuller's earth increased as the acidity increased, being almost complete at $\mathrm{pH} 0.1$. Using 3 grams fuller's earth per 100 cubic centimeters liquid ( $0.9 \mathrm{~N}$ sulfuric acid), stirring for $1 / 2$ hour, and washing with $0.9 \mathrm{~N}$ sulfuric acid resulted in removing from the filtrate 40 per cent of the total solids and organic material and producing a fuller's earth active in daily doses of 40 milligrams, carrying 6 milligrams adsorbed organic matter. No satisfactory method was found for extracting the vitamin from the fuller's earth. Several reagents were found ineffective, among which were cold barium hydroxide, alkaline to bromocresol purple; 50 
per cent alcohol containing 0.1 per cent sulfuric acid used in 2 successive extractions of 1 hour each at $60^{\circ}$ to $70^{\circ} \mathrm{C}$.; 50 per cent alcohol containing 0.1 per cent sodium hydroxide; and hydrochloric acid ( $\mathrm{pH} 6.8$ ). Under the latter condition the potency of the solids was reduced, but the extracted material was inactive. Norite appeared to be a much less satisfactory adsorbent than fuller's earth.

Concentrating an aqueous extract $(\mathrm{pH} 6.8)$ to a thick syrup and extracting this product with 50 per cent alcohol appeared to inactivate the vitamin completely.

Eighty-eight per cent of the vitamin $B_{2}(G)$ in the filtrate resulting from the decomposition of the lead acetate precipitate remained in the supernatant liquid and washings when the fraction was brought to a concentration of 50 per cent alcohol. When the concentration of alcohol was increased to 70 per cent, the supernatant fluid was inactive and the precipitate contained most of the vitamin (active in daily doses of 6 milligrams organic matter).

These investigators found no appreciable destruction of vitamin $B_{2}(G)$ when the yeast extract was boiled with 10 to 15 per cent sulfuric or hydrochloric acid for 24 hours, or treated with 10 to 15 per cent barium hydroxide at $110^{\circ}$ to $120^{\circ} \mathrm{C}$. for from one to three hours. The vitamin appeared to be stable to hydrogen peroxide and to nitrous acid. It seemed very soluble in water and in 50 to 60 per cent alcohol, but only sparingly soluble in alcohol of higher concentrations and practically insoluble in higher alcohols, as butyl.

None of the following substances was found active as vitamin $G\left(B_{2}\right)$ in doses of 1 to 5 or 1 to 10 milligrams daily: yeast nucleic acid, guanine hydrochloride, adenine sulfate, Funk's compound of m. p. $234^{\circ} \mathrm{C}$, , betaine hydrochloride, inositol, potassium pyrophosphate, or the non-saponifiable fraction from yeast fat.

Both Chick and Roscoe (1928) and Williams, Waterman and Gurin (1929) pointed out that although vitamin $B_{2}(G)$ of yeast is more heat stable than vitamin $B_{1}(B)$, the former is by no means entirely thermostable, especially under alkaline conditions. Chick and Roscoe (1930) reported further that vitamin $B_{2}(G)$ is comparatively stable to prolonged heating when the reaction is acid ( $\mathrm{pH} 5.0$ to 3.0 ), about half remaining after heating for 4 hours at $122^{\circ}$ to $124^{\circ} \mathrm{C}$. When the reaction was alkaline ( $\mathrm{pH} 8.0$ to 10.0 ), about 30 per cent of the activity was lost in 10 days at room temperature; 50 per cent upon steaming for 2 hours; and 75 to 100 per cent upon heating at $122^{\circ} \mathrm{C}$. for 4 to 5 hours.

Levene (1930) described a method of separating vitamin $G\left(B_{2}\right)$ 
from $B\left(B_{1}\right)$ as follows: "Fraction $B_{1}$ is adsorbed on silica gel at $\mathrm{pH} 3$. The filtrate is rich in vitamin $B_{2}$, but still contains some vitamin $B_{1}$. By precipitation with acetone a material is obtained of which daily doses of 0.015 gram in addition to 0.0002 gram of $B_{1}$, both added to the standard diet, suffice to maintain normal growth of white rats. By repeating the extraction six times, a material is obtained from the filtrate of which daily doses of 0.005 gram are required. Finally, when this material is dissolved in water and precipitated with alcohol containing 1 per cent of hydrochloric acid, a material is obtained of which daily doses of 0.0007 gram suffice to maintain normal growth of white rats."

\section{Quantitative Determination of Vitamin G}

When experimental animals used in vitamin research attain normal growth, it must be remembered that the nutritional essentials consciously provided in the basal diet may be supplemented (a) by unrecognized constituents in the basal diet or (b) in the test materials carrying the particular factor directly under observation, (c) by bodily reserves of the experimental animal, or (d) by a combination of these. Sudden and marked changes in the rate of growth, unequal effects upon growth of doubling the vitamin intake from different source materials, and supplementary effects in growth-promoting values of two materials are some of the indications that the basal diet is not complete qualitatively or quantitatively in all of the nutritional essentials other than the factor under consideration.

In the fractionation of the vitamin $\mathrm{B}$ complex it has become increasingly evident that the effects formerly ascribed to "water-soluble B" are due to more than two chemical entities.

Possible Interfering Factors in Vitamin Determinations.-In studies conducted before the report of Steenbock, Sell and Nelson (1923) to the effect that coprophagy diminished by half the apparent vitamin B requirements of rats, Palmer and Kennedy had used, with good results, a hot alcoholic extract of wheat embryo as a source of the water-soluble vitamins. But in papers published in 1927 and 1928 they pointed out that when coprophagy is prevented and a highly purified casein serves as the source of protein, this ration fails to support the growth of young rats for more than six or seven weeks, or the maintenance of adults except for a few weeks. They believe that this ration contains sufficient of both vitamins $\mathrm{B}$ and $\mathrm{G}$ inasmuch as it is not improved by the addition of Osborne and Wakeman's yeast concentrate "fraction II," Harris yeast powder, or by a cold alcoholic extract of yeast. The facts that 
the ration can be supplemented by untreated or autoclaved brewers' yeast ( $21 / 2$ hours at 15 pounds pressure), and by the residue left after preparing the Osborne-Wakeman fraction II from fresh brewers' yeast (a product deficient in both vitamins $B$ and $G$ ) led them to believe that these latter materials contain a new factor only slightly soluble in water or in hot 85 to 90 per cent alcohol, a factor similar to but not identical with that discovered by Smith and Hendrick (1926) and by Goldberger and his colleagues (1926). It appeared to be present in dried boiled beef, egg white, summer milk, and the casein and butter of summer milk, but not to any extent in wheat embryo or yeast extracts. According to their work, this material can be extracted from the feces of rats by hot 90 per cent alcohol in 24 to 32 hours, appears to be insoluble in ether, and seems less stable to heat in feces than in yeast.

Hunt (1928b) also reported the presence of a factor in the autoclaved residue of washed autolyzed yeast which supplements the growth promoting value of vitamin $B$ and vitamin $G$ concentrates.

Reader (1928, 1929, 1929a, 1930) has demonstrated the existence (in a yeast preparation and in the mercuric sulfate precipitate of the Kinnersley and Peters' process of concentrating vitamin $\mathrm{B}$ ) of a factor alkali-labile and more thermolabile than vitamin $B\left(B_{1}\right)$ without which the growth of young rats fails in 4 or 5 weeks when placed at weaning on a basal diet reinforced with vitamins $A, B_{1}(B), B_{2}(G)$, and $D$.

Coward and her coworkers (1929) announced the existence of a factor distinct from vitamins $A, B_{1}(B), B_{2}(G), D$, and $E$, not present to an appreciable extent in dry yeast or marmite but associated with fresh milk, certain caseins, grass, ox muscle, ox liver, and wheat embryo. This factor appears to be relatively insoluble in cold 2 per cent acetic acid, 97 per cent alcohol, or ether, but some of it was extracted from certain caseins and to a greater degree from wheat embryo, by four successive extractions of 6 hours each with hot alcohol or ether. Simmering fresh milk for 15 minutes appeared to reduce its potency; the value of dry casein was somewhat lowered by heating for seven days at $107^{\circ} \mathrm{C}$., but not (measurably) by such heating for one day. The appearance of a deficiency of this factor was evident at an earlier age in animals used in vitamin $B$ experiments than in those used in vitamin A studies.

Chick and Roscoe (1929) observed that egg white although a good source of vitamin $G\left(B_{2}\right.$, antidermatitis factor) lacks some growth essential present in autoclaved yeast. In a few instances when egg white was used as a source of vitamin $G\left(B_{2}\right)$ the growth of rats was inhibited by the sixth or seventh week of age, but it usually remained 
unaffected until 9 to 13 weeks of age, by which time the animal had attained a body weight of 100 to 130 grams. No symptoms of ill health were found in rats observed 3 to 4 months after growth had become subnormal. These findings indicate the non-identity of the "antipellagric" and a second heat-stable growth factor, or in other words, the existence here of another mammalian growth essential beyond vitamin G.

Salmon, Hays and Guerrant (1928) also reported that certain discrepancies observed in their laboratory point to the non-identity of the "pellagra-preventing" factor and some factors other than vitamin B $\left(B_{1}\right)$ needed for growth. Williams and Eddy (1929) are studying this problem by examining the physiological properties of various preparations made by fractioning an autolyzed yeast extract.

Each investigator must assure himself that no factor other than vitamin $B\left(B_{2}\right)$ is limiting growth in experiments designed to measure vitamin $G\left(B_{2}\right)$ potency. This may be done either by incorporating these additional factors in the basal diet, or by limiting the experimental period to that time during which the bodily store of these additional factors suffices to maintain the desired rate of gain. So far as can be judged from the available evidence, the bodily store of these additional factors usually far exceeds the store of vitamins B and G. Studies on the problem of amply insuring the basal diet in these factors are in progress. Until this can be accomplished the term vitamin $G\left(B_{2}\right)$ may occasionally designate not a single substance, but the mixture of factors differentiated from the antineuritic vitamin but not yet fully differentiated from each other.

Quantitative measurement of vitamin $\mathrm{G}\left(\mathrm{B}_{2}\right)$ by the rat-growth method requires supplementing a basal diet free from all water-soluble vitamins with a source of the relatively heat-labile factors comparatively deficient in the water-soluble, more heat-stable factors. Chick and Roscoe (1927) found Peters' antineuritic concentrate (Peters 1924; Kinnersley and Peters 1925) to be such a product. They used the fraction obtained by extracting with 50 per cent acidified alcohol the charcoal concentrate made from an aqueous yeast extract previously treated with lead acetate, mercuric sulfate and hydrogen sulfide. This preparation was concentrated at a low temperature under reduced pressure, and the residue was taken up in water so that 0.1 cubic centimeter corresponded to 0.6 gram of dry yeast, an amount which they found amply sufficed for the daily needs of the rat for vitamin $B_{1}$.

Evans and Burr (1928c) used tikitiki, a dilute-alcohol extract of rice polishings, as a source of the antineuritic vitamin with little of the other water-soluble growth-promoting vitamins. 
Goldberger and his coworkers (1926) found an 85 per cent alcohol extract of corn meal, and Sherman and Sandels (1929) an 80 per cent alcohol extract of ground whole- wheat, good sources of vitamin $B$ $\left(B_{1}\right)$ very deficient in vitamin $G\left(B_{2}\right)$.

In developing their method for the determination of vitamin $G$ $\left(B_{2}\right)$ Chick and Roscoe (1928) obtained evidence that casein purified by washing with acidulated water and prolonged heating at $120^{\circ} \mathrm{C}$. still contains considerable amounts of vitamin $G$, an observation confirmed by the report of Goldberger and his associates (1930) to the effect that casein still may have pellagra-preventing value after being leached in acidulated water for a week with the water changed daily. By prolonged extraction with 0.05 per cent acetic acid, followed by extraction with 50 or 70 per cent acidified alcohol, drying and roasting for 3 days at $120^{\circ} \mathrm{C}$., Chick and Roscoe believe they can render their casein practically free from vitamin $G\left(B_{2}\right)$.

These investigators prepare their test animals for vitamin $G$ measurements by confining them to a diet presumably free from all watersoluble vitamins for a week to 10 days, after which they transfer them to separate cages with raised screen bottoms and supply a daily dose of Peters' antineuritic concentrate, to which the animal generally responds by a temporary increase in weight. After 3 or 4 weeks from the beginning of the experiment, the material to be tested for vitamin $G$ is given in graded doses, and the minimum dose permitting an average weekly increase of from 10 to 12 grams during a 2- to 4-week experimental period, is taken as a standard of comparison.

In the experience of Chick and Roscoe concordant results can be obtained so long as the animals used have not been kept longer than 6 to 7 weeks on the deficient diet, and they held that each animal could be satisfactorily used for two successive test periods of 2 or 3 weeks each one after the other if the second dose of vitamincontaining material were larger than the first or after a 2-week depletion period in case the succeeding dose were smaller than the first.

Aykroyd and Roscoe (1929) noted that young rats when first caged singly and put on a strange diet may make very little growth during the first week, even on a good diet. They therefore disregard the first week's growth, and use as the standard for comparison the average weekly growth during the following four weeks, the relative vitamin $G\left(B_{2}\right)$ values of foodstuffs being estimated from the minimum amounts required daily to maintain a weekly gain of $11-14$ grams during those weeks. They use male and female test animals in approximately 
cqual numbers and follow the general technique of Chick and Roscoe (1928).

In the method developed by Bourquin (1929) from the ShermanSpohn (1923) method described in Chapter II vitamin B $\left(B_{1}\right)$ is supplied, as suggested by the work of Sandels, by incorporating into the basal diet an alcoholic extract of ground whole wheat. This extract is prepared as follows:

Eight hundred grams of freshly ground wheat is shaken with 1.5 liters of 80 per cent alcohol by weight, for an hour and a half. The extract is then filtered through a Büchner filter with suction. The residue is again treated with a liter of 80 per cent alcohol, shaken for an hour, filtered and the residue washed with 300 cubic centimeters of the alcohol. The combined extracts are concentrated in vacuo for an hour to an hour and a half or until about one-fourth the original volume, poured upon 300 grams of cornstarch and dried under an electric fan at room temperature. In the preparation of the alcohol allowance is made for the water content of the wheat (average about 10 per cent).

Her modification of the Sherman-Spohn diet has the following composition: purified casein, 18 per cent; Osborne-Mendel salt mixture, 4 per cent; butterfat, 8 per cent ; cod-liver oil, 2 per cent ; cornstarch, 68 per cent, a part of which carries the alcoholic extract from 50 grams of whole wheat for each 100 grams of diet.

For use in these experiments are recommended young normally growing animals (rats) 28 to 29 days old, reared by mothers on a diet consisting of two-thirds finely ground whole wheat, one-third whole milk powder, and sodium chloride to the extent of 2 per cent of the wheat with a daily addition of about 5 grams of fresh lean beef per adult. They are confined in cages with raised screen bottoms and fed the basal diet alone until they cease to grow (14 to 18 days), at which time the test period proper is begun. For quantitative comparative studies all the precautions necessary to insure accuracy as discussed in connection with vitamin $\mathrm{A}$ and vitamin $\mathrm{B}$ determinations should be observed.

Animals receiving the basal diet alone, "negative controls," almost invariably live throughout an 8-week experimental period, losing weight very slowly but steadily. The work of several independent investigators in our laboratory shows that under the conditions briefly outlined here the gain in weight over the negative controls is proportional to the allowance of vitamin $\mathrm{G}$ within those amounts permitting from 2 to 5 grams gain per week for an 8-week period, when natural food materials 
serve as carriers of the vitamin, even though the concentrations of the vitamin vary widely. When growth is thus restricted the response by gain in weight to small additions of vitamin is quite delicate and the bodily vigor of the animal is sufficiently maintained that the physiological variation does not vitiate average results. Average curves of growth at different levels of feeding are shown in Fig. 6.

In the Bourquin experiments summarized in Fig. 6, the vitamin $G$ was fed in the form of milk which contains all the known vitamins and appears to be relatively rich in both or all of the heat-stable factors of the vitamin-B complex (i.e., of the other factors mentioned at the

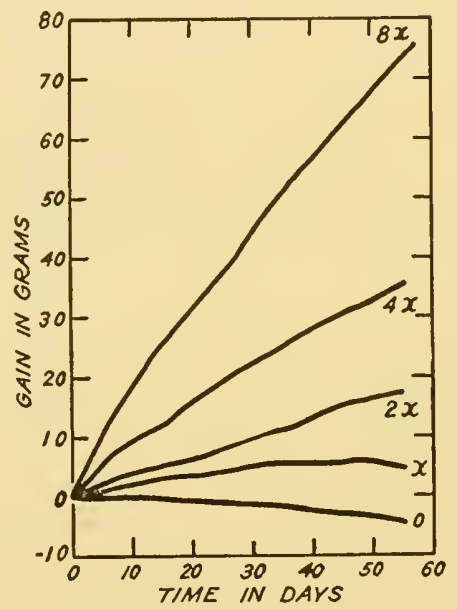

FIG. 6.-Average growth curves of rats receiving different amounts of vitamin G. The lowest curve shows the gradual loss of weight on the Bourquin basal diet alone; the others, the effects of feeding $x$ amount per day of vitamin $\mathrm{G}$ in the form of milk, and 2, 4, and 8 times this amount. (Courtesy of Dr. Anne Bourquin.)

end of this chapter as well as of vitamin $G-G_{1}, B_{2}$ itself). This fact, together with the use of test animals whose previous diet had given them opportunity to gain good bodily stores of the newer factors, helped to permit the steady gains shown in these curves. Where either of these two conditions is less favorable, there is apt to be a flattening of the curves. This is more fully discussed elsewhere in this chapter.

Numerous data not yet published indicate that if test animals of a less satisfactory nutritional history are employed, particularly when fractions of food products rather than the natural materials are being tested for their vitamin $G$ values, quantitative estimations of the vitamin content should be based on experiments terminated after 4 or 5 
weeks. In one investigation conducted under these modified conditions, between 80 and 90 per cent of the animals (124 cases) maintained fairly uniformly the restricted rate of growth during the first four weeks, but only about 20 per cent could maintain this same rate of gain throughout an 8-week experimental period. Usually between the fifth and seventh week of the experimental period, the growth curve tended to flatten; often the animals thereafter merely maintained their body weight, or suffered a decline. In view of this fact, and the findings that the least variability in response is encountered in the early weeks of the experimental period, it is tentatively suggested that all quantitative determinations be based on a 4 - or 5-week experimental period. If, as sometimes happens, the animal declines in weight during the first week or so of the test period, while adjusting itself to new conditions, it is suggested that the animal be weighed two or three times weekly, until the minimum body weight has been found, and the experimental period be counted as beginning at that point and continued throughout the following four weeks, the rate of growth being restricted by vitamin intake, as suggested by Bourquin, to three or five grams per week.

\section{Quantitative Studies of Some Properties of Vitamin G}

Sherman and Sandels $(1929,1931)$ studied quantitatively the solubility of vitamin $G$ in neutral alcohol of certain concentrations. As their results showed that relatively more of this vitamin can be extracted from ground whole wheat than from dried bakers' yeast by 80 per cent (by weight) alcohol, it would seem necessary to consider the physical and chemical nature of the source material as well as the solvent and method of extraction in evaluating the evidence regarding the solubilities (more strictly speaking, extractabilities) of these vitamins.

The essentials of their method of extraction were as follows: 400 grams of air-dry material was treated with 1,500 cubic centimeters of alcohol, thoroughly stirred and allowed to stand at room temperature $\left(20^{\circ}\right.$ to $25^{\circ} \mathrm{C}$.) for 24 hours; then filtered with suction and the residue washed on a Büchner filter with 750 cubic centimeters of alcohol of the same strength; again stirred with 1,500 cubic centimeters of the alcohol, allowed to stand 24 hours, filtered and washed as before. The residue was dried in the air at room temperature. The extract obtained by combining the two filtrates and the washings was concentrated on the steam bath and evaporated at room temperature on cornstarch.

This treatment of dried yeast with 95 per cent alcohol did not measurably diminish its vitamin $G$ potency; the residue appeared as 
active as the untreated yeast. Extraction with 60 per cent alcohol removed between one-half and three-fourths of the vitamin $G$ (assuming no destruction of vitamin in the residue). The extract in the form in which it was fed was much less potent than the residue; it appeared that over half of the vitamin thus extracted and dried was inactivated. Hence in interpreting their results, the degree of solubility was estimated largely from the vitamin potency of the residual extracted material. From this viewpoint it could be shown that there is progressively greater solubility of vitamin $\mathrm{G}$ in the more aqueous solutions of alcohol. From dried bakers' yeast, alcohol 95 per cent by weight extracted no measurable amount of vitamin G; alcohol 80 per cent by weight extracted about one-quarter; and alcohol 60 per cent by weight, about one-half of this vitamin.

As noted above, considerable of the extracted vitamin $G$ was inactivated under these experimental conditions. Bisbey (1930) found that such loss could be largely obviated if the exposure to air was reduced to the minimum during the extraction and concentration processes. In her work it was found that absolute alcohol at room temperature $\left(21^{\circ}\right.$ to $25^{\circ} \mathrm{C}$.) extracted only a small proportion of the vitamin $\mathrm{G}\left(\mathrm{B}_{2}\right)$ from skimmed milk powder, whereas in parallel experiments 80 per cent alcohol extracted about half.

Alleman (unpublished data) interpreted the finding of McCollum and Kruse (1926) that a more potent extract of water-soluble B could be obtained, if the alcohol were acidified with gallic acid, not as due to superior solvent property associated either with the acidity or molecular structure of the acid used as suggested by them, but as due to a conservation of the extracted vitamin, perhaps protected by a higher reduction potential of the gallic acid present. Both Bisbey and Alleman observed that acid in an alcoholic medium appears to catalyze the inactivation of the vitamin, thus confirming similar observations of Chick and Roscoe (1929) and Narayanan and Drummond (1930).

Halliday (1929) found that vitamin G of "protein-free milk" was adsorbed to about the same extent whether the adsorption took place at $\mathrm{pH} 3$ or $\mathrm{pH} 4$, and with widely varying proportions of Lloyd's reagent. Under the conditions employed, about one-half the vitamin was inactivated, probably due in part at least to oxidation, and of the remainder about two-thirds was found on the adsorbent and the rest in the filtrate. This relationship appeared to hold fairly constant regardless of the amounts of adsorbent used within the limits investigated ( 5 to 80 grams per liter). 


\section{Distribution of Vitamin $G\left(B_{2}\right)$ in Food Materials}

In the course of work demonstrating the multiple nature of the vitamin $\mathrm{B}$ complex some indications of the distribution of vitamin $\mathrm{G}$ $\left(B_{2}\right)$ have been given. It has been found in yeast, fresh or autoclaved, in milk, lean meat, and green leaves.

In studies of the relative vitamin $G\left(B_{2}\right)$ content of foods Aykroyd and Roscoe (1929) used as a basis of comparison the minimum amounts of food materials which when incorporated into, or added to a vitamin G-deficient basal diet, provide enough vitamin $G\left(B_{2}\right)$ to permit an 11- to 14-gram weekly increase of body-weight in the experimental rat. From the work of our laboratory it appears that this rate of growth requires about three times as much vitamin $G$ as is required to maintain a rate of gain of about 3 grams per week, at which level our quantitative comparisons are made. Thus the Aykroyd-Roscoe "units" are about 3 times as large as the Bourquin units. In so far as possible the vitamin $G\left(B_{2}\right)$ values of foodstuffs in the following text will be expressed in one or the other of these terms.

Cereals.-To furnish one Aykroyd-Roscoe unit, cereal products formed a large part of the diet: whole English wheat, 50 per cent; Manitoba wheat, whole grain, 30 to 50 per cent, embryo, bran or pollard, 15 to 30 per cent, patent or household flour, 65 per cent; white African maize, 50 per cent; yellow South American maize, more than 50 per cent; germ meal 30 to 60 per cent; endosperm grits or Italian polenta, more than 65 per cent (Aykroyd and Roscoe, 1929). Bourquin (1930) reported 1.5 of her units of vitamin G per gram of ground whole winter wheat.

Maize, long associated with the occurrence of pellagra was reported by Goldberger, Wheeler, Lillie and Rogers (1928) to contain little of the black-tongue preventive factor; wheat to contain somewhat more, and extracted commercial wheat embryo at least twice as much.

Legumes.-Thirty to 40 per cent of dried peas in the ration furnished one Aykroyd-Roscoe unit (1929). Goldberger and his coworkers found that cowpeas, while containing some of the black-tongue preventing factor, perhaps half as much as did wheat germ, was a poor preventive of black-tongue and pellagra (Goldberger and Wheeler, 1927a). Soy beans, fed in large amounts, showed the presence of blacktongue preventive factor (1928).

Yeast.-Dried yeast was reported by Aykroyd and Roscoe (1929) to contain 5 to 10 Aykroyd-Roscoe units per gram; Quinn, Whalen and Hartley (1930) found 10 to 15 Bourquin units per gram (5 
samples). In the work of Goldberger and colleagues (1925, 1928), 11 grams dried brewery yeast per day exercised definite, almost complete black-tongue preventive action; and Goldberger and Tanner (1925) found yeast very effective in pellagra. One to two cakes of compressed yeast daily appeared, however, to have little effect on the UnderhillMendel deficiency disease in dogs.

Milk.--In fresh whole milk (London winter supply) Aykroyd and Roscoe reported 0.2 to 0.3 of their units per cubic centimeter. On the basis of milk solids, evaporated and condensed milks appear about equally potent. Skim milk powder contains from 3 to 5 Bourquin units per gram. About 30 cubic centimeters fresh skim milk per kilogram did not quite protect dogs against black-tongue (Goldberger et al., 1928) ; somewhat less than 20 cubic centimeters buttermilk per kilo had protected humans against pellagra. (Goldberger and Tanner, 1924.)

Fats.-Symptoms of vitamin G deficiency can be produced in rats receiving as much as 8 per cent butterfat in the diet, or as much as 15 per cent hydrogenated vegetable oil. In the work of Goldberger et al. (1928) butter appeared to contain some black-tongue preventive value when fed in liberal amounts ( 5 to 7 grams per kilo dog). Since these authors calculated that at least 775 grams butter daily would have to be ingested per adult male per day to prevent pellagra they concluded that butter is a relatively poor source of the pellagra-preventive factor; but in the prevention of the canine deficiency disease which Goldberger considers analogous to pellagra, Underhill and Mendel found butter to be quite effective. Cod-liver oil was found ineffective by Goldberger and Tanner (1924) for preventing human pellagra when ingested at a daily rate of 2 grams per kilogram body weight, and it was ineffective in preventing black-tongue in dogs at a level of over 4 grams per kilo. (Goldberger et al., 1928.) Cottonseed oil appeared equally ineffective.

Eggs.-Aykroyd and Roscoe (1929) found 0.5 to 1 of their units of vitamin $G\left(B_{2}\right)$ per gram of cooked egg yolk (2 to 4 units per gram of dried egg yolk). Goldberger and coworkers reported (1928) cooked dried egg yolk inferior to wheat germ, fresh beef or pork liver per unit weight as a preventive against black-tongue (12 yolks per 2,400 calories did not always afford protection to dogs).

Meat and Fish.-Aykroyd and Roscoe (1929) found dried steak to contain one and one-half to 2 units of vitamin $G\left(B_{2}\right)$ corresponding to one-half to one unit per gram of fresh lean beef; and ox liver appeared to be from 7 to 10 times more potent. Day (unpublished data) finds liver 10 times as rich in vitamin $G$ as is muscle. This relationship between muscle and liver tissue agrees with the finding of Carlsson 
(1929), who observed liver and kidney tissue (rat) to be approximately 10 times as rich in vitanin $G$ as voluntary muscle. Carlsson further found cardiac muscle five times, brain two and one-half times, spleen three times and blood one-third as rich per gram fresh tissue as voluntary muscle.

A few unpublished experiments by Stiebeling (personal communication, 1930) indicate that fresh lean beef contains approximately one Bourquin unit per gram. Lean beef muscle was demonstrated by Goldberger and his colleagues $(1920,1924,1926)$ to be a good source of the pellagra-preventing factor. When fed to the extent of 50 grams protein in a 2,400 Calorie ration, either fresh or cooked and dried, it contained more than minimal amounts to prevent black-tongue in dogs; pork liver, incorporated into the diet to the extent of 43.5 grams protein in a 2,400 Calorie diet appeared to be a good source of the blacktongue preventive. Three hundred grams canned salmon per 2,400 Calories afforded complete protection to the dog (Goldberger, 1928); six ounces daily (Goldberger and Wheeler, 1929) prevented human pellagra.

Hoagland and Snider (1930), using an alcoholic extract of white corn meal as the vitamin $B\left(B_{1}\right)$ supplement for the basal vitamin $B$ free diet, secured from good to excellent growth in rats on the addition of moisture-free commercial beef extract as the source of vitamin $G\left(B_{2}\right)$. In similar experiments with dried lean beef as the source of vitamin G, 25 per cent sufficed for excellent growth. From these results it was estimated that 1 pound of concentrated beef extract contains approximately as much vitamin $G$ as 11 pounds of fresh lean beef.

More recently Hoagland and Snider (1930a) have reported that beef, pork, and lamb muscle contain approximately the same amounts of vitamin $G$, from 15 to 25 per cent of the dried material sufficing for excellent growth. Beef spleen was about as rich, and beef and pork liver and beef kidney from 5 to 8 times as rich as muscle meats in vitamin $\mathrm{G}$.

Vegetables.-According to unpublished data of Hartley (personal communication, 1930), the juice from canned tomatoes contained 0.16 of a Bourquin unit of vitamin $G\left(B_{2}\right)$ per cubic centimeter. According to Goldberger and Wheeler (1927) forty ounces of this material daily prevented pellagra. Goldberger, Wheeler, Lillie and Rogers (1928) found that upwards of 30 cubic centimeters per kilogram may be needed to protect dogs from black-tongue. They further report that 400 to 450 grams cooked carrot daily was found inadequate to prevent human pellagra; and only when the allowance exceeded 40 grams per 
kilogram were carrots protective for dogs. Rutabagas appeared even less effective.

The fair agreement in the distribution and relative concentration of the factors in various food materials preventive of the vitamin $\mathrm{G}\left(\mathrm{B}_{2}\right)$ deficiency in rats, pellagra in man, and black-tongue in dogs respectively, led both Aykroyd and Roscoe (1929) and Goldberger and his collaborators (1928) to express their belief in the fundamental identity of these three conditions.

\section{Relation to Nutrition}

Many of the observations upon the nutritional deficiency which develops when vitamin $G$ is lacking or insufficiently supplied have been mentioned earlier in the chapter in the account of the differentiation of vitamins B and G. It was there noted that as early as 1926 Goldberger and his colleagues pointed out the probable connection between the pathological conditions observed in human pellagra, canine blacktongue, and the dermatitis of rats resulting from a deficiency of the more thermostable part of the vitamin B complex. Chittenden and Underhill (1917) had experimentally induced by diet a pathological condition in dogs, in many respects similar to pellagra. Underhill and Mendel $(1925,1928)$ made a further study of this condition.

When the animals first showed signs of loss of appetite, the mouth revealed an inflamed condition of the gums as well as the inside of the lips, and a characteristic red line extending around the teeth. When autopsied, Dr. Robert A. Lambert reported characteristic acute pharyngitis, also lesions of the esophagus, stomach and the upper portion of the small intestines. These findings, though less severe in character than those reported by Chittenden and Underhill in 1917, corroborated the earlier symptoms which were reported as pellagra-like.

Neither compressed yeast in amounts sufficient to supply vitamin B requirements of the dog nor cod-liver oil protected against the incidence of the disease nor prolonged life. Meat and pig's liver prolonged life. Butter ( 5 per cent of the ration) cured and maintained the animal in good condition over long periods of time. Egg yolk, though containing the potent factor, seemed less effective than butter; boiled unpeeled carrots were more potent, and a lard extract of carrots also alleviated the symptoms. The effective agent in butterfat was apparently quite labile and associated with the yellow coloring matter, for the butter lost its potency as well as some of its color during a period of from 9 to 11 months of cold storage. These two facts led to a further investigation of the relationship between the effective agent and carotinoids 
in which it was found that carotene, prepared from carrots, recrystallized six times and fed either dissolved in cottonseed oil or as solid mixed with ground meat could cure the diseased dogs when given in relatively small doses.

Underhill and Mendel prefer to treat this deficiency disease of dogs as such and state definitely that they make no attempt to correlate this disease with human pellagra or even to establish its identity with blacktongue.

Until the complete report of Underhill and Mendel was published in 1928, Goldberger and his associates had been of the opinion that the pathological conditions studied in the two laboratories were probably identical; but in commenting on the divergent results obtained with similar foods, Goldberger and his coworkers (1928) called attention to certain differences in the two clinical pictures of mouth disease in dogs. They wrote: "A troublesome skin rash appears to have been common in the condition with which Underhill and Mendel have worked. We have encountered none such in our dogs. On the other hand, while we have observed a pellagra-like dermatitis of the scrotum in some 40 to 50 per cent of attacks in our male dogs, there is no mention of the occurrence of such an eruption in the condition studied by Underhill and Mendel. Again in the condition studied by us,namely, black-tongue,--there is a definitely marked febrile stage which does not seem to occur in the Chittenden-Underhill syndrome studied by Underhill and Mendel.- These clinical differences seem to us of such importance that, in spite of the striking clinical similarity in certain other respects, doubt now arises as to the identity of the Chittenden-Underhill "pellagra-like" syndrome. Until this doubt is definitely resolved one way or another it would seem premature to discuss, and we therefore do not attempt to discuss, the differences between the results reported by Underhill and Mendel and those recorded by ourselves."

In describing the pellagra-like condition of the rat resulting from the deficiency of vitamin G, Goldberger and Lillie (1926) called attention to a characteristic abnormal condition around the eye, loss of fur, a bilaterally symmetrical dermatitis, occasionally a linear fissuring or ulceration at the angles of the mouth, and an ulceration of the tongue and anterior part of the floor of the mouth. These symptoms resemble clinically certain types of pellagra, and the authors pointed out that the identity suggested by this clinical similarity is supported by the apparent identity of the dietary conditions associated with the development of the respective conditions. 
Chick and Roscoe (1927) described the condition of rats on a diet deficient in vitamin $G\left(B_{2}\right)$ as follows: "The body weight was usually maintained, but the animals were in poor condition; they were thin and active, with rough coat and with urine slightly blood stained. Loss of hair took place, and red inflamed patches of skin appeared on the nose and on the backs of the fore feet, which later became edematous. There was a marked conjunctivitis and in some cases the ears also became red and thickened. The inflamed areas spread and often involved the hind legs and whole areas around the nose and mouth; sometimes the chest became bare and inflamed. The animals survived 2 to 3 weeks or longer in this condition before death occurred. On post mortem examination there were no definite macroscopic signs except a very unhealthy condition of the whole alimentary tract, especially in the small intestine which showed signs of inflammation with atrophy of the mucus membrane and often contained blood-stained mucous.

"This pellagrous condition was cured slowly but surely by the addition to the diet of a small daily ration of autoclaved yeast, and at the same time growth was restored. Several days, however, usually elapsed before there was definite improvement. The skin lesions gradually healed, desquamation took place on chest and paws and round nose and mouth; thickened cheesy layers peeled from the ears leaving them thin and papery. In about 2 weeks the skin was healed and hair beginning to grow."

In a further attempt to differentiate the symptoms of a deficiency of vitamins $B$ and $G$ from those formerly ascribed solely to vitamin $B$, Findlay (1928) fed four groups of 10 rats, from weaning time, upon a basal diet of purified casein 25, rice starch 70, and McCollum and Davis salt mixture 5 parts, with 0.2 gram of cod-liver oil per rat per day. Group $A$ received no addition to this diet; group $B, 0.5$ gram per rat per day of yeast autoclaved at $120^{\circ} \mathrm{C}$. for 5 hours; and group $C, 0.2$ cubic centimeter of the Kinnersley-Peters antineuritic vitamin concentrates.

The rats in group $A$ (strikingly deficient in all water-soluble vitamins) showed loss of appetite, body weight, and temperature, but only 2 showed symptoms of polyneuritis. The average duration of life was 34 days. In group $B$ (deficient in heat-labile $\mathrm{B}$ vitamins) the symptoms resembled those in group $A$ except that paralysis was present in 6 of the 10 rats. The average duration of life was 37 days. In group $C$ (shortage of vitamin G) moderate growth occurred for from 4 to 6 weeks, followed by a period of slight but gradual loss in weight, during which time cutaneous changes developed, preceded in some cases by a sudden change in behavior, the animals becoming very irritable. The 
body temperature remained normal until shortly before death, and, though the food intake was gradually reduced, complete loss of appetite was not evident, nor was there any appearance of the characteristic nerve symptoms of polyneuritis. The skin changes, which are described in detail in the original paper (Findlay, 1928), were bilaterally symmetrical and were regarded by Findlay as very similar to those of pellagra in man.

Chick and Roscoe (1928) reported that, on a basal diet supplemented with Peters' antineuritic concentrate but free from vitamin G, young rats were maintained for three months or more at practically constant weight, with fair appetite and no outward symptoms with the exception of an abnormality of the skin which usually but not always developed after the fifth or sixth week. The most constant features of the skin condition were: (1) Dermatitis and loss of hair from the eyelids, which may become stuck together; if the eyelids are loosened by bathing with warm water, the eyes, though much sunken, appear to be healthy; (2) front paws stained with blood, caused by rubbing the inflamed margins of the nostrils; wetting of the lower portion of the abdomen with blood stained urine; (3) dermatitis and loss of fur on head, round the nose and mouth and on the abdomen.

The irregularity in the occurrence of the skin symptoms and the occasional instances of animals deprived of vitamin $G\left(B_{2}\right)$ remaining stunted in growth but not exhibiting skin lesions suggested to Chick and Roscoe the possibility of more than one dietary deficiency.

These authors did not observe the irritability in the rats as noted by Findlay nor the loss of appetite which he recorded. On the addition of vitamin $G$ to the diet there was a rapid response in growth and improvement in the dermatitis.

Salmon, Hays and Guerrant (1928) found that on their basal "pellagra-producing" ration, young rats made little or no increase in weight, but showed no particular symptoms for from 2 to 4 weeks after the preliminary depletion period of 2 weeks. The symptoms which then developed gradually were alopecia, dermatitis, stomatitis, ophthalmia, and arthritis, and usually cachexia. The internal lesions of advanced cases were, "hemorrhagic gastroenteritis, atrophy of the spleen, fatty infiltration or degeneration of the liver, cloudy swelling of the kidneys, and often cystitis. There is a relation between the character of the diet and the occurrence of the syndrome. A mild dermatitis has been found among mature rats receiving a diet which is adequate for excellent growth and reproduction. The severe form has been produced only on restricted diets." They further report that bac- 
teriological examination of the lesions has revealed the invariable presence of a Gram-positive coccus about one micron in diameter. This organism under ordinary conditions is not pathogenic. It is incapable of producing typical pellagrous lesions by injection, but when fed in massive doses produces the characteristic syndrome readily in about 80 to 85 per cent of the test animals and eventually in all of them; also that concentrates of the preventive factor prepared from kudzu leaves proved effective in prophylactic and curative tests, and have been demonstrated to inhibit the growth of the causative organism in culture medium. The amount of the protective factor required for complete protection they thought to depend to some extent on the number and virulence of the organisms ingested and the age and individual susceptibility of the animal. Young animals are much less susceptible than older ones. They suggested that the pellagra-preventive factor may not be identical with vitamin $G$, since the correlation between the degree of protection against infection and the rate of growth was not always good.

Sherman and Sandels $(1929,1931)$ reported that, within certain limits, the restriction of vitamin $G\left(B_{2}\right)$ resulted in pellagra-like symptoms such as have been described by Goldberger and his coworkers and by Chick and Roscoe, but in cases of almost complete deprivation of vitamin $G\left(B_{3}\right)$ death sometimes occurred without the development of conspicuous skin symptoms. In general their experiments indicated that in the condition resulting from almost complete deprivation of this vitamin, the eyes, nose, mouth, alimentary tract, and perhaps kidneys were apt to show more pronounced abnormalities than the skin; whereas with a less complete deprivation and a somewhat longer course of the deficiency disease the abnormalities of the skin were apt to become more prominent. They described their observations essentially as follows: When vitamin $G$ is decidedly lacking in the diet of rats of an initial age of 4 weeks, growth is quickly retarded, usually within two weeks, but the time interval is subject to considerable variation, depending upon the completeness with which the diet has been freed from the factor in question and also, doubtless, on the previous diet of the animal, inasmuch as the body is able to store appreciable amounts. Several weeks later, soreness of the eyes appears with abnormal secretion and a tendency to sticky lids, and to frequent rubbing and occasional scratching of the lids by the animal; in many cases the fur falls out around the eyes, leaving bald inflamed lids; usually the mouth becomes sore, first with indications of excessive salivation, then inflammation at the corners, sometimes progressing to an inflamed or 
ulcerated condition of the entire lower lip; in many cases soreness of the nose develops with occasional bleeding; the animal becomes weak and tends to remain in a humped posture; in advanced stages diarrhea is common and there may be bloody discharges, both in feces and urine.

In less rapidly fatal cases in which abnormalities of the skin were more common, the fur becomes dry and pulls out readily, often a definite saddle-like pattern appearing on the shoulders or back. Small dry cream-colored scales may appear on the affected areas of the skin, which later may be replaced by larger yellowish crusts, tending to appear symmetrically on the sides of the back, shoulders and chest. Occasionally rough reddened areas are found on the paws and inside of the fore legs.

While the animals showed individual variation, yet on the whole the symptoms observed were consistent with those reported by Goldberger and his coworkers and by Chick and Roscoe.

Vitamin $G$ is evidently a substance of coördinate importance with the longer known vitamins as an essential factor in normal nutrition and deprivation or serious shortage of this substance results in widespread injury to the body. Conversely, the liberal feeding of this substance may be expected to play a significant part in inducing a better-than-average nutritive condition.

Hartwell (1924a) had observed the increased need of the lactating rat for the "vitamin B complex." Evan and Burr (1928a) and Sure (1928f) have reported that the vitamin sed is much greater for successful lactation and reproduction than for rapid growth.

Hassan and Drummond (1927) noted that the heat-stable alkaliresistant fraction of the "vitamin B complex" is the one primarily concerned in the relation of yeast extract to protein metabolism, rendering high-protein diets adequate for growth, thus clarifying Hartwell's (1924a) observation of a relation between the amount of protein in the diet and the need for vitamin B complex in a lactating rat's diet, the amount of vitamin needed increasing with the protein intake.

Kon (1929) found the carbon-nitrogen ratio of the urine of rats deprived of vitamin $G$ to be higher than that of rats receiving a normal diet, an indication of some important relation of vitamin $G$ to the metabolic processes of the body.

In part also to vitamin $G$ probably belongs the improved nutrition resulting from an increased proportion of milk in an already adequate diet, as summarized by Sherman and Campbell in 1924. This improvement was evidenced by more rapid and efficient growth during the 
period following weaning; somewhat larger size at all ages; earlier maturity as shown by age of female at birth of her first young; longer duration of the reproductive life, old age being deferred in the same individuals in which earlier maturity had been induced; greater success in rearing young, as shown by increase both in numbers and in percentage of young reared, and by decrease in percentage of females failing to bear and rear young; better growth of young during the suckling period as shown by larger average weight at the uniform weaning age adopted.

Whalen (unpublished data) has studied the effects upon growth, reproduction and lactation of food mixtures containing graded amounts of vitamin G. Her results confirm those of Sherman and Campbell in indicating that the amount of vitamin $\mathrm{G}$ in the diet, particularly during early life, affects the rate of growth, and influences body vigor at all ages. Liberal intake of vitamin $G$ was found to bear an important relation to lactation and to the growth and development of the suckling young. An abundance of vitamin $G$ favored early maturity, indicating excellence of general nutritive condition.

The significant improvement in longevity found by Sherman and Campbell $(1928,1930)$ resulting from improving a diet already adequate by increasing the proportion of milk in the ration, is probably due in part to the higher content of vitamin $G$ as well as of vitamin $A$ and calcium in the better diet.

The amount of vitamin $G$ stored by the young is influenced by the vitamin $\mathrm{G}$ content of the diet of the mother. This is indicated by the length of survival and the time of onset and severity of symptoms of G-avitaminosis when the young drawn from families of different nutritional histories are placed on a vitamin $\mathrm{G}$ deficient diet (Whalen, unpublished data).

A study of the distribution of vitamin $G$ in the various tissues of the body was made by Carlsson (1929). Liver and kidney tissue of adult animals subsisting on a food mixture consisting largely of onethird whole milk powder and two-thirds ground whole wheat, appeared to be about equally rich in vitamin $G$ and both about ten times as rich in vitamin $G$ as muscle. Cardiac muscle was five times, brain tissue two and one-half times, spleen about three times and blood one-third as rich as voluntary muscle. She further found that the vitamin G content of the tissues may be measurably influenced by the abundance or paucity of this vitamin in the food. Vitamin $G$ does not appear to be especially stored in the liver to such a conspicuous degree as is vitamin $\mathrm{A}$, for in animals equally well nourished the liver is only about 
ten times richer than muscle in vitamin $G$, whereas it was found to be 200 to 400 times richer in vitamin A.

\section{Other Factors}

That other factors are operative in the phenomena of mammalian nutrition which may soon be fully recognized as seventh, eighth and perhaps ninth (or more) mammalian vitamins now seems highly probable in view of the work of Coward et al. (1929), Hunt (1928b), Palmer and Kennedy (1928-29), Sherman and Stiebeling (unpublished data of 1930), Peters (1930), Reader (1929a, 1930), and others. Development of knowledge in this direction may soon permit of better interpretation of much of the work upon vitamins $\mathrm{B}$ and $\mathrm{G}$. 


\section{CHAPTER IV}

\section{VITAMIN C}

Theobald Smith in 1895 noted that guinea pigs kept upon a diet of oats developed a hemorrhagic disease, but the importance of this fact was not realized until several years later.

At about the time that Eijkman and others were showing that beriberi could be induced in fowls as the result of a diet lacking in some definite though unidentified substance, Holst and Frölich (1907, 1912) of Christiania undertook similar experiments with different laboratory animals in the hope of obtaining light upon the disease known as ship-beriberi which was seriously prevalent among Norwegian sailors when on long voyages or fishing trips. Feeling that their experiments would be more directly applicable to human experience if performed upon mammals rather than pigeons or fowls, they gave particular attention to feeding tests upon guinea pigs. These were found to differ greatly from fowls in their reaction to a polished rice diet. Instead of showing experimental beriberi, the guinea pigs developed symptoms which Holst and Frölich considered to be "identical in all essentials with those of human scurvy." A diet of other grains or of bread alone resulted in the same symptoms. Among these symptoms are loss of weight, soreness of joints, hemorrhages around rib junctions and knee joints, soreness and hyperemia of the gums leading to looseness of teeth, separation of epiphyses from long tubular or medulated bones (especially the upper epiphysis of the tibia), characteristic changes of bone marrow, and sometimes hemorrhages into the skin.

Among the foods showing antiscorbutic properties in varying degrees were raw cabbage, dandelion, lettuce, endive, sorrel, potatoes, carrots, cloudberries, bananas, and apples.

Cabbage and dandelion juices apparently lost their antiscorbutic properties rapidly when kept at room temperature or even in the ice box, while fruit juices and sorrel juice proved more stable. That the stability of the active constituent was greater in an acid than a neutral or alkaline medium was shown by these findings and also by the observation that acidulated cabbage or dandelion juice retained its antiscorbutic property better than the corresponding natural juice. 
A study of the effect of cooking on the antiscorbutic property of these materials showed that loss in activity varied with the food and the time of heating. In general, heating for one-half hour at $100^{\circ} \mathrm{C}$. proved much less destructive than for one hour at the same temperature. Cooking cabbage at $110^{\circ} \mathrm{C}$. for one-half hour did not entirely destroy its antiscorbutic properties, but after cooking for one-half hour at $100^{\circ} \mathrm{C}$. and then for one hour at $120^{\circ} \mathrm{C}$. its antiscorbutic properties were almost negligible. Berries retained their efficiency after cooking to a very marked extent. Raspberry juice seemed to be but little injured by heating for one hour at $100^{\circ} \mathrm{C}$. or even $110^{\circ} \mathrm{C}$. Cloudberries heated in the same way retained their antiscorbutic properties for at least three months after cooking.

An interesting observation in this paper was that the influence of cooking was apparently less when the air was excluded during the heating. (Compare later work on destruction of antiscorbutic vitamin by oxidation.)

In most cases vegetables lost the greater part of their antiscorbutic value on drying; but it was observed that such of the antiscorbutic property of cabbage as was not lost in the process of drying was subsequently better preserved in the dry state than when the vegetable was simply stored under ordinary room conditions.

Holst and Frölich in this same article (1912) described the production of scurvy in swine by feeding either (1) rye bread, (2) rye bread and cooked beef, or (3) rice and dried cooked fish. In most of these animals a marked polyneuritis developed in addition to the scurvy. This same combination of the symptoms of beriberi and scurvy characterizes the "ship-beriberi," the disease which had led Holst and Frölich to undertake these experiments. Thus it appeared that shipberiberi might be regarded as scurvy complicated with beriberi, and that scurvy might now be studied experimentally in the guinea pig as beriberi had been in fowls and pigeons.

Holst and Frölich showed that scurvy develops in about the same manner when any of the ordinary grains or its mill product is fed. Fürst (1912), in the same laboratory, showed that dried peas, lentils or almonds, although differing markedly from the cereal grains in percentage composition as shown by ordinary analysis, were like the cereals and similar among themselves in their lack of the antiscorbutic substance when fed after ordinary cooking. When peas or lentils were fed raw, scurvy appeared less quickly and in a less severe form, indicating that these seeds contain some of the antiscorbutic substance, but that this is destroyed (or reduced to negligible proportions) by 
heating to the extent required for the thorough cooking of these dry legumes. Fürst also experimented with mixtures of seeds and concluded that "there is no advantage in numerous foods when none contains the needed substance"-an observation which might well become an aphorism for the guidance of menu makers.

Fürst found further that these seeds developed antiscorbutic properties when soaked and allowed to sprout.

From this paper by Fürst, as well as from those of Holst and Frölich, one gets the impression that these authors believed in more than one antiscorbutic substance since the antiscorbutic property seemed to differ in stability in different foods. Similar suggestions have since been advanced by Bezssonoff (1926a, 1927a) and Randoin and Lecoq (1927) but have not received very much attention. From our present point of view it seems more natural to assume that these differences in behavior may be due to differing conditions such as hydrogen-ion activity, oxidation potential of the medium, and to colloids whose presence or absence would result in the vitamin being contained sometimes in a heterogeneous and sometimes in a homogeneous system. Nevertheless we know of no evidence adequate to constitute a conclusive demonstration that there is only one antiscorbutic vitamin.

Frölich (1912) held that (under the conditions existing in Norway) infantile scurvy was most common among children living under good hygienic conditions, and that this was probably due to their being fed too largely upon prepared foods and well-sterilized milk, whereas children less sedulously cared for were likely to eat a greater variety of foods, some of them raw, and thus were less liable to scurvy. Since on the other hand the epidemics of scurvy among adults had so often developed under conditions of hardship and insanitary living, there had been a misleading appearance of these diseases (adult and infantile scurvy) being different, whereas in reality they might be essentially identical.

Frölich showed that milk normally contains enough of the antiscorbutic substance to afford complete protection from scurvy when raw milk constitutes the sole or chief food. Such heat treatments as are ordinarily involved in pasteurization of milk, were found in his experiments to diminish but not entirely to destroy the antiscorbutic property so that whether pasteurized milk will furnish enough of the antiscorbutic substance to make the diet safe will depend both upon the treatment to which the milk has been subjected and upon the amount of milk consumed. Pasteurized milk may prevent scurvy in the same manner that raw milk does, provided the pasteurization has been prop- 
erly carried out and the pasteurized milk is used in sufficiently liberal amounts. His position on both these points has been amply confirmed by later work.

Holst and Frölich (1913) found that cabbage dried quickly in an oven at $37^{\circ} \mathrm{C}$. lost little of its antiscorbutic property, and that on keeping it in a desiccator the property was better preserved than when the material was stored in a closed, moist receptacle for the same length of time, thus indicating that destruction of the antiscorbutic substance occurred more readily in the presence of moisture than in its absence. Attempts to extract the antiscorbutic constituent from cabbage by means of neutral ether were unsuccessful, but it was found to be soluble in alcohol acidulated with 0.5 per cent of citric acid.

A further study of the effect of dehydration upon the antiscorbutic properties of cabbage was reported by Holst and Frölich in 1916 but escaped general notice until reported in English several years later (Holst and Frölich, 1920). Slices of cabbage dried for one week in an oven at $37^{\circ} \mathrm{C}$., further dehydrated at the same temperature by the action of phosphoric anhydride, and then placed in vacuum bottles and stored at the same temperature showed very pronounced antiscorbutic properties after from 18 to 26 months. Cabbage dried at $37^{\circ} \mathrm{C}$., but not further dehydrated, lost most of its activity when kept for 18 months at $37^{\circ} \mathrm{C}$. in closed vessels but retained it to a certain extent when kept at from $4^{\circ}$ to $12^{\circ} \mathrm{C}$. Samples kept at $4^{\circ} \mathrm{C}$. in closed vessels without being previously dried showed moderate antiscorbutic properties at the end of 18 months.

Darling (1914) showed in an interesting way by tabulation and diagrams the overlapping of the symptoms of beriberi, scurvy and other deficiency diseases and the extent to which the symptoms of experimental beriberi in fowls and experimental scurvy in guinea pigs correspond with those of the diseases as observed in man.

The interrelationship of scurvy, beriberi, rickets, and other deficiency diseases, the relative susceptibility of different animals to scurvy, and the striking resemblance between guinea pig and human scurvy have also been discussed quite fully by Hess in his monograph on Scurvy, Past and Present (1920). In calling attention to the fact that a diet of polished rice or other decorticated grain will lead to the development of scurvy in the guinea pig, to polyneuritis in the pigeon or fowl, or to a combination of these disorders in the hog, he suggests that the difference between susceptible and non-susceptible animals is relative rather than absolute. Guinea pig and human scurvy are considered practically identical, although the guinea pig is far more sensitive to 
scurvy than man. "This does not indicate that the guinea pig is an unsuitable experimental animal, any more than the fact that the pigeon is more susceptible to polyneuritis than man indicates that it is unsuited to investigations of beriberi."

In the study of vitamin problems through the evidence obtainable from observations upon disease, two points which are not always clearly distinguished should be kept carefully in mind. From the standpoint of pathology and, to a less extent, of prevention it is of course of great importance that the experimental disease induced in a laboratory animal shall really be the counterpart of the pathological condition observed in man. At the same time it must not be forgotten that the clinical picture tends to grow with the literature of the disease and that the complete clinical picture may easily include symptoms arising from concurrent infections or other causes aside from the deficiency of the vitamin, whereas in laboratory experimentation it is usually sought to study only one variable factor at a time. Thus, in experimental scurvy, we may have a picture not showing all of the features which have sometimes been considered as belonging to typical cases of scurvy in man, yet partly for this very reason the experimental form of the disease may be better adapted to the study of the vitamin problem than is its clinical form.

Another important factor in the study of scurvy as a deficiency disease through both experimental and clinical evidence is the possibility that the disease may exist in a latent or sub-acute or partially developed form in which case the patient does not show the complete or typical clinical picture yet may be demonstrably improved by the same measures which are clearly shown to prevent or cure the disease in experimental animals. This is strikingly true in Hess's experience with infantile scurvy. Hess and Fish (1914) described outbreaks of scurvy occurring in an orphan asylum among children who had been fed with milk heated at $165^{\circ} \mathrm{F}$. for 20 minutes or at $145^{\circ} \mathrm{F}$. for 30 minutes. No orange juice or other antiscorbutic had been fed. These cases in general did not present a fully developed or clear picture of scurvy but represented only an early stage or a partially developed or subacute form of the disease which was, however, responsible for much restlessness, irritability, evident discomfort, and retardation in the growth and development of the children. The condition was cured by the addition of small amounts of orange juice, which, it seems certain, must have been effective because of its antiscorbutic property. Cures were also effected, but less rapidly, by the substitution of raw for pasteurized milk. Modification of the pasteurized milk by the use 
of mashed potato made into a thin gruel instead of cereal gruel also served to cure and to prevent the disease. In most of the cases in which the disease developed in children fed pasteurized milk, the milk constituted about two-thirds, and cereal gruel one-third, of the food mixture. On such a diet some of the children developed scurvy at least to the extent here described, while others in the same ward did not, illustrating the fact that susceptibility to scurvy is subject to considerable individual variation or (to state what is probably the same thing from a different angle) that the antiscorbutic requirement for the maintenance of normal nutrition and growth is higher in some children than in others. In this paper and in another published by Hess in the following year (1915) the pathology of the disease was fully described.

Ingier (1915) studied the effect of a scorbutic diet upon pregnant guinea pigs and their unborn young. When the mothers received the scorbutic diets in the early stages of pregnancy, the young were born dead, often prematurely, and on examination showed evidence of impeded growth. When the scorbutic diet was given only during the latter part of pregnancy the young were born alive and apparently fully developed, but with latent scurvy which soon became acute if the mother were continued on the scorbutic diet and the young were dependent upon her milk. It was also observed that pregnant females more quickly succumb to a scorbutic diet than do animals not subject to the demands of pregnancy or lactation.

Commenting upon these results of Ingier, Hess (1920, pp. 126-127) concludes: "In view of the similarity between human and guinea pig scurvy, we should expect not only miscarriages and still-births to result (when the mother's diet is deficient in antiscorbutic food) but cases of congenital scurvy, especially of the latent or rudimentary type."

An example of the extent to which the growth and development of an infant may be retarded by lack of antiscorbutic vitamin before appearance of distinct scurvy symptoms is afforded by the following case described by Hess (1920, p. 213): "An infant which had been fed to an age somewhat over nine months without the use of raw milk or other antiscorbutic food was observed to be practically dormant as regards growth, having gained only about one-half pound in four months. It was then fed orange peel juice as an antiscorbutic and gained two pounds within a month. There were no other symptoms of scurvy, but the failure of growth followed by prompt resumption when antiscorbutic was given were held to show that the baby was suffering from a progressive scurvy, due to lack of antiscorbutic vitamin 
and curable by its administration, but not recognizable by any of the classic scorbutic signs."

Jackson and Moore (1916) described experimental studies of scurvy developing in guinea pigs kept under laboratory conditions on a considerable variety of diets some of which contained foods supposed to be more or less efficient as antiscorbutics. Post-mortem examinations showed hemorrhages in the muscles, ends of bones, bone marrow, skin and tooth pulp; swollen tender joints and fragile bones were also common. The results of a bacteriological study of these cases were described simultaneously by Jackson and Moody (1916). In the postmortem examinations which had been made, the staining of certain tissues had suggested the presence of bacteria. Cultures from the affected tissues showed diplococci of low virulence. Rabbits proved more susceptible to inoculations with this organism than guinea pigs. Only one rabbit died but all showed some stiffness and emaciation. Similar bacteria were recovered from the lesions of inoculated animals as long as 40 days later. When introduced into a second group of animals these organisms failed to produce the disease. Cultures of the heart blood did not show the organisms and blood transfused from diseased to normal animals produced no changes. Animals which had been fed adequate amounts of antiscorbutic food were less susceptible to inoculations with these bacteria than poorly fed animals. These results would seem to illustrate the likelihood that some of the symptoms observed in scurvy may be more or less influenced by the effects produced by infectious organisms which may be in some degree characteristic in the sense that they more readily develop upon a host weakened by scurvy. The fact, however, that a much clearer picture of scurvy develops with greater certainty as a result of purely dietary causes, confirmed as it is by the great amount of work which has been done upon experimental scurvy, leaves no doubt that the disease should be regarded as essentially due to dietary deficiency, though perhaps often complicated by infections and possibly by a particular infection to which the animal becomes more susceptible as a result of the deprivation of the antiscorbutic vitamin. See also Findlay (1923) and Werkman, Nelson and Fulmer (1924).

Chick and Hume (1916-17) in a study of the distribution among foodstuffs, especially those suitable for the rationing of armies, of the substances required for the prevention of beriberi and scurvy, emphasized the fact that the maintenance of adult human beings in health requires an adequate intake of antiscorbutic food as Hess had shown to be the case with children. They distinguished clearly, as 
Holst and Frölich and Hess had previously done, between two classes of vitamins concerned respectively with beriberi and scurvy, each of which they pointed out has its individual rôle in metabolism possessing properties differing from the other. They also emphasized the difference in distribution of the two substances among natural and commercial articles of food, through a comparison of foods as sources of antineuritic vitamin by experiments upon pigeons, and as sources of antiscorbutic vitamin by experiments upon guinea pigs, scurvy being induced in the latter by rations of cereals either alone or with sterilized milk. They emphasized the occurrence of the antiscorbutic vitamin in active, living, vegetable tissues, and in smaller degree in corresponding animal tissues. All the dried foods which they examined, including desiccated vegetables, they reported to be deficient in antiscorbutic vitamin. Dried pulses (legumes) and cereals, though rich in antineuritic, proved too poor in antiscorbutic vitamin to give any protection from scurvy, but if moistened and allowed to germinate the antiscorbutic property was found to be regenerated with the beginning of active cell life in the seed, as previously demonstrated in Holst's laboratory. They therefore recommended that such seeds be made a part of the army provisions, to be sprouted and added to the ration when other antiscorbutic food was not available in sufficient quantities. They gave full directions for sprouting the seeds in order to develop the antiscorbutic property and emphasized strongly the fact that the antiscorbutic vitamin is more sensitive to drying and to high temperatures than is the antineuritic.

Chick and Hume (1917) discussed further the independent need of both antineuritic and antiscorbutic substances in the diet at all ages, the absence of antiscorbutic in dry cereals and legumes, its development in either of these groups of seeds when germinated, and its occurrence in varying proportions in different fruits and vegetables. This paper includes a tabulation of different articles of food with notations of their relative antiscorbutic value by means of zero marks or one or more plus signs, a device which has been made increasingly familiar by the successive publications of the English workers and especially by the British Committee Report whose table forms the basis of many similar tables.

Cohen and Mendel published in 1918 the results of a very thorough and systematic investigation of the relation of diet to the production of scurvy in the guinea pig. They determined the results of feeding diets restricted to a single seed such as oats, barley or soy bean, and diets in which one or more seeds were fed together with supplements 
designed to furnish adequate amounts of the different factors known to be needed for normal nutrition. The conditions resulting in the experimental animals were fully described both as to symptoms and autopsy findings. The symptoms regarded by these authors as most characteristic are tenderness of joints developing usually in the wrists, ankles, and knees in this order, followed in two or three days by swelling of joints or sometimes spontaneous fracture. Fractures were more common among the younger animals. In older ones stiffening or paralysis of the hind legs may occur. The so-called scurvy positions are more or less characteristic. Swellings of the joints may disappear when antiscorbutic is given or they may harden. Hemorrhages of the gums in the experience of these investigators was relatively rare, but loosening of the teeth more common. With a well chosen diet the scorbutic animal may still eat well and gain in weight, at least for a time, though at a more advanced stage of the scurvy loss of appetite and of body weight occurs. Susceptibility was found to depend upon age and diet. Guinea pigs weighing from 110 to 250 grams succumb rapidly and the more so the poorer the diet as regards the character of its protein and mineral content.

Animals weighing 300 to 500 grams were considered to be better for the study of most phases of the scurvy problem since younger ones died too quickly. When such animals were kept on a diet of oats and milk, the latter the sole antiscorbutic, it was found that 50 cubic centimeters of milk per capita per day delayed the onset of scurvy for about 75 days, while 85-135 cubic centimeters resulted in complete protection. Orange juice, fresh cabbage, fresh carrots, adequate amounts of cabbage which had been carefully dried at a temperature of $70^{\circ}$ to $80^{\circ} \mathrm{C}$., germinated oats and barley were all shown to afford protection from scurvy.

Thus it was clearly shown by Cohen and Mendel that experimental scurvy of guinea pigs was demonstrable at will with a suitable diet, that it is induced by an exclusive diet of cereal grains as ordinarily fed, but delayed or prevented when the grains are germinated before feeding. It was rapidly produced by a diet of soy beans supplemented with additions to ensure adequate amounts of both the $\mathrm{A}$ and $\mathrm{B}$ vitamins together with a suitable mineral content and adequate roughage in the form of added cellulose. In general the findings of Holst and Frölich regarding the antiscorbutic properties of milk and of typical fruits and vegetables were confirmed and it was shown that cabbage could be so dried as still to retain a significant amount of the antiscorbutic substance. 
In a paper published simultaneously with that of Cohen and Mendel, Hess and Unger (1918a) furnished further evidence that, in the guinea pig, scurvy develops upon diets containing both "fat soluble A" and "water soluble B" in sufficient amounts to guard against deficiency of either of these vitamins.

\section{The Vitamin C Requirements of Different Species}

The universal acceptance of the vitamin hypothesis as related to scurvy was doubtless retarded by the fact that rats, which require both vitamin $A$ and vitamin $B$ for successful nutrition, do not show symptoms of scurvy when subsisting on diets which in human subjects, monkeys and guinea pigs cause this disease. Harden and Zilva (1918c), however, from studies of the growth and health of rats kept upon diets lacking each in turn of the three vitamins, concluded that "rats existing on a scorbutic diet, although capable of gaining in weight and reproducing themselves, without any apparent manifestation of pathological symptoms for months, do not thrive so well as animals which have their diets supplemented with an antiscorbutic. This suggests that although rats are not very susceptible to scurvy they cannot absolutely dispense with antiscorbutics without restriction of their normal development."

These conclusions were confirmed by Drummond (1919) in feeding experiments with rats through two generations. Larger numbers of litters of young were obtained from the females which received the added antiscorbutic, which was given in the form of orange juice. Drummond concluded from his observations that "the rat requires the antiscorbutic factor in order to achieve a normal development, and that although the requirements of this species are of a very much smaller order than those exhibited by man, the monkey, or the guinea pig, they are sufficiently well marked to dispel any idea that there exists a fundamental difference in the nutritive requirement of the two types of animal."

This argument might also apply to the evidence reported by Findlay (1921d) concerning the need of rabbits for vitamin C. Rabbits when fed oats, bran, and water did not develop typical symptoms of scurvy, although there was some evidence of congestion of the internal organs on autopsy. This condition, together with the fact that the animals lost weight on this diet, but showed prompt gain in weight if rutabaga juice was administered as an antiscorbutic, led Findlay to conclude that the nonsusceptibility of the rabbit to scurvy must be looked upon as relative rather than absolute. 
Osborne and Mendel (1920c) and Parsons (1920) were of the opinion however that the evidence upon which Harden and Zilva and Drummond based their conclusions was not convincing inasmuch as the orange or lemon juice used as antiscorbutics may have functioned merely as additional sources of vitamin $B$.

The susceptibility of the monkey to scurvy and its requirement for vitamin $C$ as compared with the requirements of the guinea pig and of man were studied by Harden and Zilva $(1919,1920)$. In the first of two papers on this subject (1919) three experiments were reported in which scurvy was induced in the monkey by the following diets: First, fresh beer, steamed wheat germ, and autoclaved bread, rice and peanuts; second, autoclaved rice, bread and autoclaved milk; and third, a diet similar to the first with the substitution of autoclaved milk for the beer. In all cases an acute scorbutic condition developed in from three to four months, the symptoms in the first two cases being confirmed by the histological changes noted on autopsy. In the third experiment the animal was cured in less than a week by the feeding of lemon juice. The minimum protective dose of orange juice for monkeys was later (1920) found to be between 1 and 2 cubic centimeters per day while doses of 2 to 5 cubic centimeters proved an adequate prophylactic. "A monkey therefore of 2 or 3 kilos is protected from scurvy by about the same quantity of orange juice as a guinea pig weighing 300 to 400 grams. It is interesting to note that while the minimum dose of antiscorbutic required by the two animals to protect them from scurvy is of the same order, the time taken for the development of the disease is very different, being about two months for a monkey and three weeks for a guinea pig. This suggests that the monkey possesses a higher store of the antiscorbutic factor than the guinea pig, while their daily requirements for metabolism are equal."

It is estimated that an average child of 8 to 10 kilos needs as much antiscorbutic vitamin as is furnished by about 15 cubic centimeters of orange juice per day. This indicates about the same requirement per unit of body weight in the child as in the monkey, while the guinea pig requirement per unit of weight is about five times larger. This is probably only a very rough approximation.

While it is generally conceded that the antiscorbutic vitamin can not be stored in the body to the extent that it is possible with vitamin A (Hess, 1920, p. 275) the suggestion of Harden and Zilva as to the relative storage of the antiscorbutic vitamin in the monkey and the 
guinea pig is of interest as offering a possible explanation of the varying requirements of different species of animals.

Of great importance in this connection was the demonstration by Parsons (1920) of the persistence of the antiscorbutic substance in the liver of the rat after long intervals on a scorbutic diet. In an effort to explain the apparently marked differences in antiscorbutic requirement of the two rodents, the guinea pig and the rat, a comparison was made of the antiscorbutic content of the rat's body tissues (1) when a typical scurvy diet was fed and (2) when the diet was high in antiscorbutic substances. The liver and muscle tissues of both groups were fed to scorbutic guinea pigs and for purposes of comparison fresh fish tissue was also used. The muscle tissues could not be fed at a high enough level to effect a cure but the liver tissues of both groups of rats showed a high content of antiscorbutic substance, practically no differences in the growth curves or alleviation of scorbutic symptoms of the guinea pigs on the two diets being noticeable.

These results were thought to indicate the need for the antiscorbutic vitamin by the rat, thus confirming in part the conclusions of Harden and Zilva and of Drummond, and to suggest the possibility that the rat is capable of synthesizing this substance. Other hypotheses advanced by Parsons at this time were as follows: "The antiscorbutic substance may pass into a modified form in foods on ageing, drying, etc., and this form may not be utilizable by the guinea pig but be utilized by the rat. The rat's requirement for antiscorbutic substances may be strikingly low. The rat's requirement for antiscorbutic substances may approximate that of the guinea pig from the standpoint of metabolism but there may be less waste of the substance due to a different rate of excretion."

An extension of these observations, using diets more carefully freed from the antiscorbutic substance and continuing the feeding into the second generation, was reported simultaneously by Lepkovsky and Nelson (1924) and Parsons and Hutton (1924), who found that the livers of second generation rats on these highly purified diets showed no diminution in antiscorbutic substance. This was thought to disprove (or weaken) the suggestion that the rat's requirement for the antiscorbutic factor is very low. In striking contrast with these observations was the discovery by Parsons and Reynolds (1924) that the livers of guinea pigs on a scorbutic ration contain inappreciable amounts of vitamin $C$ although the livers of normal guinea pigs contain it in abundance. This discovery was thought to emphasize the reasonable- 
ness of the hypothesis that the rat produces vitamin $\mathrm{C}$ in metabolism from some source not available to the guinea pig.

Fowls and other members of the avian species resemble rats in having little apparent need for vitamin $\mathrm{C}$ in their diet: Hart, Halpin, and Steenbock (1922); Emmett and Peacock (1923); Mitchell, Kendall, and Card (1923) ; Plimmer, Rosedale, and Raymond (1923); and Suguira and Benedict (1923). Following the method first used by Parsons, Carrick and Hauge (1925) and Hart, Steenbock, Lepkovsky and Halpin (1925) demonstrated the presence of vitamin $C$ in the livers of chicks which had been fed for a long time on diets devoid of vitamin $\mathrm{C}$ and concluded that the chick like the rat must produce the antiscorbutic substance in metabolism from sources not available to the guinea pig. Hauge and Carrick (1926a) attempted to determine the source of the supply by hatching chicks from eggs known to be lacking in vitamin $\mathrm{C}$ and testing the livers and kidneys of the chicks for this vitamin. Both the yolks and the whites of eggs laid by hens which had been on an antiscorbutic ration proved to contain no demonstrable amount of vitamin $\mathrm{C}$ but the livers and kidneys of five-weeks-old chicks hatched from these eggs and fed scorbutic rations contained an abundance of this vitamin.

Thurston, Eckles and Palmer (1926) found that calves grew as well on a ration extremely deficient in vitamin $\mathrm{C}$ as on the same ration supplemented with tomato juice as a source of this vitamin and showed no scorbutic symptoms on autopsy. Hughes, Aubel and Lienhardt (1928) reported that absence of vitamin C in no way affected the growth and reproduction of swine.

Thurston, Palmer and Eckles in 1929 reported an extension of their investigation of the rôle of vitamin $\mathrm{C}$ in the nutrition of calves. The livers of two of the calves on the vitamin-C-free ration were found to contain vitamin $\mathrm{C}$ as did also the milk from a heifer which had never received any demonstrable amount of vitamin C. Attempts to trace the synthesis of the vitamin were unsuccessful although negative results in tests of the antiscorbutic value of the stomach contents and feces of calves fed the scorbutic ration suggest that the synthesis occurs elsewhere than in the digestive tract. Based upon the enlargement of the adrenal glands in guinea pig scurvy, the possibility was suggested that perhaps the adrenals of the bovine, rat, and chicken are capable of synthesizing vitamin $\mathrm{C}$ while failing of this function in man, the guinea pig, and monkey.

Consistently with the fact above noted that guinea pig liver is not constant in antiscorbutic value, Anderson (1929) has demonstrated 
a slight but theoretically interesting storage of vitamin C in guinea pigs. Four groups of about 25 animals each were continued from the age of one month until death on a basal diet adequate in every respect except for vitamin C, and supplemented for 4 weeks only during the period of most active growth with orange juice in amounts of 3,6 , 12 , and 24 cubic centimeters per guinea pig daily. By observing the signs of scurvy quantitatively, together with survival periods, growth curves, and general behavior of the animals, it was possible to judge closely when scurvy occurred and to what degree in each group. The group which had received the smallest amount of vitamin $\mathrm{C}$ showed an earlier loss in weight and a slightly higher average "scurvy score" than those receiving the larger amounts. With increasing dosage up to 12 cubic centimeters, there was an increase in the average survival period, but beyond this point increasing dosage appeared to have no effect. And at most the guinea pig seems able to store no more vitamin $\mathrm{C}$ than he uses in a short time. The difference between the rat and the guinea pig in regard to the storage of vitamin $C$ thus appears to be quantitative rather than qualitative. The relatively small degree of storage of vitamin $\mathrm{C}$ in the body of the guinea pig as compared with the storage of vitamin $\mathrm{A}$ in the body of the rat is not unlike the relative difference between the storage of fuel in the form of carbohydrate and of fat in the human body.

\section{Vitamin $\mathbf{C}$ in Nutrition and Health}

The presence of vitamin $C$ in certain organs of animals which apparently do not require it in their food, no less than the characteristic symptoms which develop in other animals following deprivation of this vitamin, points to a distinct need of it for metabolic processes. Many of the studies which have been reported in the literature on the effects of vitamin $\mathrm{C}$ deficiency are of little value, however, on account of the fact that the basal diets employed were markedly deficient in other factors as well as in vitamin $\mathrm{C}$, thus making it impossible to attribute the findings to lack of this vitamin alone.

As is the case of vitamin B, and as will be shown later of the other vitamins, vitamin $\mathrm{C}$ may be taken in the food in sufficient amounts to prevent the development of the typical symptoms of the deficiency disease, yet not to supply the full needs of the body for the vitamin.

In such cases there may develop, especially in the growing child, the condition described by Hess as latent or subacute scurvy and there may also result a more or less serious injury to the teeth. Since human dietaries are not likely to be completely lacking in vitamin $\mathrm{C}$, the 
symptoms developing under conditions of partial deprivation or in the earlier stages of complete deprivation of the vitamin are probably of greater significance than those of typical scurvy.

Hess and Unger (1920a), from clinical observations and postmortem examinations and from the literature on scurvy and rickets, concluded that beading of the ribs, the so-called rachitic rosary, is not the result of rickets alone, but is one of the typical signs of scurvy and has also been noted in connection with beriberi and pellagra in children. The belief that this phenomenon is attributable solely to rickets is thought to be responsible for the misinterpretation of many cases of latent scurvy. On the other hand there is the possibility, as noted before, of attributing certain phenomena to lack of vitamin $C$ when in reality a deficiency in another factor or factors is responsible. Tozer (1921a) compared histologically the condition of the bone marrow of guinea pigs suffering, respectively, from lack of vitamin $\mathrm{C}$, of vitamin $\mathrm{A}$, and of both of these factors and concluded that unless the scurvy animal is protected from the effects of an insufficiency of vitamin A the results will be complicated and difficult of diagnosis. It was the recognition of the multiple deficiencies of a diet of cereal grains and water alone that led to the use of autoclaved milk in basal diets for the vitamin $\mathrm{C}$ studies.

Zilva and Wells (1919) compared microscopic sections of teeth from normal guinea pigs which had received subnormal amounts of antiscorbutics in their food and came to the conclusion that the tooth is one of the first if not the first part of the system to be affected by a deficiency of antiscorbutic material and that changes of a profound nature may occur in the teeth even when the ordinary scorbutic symptoms are still so slight as to be almost unrecognizable. They described the effect upon the teeth as a fibroid degeneration of the pulp, the fine cellular connective tissue of the normal tooth being replaced by a fibrous structure devoid of distinct cells. Similar radical changes were also noted in monkeys on a scorbutic diet. In discussing the application of these results to human nutrition, they suggested the possibility that dietary deficiency is an important factor in the great prevalence of tooth decay in civilized communities. They pointed out that cases of scurvy so mild as to escape detection may occur more often than is usually suspected and may reasonably be expected to influence dentition.

Howe (1920) also found that scorbutic diets have a very marked effect upon the teeth. He noted in guinea pigs in a state of chronic scurvy a marked decalcification and loosening of the teeth, with extensive absorption of the alveolar processes similar to pyorrhea. The 
gums bled easily and there was a copious flow of pus. If the condition was not too far advanced it could be checked promptly, with complete recovery, by supplementing the diet with orange juice. By alternating deficient with normal diets it was possible to produce distinct markings in the enamel of the teeth. Other decalcified areas were noted in the maxillary bones and ribs and in the bones of the head and legs.

Robb, Medes, McClendon, Graham, and Murphy (1921) reported a rapid loss of calcium from the bodies of guinea pigs on a scorbutic diet. The teeth on sectioning showed marked hyperemia of the pulp, with some hemorrhage. The odontoblasts were considered to secrete osteodentin which in some cases nearly filled the space normally occupied by the pulp, the change described by Zilva and Wells (1919) as fibroid degeneration.

Toverud (1923) from histological studies of the teeth of the scorbutic guinea pigs in Howe's investigation confirmed in general the conclusions of Zilva and Wells and of Robb et al. concerning the degeneration of the pulp tissue. The process was found to begin with hemorrhages in the upper part of the pulp and to extend down towards the apex of the tooth. In the more severe cases the entire pulp cavity became filled with bone or osteodentin.

Findlay (1921c), as the result of an investigation of the changes in the blood and vascular system in guinea pigs in progressive stages of scurvy induced by a diet of oats and bran supplemented with autoclaved milk, came to the conclusion that the essential lesion in scurvy is the interference with the nutrition of the capillary endothelium, resulting in stagnation of the blood in the capillaries, followed by insufficient oxygenation and death. The occurrence of hemorrhage was considered merely incidental to the wearing out of the intracellular substance. Hemorrhagic foci were most common in the kidney, bladder, intestines, liver, adrenals, bone marrow, and spleen in increasing order. Later Findlay (1923) produced a condition of chronic or latent scurvy in guinea pigs by the use of the same basal diet of oats, bran, and autoclaved milk, but supplemented with a very small amount (2 cubic centimeters every third day) of orange juice, and determined the resistance of these animals to infection with four species of pathogenic bacteria. In all cases the animals suffering from chronic scurvy succumbed to smaller infecting doses than did the controls on a complete diet. It is of interest that prior to the infection the guinea pigs on the deficient diet showed no definite clinical symptoms beyond failing to grow and appearing less active than usual-a condition not unlike that described by Hess in children suffering from latent scurvy. 
An examination of the blood, bone marrow, and lymphoid tissues of the guinea pigs with chronic scurvy showed more degeneration of the bone marrow than of the blood or lymphoid tissue, the changes being of the same nature as described by Zilva and Wells (1919) in the tooth pulp. The lowered resistance to infection of these animals was thought to be due, in part at least, to the degenerative changes in the bone marrow.

Mouriquand, Michel, and Bernheim (1924, 1924a) also noted an abnormal condition of the bone marrow in scorbutic guinea pigs and attributed the increased sensitivity to lack of vitamin $C$ of guinea pigs recently cured of scurvy to the persistence of these lesions. Werkman, Nelson and Fulmer (1924) reported a definite though not a marked decreased resistance to infection in scorbutic guinea pigs. Since this was accompanied by no change in the agglutinin production and phagocytic activity of the blood, the suggestion was made that the decreased resistance to infection was due chiefly to lowered body temperature. No study was made of the bone marrow.

In 1924 there was published as a supplement to the Swedish journal Acta Paediatrica a report by Höjer of his extensive investigation of experimental scurvy. As was the case with Holst and Frölich, Höjer's studies did not become fully effective until the publication of later papers in more accessible journals.

In reviewing earlier vitamin $\mathrm{C}$ studies, Höjer emphasized the necessity of having the basal diet complete in every respect except the antiscorbutic and in conducting the feeding tests quantitatively. The stock diet which he used consisted of crushed oats, bran, unboiled lightly skimmed milk, swedes (rutabagas), and green leaves, chiefly dandelion; and the basal scurvy-producing diet was crushed oats and bran ad libitum, supplemented with 60 cubic centimeters of lightly skimmed milk boiled for half an hour with air bubbling through it, and 0.5 cubic centimeter cod-liver oil per guinea pig per day. Occasionally 1 cubic centimeter of a 50 per cent yeast emulsion was administered by pipette to ensure a sufficiency of vitamin B. On this diet guinea pigs weighing from 200 to 500 grams showed the first outward symptoms of scurvy in about 16 days and died in from 18 to 38 days, the average survival period being about 28 days. Realizing that in human scurvy the latent form probably plays a more important rôle than the acute, Höjer made a careful study of the effects of diets containing varying suboptimal doses of orange juice as antiscorbutic as well as of diets supposedly free from vitamin $\mathrm{C}$. He found that even on the latter diet at least two and often four weeks elapsed before growth ceased entirely, but 
that changes could be noted in the teeth during the second week and in the lower jaw and ribs during the third week. The progressive changes taking place in the teeth were considered to be as follows: (1) Gradual changes of the odontoblast layer; (2) Amorphous calcification of the predentin; (3) Widening of Tome's canals in the dentin formed before the onset of scurvy; (4) New formation of bone instead of dentin, the new bone first forming a thin layer inside the calcified predentin and then extending toward the center of the pulp as a spongy porous mass; (5) Dilatation of the vessels and, in the early stages, hyperemia with occasional hemorrhages into the pulp; (6) Atrophy and resorption of the pulp tissue, sometimes going so far as to leave large hollows filled with fluid.

In Höjer's opinion the tooth picture is determined not so much by the time during which the guinea pig has been deprived of vitamin $\mathrm{C}$ as by the extent of the deprivation. The changes in tooth structure evident in guinea pigs which had received about seven-tenths of the minimal protective dose of orange juice for 22 and 92 days, respectively, were similar, but the condition was unlike that in other guinea pigs which had received eight-tenths of the protective dose for similar periods of time. In Höjer's opinion the condition most likely to be met in human beings is that developing when at least half of the necessary amount of antiscorbutic is provided. In guinea pigs on such a dosage the dentin is irregular with ridges of hard tissue. This is also the condition during the early stages of healing of severe scurvy.

Discussing the opinions of other investigators concerning the changes taking place in the teeth, Höjer agreed with Zilva and Wells and with Toverud that the changes begin in the crown part of the pulp and spread toward the root, but took exception to their theory of fibroid degeneration. In his opinion a few cells may degenerate, but the change is primarily from active odontoblast to active osteoblast cells-not necrosis but newly formed bone. In Höjer's opinion this term should be applied only to the irregular dentin formed when the tooth is being reorganized by reappearing odontoblasts or under conditions of partial deprivation of antiscorbutic.

The changes noted in the bones, although appearing later than those in the teeth, were thought to be of similar character. The new pathological bone, although porous and spongy, showed less differentiation than normal. No evidence could be obtained, however, of an altered proportion of calcium in the bones. In healing, the osteoblast layers gradually resume their normal appearance provided the dosage of antiscorbutic 
is ample. If insufficient, the scurvy may pass from the acute to the latent stage.

No primary derangement in calcium metabolism was thought to occur in either latent or acute scurvy. In the earlier stages bone formation is inferior quantitatively, though not qualitatively. This results in a surplus of calcium with increased excretion and possible kidney damage. Later, when the tissues become more or less necrotic, calcification takes place in the muscles and liver, resulting in a positive calcium balance. Healing is accompanied by an increased excretion of calcium. The most pronounced difference in the bone system between scurvy and rickets was thought by Höjer to be in regard to calcification. In rickets an osteoid substance is formed which is not calcified, while in scurvy bone is formed to a limited extent and rapidly calcified.

Most important among the scorbutic changes noted in the skeleton musculature and in the tissues of various organs, including the heart, liver, spleen, kidneys, adrenals, and salivary glands, was general atrophy. The condition in the skeleton musculature is described as "a general hyperemia, the appearance of certain giant nuclei cells, a great tendency towards necrosis, and the impregnation of the necroses with calcium and hemorrhages in the places exposed to mechanical strain or traumata." These changes, while taking place a little later than those in the teeth and bone marrow, set in during the chronic or so-called latent stage of scurvy. Contrary to most investigators, Höjer considered the change in the adrenals to be of no greater significance than in any other organ and to be simple atrophy rather than hypertrophy. Hemorrhage, one of the characteristic symptoms of scurvy, is attributed chiefly to weakness in the vascular wall and not to changes in the blood, although dilatation of the capillaries and veins is sometimes so strong as to give the impression of hemorrhage.

In 75 per cent of Höjer's animals with acute scurvy no signs of infection could be detected, but more than half of the animals with latent scurvy suffered from infection of one kind or another. This lowered resistance to infection is attributed chiefly to weakness of the lymphoid tissues.

Throughout this entire report and a later one by Höjer and Westin (1925) dealing specifically with the condition of the jaws and teeth of guinea pigs on graded doses of vitamin C, the significance is emphasized of latent scurvy accompanied by no microscopic or macroscopic changes. It is thought that even in animals receiving the amount of antiscorbutic vitamin hitherto considered the minimum preventive dose. there may be indistinct signs of general ill health resulting from a 
lowered power of reaction of the cells. Höjer's papers have been reviewed in considerable detail on account of their quantitative aspect and their emphasis on the significance of slight as well as marked deficiencies of vitamin C. Quite recently they have received more attention than when first published on account of awakened interest in the practicability of the method of tooth examination as a rapid quantitative method in vitamin $\mathrm{C}$ determination. (See beyond.)

Wolbach and Howe $(1925,1926)$, reporting upon an investigation planned and begun before the appearance of Höjer's monograph, confirmed some of Höjer's observations, but were of the opinion that Höjer's diets were not completely deficient in vitamin $C$ and consequently the feedings were not always comparable. With the basal diet employed by them the earliest changes in the teeth were observed in from 6 to 7 days. The most striking of these changes are described as the separation of the layer of odontoblasts from the dentin, with the filling in of the space between them with a liquid. The odontoblasts themselves underwent changes in size, arrangement and staining reaction. The changes noted in the bones were cessation of bone formation, with accumulation of osteoblasts in certain localities, particularly under the periosteum. The effect of the addition of an antiscorbutic was very rapid. In the teeth, formation of new dentin to fill in the space caused by the separation of odontoblasts was evident within 48 hours, while in the bones a single dose of orange juice or other antiscorbutic substance was followed by the prompt appearance of bone matrix between the cells. Artificial bone lesions showed no repair until the addition of antiscorbutic substances to the diet, after which there was prompt formation of new bones. In the opinion of Wolbach and Howe: "The scorbutic state may be characterized as one affecting supporting tissues in which the cells are unable to produce and maintain intercellular substances. This condition affects various supporting substances to a different degree, and is most marked in those in which the intercellular substance is calcified, as the dentin of teeth and the matrix of bone. The characterization applies to cartilage and connective tissue, and, by inference, to other intercellular substances, including that of blood vessels. The hypothesis is entertained, based upon the study of repair in incisor teeth of scorbutic guinea pigs, that in the formation of intercellular substances there is a change of the material from a liquid to a solid or jell state, and that the missing factor in the scorbutic condition is one affecting the jelling or setting of the liquid product."

This theory corresponds closely with the conclusion of Aschoff and Koch (1919) on the basis of an extensive study of scurvy in man that 
the scorbutic condition is due to a lack of, or to a failure in the formation of, cement substance (Kittsubstanz). At first glance the recent observations of Meyer and McCormick (1928, 1928a) of widely distributed liquefaction of the cytoplasm and cell walls in experimental scurvy would also seem to be in line with this hypothesis. As an extreme illustration of this liquefaction Meyer noted that if a very loose molar tooth is extracted from a scorbutic guinea pig, a drop of gelatinous material is usually found adhering to what remains of the root or occupying the place of it. He attributes the phenomenon, however, to a chemical and not a directly cellular activity-intra- rather than intercellular changes.

Hanke (1929), working in coöperation with the Chicago Dental Research Club, made a very thorough study of the dietary habits with relation to dental disorders in 114 patients from 6 to 60 years of age. Classified according to the type of disorder, 47 of the patients investigated showed uncomplicated dental caries; 25 , caries associated with inflamed gums or pyorrhea; and 25, gum inflammation and pyorrhea with no caries. The diets of 27 in the first group and 14 in each of the other two groups were deficient in vitamin $\mathrm{C}$ alone. Even more striking was the prompt response in the arrest of caries and in improvement in the gum tissue and tightening of the loose teeth in the pyorrhea cases following dietary changes involving a marked increase in vitamin C. For adults 1 pint daily of orange juice containing the juice of one lemon, together with one-fourth to one-half head of lettuce or its equivalent in raw cabbage is recommended for the vitamin $C$ supply. More recently Hanke (1930) reports confirmation of his findings on extending his observations to a larger group of patients.

\section{Quantitative Determination}

As Hess points out (1920, p. 173), "For well over a hundred years it was generally known that scurvy could be cured by fruits and vegetables, and yet no further progress was made toward a more complete understanding of the value of these foodstuffs. . . . Decided advance has been made only in the past few years since these foodstuffs have been studied from the quantitative viewpoint."

The beginnings of our modern knowledge of the quantitative determination of antiscorbutic values, as of so many other aspects of scurvy and the antiscorbutic vitamin, are to be found in the work of Holst and Frölich (1912), who made comparisons of the antiscorbutic values of foods by determining whether or not certain empirically chosen quantities of foods which they fed would suffice to prevent the appearance 
of scurvy in guinea pigs. They tabulated fully the results of experimental feeding with and without the antiscorbutics and made some progress toward the standardization of the test animals, but in general they fed the foods to be tested only at one level of intake, and the basal diet which they employed, consisting usually of oats and water only, was deficient in other respects as well as in antiscorbutic vitamin.

Cohen and Mendel (1918) devised a new basal ration in the form of a "soy bean cracker" made of cooked soy bean flour, cellulose, sodium chloride, calcium lactate, dried brewers' yeast, and heated milk, planned to provide all necessary nutrients except the antiscorbutic. They showed that experimental scurvy could be induced in the guinea pig at will, and formulated more fully the criteria for the recognition of this condition.

Hess and Unger (1918a) employed chiefly a basal ration of oats, hay and water. This has also been used in other laboratories, alfalfa hay being especially advocated.

Chick, Hume and Skelton, Delf, and other workers at the Lister Institute have used a basal diet of oats and bran ad libitum with a liberal allowance (usually 60 cubic centimeters per guinea pig per day) of milk autoclaved at $120^{\circ} \mathrm{C}$. for one hour. This addition of milk to the basal diet was found to result in a much better general condition of the animals as would be expected in view of the extent to which the diet is thus improved in its mineral and vitamin A content, and the nutritive efficiency of the protein mixture which the combination of grain and milk affords. As Hess points out, however (1920, p. 117), this milk still retains a small amount of antiscorbutic vitamin; and from the results of other work we know that the amount of vitamin C, thus introduced into the basal diet which should be free from it, while small, is variable and therefore must detract from the quantitative accuracy of work in which this basal ration is used.

Building upon the experience of previous investigators as well as upon the results of their own studies of the above-mentioned basal diets and several modifications of them, Sherman, La Mer and Campbell (1922) still further developed the basal ration to ensure its freedom from vitamin $\mathrm{C}$ and its entire adequacy in all other respects. Their basal diet consisted at first of sound whole oats ground in the laboratory as needed 59, skimmed milk powder, heated in open trays at $110^{\circ} \mathrm{C}$. until all of the antiscorbutic vitamin is destroyed, 30, freshly prepared butterfat 10, and sodium chloride 1 per cent. Later the allowance of whole oats was replaced by a mixture of rolled oats 39 , and bran 20 
per cent of the ration. This diet supports excellent growth up to the time of the onset of scurvy.

Sound mature oats show no antiscorbutic property when fed to guinea pigs and are eaten readily by them. By using heated skimmed milk and fresh butterfat instead of heated whole milk, the absence of antiscorbutic vitamin is at least equally well ensured and the palatability of the diet and its fat-soluble vitamin content are improved. The heat treatment necessary to ensure complete destruction of vitamin $\mathrm{C}$ in the skimmed milk powder should be determined by each investigator for his own material and technique, keeping in mind the likelihood of variation in antiscorbutic vitamin content of milk with the season and the importance of eliminating this vitamin completely from the basal ration without subjecting the food to such excessive heating as would give it a burnt taste and prevent its being eaten readily by the experimental animals. In these experiments, two hours' heating at $110^{\circ} \mathrm{C}$. in shallow trays freely exposed to the air of the oven was found to be sufficient, as determined by controlled feeding experiments. After such heating the milk powder is of a light brown color.

The butterfat, prepared by melting butter of good quality at the lowest possible temperature and freeing from water and curd by decantation and filtration through paper, is intimately mixed with the heated milk powder, the salt, and the freshly ground oats, so that the constituents of the mixture cannot be separated by the experimental animal while eating. The food mixture should be kept in a refrigerator in well-filled, air-tight containers; fresh portions fed daily, and feeding cups thoroughly cleansed at least twice a week. Even the slightest incipient rancidity may result in failure of the animals to eat the food readily, in which case the interpretation of results will be difficult and probably of very doubtful value.

Selection and Care of Experimental Animals.-The experimental animals should either be bred by the investigator or purchased at an early and known age. They should be known to be growing at a normal rate. For about a week before the beginning of the experimental period they should be housed in the experimental cages or pens and fed with the above basal ration plus green food, both ad libitum. The experiment proper is then begun by simply discontinuing the green food with or without the feeding of a measured amount of antiscorbutic in addition to the basal ration. Guinea pigs six to eight weeks old and weighing 300 to 350 grams are best used for this purpose. Placed at this age and size upon the above basal diet with food and water always available they usually eat about 18 to 20 grams of the dry food mixture per 
day and continue to grow for about 15 days, then lose weight rapidly and die of scurvy in from 26 to 34 days after being deprived of antiscorbutic food. If the animals are much younger, the results are somewhat less regular; if much older, the animals are somewhat less susceptible, and also less likely to show good growth up to the time of onset of scurvy symptoms.

Symptoms, Survival Period, and Autopsy Findings.-With animals such as have been described, the first symptoms of scurvy appear after about 12 days on the above basal diet. The nature and sequence of the symptoms have been so fully and clearly described by Cohen and

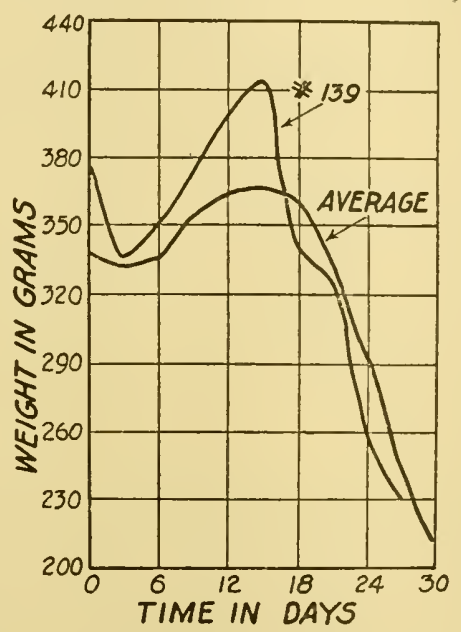

FIG. 7.-Weight curves of guinea pigs receiving basal ration adequate in all other respects but lacking vitamin C. Average curve and curve of one animal showing in pronounced degree the initial loss in weight at the beginning of the experimental period as described in the text.

Mendel (1918) and by Hess (1920, pp. 135-140) as not to require detailed discussion here. Loss of weight usually begins soon after the appearance of the first symptoms, though some animals begin to lose weight earlier. On the first or second day of the experimental period there may occur a decrease of body weight due to elimination of bulky intestinal contents from the green food of the fore-period. In such cases the minimum weight of the first or second day is taken as the initial weight of the experimental period. As an accurate weight curve is helpful in the interpretation of results, each animal should be weighed at least once in three days throughout the experimental period. Fig. 7 shows the average weight curve of 10 guinea pigs kept on the basal diet above described. Since the time of reaching a maximum and begin- 
ning to lose weight differs with individuals, the effect of averaging the results is to blunt the typical peak of the weight curve. For this reason there is also given in Fig. 7 the weight curve of an individual guinea pig which shows in pronounced degree the initial fall in body weight upon being placed on the basal diet (i.e., upon discontinuing allowance of green food), followed by rapid growth in weight to a sharp maximum, then followed by rapid loss of weight after the onset of scurvy. The weight curves in the majority of cases may be expected to be intermediate in character between the two curves shown in Fig. 7.

The survival periods of 15 successive animals kept upon the above basal diet only were as follows: $33,26,27,28,34,28,27,34,26,29,32,32$, $32,31,34$ days. At autopsy the findings which proved most significant were: looseness of teeth, fragility of bones, enlargements and hemorrhages of joints and rib junctions. For full description of the pathology of experimental scurvy the reader is referred to the monograph of Hess (1920, pp. 122-125, 129-130).

Quantitative Rating of Results.-The results obtained by Sherman, La Mer and Campbell (1921a) on feeding the above-described basal ration alone and with the addition of filtered juice of canned tomatoes in measured amounts calculated to the basis of 300 grams of guinea pig were in brief as follows:

With no antiscorbutic there was good initial growth followed by onset of scurvy symptoms, cessation of growth and great loss of weight before death from scurvy which occurs at 26 to 34 days. Autopsy reveals in severe form all the typical signs of scurvy, notably hemorrhages, fragility of bones, and looseness of teeth. (See table below.)

With 1.0 cubic centimeter of tomato juice the duration of life is prolonged and becomes less uniform than on the completely scorbutic basal diet. The animals become lame and stiff before death and at death show severe hemorrhages, fragile bones and loose teeth.

With 1.5 cubic centimeters tomato juice the animals usually live out the experimental period of 70 to 90 days after which it is unlikely that death from scurvy will occur. Scurvy symptoms develop with soreness and stiffness in the joints and usually with loss in body weight. Hemorrhages and enlargements of rib junctions may become quite as pronounced as in the previous cases. (Since the animals live longer there is more time for these abnormalities to develop.) Fragility of the bones and looseness of teeth are less marked than when less antiscorbutic is given.

With 2.0 cubic centimeters tomato juice growth after 15 days is subnormal and animals show soreness of joints without noticeable stiff- 
ness. When the animals are killed and examined after 70 to 90 days on this diet they show hemorrhages but not to a pronounced degree. Jaws and teeth appear normal and bones usually so.

With 3.0 cubic centimeters or more tomato juice there is complete protection from scurvy as judged by examinations both during life and at autopsy. Growth is fully normal in all animals that eat the basal diet well.

The accompanying table shows the protocols of typical cases of guinea pigs kept on the basal diet alone or with measured amounts of tomato juice as antiscorbutic. In all cases the volume of tomato juice as stated in the table is the amount which was fed per 300-gram guinea pig. This reduction of the dosage of antiscorbutic to a uniform basis of body weight of standard animal has been found to add considerably to the quantitative significance of the results. Following the precedent of Holst and Frölich the severity of autopsy findings such as hemorrhages and fragility of bones and looseness of teeth are indicated by - (no different from normal), ? (doubtful), tr (trace), and,+++ , +++ for increasing degrees of severity. From this series of observations upon animals receiving no antiscorbutic vitamin and with different measured amounts up to the amount which affords complete protection and permits optimal growth, it becomes possible to interpret the symptoms and autopsy findings in terms of the percentage of the required amount of antiscorbutic which was actually received by the animal in any individual case. This means that animals fed on this basal diet and receiving some antiscorbutic but not enough for complete protection can be given a quantitative rating based on the weight curve, survival period, and severity of the symptoms and autopsy findings.

This method was applied in studying the "heat destruction" of the antiscorbutic vitamin quantitatively, with feeding experiments to determine how much more of the heated juice must be fed in order to get the same results as with a known quantity of raw juice. Comparison of the quantities of the two juices necessary to give the same degree of protection then enables one to calculate the percentage of antiscorbutic which had been destroyed during the heating.

The last four lines in the accompanying table give the results of experiments of that kind. Comparing the findings in the case of No. 155 with results given previously, it was considered that on the whole 3.9 cubic centimeters of tomato juice which had been heated for one hour at $100^{\circ} \mathrm{C}$. showed practically the same antiscorbutic effect as 2 cubic centimeters of the unheated tomato juice and therefore that approximately one-half of the antiscorbutic vitamin of the tomato juice 


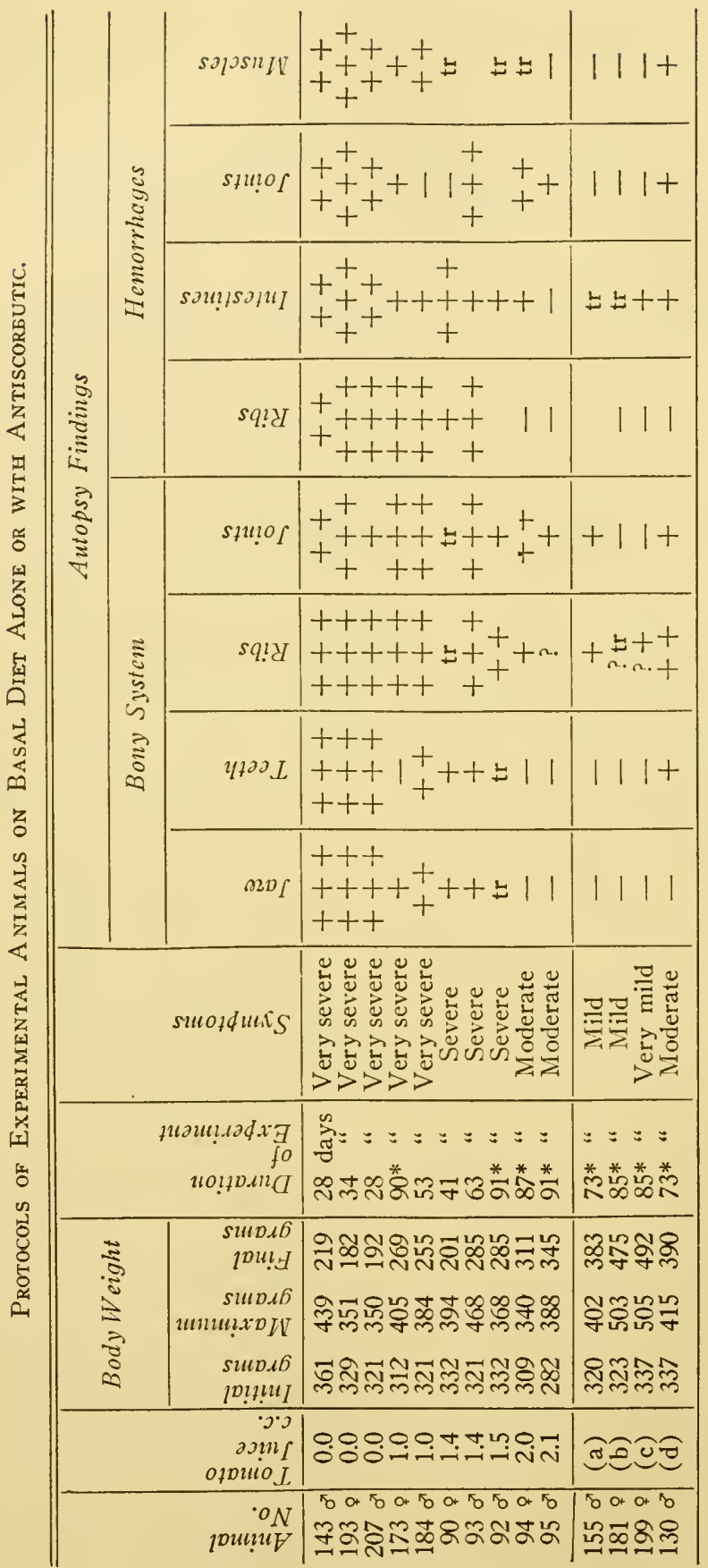

نั. ن.ّ. 君㗏记 प्येक्ष :0: 每 \&\&점준

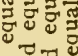

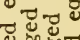

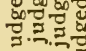
نu? ن 8요요 तथत्ण तो क ल 도옹 0 吉台。 두웜 $\exists \widetilde{\Xi}$

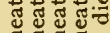

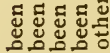

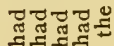
동동동등 흔

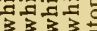
을 ㅇํㅇํㅇํㄹ

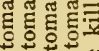
88084 micom ชี צะ ณัฒ

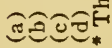


had been destroyed by the heat treatment. The average of a considerable number of such experiments was practically 50 per cent.

In the next case (No. 181) the animal received 7 cubic centimeters of tomato juice which had been heated at $100^{\circ} \mathrm{C}$. for 4 hours. The results indicated that this amount of heated juice was equivalent in antiscorbutic value to 2.5 cubic centimeters raw juice, and the average of a number of such experiments indicated that heating for four hours at $100^{\circ} \mathrm{C}$. resulted in the destruction of about 68 per cent of the antiscorbutic vitamin present in the raw juice. The results of experiments on Nos. 199 and 130 (notes "c" and "d") show results of heating at $60^{\circ} \mathrm{C}$. with, of course, a lower rate of destruction than at $100^{\circ} \mathrm{C}$.

It is plain that in the same way one may compare the antiscorbutic potency of a measured amount of any other material with that of any of the different amounts of canned tomato juice representing any degree of protection up to the complete protection afforded by 3 cubic centimeters and can thus determine the relative amounts of antiscorbutic vitamin in different foods both from experiments in which the exact minimum protective dose is found and from those in which there is a definite partial protection to which a quantitative rating can be given. This method possesses the advantage of the method of minimum protective doses, and in addition permits the use of a numerical value for each individual experiment of a series, the average of which should yield a more trustworthy result than when only those animals receiving exactly the minimum protective dose are taken into account.

In later studies (Kenny, 1926) the severity of the autopsy findings has been expressed quantitatively by a scurvy score which is the total number of + signs at the end of the experimental period of 70 to 90 days as may be determined upon. Death from scurvy before the end of this period is "scored" by dividing the autopsy score by the fraction of the period that the animal lived.

Other basal diets have been proposed from time to time. LopezLomba and Randoin (1923, 1923b), objecting to the use of milk as furnishing some vitamin $\mathrm{C}$, recommended a diet consisting of meat peptone, starch, granulated brewery yeast, butterfat, salt mixture, and filter paper.

As already noted, Höjer (1924) used oats and bran supplemented with 60 cubic centimeters daily of milk boiled with aeration to destroy vitamin C.

Bezssonoff (1926a), objecting to the inclusion of milk in any form in the basal ration, suggested fresh egg yolk as a substitute, the ration consisting of 900 grams of oats mixed with 100 grams of bran, 
40 grams of fresh yeast, and 75 grams of egg yolk emulsified with 125 cubic centimeters of water. On this, guinea pigs are said to succumb to scurvy without exception before the thirty-third day unless the diet is supplemented with an antiscorbutic, 3 cubic centimeters of lemon juice daily being the minimum protective dose for a 400 gram animal.

Eddy (1929) modified the Sherman-La Mer-Campbell basal ration by substituting 1 per cent of cod-liver oil for the equivalent amount of butterfat in order to insure against vitamin D deficiency and by feeding yeast separately as an additional source of the vitamin B complex.

Along with lack of uniformity in the basal diets are corresponding variations in methods of recording results. One basis for the expression of the relative antiscorbutic value of foods is the "minimum protective dose" which represents the daily allowance of the food in question which just meets the need of the guinea pig for complete protection from scurvy. Another way is to express the antiscorbutic value of the food in comparison with that of some well-known antiscorbutic such as orange juice, which for convenience of comparison may be given an arbitrary value of 100 .

The unit method of calculating vitamin values as described for vitamin $B$ (Ch. II) affords perhaps the most convenient mode of expression. The unit of vitamin $C$ suggested is that amount which when fed as a daily allowance just suffices to afford complete protection from scurvy (as the term is here used) to a standard guinea pig as described in the method for quantitative determination of vitamin $\mathrm{C}$ above described. This is essentially what was proposed by Chick and Hume who called it the minimum protective dose (m.p.d.).

The use of scurvy scores to form a judgment of the fraction of complete protection an animal has received and from this to calculate the amount required for complete protection should be attempted only by skilled and experienced observers, but in such hands it has the advantage of bringing into the quantitative interpretation a larger proportion of the test animals than simply those which received exactly the minimum protective dose.

Bezssonoff (1921b, 1923a) claimed to have discovered a color test for vitamin $\mathrm{C}$ sufficiently specific to be of value as a substitute for the feeding method. The reagent is essentially the Folin-Denis phenol reagent modified by reducing the proportion of concentrated phosphoric acid to one-third the original amount and adding without heating an equal volume of normal acid (sulfuric, hydrochloric, or nitric). This was said to give a slate gray color turning to blue with various extracts of known antiscorbutic properties but not with the 
same extracts after destruction of vitamin C. Of various phenols tested, quinol alone was said to give the blue color. Kay and Zilva (1923) applied the test to a large number of substances which were tested simultaneously on guinea pigs and found the relationship not to hold true in all cases. Some antiscorbutically active substances failed to give the color reaction and other inactive substances gave a positive reaction. Wedgewood and Ford (1924) also reported a lack of specificity in the test. In later papers Bezssonoff (1924a, 1924b, 1926) outlined various conditions which must be fulfilled to obtain satisfactory results, but the method has never received much favor.

Höjer (1926) proposed a new method based on the histological changes in the teeth of guinea pigs on diets deficient in vitamin C. Since changes are observable in the teeth earlier than in any other part of the body, the feeding period is considerably shortened, from 10 to 14 days being considered sufficient.

The technique as originally described by Höjer is as follows: "As previously, young guinea pigs from a certain day on are given a basal diet free from antiscorbutic, but otherwise complete, and to this diet are added quantitatively daily doses of the juice to be examined. For a sharp determination of the fully protective dose it is advisable to have several animals on different doses. In addition there ought to be two control animals on the basal diet alone, and two on the basal diet with a fully protective dose of a known antiscorbutic. After a period of 10-14 days all the animals are killed. The one-half of the lower jaw is taken out and decalcified in a 5 per cent tri-chloracetic acid solution. It is then embedded and sectioned after one week. The section is made through the foremost molar at right angles to the longitudinal axis of the jaw. Some sections are stained with haematoxylin-eosin, and others with tri-oxyhaematin according to the method of Hansen."

The principal examination is made of the cross section of the incisor roots. It is stated that on a fully protective antiscorbutic dose the section stained with hematoxylin-eosin shows a broad uniformly stained pink layer of dentin, a white uncalcified predentin, a layer of long, slender parallel odontoblasts, and a pulp of star-shaped cells. When about 0.9 of the minimum protective dose of vitamin $C$ is given, the odontoblast layer is distinctly narrower; with still less, the uniformity of the dentin staining has disappeared, the freshly made dentin having a lighter color and less collagen. When vitamin $C$ is completely lacking the dentin is rather thin, the predentin is stained very dark because it is calcified, the odontoblasts no longer exist as a continuous layer. "They seem to be on a disordered march into the hyperemic pulp and do not 
deserve the name of odontoblasts any longer, as they are not forming dentin, but bone in the form of an irregular network." Similar changes except for a difference in color are apparent in the sections stained by the Hansen method.

In Höjer's opinion the error in the determination is not over 10 per cent and fairly accurate estimates may be made in tenths of the protective dose, although it is advisable to determine the minimum protective dose, since this is more sharply defined. The chief advantages of the new method as compared with the old are considered to be the accuracy* in fixing the fully protective dose and the saving in time and expense.

Goettsch (1928) subjected the Höjer method to critical examination, particularly to determine whether the histological changes in the teeth are specific for scurvy and how the results obtained by this method compare with those of the method in general use. Her conclusions were that the changes are specific for scurvy and that it is possible by this method to determine the minimum protective dose, but that such variations occur within a group of animals on an inadequate dose that its quantitative value can be determined only with the use of large numbers of animals, using orange juice as the standard antiscorbutic, she found that twice as much was required for the minimum protective dose by the new method as by the old. Another advantage mentioned by Goettsch in the new method as compared with the old is that with the time of feeding shortened to two weeks there is less danger of the animals dying from other causes than scurvy during the course of the test. For those interested in this test, this paper of Goettsch, which includes an appendix by K. M. Key giving the details of the technique, is recommended as furnishing more explicit directions for conducting the test than are given in Höjer's paper.

Eddy (1929) has recently reported his experience in comparing the Höjer method with that in general use. Using canned string beans, orange juice, and bananas as the materials to be tested by the two methods, he found, in agreement with Goettsch, that almost exactly twice as much is required for the minimum protective dose for the teeth as for protection against scurvy as previously understood. Commenting upon this, Eddy states: "It seems hardly possible that this ratio is entirely fortuitous. If further tests establish it as the true difference between tooth and general body requirements it will be a

\footnotetext{
* It is to be remembered, however, that this "accuracy" may mean delicacy rather than dependability, since it rests on a single criterion whereas the method previously described employs the broader basis of a composite of criteria as the basis of judgment of the degree of scurvy, or of its absence.
} 
simple matter to convert antiscorbutic values that have been reported since the general adoption in this country of the Sherman method into tooth protective values. We will need only to multiply by two.

"What the true values are in terms of teeth obviously requires much more extended study. I believe, however, that the results reported herewith justify considerable faith in the Höjer method, and its speed, its relative simplicity, its greater freedom from the danger of contaminating disease factors in the longer test all commend it to our careful appraisal."

Dalldorf and Zall (1930) have recently recommended using the rate of growth of the incisor teeth of guinea pigs in testing foodstuffs for th:eir antiscorbutic value. In their opinion "under standard experimental conditions used in the testing of foodstuffs for antiscorbutic value, the rate of tooth growth would appear to be a precise indication of the degree of scurvy, being more delicate than the Sherman (Kenny, 1926) score, and more constant as well as more simple, than the Höjer method."

\section{Occurrence and Stability of Vitamin C in Food Materials}

Impetus to a systematic study of the occurrence of the antiscorbutic vitamin in food materials and its stability under different treatment was given by the tremendous problem of civilian and army rationing during the World War. A striking illustration of the relationship of beriberi and scurvy to particular dietary deficiencies is afforded by the reports of Willcox $(1917,1920)$ and of Hehir $(1919,1919 a)$ of the outbreaks of beriberi in the British troops and scurvy in the Indian troops in Mesopotamia in 1915 and 1916, particularly during the siege of Kut-el-Amara. Hehir reported that during this siege there were about 1,050 cases of scurvy, all but one of which were in the Indian troops, while beriberi appeared among the British, and not among the Indian troops.

The high incidence of scurvy among the Indian troops and its almost complete absence among the British troops was traced to the use by the latter of fresh meat toward the end of the siege when the bullocks, horses, and mules were killed to eke out the diminishing food supplies. The Indians who did not overcome their scruples against eating such flesh were the worst sufferers from scurvy. In the last period of the siege, although the men were rapidly losing weight on a starvation diet, the disease declined coincident with the use of about 3 ounces per man per day of green herbs collected from the plains. It was emphasized that fresh meat alone without vegetables will not indefinitely 
postpone scurvy but seems to delay its appearance. An increase in the meat rations for the Indians and the development of vegetable gardens for the stationary troops caused scurvy practically to disappear from Mesopotamia. Willcox in commenting on this same outbreak attributed the greater immunity to scurvy of the white troops in part to the slight antiscorbutic value of the fresh meat which they consumed and in part to the fact that they had previously been accustomed to a varied diet containing ample antiscorbutics whereas the previous food habits of the Indian troops had resulted in their being in many cases already on the border line of scurvy. While the fresh meat was believed to have undoubted value it was of course here as in much other experience recognized as being but a poor antiscorbutic.

The occurrence of beriberi among the white troops and the complete absence of it in the Indian troops during the same siege was attributed chiefly to the use of ration biscuits or white bread by the former and atta (flour containing the wheat embryo) by the latter troops. One of the measures taken to improve the dietary of the British troops was the incorporation of 25 per cent of atta in the bread.

The urgent need of more exact knowledge of the antiscorbutic values of foodstuffs of practical war-time utility led to a systematic survey (principally at the Lister Institute, London, and simultaneously to a lesser extent in other laboratories) of the commoner foodstuffs with respect to their relative value as antiscorbutics, and to studies of methods of stabilizing and concentrating the vitamin for practical use.

The methods used at the Lister Institute were essentially a development of those of Holst with the exception that a daily ration of 60 cubic centimeters of milk autoclaved at $120^{\circ} \mathrm{C}$. for one hour was added to the basal ration of oats and bran. This addition was found to improve greatly the general condition of the guinea pigs without greatly influencing the onset of scurvy. In view of later findings it appears (Hess, 1920) that small but varying amounts of the antiscorbutic vitamin may remain in such autoclaved milk which would detract somewhat from the exact quantitative value of the results.

Fruits and Vegetables.-A study by these methods of the relative antiscorbutic values of the juices of limes and lemons (Chick, Hume, and Skelton, 1918b) led to the conclusion that lemon juice is about four times as rich in antiscorbutic vitamin as is lime juice, and that preserved lime juice is not to be depended upon for prevention of scurvy. These results are of interest in view of the traditional use of "lime juice" as an antiscorbutic. Historical investigation (A. Henderson Smith, 1919) indicated that the actual material to which the term 
"lime juice" was applied was frequently the juice of lemons and not of limes and that it was really to lemon juice that the practical elimination of scurvy from the British navy and merchant marine had been due. As a striking instance of this the case is cited of two British expeditions into the Arctic, that of the Investigator in 1850 which reported wonderful immunity to scurvy throughout two years of privation during which, however, lemon juice was available as antiscorbutic and that of the Alert and the Discovery in 1875 which used lime juice and had trouble with scurvy before the end of the first winter.

Harden and Zilva (1918a), selecting lemon juice as a convenient material for studying the concentration of the antiscorbutic vitamin, found that the liquid remaining when citric acid had been precipitated from the juice by the addition of calcium carbonate appeared to contain most if not all of the vitamin. The method used was as follows: To the fresh lemon juice was added with constant stirring an excess of precipitated calcium carbonate followed by two volumes of absolute alcohol. The mixture was then filtered, the residue pressed in a hand press and the liquid thus obtained filtered; the filtrates were combined, evaporated in vacuo at $35^{\circ} \mathrm{C}$. to remove all alcohol, and made up to the original volume with distilled water. The solution thus obtained, which is sometimes referred to as "treated juice," was yellow, acid to litmus, sweet to the taste and lemon flavored. This liquid showed some deterioration after storage in the cold for about a fortnight and marked deterioration on evaporation at $30^{\circ}$ to $40^{\circ} \mathrm{C}$. in vacuo. If, however, the treated juice was slightly acidified by the addition of citric acid and then evaporated in vacuo at $30^{\circ}$ to $40^{\circ} \mathrm{C}$. as before, a residue of high antiscorbutic potency was obtained.

By the use of this preparation it was possible to feed larger doses of antiscorbutic than in the form of ordinary foods. The administration of liberal doses of treated lemon juice previous to depriving guinea pigs of the antiscorbutic factor did not seem to prevent nor delay the onset of scurvy, thus indicating that the antiscorbutic vitamin is not stored to any extent in the body. The treated juice not only proved potent as an antiscorbutic for guinea pigs and monkeys (Harden and Zilva, 1918a, 1919), but brought about prompt recovery from scurvy in children seven months to one year old (Harden, Zilva, and Still, 1919). The rapidity of recovery was attributed to the fact that it was found possible to give the antiscorbutic in amounts equivalent to the juice of from 6 to 12 lemons daily without any gastrointestinal disturbances, even in the case of children less than a year old. 
Later Zilva (1924) prepared a still more concentrated extract by evaporating the decitrated lemon juice to one-tenth of its volume and acidifying it by the addition of seven grams of citric acid to one liter of juice. No loss in potency of this product could be detected after storage under laboratory conditions in a dark cupboard for five months. The concentrated product was also used after six months' storage in the treatment of a very severe case of infantile scurvy. The treatment was very intensive, the equivalent of about fifteen lemons being given during the first twenty-four hours. The patient was discharged cured in ten days after the equivalent of about forty-eight lemons had been administered. Decitrated lemon juice has been used to a considerable extent as the basis for the preparation of concentrates for prophylactic and therapeutic treatment and also in experimental work on the antiscorbutic vitamin.

Another method of preparing a lemon juice concentrate in convenient form was described by Bassett-Smith (1920). The fresh juice is filtered through muslin and then through filter paper under reduced pressure. The filtered juice is evaporated in vacuo over sulfuric acid at ordinary temperature and the sirupy residue made into as stiff a paste as possible with a mixture of 97 per cent anhydrous lactose and 3 per cent gum tragacanth. The paste is cut into sections, each containing the juice of half a lemon. These are then rolled and pressed into the shape of lozenges. Experiments were reported in which these tablets were used both for the prevention and for the cure of scurvy in guinea pigs with excellent results, one-fifth of a tablet, equivalent to 4.8 cubic centimeters of fresh lemon juice, representing the minimum preventive dose. It was estimated that one tablet, the equivalent of half a lemon, would be ample as a daily dose to prevent scurvy in adult human beings.

As these tablets have been found to retain their antiscorbutic properties for over a year when stored at room temperature (Bassett-Smith, $1920 \mathrm{a}, 1921$ ) they afford an antiscorbutic of small bulk eminently suited for long voyages. Macklin and Hussey (1921) state that the antiscorbutic substances used on board ship in the Shackleton polar expeditions included lemon juice concentrated by this method but not made into tablets, while for sledging conditions lemon juice tablets are used.

Harden and Zilva (1918) also made use of orange juice in their studies of the behavior of antiscorbutic substances. On treating a mixture of autolyzed yeast and orange juice with various adsorbents and testing the filtrates on polyneuritic pigeons and scorbutic guinea pigs, 
they found that while fuller's earth and dialyzed iron adsorbed almost all of the antineuritic vitamin, such treatment did not affect the antiscorbutic activity. Similarly, orange juice did not lose its activity on filtration through a Berkfeld candle.

Harden and Zilva (1918d) also studied the susceptibility of the antiscorbutic substance to alkalinity hy experiments upon guinea pigs receiving oats, bran and autoclaved milk with measured addenda of orange juice which was fed in some cases fresh and unchanged, in other cases neutralized immediately before feeding, while in still other cases the juice was made alkaline and allowed to stand for some time before being fed. Of freshly expressed orange juice, from 3 to 5 cubic centimeters was always sufficient to keep guinea pigs in good condition and free from any symptoms of scurvy. Three guinea pigs receiving respectively 3,5 , and 7 cubic centimeters of orange juice which had just been rendered neutral to phenolphthalein were also protected from scurvy. On the other hand, doses of 3,5 , and 7 cubic centimeters of an orange juice which had been made twentieth-normal alkaline by addition of sodium hydroxide and then allowed to stand 24 hours in a cold room before feeding did not protect. That this was due to destruction of the antiscorbutic vitamin and not to any effect of the alkali upon the animal was shown by the fact that the results were unchanged when the juice which had been thus treated was reacidified immediately before being fed. In a final experiment to test the effect of less drastic treatment, the juice was made fiftieth-normal alkaline by addition of sodium hydroxide and later in the same day fed in doses of 3,5 , and 7 cubic centimeters respectively to three guinea pigs. All died of scurvy, though not so quickly as those receiving the basal diet only, showing that a considerable part but not all of the antiscorbutic vitamin was destroyed by this treatment.

Hess (1918) reported that orange juice, boiled and slightly alkalinized, constitutes an excellent antiscorbutic agent for intravenous use in infantile scurvy, but that in such cases the juice should be used immediately and not kept in the alkaline condition.

Hess and Unger (1918a) in a study of guinea pig scurvy reported that orange juice lost some of its antiscorbutic power after storage in a refrigerator for three months, that the antiscorbutic substance of orange juice could be extracted with 95 per cent alcohol, and that orange peel after being dried and kept dry for three months still showed antiscorbutic properties. Prunes seemed, in their experiments, to have no value as an antiscorbutic, and the question naturally suggests itself whether the practice of dipping prunes in alkaline solution in connec- 
tion with the drying process is the cause of their deficiency in vita$\min \mathrm{C}$.

Harden and Robison (1919) showed that the antiscorbutic substance in orange juice is not volatilized nor largely destroyed when the juice is distilled at $40^{\circ} \mathrm{C}$. under reduced pressure. The solid residue obtained by this process was found to have strong antiscorbutic properties which were not appreciably diminished on keeping the substance in a dry atmosphere at room temperature for six months. Later tests $(1920,1921)$ of the dried orange juice stored in a desiccator at room temperature showed a gradual destruction of the antiscorbutic properties amounting to about 50 per cent in 15 months.

Givens and McCluggage (1919a) dried orange juice in two different ways as follows: For one product, the juice was expressed from the oranges and strained through several layers of cheesecloth until a clear filtrate was obtained. This was concentrated to about two-thirds the original volume by heating in a drier at from $55^{\circ}$ to $60^{\circ} \mathrm{C}$., using 100 cubic centimeter portions in small shallow dishes. Soy bean flour, previously heated under 20-1bs. pressure for one-half hour, was then added in the proportion of 12 grams to 100 cubic centimeters of the original juice and mixed thoroughly. The mixture was kept in the drier at from $55^{\circ}$ to $60^{\circ} \mathrm{C}$. until thoroughly dried, the whole process requiring about 50 hours. For the other product, the juice was dehydrated by a commercial process employed for making milk powder. The juice was pressed from the oranges, strained, mixed with corn sirup, and dried almost instantaneously by spraying into a chamber kept at from $75^{\circ}$ to $80^{\circ} \mathrm{C}$. In the case of the second product a dose equivalent to 3 cubic centimeters of the fresh juice was sufficient to protect a guinea pig against scurvy while over 12 cubic centimeters of the first was required for protection. Both products retained their antiscorbutic properties after three months' storage. It was suggested at this time that dried orange juice would be a convenient antiscorbutic for use in infant feeding, on polar expeditions, and in navy and army rations.

Further studies of the juices of the orange, lemon and lime were reported by Davey (1921). The minimum daily doses of the three citrus fruits needed to protect the guinea pig from scurvy were established as lemon and orange 1.5 cubic centimeters, and lime 5 cubic centimeters. These values were used as a basis for comparing the antiscorbutic properties of the juice of oranges and lemons kept for varying times at different temperatures, alone, with the rind oil, and in the case of lemon juice with the addition of sodium sulfite. Preservation 
with sulfite appeared to be satisfactory at low temperatures, uncertain at room temperature, and unsatisfactory at $37^{\circ} \mathrm{C}$. Preservation with the rind oil was satisfactory and reliable at about $0^{\circ} \mathrm{C}$. and room temperature, but unsatisfactory at $37^{\circ} \mathrm{C}$. Experiments on the preservation of oranges and lemons in cold storage were not particularly satisfactory on account of the fact that the fruit did not keep well in these particular cases for any length of time, but the results indicated that the antiscorbutic property was not seriously diminished so long as the fruit remained edible.

This investigation was extended by Delf (1925) who determined the antiscorbutic properties of the whole fruits and fruit juices after storage for five years. The whole fruits (oranges and lemons), as in the earlier study, retained their antiscorbutic value when kept in cold storage as long as the material remained in good condition. Orange and lemon juices stored in a frozen condition for five years retained about half of their original antiscorbutic value. Oranges canned by heating gradually to $100^{\circ} \mathrm{C}$. and holding at this temperature for five minutes, the whole process occupying from twenty to thirty minutes, showed no loss of antiscorbutic property when first canned and only a slight decrease in activity after four years. Lemon juice preserved with potassium bisulfite retained its flavor but lost about five-sixths of its antiscorbutic value after storage for four and one-half years at room temperature. Orange and lemon juices preserved in rind oil retained their activity after from one to three years.

The possibility of utilizing surplus and cull citrus fruits for the manufacture of vitamin $\mathrm{C}$ concentrates led to an investigation by Goss (1925) at the California Agricultural Experiment Station of the antiscorbutic properties of various commercial orange juice concentrates. The products tested included concentrated whole juice, prepared from ripe navel oranges by processing for about four hours under high vacuum at a temperature between $40^{\circ}$ and $45^{\circ} \mathrm{C}$. $\left(104^{\circ}\right.$ to $113^{\circ} \mathrm{F}$.) ; clarified orange sirup prepared from whole fruit by heating for thirty minutes at $185^{\circ} \mathrm{F}$., filtering slowly through Filter-cel, allowing it to stand overnight, adding a small amount of sugar and concentrating in glass enamel vacuum pans for about seven hours under a vacuum of 28 inches of mercury; desiccated orange juice, prepared by a spray drying process with the addition of a little cane sugar; and dried ground whole orange, a product prepared from sound cull oranges by grinding the fruit, allowing it to stand overnight and then drying in a dehydrator for about eight hours at a temperature of from $155^{\circ}$ to $175^{\circ} \mathrm{F}$. A concentrated lemon juice, prepared similarly to the orange 
concentrates except that the juice was held for 24 hours preserved with six ounces of potassium metabisulfite to 100 gallons was also used. The concentrated whole juice retained practically all of the antiscorbutic value of the original juice. The clarified sirup showed slight but not significant destruction of vitamin $\mathrm{C}$. The desiccated juice, although two years old at the time of testing, was found to have retained a good proportion of its antiscorbutic properties and the dried whole orange proved to be a highly concentrated source of vitamin C. The concentrated lemon juice was not relished by the guinea pigs but was apparently a rich source of vitamin $\mathrm{C}$.

The value of conmercial orange sirup as an antiscorbutic was also demonstrated by Priston (1926) in studies conducted in England in behalf of the British Admiralty. The sirup, when first tested after storage from ten to sixteen weeks at warehouse temperature and from five to twenty-four weeks in the icebox at $8^{\circ} \mathrm{C}$., was protective in three out of four cases in a dosage of 0.3 cubic centimeter of undiluted sirup, or about one-third of the amount required of fresh orange juice. Prolonged storage at icebox temperature did not reduce the potency to any marked extent, but storage at $19^{\circ} \mathrm{C}$. for 42 weeks reduced it to about one-third, and for 24 weeks at temperatures varying from $8^{\circ}$ to $37^{\circ} \mathrm{C}$. to one-tenth the original value. Priston estimated that 50 gallons of the concentrate would probably contain sufficient vitamin $C$ to supplement the supply available in the tropical war diet of 1,200 men for three months and that economy could be effected by substituting this concentrate for the lime juice then being issued.

Willimott and Wokes (1927c) are of the opinion that there is some connection between the biochemical activities taking place in the flavedo (outer peel) of citrus fruits and the constituents of both peel and juice. A comparative study of the flavedos of oranges (California Navel), lemons (fresh Palermos) and grape fruit (Florida) showed the presence of vitamin $\mathrm{C}$ in the flavedo of the orange but not of the lemon or grapefruit. In their experiments one gram of fresh orange peel, or 1.5 cubic centimeters of fresh orange or lemon juice appeared to be adequate protective doses for guinea pigs. Grapefruit was not quite as rich in vitamin $\mathrm{C}$ as lemons or oranges. In view of the known sensitivity of both vitamin $\mathrm{A}$ and vitamin $\mathrm{C}$ toward oxidizing agents the flavedo and juice of the three fruits were tested for oxygenase and peroxidase. Oxygenase was always absent but peroxidase was present in both flavedo and juice. In the flavedo the concentration was higher in grapefruit than in the oranges-thus affording a possible explanation of the relative amounts of vitamin $\mathrm{C}$ in the two fruits. It would 
be interesting to see if a similar explanation would hold for the low content of vitamin $\mathrm{C}$ in limes.

Grapes appear to have received surprisingly little attention. Chick and Rhodes (1918) report that scurvy developed in three or four guinea pigs receiving 20 grams each of grapes daily. This would therefore appear to approximate the minimum protective allowance and to indicate that the concentration of vitamin $\mathrm{C}$ in grapes is about one-tenth as great as in oranges. Givens and Macy (1921) state that grape juice dried by the spray process shows no antiscorbutic properties.

The tomato, while ordinarily referred to as a vegetable, is more properly a fruit and may well be considered at this point because of the similarity of tomato juice to orange juice as an antiscorbutic. Hess and Unger (1918) called attention to the importance of tomatoes whether fresh or canned as antiscorbutics and showed that canned tomato juice is a very efficient, readily available, and inexpensive antiscorbutic which may be used in the same manner and with practically the same results as orange juice, even in the feeding of infants. Hess (1920) says: "In our experience there is no contra-indication to the giving of orange juice or of strained canned tomato, the two antiscorbutics with which we have had a large experience, to babies one month of age or even younger." And again "Tomatoes ... are regarded with suspicion amounting almost to superstition by mothers and nurses as a food for children. In spite of this fact, it may be stated without hesitation that they are fully as well borne by infants a few weeks or months of age as orange or lemon juice. . . The dose is two tablespoonfuls for babies over three months of age. The tomatoes are merely strained through a colander and warmed (not cooked). To illustrate their innocuous character, it may be added that as much as 6 and 8 ounces a day of this juice have been given to a baby under one year of age without producing untoward symptoms. This antiscorbutic should have a wide applicability, especially in the United States."

It is probable that the antiscorbutic property of the canned tomato was a factor (even though not consciously recognized) in the popularity of this food among, for example, the city poor as well as among those economically able to command a more diversified diet. A similar significance may be attached to the provision introduced into the United States Army Regulations in 1895 permitting requisition of canned tomatoes "in lieu of an equal quantity of potatoes not exceeding twenty per cent of the total issue." Hess records $(1920$, p. 231) his inability to ascertain whether or not the canned tomatoes were thus provided for specifically as an antiscorbutic. 
Largely no doubt on account of the natural high acidity and the low oxidation potential of their juice tomatoes may be canned without apparent loss of antiscorbutic value, and may even retain a relatively high antiscorbutic value after drying.

Givens and McClugage (1919) found that when 1 gram of dried tomato which had not been heated above $40^{\circ} \mathrm{C}$. was fed per guinea pig per day no scurvy developed. Since this may have been considerably more than the minimum necessary for complete protection, these experiments show clearly that tomatoes may retain a considerable amount uf antiscorbutic vitamin after drying; but do not indicate what proportion of the vitamin originally present may have been destroyed by the drying process.

In the experience of Hess and Unger (1918), 4 cubic centimeters and in that of Sherman, La Mer and Campbell (1921), 3 cubic centimeters daily of canned tomato juice was found to afford the guinea pig complete protection from scurvy. In tomato juice of natural acidity, the antiscorbutic vitamin was found to be fairly stable to heat, boiling for one hour destroying only about one-half; and for four hours only about two-thirds of the vitamin originally present. When the acidity was partially neutralized, and still more when the tomato juice was rendered alkaline, the destruction of the vitamin by heat became more rapid. (Sherman, La Mer, and Campbell, 1921, 1922.) Kohman (1928) has reported some loss in vitamin C in the preparation of commercial tomato concentrate (puree), but not in proportion to the concentration. Weight for weight the tomato pulp or puree is thus richer in vitamin $C$ than raw tomatoes. The rate of destruction of the antiscorbutic vitamin of tomatoes at different temperatures and concentrations of hydrogen ion will be referred to again in connection with the summary of chemical and physical properties of vitamin $\mathrm{C}$ toward the end of this chapter.

The increasing use of ethylene gas in the ripening of fruits and vegetables has led to investigations in several laboratories of the relative content of vitamin $\mathrm{C}$ in tomatoes ripened in various ways. Studies by Clow and Marlatt (1930) led to the conclusion that ripe tomatoes are much richer in vitamin $C$ than green tomatoes even when the tomatoes are ripened in the dark after removal from the vines and that ethyleneripened tomatoes are richer in vitamin $\mathrm{C}$ than green tomatoes, but not quite so rich as tomatoes thoroughly ripened by standing at room temperature until the tissues are considerably softened. In these reports the impression is given that the method of ripening is not so important a factor as the extent of ripening for it is noted that ripe greenhouse 
tomatoes do not ordinarily contain as much vitamin $\mathrm{C}$ as field-ripened tomatoes on account of the fact that they are not as ripe when used, but if allowed to ripen thoroughly give comparable results. Green tomatoes ripened at $70^{\circ} \mathrm{F}$. after picking were reported to contain as much vitamin $\mathrm{C}$ as the vine-ripened fruit. House, Nelson and Haber (1929) on the other hand found vine-ripened tomatoes to be richer in vitamin $C$ than air-ripened or ethylene-ripened, although this was not the case with vitamin A or B. No differences could be detected in their experiments between the vitamin $\mathrm{C}$ content of air-ripened or ethylene-ripened fruit.

Jones and Nelson (1930), using in their tests only that portion of the tomato which passed through a fine muslin cloth, concluded that naturally ripened tomatoes contained the most vitamin $\mathrm{C}$, followed by full grown green tomatoes and then by the small immature fruit. Ethylene treatment of the green fruit produced no change in the vitamin $\mathrm{C}$ content. No tests were reported for air-ripened fruit.

Berries are much sought as antiscorbutics in northern regions but appear to have been little studied. Holst and Frölich (1912) reported raspberries and cloudberries to be good antiscorbutics and state that their juices can be boiled, canned and kept for months with relatively little loss of antiscorbutic potency.

Kohman, Eddy and Halliday (1928) found strawberries to be as rich as tomatoes in vitamin $\mathrm{C}$ and to show no appreciable loss in this vitamin when canned by the usual commercial methods. In their experiments, from two to three grams daily of the fresh berries, or the equivalent in canned, provided sufficient vitamin $\mathrm{C}$ for protection of guinea pigs against scurvy. Scheunert (1929) considered strawberries to be a good source of vitamin C. In his experiment, protection was secured with five grams daily. No appreciable differences were noted between different varieties, and canning or preserving brought about only a slight decrease in the vitamin $\mathrm{C}$ content. Raspberries, red and white currants, and gooseberries were also reported by Scheunert to be good sources of vitamin $\mathrm{C}$ and in general to retain their antiscorbutic properties when canned or preserved. Commercial fruit juices (manufactured according to the German Pharmacopoeia) were very low in vitamin C.

Other fruits tested by Scheunert included apples which he considered a fairly good source of vitamin $C$ on account of their being eaten so extensively raw; pears, apricots, peaches and plums all of which were low in vitamin $C$, and cherries, a good source of vitamin $C$ when raw but poor when cooked or canned. 
Lewis (1919) concluded that, with a basal diet adequate in all respects except vitamin $C$, from 10 to 15 grams of raw banana per guinea pig per day sufficed for growth and protection against scurvy. Givens, McClugage and Van Horne (1921) considered 10 grams to be the minimum protective dose and the same amount was reported by Jansen and Donath (1925) for two varieties of Indian banana. Eddy (1926) considered 5 grams of banana to be the minimum protective dose and from 8 to 10 grams the optimum dose for growth stimulation as well as scurvy prevention in guinea pigs. In the senior author's laboratory raw bananas have appeared to contain about one-third as much vitamin C as oranges or tomatoes. Eddy and Kellogg (1927) found that bananas baked in the skin retain their antiscorbutic properties better than when baked without the skin, probably on account of the protective action of the skin against oxidation. They reported at this time the cure of scurvy in an eight months' old baby by a bananamilk mixture made by whipping 200 grams of ripe, raw banana into 570 cubic centimeters of milk. This was fed in 120 cubic centimeter portions every four hours with no resulting digestive disturbances.

Fresh raw apples are in general about half as rich in vitamin $\mathrm{C}$ as bananas, and there may be considerable loss of this vitamin when apples are kept in storage or cooked by the usual household methods. Kohman, Eddy and Carlsson (1924) found that apples held in cold storage from October to March showed a gradual deterioration in vitamin C content, that very little of the vitamin could be demonstrated in freshly prepared apple sauce, baked apples, or apples canned without any special treatment, but that there was no appreciable loss in vitamin $\mathrm{C}$ in apples canned according to the commercial practice of allowing them to stand in salt solution long enough to exhaust their respiratory oxygen before the usual processing. With this provision, the time of processing within the limits followed in commercial practice was immaterial. These findings were considered to indicate that the amount of respiratory oxygen in the cells of apples, and presumably other fruits, is an important factor in the destruction of vitamin $\mathrm{C}$ not only in canning but in storage.

A recent investigation by Bracewell, Hoyle, and Zilva (1930) of the vitamin $\mathrm{C}$ content of a number of varieties of apples grown in England and several of the colonies showed wide differences according to the variety. Of all the varieties tested, the English cooking apple known as Bramley's Seedling was found to be decidedly the most active, complete protection against scurvy being secured with 3 grams daily. The relatively high activity of this particular apple appeared not 
to be related to the soil in which it was produced, the age of the tree, or the season. Apples picked before complete maturity were as active as those picked from the same tree 14 days later. Next in order of vitamin $C$ value was the Dabinett, a cider variety, while Cox's Orange Pippin, a dessert variety, ranked last. Canadian dessert apples had about the same vitamin $C$ value as the corresponding English varieties, but Australian and New Zealand apples were less potent, probably on account of increased time elapsing between the picking and testing of the fruit. Little loss in vitamin $C$ value was demonstrated in the English grown apples during three months' storage in air at $1^{\circ} \mathrm{C}$. Storage in a mixture of carbon dioxide, nitrogen, and oxygen at $10^{\circ} \mathrm{C}$. for the same period resulted in a slightly greater loss in activity. Apples of the Bramley variety were baked for 50 minutes in their skins without noticeable loss in antiscorbutic properties. The remarkably high content of vitamin $\mathrm{C}$ in this one variety of apple was not accompanied by any detectable differences in such factors of chemical composition as are shown by analysis in vitro, with the possible exception of the nitrogen content which appeared to be higher in the variety of high vitamin $\mathrm{C}$ content.

Kohman, Eddy, Carlsson and Halliday (1926) found that 5 grams of raw peaches purchased in the New York market during August, September and October was about the minimum protective dose. The same peaches cooked by the open kettle method contained only about one-fifth as much vitamin $\mathrm{C}$ as the fresh peaches, but peaches canned in California by commercial methods appeared as rich as the fresh New York City market peaches. Unlike apples the elimination of the oxygen from the peaches previous to processing was without appreciable effect on the vitamin $\mathrm{C}$ content of the canned product.

In a recent study by Morgan and Field (1929) of the effect of various methods of drying upon the antiscorbutic properties of fruits, the minimum protective dose of fresh Muir peaches was given as eight grams daily. Unsulfured sun-dried or dehydrated peaches retained no detectable amount of vitamin $\mathrm{C}$, but the sulfured fruit dried by either method showed no measurable loss in vitamin C. In commenting upon the protective action of sulfur dioxide upon vitamin $\mathrm{C}$ as thus demonstrated the authors state:

"The mechanism of protection of vitamin C by the sulfur dioxide treatment in these fruits is of interest and is being studied in this laboratory. Whether the result is due to decreased oxidation because of a surface coagulation of the fruits, or because of the reducing action of the sulfur dioxide, or to greater stability of the vitamin in the possibly more acid medium provided by the sulfurous acid, or to some other cause remains to be discovered. In any case, a definite advantage 
in an important phase of dried fruit nutritive value, appears to be derived from the sulfur dioxide treatment during drying."

Craven and Kramer (1927) reported no difference in the vitamin C content of ripe Kieffer pears grown locally (Kansas) or obtained from California, and no loss of potency on storage in a fruit storage room at a temperature of $40^{\circ} \mathrm{F}$. from February to April. They found the minimum protective dose of the raw pear to lie between 10 and 15 grams and concluded that pears of this variety are about one-fourth as rich in vitamin $\mathrm{C}$ as oranges. Apparently complete destruction of vitamin $C$ resulted from the open-kettle method of home canning and considerable destruction by the cold-pack method.

In a later coöperative study Kramer, Eddy and Kohman (1929) found that Kieffer pears canned by commercial processes while still hard and green retained most of their vitamin C. The fully ripened pears when canned did not afford full protection against scurvy in doses as high as 30 grams. Bartlett pears were even lower in vitamin $\mathrm{C}$ in the ripe state than Kieffer pears and no great difference could be noted between the potency of the raw and canned product. A considerable loss in vitamin $\mathrm{C}$ was attributed to the ripening and mellowing process.

Chick, Hume and Skelton (1919) were led to investigate the value of some Indian dried fruits through the fact that these foods enjoyed the reputation of antiscorbutics in India and have been relied upon to some extent by native Indian troops. They concluded that dried tamarinds, cocum and mango possess significant but not large quantities of the antiscorbutic vitamin, that weight for weight these fruits are superior to carrot, cooked potato, or raw meat juice, but greatly inferior to raw cabbage, germinated pulses or the juice of Swedish turnips, oranges or lemons.

In an investigation carried out at the South African Institute for Medical Research, Johannesburg, to determine the relative antiscorbutic values of the foodstuffs commonly used in the dietary of native workers in the Rand Mines, Delf $(1921,1922)$ found the native navel oranges and tangerines to be as rich in vitamin $C$ as hitherto reported for oranges, 1.5 cubic centimeters daily being the minimum protective dose for guinea pigs. Corresponding values for other fruits were 2.5 cubic centimeters of pineapple juice, representing 9 grams of the whole fruit and 5 cubic centimeters of papaya (Carica papaya) juice or 10 grams of the fresh fruit. From two to three cubic centimeters of the juice of peaches afforded protection early in the season, but as the peaches ripened they proved less effective. 
The antiscorbutic value of Hawaiian-grown pineapples has been determined by Miller (1925), who found that 5 grams daily of the fresh fruit was sufficient in curative tests. Canned pineapple was somewhat less effective, but a mixture of 7 grams of canned pineapple and 5 cubic centimeters of the canned juice, the whole corresponding to 7.7 grams of fresh pineapple, sufficed for renewal of growth and prevention of scurvy. The apparent slight destruction of vitamin $C$ in the canned product could not be attributed to a decrease in hydrogen-ion concentration for the $\mathrm{pH}$ values of both fresh and canned juice lay between 3.5 and 4 , an acidity higher than that of canned tomatoes. Miller (1926) also gave a somewhat higher value than Delf to the papaya as a source of vitamin C. Although the minimum dosage was not determined, three grams daily appeared to give as complete protection and to cause as good gains in weight as 5 grams.

The Chinese persimmon (Diospyros kaki) was reported by Embrey (1923) and Hsu (1928) to be low in vitamin C, the minimum protective dose being from 25 to 30 grams of the fresh fruit. According to Iwasaki (1927), in a Japanese report with English summary, the same fruit, known in Japan as kaki, in amounts of 10 grams daily cures guinea pig scurvy completely in 30 days.

Among vegetables, raw greens and some of the roots and tubers enjoy high reputation as antiscorbutics.

Roots and Tubers.-The Swedish turnip, rutabaga or "swede" was studied by Chick and Rhodes (1918), who found that the juice obtained by filtering the freshly grated pulp through a muslin bag approximated orange juice in antiscorbutic potency. A daily ration of 2.5 cubic centimeters of this juice fully protected a guinea pig against scurvy while to afford approximately the same amount of protection required about 20 cubic centimeters of carrot juice and still more of beet juice. Raw "swede" juice has therefore been widely advocated by the English workers as an inexpensive source of vitamin $C$ particularly in infant feeding.

Delf (1925) found that rutabagas, like oranges and lemons, retained their antiscorbutic properties in cold storage as long as they remained in good condition. The stability of the frozen juice was not as great, however, for the juice became entirely inactive after about 15 months. The emphasis by the English workers on yellow turnips or rutabagas as antiscorbutics has led to the general impression that they are much richer in vitamin $C$ than white turnips, but it is doubtful whether any such distinction holds. Scheunert (1929) has reported almost as high values for early turnips as for rutabagas. In his experi- 
ments, guinea pigs were fully protected against scurvy by 1 gram daily of rutabagas and from 1 to 2 grams of the early turnips.

Radishes, which belong to the same family as the turnip and cabbage, have received little attention as to vitamin content, but Shinoda, Fujimaki and Saiki (1927) in a study of the vitamin content of Japanese food products secured protection against scurvy in guinea pigs with 5 cubic centimeters daily doses of radish juice and noted a higher content of vitamin $\mathrm{C}$ in the peelings than in the peeled fruit.

Carrots are reported to be less rich in vitamin $C$ than turnips. Hess and Unger (1919) called attention to distinct differences in the antiscorbutic properties of old, as compared with fresh young carrots particularly after cooking. It was found that while 35 grams of old carrots were sufficient to protect a guinea pig from scurvy when fed raw, after cooking for three-quarters of an hour their addition to the dietary proved insufficient for protection. In a parallel test with fresh young carrots 25 grams proved adequate for complete protection even after cooking. It was pointed out that in such cases as this the fresh young vegetable may have a double advantage over those which are older and tougher, since in the first place the younger or fresher specimens may be richer in antiscorbutic vitamin to start with and as the older, tougher vegetables require more prolonged cooking they are apt to undergo a greater loss of antiscorbutic during the cooking process.

Hess also pointed out (1920, p. 160) that the loss of antiscorbutic value in the boiling of carrots is due to destruction and not merely to extraction of the antiscorbutic vitamin since the water in which the carrots had been cooked was found by feeding experiments to have little if any antiscorbutic value. This is in contrast to observations showing considerable amounts of vitamin B in the "water" of cooked or canned vegetables. The difference is probably due to the greater susceptibility of the antiscorbutic vitamin to heat, which would result in its destruction rather than its accumulation in the boiling water surrounding the cooking vegetable, whereas the equally soluble and more stable vitamin $B$ would accumulate in the cooking water to a much larger extent. Less easily understood is the further finding by Hess that the acidulation of the cooking water with vinegar did not reduce the loss. Foods naturally acid seem always to retain their antiscorbutic property relatively well under heat treatment, while attempts to conserve the antiscorbutic vitamin of other foods by addition of acid seem to give variable results.

Largely as the results of his experience with carrots, Hess (1920, pp. 160,161$)$ laid much emphasis upon the view that vegetables must 
be expected to vary considerably in their content of antiscorbutic vitamin according to their freshness and age. While young carrots were much superior to old carrots in antiscorbutic value, he found that slightly green tomatoes contained less antiscorbutic vitamin than did those which were fully ripe. So far as fruits and vegetables are concerned, our present limited knowledge would seem to suggest that the degree of maturity at which the fresh food is most prized will probably approximate that at which it has greatest vitamin value.

Dried carrots were found by Hess and Unger to have lost antiscorbutic vitamin to a serious extent but not necessarily entirely. In the quantities which they fed, the carrots which had been allowed to age before drying, failed after drying to prevent scurvy, whereas protection was afforded by an equal amount of carrot which had been quickly dried when young and fresh.

The emphasis laid by Hess upon the importance of the time factor in problems connected with the destruction or conservation of antiscorbutic vitamin will be discussed in connection with other experiments to be described beyond.

Potatoes while containing the antiscorbutic vitamin in distinctly lower concentration than do oranges, lemons and tomatoes, are yet of great importance as antiscorbutics because of the quantities in which they are consumed. Hess (1920) estimated that in the countries of the temperate zone the consumption of potatoes is probably twice that of all other vegetables combined and that for this reason our protection from scurvy actually rests very largely upon the potato. "Therefore if this crop fails scurvy will develop in the spring." Weight for weight, cooked potatoes are comparable as antiscorbutics with apples and bananas and have only around one-fourth of the concentration of vitamin $\mathrm{C}$ which we find in oranges, lemons and tomatoes.

Givens and Cohen (1918) reported a number of experiments with potatoes cooked in different ways and in some cases dried before feeding. From the variable results obtained the authors were led to suggest the possibility that the factors involved in the destruction of the antiscorbutic vitamin are not only the degree of heat and the duration of the heating but also the enzyme content and the reaction of the food being dried. By employing a high temperature for a short time, as in the case of baked potatoes, the enzymes are destroyed, while at any temperature below $80^{\circ} \mathrm{C}$. enzymes may still function and probably play an important rôle in the destruction of the antiscorbutic vitamin. (It is presumably the oxidizing enzymes that would be chiefly destructive of vitamin C.) 
In a later paper Givens and McClugage (1920a) reported further that a daily allowance of 10 grams of raw white potato was apparently more than sufficient to protect the growing guinea pigs from scurvy for the duration of the experiment, 129 days. Cooking the potatoes in water at $100^{\circ} \mathrm{C}$. for 15 minutes caused only a slight reduction in antiscorbutic value, while cooking for an hour at the same temperature reduced the vitamin content to such an extent that the disease could not be arrested by feeding 15 grams daily of the product. Scurvy was checked in two animals by feeding 10 grams of potatoes cooked in 0.5 per cent citric acid for 1 hour.

With potatoes dried at $35^{\circ}$ to $40^{\circ} \mathrm{C}$., death from scurvy was slightly delayed by an amount (2.5 grams) equivalent to 10 grams of the fresh product, while with double the amount life was prolonged still further. One out of four animals on a daily dose of 2.5 grams dried at $55^{\circ}$ to $60^{\circ} \mathrm{C}$. and one out of four animals on a daily dose of 2.5 grams dried at $75^{\circ}$ to $80^{\circ} \mathrm{C}$., showed signs of scurvy at death. On heating at $100^{\circ} \mathrm{C}$. for 1 hour the products dried at these temperatures, no protection was secured in any case.

Scheunert (1929) found old sprouted potatoes to be somewhat lower in their content of vitamin $\mathrm{C}$ than new potatoes, to which he gave much higher values than usually reported. According to him 3 grams of new white potatoes fed raw and 4 grams cooked with or without skins sufficed for the best development of guinea pigs. Steaming or ordinary cooking in water appeared to be less destructive than cooking in the pressure cooker for even a very short time, 12-14 minutes.

Sweet potatoes are probably a fairly good source of vitamin C. Delf $(1921,1922)$ in the report of her investigation of South African foods recommended the introduction of sweet potatoes into the ration of the Rand miners when fruits such as oranges, pineapples and tangerines were not available. The minimum protective dose in her experiments lay between 4 and 5 cubic centimeters of the juice or about 13 grams of the raw vegetable.

The dasheen or taro, a staple root food of the Pacific Islands and coming into more general use in certain sections of the country as a substitute for the potato, is low in vitamin $\mathrm{C}$ according to Miller (1927) who worked with the steamed vegetable and poi, the fermented paste prepared from it according to methods followed in Hawaii.

Stems such as asparagus and celery would seem to be worthy of attention as regards antiscorbutic value but have not been studied to any extent. Rhubarb was shown to possess antiscorbutic power by 
Pierson and Dutcher (1920). Scheunert (1929) considers it to be a good source of vitamin $C$ in the raw state but to lose more than half of its antiscorbutic properties when heated with equal parts of water and sugar and sealed. Other stem vegetables tested by Scheunert include kohlrabi reported to be a good source, and asparagus a very good source of vitamin $\mathrm{C}$ when tested raw. Two grams daily of raw asparagus afforded complete protection against scurvy but cooked asparagus was of little value.

Onions are reported good antiscorbutics by the British Committee. See also Shorten and Roy $(1919,1921)$.

Leaves are apparently good antiscorbutics, particularly if they can be eaten raw, but until quite recently only a few of them have been studied. Lind gave directions for the use of fir tops as an antiscorbutic in time of need, and referred to "scurvy grass," cress, and spinach as foods of known antiscorbutic value. Holst and Frölich (1912), as noted previously, demonstrated antiscorbutic properties in cabbage, dandelion leaves, lettuce, endive and sorrel.

Cabbage has been more extensively studied than any other leafy vegetable, doubtless because it is quantitatively the most important of this class of vegetables and perhaps in part also because it is so commonly fed to, and so readily eaten by, the guinea pig, which usually serves as the experimental animal in studies of the antiscorbutic vitamin.

Reference has already been made to the experiments of Holst and Frölich with raw, cooked, and dried cabbage. The work of Delf (1918) and of Delf and Skelton (1918) indicated that with a good basal diet from 1.5 to 2 grams of raw cabbage will suffice to prevent scurvy in a guinea pig, thus pointing toward as high a concentration of the antiscorbutic vitamin in the raw leaf as in the juice of the orange or lemon and somewhat higher than in the tomato. In practice, however, cabbage is probably a less important antiscorbutic than the tomato, because the cabbage loses a much larger fraction of its vitamin $\mathrm{C}$ in cooking than does the tomato. (See, also, the section on chemical behavior beyond.)

Delf (1918) found that five grams of cabbage cooked for one hour at $60^{\circ} \mathrm{C}$. or for twenty minutes at $100^{\circ} \mathrm{C}$. was equivalent in antiscorbutic properties to one gram of raw cabbage. This suggests a rather low temperature coefficient of destruction of the vitamin, since a temperature increase of about $40^{\circ} \mathrm{C}$. apparently increased the rate of destruction only about three-fold. In experiments in which cabbage was heated at temperatures of from $100^{\circ}$ to $130^{\circ} \mathrm{C}$. the destruction of anti- 
scorbutic properties, though extensive, was less complete than might have been expected from the results at lower temperatures.*

Applying these results to a discussion of methods of cooking vegetables, Delf pointed out that slow cooking at a low temperature is likely to be more deleterious than more rapid cooking at high temperature. The prolonged cooking of vegetables in a fireless cooker might thus be more destructive of vitamin $\mathrm{C}$ than actual boiling for a short time. Contrary to the results of Holst and Frölich with cabbage and of Harden and Zilva with lemon juice, the addition of dilute citric acid to the cabbage before boiling did not prevent the destruction of vitamin C. In view of these results and previous observations of the instability of vitamin $\mathrm{C}$ to alkalinization, Delf concluded that in cooking vegetables it is better to add neither acid nor alkali to the water in which they are cooked.

Delf and Skelton (1918) found a loss in antiscorbutic potency of more than 93 per cent when cabbage was dried at a low temperature $\left(60^{\circ} \mathrm{C}\right.$.) and stored subsequently for two to three weeks at laboratory temperatures. This loss increased with prolonged storage until at the end of three months nearly all the protective value of the fresh material was lost. By plunging the cabbage into boiling water before drying, the residual amount of antiscorbutic factor was distinctly greater, indicating that "killing the cells" (coagulation of protoplasm with destruction or inactivation of enzymes including oxidases) by heat before drying is beneficial in lessening the loss of vitamin $\mathrm{C}$ taking place during drying.

Givens and Cohen (1918) reported that cabbage dried in a blast of air at $40^{\circ}$ to $52^{\circ} \mathrm{C}$. retained some of its antiscorbutic value, but heated in an oven for 2 hours at $75^{\circ}$ to $80^{\circ} \mathrm{C}$. and then dried at $65^{\circ}$ to $70^{\circ}$ C. afforded no protection. Ellis, Steenbock, and Hart (1921) attempted to prevent the destruction of vitamin $\mathrm{C}$ in cabbage on drying by heating the material in a vacuum oven in an atmosphere of carbon dioxide at $65^{\circ} \mathrm{C}$., the process requiring 35 hours. Practically no protection was afforded by the dried cabbage when fed to guinea pigs in 1.5 grams daily amounts.

In recent studies by Clow, Marlatt et al. (1929) fresh raw sauerkraut was found to contain about half as much vitamin $\mathrm{C}$ as the cabbage from which it was prepared. An extension by Clow, Parsons, and Stevenson (1930) of these studies to six brands of commercially canned sauerkraut put up in tin showed wide variations in the content of

* From the point of view of newer knowledge, briefly summarized in the section on chemical behavior (beyond), the interpretation of these results should also take account of oxidation potentials and of possible destruction of oxidases. 
vitamin $\mathrm{C}$ in the different brands, with the best equalling, and the poorest containing about half as much vitamin $\mathrm{C}$ as, the freshly prepared sauerkraut. Because of lack of uniformity in the methods used in the manufacture of the sauerkraut tested, it was not possible to determine what factors were responsible for the loss of vitamin $\mathrm{C}$ in the poorer samples.

Campbell and Chick (1919) studied the effect of canning upon the vitamin $\mathrm{C}$ content of cabbage and string beans. The vegetables were washed in cold water, blanched, cold dipped, and packed into lacquered cans, which were then nearly filled with boiling water, hermetically sealed and sterilized by exposure to steam at $100^{\circ} \mathrm{C}$. for one and one-half hours in the case of cabbage, and on two successive days, to a total period of two and one-half hours in the case of the beans. The cabbage was tested two weeks, and the beans three months after canning. The conclusions drawn from this study were summarized as follows: "In the process of canning vegetables the greater part of the original antiscurvy value of the raw vegetable is destroyed. In the case of runner bean pods (string beans) the loss is estimated at about 90 per cent of the original value; in the case of cabbage at about 70 per cent of the original value. . . This loss is primarily due to the destruction of antiscurvy material occurring during the heating involved in the process of canning. A further loss may be expected to take place during the period of storage.

Delf (1920) compared the antiscorbutic properties of the freshly expressed juices of the cabbage, the "swede" (rutabaga), and the orange in the raw state and after heating. With the basal ration used, the minimal daily doses of the raw juice required for the adequate protection of the young guinea pigs was about 1 cubic centimeter of cabbage, 2.5 cubic centimeters of rutabaga and 1.5 cubic centimeters of orange juice, respectively. After the juices had been heated at $100^{\circ} \mathrm{C}$. for an hour in small flasks stoppered with cotton wool plugs the orange juice showed no loss in potency, while there were required twice as much of the juice of the rutabaga and at least 7.5 times as much of the juice of the cabbage as before heating. Almost as much loss in potency of the cabbage juice seemed to have occurred after heating only twenty minutes. The rutabaga and orange juices were also heated for an hour in the autoclave at $130^{\circ} \mathrm{C}$.; protection was then secured with 10 cubic centimeters of the rutabaga and 3 cubic centimeters of the orange juice. The relatively high stability of the vitamin $C$ of these juices at $130^{\circ} \mathrm{C}$. in the absence of air led Delf to suggest at this time "that there may be advantage in adopting methods of canning fruit or 
vegetables at temperatures above boiling-point for as short a time as possible to insure sterility."

Eddy and associates (1923) reported a 95 per cent destruction of vitamin $C$ in cabbage when cooked until palatable either by boiling in an open kettle or by cooking in a pressure cooker. Cabbage canned by ordinary commercial methods was found by Eddy and Kohman (1924) to have lost only about one-fourth of its vitamin C potency. The method followed consisting of packing the cabbage in No. 2 cans, filling the cans with hot water, passing live steam through for 2 or 3 minutes, and then sealing and processing them. The times and temperatures of processing included 30 and 60 minutes at $100^{\circ} \mathrm{C} ., 15,30$, and 45 minutes at $115^{\circ} \mathrm{C}$. and 30 minutes at $126^{\circ} \mathrm{C}$. These variations apparently had no important effect upon the antiscorbutic properties of the cabbage, indicating that the slight destruction of vitamin $\mathrm{C}$ took place mainly during the initial treatment with steam. In this investigation a slight increase in the potency of raw cabbage on storage was noted.

In a similar study of spinach, Eddy, Kohman, and Carlsson (1925) found that, on a strictly vitamin-C-free basal ration, guinea pigs were protected by 1 gram daily of the fresh raw material while on a basal ration later found to be not entirely free from vitamin $\mathrm{C}$ a dosage of 0.25 gram of the raw material proved sufficient. With the second basal ration it required the equivalent of 10 grams of home-cooked spinach or of 4 grams of commercially canned spinach to afford the same protection. For the home-cooked materials the standard household method was followed of cooking the freshly washed material for 15 minutes without additional water. The canned spinach was prepared according to the commercial methods by blanching in hot water for two minutes and processing for 70 minutes at $115^{\circ} \mathrm{C}$., using No. $2 \mathrm{r} / 2$ cans, and also by blanching 5 minutes in water and 1 minute in steam and processing for 70 and 120 minutes, respectively. There was some evidence that the 5 minute blanch was more destructive than the two or one minute blanch. Later studies at the South Dakota Agricultural Experiment Station (1929) have indicated a greater destruction of vitamin $\mathrm{C}$ in spinach in blanching than in processing.

In discussing their results Eddy and his associates state:

"The results obtained by the writers in their studies of cabbage, apples, and spinach all tend to confirm the view that oxidation factor is more important than the heat factor. There is no question but that acidity or alkalinity is a factor and that heat accelerates destruction, but when these other factors are constant wide variation in the destruction may be obtained by controlling the oxygen factor alone. It is possible that this factor is not eliminated by mere exclusion of air; 
in fact, in the writers' apple experiments the presence or absence of oxygen carriers is suggested as important in this connection. Practically, the combined results show that in the canning process as evolved commercially the antiscorbutic factor in foods is preserved to the extent of making such canned foods important sources of this vitamin. In the case of vegetables stuch as cabbage and spinach it is reassuring to learn that one may employ temperatures and periods of heating necessary to proper sterilization without at the same time eliminating one of the values of the foodstuffs as a nutrient."

While thus emphasizing the value of commercially canned products, these authors call attention to the fact that even home-cooked spinach may be considered an excellent antiscorbutic in view of the richness of the raw material in vitamin $\mathrm{C}$ and the size of portion usually eaten by the consumer.

The extensive use in the Southern states of collards and turnip greens has led to studies of these at the Alabama and Georgia Agricultural Experiment Stations. Burton (1928) at the Alabama station, considered 1 gram of raw collards and slightly more than 1 gram of raw turnip greens to be the minimum vitamin $\mathrm{C}$ protective dose. The collards required cooking for about two hours and the turnip greens 30 minutes for palatability. Under such conditions the collards showed about 10 per cent destruction of vitamin $\mathrm{C}$ and the guinea pigs refused to eat sufficient turnip greens for protection. From these admittedly incomplete experiments Burton concluded that collards are higher in vitamin $\mathrm{C}$ than turnip greens. At the Georgia station, however, turnip greens have been reported to be exceptionally high in vitamin $\mathrm{C}, 0.3$ gram daily of the raw material being sufficient for protection. Boiling the greens for 45 minutes with all of the water cooked back caused a loss of about 85 per cent in vitamin $\mathrm{C}$ potency. Considering the reported richness of the raw material in vitamin C, cooked turnip greens even with this loss in potency may be considered a good source of vitamin $\mathrm{C}$.

Watercress resembles cabbage, lettuce and other leafy vegetables in its high content of vitamin C. Coward and Eggleton (1928) reported that 1 gram daily of fresh watercress is sufficient to protect a guinea pig completely for a period of 70 days.

Scheunert (1929) considers kale and brussels sprouts to be very good sources of vitamin $C$ when tested raw and good sources when cooked. Red cabbage resembled white cabbage in its high content of vitamin $C$ in the raw state and low content after cooking for half an hour. Sauerkraut was found by Scheunert to have as high a content of vitamin $\mathrm{C}$ as cabbage but to be deficient in it after cooking. Head lettuce was completely protective in doses of 1 to 2 grams daily.

Seeds and Seedpods.-Sound mature seeds, whether cereals or le- 
gumes, are either entirely devoid of vitamin $\mathrm{C}$ or contain so little of it as to show practically no antiscorbutic value in experiments upon the guinea pig. However, with the sprouting of the seed a development of antiscorbutic property occurs which must mean that the resting seed contains some substance so closely related to vitamin $\mathrm{C}$ as to be transformed into it in the process of germination of the seed. Fürst (1912), working in the laboratory of Holst and Frölich, demonstrated this fact in the cure of guinea pig scurvy with germinated seeds. Chick and Hume $(1917,1917 \mathrm{a})$ confirmed his findings with germinated peas and lentils and suggested that in case of unavoidable shortage of fresh fruit and vegetables in army rations a substitute could be quickly made available by germinating the pulses included in the usual army field rations. Practical directions were given for the germination of the pulses under active service conditions.

Acting on their suggestion, Wiltshire (1918) compared germinated beans with lemon juice as antiscorbutic for scurvy patients (Serbian soldiers) in a war hospital in London. Thirty patients were treated with lemon juice and twenty-seven with germinated beans; the two groups of patients having been selected as being in as nearly the same condition as possible. Those treated with lemon juice received 4 ounces of the fresh juice daily. In comparison with those, each patient in the other group received, as his daily allowance of antiscorbutic, the product resulting from 4 ounces of beans weighed dry, soaked 24 hours, germinated for 48 hours at a temperature of $60^{\circ}$ to $70^{\circ} \mathrm{C}$. and then cooked for 10 minutes. Of the patients receiving the germinated beans, 70 per cent recovered within four weeks as against 53 per cent of recoveries within the same length of time among those receiving the lemon juice. A better standard of comparison in Wiltshire's report was the time required for the return of the gums to normal condition, which averaged 3.1 weeks for the patients receiving germinated beans and 3.4 weeks for those receiving lemon juice. Judged from either standpoint it is evident that in this case the germinated beans were fully as efficient as the lemon juice in the quantities used.

Simple infusions of malted (sprouted) grains may possess sufficient antiscorbutic vitamin to be an important factor in the prevention of scurvy, as is indicated by experience as early as that of Captain Cook and as late as that of the South African negro labor camps in France during the World War (Hess, 1920); McClendon, Cole, Engstrand and Middlekauff (1919) have also reported experiments demonstrating the antiscorbutic value of germinated barley. Clarified beer, however, has failed to show antiscorbutic value. 
In a more extensive investigation of the development of antiscorbutic properties in legumes on germination Chick and Delf (1919) found, as had Holst and Frölich, that dry legumes had very slight antiscorbutic value since scurvy developed only after a longer time and in a less acute form with these seeds than with cereals. Dry green peas after soaking for 24 hours in water at room temperature showed no increase in antiscorbutic properties. Soaked lentils were slightly higher in antiscorbutic value than soaked peas. In this connection it was noted that in a given weight of lentils there are many more individual seeds than in the same weight of peas. After 48 hours of germination at room temperature the antiscorbutic value of the soaked seeds was increased from 3 to 6 times. Some degree of protection was secured with 2.5 grams daily (dry weight 1.29 grams) of both lentils and peas, and complete protection with 10 grams daily. Cooking the germinated lentils in water even for as short a time as 15 minutes caused a loss of about 75 per cent in antiscorbutic value.

Even with the unavoidable losses in cooking, germinated seeds continued to be considered an important antiscorbutic. In the memorandum on the Importance of Accessory Factors in the Food issued by the British Committee on Accessory Food Factors in June 1919 for the guidance of those engaged in the administration of food relief to famine stricken districts, the use of germinated seeds in the prevention of scurvy is given prominence. "If fresh vegetables or fruit are scarce or absent, an anti-scorbutic food can be prepared by moistening any available seeds (wheat, barley, rye, peas, beans, lentils) and allowing them to germinate... The seeds should be soaked in water for 24 hours and kept moist with access of air for from one to three days, by which time they will have sprouted. This sprouted material possesses an antiscorbutic value equal to that of fresh vegetables and should be cooked in the ordinary way for as short a time as possible." The British Committee points out that the legume dhall is a staple Indian article of food, and had it been known that this could be made an effective antiscorbutic through simple sprouting, the great losses and suffering due to scurvy among Indian troops in the Mesopotamian campaign might have been avoided.

Renewed interest in the synthesis of vitamin $C$ in seeds on germination was occasioned by the observations of Parsons of which one interpretation is that rats and possibly other species of animals may synthesize vitamin C. Inasmuch as grains constituted part of the diets of the rats used in the experiments upon which these observations were based, it occurred to Honeywell and Steenbock (1924) that possi- 
bly the rats synthesize vitamin C from certain constituents of grains, in a manner similar to its synthesis on germination. In an attempt to determine whether or not the entire process of germination is essential for the synthesis of vitamin $\mathrm{C}$, they compared the antiscorbutic properties of barley fed dry, soaked for 24 hours, soaked for the same length of time and germinated in the dark for 3 days, and soaked for 96 hours, respectively. The soaking was carried on in the absence of oxygen. During the germination the seeds were kept moist and allowed plenty of air. With the soaked, but not germinated, barley as the source of vitamin $C$ the loss in weight and symptoms of scurvy occurred in from 28 to 30 days in the case of the 24-hour samples and in about 37 days with the 96-hour samples. The germinated barley, although practically free from green pigment, afforded complete protection against scurvy. It was concluded that vitamin $C$ though easily destroyed by oxidation nevertheless requires oxygen for its synthesis by the germinating seed.

Kučera (1928) has reported that the presence of vitamin C can be demonstrated in wheat after only 15 hours' soaking, in rye and barley after 24 hours, and in oats not until from 72 to 96 hours. Unfortunately the available reports of his studies do not indicate under what conditions the seeds were soaked. Simonik (1929), working in Kučera's laboratory, traced the development of vitamin $\mathrm{C}$ in various legumes (beans, vetch and peas) during germination and reported demonstrable increase of vitamin $\mathrm{C}$ in as short a time as six hours.

$\mathrm{He}$ concluded that conditions are more favorable for the synthesis of vitamin $\mathrm{C}$ on germination in legumes than in cereals. Luettmerding (1929), on the theory that vitamin C is preformed in the ripened seed and becomes activated when the seed swells in soaking, tested the effect of rapidity of imbibition on the formation of vitamin $C$ by soaking wheat, oats, and peas in distilled water and in 0.05 per cent and 0.1 per cent solutions of citric acid for varying lengths of time and determining the time required for the development of sufficient vitamin $\mathrm{C}$ for the protection of guinea pigs in daily doses of from 20 to 30 grams. The wheat grains required 15 hours' soaking in distilled water, 12 in 0.05 per cent citric acid, and 6 in 0.1 per cent citric acid. Oats showed no evidence of vitamin $\mathrm{C}$ after soaking for 48 hours in distilled water, but sufficient for protection after soaking for the same length of time in 0.05 per cent citric acid and for 24 hours in 0.1 per cent citric acid. Peas developed sufficient vitamin $\mathrm{C}$ for protection after soaking for 3 hours and the addition of citric acid did not hasten the process. Luettmerding also tested wheat and peas soaked 
in water after being ground to a coarse powder. Evidence of vitamin C was noted after 15 hours for the peas, but on prolonging the time of imbibition the activity disappeared.

It is unfortunate that these studies from Kučera's laboratory are not reported in greater detail for the question of the development of appreciable antiscorbutic properties in cereal grains during the soaking which sometimes precedes the application of heat in cooking is of interest both in theoretical consideration of the mode of synthesis of vitamin $\mathrm{C}$ and in interpreting popular impressions. Among Russian peasants there is a belief that their black bread prepared from whole grain flour (including the embryo) by a slow process in which there is long soaking and an opportunity for considerable enzyme action to take place, has some value as an antiscorbutic, while white bread made from the endosperm only and by a quicker process has none.

Seed pods have not been studied extensively, though by analogy to fruits it might be expected that they should show antiscorbutic properties in the succulent stage of their development, and this has been found to be true in striking degree by Quinn, Burtis and Milner (1927) in their studies of string beans and of green peppers. Delf $(1921,1922)$ tested the pumpkin and vegetable marrow: 15 to 20 cubic centimeters of juice from 75 to 100 grams of raw pumpkin proved to be the minimum protective dose. The results obtained with vegetable marrow were inconclusive although the juice of the young green marrow was more active than that at the end of the season. This does not suggest a very high content of vitamin $\mathrm{V}$ in this class of vegetables. The cucumber enjoys the reputation of a good antiscorbutic in Russia but has received little attention in studies of vitamin $\mathrm{C}$ content. Thurman and Vahlteich (1928) found that 4 grams daily of fresh cucumber gave complete protection to 300 grams guinea pigs. Smaller amounts were not tested, since an earlier study by Embrey (1923) had given 10 grams as the minimum protective dose. Thurman and Vahlteich also found that fresh cucumber pickles made by cooking sliced fresh cucumbers with vinegar, sugar, salt, and spices according to a modern commercial method, gave complete protection in 6 grams daily amounts, while pickles prepared in the same way from salt stock cucumbers afforded no measurable protection in 16 grams daily doses.

Munsell (1930) has recently reported that the watermelon is a good source of vitamin C. In her studies 6 grams daily of the red or pink fleshy portion of ripe watermelons just failed to protect and 8 grams afforded complete protection to guinea pigs during the custo- 
mary 90-day period. This indicates that watermelon is at least one-third as rich in vitamin $\mathrm{C}$ as the tomato.

The immature seed-pod of the string bean has been found comparable with many leaves and fruits in its content of vitamin C. Campbell and Chick (1919) considered 5 grams of the green pod to be the minimum protective dose. Quinn, Burtis and Milner (1927) found that 4.3 grams daily gave complete protection from scurvy and allowed considerable growth. They used green string beans purchased in retail markets and were careful in their feeding tests to include not only the flesh of the pod but the immature seeds. Scheunert (1929) found wax beans to be as rich in vitamin $\mathrm{C}$ as green beans, both being considered "good" sources of this vitamin.

Quinn and his associates also tested green peppers but in this case discarded the seeds and cores and used only the flesh of the pods. The peppers proved to be about 4 times as rich in vitamin $C$ as the string beans. An allowance of 0.9 gram daily gave complete protection from scurvy and produced good growth. "This concentration is comparable with the highest which has been reported for any plant material, such as the raw leaf tissue of cabbage or spinach or the juice of orange or lemon."

MacLeod and Booher (1930) found that canned pimiento pulp protected guinea pigs in 2 grams daily doses, thus showing that its vitamin $\mathrm{C}$ potency is of the same order of magnitude as that of lemon or orange juice or tomatoes.

Immature seeds alone have been studied in the case of green peas. Eddy, Kohman and Carlsson (1926) found that 1 gram daily of ungraded raw green peas from the city market was not protective, but that 2 grams daily afforded complete protection against scurvy in guinea pigs for periods of 90 days. In their opinion raw peas are about as rich as tomatoes and oranges in vitamin C. It required 5 grams of home-cooked peas (12 to 15 minutes at $100^{\circ} \mathrm{C}$.) to afford the same protection as 2 grams of the raw peas. The commercially canned peas tested were of three sizes: Nos. 1, 3, and 6, representing "Petit pois," medium size, and large or marrow-fat type. On the basis of protection against scurvy with smaller amounts of the peas from No. 1 and 3 grades than from No. 6 , it was concluded that immature peas are richer in vitamin $C$ than mature. From the relative richness in vitamin $\mathrm{C}$ of the immature seed and the flesh pod which feeds it, as shown in these studies on green peas, string beans, and peppers, and the disappearance of the vitamin as the seed matures and its reappearance when the seed sprouts, it is plainly suggested that in the mature resting 
seed there must be present something which plays the part of a "resting stage" or reserve form of the antiscorbutic vitamin and which in turn is transformed into vitamin $C$ when the seed sprouts.

Vitamin C in Animal Tissues and in Milk.-In the work of Parsons (1920) previously cited and in other investigations it has been made clear that the liver may be much richer in vitamin $C$ than the muscles. Thus Parsons found that the livers of rats which had been for a long time on "scorbutic" food contained enough antiscorbutic vitamin to be readily demonstrable in experiments with guinea pigs, whereas their muscles did not, nor could any antiscorbutic effect be obtained in parallel experiments in which fish muscle was fed.

Blood probably also carries more vitamin $C$ than does muscle. Hess (1920) suggests that blood may be comparable with milk in its vitamin $\mathrm{C}$ content. This is not a sufficiently high concentration to make blood transfusion an important means of supplying the vitamin to a scurvy patient (Hess, 1920, p. 76). In fact the body as a whole seems to have only a very limited capacity for storage of vitamin C. Previous liberal feeding with antiscorbutic food does not enable guinea pigs to survive much longer when subsequently placed upon a scorbutic diet than do animals which have received merely an ample amount for the maintenance of health. (Harden and Zilva, 1918b; Hess, 1920, p. 75.) This is not inconsistent with the fact that a man or animal whose food has been deficient in vitamin $\mathrm{C}$ and who is therefore already in a condition of latent scurvy may develop the disease more quickly when placed upon a wholly scorbutic diet than does an individual whose previous food supply has been normal. It is evident that in general we must look to the daily food supply rather than to any stores carried in the body for the antiscorbutic vitamin needed in nutrition.

Muscle tissues, ordinary meats, are so poor in antiscorbutic vitamin that attempts to show its presence by experiments upon guinea pigs have given negative results. Chick, Hume and Skelton (Hess, 1920, p. 168) found that 10 cubic centimeters daily of raw beef juice failed to protect guinea pigs. Dutcher, Pierson and Biester (1919) were not able to observe any antiscorbutic effect from raw lean beef fed to guinea pigs. On the other hand, observations upon human scurvy have sometimes indicated that meat, if eaten sufficiently fresh, raw, or "rare," and in large quantities, has an appreciable though small antiscorbutic value. Thus the fresh beef and horse meat eaten by the British troops in Mesopotamia is believed to have been largely responsible for the fact that they did not develop scurvy as did the Indian troops serving with them. In 1877 the British Arctic Survey Committee 
reported in regard to the outbreak of scurvy in the Polar expedition of $1875-76$, that "although the scurvy was due to the absence of lime juice from the sledge dietaries, meat in large amounts is able to prevent the disease." Stefánsson $(1918,1918 \mathrm{a})$ reported avoidance of scurvy during his Arctic explorations by the use of large quantities of meat from freshly killed game, this meat being usually eaten raw. In view of the fact that even when eaten in very large amounts meat can be expected to prevent scurvy only if eaten raw or nearly so, we must conclude that cooked meat, as ordinarily eaten, probably furnishes but insignificant amounts of the antiscorbutic vitamin.

There is a somewhat higher concentration in the glandular organs and perhaps also in the blood. As has been noted previously, the presence of vitamin $\mathrm{C}$ has been demonstrated in chicken liver (Hart, Steenbock, Lepkovsky and Halpin, 1925) and calve's liver (Thurston, Palmer and Eckles, 1929) even although these species do not require vitamin $C$ in their food. The absence of vitamin $C$ in the liver of guinea pigs on a scorbutic ration and its presence in considerable amounts in the livers of normal guinea pigs (Parsons and Reynolds, 1924) suggest that in those species requiring vitamin $C$ the concentration in the liver depends largely upon the amount present in the food supply.

The concentration of vitamin $\mathrm{C}$ in milk is likewise largely dependent upon the food of the nursing mother or lactating animal. Chick, Hume and Skelton (1918) found that with fresh cow's milk as the sole source of vitamin C complete protection was secured when guinea pigs were fed an average of 85 cubic centimeters of milk per capita per day. On the usual assumption that a baby requires about five times as much vitamin $\mathrm{C}$ as a guinea pig, this result agrees well with the general experience of Hess (1920, p. 152) that an infant will receive sufficient antiscorbutic vitamin from one pint of fresh milk per day. These estimates may be considered to apply to milk of average or nearly average antiscorbutic value. If the value is materially lessened by heating or ageing of the milk or by faulty feeding of the cow more milk will be required; and conversely fresh milk from a cow properly fed may be considerably richer in vitamin $\mathrm{C}$ than these studies would indicate.

The influence of the feed of the cow upon the antiscorbutic value of the milk produced was investigated by Hart, Steenbock and Ellis (1920). The samples of milk tested included dry feed milk, obtained from a herd of 18 cows which had never been fed any fresh vegetable tissues but only air-dried roughages and grains; summer pasture milk from cows which during part of the day grazed on a timothy, blue grass, clover pasture; and winter-produced milk from cows fed on 
dried grains and hays, supplemented in one case by a silage made from corn that had well matured and partly dried but had not been frozen, and in another case by a small amount of silage and a considerable amount of hybrid sugar mangels. Each variety of milk was tested by feeding it to guinea pigs as a supplement to a basal scurvy-producing ration of heated ground alfalfa hay, rolled oats and common salt. The amounts fed varied from 15 to 50 cubic centimeters daily of the summer pasture milk and from 15 to 100 cubic centimeters of the dry feed milks. Full protection was secured by the daily consumption of 50 cubic centimeters of the summer pasture milk and 75 cubic centimeters of the dry-feed milk.

Dutcher, Eckles, Dahle, Mead and Schaefer (1920) determined in a similar manner the vitamin $C$ content of the mixed milk of two cows, first on dry feed and later smaller amounts of the dry feed supplemented by pasture feeding. Greater protection against scurvy was secured with 20 cubic centimeters of the summer milk than with 60 cubic centimeters of the winter milk as thus produced. The response to pasture feeding was very rapid, being noticeable within two weeks after the cows were put to pasture. Hess, Unger and Supplee (1920) also showed that the milk of cows on pasture is much richer in vitamin $\mathrm{C}$ than dry fodder milk and that the antiscorbutic vitamin in the feed is rapidly secreted into the milk rather than stored in the body.

That winter-produced milk under certain conditions may be as rich as summer-produced has been demonstrated by Kierferle and Zeiler (1926) and by MacLeod (1927). The former compared the antiscorbutic properties of milk produced by cows on three different rations, one consisting largely of silage prepared from young second crop grass of good quality, another of hay and another of brewers' grains. On a basal diet of oats and autoclaved powdered alfalfa with one per cent of common salt, guinea pigs were completely protected from scurvy by 45 cubic centimeters daily of the silage milk and by 60 cubic centimeters of the dry feed milk, while 60 cubic centimeters of the milk from cows fed brewers' grains did not afford complete protection.

MacLeod (1927) determined the vitamin C content of milk from cows receiving a ration of mixed grains, alfalfa hay, corn silage, and beet pulp. The cows were allowed exercise in a yard but had no access to pasture throughout the year. Of the milk thus produced 30 cubic centimeters fed 6 days a week in two or three feedings a day afforded considerable protection since none of the guinea pigs receiving this amount died before the end of 90 days. For complete protection 50 cubic centimeters was found sufficient. The experiment was continued 
for a year during which time there was no appreciable variation in the vitamin $\mathrm{C}$ content of the milk. MacLeod concludes that by including good quality silage in the ration of stall-fed cows it is possible to prevent seasonal variations in the antiscorbutic value of milk.

Milk scalded by being heated rapidly to the boiling point over a gas burner and then quickly cooled was found by Barnes and Hume (1919) to retain about one-half of its original antiscorbutic value. Hart, Steenbock and Smith (1919) reported that milk sterilized for 10 minutes at $120^{\circ} \mathrm{C}$., commercial evaporated milk and commercial milk powder did not show antiscorbutic properties when fed in amounts corresponding to protective doses of the raw milk. Hess (1920) stated that in his own experience in infant feeding "dried milk not only does not lead to scurvy but may contain sufficient antiscorbutic vitamin to cure this disorder." He attributed the unfavorable results obtained by Hart, Steenbock and Smith with dried milk to lack of uniformity in the product and suggested that "For milk to retain its antiscorbutic value, notwithstanding drying, it must have been rich in the vitamin before desiccation, it must have been dried quickly, and packed within the shortest possible interval in air-tight, preferably hermetically sealed, containers. As in relation to the heating of milk so in regard to its drying it is not the degree of heat to which it is subjected which is all important but rather the associated conditions. The merits of each process will have to be tested individually and perhaps even each particular brand of milk."

We have seen that foods of high initial acidity such as tomatoes and orange and lemon juice can be dried and still retain marked antiscorbutic value, while attempts to conserve vitamin $\mathrm{C}$ during the drying of less acid foods, and for those of less favorable oxidation-reduction potential, such as cabbage and potatoes have been less successful. The drying of milk is perhaps more highly developed from the mechanical standpoint than that of any other food and since it is nearly neutral in reaction and largely used in infant feeding, special interest attaches to the question of retention of the antiscorbutic value of milk during drying. In view of the fact that the initial concentration of vitamin $\mathrm{C}$ in milk may vary widely the actual destruction of the vitamin can be measured quantitatively only by determining the vitamin $C$ content of the raw milk employed as well as of dried product. Such an investigation was undertaken by Cavanaugh, Dutcher and Hall (1923). The milk was taken from the mixed milk of 175 dairies, representing 1,276 cows. The samples to be used raw were cooled and shipped to the laboratory in sterilized vacuum bottles, while more from the same lot 
was dried by a spray process in which the milk is first evaporated in a continuous vacuum evaporator, pasteurized in a closed glass-lined tank at $145^{\circ} \mathrm{F}$. for 30 minutes and then dried instantaneously by being forced into a heated chamber under very high pressure. The freshly prepared milk powder showed no destruction of vitamin C. This was attributed to the rapidity of the drying process and the absence of oxygen during the pasteurization. No claims were made for the vitamin $\mathrm{C}$ content of other milk powders.

Human milk and goats' milk undoubtedly vary in their vitamin $\mathrm{C}$ content in the same manner as cows' milk and, according to the limited amount of evidence available, are not likely to be much richer in vitamin $\mathrm{C}$ than cows' milk produced under the best feeding conditions. It is probably best to recommend that all infants be given some such antiscorbutic food as orange juice or tomato juice. If this is done the possible variations in vitamin $\mathrm{C}$ content among the different forms of milk are of little concern.

\section{Chemical Behavior of Vitamin C}

The pioneer studies of Holst and Frölich demonstrated the destruction, wholly or in part, of antiscorbutic properties in foods when heated under the conditions of their experiments; and in other investigations which followed, evidence of a more or less quantitative nature was obtained regarding the instability of vitamin C. Thus La Mer, Campbell and Sherman $(1921,1922)$ in an investigation of the effect of temperature and of hydrogen-ion activity upon the rate of destruction of vitamin $\mathrm{C}$, established time curves for destruction of the vitamin at $60^{\circ}, 80^{\circ}$, and $100^{\circ} \mathrm{C}$. in an aqueous medium of the acidity of natural tomato juice and some effects of other variables under stated conditions. The results of the study may be briefly summarized as follows:

Effect of Heating in Acid Solution.-In the case of tomato juice of natural acidity, $\mathrm{pH}=4.3$, it was found that boiling for one hour destroyed practically 50 per cent, and for four hours practically 68 per cent of the antiscorbutic vitamin present. The time curve of the destructive process is, therefore, much flatter than that of a unimolecular reaction or of a reaction proceeding according to the square root rule of Schütz. Similar flattening of the time curves of the destruction of the vitamin were found also in experiments in which the tomato juice was heated at temperatures lower than boiling, viz. at $60^{\circ} \mathrm{C}$. and at $80^{\circ} \mathrm{C}$. Comparisons of the data obtained at $60^{\circ}, 80^{\circ}$, and $100^{\circ} \mathrm{C}$. show relatively low temperature coefficients:

$$
Q_{10}\left(60^{\circ}-80^{\circ}\right)=1.23 ; Q_{10}\left(80^{\circ}-100^{\circ}\right)=1.12 \text {. }
$$


Doubtless this is due in part to the lowering, during the period of experimental heating, of the initial oxidation potential, through the consumption or expulsion of the residual dissolved oxygen or the destruction of labile oxidizing substances, or both.

Effect of Reduced Concentration of Hydrogen Ions (Hydrogen-ion Activity).-In experiments in which the natural acidity of the tomato juice was first neutralized in whole or in part, the juice then boiled for one hour and immediately cooled and reacidified, it was found that at $\mathrm{pH} 5.1$ to 4.9 the destruction during one hour's boiling was increased to 58 per cent (as compared with 50 per cent at natural acidity, $\mathrm{pH} 4.3$ ). Neutralization of a larger proportion of the natural acidity increased the rate of destruction of the vitamin. When alkali was added to an initial $\mathrm{pH}$ of 11 , which fell to about $\mathrm{pH} 9$ during the hour of heating (doubtless because of reaction of the alkali with the sugars, proteins, etc., present), the destruction found by feeding of the juice thus treated, but immediately cooled and reacidified, was about 65 per cent.

Heating at $100^{\circ} \mathrm{C}$. for one hour at $\mathrm{pH} 11$ to 9 as described above, followed by standing for one to five days in stoppered but only partially filled bottles in a refrigerator at $10^{\circ} \mathrm{C}$. at an alkalinity of only $\mathrm{pH} 9$ was found to destroy 90 to 95 per cent of the antiscorbutic vitamin, as compared with 65 per cent when the solution was reacidified after heating; thus confirming, extending and bringing into more quantitative form the observations of Harden and Zilva (1918d) and of Hess and Unger (1919) upon the susceptibility of this vitamin to alkalinity even at low temperatures.

Effect of Oxidation and Reduction.--In all of the foregoing experiments the heating was performed in cotton-stoppered, narrow-necked flasks from which air was probably very largely displaced by water vapor early in the heating. When samples of the juice at $\mathrm{pH} 3$ and 8.3 were heated at $100^{\circ} \mathrm{C}$. for one hour with oxygen bubbling through the liquid, practically complete destruction of vitamin $\mathrm{C}$ took place in both acid and alkaline preparations. Kenny (1926), continuing the work of La Mer, Campbell and Sherman, showed that when the tomato juice of natural acidity was heated in a most rigorously maintained anaerobic environment, the previously observed rate of destruction was reduced by about two-thirds; and by strictly parallel experiments with the juices of tomato and cabbage, he showed that the more rapid destruction of the vitamin $C$ of the latter could be correlated with, and is presumably a function of, its higher oxidation potential. At $\mathrm{pH} 4.2$ he found $E_{h}=+0.137$ volts for tomato juice and $E_{h}=+0.362$ volts for cabbage juice; at $\mathrm{pH} 6.2, E_{h}=+0.045$ volts for tomato juice and 
$E_{h}=+0.248$ volts for cabbage juice. Thus the work of Kenny clarifies the earlier work of Delf as well as that of La Mer, Campbell and Sherman.

Kohman (1923) called attention to the fact that fruits and vegetables, as well as the water in which they are cooked, may contain sufficient dissolved oxygen to become an important factor in the destruction of vitamin $\mathrm{C}$. Interested in the practical problem of the preservation of vitamin $\mathrm{C}$ in canning processes he suggested various methods of removing oxygen from liquid and solid foods without the application of heat, including vacuum exhaust and for certain fruits and vegetables preliminary respiration. These suggestions were put into effect in the later studies of Kohman, Eddy, and associates as noted elsewhere.

\section{Concentration Experiments}

Zilva $(1921,1922)$ in the first of his series of studies on the stability and chemical nature of vitamin $C$, used, as his source of vitamin $\mathrm{C}$, lemon juice from which the organic acids had been removed by precipitation with powdered chalk. The "minimum protective dose" of this decitrated lemon juice was from 1.5 to 2 cubic centimeters daily. After air had been aspirated through the solution for 12 hours at laboratory temperature, daily doses of 3 and 5 cubic centimeters proved insufficient to prevent, but delayed slightly, the onset of scurvy and 7 cubic centimeters was inadequate for promoting growth. After the decitrated juice had been boiled for an hour with constant aeration, a daily dose of 7 cubic centimeters were inadequate to prevent or delay the onset of scurvy, while 1.5 cubic centimeters of the juice boiled for 2 hours in an atmosphere of carbon dioxide provided ample protection. Hydrolysis with hydrochloric acid for 5 hours impaired but did not entirely destroy the vitamin $C$ value of the juice.

In an attempt to correlate the effects of the reaction of the medium and of oxidation on the stability of vitamin C, Zilva (1923) adjusted portions of decitrated lemon juice to $\mathrm{pH} 6.6$ to $6.8,12.12$, and 2.2 to 2.4. Portions of the alkaline solution $(\mathrm{pH} 12.12)$ were allowed to stand for 24 hours under aerobic and strongly anaerobic conditions, while the strongly acid solution ( $\mathrm{pH} 2.2$ to 2.4 ) was aerated for 1 or 2 hours and the nearly neutral solution for 1 hour at $100^{\circ} \mathrm{C}$. The alkaline solution was acidified with citric acid immediately before being tested and all the preparations were tested on guinea pigs which had been for about 14 days on a ration of oats and bran supplemented by not more than 40 cubic centimeters of autoclaved milk daily. As thus tested, the alkaline solution was found to have lost about 80 per cent 
of its antiscorbutic properties in 1 hour when exposed to air at room temperature, while a solution of the same reaction kept for 24 hours at room temperature in the absence of air showed no deterioration. Zilva (1923a) reported no loss in activity in slightly acid juice after storage for 3 months in an ordinary conical flask in an evacuated bell jar.

In later tests (Zilva, 1924a) the decitrated lemon juice was concentrated to one-tenth its volume, acidified by the addition of 7 grams of citric acid to 1 liter of juice, and placed in 50 cubic centimeters amounts in a series of flasks which were then evacuated, sealed, and stored in a dark cupboard for 3 and 5 months, with no detectable loss in potency. The concentrate was also used after 6 months' storage in the treatment of a severe case of infantile scurvy. The treatment was very intensive, from 2.5 to 6 drams being given every 3 hours during the first 2 days and 1 dram every 6 hours on the following day. The patient was discharged cured in 8 days, during which time the equivalent of about 48 lemons had been administered.

On subjecting decitrated lemon juice to fermentation with yeast in the presence of carbon dioxide, Zilva (1924) was able to bring about further concentration of the fraction containing the antiscorbutic vitamin through destruction of the sugar present. The active fraction thus obtained contained less than half the dry matter of the decitrated juice and had a nitrogen content varying from 0.004 to 0.014 per cent. It was said to give negative biuret, sulfur, and tryptophane tests, and a faint murexide reaction. Precipitates were obtained with mercuric sulfate, basic lead acetate, and Millon's reagent and occasionally but not invariably with phosphotungstic acid. The solution reduced ammoniacal silver nitrate and gave a marked Pauly reaction.

Zilva (1924c) next found that much of the inactive material could be removed from decitrated lemon juice by precipitation with alcohol and that the active material was precipitated by basic lead acetate. His method at this time was essentially as follows:

The lemon juice was first decitrated with excess calcium carbonate and the filtrate concentrated in vacuo at $50^{\circ} \mathrm{C}$. to about one-fifth its original volume. A volume of alcohol equal to about one-half the original volume of juice was then added gradually, the inactive precipitate filtered off and washed with a little alcohol and the filtrate and washings distilled in vacuo at $50^{\circ} \mathrm{C}$. to remove the alcohol. The residue was then made up to the original volume with distilled water, fermented as previously described, concentrated to small volume, and treated with several volumes of absolute alcohol. After removing the inactive precipitate and excess alcohol as before, basic lead acetate was added to complete precipitation, the active precipitate quickly centrifuged out and dissolved in acetic acid. Magnesium sulfate and absolute alcohol were added to remove the lead and other inactive material and the filtrate finally distilled in vacuo at $50^{\circ} \mathrm{C}$. 
The concentrate as thus prepared, when diluted to the original volume contained from 0.03 to 0.05 per cent of total solids and was capable of preventing scurvy in guinea pigs in doses as low as 1.5 cubic centimeter daily. It reduced ammoniacal silver nitrate in the cold and decolorized potassium permanganate.

In spite of the close association of reducing and antiscorbutic properties, Connell and Zilva (1924) found that destruction of vitamin C and of reducing properties did not proceed at the same rate. The material responsible for the decolorization of potassium permanganate was very little affected by heat or alkalinity. The ability to reduce ammoniacal silver nitrate was somewhat decreased, but in much less degree than the inactivation of antiscorbutic properties. It was concluded that a part at least of the reducing material in vitamin $\mathrm{C}$ concentrates is chemically independent of the vitamin itself.

Bezssonoff (1925) also made use of basic lead acetate as a precipitant of vitamin C. He had earlier (1921) used neutral lead acetate to remove some of the inactive material from cabbage juice, concentrating the filtrate, after removal of the lead, to a hygroscopic yellowish white powder which was said to furnish sufficient vitamin C for 600 gram guinea pigs in 0.1 gram doses.

In the modified method, 100 liters of fresh cabbage juice was treated with 40 liters of water containing 320 grams of lead acetate and 1,600 cubic centimeters of acetic acid. The filtrate, without being freed from lead, was brought to $\mathrm{pH} 8.2$ with sodium hydroxide with the formation of a precipitate containing the active material. This was dissolved in acetic acid and the process repeated twice. The final precipitate was dissolved in acetic acid and treated with hydrogen sulfide under pressure to precipitate the lead. The filtrate was subjected to four evaporations in vacuo at $25^{\circ} \mathrm{C}$. with subsequent dilution with distilled water and finally extracted successively with absolute alcohol and acetone.

The extract as thus prepared yielded on evaporation colorless needle-like crystals which were very hygroscopic and easily oxidized, "forming a yellowish brown quinone." The oxidation product, but not the original crystals, gave the blue color reaction considered by Bezssonoff (1921b, 1923a) to be characteristic of vitamin C. Two cryoscopic determinations of the molecular weight of the crystalline substance gave values of 200 and 230 . It melted at $47^{\circ} \mathrm{C}$. and contained 45.57 per cent carbon, 48.24 per cent oxygen and 6.19 per cent hydrogen. It was soluble in water in all proportions and insoluble in ether, benzene, and toluene. The minimum protective dose for guinea pigs was less than 2 milligrams daily.

In view of the elementary analysis reported by Bezssonoff for his most active vitamin C concentrate, it is of interest that Zilva (1925a) reported at about the same time that his vitamin $C$ concentrates always 
contained small quantities of amino and amide nitrogen. The latter was definitely proved to have no connection with antiscorbutic properties, but doubt was expressed concerning the former. Later Daubney and Zilva (1926) found nickel, cobalt, and boron to be absent from the ash of the concentrate and iodine to be present in amounts corresponding to 0.1 to 0.2 milligrams per liter. This was excluded as being a part of vitamin $\mathrm{C}$ itself, as it did not diffuse through collodion membranes permeable to the active material. Phosphorus appeared to be present in amounts of from 0.0005 to 0.0009 per cent of the concentrate. Later analyses by Hoyle and Zilva (1927) gave slightly lower figures for phosphorus, with traces of iron and sulfur but no magnesium.

Daubney and Zilva (1926) inactivated decitrated lemon juice by aeration and attempted to reactivate it by the use of various reducing agents including hydrogen in the presence of platinum black, nascent hydrogen obtained by the action of citric acid on magnesium, and electrolysis. Nonaerated juices were subjected to the same treatment and both series of samples were tested on guinea pigs. No protection was secured with the aerated samples, indicating that vitamin $\mathrm{C}$ when destroyed or inactivated by oxidation cannot be regenerated by simple reduction.

The risk of inactivation of vitamin $\mathrm{C}$ by excess alkalinity in the precipitation with basic lead acetate led Zilva (1927) to make a study of the range of hydrogen-ion activity within which the precipitation takes place. This was found to lie between $\mathrm{pH} 5.4$ and $\mathrm{pH}$ 7.2. A convenient method of bringing about the precipitation was found to consist in treating the decitrated lemon juice with an excess of neutral lead, centrifuging the inactive precipitate, and adjusting the hydrogen-ion activity of the supernatant solution by the cautious addition of dilute ammonia to $\mathrm{pH}$ 7.2. The fraction thus precipitated, while smaller in bulk and containing much less total solids and reducing sugar than that hitherto obtained, was of the same order of activity.

Continuing his investigation of the relationship between reducing and antiscorbutic properties, Zilva (1927a) determined the reducing capacity, for (toward) the indicator phenolindophenol, of various lemon juice fractions with their antiscorbutic property. The two properties were found to be very similar with respect to the destructive influence of alkalies and of aeration, but quite unlike in their behavior toward lead acetate as a precipitating agent. Only about 30 to 50 per cent of the reducing substance or substances could be accounted for in the 
fraction precipitated at $\mathrm{pH} 5.4$ to 7.2 which contained almost the entire amount of the vitamin $C$ in the original juice, while the fraction precipitated by neutral lead acetate at $\mathrm{pH} 5.4$, although totally inactive in antiscorbutic properties, showed an even higher reducing capacity than the active fraction. On adding phenolindophenol to decitrated lemon juice until the indicator was no longer reduced and testing the treated solution immediately for vitamin $C$ no appreciable loss in antiscorbutic activity was observed. These findings were thought to furnish additional proof that there is no direct connection between the reducing and antiscorbutic activity, or at least that the antiscorbutic factor is of a lower reducing intensity than the reducing agency. Attention was then directed to the possible function of the reducing agency as a stabilizing agency for the antiscorbutic factor. This possibility seems to be borne out by the following observations:

Decitrated lemon juice purified by precipitation with alcohol was tested for both reducing and antiscorbutic properties after storage for 24 hours and for one week. The deterioration of the two properties was more or less of the same order, although there was possibly a greater loss of reducing than of antiscorbutic properties. Decitrated lemon juice adjusted to $\mathrm{pH} 7$ and heated for 1 hour in a steam autoclave under a pressure of one atmosphere showed little loss in either antiscorbutic or reducing properties when tested immediately, but almost complete loss in both after storage in the cold for a week. In commenting upon these observations Zilva stated: "It would almost appear as if the stability of the antiscorbutic factor depends on a chain of reactions which are kept in equilibrium in the living cell and that on damaging the cell the equilibrium is disturbed and the individual links are progressively damaged with the ultimate inactivation of the vitamin. The presence of some impurities would, according to this view, be a necessary condition for establishing the antiscorbutic potency, since the removal of such stabilizing substances would inactivate the vitamin before the preparation could be tested."

Zilva (1928) soon presented further evidence favoring the hypothesis that in the chemical fractionation of vitamin $\mathrm{C}$ a reducing substance is removed which in its natural medium exerts a protective action on the vitamin.

After the reducing substance in decitrated lemon juice had been neutralized with phenolindophenol and the solution adjusted immediately to $\mathrm{pH} 7$ the antiscorbutic activity of the juice disappeared within 24 hours. Concentrates, prepared as previously described, lost their antiscorbutic property much more rapidly than did decitrated lemon juice 
of similar activity, thus suggesting that a protective substance had been removed during fractionation.

Decitrated lemon juice submitted to dialysis in a collodion thimble previously soaked in 83 per cent alcohol retained most of its antiscorbutic property and slightly less than half its reducing property, while similar juice dialyzed in a thimble soaked in 92 per cent alcohol retained none of its antiscorbutic or reducing properties.

Previous dialysis experiments by Zilva and Miura (1921) and Connell and Zilva (1924) had suggested the probability that the dimensions of the vitamin $\mathrm{C}$ molecule were not far different from those of the hexoses, since an 87 per cent membrane was impermeable to both the sugar and the vitamin in decitrated lemon juice, while an 88.5 per cent membrane allowed the slow passage of the sugar but not the vitamin. The different rates of diffusion of the reducing and antiscorbutic materials now demonstrated were thought by Zilva to suggest the probability that the vitamin $\mathrm{C}$ molecule is larger than hitherto surmised and that its apparent inactivation on dialysis noted in the earlier studies was due to diffusion of the protective reducing agent.

Zilva (1929) studied the effect of heating at temperatures lower than $100^{\circ} \mathrm{C}$. Decitrated lemon juice adjusted to $\mathrm{pH} 7$ was heated in air-exhausted, nitrogen-filled ampoules at temperatures of $55^{\circ}$ to $58^{\circ}$, and $80^{\circ}$ to $85^{\circ}$, respectively, for 1 hour, adjusted if necessary to $\mathrm{pH} 7$, and kept in the cold for 7 days before being tested for vitamin C. With the exception of one series in which the juice was heated to $55^{\circ} \mathrm{C}$. there was no more deterioration of vitamin $\mathrm{C}$ in the heated than in unheated juices in corresponding periods of storage. The destruction of the reducing principle was likewise of the same order as that of unheated solutions.

The stability of the vitamin after autoclaving was not increased by the addition of decitrated lemon juice previously inactivated by aeration at ordinary temperatures, or of the inactive precipitate obtained by treating ordinary decitrated lemon juice with alcohol, or of the neutral lead acetate fraction, although it was noted that occasionally considerable antiscorbutic activity could be demonstrated in the neutral lead acetate precipitate. Decitrated lemon, cabbage, tomato, and rutabaga juices were all found to contain a peroxidase, the activity of which persisted after the vitamin had been partially or entirely destroyed. Autoclaving decitrated lemon juice under anaerobic conditions diminished somewhat its capacity for reducing phenolindophenol, but increased its capacity for decolorizing iodine. This increased capacity 
for decolorizing iodine was not observed after decitrated lemon juice had been heated anaerobically at $58^{\circ}$ or $85^{\circ} \mathrm{C}$.

These observations suggested the possibility that the decrease in stability of vitamin $\mathrm{C}$ produced by autoclaving is due to the formation of a substance which hastens the destruction of the vitamin rather than to the destruction of a thermolabile stabilizing factor. This suggestion received confirmation in the decreased antiscorbutic properties and capacity to reduce phenolindophenol of autoclaved decitrated lemon juice when mixed with unheated juice and stored for 7 days. The addition of quinol to decitrated lemon juice had the same effect.

The use of butyl alcohol to remove the yellow coloring matter and wax from lemon juice, in the process of preparing vitamin $\mathrm{C}$ concentrate has been introduced by Grettie and King (1929). Their method, based in other respects upon the procedure of Zilva with modifications, is essentially as follows:

The juice is decitrated with basic lead carbonate instead of with chalk as in the Zilva method. After stirring slowly until the evolution of carbon dioxide has ceased, 2 or 3 hours, and cooling, the crystalline precipitate is filtered off and the filtrate, without concentration or alcohol treatment as in the Zilva method, is fermented with yeast. This required, in their experiments, about 24 hours, but could be hastened by adding a few drops of 20 per cent phosphoric acid and centrifuging before the yeast was added. After the fermentation is complete, the yeast is centrifuged out and 17 cubic centimeters of a saturated solution of neutral lead acetate added to each 100 cubic centimeters of the decitrated juice. The white inactive precipitate is removed by centrifuging, the filtrate brought to a $\mathrm{pH}$ of 7.2 to 7.4 with dilute ammonia, and the active precipitate removed by centrifuging. This is dissolved in dilute acetic acid made up to half the original volume with water and the precipitation with ammonia repeated. The second precipitate is treated with dilute hydrochloric acid in sufficient excess to convert the yellow lead precipitate to white lead chloride and is then extracted three times with 10 cubic centimeters portions of butyl alcohol which precipitates the waxy matter. Sufficient ethyl alcohol is then added to bring the alcohol concentration to 90 per cent, the precipitated lead chloride is removed, and the filtrate is evaporated to dryness and made up to one-fifth the original volume of the lemon juice with water containing traces of acetic acid.

The extract thus prepared was found to retain practically all of the antiscorbutic value of the original lemon juice. In an attempt to purify it still further acetone was added to a concentrated water solution of the extract. By precipitating the ammonium chloride present this reduced the total solids to from 0.5 to 1 milligram per cubic centimeter of lemon juice equivalent. The filtrate from this precipitation was carefully dried in vacuo and the dried material extracted with 50 cubic centimeters of absolute acetone for 24 hours. Feeding tests showed that the active material is definitely soluble in absolute acetone. Analyses of the extract gave a content of 0.38 to 0.6 milligram of the total solids per cubic centimeter of lemon juice. Total nitrogen constituted 
3.46 per cent, ammonia-nitrogen 1.9, and reducing sugars as dextrose 19 per cent of the total solids. "The final active material gave a faint carbylamine test for amino nitrogen, reduced potassium permanganate and ammoniacal silver nitrate quickly, and gave a faint coloration with ferric chloride. Bromine water produced a slight cloudiness. The phthalic anhydride and Liebermann reactions for phenols were too faint to be considered positive. Further extraction of the dried acetonesoluble material with absolute ethyl ether, in which the vitamin is practically insoluble, reduced the total actıve solids to 0.28 milligram per cubic centimeter of lemon juice."

A slightly different method was described by Sipple and King (1930). The lemon juice was decitrated with neutral lead acetate as in the method of Grettie and King and was immediately adjusted to $\mathrm{pH} 7.4$ to 7.6 with diluted ammonium hydroxide. The yellow active precipitate was centrifuged quickly, dissolved in dilute acetic acid and the process repeated, beginning with the addition of dilute ammonium hydroxide. An aqueous solution of the active precipitate was then treated with two successive portions of $n$-butyl alcohol as in the method of Grettie and King, the filtrate concentrated, dissolved in 98 per cent alcohol, and treated with one volume peroxide-free absolute ethyl ether which removed more inactive material in the form of a white crystalline precipitate.

The active liquid was found to contain 0.56 milligram of total solids per cubic centimeter of the equivalent lemon juice, with an inappreciable amount of ash. Reducing substances calculated as glucose amounted to 0.45 milligram per cubic centimeter; ammonium salts expressed as nitrogen, 0.045 milligram; and total nitrogen as determined by the micro-Kjeldahl method, 0.145 milligram of nitrogen per cubic centimeter. The ferric chloride test for phenols was negative and the carbylamine reaction mildly positive. Ammoniacal silver nitrate was reduced slightly.

Attempts to use barium acetate in place of lead acetate and sodium hydroxide in place of ammonium hydroxide gave negative results. This is interpreted as indicating that the precipitation of the vitamin by lead at a $\mathrm{pH}$ of 7.4 to 7.6 is a more or less specific adsorption phenomenon.

The alcohol-ether preparation was finally purified still further by evaporating to dryness in vacuo and extracting the residue with absolute ether. This removed a significant amount of amorphous material, leaving the total solids of the active fraction equivalent to 0.28 milligram per cubic centimeter of lemon juice. 


\section{CHAPTER V}

\section{VITAMIN A}

While the conception of the presence in certain food materials of the water-soluble vitamins $\mathrm{B}$ and $\mathrm{C}$ developed primarily from observations upon disease, the conception of the fat-soluble vitamin $\mathrm{A}$ arose, as has been noted in Chapter I, from the failure to secure normal growth in experimental animals for a long period of time on purified food materials furnishing adequate proteins, fats, carbohydrates, and salts. The plan of studying the nutritional needs of animals through the feeding of mixtures of purified foodstuffs instead of natural foods had early impressed itself as a logical method of procedure but one beset with difficulties. Voit (1881) in his treatise on nutrition in Hermann's Handbuch der Physiologie wrote: "Unquestionably it would be best for the purpose if one could feed only pure chemical compounds (the pure foodstuffs), for example, pure protein, fat, sugar, starch, ash constituents, or mixtures of the same. However, inasmuch as men and animals only rarely tolerate continuously such tasteless mixtures, it is necessary in most cases to choose foods as they are provided by nature." Several workers contributed links to the chain of evidence of the existence in natural food materials of previously unknown growth-promoting substances as noted in the introductory chapter. Lunin's experiments conducted primarily to determine the nutritional significance of certain inorganic substances led to the suggestion that milk must contain unknown substances indispensable in nutrition. In 1909, Stepp published the first of a series of experiments concerned with the indispensability of lipoids for normal nutrition. His procedure was to feed mice with milk-bread subjected to prolonged extraction with alcohol and ether for the purpose of removing the lipoids. In commenting on the use of this food instead of an artificial, "synthetic," lipoid-free mixture he stated that, as there was no exact knowledge of the materials necessary for life, nutritive failure on a "synthetic" ration lacking in lipoids might be attributed not to lack of lipoids but of other (unknown) substances. Stepp found that mice could not live on the extracted bread but that, if the total extract were fed with the bread, normal growth ensued. In the light of our present 
knowledge of vitamins, the results obtained by Stepp in his effort to identify the indispensable substances removed from the food materials by extraction with alcohol and ether, as reported in this and later studies, can readily be interpreted from the known properties of the watersoluble and fat-soluble vitamins, both soluble in alcohol and the latter in ether, and both consequently removed from the materials used in his experiments. Unable to identify the removed indispensable component as any of the known lipoids, Stepp (1911, p. 150) called attention to the property of lipoids of influencing the solubility of other materials to such an extent that they also would be removed during the extraction. "So wäre es nicht undenkbar dass gemeinschaftlich mit den Lipoiden irgenwelche unbekannte lebenswichtige Stoffe in Lösung gehen und dass so die Lipoide gewissermassen zu Trägern für diese Stoffe würden, dass mit anderen Worten bei der Entfernung von Lipoiden die unbekannten Körper mit enfernt und bei Zusatz von Lipoiden mit diesen zugesetzt werden. Ein Hinweis auf eine derartige Möglichkeit erscheint notwendig, solange es nicht gelingt, die Versuche mit chemisch reinen Körpern durchzuführen."

The work of Osborne and Mendel during this same period was directed largely to the question as to whether normal nutrition could be secured upon a dietary containing a single individual protein instead of mixtures of proteins such as ordinary dietaries comprise. They soon came to emphasize also the importance of factors other than protein, and discovered that mixtures of purified foodstuffs which were capable of maintaining for some time the life of full-grown rats were unable to support the growth processes in young animals. Favorable experiences with milk and milk powder led to many unsuccessful attempts to modify the inorganic and non-protein ingredients of the diet of purified foodstuffs and finally to the use of the so-called protein-free milk which was prepared by removing as far as possible by precipitation and coagulation the proteins from the fat-free milk, evaporating the filtrate and grinding the residue to powder. This material, which was added to the artificial dietaries in liberal amounts (about 28 per cent of the total food mixture), was found to promote rapid growth in rats.

Shortly after the publication of Osborne and Mendel's work involving the use of protein-free milk, Hopkins (1912) published the results of his experiments conducted in 1906 and 1907 and showing a remarkable effect produced in the growth of rats on a diet of purified food substances by the addition of small amounts of milk or the alcoholsoluble portion thereof. That his observations had at an even earlier date 
suggested the idea that natural food materials contain other essential ingredients than those hitherto recognized is shown by his statement in 1906 noted in Chapter I. The delay in publishing the remarkable results which he had obtained was explained in a note regarding his early experiments in which Hopkins (1921) stated, "The experiments which I described in 1912 followed upon a long experience of the effects of adding tissue extracts and especially fractionated yeast preparations to purified diets. Looking back to one's experience in these years during which startling successes were mingled with puzzling failures-failures which led to delay in publication, I realize that the absence of all knowledge concerning the factor associated with fats was the cause of any experimental contradictions. Had I possessed the scientific vision which afterwards led McCollum and Osborne and Mendel to recognize the existence of this, I should have reached full conviction as to the reality of my results long before I did. In the synthetic diets employed by me the proteins and the carbohydrates were purified to the utmost, but I used little or no discrimination with regard to the fats."

The work of McCollum and of Osborne and Mendel to which Hopkins here refers, appeared almost simultaneously in 1913. McCollum and Davis employed rations of purified casein, carbohydrates, and various salt mixtures; and the same rations in which part of the carbohydrate was replaced by lard. It was found that with certain proportions of the various ingredients normal growth of the animals for periods varying from 70 to 120 days often resulted, while beyond that time little or no increase in body weight could be induced, although the animals remained in apparently good nutritive condition for some time. After numerous attempts to promote further growth by adjustments between the various ingredients of the food mixture, it was found that resumption of growth occurred quite promptly after the introduction into the diet of the ether extract of egg or butter. Negative results were obtained with lard and olive oil, thus showing that the suspension of growth was not due merely to the absence of fat from the diet. The conclusions drawn at that time, as taken from the authors' discussion, are as follows: "Whether the resumption of growth is the result of supplying in the ether extract of egg or of butter some indispensable organic complex of the chemical nature of the lipins, or is the result of a stimulating action of some substance accompanying the lipins can not be decided from the data available. . . . Our observation that ether extracts from certain sources improve the condition of animals on such rations strongly supports the belief that there are certain accessory 
articles in certain foodstuffs which are essential for normal growth for extended periods."

Similar conclusions published by Osborne and Mendel (1913) during the same summer were arrived at from an attempt to explain the great superiority of diets employing milk over purely artificial food mixtures or even mixtures containing protein-free milk. A mixture of milk powder 60 , starch 12 , and lard 28 per cent proved to be adequate for growth and maintenance while "protein-free milk" food consisting of protein in the form of edestin or casein 18 , starch 26, lard 28, and "protein-free milk" 28 per cent failed to support growth for more than about 100 days. The essential difference evidently lay in the absence from the protein-free milk foods of those components of milk which are separated in the process of centrifugation. This led to the substitution for part of the lard in the protein-free milk food of a corresponding quantity of butterfat, a change which brought about prompt recovery and rapid growth of the experimental animals and which justified the authors in concluding: "It would seem, therefore, as if a substance exerting a marked influence upon growth were present in butter, and that this was largely, if not wholly, removed in the preparation of our natural 'protein-free milk.' Whether or not the latter is wholly deficient in this substance can not be determined as yet from any data we possess. It is true that young rats are able to make very considerable growth when fed on the natural 'protein-free milk' diet, but possibly this is accomplished at the expense of some reserve substance stored in the cells of the young animal." The ability of the animal body to store the fat-soluble vitamin, thus early suggested by Osborne and Mendel, has proven to be a matter of much importance.

Further study by Osborne and Mendel on the influence of butterfat on growth, reported later in the same year (1913a) showed that the active ingredient was contained in the clear fat fraction of butter, was essentially free from nitrogen and phosphorus and devoid of any ashyielding material, and evidently not destroyed when steam was passed through the melted butterfat. In discussing the nutritive superiority of butterfat over lard, the authors note at this time that failure at certain periods of the year, particularly in the summer months, to secure satisfactory growth on dietaries which had proved adequate at other times could be averted by the addition of butterfat to the usual protein-free milk food mixtures and further that a "type of nutritive deficiency exemplified in a form of infectious eye disease prevalent in animals inappropriately fed is speedily alleviated by the introduction of butterfat into the experimental ration." This characteristic eye condition (Fig. 8) 


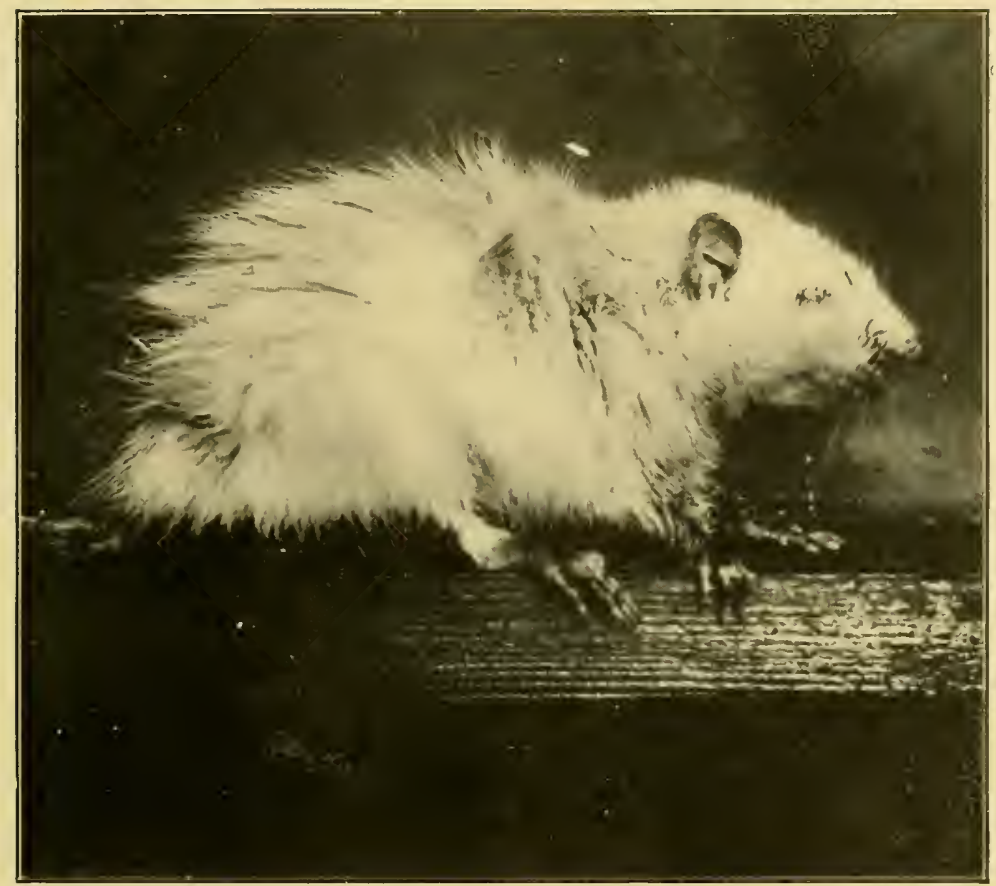

FIG. 8.- Photograph of rat showing effects of diet lacking vitamin A. In this case the effects are apparent in the eye, ear, nose, feet and coat. See accompanying text and discussion later in this chapter. (Courtesy of Doctors Osborne and Mendel.) 

will be discussed more fully later. Further work of Osborne and Mendel on this fat-soluble, growth-promoting substance (vitamin A) showed it to be present in cod-liver oil, but absent in freshly pressed almond oil (1914), and to be present in small amounts in beef fat (1915a). When butterfat and beef fat were subjected to fractional crystallization from alcohol, the active substance was found to be concentrated in the mother liquor or oil fractions rather than in the fractions containing fats with high melting points. Heating butter with live steam two and one-half hours did not destroy its potency in this connection, nor did storage under ordinary conditions (1916). The butter oil, in which the growth-promoting substance is more concentrated than in the original fat, showed gradual deterioration, losing most of its potency in a year.

Meantime McCollum and Davis (1914) had shown that the growthpromoting substance in butter was sufficiently stable to withstand the saponification of the butterfat in alcoholic potassium hydroxide and that it could be shaken out from the saponified material by olive oil. In an attempt to determine whether the substance was carried by vegetable materials as well as animal fats (1915) rats which had been brought to a state of emaciation on a fat-free diet of casein, milk sugar, dextrin, agar, and salts were brought back to a normal condition by the substitution of corn meal for 50 per cent of the fat-free diet. Similar results were obtained with wheat embryo (1915a), but less favorable with whole wheat flour, rye flour, and oats. Reproduction was not secured in any of these experiments. In the following year McCollum, Simmonds, and Pitz (1916b) published results which indicated that oils such as maize, cottonseed, linseed, olive, sunflower seed, and soy bean oils in amounts up to from 10 to 20 per cent of the ration do not furnish fat-soluble vitamin in appreciable amounts. On the other hand, alfalfa and cabbage leaves were found to be excellent sources of the vitamin. The cereal grains, while containing a small amount, were markedly inferior to the alfalfa leaves. The authors concluded at that time that "the superiority of the forage portion of the plant over the seed with respect to its content of the fat-soluble $\mathrm{A}$ is of considerable interest when viewed in the light of the dietary habits of lower animals. Those which consume the forage rations grow successfully from generation to generation on a strictly vegetarian diet, while the seed-eating animals, so far as we have been able to learn, normally vary their diet to a considerable degree by addition of green leaves, worms, insects, etc." They further concluded from the observation that the ether-extracted residue of corn meal is more effective in causing growth than is corn oil, 
that "ether extraction of plant tissue does not remove the substance essential for growth which is contained in butterfat. The obvious working hypothesis must for the present assume that the fat-soluble $\mathrm{A}$ is in chemical union in the plant tissues, and in a complex which is not soluble in fat or in ether. In digestion and absorption it is set free and being readily soluble in fats, thereafter accompanies the fats in the animal body."

That neither vitamin A nor B could be synthesized by the animal was indicated by the failure of rats on diets deficient in one or the other of these vitamins successfully to suckle their young except through a sacrifice of their own tissue and then to only a limited extent (McCollum, Simmonds and Pitz, 1916c).

The foregoing sketch attempts to review the most noteworthy features of the literature of vitamin $\mathrm{A}$ in chronological sequence to the end of 1917. Later investigations have in many cases proceeded along such distinct lines that it seems best to consider them under separate phases rather than to continue further the chronological development of the whole subject.

\section{Physical and Chemical Properties of Vitamin A and Progress toward its Isolation}

Solubility and Extraction.-As noted above, an apparent difference between the solubility of vitamin $A$ as it occurs in animal fats and in plant tissues was early reported. The data, however, do not seem entirely concordant.

We have seen that the substance was discovered through its presence in butterfat, egg-yolk fat, and cod-liver oil. In all these (and doubtless many similar) cases, the vitamin A dissolves readily in ether along with the accompanying fat. Osborne and Mendel (1919, 1919d) obtained vegetable fats which appeared to be rich in vitamin $A$ when they extracted, with U.S.P. ether, spinach leaf or young clover, previously dried in a current of air at $60^{\circ} \mathrm{C}$. This ether extract was a dark green, greasy or oily mixture evidently containing the fat of the plant tissue and such other material as was soluble in the ether used as solvent, or in the mixture of ether and fat which must have been present so long as fat was still being extracted from the dried tissue by the ether. It is well known, but not always remembered, that an ether extract may contain substances which are not appreciably soluble in pure ether but are dissolved by the mixture of fat and ether which results when a plant or animal tissue containing fat is subjected to ether extraction. This source of possible confusion should be kept clearly in mind when dealing with 
observations which apparently yield discordant data as to whether vitamin $\mathrm{A}$ is soluble in ether, and as to whether it occurs in nature in different forms having different solubilities. As yet, however, there seem to be differences among the recorded observations which are only in part explainable on this basis alone. Thus the observations of Osborne and Mendel just mentioned would seem to call for a revision or reinterpretation of the extraction experiments of McCollum, Simmonds and Pitz (1916b) mentioned above, and are also difficult of coördination with observations subsequently recorded by Steenbock and Boutwell (1920b) who attempted to concentrate the fat-soluble vitamin* from carrots, alfalfa, and yellow corn by fat solvents with the following results:

The fat-soluble vitamin in carrots was not removed to any extent by saturation with corn oil and extraction with ether, and apparently not at all by similar saturation with lard. The vitamin appeared to be slightly soluble in ether, somewhat soluble in chloroform and carbon disulfide, and quite soluble in alcohol and benzene. With alfalfa, more satisfactory results were obtained with ether, benzene, and alcohol, sufficient fat-soluble vitamin being obtained in the ether extract to permit satisfactory growth when fed to rats at a level corresponding to 20 per cent of alfalfa in the food mixture. These authors also employed with the alcohol extract from alfalfa meal the method commonly used for the separation of carotinoids. The extract was saponified with alcoholic potassium hydroxide and the mixture extracted repeatedly with ether until the yellow pigments were completely removed. The ether extracts, after washing with water to remove alkali and salts, were evaporated on a water bath to a small volume. This extract proved to be rich in fat-soluble vitamin. A further concentration of the vitamin was brought about by taking up a similar extract in a mixture of alcohol and petroleum ether and subjecting it to fractional extraction. The petroleum-ether-soluble carotene fraction was found to contain an abundance of the vitamin, while the alcohol-soluble xanthophyll fraction contained little or none of it.

About the same time Zilva (1920) reported experiments in which fractions active in vitamin $A$ were obtained from fresh vegetables (carrots and cabbage) by extraction with alcohol. Absolute alcohol extracted in the form of a sirup about 7.5 per cent of dry matter from

* Inasmuch as Steenbock found suhsequently that under the conditions of testing used in his laboratory there was danger of confusion between vitamins $A$ and $D$, it is conceivable that vitamin D may have been the growth-limiting factor in the experiments of Steenbock and Boutwell, whereas Osborne and Mendel were dealing with vitamin A. Investigations upon the quantitative differentiation of vitamins $A$ and $D$ are discussed further on in this chapter and in the chapter which follows. 
fresh carrots or about one half of the total solids. This sirupy extract given daily in amounts equivalent to from 10 to 12 grams of fresh carrots provided sufficient vitamin A to promote normal growth in rats subsisting on a diet otherwise lacking this vitamin. On extracting with ether the concentrated extract obtained by evaporation of the alcohol, an oily residue remained which, in amounts equivalent to 25 grams of fresh carrots, promoted recovery and renewed growth in rats declining on a basal diet lacking in vitamin $\mathrm{A}$.

Thus, the evidence indicates that vitamin $A$ may be removed from either animal or vegetable material by extraction with alcohol. This has led to the custom of subjecting materials to be used in a basal ration free from vitamin A to prolonged extraction with alcohol or alcohol and ether. Drummond and Coward (1920) have emphasized the necessity of such extraction, stating that in their opinion "it is frequently the composition of the basal dietary which is responsible for the many misleading and contradictory statements which tend to confuse the literature on the vitamins at the present time."

The present writers concur fully in the view that careful extraction of possible traces of vitamin A (preferably by hot strong alcohol) should be a universal precaution in the preparation of experimental rations which are designed to be devoid of this vitamin; but believe that the "misleading and contradictory statements which tend to confuse the literature" are due largely to the further factor of storage of vitamin A in the body of the experimental animal, so that whether the vitamin causing the confusion came from the food or the animal's body has not always been clear. We now use experimental animals which have been carefully depleted of any bodily surplus of vitamin A, and to ensure freedom of food materials from this vitamin we extract them repeatedly with hot strong alcohol in the manner recommended by Osborne and Mendel (1921) and described in full in connection with methods for the quantitative determination of vitamin $\mathrm{A}$ further on in this chapter.

Stability to Heat and Oxidation.--Similar confusion has existed in regard to the stability of vitamin $\mathrm{A}$. In their early work McCollum and Davis reported that the factor was present in the ether extract of boiled eggs and Osborne and Mendel (1915a) found that butterfat treated with steam for two and one half hours did not appear to have lost its growthpromoting properties. The fat-soluble vitamin was generally considered a thermostable substance and in experimental work on its occurrence in foods but little thought was given to the possibility that the manipulation involved, such as shaking of the butterfat with water, or subjecting materials to prolonged drying with artificial heat, might destroy their 
activity. In 1918, however, Stcenbock, Boutwell, and Kent, in the first of a series of studies on the fat-soluble vitamins, reported that vitamin A was comparatively labile to heat. Melted butterfat shaken with water for 12 hours became inactive as did butter aerated for 12 hours at $100^{\circ} \mathrm{C}$. Against the possibility that the destruction was due to oxidation, they cited similar results obtained when butter was shaken with carbonated water. Butter heated in jars at $100^{\circ} \mathrm{C}$. in a Freas oven also lost its activity as did butter kept unsalted under poor storage conditions for three weeks. The authors concluded that "the failure of other investigators to note this destructive action was undoubtedly due to the high initial content of fat-soluble vitamin in the material studied. The destructive process is evidently a reaction of low velocity. With large amounts of vitamin present and with heat treatment for a limited period of time sufficient amounts of the vitamin remained to satisfy all the requirements for normal growth in the experimental animals."

Shortly afterward, Drummond (1919a), in an investigation of the effects of hydrogenation on the vitamin content of oils, found that vitamin $\mathrm{A}$ was destroyed in experiments with whale oil, both during the hardening process involving exposure to hydrogen gas at $250^{\circ} \mathrm{C}$. for four hours or more, and also upon heating the oil to $100^{\circ} \mathrm{C}$. or more for four hours. These results were thought to demonstrate beyond any doubt that the fat-soluble vitamin $\mathrm{A}$ in the form in which it occurs in natural animal fats is much less stable to high temperatures than had previously been assumed. "Such an observation necessitated a complete revision of all the previous experiments to isolate and identify the factor in many of which high temperatures had been employed." In a series of experiments reported at the time on the effect of heat on the vitamin present in certain oils, the results obtained supported the conclusions of Steenbock that the vitamin was comparatively easily destroyed by heat. The conclusion drawn at the time was that "the chief agent in the inactivation of fat-soluble $\mathrm{A}$ is temperature. It must, however, be borne in mind that all these speculations refer solely to the factor as present in secondary sources such as the animal oils. It is quite possible that the factor occurs in a different form in its primary environment in the plant tissues."

That vitamin A as it exists in animal fats is stable to heat under certain conditions was shown by Osborne and Mendel (1920c) who published a confirmation of their earlier work indicating that the vitamin present in butter is notably stable at relatively high temperatures. Butterfat treated with steam and also heated at $96^{\circ} \mathrm{C}$. for 15 hours retained its growth-promoting activity. The explanation of these con- 
flicting results came in papers published simultaneously by Hopkins (1920a), by Drummond and Coward (1920b), and by Zilva (1920a), all of which presented evidence that the destruction of vitamin A takes place rapidly at high temperatures when oxygen or an oxidizing agent is present, but not otherwise.

The method adopted by Hopkins was to feed young rats of the same age, weight, and sex, a ration free from vitamin $A$ to which was added 15 per cent of filtered butter. The butter fed to half of the animals in each group had been heated in the autoclave for different lengths of time, while that fed to the other half had undergone heating at the same temperature but with aeration. In all of these experiments growth was normal on the unaerated fat; but on the aerated fat growth ceased and in 60 per cent of the cases xerophthalmia developed. As judged from the growth curves, 4 hours' exposure to a temperature of $120^{\circ} \mathrm{C}$. in the absence of air did not appreciably affect the vitamin content of butter, at least when fed at 15 per cent of the food intake; 12 hours' exposure under the same conditions involved slight destruction, aeration at $120^{\circ} \mathrm{C}$. for 4 hours destroyed the greater part, and for 12 hours practically all of the vitamin; aeration at $80^{\circ} \mathrm{C}$. brought about quite rapid destruction; and exposure to air at ordinary temperatures for a week, almost complete destruction. Of significance in this report was the fact that identical figures were obtained for the iodine value of the fatty acids of the butter before and after heating for 4 hours in a stream of air at $120^{\circ} \mathrm{C}$.

The plan adopted by Drummond and Coward (1920b) differed from that of Hopkins in that the butterfat was fed in small amounts (0.2 gram) as a supplement to the daily vitamin A-free basal ration to rats whose growth had been completely inhibited by this ration. The temperatures and times of aeration were: exposure to a current of live steam for 6 hours, and heating at $96^{\circ} \mathrm{C}$. for 15 hours, at $50^{\circ} \mathrm{C}$. for 6 hours, and at $37^{\circ} \mathrm{C}$. for 3 weeks with and without exposure to air. The results of these studies also indicated that destruction of vitamin $\mathrm{A}$ takes place rapidly at high temperatures and to a considerable extent at temperatures as low as $37^{\circ} \mathrm{C}$. in the presence but not in the absence of air.

The confirmatory work of Zilva (1920a) published at the same time resulted from an earlier observation that the vitamin $A$ value of butterfat is lost or lowered by exposure to ultra-violet rays (Zilva, 1919). In a further study to determine whether this was due to the action of the rays or to ozone produced by the mercury quartz lamp, cod-liver oil was exposed in thin layers to the action of ultra-violet 
light in an atmosphere of carbon dioxide gas for 16 hours, at the end of which time it was found to have lost none of its growth-promoting activity. On exposure for from 6 to 10 hours to a current of ozone in a bottle impervious to light, it became so inactivated that large doses failed to promote growth in rats on a diet otherwise deficient in vitamin $\mathrm{A}$, showing that ozone destroys or inactivates the vitamin $\mathrm{A}$, a finding in complete agreement with the observations noted above, the action of ozone being more drastic and therefore more rapid than aeration.

Accepting these findings as conclusive, the discrepancies in earlier conclusions concerning the stability of vitamin A are readily understood. In cases where stability was reported as in the exposure of butterfat to live steam, the conditions of the experiment gave an approximately airfree medium, while in such experiments as the removal of vitamin A from butterfat by repeated shaking with hot water, in the presence of air, the destructive action was probably one of oxidation. It should be noted, however, that in all the experimental work reviewed above, animal fats, sometimes referred to as the secondary source of vitamin $A$, have been used.

Steenbock and Boutwell (1920a) determined the stability of the fat-soluble vitamin in plant materials by means of feeding experiments in which the vitamin-containing material was dried at room temperature, soaked in water, autoclaved for 3 hours at approximately 15 pounds pressure, and again air-dried before being incorporated into the ration. This treatment appeared to cause no noticeable destruction of the fatsoluble vitamin of yellow maize, chard, carrots, sweet potatoes, and Hubbard squash. The results, in growth of rats, obtained with dried alfalfa were not so favorable, although this was fed at a level at least twice as high as is usually necessary for normal growth. The rats on this diet after a normal growth for 3 or 4 weeks declined and died, some showing signs of xerophthalmia. While at this time the effect of heat rather than possible destruction by oxidation through exposure to air in the drying process was the one chiefly considered, the authors called attention to the fact that the alfalfa meal used was a commercial product, the history of which was not known, and that possibly while the fatsoluble vitamin was not destroyed by the heat treatment itself, it was made more susceptible to destruction-possibly by liberation from combinations-by the agents operative in the aging process.

That vitamin $\mathrm{A}$ as it exists in plant tissues is not in a form to be as easily acted upon by oxidation as it is in animal fats is further indicated from the ability to prepare highly potent materials by extraction of airdried vegetables as illustrated by work of Osborne and Mendel (1920c), 
Alfalfa, clover, timothy, and spinach, freshly dried at $60^{\circ}$ C. apparently furnished, in amounts of 0.1 gram of the dried substance, as much vitamin $A$ as did 0.1 gram of butterfat; while with tomatoes more rapid growth resulted from the addition of 0.1 gram of the dried material than of the same amount of butterfat. In this case, however, the rapid growth is attributed partly to the richness of the tomato in vitamins $\mathrm{B}$ and $\mathrm{C}$.

Experiments were later made by Sherman, Quinn, Day and Miller (1928) to determine the relative stability of vitamin A from plant and animal sources when heated under like conditions, and also to ascertain the influence of such conditions as the nature of the solvent and the hydrogen-ion activity $(\mathrm{pH})$ of the medium upon the rate of destruction or inactivation.

In these experiments, albino rats were placed on a vitamin A-free diet (described in the section on quantitative determination beyond) at 28 days of age and the feeding of this diet continued until the surplus vitamin A stored in the animal's body was depleted, as evidenced by cessation of growth and by the incipient appearance of other symptoms characteristic of vitamin A deficiency. Graded portions of the test foods whose relative vitamin $A$ values were to be compared were then fed as supplements to the vitamin A-free diet. All of the feeding experiments were made in pairs upon litter mates of essentially the same weight, one being fed one material, the other the same material but differently treated or a different material similarly treated, as the case might be. Those portions of the two samples which, when fed daily during an 8-week experimental period, gave the same small but definite gain in weight, i.e., those portions which contained the same amount of vitamin A, were thus determined ; and from these values the amount of destruction of the vitamin due to any particular treatment was calculated.

First, a study was made of the stability of vitamin $\mathrm{A}$ in a plant material (filtered tomato juice) when heated for 4 hours at $100^{\circ} \mathrm{C}$. under anaerobic or aerobic conditions.

For the study of the stability of the vitamin in the absence of oxygen the juice was placed in a 1-liter flask provided with a rubber stopper through which passed a reflux condenser with its open end protected from the air by a water seal, and nitrogen, freed from oxygen by passing through alkaline pyrogallol and then over 12 inches of red-hot copper gauze, was bubbled through the liquid for 2 hours. At the end of this 2-hour period, half of the juice was withdrawn through a properly arranged delivery tube to an evacuated bottle and the remainder heated on a steam bath for 4 hours at $97^{\circ} \mathrm{C}$. $\pm 2^{\circ}$. Nitrogen was permitted to 
bubble through the liquid during the heating process at the rate of about one bubble per second. At the end of 4 hours the flask was cooled with running water and the tomato juice then removed to an evacuated bottle. Both the unheated and heated samples were stored in a refrigerator. When portions were removed for the feeding tests the amount withdrawn was displaced by nitrogen.

With the samples obtained under rigorous precautions for excluding atmospheric oxygen, it was found that 7.0 cubic centimeters of heated juice daily were required to produce the same gain in weight as 5.8 cubic centimeters of unheated juice daily. Thus, with the greatest care taken to exclude oxygen from the system in which the vitamin was heated, about 17 per cent of the vitanin A was destroyed in 4 hours heating at $97^{\circ} \mathrm{C} . \pm 2^{\circ}$, at the natural acidity of the tomato juice; viz., about $\mathrm{pH} 4.2$.

To determine the effect of heating the vitamin in the presence of oxygen, experiments were undertaken in which the tomato juice was heated on a steam bath under a reflux condenser for 4 hours at $97^{\circ} \mathrm{C}$. $\pm 2^{\circ}$ with air aspirated through the sample at the rate of about one bubble per second throughout the heating. The animals receiving 7.0 cubic centimeters of this heated juice daily made approximately the same growth as the animals receiving the 5.8 cubic centimeters of the unheated juice daily. Thus no measurable difference in destruction of vitamin A was found between the experiments in which the vitamin was heated in the strict absence of oxygen and those in which oxygen was present abundantly throughout the period of heating.

Evidently vitamin $\mathrm{A}$ in the form in which it exists in this primary (vegetable) source is less susceptible to oxidation than had generally been supposed in recent years in view of the experiments upon heating and aerating animal fats, as outlined above.

As it had been found that vitamin B in tomato juice is more rapidly destroyed if the acidity of the medium is decreased or if the juice is made faintly alkaline, experiments were made to determine whether a similar change of hydrogen-ion activity $(\mathrm{pH})$ would increase the destruction of vitamin $\mathrm{A}$ as it exists in tomato juice.

The portion to be subjected to the heat treatment under changed hydrogen-ion activity was brought to the desired $\mathrm{pH}$ of 9.2 . The juice thus prepared was heated in parallel with juice of natural acidity (approximately $\mathrm{pH} 4.2$ ) on a steam bath for 4 hours at $98^{\circ} \mathrm{C} . \pm 2^{\circ}$ under an atmosphere of nitrogen as already described. The juice in the two flasks was then quickly cooled and, to the sample whose $\mathrm{pH}$ had been changed, hydrochloric acid was added in amount just sufficient to 
neutralize the sodium hydroxide that had previously been introduced. Two cubic centimeters daily of the juice heated at $\mathrm{pH} 4.2$ were fed to rats and a comparison of the growth resulting was made with the growth of litter mates receiving an exactly equivalent amount (correction being made for increase in volume due to acid and alkali added in adjusting the $\mathrm{pH}$ ) of the juice heated at $\mathrm{pH}$ 9.2. The total average weight gain for the 8-week period was 47 grams for test animals fed the juice which had been heated at $\mathrm{pH} 4.2$ and 45 grams for those receiving the juice which had been heated at $\mathrm{pH}$ 9.2. Thus the change of hydrogen-ion activity from $\mathrm{pH} 4.2$ to $\mathrm{pH} 9.2$ did not appreciably influence the rate of destruction of vitamin $\mathrm{A}$.

Since results of the first series of experiments had shown vitamin A in a plant material to be relatively stable, it seemed desirable to compare the stability of the vitamin from animal and plant sources when heated under conditions as nearly identical as possible, including the use of the same solvent. It was found that an extract fairly rich in vitamin A could be prepared by shaking dry powdered spinach leaves with olive oil. Since a solution of butterfat in olive oil could readily be obtained, these two olive oil preparations were used as materials for the study.

These olive oil solutions containing the vitamin A of spinach leaves and of butterfat, respectively, were heated for 4 hours at $97^{\circ} \mathrm{C} . \pm 2^{\circ}$ under anaerobic conditions, essentially as described above. Frequently repeated quantitative determinations of the vitamin $\mathrm{A}$ values by the feeding method already described, showed that of the vitamin A of the spinach about 20 per cent, and of that of the butter about 33 per cent, were destroyed during the 4 hours heating.

Cady and Luck (1930) have also noted greater stability of the vitamin $\mathrm{A}$ in plant than in animal materials. On bubbling purified sulfur dioxide through cod-liver oil at temperatures of from $20^{\circ}$ to $100^{\circ} \mathrm{C}$. for periods of 15 minutes to 2 hours, the vitamin A activity was completely destroyed, while similar treatment of a concentrated alcoholic extract of alfalfa was without appreciable effect. Butter occupied an intermediate position, the destruction of vitamin A being reported very slight. In a further study of the stability of vitamin A in cod-liver oil toward various reagents, complete destruction was demonstrated with phosphorus pentachloride, chlorine, acetyl chloride, nitrous acid fumes, Benedict's alkaline copper reagent, and after prolonged treatment with sodium bisulfite. Hydrogen sulfide, ethylene, ammonia, and Benedict's reagent after neutralization had no, and formaldehyde very slight, destructive effect, while hydrogen peroxide brought about a partial loss of vitamin A activity. Attempts to regenerate the vitamin A potency of 
oils that had been inactivated by treatment with sulfur dioxide and oxygen were unsuccessful.

Attempts to Isolate Vitamin A and Determine Its Chemical Nature. -It was early found that vitamin A belongs to the unsaponifiable fraction of natural fats rather than to the triglycerides themselves. The unsaponifiable matter consists chiefly of unsaturated monatomic alcohols of high molecular weight which are solid at ordinary temperatures and are therefore called "sterols." The best known members of the sterol group are cholesterol and phytosterol occurring in animal and vegetable fats respectively. Animal fats have served as the starting point in most of the attempts to isolate vitamin A so that references to its properties are often in terms which suggest comparison or contrast with cholesterol. In 1924, Drummond reported the first results of his studies in this field, which showed that vitamin A could be separated from cholesterol and could be distilled in superheated steam without losing its vitaminic activity. He succeeded in effecting a 1,000-fold concentration of the vitamin A of cod-liver oil. Description of his actual procedure is omitted here as the method in its more fully developed form is outlined below in discussing the work of Drummond, Channon and Coward (1925). The purified material which Drummond described in 1924 appeared to consist chiefly of an unsaturated sterol. Similar results were reported from Japan in the following year (Takahashi, Nakamiya, Kawakami and Kitasato, 1925). Takahashi et al. believed their preparation to consist essentially of vitamin $A$ in a practically isolated condition. This they described as showing the properties of a sterol and proposed to designate as "biosterol" or "biosterin."

A critical discussion of this work together with additional experimental evidence from their own investigations has been offered by Drummond, Channon and Coward (1925), who believe that the "biosterin" or "biosterol" of the Japanese investigators cannot be regarded as a pure substance nor as approximating closely to an actual isolation of vitamin A. Drummond, Channon and Coward here reported work of their own in which they effected essentially the same concentration of the active substance as did Takahashi et al., but with a resulting product which they describe as being still a mixture.

The methods and results of Drummond and his coworkers may be briefly outlined as follows: Cod-liver oil is poured slowly with vigorous stirring into a hot solution of potassium hydroxide in alcohol, the mixture boiled for about 30 minutes (the alkali being present in an excess of about 10 per cent over the amount theoretically necessary for saponification of the oil), then cooled, diluted with 10 volumes of water 
and the unsaponifiable matter extracted with ether. This was then treated with sodium ethylate for the removal of any remaining saponifiable matter, nitrogen being used to displace air during this process and prevent loss of vitamin A by oxidation. Complete removal of fatty acid esters of cholesterol as well as of glycerol is important and was always assured before proceeding to the next step, which was the extraction of the unsaponifiable substances by means of ether and their recovery, on evaporation of the solvent, as "a semi-solid crystalline mass with a waxy appearance and varying in color from pale yellow to deep orange according to the color of the original oil." The crystalline appearance was found to be due to cholesterol which constituted about one-half of the material at this point; but which was removed, mainly by freezing out from solution in methyl alcohol, and completely by subsequent precipitation with digitonin, an atmosphere of nitrogen being maintained throughout the process to prevent loss of vitamin A by oxidation. The cholesterol digitonide was removed by filtration, the filtrate evaporated at low temperature and pressure to remove the solvents, the residue dissolved in ether, and the small excess of digitonin washed out by repeatedly shaking the ethereal solution with water in an atmosphere of nitrogen. "Finally the ethereal solution is separated and dried with anhydrous sodium sulfate and the solvent removed at low pressure. The product thus obtained is a deep reddish orange oil possessing the characteristic smell of the original unsaponifiable fraction. If it is carefully prepared it shows a vitamin A value proportionate to the cod-liver oil from which it was prepared."

In a typical case, 10 liters of light-colored cod-liver oil yielded 30 grams of orange-colored product as just described; and this, when distilled in a current of super-heated steam and nitrogen, was separated from its resinous matter and yielded a distillate of 25 grams of a clear, pale yellow oil. On distillation at 3 millimeters pressure this oil yielded the following fractions:

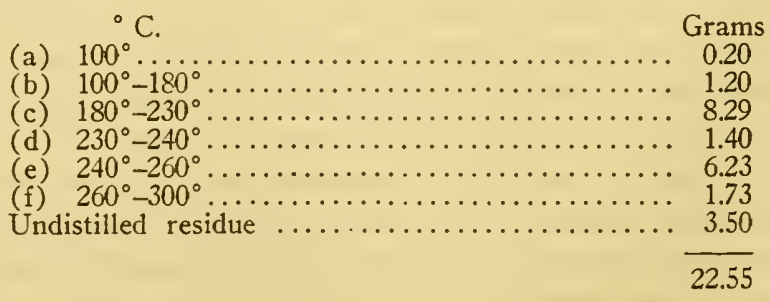

Fraction (a) was considered to consist of impurities from the ether previously used as solvent. Feeding experiments with fractions (b) to 
(f) "indicated that vitamin A passed over mainly between $180^{\circ}-220^{\circ}$ at 2-3 mm."

Even the most active distillate, however, could not be regarded as an isolated vitamin $\mathrm{A}$ for it appeared to be a mixture of at least three substances. "Chemical examination of active distillates indicated the presence of (a) a saturated solid alcohol (m.p. about $60^{\circ}$ ), (b) the unsaturated hydrocarbon spinacene, (c) one or more than one unsaturated alcohol, boiling about $200^{\circ}$ at $2-3 \mathrm{~mm}$." (Drummond, Channon and Coward, 1925.) Separations of these substances could not be effected either by re-distillation, crystallization from solvents, or attempts to fractionate through differential solubilities. In the judgment of these investigators, the final products obtained, both in their own attempts at isolation and in those of Takahashi and his coworkers, must still be regarded as mixtures.

The authors considered that this work conclusively proves that vitamin A can be distilled either with super-heated steam or directly under reduced pressure, and still retain its characteristic activity; but that it is not definitely established whether the vitamin activity is due to an unsaturated sterol or not. "No satisfactory answer to this question can be given until the active constituent has been isolated in pure condition or as a derivative suitable for purification. It was of interest to observe that, during the course of exhaustive experiments to obtain solid derivatives suitable for purification and examination, the vitamin activity of our fractions survived certain chemical treatments of the hydroxyl group of the alcohols present, e.g., acetylation, benzoylation, and treatment with phthalic anhydride or substituted benzoyl chlorides, but in no case did it survive exposure to reagents that affected the unsaturated linkages, e.g., bromination, reduction."

The observations just mentioned, taken in conjunction with the fact that the unsaturated hydrocarbon spinacene is without vitamin A action, plainly furnish another indication of the possibility that vitamin A may prove to be an unsaturated alcohol of the "sterol" group; but the authors concluded that, "It has not been possible to decide whether vitamin A is identifiable with one of the unsaturated alcohols. ... The unsaturated alcohols, oleyl alcohol $\left(\mathrm{C}_{18} \mathrm{H}_{36} \mathrm{O}\right)$ and selachyl alcohol $\left(\mathrm{C}_{20} \mathrm{H}_{40} \mathrm{O}_{3}\right)$, which have been described as constituents of other liver oils, are not identifiable with vitamin A. The unsaturated alcohol, phytol $\left(\mathrm{C}_{20} \mathrm{H}_{40} \mathrm{O}\right)$, which is present in the chlorophyll molecule, is also devoid of activity." (Drummond, Channon and Coward, 1925, p. 1066.)

Drummond and Baker (1929a) concluded from further studies of the chemical nature of vitamin $\mathrm{A}$ that the active substance is present in 
liver oil concentrates in amounts so minute that direct attempts at its isolation by ordinary chemical methods are of little use. They were unsuccessful in separating by fractional distillation at pressures of about 0.01-2 $\mathrm{mm}$. the vitamin $A$ present in the unsaponifiable matter of certain liver oils. The unsaponifiable fractions of cod-liver oil and sheepliver oil tend to decompose on distillation in high vacuum, and are not readily reduced by hydrogen in the presence of platinum or palladium catalysts. The unsaponifiable fraction of shark-liver oil can be distilled with little destruction of the vitamin A, owing, it is thought, to the small proportion, or absence, of the complex alcohols and hydrocarbons of the terpene series. From 90 to 95 per cent of the unsaponifiable fraction of these latter oils consists of selachyl, batyl, chimyl and oleyl alcohols, with small amounts of cholesterol and squalene. Probably less than 1 per cent of the non-saponifiable matter of shark oil with vitamin activity comparable to that of good cod-liver oil, is vitamin A.

Clenshaw and Smedley-MacLean (1929) worked extensively with plant materials (unsaponifiable matters of spinach leaves and green cabbage) and discussed carotene (carotin) and some other substances as possibly related to vitamin $\mathrm{A}$.

Color Reactions and Spectrographic Studies.-Drummond and Watson (1922a) suggested that the striking purple color of the sulfuric acid reaction of liver oils might be due to vitamin $\mathrm{A}$ or some related substance, since there seemed to be a parallelism between the intensity of the color reaction given by an oil and its vitamin $\mathrm{A}$ value.

Later Rosenheim and Drummond (1925) developed the same idea more fully in connection with the arsenic trichloride reaction. When this reagent is mixed with a liver oil a color series follows, which is first blue, then turns purplish, and gradually fades. This reaction was found to be about 20 -fold more sensitive than the sulfuric acid test, and was given with progressively greater intensity (or at progressively greater dilution) by all materials tested at the time, in which a concentration of vitamin $A$ had been effected. The highly potent fraction obtained upon the removal of cholesterol from the unsaponifiable matter of cod-liver oil was found to give this reaction at a dilution of $1: 2,000,000$. The chromogenic factor also responded as does vitamin $\mathrm{A}$ to steam distillation and oxidation, which adds weight to the suggestion of intimate relationship.

Over 30 oils and fats were tested as to growth-promoting property and reaction with arsenic trichloride, with results that showed a general parallelism. Furthermore, irradiated cholesterol, although strongly antirachitic, did not show the arsenic chloride test. 
Fearon (1925) proposed the substitution of phosphorus pentoxide for sulfuric acid, and discussed at length the results yielded by these tests. He also developed a color test using pyrogallol and trichloroacetic acid, which he believed to be due to the same substance as the former tests.

Willimott and Moore (1926) found that Fearon's pyrogallol test is not specific for vitamin A, however. A sample of oil which had been exposed to ultra-violet light gave a positive Fearon test after $31 / 2$ hours, but failed to respond to the arsenic chloride test after only $11 / 2$ hours exposure. A sample of oil shaken with concentrated sulfuric acid and then washed with water had no vitamin A potency when fed to rats, but gave a positive test with pyrogallol.

Further Rosenheim and Webster (1926b) stated "Since we found ... that Fearon's color reaction is not given by the unsaponifiable fraction of cod-liver oil, it is evident that this reaction has no relation to vitamin A."

Carr and Price (1926) investigated a number of condensing agents, and found antimony trichloride to be the most suitable and convenient of all the reagents tested for use in color tests of vitamin A. The conditions which they adopted for its use are essentially as follows: Antimony trichloride washed with chloroform and dried is dissolved in chloroform to make a 30 per cent solution (weight in volume). After standing, the clear solution is decanted and used from a burette. The oil to be tested is dissolved in chloroform (20 per cent solution) and to 0.2 cubic centimeter delivered from a 1 cubic centimeter burette, 2 cubic centimeters of the antimony trichloride solution is added. The liquid is at once transferred to a cell and the blue color intensity measured against standard glasses in a Lovibond tintometer.

Willimott and Moore (1926) also recommended antimony chloride as more suitable than arsenic chloride, as it gives colors which persist longer. Either test, however, appeared to be more sensitive than the sulfuric acid or phosphorus pentoxide test.

The literature on color tests for vitamin A was reviewed briefly by Willimott and Wokes (1927b) and a summary was given of the results obtained in attempts to apply several of the tests to cod-liver oil, foodstuffs, and various natural products from which interfering pigments had been removed by shaking the material in petroleum ether solution with an adsorbent charcoal, preferably norite. Tables were given of the results obtained with the concentrated sulfuric acid, phosphorus pentoxide, arsenic trichloride, and antimony trichloride color tests, together with an estimation of the activity of the materials tested by rat feeding 
experiments. Of the color reagents, arsenic trichloride and antimony trichloride proved most satisfactory. It was found that vitamin D apparently does not interfere with the test nor give a color reaction. Olive oil and oleic acid interfered with the test, and their use as diluents of cod-liver oil in the color tests is discouraged. Natural pigments in cod-liver oil could not be removed completely by treatment with the charcoal. Decolorized orange rind oil gave a strongly positive test, as well as showing marked activity in animal feeding tests. Another very potent material, as judged by both color and feeding tests, was decolorized spinach extract. Positive results were also obtained with decolorized extracts of carrots and yellow corn.

These authors concluded that the successful application of color tests for vitamin A to pharmaceutical materials and foodstuffs requires the careful observation of a number of precautions, but urged that the tests be tried on as many natural products as possible in order to establish definitely the specificity of the tests.

As had been noted by Takahashi et al. (1925), Morton and Heilbron $(1928,1928 \mathrm{a})$ reported that oils or concentrates rich in vitamin A exhibit a well marked absorption band with a maximum at about $328 \mu \mu$; and these latter workers found a parallelism between its intensity and vitamin activity. Although it has been shown by Bourdillon and coworkers (1929) that marked absorption in this region is also characteristic of dehydroergosterol, a substance unable to serve as vitamin A for the growing rat or to produce the blue color reaction with antimony trichloride. Morton, Heilbron and Spring (1930) found that the band of dehydroergosterol is more complex and much less intense than that for vitamin $\mathrm{A}$, and states that in order to demonstrate from a study of an inactive substance that the band at $328 \mu \mu$ is not due to vitamin $\mathrm{A}$, it is necessary to show that such a substance as dehydroergosterol accounts quantitatively for the absorption shown by vitamin A-rich material.

Drummond and Morton (1929) reported good agreement for a number of oils of marine origin in an extensive comparison of vitamin A determinations by spectroscopic, colorimetric (Carr-Price modification of Rosenheim-Drummond test) and feeding methods.

But within the last two years very contradictory evidence has accumulated with reference to the specificity of the Rosenheim-Drummond test. Drummond and Morton (1929) believe that for cod-liver oil at least, the color test and the relative intensities of the absorption band $(328 \mu \mu)$ are more accurate measures of vitamin $A$ than the biological tests. Norris and Danielson (1929a) point out that the intensity of color 
is approximately proportional to the vitamin A values only when the oil is used at very low concentrations, while Towle and Merrill (1928) indicate that the working temperature influences the rate of the development of the blue color and its change to red. At $10^{\circ} \mathrm{C}$. or below the rate of change is about one-twentieth of that at $25^{\circ} \mathrm{C}$. Jones, Briod, Arzoomanian and Christiansen (1929) reported that five samples of cod-liver oil out of 13 responded to colorimetric assay within 15 per cent of the value determined by the biological method, while of the remaining 8,6 exceeded the biological assays by 20 to 600 per cent. Drummond and Morton (1929) attached little significance to these latter results as they were not assured that sufficient vitamin D was furnished to the test animals during the period in which they were depleted of their vitamin A reserves. This should tend to render the biological values too high rather than too low, however. They dismissed the adverse criticisms of Steudel $(1927,1929$ a) regarding the specificity of the blue color reaction for vitamin $\mathrm{A}$ on the grounds that the antimony-chloride test is not sensitive enough to be employed with preparations as poor in vitamin A as was Steudel's product. In investigating relatively poor source materials they state that it is necessary to concentrate the chromogenic substance in a non-saponifiable fraction before applying the color test. Hawk (1929) found that samples of oil exposed to the atmosphere gave a deeper blue color with antimony chloride than did those kept in the dark to preserve their vitamin potency. These results are not concordant with those of Drummond and Morton, who have "not encountered a single instance in which there has been disagreement between the animal tests and the intensity of the blue colour showing maximum absorption near $608 \mu \mu$."

Similarity in color of the reaction products of antimony trichloride with carotene and with liver oil or other vitamin A concentrates of animal origin proved a stumbling block in the acceptance of the color test as specific for vitamin A. Dulière, Morton, and Drummond (1929) found, however, that the absorption band of the blue reaction product with carotene was at $590 \mu \mu$, while that of the product obtained with liver oil was at approximately $610 \mu \mu$. Spectrographic analyses of the color products, as well as of the original materials, were thus considered essential in determining the specificity of the color test. In the opinion of Moore (1929a), "for the present it seems safe to assume that materials which give no blue coloration with antimony trichloride, even after the removal of saponifiable matter, must be devoid of vitamin A activity. Materials of liver oil origin giving color reactions characterized by absorption at $610 \mu \mu$ may be considered active. But, on the other hand, 
materials which give color reactions characterized by absorption at other positions may be either active or inactive, or mixtures of inactive and active chromogens, and the biological technic still remains the only satisfactory method of assay."

Recently Wokes has observed a similar range of absorption bands in the blue products obtained by treating with antimony trichloride certain cholesterol preparations which are devoid of vitamin A potency, and he considers it doubtful that the blue colors given by arsenic and antimony chloride with cod-liver oil and other sources of vitamin A are directly due to that vitamin. Even though spectroscopic measurements may distinguish between the colors shown by vitamin A and other chromogenic substances, the fact remains that lipochromes disturb the direct method of applying color tests to food products, such as butter, for the estimation of vitamin A.

Various modifications of early technique were reported by Bertram (1929), Evers (1929, 1929a), and Andersen and Nightingale (1929). The last-named investigators consider it essential to prepare the unsaponifiable fraction of the material to be tested and to dilute the chloroform solution of this fraction to such a point that the blue color with antimony trichloride is just perceptible. Under the conditions which they prescribe they state that "hundreds of tests have confirmed the accuracy of the method from a comparative viewpoint. In addition, many duplicate tests have been made by independent laboratories, and in all cases very reasonable agreement has been obtained. That it is reliable when compared with feeding tests on experimental animals, under the strictly defined conditions now adopted for such tests, has been confirmed by numerous biological tests. The vitamin potencies determined by it have been in satisfactory agreement with the results obtained in the biological tests conducted by the Pharmacological Laboratories of the Pharmaceutical Society and by other highly qualified observers." This is doubtless to be taken as referring to liver oils and not to miscellaneous food materials.

Norris and Church (1930), following the technique developed by Norris and Danielson (1929a), tested the unsaponifiable fractions of cod-liver oil and found that the blue color of the extracts was (within limits) a linear function of the percentage concentration, while this had not been true of the original oils. Unsaturated oils and oleic acid added to the extracts were found to accelerate the rate of fading of the blue color, but on dilution of the extract a point was reached at which there was no longer interference. This was thought to explain the findings of Norris and Danielson that at sufficiently low color values 
the curve for cod-liver oils appears to be a linear function. They concluded that "quantitative comparison of the color values between different oils or between colorimetric and feeding experiments can only be made at a sufficiently low value so that the dilution curve approaches a linear function, or be made on the unsaponifiable portion."

Continuing their study of the specificity of the antimony trichloride color reaction for vitamin A, Norris and Church (1930a) obtained dilution curves for five samples of good grade cod-liver oil and one of ratfish liver oil. The intensity of the blue color produced with the different oils was in no case a linear function of the concentration of the oil nor were the type of curve and the deviation from a linear function the same for the different oils. The original paper should be consulted by those concerned with such testing.

Attempts were made to extract the unsaponifiable substance completely from a saponified cod-liver oil. Chloroform solutions of the unsaponifiable substance representing various dilutions of the original oil when tested with the antimony trichloride reagent gave straight line dilution curves, but a complete extraction of the unsaponifiable matter with no losses was never obtained.

Probable Relationship of Vitamin A to Certain Plant Pigments.-As the list of materials containing fat-soluble vitamin increased, a certain analogy was noted and first pointed out by Steenbock (1919) between the simultaneous presence of the carotinoid pigment and fat-soluble vitamin in certain foods and their absence in others. Palmer had earlier shown $(1915,1916)$ that the plant carotinuids are the source of the so-called lipochromes of the higher animals by direct transfer of the pigments of the diet. Experimental work reported by Steenbock and Boutwell (1920) shortly after the preliminary statement of the hypothesis of a relationship between the fat-soluble vitamin and the yellow pigment carotene, showed that yellow corn furnished enough of the fat-soluble vitamin to allow growth at the normal rate to take place in the rat and to make possible reproduction but not rearing of the young; while the feeding of white corn under similar conditions resulted in nutritional failure. Red corn with a white endosperm gave the same results as white corn, while that with a yellow endosperm gave results approximately the same as those from yellow corn. Later papers by the same author and his coworkers furnished many data in harmony with the theory of a relationship between vitamin $\mathrm{A}$ and lipochrome pigment. As noted above, fractionation of the unsaponifiable matter from alfalfa hay brought down the fat-soluble vitamin in the carotene-rich fraction. 
The above theory at first received the support of Rosenheim and Drummond (1920). The failure of lipochromes to serve as sources of fat-soluble A as previously noted by Drummond (1919a) was considered to justify the conclusion that the lipochrome pigments and vitamin $\mathrm{A}$ are not identical but not to refute the theory of a close relationship between them. Later Drummond and Coward (1920a) subjected this theory to the test of comparing a large number of animal and vegetable oils as to their relative richness in vitamin $A$ and in lipochrome pigments. The lack of relationship between the pigmentation of the 24 fats examined and their richness in vitamin A led them to conclude that "unless we assume the existence of a leuco-form it does not appear probable that the fat-soluble vitamin is a member of the lipochrome class of pigments. The frequent association of the growth factor with pigments of that type must therefore be regarded as accidental." In this they agreed with the conclusions of Palmer and Kempster $(1919,1919 a, 1919 b)$, who reported success in raising a large number of White Leghorn fowls from hatching to maturity on rations which contained at the most mere traces of carotinoids. Hens on this ration laid eggs which were practically free from pigments and from which normal chickens were hatched. Furthermore Stephenson (1920) found that a crude (alcohol-light petroleum) extract of dried carrot when added to a fat lacking in vitanin A conferred upon it growthpromoting properties and protected rats from xerophthalmia while pure carotene extracted from carrots was without effect. Moreover, butter from which the coloring matter had been completely removed by filtration through charcoal did not lose its growth-promoting properties.

The earlier literature on this subject was subjected to an extensive and critical review by Palmer, Kennedy and Kempster (1921) who opposed the idea of identity or relationship between vitamin A and carotinoids. They not only reported a complete absence of carotinoid pigments in the albino rat, the experimental animal used in most of the biological studies on vitamin $\mathrm{A}$, but were able to report growth and reproduction of rats with ewe milk fat containing only 0.00014 per cent carotene, as the sole source of vitamin $\mathrm{A}$, the ration showing the best results containing only 0.0000126 per cent carotene. Growth and reproduction were also obtained with rats using carotinoid-free egg yolk as the sole source of vitamin A. Quantitative comparisons were made of the carotene content and vitamin A efficiency of various rations as reported in the literature, and the conclusion was drawn that carotene and vitamin $\mathrm{A}$ are not quantitatively associated in the plant tissues in which both were presumed to be synthesized. The existence 
of a leuco-form of the vitamin as suggested by Steenbock (1919) to cover the exceptions to the association of pigment and vitamin was thought by Palmer to be unlikely in view of the fact that the only leucoforms of carotinoids so far produced are oxidation products and that oxidation destroys the efficiency of the fat-soluble vitamin.

Later, however, Steenbock and his associates published further evidence of a tendency toward greater richness in vitamin A in association with higher carotene or lipochrome pigmentation. Thus Steenbock, Sell and Buell (1921) pointed out that "with the diversification of metabolic processes which obtain in the plant and animal kingdom, it was to be expected that sooner or later the fat-soluble vitamin would be found to be present in a menstruum entirely free from pigments of the carotinoid type. To run across such an instance, appears to have been the good fortune of Palmer and Kempster (1919) who demonstrated that pork liver, rich in the vitamin, contained no pigments of the aforementioned character." . . "Nevertheless, as far as studies in this domain have been pursued, both in regard to distribution of vitamin and pigment and in regard to their physical and chemical properties, there is left no doubt but that chemically and physiologically they are related." These authors then gave experimental results of their own showing high vitamin A value with very low pigmentation in cod-liver oil, and further noted that "The fat-soluble vitamin content of butterfat does not run closely parallel to the yellow pigment; yet in general, due to determination by their content in the feed, butters highly pigmented are rich in the vitamin; butters low in pigment should be looked upon with suspicion. In beef fats the relations are somewhat similar; those most pigmented are also generally richest in their fatsoluble vitamin content."

In the next paper of the series, Steenbock, Sell and Boutwell (1921) recorded feeding experiments with six varieties of peas showing that "those of a green color, also carrying considerable yellow pigment, were far richer in their fat-soluble vitamin content than yellow peas which contained much less yellow pigment."

In 1927 Willimott and Moore reported that vitamin A is not identical with purified xanthophyll.

Quinn, Burtis and Milner (1927) demonstrated the relationship of greenness to high vitamin $\mathrm{A}$ content in plant tissues other than leaves, string beans and green peppers being found of high vitamin A value. More recently, Crist and Dye have shown both with head and leaf lettuce (Dye, Medlock and Crist, 1927) and with bleached and green asparagus (Crist and Dye, 1929) a positive correlation between the 
degree of greenness in edible plant parts and their content of vita$\min \mathrm{A}$.

Quinn and Cook (1928) in quantitative studies of the vitamin A content of white and yellow yautia showed the latter to be over ten times as rich in vitamin $\mathrm{A}$ as the white variety.

Hauge and Trost $(1928,1930)$ reported a close physiological relation between vitamin $\mathrm{A}$ and the yellow endosperm kernel character in maize. In the process of crossing and segregating yellow and white dent maize they found vitamin $\mathrm{A}$ associated exclusively with the yellow endosperm, and not with the white, even in kernels of corn secured from the same ears. The genetical factors responsible for yellow pigmentation and vitamin A occurrence could not be separated as it was not possible to secure any demonstrable transfer of vitamin A to grains possessing pure white endosperm. This association was further confirmed when in hybrid red maize, vitamin A was found only in kernels possessing yellow endosperm. The color of the pericarp was found to be without effect in the formation of vitamin $\mathrm{A}$ in the kernel. It appears that the genetical factor responsible for carotinoid pigmentation is also responsible for vitamin A formation. In testing two types of corn, YYY and Yyy, possessing as wide a range of yellow endosperm character as possible, it was found that the vitamin A content parallels very closely the yellow endosperm character. "The critical level for Yyy corn is approximately 15 per cent while that of the YYY corn is about five per cent. Seven per cent of YYY corn gave good growth while it took 20 per cent of the Yyy corn to give comparable results. It is apparent that the YYY corn contains approximately three times as much vitamin $\mathrm{A}$ as the Yyy corn which is also the same ratio as the genetical character of the yellow endosperm. These studies show that the physiological relationship between yellow endosperm character and vitamin A content is very close in maize."

Pointing out that the early negative results of tests of the vitamin A activity of pigments had been obtained before the recognition of vitamin D, v. Euler and his colleagues $(1928,1929)$ reported that of the lipochromes yielding a blue color reaction with arsenic chloride, two -carotene and dihydro- $\alpha$-crocetin-promoted growth in vitamin-Adepleted rats. It is of special interest that the active dihydro- $\alpha$-crocetin had been prepared by reducing the biologically inactive $\alpha$-crocetin with titanous chloride. Moore (1929) also found carotene (m. p. $174^{\circ} \mathrm{C}$. in air) active and did not regard the active material as a mere contaminant. A carotene (m. p. 174 to $178^{\circ} \mathrm{C}$; ; iodine number 330 ) obtained by Collison, Hume, Smedley-MacLean and Smith (1929) from 
green cabbage leaves was potent in restoring growth in rats in doses of 0.003 milligram per day. The non-saponifiable material obtained from green cabbage leaves was more abundant, more unsaturated and many times richer in vitamin A than the similar product from white cabbage leaves.

Dulière, Morton and Drummond concluded in 1929: "We are inclined to accept as the most plausible explanation of the discrepancy between our results and those of the investigators who have obtained a growth-promoting action with carotin, that their preparations were contaminated with a trace of exceedingly potent growth-promoting factor which need not necessarily be different from the classical vitamin A. ... The activity of carotin decreases enormously with purification ... the growth-promoting action of liver oils is not due to carotin; the results further indicate that the classical vitamin $\mathrm{A}$ must be a colourless substance. Its intrinsic potency must be very much higher than that of any sample of carotin or dihydro- $\alpha$-crocetin for which data have so far been published. . . .

"Whilst in our view it is undesirable prematurely to assume the existence of a plurality of vitamins $A$, it must not be forgotten that the identity of the growth-promoting principle in vegetable matter with that present in liver oils has never been satisfactorily demonstrated. The investigation of growth promotion from the points of view of liver fats and vegetable extracts may well be parallel rather than converging lines of study."

The question of these divergent results being attributable to technique in the biological tests was discussed by several investigators. Moore and Hume had each noted that the investigators obtaining negative results used basal diets which contained no fat, whereas those obtaining positive results used basal diets containing fat. Upon investigating the matter Hume and Smedley-MacLean (1930) satisfied themselves, however, that the differences could not be attributed to the presence or absence of fat in the basal diet but more probably to the nature of the solvent in which the carotene is administered. Carotene appears fairly stable when dissolved in natural oils, but loses color rapidly when in contact with oleic acid or ethyl oleate, the solvent used by Drummond. Hume and Smedley-MacLean believed that this rapid oxidation of the highly unsaturated carotene in ethyl oleate was a plausible explanation for the negative results of Dulière, Morton and Drummond.

Drummond, Ahmad, and Morton (1930) later confirmed the conclusions of Hume and Smedley-MacLean concerning the destruction of 
carotene by ethyl oleate and the growth-promoting properties of pure carotene. They were, however, unable to confirm the claims of v. Euler that dihydro- $a$-crocetin shares with carotene the power to replace vita$\min \mathrm{A}$ in the diet.

Following the announcement of v. Euler et al. of the activity of carotene as a source of vitamin A, Moore (1929) confirmed their results and in a series of important studies obtained evidence which appears to throw light upon the differences between vitamin $A$ in plant and animal materials and to mark an important advance in the chemistry of this vitamin. In the preliminary note in which he first reported the confirmation of v. Euler's results, Moore (1929) called attention to the fact that the Japanese vitamin A concentrate biosterin was stated by Takahashi et al. (1925), confirmed by Morton and Heilbron (1928) to be characterized by marked selective absorption at $320 \mu \mu$, but no absorption in the visible spectrum. Carotene on the other hand exhibits selective absorption in the visible spectrum and no marked bands in the ultra-violet region. In spite of these differences, the minimum physiological dose for rats of each of the two materials appears to be about the same, in the neighborhood of 0.005 milligram daily. In endeavoring to reconcile these difficulties, Moore suggested two possibilities: "(1) Both carotin and the classical 'vitamin A' may independently possess the same physiological action or (2) physiological action may be due to an inactive impurity present in each in amounts so minute as not to affect the physical properties of the materials in bulk."

A few months later, however, Moore (1929b) reported preliminary experiments suggesting the possibility that carotene, while not identical with vitamin A, may be the precursor from which the vitamin is formed in vivo. These experiments consisted briefly in feeding rats depleted of their vitamin A reserves graded doses of carotene, from 0.0001 to 0.75 milligram daily, and after 36 days killing the rats and examining their liver fats for carotene and for vitamin A by colorimetric and spectrographic methods.

It was noted first that although the largest amount of carotene was sufficient to color the whole rat intensely yellow if absorbed unchanged, the body fat was found to be as colorless as that of the controls and the liver to show only feeble yellow pigmentation. On the other hand, an intense blue color was given with antimony trichloride and on spectrographic examination the absorption band was found to be at 610 to $630 \mu \mu$, characteristic of vitamin A, rather than $590 \mu \mu$ characteristic of carotene. A comparison of the blue and yellow units 
showed that in cases where the amount of carotene fed was below the minimal physiological dosage for vitamin $\mathrm{A}$, the blue units did not rise above those of negative controls, while, in the case of all three rats receiving carotene in excess of the minimal dose, higher values in the blue units were noted, with no corresponding changes in the yellow such as would have been the case if carotene had been stored unchanged.

In the detailed report of this important development concerning the relationship of carotene to vitamin A, Moore (1930) has cleared up many doubtful points raised by various investigators and has presented apparently convincing evidence that the carotene in plant materials is more or less completely converted into vitamin A in the animal body. Some features of the conversion are summarized by Moore as follows:

Carotene

Synthesized in plant

Intensely yellow

$328 \mu \mu$ absorption band absent

Greenish blue $\mathrm{SbCl}_{3}$ reaction at $590 \mu \mu$.
Vitamin $A$

Stored in animal

Almost colorless

$328 \mu \mu$ absorption band developed

Vivid blue $\mathrm{SbCl}_{3}$ reaction at 610 $6.30 \mu \mu$.

In this connection Moore remarks that "since carotene and the liver oil vitamin A both give positive results when tested biologically it is unavoidable that recourse to colorimetric data should be made in studying the conversion of one into the other." It is noted that the natural yellow color of carotene in chloroform solution compared with the blue color formed when it is dissolved in the same volume of the antimony trichloride reagent has a yellow to blue ratio of about 11 to 1 , while the faintly yellow color of cod-liver oil concentrates compared with the intensely blue color of its antimony trichloride reaction has a corresponding ratio of about 1 to 100 . Thus when one constituent is present in a given material in predominant amount, the yellow to blue ratio suggests at once whether this constituent is a carotinoid or the almost colorless vitamin, while in the case of equal mixtures a rough working approximation of the probable proportion is afforded. This paper contains a note added in proof as follows:

"In a recent communication accessible to the present author only after the above paper went to press, Euler and Rydbom (Svensk. Kemisk. Tidskrift, 1929, 41,223 ) have shown that when rabbits are fed carrots or carrot extracts, a 'pale yellow-colored carotinoid,' and not carotene, is stored in the liver. Although no attempt has been made to identify this 'carotinoid' with the classical vitamin A, it is obvious that this result is in complete agreement with the experimental findings described in the present paper in the case of the rat."

Evidence along similar lines has also been reported by Wolff, Overhoff, and van Eckelen (1930) who state that they have demonstrated 
the synthesis of vitamin $\mathrm{A}$ in the livers of rabbits following the administration of carotene. They have attempted to distinguish between carotene, xanthophyll, and vitamin A in food materials by means of (1) spectrographic analysis of color produced by the antimony trichloride reaction, (2) the intensity of color of the materials, and (3) separation of carotene from vitamin $\mathrm{A}$ and xanthophyll by shaking a petroleum ether solution of the material with 90 per cent alcohol, the carotene remaining in the petroleum ether and the xanthophyll and vitamin $\mathrm{A}$ being taken up by the alcohol. Of the materials tested, all of which were of known vitamin $A$ activity by biological tests, egg yolk gave positive results by the methods followed for carotene, xanthophyll, and vitamin $\mathrm{A}$; butter for carotene and vitamin $\mathrm{A}$; and extracts of carrots, green cabbage, and spinach for carotene only.

Meantime continued confirmation of the ability of purified carotene to function as vitamin A was reported from various laboratories. Kawakami and Kimm (1929) reported that carotene purified by repeated crystallization from carbon disulfide and ethyl alcohol cured xerophthalmia in rats in doses of between 0.03 and 0.05 milligram daily. Javillier and Emerique (1930) found that a sample of spinach carotene which had been preserved for 40 years in an atmosphere of hydrogen in a sealed tube in diffuse light was effective as a source of vitamin $\mathrm{A}$ in a dosage of 0.01 milligram per 100 grams weight of rat.

Green and Mellanby (1930) reported complete protection of rats against infection with purified carotene, m. p. $174^{\circ} \mathrm{C}$, , in doses of 0.02 milligram daily and stated their belief that carotene itself is the specific substance responsible for the vitamin A activity of green vegetables, carrots, and butter, and possibly of egg yolk. In regard to liver, the possibility was suggested of the preșence of a "highly active leuco-form of carotinoid which may be reconverted into carotene and liberated into the circulation as required."

\section{Formation of Vitamin $\mathbf{A}$ in Nature}

Acceptance of the hypothesis that vitamin A occurs in plant material in the form of its precursor carotene necessitates either a restatement of findings as to the origin of this vitamin, or conscious acceptance of the fact that in actual usage the term vitamin A may (and often does) cover both the "primary" or precursor form (carotene) and the "secondary" form immediately active in animal nutrition (vitamin A in the spectrographic sense). The latter alternative is much more accordant with present (1930) usage, and is therefore employed in this monograph. In this sense there is no evidence of the formation of this 
factor de novo in animals; but both land and water plants synthesize the vitamin (at least in its precursor form) and from them the animals of the land and sea, including the fishes, derive their supplies-either directly or by preying upon other species.

Coward and Drummond (1921) attempted to trace the origin of vitamin A in plants by feeding tests with seeds, germinated seeds, etiolated seedlings, green seedlings and older green plants for the relative content of vitamin A. No increase in the amount of vitamin A in the barely germinated seeds could be detected. Their experiments also indicated that the content of vitamin A was not appreciably greater in the etiolated seedlings than in the original seeds. Green shoots of turnip, maize, and peas (soil and sand grown) were, however, decidedly richer in vitamin $A$ than the original seeds or etiolated seedlings. A more detailed study with the sunflower confirmed these results. The dry seeds and etiolated shoots were relatively inactive, while the green shoots were very active as a source of vitamin A, thus suggesting that the formation of large amounts of vitamin $\mathrm{A}$ in green leaves requires the influence of light.

Evidence was also furnished in this contribution that vitamin A can be produced by the green plant from inorganic sources as shown by its high content in the green shoots of Tradescantia (Wandering Jew) grown in water; that green cabbage is much richer in vitamin A than white cabbage, that mushrooms contain only a small amount of vitamin $\mathrm{A}$, and that common green seaweeds (Ulva and Cladophora) are as potent in vitamin $\mathrm{A}$ as the green land plants such as cabbage, and are much richer in this vitamin than are red seaweed (Polysiphonia) and Carrageen moss (Chondrus crispus).

Jameson, Drummond and Coward (1922) reported the work begun by the late Dr. Jameson upon the project of developing the complete cycle of transfer of vitamin from the minute diatomaceous seaweeds through the crustacea and smaller fish to the cod. In the diatomaceous and other green seaweeds they found evidence of formation of vitamin A which they correlated with the presence of chlorophyll or related coloring matter. Later Coward (1925) extended this work to the study of a fresh water alga which she found to synthesize vitamin $A$ when grown in pure cultures. As little as 0.003 gram per day of the dry weight of this alga proved effective as a source of vitamin $A$ in her work.

There is no reason to doubt that the green plants of the water as well as those of the land, synthesize this vitamin under the influence of sunlight. Many of these diatoms, minute algae, and other tiny aquatic 
plants become food for the group of small sea organisms known as plankton and this in turn for larger species up to the crustaceans and small fishes on which codfish feed. In such ways the synthesis of vitamin $\mathrm{A}$ in the small green plants of the sea presumably becomes the source of the abundant supplies of this substance which we find in cod-liver oil; the cod, it will be remembered, having very little fat elsewhere in its body.

In 1922, Wilson published experiments indicating that etiolated wheat shoots showed evidence of a synthesis of vitamin $A$ at this stage in the development of the young plant.

In 1927, Coward extended her studies of the problem of the development of vitamin A in the sprouting seed and young growing shoot. She concluded that "seedlings of wheat which have germinated for two days and have only made three rootlets and no shoot or only a very short one, do not contain any more vitamin $A$ than similar ungerminated seeds." And, on the other hand that: "Etiolated shoots of wheat and white and yellow corn may contain small quantities of vitamin A when grown to a height of about 10 to $12 \mathrm{~cm}$. above the ground."

Hence there may be a slight development of vitamin A during the growth of the etiolated shoot; but it is certain that the main synthesis of vitamin $\mathrm{A}$ in the plant takes place only under the influence of light. In nature, of course, this means ordinary sunlight with its mixture of rays of various wave-lengths. Such evidence as is thus far available seems to indicate that the short ultra-violet rays are neither essential nor deleterious to the synthesis and accumulation of vitamin $A$ in the normal living plant tissue. Coward (1927) concluded that radiation from a mercury-vapor quartz lamp is effective; but also, that in the presence of an abundance of visible radiation the addition of ultraviolet rays has no influence on the ultimate amounts of vitamin A contained in the plant tissues. "Within reasonable limits, excessive illumination of the living plant tissue does not seem to have any deleterious effect on the vitamin A formed in it."

In a study of the persistence of vitamin $\mathrm{A}$ in plant tissues, Coward (1925a) found that vitamin A is not used up in any process carried on by living plant tissue in the dark; that the vitamin A content does not decrease when a leaf loses its green color and becomes yellow; that it is completely destroyed when the leaf dries up, becomes brown, and dies; and that it does not diffuse appreciably into water from the cut ends of shoots.

Morgan and Smith (1928) found the vitamin A content of tomatoes to increase during ripening. 
House, Nelson and Haber (1929) also found a lesser amount of vitamin A in green "mature" tomatoes than in the fully ripened fruit; but the same quantity was finally developed whether they were allowed to ripen on the vines, at $22^{\circ}$ to $25^{\circ} \mathrm{C}$. in the laboratory, or by treatment with an ethylene air mixture $(1: 800)$. Jones and Nelson (1930), however, consider vine-ripened tomatoes to be richer in vitamin A than green, air-ripened, or ethylene-ripened tomatoes.

\section{Measurement of Vitamin A Values}

The starting point of our knowledge on this subject was the discovery by McCollum and Davis (1913) that rats could not be nourished satisfactorily upon certain food mixtures of which lard was the sole fat; but the use of butterfat sufficed to make the food mixture adequate. Further experiments showed that, with the samples used, no more than 5 per cent of butterfat need be contained in the food mixture to make it adequate in this respect, whereas even 28 per cent of lard did not suffice. Evidently, then, butterfat is rich in this nutritive essential (vitamin A) and lard either lacks it entirely or contains so little as to show no evidence of its presence in such experiments.

Through the work of Osborne and Mendel and of McCollum and his associates it was shown that egg fat, cod-liver oil, and the fat of pigs' kidney resembled butter in serving as efficient sources of vitamin A while cottonseed oil, olive oil, almond oil and other commercial vegetable fats seemed, like lard, to lack it. The skeletal muscles (ordinary meats) seemed to contain but very little vitamin A; heart muscle, somewhat more, but not so much as liver or kidney. As shown by Osborne and Mendel (1915a) beef fat, while not so rich as butter, yet contains this vitamin in significant amounts. Daniels and Loughlin (1920) and Drummond, Golding, Zilva and Coward (1920) have published experimental evidence from which they conclude that lard and probably some other commercial forms of fat previously regarded as devoid of vitamin A are not wholly so, the vitamin value of lard (as probably also that of beef fat and butter) depending largely upon the food of the animal.

That vitamin A may be stored to an important extent in the body is indicated not only by the results of feeding body fats and glandular organs as sole sources of this vitamin in the diet, but also by the fact that when growing animals previously well fed are placed upon diets certainly deficient in vitamin A and often apparently devoid of it, they usually continue to grow for some time, often doubling their body weights before signs of nutritive deficiency appear. See the accompany- 
ing growth charts of rats fed by Osborne and Mendel (1921) upon diets made up of foods which had been especially extracted to free them from this vitamin (Fig. 9).

When an animal placed upon a diet devoid of vitamin A ceases to grow at once, as has been reported in some cases, it may be because of insufficient food intake or because the previous food of the animal had not been such as to induce a storage of vitamin $\mathrm{A}$ in the body. (Storage of vitamin A in the body and in its different parts is discussed more fully further on in this chapter.) As we now have much evidence that such storage is a very important factor and that it depends largely upon the food consumed, we must expect that the vitamin A content of

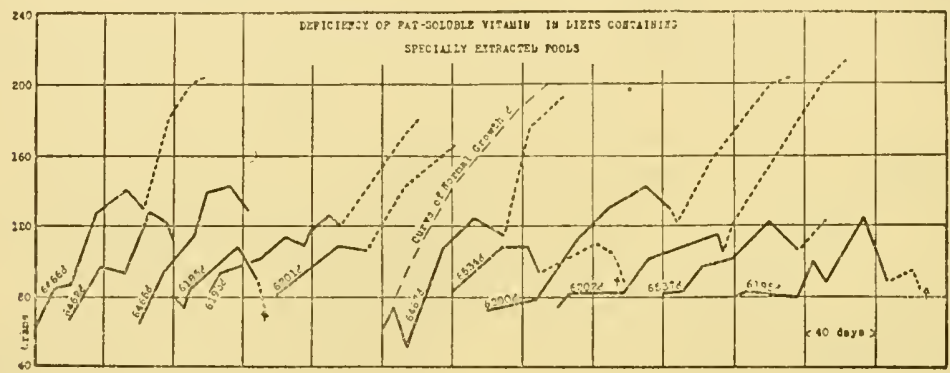

FIG. 9.-Weight curves of rats on diets lacking vitamin A, from experiments by Osborne and Mendel (1921). The curves show that growth continued for differing lengths of time at different rates after the animals were placed upon food which had been carefully freed from vitamin A. The interrupted (broken) lines show periods of feeding with vitamin A after growth had stopped and animals had shown pathological effects from lack of this vitamin. In most but not all of these cases cures were effected and normal growth resumed. (By permission of the Journal of Biological Chemistry.)

the body may vary considerably with different individuals of the same species. This means much in the quantitative determination of vitamin A inasmuch as variable stores of the vitamin in the bodies of the test animals according to their age, previous feeding, and possibly other conditions, will render results unreliable if they are not compensated. Typical cases are shown in Fig. 10.

The storage of vitamin A in the body is undoubtedly a very important factor in the relations of this vitamin to health and will be discussed further in that connection later in this chapter.

Although much of the literature upon the occurrence of vitamin A antedates the publication, or at least the general use, of quantitative methods, yet it may help to clarify subsequent discussion if the consideration of method be taken up at this point and from the quantitative point of view. 
Quantitative Determination of Relative Amounts of Vitamin A by Means of Feeding Experiments.-The foundations for quantitative work in this field were laid by Osborne and Mendel (1920c, 1921), by Steenbock and associates (Steenbock, Boutwell and Kent, 1918; Steenbock and Gross, 1919; Steenbock, Nelson and Black, 1924) and by Drummond and Coward (Drummond, 1919; Drummond and Coward, 1920).

In 1919 Drummond described the technique used in his laboratory to compare the vitamin A values of substances quantitatively as follows: Young healthy rats selected from home-bred stock and weighing about

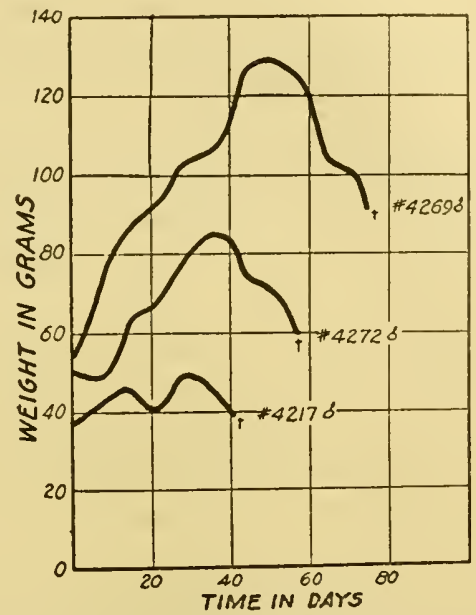

FIG. 10.-Showing the influence of previous feeding upon growth on diets lacking vitamin A. Rat 4217 had received a diet relatively low in vitamin A ( $\%$ whole wheat and $1 / 6$ dried whole milk). Rat 4272 had received a diet richer in vitamin A ( $2 / 3$ whole wheat and $1 / 3$ dried whole milk). Rat 4269 had received a diet still richer in vitamin A ( $1 / 3$ whole wheat and $2 / 3$ dried whole milk). All were placed upon a diet devoid of vitamin $\mathrm{A}$ at the same age, 28 days. Growth and duration of life upon this diet was evidently determined by the preëxperimental bodily store of vitamin A, as determined by the richness of their previous diets in vitamin A. (From experiments by Sherman and Boynton.)

50 grams each, are fed a ration consisting of purified caseinogen 20, purified starch 50 , salt mixture 5 , yeast extract 5 , butterfat 15 and filtered orange juice 5 parts. The animals which give evidence of a normal power of growth are removed from the complete ration when they have attained a body weight of 70 to 80 grams, and are given a similar ration in which an equivalent amount of hardened linseed oil is substituted for the butterfat. When it is definitely established that growth is inhibited by this deficiency in vitamin A, the linseed oil is wholly or partially replaced by the substance to be tested, and the be- 
havior of the animal is closely watched for a period of from 5 to 6 weeks. Absence of vitamin $\mathrm{A}$ is indicated by the failure to grow followed by a decline in health accompanied by the characteristic eye condition. That there was an individual variability in reaction to this standardized technique was noted in the observation that whereas experimental animals usually soon cease growing when fed this diet deficient in vitamin A, "occasionally a very vigorous individual will continue to grow for some weeks after the deficiency has been introduced, and such animals should not be employed in experiments of a quantitative nature."

Certain improvements in this technique were soon reported by Drummond and Coward (1920). Rice starch was substituted for the wheat starch as the former in the crude state was found to be almost devoid of vitamin $\mathrm{A}$, and hence could be more readily purified than the wheat starch; and refined fully hydrogenated vegetable oil (usually cottonseed) replaced the linseed oil. On this ration it was reported that young rats of 50 to 70 grams weight should show only slight growth, becoming stationary after a week or two. Definite weights of the substances to be examined for vitamin A were administered daily before the basal ration to rats, the growth of which had been suspended for from 10 to 14 days, and which did not weigh more than 80 to 120 grams. Later workers have confirmed Drummond's view that reasonable uniformity of size of experimental animals is important to the quantitative accuracy of the results.

The quantitative method thus introduced by Drummond and Coward has been developed by Sherman and Munsell (1925) and Sherman and Burtis (1928a) into the following form:

\section{Feeding Method as Used in 1930}

The Basal Diet.-Much attention has been given to the development of a basal diet which shall be free from vitamin A but fully adequate in all other respects and which can be duplicated with certainty.

As the protein component of our basal diets we have used both casein and meat residue with satisfactory results. In either case, our experience leads us to depend upon thorough extraction with hot alcohol for removal of vitamin A rather than to attempt to free the material completely from this vitamin by heating in the air. Casein is perhaps preferable to meat residue as a component of a standardized basal diet because it is more definite chemically. We conduct the extraction of the casein essentially as described by Osborne and Mendel (1921). The 
following details of procedure in this extraction have been found convenient and effective:

Place 200 grams of fine-grained, air-dry, high grade casein in a 2-liter Pyrex flask with 500 cubic centimeters of 95 per cent alcohol, boil for one hour on a steam bath under a reflux condenser and then transfer the contents of the flask rapidly without cooling to a suction filter in a Büchner funnel of about 15 centimeters diameter. (A tincture press may be used when working with larger amounts.) After thorough removal of the hot alcohol, return the casein to the flask and add 500 cubic centimeters of fresh 95 per cent alcohol, boil and filter as indicated above; then repeat the process again. In this way the casein receives three successive one-hour extractions with boiling alcohol. Finally it is dried in the air at room temperature.

Numerous feeding experiments showed that the casein thus extracted was free from any trace of vitamin A detectable by present methods and was indistinguishable in this respect from other portions of casein which had been subjected to still longer extraction with hot alcohol.

Cornstarch is used as the chief source of energy.

Dried brewery yeast is used as the source of vitamins $B$ and $G$ in the basal diet. Since Osborne and Mendel (1921) have shown that such yeast, even when fed to the extent of 42.5 per cent of the total food consumed, does not furnish any detectable amount of vitamin $\mathrm{A}$, it seemed safe to assume that in feeding about one-fourth of the quantities fed by them no appreciable amount of vitamin $\mathrm{A}$ would be introduced into the basal diet. This has been confirmed by many years' experience with the use of basal diets of the type here described. However, each laboratory should carefully test the yeast that it uses.

Mineral elements are supplied by the use of the complete salt mixture prepared according to the directions of Osborne and Mendel (1919). Additional sodium chloride to the extent of 1 per cent of the dry weight of the food mixture is also included in the basal diet, as this seemed to result in the diet being somewhat more uniformly wellrelished by the experimental animals of our colony.

As a source of the antirachitic factor, direct irradiation* of the animal body or the inclusion in the diet of irradiated ergosterol in some form appears to be equally effective. We use an irradiated preparation free from vitamin $\mathrm{A}$ and of tested antirachitic potency.

Fat, other than the small amount contained in the yeast, is not in-

* For convenience we use the simple term "irradiation" meaning, in this connection, exposure to the rays of the mercury-vapor quartz lamp or other suitable source of ultra-violet rays. The relation of wave-lengths to antirachitic effects is discussed in the following chapter. 
cluded in the basal diet, as our animals (containing considerable body fat) seem to thrive as well without it (for the time covered by these tests, at least) and its omission avoids any possible uncertainty as to its freedom from vitamin $\mathrm{A}$.

The basal vitamin A-free diet which we now use consists of purified casein 18 , salt mixture 4 , dried brewers' yeast 10 , sodium chloride 1 and cornstarch 67 parts with the addition of 0.500 grams irradiated commercial cholesterol per kilogram of the basal ration or some equivalent form of irradiated ergosterol.

Selection and Standardization of Experimental Animals.-The experimental animals are young albino rats reared under the supervision and control of the experimenter. Sherman and Kramer (1924) found that animals reared on food mixtures of dry whole milk and ground whole wheat with increasing proportions of milk and therefore of vitamin $\mathrm{A}$, possessed correspondingly increased body stores of vitamin $A$, even at four weeks of age, as was apparent from the increasing average survival periods, when placed on a vitamin A-free diet. Along with the difference in survival period, the differing store of vitamin A in the body resulted also in markedly different growth curves on the same vitamin A-free diet. Hume and Smith (1928) suggest that vitamin A potency can best be evaluated by prolonged tests, dispensing with a depletion period. But unless the factor of varying storage in the body of the young rat is very carefully safeguarded it is likely to be an extremely important source of error in studies of vitamin A and especially in attempts to make such studies quantitative.

Because of the large differences shown by rats of the same age when placed on the same diet, partly individual and partly due to previous diet or family history, we follow the practice of Drummond and his coworkers in beginning each experiment by keeping the rat upon a vitamin-A-free diet until his stored surplus of vitamin A is exhausted as indicated by cessation of growth, before beginning the feeding of the food to be tested.

Too large a bodily store of vitamin A is inconvenient, for during a long preliminary period the animals become too large to be the most sensitive reagents to measure growth. On the other hand, a previous diet so restricted as to result in a very small bodily store also appears to be undesirable; Sherman and Burtis (1928) have shown that the young reared to 28 days by mothers receiving a ration of $1 / 6$ whole milk powder and $5 \%$ ground whole wheat are more susceptible to infection during the vitamin A test period than those reared by mothers receiving twice as much milk, even though the variations in the surplus 
store of vitamin A were eliminated by means of a preliminary period. As a ration for the stock colony from which experimental animals are to be drawn, a diet consisting of two-thirds ground whole wheat and one-third whole milk powder with sodium chloride added to the mixture in the proportion of two per cent of the weight of the wheat has been extensively used with fairly satisfactory results.* This diet is not recommended as an optimal food either for growth or for reproduction. It has long been known that the addition of suitable fresh foods, e.g. 5 grams of raw lean beef per adult rat per day, often results in somewhat more rapid growth and earlier and more abundant breeding. Osborne and Mendel (1926) have shown that more rapid growth can also be obtained with certain other dry food mixtures. The diet here described contains, however, ample vitamin A to permit successful reproduction and lactation, with the production of young possessing at 28 days of age a moderate bodily store of vitamin $A$. This breeding diet also contains sufficient of the antirachitic factor not only to prevent the development of rickets at any time of the year in animals kept on the stock diet and living in a laboratory lighted through glass, but also to endow their young by 28 days of age with considerable antirachitic reserves. Steenbock and Nelson (1923), Drumnond, Coward and Handy (1925) and Chick (1926) found that the young reared on their stock diets have larger bodily reserves of vitamin $A$ than of vitamin $D$; but we find that 2S-day old animals reared by mothers on the ration here described have greater bodily reserves of vitamin $D$ than of vitamin $\mathrm{A}$, relative to their needs when transferred to our fat-solublevitamin-free diet. In laboratories using animals thus reared, and employing a low rate of growth during the test period, quantitative studies conducted before the differentiation of vitamins $A$ and $D$ were probably not invalidated by shortage of vitamin D. (Sherman and Hessler, 1927; Sherman and Burtis, 1928a; Sherman and Stiebeling, 1929.) However, we recognize that the vitamin D content of whole milk powder, the source of this vitamin for our breeding colony, and indirectly for any bodily reserves in their young, may be variable, and hence, subsequent to Steenbock's demonstration that vitamin D is necessary for growth, we have recommended and employed measures to provide vitamin D liberally in all vitamin A experiments. It is well to conduct all quantitative determinations of vitamins under conditions which amply provide all known nutritional essentials except the one under investigation.

\footnotetext{
* Practically the same diet may be conveniently stated in percentage form as follows: Finely ground whole wheat, 66 per cent; whole milk powder, 33 per cent; sodium chloride, 1 per cent.
} 
Experimental Procedure.-The discussion which follows relates to young albino rats which, unless otherwise stated, were from families fed upon a diet of one-third dried whole milk and two-thirds ground whole wheat, with sodium chloride in the proportion of 2 per cent of the weight of the wheat, with or without the addition of 30 to 60 grams per week of fresh lean beef per adult. This addition of lean beef was found to have no appreciable effect upon the amount of vitamin A stored in the body of the young rat as judged from the length of time it was able to survive when placed upon vitamin-A-free diet.

When such 21- to 29-day old rats of an initial weight of 35 to 50 grams are placed upon a diet well adapted to their needs in other respects but devoid of vitamin $\mathrm{A}$, such as our basal vitamin-A-free diet described earlier, growth usually continues for from four to five weeks during which period our rats usually gain from 35 to 50 grams. Around the time when cessation of growth is anticipated the animals should be weighed every other day and finally daily. Having decided upon a definite and uniform diet for the families from which the young are to be drawn, and a uniform age at which to place the young upon the vitamin-A-free diet, one can learn by experience about when to expect cessation of growth, and will also be guided by the general appearance of the animals in determining when the fore-period should end and the test period begin. This should be after the animals have certainly used up their previous reserve store of vitamin A but before they have been permanently injured by the vitamin-A deficiency.

As soon as the body weight ceases to increase, and/or the first signs of eye disease appear (slight swelling of the eyelids and an accumulation of exudate in the corners), usually accompanied by a barely perceptible tendency to flabby musculature and slightly unkempt fur, the depletion period should be ended.

Individuals or litters which appear too weak or too vigorous to be comparable to the general average should be discarded. The others are placed in individual cages with raised screen bottoms to prevent access to excreta, and at least one of each litter is continued on the basal vitamin-A-free diet until death, as a "negative control," while the others are fed graded portions of the food to be tested, as their sole source of vitamin A, daily or at other suitable intervals during the test period in addition to the basal diet and distilled water ad libitum. Any advantage in survival period or maintenance or gain in weight shown by a test animal over its "negative control" must be attributed to the vitamin A received from the weighed portions of the food or other material which is being tested, provided the experiments are conducted properly 
and in sufficient numbers to avoid vitiation of results through individual variability of the animals. Hence, animals making equal gains in weight during the experimental period may be regarded as receiving equal amounts of vitamin $\mathrm{A}$, and the richness of different foods in vitamin A may be taken as inversely proportional to the amounts of the foods required to furnish that fixed amount of the vitamin which will produce a given result in a standard experimental animal in or for a given time, as, for example, a given rate of gain in weight during the experimental period in a rat that has been prepared for the purpose in the manner described above.

It is plain, as Steenbock and Coward (1927) point out, that the cure of ophthalmia is more specific than response in growth; but it has not been demonstrated that this criterion lends itself so well as growth to accurate quantitative measurement of the vitamin.

Weekly records of weights, food consumption and careful notes on the condition of the animals should be kept. Only clean sterilized apparatus should be used. Laboratory conditions should be controlled so that dust, fumes, noise and fluctuations in temperature are reduced to a minimum. At death or at the end of the experimental period all animals should be autopsied. This procedure serves to detect any possible cases of abnormality due to causes unrelated to the vitamin deficiency; and to confirm the conclusions drawn from the weight curves as to the extent to which the vitamin deficiency, whether absolute or relative, has affected the life and growth of the experimental animal.

It is suggested that each animal be autopsied with all these points in mind. If done thoroughly, the autopsies will acquaint the worker with the characteristic effects of the vitamin A deficiency and will help to increase our knowledge in this direction.

In the experience of the Chandler Laboratories of Columbia University, about 85 per cent of the rats placed, when 4 weeks old, upon the vitamin-A-free diet have developed the ophthalmia first noted by Osborne and Mendel (1913a) as characteristic of the results of this dietary deficiency. Pus in one or more of the glands near the base of the tongue was found in 76 per cent of the "negative controls" examined for this sign, which was, therefore, almost as characteristic of the vitamin A deficiency as was the ophthalmia. These animals, placed at an early age upon a diet entirely devoid of vitamin A and dying usually within about two months thereafter, did not show the frequency of lung trouble stated by Steenbock and Nelson (1923) to be one of the characteristic effects of vitamin A deficiency. There is, however, probably no real discrepancy between the work of the two 
laboratories in this respect. It is probably because of the complete deprivation and consequent early death of our animals that the lung trouble did not show itself in them. Where the diet is deficient in, rather than devoid of, this vitamin, we fully confirm the findings of Steenbock and Nelson that vitamin A deficiency leads to marked increase in the incidence of lung disease.

For quantitative estimates of relative amounts of vitamin $\mathrm{A}$ in which the highest degree of accuracy is desired it is necessary to conduct the comparative studies in strict parallel with enough experiments to eliminate the effect of individual variability, and with rigid control of all factors such as the previous nutritional history, the weight and sex of experimental animal, litter variability, and rate of growth during the test period. So far as possible, rats of the same litter should be used for those tests the results of which are to be most directly compared with one another. Rats from one litter behave more uniformly than a miscellaneous selection even though the latter appear to be in a similar condition.

There is clearly a tendency toward an inverse relationship between the body weight of the animal and its rate of gain upon a given limited allowance of vitamin A. Undoubtedly, too, this may also be stated conversely; i.e., that among animals of the same species and age, the amount of vitamin A required to support a given rate of gain tends to vary with the size of the individual. This corresponds with the similar relationships previously established in the case of vitamin $B$. Therefore, only such animals as do not differ greatly among themselves in size at the end of the depletion period should be used. For conditions such as those of our laboratory, a minimum limit of 70 or 75 grams and a maximum limit of 100 grams at the end of the depletion period would seem to be desirable.

The early experience of several workers, which indicated that male and female rats may be used interchangeably in determinations of vitamin A by the method here employed, with gains of about 3 grams per week in the test period, has now been confirmed by the data of large numbers of experiments made in such manner as to permit of direct comparison of the quantitative results yielded by the two sexes. Thus the average gain shown during an 8 -week test period by 148 males was 24.6 grams, and by 148 directly comparable females was 22.3 grams. Statistically, the average difference of 2.3 grams (or slightly less than 0.3 gram per week) is probably, though not certainly, significant. Practically, it seems so small as to be safely negligible, although for investigations in which an extraordinary degree of refinement is sought it 
might be slightly preferable to work entirely with animals of the same sex.

Probably any possible slight advantage theoretically attributable to discrimination between the sexes will actually, in experiments of this kind, be outweighed by the advantages of being able to make greater use of litter mate controls when both sexes are employed.

The very slight tendency toward more rapid growth on the part of males than of females of the same age and nearly the same size, will in practice tend to be offset by the larger size which the males will usually have attained at the beginning of the test period, when the animals will usually be about 2 months old. This equalizing influence can readily be further availed of, at the cost of rejection of very few animals, by specifying a slightly larger standard size for male than for female rats at the end of the depletion and beginning of the test period. Any laboratory might, for example, decide that only females between 70 and 95 grams or males between 75 and 100 grams should be used interchangeably as standard test animals for quantitative determinations of vitamin A by the rat growth method.

It should, however, be carefully noted that all the foregoing discussion of the relation of sex to rate of gain, refers to experiments in which the level of feeding of the vitamin A-containing food or other material to be tested is such as to induce a gain in weight of only about 3 grams per week during the test period. Much larger differences are to be expected if the level of vitamin A feeding is considerably higher, for in this case something approaching a normal rate of growth may become possible, and, as is well known, the normal growth curve of the male rat is distinctly steeper than that of the female at this age. Thus when Sherman and Burtis (1928a) fed about twice the amount of vitamin-A-containing food required for 3 grams gain per week, the average gain during the 8-week test period was: for 117 males, 57 grams; for 117 females, receiving the same amount of vitamin $\mathrm{A}$, 42 grams.

Drummond and Coward (1920) and Zilva and Miura (1921b) pointed out that a definite small gain in weight furnishes the best basis for quantitative comparison. Further work has resulted in the general adoption of an average gain of about 3 grams per week during an experimental period of five to eight weeks, as a standard rate of gain or "unit growth." A lower rate of gain has been found to give more variable results, some animals dying before the completion of the test period.

"Normal growth" is undesirable as a basis of quantitative compari- 
son for several reasons. The term means various rates in different laboratories, and it does not permit the results to be verified by means of larger as well as smaller gains in weight resulting from feeding correspondingly larger or smaller amounts of vitamin-A-containing food. Moreover, with foods poor in the vitamin it is frequently difficult and sometimes impossible to feed enough to induce fully average normal growth.

In general, the less one need feed of the food under test, the less is the danger that the rate of growth may be influenced by changes in other factors through partial displacement of the basal diet by the test food, and hence the better is the prospect that the resulting weight curves will furnish an accurate quantitative measure of the vitamin value of the material under investigation.

While the procedure here recommended aims to secure a gain of weight of 3 grams per week during the test period, it is recognized that, in the judgment of some investigators, it may be possible to secure more uniform results at a somewhat higher level of feeding and rate of growth because the animals will then be more vigorous. Dutcher et al. (1927) while recognizing that quantitative differences may be more discernible at a rate of growth of about 3 grams per week are inclined to favor the Javillier (1925) standard of half-normal growth (about 6 grams per week) since under this condition they encountered less infection among their test animals. Sherman and Burtis (1928a) studied variability with the following results: In 104 cases, when the level of vitamin intake during the experimental period was twice that needed for a gain of 3 grams per week, the data showed coefficients of variability of 32 and 30 per cent at the ends of the fifth and eighth weeks, respectively, when the influence of differing response of the sexes was ruled out; while at a level of vitamin intake inducing a gain of 3 grams per week, the coefficients of variability in the same number of cases were 45 and 26 per cent respectively. The variability is slightly (perhaps not significantly) greater at the higher level when the tests are continued for 8 weeks, but less when the test period is shortened to 5 weeks.

While undoubtedly the higher level of vitamin A intake does result in more vigorous animals, the rates of gain are not increased in full arithmetical proportion to the vitamin intake as was plainly shown by data of Sherman and Munsell (1925), so that quantitative comparisons at higher rates of gain necessarily make the method less delicate. Also, the higher rates of growth are attended with much less uniform results between male and female test animals. 
On the whole, we find no certain gain in uniformity to balance the undoubted loss in delicacy when the higher rate of growth is employed as a standard by means of which to make quantitative comparisons of vitamin $\mathrm{A}$ values.

In determining the relative vitamin potency of two or more materials, we recommend the determination by a suitable number of controlled experiments, of the quantity of each material which contains respectively, the amount of vitamin A needed to induce (say) 3 grams gain per week. The relative vitamin contents of two given test materials are inversely proportional to the weights of the material required to support this arbitrary rate of gain.

For an accurate quantitative determination, such as should be expected when the effect of some treatment or variation of condition upon the vitamin value of a product is being studied, it would seem best to feed in parallel 10 or more animals on each of the amounts which are being used for direct comparison, but the number logically should depend on the uniformity of the results. The average deviation of gains in body weights in an 8-weeks experiment which aims at a total gain of 24 grams should never be over 10 grams. If all conditions are of the best this can be reduced to about 5 grams.

The results of experiments carried out as here described may be expressed in terms of the conventional standard units, a unit of vita$\min A$ being defined as that amount which when fed daily to a standard test animal prepared as described, will suffice to support an average gain in body weight of 3 grams per week during the test period of 4 to 8 weeks. (Obviously one may always express vitamin $\mathrm{A}$ values in terms of this standard unit, and yet may state the number of such units contained in one gram, or one kilogram, one ounce, one pound, or in a 100-Calorie portion of the food, or in whatever other amount of food may be regarded as convenient-as for example, the number of units of vitamin $\mathrm{A}$ in a dozen eggs. In this monograph vitamin $\mathrm{A}$ values are expressed in terms of units per gram.)

However, it is to be kept in mind that one who desires may use a higher level of feeding and make comparisons at rates of growth higher than 3 grams per week, and yet may express the vitamin A values thus found in terms of the now-recognized standard units by establishing (or accepting as established) the relative amounts of vitamin $\mathrm{A}$ which must be fed to induce the two rates of gain in question, i.e., a gain of 3 grams per week and the higher rate at which the experimenter has worked or proposes to work.

Occasionally also, there are practical difficulties which make it im- 
possible to feed, daily, enough of the material under test to carry a unit of vitamin A. By comparing the curves resulting from experiments carefully conducted on the test material with any adequately established set of weight curves representing different levels of feeding, the quantity of the test material which would contain a unit of the vitamin may be estimated approximately, if somewhat less accurately than when experiments are conducted under the "standard" conditions.

Without some such experimentally established basis of reference, it is obviously misleading to try to compare quantitatively the vitamin potencies of two products which, when tested, have shown different rates of gain. The plan should be to compare the quantities of each necessary to induce the same rate of growth, which rate should be subnormal, and should be decided upon in the light of the considerations discussed above.

The Length of the Experimental Period.-The practice of many laboratories has been to continue the test period for 8 weeks after the depletion period. Laboratories devoted to control work upon cod-liver oil commonly use a test period of 5 weeks and this was, therefore, incorporated in the United States Pharmacopoeia method of determining vitamin $\mathrm{A}$ values. With both practices in use, and resulting data recorded in terms of the same unit, it becomes important to know whether this difference in length of test period will influence either the uniformity of the results obtained or their average numerical values.

Averaging the results of 104 of their experiments in which the test animals showed about unit growth, Sherman and Burtis (1928a) find at the end of the usual 8 -weeks test period a gain of 25.89 grams (or 3.24 grams per week) with a coefficient of variation of 26 per cent. These same experiments averaged at the end of the fifth week of the test period show a gain of 18.12 grams (or 3.62 grams per week) with a coefficient of variation of 45 per cent. Thus in this series of determinations the shortening of the test period to 5 weeks would have resulted in somewhat higher and more variable findings. The average difference of 0.38 gram per week in rate of gain is about one-sixth of that which results from doubling the vitamin intake under these experimental conditions; so that the shortening of the test period from 8 weeks to 5 weeks might be expected, as an average tendency, to increase by about one-sixth the numerical ratings of vitamin $A$ values of materials thus tested.

The slackening rate of growth encountered after 3 months of age as noted here, and emphasized by Hume and Smith (1928) may be due in part to the animal's larger need of vitamin A due to increased 
body weight, in part in the larger animal to a less-pronounced impulse to grow, and perhaps in part to a shortage of some growth factor still unrecognized, and not so abundantly provided or stored as to permit indefinite uniform growth in these vitamin A experiments.

The probability that mammalian nutrition requires more than the six well-recognized vitamins is discussed elsewhere in this monograph. In their discussion of vitamin A testing, Drummond and Morton (1929) say: "In any case the test must not be too prolonged, for even on doses producing normal or nearly normal growth in the early stages for a time, the response may later show a failure which is not made good by increasing the amount of the supplement." These authors consider it reasonably safe to use a test period of 5 or 6 weeks (after depletion).

\section{Vitamin $\mathrm{A}$ in Plant and Animal Tissues and Products}

The distribution of vitamin $\mathrm{A}$ in nature has been the subject of numerous researches in the past few years. As explained in the foregoing section, its determination has become quantitative in character and relative amounts of the factor may be expressed in terms of recognized standards or "units." This has a decided advantage over the former classification of foods as "rich," "poor," or "intermediate" sources of the vitamin in that it permits of finer distinctions and of more concrete comparisons and applications.

It is now known that in much of the early work, vitamin A has not been the only limiting factor in the growth of the experimental animal. Furthermore, certain important refinements of the method have not been employed in all cases even of recent work. But granting these facts, our knowledge of the occurrence and estimation of vitamin $\mathrm{A}$ is clearly passing out of the qualitative stage and hereafter may be organized in a more exact form. At the present time, a comprehensive survey of our knowledge of the natural distribution of vitamin A will involve the use of both qualitative and quantitative data.

Since the early experiments of McCollum and Davis (1913) and of Osborne and Mendel (1913a, 1914) showed that certain foods such as butterfat, egg fat, cod-liver oil and the fat of pig's kidney are rich sources of the fat-soluble factor and that lard and vegetable fats in general seem to lack it, the data have been extended over a very wide range of foods.

The fact that they are so numerous makes it undesirable to include all of them here; but typical foods from a wide biological range of sources may be mentioned. 
Plant products as primary sources of vitamin A will here be considered first. These may be grouped under green and other vegetables, fruits, cereals, nuts, and plant fats and oils.

Green leaves and other green vegetables are important sources of vitamin A. A rating of them in order of their content of this factor must necessarily be more or less tentative: the following estimates are made with the realization that they do not represent fixed values but with the belief that they indicate approximate relative values more nearly than do the conventional signs previously used.

Escarole, a green leafy vegetable with a vitamin A content of around 200 units per gram is the richest green vegetable which we have seen reported. Next is spinach which contains about 60 units per gram. Indications based on experiments made on the dried product are that the leaves of alfalfa, chard, clover, and cowpeas may contain amounts fairly comparable with spinach. One sample of dried alfalfa cured in the dark contained approximately 100 units per gram. Quantitative studies of vitamin-A values of hays are reported to be in progress. Vegetables (among others) reported to contain from 1 to 10 units per gram are artichoke, green string beans, brussels sprouts, celery leaves, lettuce, green peas, and red and green peppers. Successive tests in one laboratory indicated that green string beans may vary from 4 to 10 units per gram (Quinn, Burtis and Milner, 1927). Bleached celery leaves were found to contain only about one-fourth as much vitamin A as green leaves. No significant difference has been reported between canned and fresh samples of green peas and spinach. One unit per gram or less has been reported in headed cabbage, cauliflower, cucumber, and eggplant. Old cabbage, sauerkraut and white leaves seem to contain significantly lesser amounts of the vitamin than do the fresh green leaves of cabbage. The rind of the cucumber has in one instance been reported richer than the fleshy tissue. Bleached celery stems did not show detectable quantities of vitamin A. Green outer leaves of lettuce are reported to be 30 -fold richer in vitamin A than the white inner leaves from the same heads (Kramer, Boehm and Williams, 1929). McLaughlin (1929) reports the small leaves of New Zealand spinach to be especially rich in vitamin $\mathrm{A}$.

Carrots, which are reported to vary from 25 to 70 units per gram are conspicuously the richest in vitamin $\mathrm{A}$ of vegetables other than green tissues which have thus far been reported. Next are found the yellow tissues of pumpkin, sweet potatoes, yellow yautia (a Porto Rican root vegetable) and green dried peas which contain from 4 to 10 units per gram. While the indication is that yellow squash contains 
a similar concentration, this cannot be asserted with confidence. Yellow turnip on the other land, showed less than one unit per gram. White sweet potatoes, turnip and yautia contain markedly less vitamin A than do the yellow varieties. Other vegetables which have shown a value of less than one unit per gram are canned baked beans, soy beans, white beans, beets, lentils, onions, parsnips, and baked or dried Irish potatoes. None or only traces of the vitamin have been reported in dried lima beans, dasheens, mangels, mushrooms, radishes and rutabaga; but these foods were tested early and might show different results upon reinvestigation with the quantitative refinements now available. In any case in which a vegetable is prominent in the diet of any considerable community it will be of significance to know its vitamin $A$ value as accurately as may be.

Some fruits compare quite favorably with many of the common fresh vegetables which range from 1 to 10 units per gram. Fruits falling in this class are the avocado (alligator pear), banana, cantaloupe, chayote, date, plantain (Porto Rican "cooking banana"), dried prune, sapodilla, and raw and canned tomatoes. Those which have shown less than 1 unit per gram but distinctly measurable quantities are fresh apples, cooking figs, fresh grapes, orange juice, papaya (a tropical fruit), and pineapple. Grapefruit, lemon and peaches are reported to contain small amounts. As yet no one has detected a measurable quantity in raisins. The presence of the vitamin has been detected in the peel and peel oil of the citrous fruits with the indication that the orange oil furnishes greater concentrations than either that from lemon or grapefruit peel. One sample contained as much as 50 units per gram. Watermelon has recently (Munsell, 1930) been found a good source of vitamin $\mathrm{A}$.

Cereals are conspicuously poor sources of vitamin A. Yellow corn has been reported to contain about 3 to 4 units of vitamin A per gram, and yellow corn oil as much as 10. Detectable quantities but apparently less than one unit per gram have been found in samples of barley, bran, commercial bread, cottonseed, and cottonseed oil. Traces have been indicated in millet and unpolished rice, and some flours. As yet vitamin A has not been demonstrated in white corn, oats, polished rice, cornstarch and purified samples of patent flour.

Concerning nuts, few experiments of a quantitative nature have been reported but the indication is that less than one unit per gram is found in almonds, barcelona, Brazil, butternuts, walnuts, and peanuts. One reference to pecans indicated a vitamin content of around 3 units 
per gram, which if confirmed would be significantly different from nuts as a whole.

Drummond and Coward (1920a) indicated that "no hard and fast line can be drawn between animal and vegetable oils and fats when their value as a source of vitamin A is being considered" but that "taken as a class the animal fats possess a growth-promoting power superior to that of the vegetable oils." Later, concerning seed oils (which constitute the majority of oils from plant sources), Drummond and Zilva (1922) state that "the majority of the vitamin in the seed passes into the oils, producing oils of very low potency as compared with the chief animal oils and fats." As has been indicated, reports of a trace or failure to detect measurable quantities may be due to faulty methods of testing. Therefore plant oils which have been found to contain none or only a trace may be classed in one group. This includes the oil of almonds, arachis, castor beans, coconuts, cottonseed, djave, grapefruit peel, hempseed, linseed, mafurreira, mustard seed, olives, peanuts, rapeseed, sesame seed, Siak Illipe nuts, soy beans, and sunflower seeds.

The vitamin A content of animal products is now known to be ultimately dependent upon the diet of the animal but influenced by the reserve store in the body.

The distribution of the vitamin among the various tissues of the body has been studied quantitatively (Sherman and Boynton, 1925). "If adipose tissue and skin be ignored, about nine-tenths of the total vitamin $\mathrm{A}$ in the body of a well nourished rat was found in the liver and the remaining tenth about equally divided between the muscles as a whole, the blood, the kidneys, and the lungs. Weight for weight, kidney was about 40 times, lung more than 40 times, and liver between 200 and 400 times as rich in this vitamin as is muscle. Moderate differences in the vitamin A content of the food, such as are well within the range of variation likely to be encountered in human experience, resulted in large differences in concentration of this vitamin in the liver, and distinct differences in the amount of it found in lung tissue."

Therefore among the animal products which are used as food, the muscle tissues are poor in vitamin A while glandular organs, animal fats and oils, eggs, milk and milk products are usually much richer sources. Indications in reported studies on the muscle of beef, codfish, haddock, lamb, pig, poultry, rat, salmon and veal, are that they contain from traces to 0.5 unit of vitamin A per gram. On the other hand, glandular organs which have been investigated have always contained markedly more unless taken from animals whose food had been de- 
ficient in vitamin $\mathrm{A}$. The blood of the rats used in the study of the quantitative distribution of the vitamin contained from 1 to 2 units per gram. Judging from the results of that study and reports from other laboratories, kidney may be estimated to contain from 8 to 10 units per gram, lung from 10 to 15 and liver from 5 to 140 units per gram.

Some of the animal fats and oils are rich sources of vitamin A. Cod-liver oil has long been known to contain high concentrations. Indications are that storage in a cool dark place is not attended by marked destruction. Storage in warm light places for varying lengths of time has in some instances been reported to result in over 95 per cent destruction of the vitamin. Other fish liver oils which contain similar concentrations of the vitamin are those from burbot, coalfish, dogfish, haddock, hake, pollock, and topefish. Seal and whale oil are fairly rich sources of vitamin A. Fats of beef, dog, horse, mutton, pig, poultry and wool have shown measurable amounts of vitamin A. Lard, which usually seems lacking in the vitamin, has at times shown detectable quantities. Whole small fish, which serve as food for the cod are reported to contain from a few to approximately 500 units per gram.

Eggs are an important source of vitamin A. The vitamin is contained essentially in the yolk of the egg and the concentration depends, to an extent not yet fully determined, upon the food of the fowl. Undoubtedly the fowl depends upon its food for the vitamin A which the egg contains; but to what extent a shortage of this vitamin in the food results in the production of vitamin-A-poor eggs, as against a simple diminution of egg production, remains to be investigated in detail. There is little doubt that egg yolk is a more constant source of vitamin A than is liver, which alone among animal body tissues approaches it in richness in this vitamin. The egg as a whole may be expected to contain about 15 to 20 units of vitamin A per gram; and the yolk about three times this concentration.

Milk is also a rich source of vitamin A. While the number of units per gram is not conspicuously high, the extent to which milk enters into the well-planned dietary makes it one of the most important sources of this substance. It was largely because of their importance as sources of vitamin A as well as of calcium that milk and green vegetables were designated by McCollum as "protective foods," although their newly discovered vitamin $G$ values will further strengthen this classification.

In the case of milk, the vitamin A content is influenced by the abundance of the supply contained in the feed; but not directly and 
exclusively dependent upon it, because of stores in the body. The presence of an abundance of vitamin $\mathrm{A}$ in the grasses on which dairy cows are pastured in summer and in the hays fed in winter is an important factor in ensuring that the milk produced by the cow is rich in vitamin $\mathrm{A}$ at all times of the year. A factor of safety in maintaining the vitamin A value of milk is the fact that the store of this vitamin carried in the body of the cow is undoubtedly drawn upon to keep up the normal concentration in the milk in case of fluctuations in the supply received in the feed.

Of interest in this connection is the observation of Drummond, Coward and Watson (1921) that colostrum has a much higher concentration of vitamin A than the later milk. They are inclined to regard this higher value of colostrum as an indication of a mobilization of the reserves of the mother since it does not appear to be proportional to the fat content. "It is again interesting to recall that there is also a partial mobilization of the lipochrome pigments of the mother's body fat for the production of colostrum which normally contains a much higher concentration of those coloring substances than the later milk." A similar relation between vitamin A and the lipochrome pigments is suggested in the apparent tendency reported by these investigators for milk from cows of the Jersey and closely related breeds to be richer in vitamin A than that of Shorthorn or Black Angus. As a possible explanation, it is suggested that cows of the former breeds may have a higher storage capacity for vitamin A similar to their higher capacity for storing pigment.

The average vitamin A content of fluid milk is about 2 units per gram (or about 2,000 units per quart); that of condensed and evaporated milks about 4 units per gram, and of dried whole milk about 16 units per gram. To what extent milk can be enriched in vitamin A by increasing the amount of this factor in the food of the cow is still a matter of investigation.

So far as the writers are aware, there is no conclusive evidence as to how the vitamin A content of milk is divided quantitatively between its fat globules and its aqueous phase. The observations noted in the first edition of this monograph are now believed to have overestimated the proportion contained in skim-milk as compared with whole milk and butterfat. Crawford, Golding, Perry and Zilva (1930) believe that all the vitamin A (and D) of milk is in the fat.

Butter appears usually to contain about 30 to 50 units of vitamin A per gram, ranking in richness, weight for weight, with egg yolk and fresh spinach. Variations of vitamin A value of milk due to changes 
in the food of the cow must result in corresponding variations in the butter made from the milk; and to this is to be added as a further cause of variation in butter, the possibility of deterioration in vitamin value of the cream or butter under industrial manipulation or storage. It is believed that under good conditions there should be but very little loss from this cause.

Drummond, Coward and Watson (1921) from an extensive examination of storage butter concluded that the season at which the butter is placed in storage, i.e., the feed of the cows at that season, is a more important factor in determining the value of the storage butter as a source of vitamin A than the length of time the butter remains in storage, provided undue exposure to air is prevented and other conditions are good. As, under present conditions, by far the largest part of the butter placed in storage is that produced when the cows are on green pasture and are consequently giving a greater yield of butter of superior quality, and as it is kept in air-tight casks or tins during storage, it seems reasonable to assume that such butter will usually be scarcely less rich in vitamin A than fresh butter as obtained the year round. The development of acidity in butter may occur without appreciable loss of vitamin A if oxidation is prevented, while in so far as the process of renovating rancid butter offers opportunity for oxidation it may entail sufficient losses of vitamin A to render the product of materially less value in this respect.

Of interest in this connection is the statement made to one of the writers by an expert judge of butter ("butter taster") that in the winter months storage butter is to be preferred, on account of its summer flavor, to much of the fresh butter obtainable at the time.

\section{Relation to Nutrition and Health}

Some of the effects of a lack of sufficient vitamin A in the diet may now be summarized briefly as a basis for our consideration of the broader significance of this vitamin in relation to normal nutrition and the ability to resist disease.

When a diet lacking vitamin A but adequate in all other respects is fed to a young growing rat, growth may cease, or continue slowly, or may continue for as much as 10 weeks at a normal or nearly normal rate, depending upon the store of vitamin $\mathrm{A}$ in the body of the experimental animal at the beginning, and the amount of the experimental diet eaten. Under favorable conditions growth continues for some time at a nearly normal rate (as indicated by the curves shown in Fig. 10 above) during which time the surplus of vitamin A possessed at 
the beginning has presumably become exhausted or depleted. (Not necessarily lost; but "consumed" in that it is needed for, and diluted by, the growth of the animal.) Growth then slackens and usually soon ceases; often a rather rapid loss of weight begins shortly and the animal goes into a condition of general decline leading to death.

By the time the body's reserves of vitamin A have been depleted and growth ceases, the animal becomes more susceptible to bacterial infection, and this lowered resistance often shows itself in various ways - most conspicuously in the tendency of a large proportion of such experimental animals to develop a characteristic disease of the eye, variously known as ophthalmia, xerophthalmia, keratomalacia, conjunctivitis, or keratoconjunctivitis. This usually begins with a swelling of the lids of one or both eyes or with indications that the eye is becoming unduly sensitive; then there commonly develops an inflamed and catarrhal condition of the conjunctivae with a bloody or purulent discharge, the lids becoming scabby or sticky. This, with the swelling of the lids sometimes results in the eye being found completely closed. Often the inflammation extends to the cornea and if not treated may result in permanent blindness, though the animal often dies before the eye disease reaches this stage. The typical extremely severe eye condition undoubtedly involves infection and in this sense is not purely a deficiency disease, yet it is essentially so in its origin, inasmuch as the dietary deficiency so enormously increases the susceptibility of the animal to the infection as practically to determine the incidence of the disease. It is also very significant that without any other treatment whatever, the eye disease, if not too far advanced, usually disappears quickly when the animal is given any food containing a sufficient amount of vitamin A.

The relation of the diet to this eye disease and its cure was discovered by Osborne and Mendel in 1913. At that time they stated that a "type of nutritive deficiency exemplified in a form of infectious disease prevalent in animals inappropriately fed is speedily alleviated by the introduction of butterfat into the experimental rations," and in 1914 they reported "uniform success by substituting cod-liver oil for a portion of the lard in our standard diets. . . Not only was growth resumed in most cases at a very rapid rate, but all evidence of malnutrition, especially the affection of the eyes, promptly disappeared." Similar cures by feeding materials containing vitamin A have been reported repeatedly by Osborne and Mendel and others since that time so that there can be no doubt of the frequent cure of the disease by dietary treatment alone. Moreover, its cure by any other than dietary 
means is doubtful, if allowance be made for the fact that on diets only deficient in vitamin $\mathrm{A}$ and not devoid of it the animals not infrequently show the eye trouble in some degree and recover spontaneously. Bulley (1919) believed the eye trouble to be an infection controllable by sanitary precautions and local treatment with antiseptics, but Osborne and Mendel (1921) found this not to be true in their cases, and Stephenson and Clark (1920) have pointed out that the diets used by Bulley may not have been so thoroughly freed from vitamin A as they were intended to be. In their own experience, Stephenson and Clark report the development of the eye disease in 13 of 46 rats kept on diets deficient in vitamin $\mathrm{A}$. Other investigators have recorded its occurrence in a considerably larger proportion of cases. Emmett (1920) found it in 120 out of 122 such cases and in no case on other diets. Osborne and Mendel (1921a), in a critical discussion of the subject, give the results of an examination of their laboratory records of 1,000 rats representing essentially the entire group under study in their laboratory during one year. The rats were classified according to diet; and the total numbers, and numbers showing eye symptoms, were counted with the following results :

Incidence of Eye Disease in Osborne and Mendel's Rats. (Nos. 5000-5999.)

of Rats Eye Symptoms

On diets deficient in Vitamin A.............. $136 \quad 69$

On diets deficient in Vitamin B..................... 225

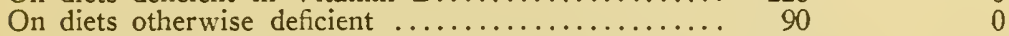

On diets experimental but presumably adequate..... $201 \quad 0$

On mixed food (stock animals) ................ $348 \quad 0$

$\overline{1,000} \quad \overline{69}$

"From this summary it will be seen that although nearly one-half of the thousand rats were on diets undoubtedly deficient, not a single case of the eye disease was observed in animals other than those experiencing a deficiency in fat-soluble vitamin in the ration. The incidence of the disease among this group is 50 per cent, somewhat higher than that observed by Stephenson and Clark, and lower than that reported by Emmett. It should be added that in observations on several thousands of rats we have never observed distinct symptoms of comparable eye disease in any animals except those which had experienced a deficiency of fat-soluble vitamin in their diet."

Among animals on diets otherwise adequate but devoid of vitamin A, the eye disease may occasionally appear before complete cessation of growth occurs. Most frequently the first slight indication of the eye 
disease very nearly coincides with the period in which the rat ceases to grow and begins to lose weight. Of the 69 cases above, reported by Osborne and Mendel, 33 showed eye symptoms at approximately maximum body weight; 15 after a decline of 10 to 20 grams from the maximum; 13 after a loss of 20 to 30 grams; 8 after a loss of more than 30 grams in body weight. The pathology of this eye disease has been studied by Wason (1921).

As the animals approach maturity their requirement for vitamin $\mathrm{A}$ appears relatively less acute because of the fact that they can remain in health for a longer time upon a diet deficient in this respect. In how far this means a real diminution in the vitamin $\mathrm{A}$ requirement and in how far it depends upon the fact that the older and larger animal, if it has previously been well fed, has a larger store upon which to draw in such emergencies is not entirely clear; and the solution of the question is complicated and difficult because our usual criteria become less serviceable as the young animal approaches maturity, since growth has now nearly ceased and it is reasonable to suppose that the conjunctival tissue will be less sensitive in an animal which has made a normal growth and development than in the young growing individual. Full grown rats when placed upon diets lacking vitamin $\mathrm{A}$ are less likely to develop the characteristic eye trouble than are young growing individuals; but, if the experiment is sufficiently prolonged, eye trouble appears in a considerable proportion of cases even of rats which had grown to maturity on good diets.

As shown more fully below, the relation of the eye disease to this dietary deficiency (lack of vitamin A) has been experimentally demonstrated in several other species as well as rats, and special interest attaches to the question as to whether the use of dietaries poor in vitamin A may be a factor in the susceptibility to eye trouble in man. Relatively early McCollum (1918) wrote, "There are several instances of the occurrence of conditions described in the literature as xerophthalmia, which seem to be beyond question, cases in which the disease has occurred in man as the result of specific starvation for the dietary essential, fat-soluble A." The reports here referred to are those of Mori (1904) in Japan whose cases of xerophthalmia occurring among children at a time of food shortage could be cured by feeding chicken livers (liver being rich in vitamin A) and those of Bloch (1917) whose cases among the children of the Danish poor also responded to the feeding of foods rich in the A-vitamin. Wells found in Roumania, and Dalyell in Vienna, cases of children suffering from eye trouble which could be cured by cod-liver oil and presumably 

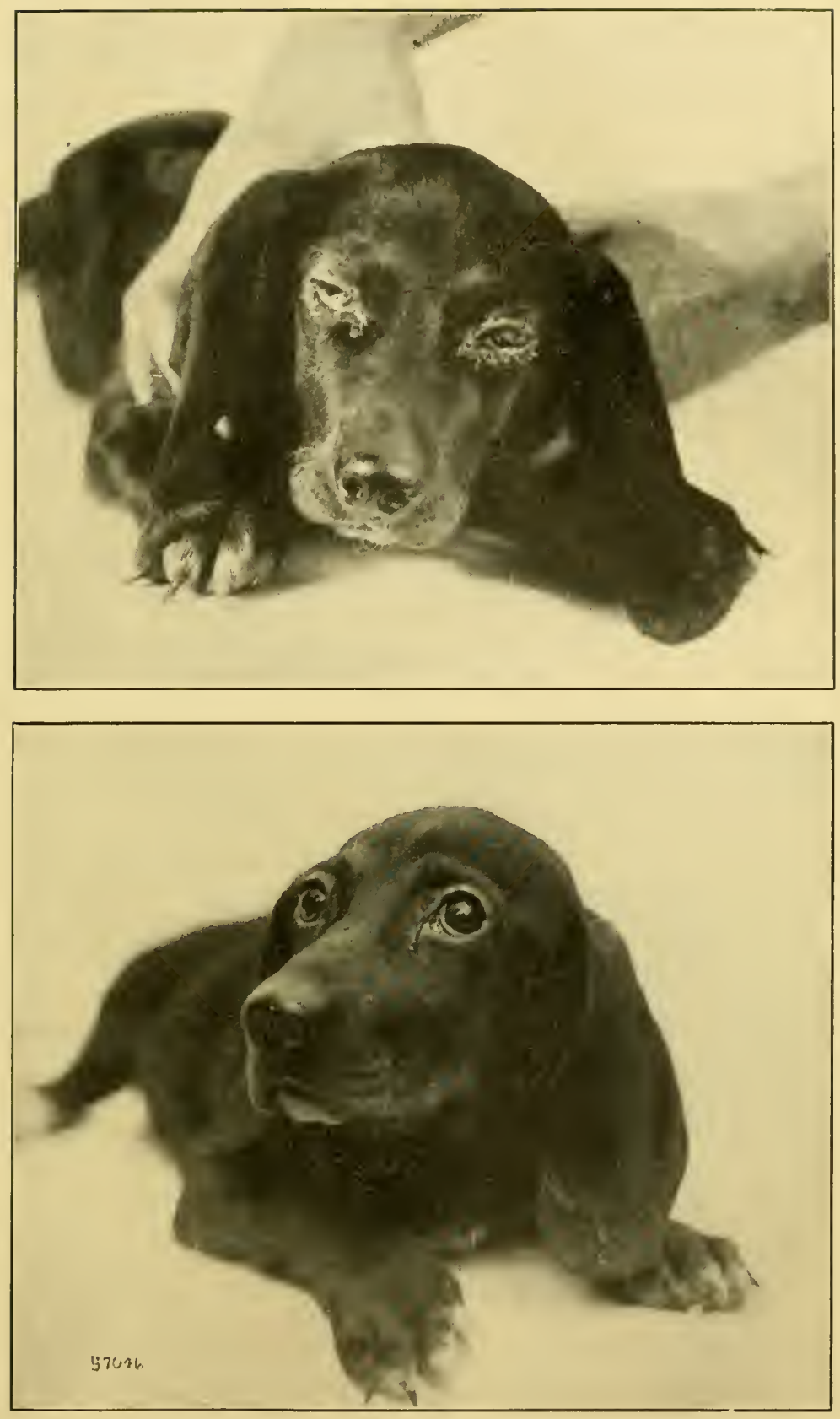

FIG. 11.-(Upper photograph.) Ophthalmia in dog as the result of diet deficient in vitamin A. (Steenbock, Nelson and Hart, 1921.)

FIG. 12.--(Lower photograph.) Same dog shown in Fig. 11 cured in 10 days by addition to his diet of 20 cubic centimeters cod-liver oil per day. (Steenbock. Nelson and Hart, 1921.) (By permission of the American Journal of Physiology:) 

arose from a deficiency of vitamin A in their food (Blunt and Wang, 1921); and McCollum, Simmonds and Parsons (1921) have attributed "night blindness," an eye trouble of frequent occurrence in Northern regions, to the use of diets poor in this vitamin. Appleton (1921), on the other hand, appears to doubt the dietary origin of night blindness. More recently Fridericia and Holm (1925) have studied the relation between malnutrition and night blindness by means of observations upon regeneration of visual purple, bleached by action of light upon the eyes of rats on normal diet or on diet deficient in vitamin $\mathrm{A}$ or in vitamin $\mathrm{B}$. They summarize their work in part as follows :

"Rats starved for A-vitamin and corresponding control rats receiving an adequate diet were examined as to the amount of visual purple in their retinae and their faculty of regenerating the color of the bleached visual purple.

"No influence of starving for A-vitamin on the amount of visual purple in the retinae of rats kept in darkness has been found.

"When the visual purple of the retinae has been completely bleached by exposure of the rats to light, the regeneration of the purple is delayed in rats starved for vitamin $\mathrm{A}$ as compared with control rats receiving an adequate diet. ... The abnormality in rats starved for A-vitamin as to the regeneration of the bleached visual purple occurs earlier than pronounced symptoms of xerophthalmia.

"The abnormality in the regeneration of the bleached visual purple has not been found in rats starved for B-vitamin. In human beings a relation between xerophthalmia and a kind of night blindness, often preceding the xerophthalmia, has been suggested by several authors. This kind of night blindness is by some authors thought dependent on a deficiency in the diet of A-vitamin, by others on the exposure of the eyes to intense light. If this kind of night blindness depends on a defect in the function of the visual purple, identical to that observed in rats starved for A-vitamin, both views contain part of the truth."

Holm (1925) reported experiments in which he found that hemeralopia develops as the joint effect of shortage of vitamin A and much exposure of the eyes to light. ". . . the hemeralopia appears very early during the avitaminosis, as is also seen in man."

The observations of Bloch referred to above, as well as some later investigations by the same author, were republished in English (Bloch, 1921) under the auspices of the British Committee on Accessory Food Factors, on account of their unusual interest when considered in conjunction with the experimental production of the disease in rats by 
a deficient diet. The xerophthalmia observed among these Danish children was regarded as one phase of their condition of malnutrition. The eye disease appears to have been quite definitely related to deficiency of vitamin $\mathrm{A}$ in the diet since it was cured by feeding with whole milk or with cod-liver oil and in 1918 the disease nearly disappeared from the community coincidently with the general introduction of butter into the dietary of the poorer people, this being brought about through government food regulations. Seasonal variation of the incidence of the disease was studied and it was found to be most prevalent in the season at which children make their most rapid growth. "As xerophthalmia is caused by the absence of something essential to growth, it is logically to be expected that the disease will predominate during the part of the year when the organism consumes the largest quantity of this lipoid material (vitamin A) for its growth, as is here found to be the case. It is also suggested that the specific lipoid (vitamin A) may be necessary for the formation of antibodies against infection and may be continually used up in this process as well as in growth."

Still more recently, Bloch (1926) has reported observations upon two patients, a brother and sister, who came under his care both suffering from xerophthalmia and night blindness. Light baths had no curative effect whereas the girl recovered when given cod-liver oil and other vitamin-A-containing food. Here it was quite certain that the human eye disease was curable by dietary treatment and that the curative substance was vitanin $\mathrm{A}$ and not the antirachitic factor nor anything which the light-treatment could supply. The brother apparently could not absorb vitamin A normally from his digestive tract, for he was not cured until given cod-liver oil subcutaneously.

That lack of vitamin A may lead to weakness or abnormality of other tissues as well as those of the eye, has been shown by the work of several investigators. Osborne and Mendel (1921a) refer to diarrhea and diminished appetite as frequently resulting from this lack; and they have definitely correlated it (1917d) with the occurrence of phosphatic renal calculi among their experimental animals. McCollum (1917) and also Drummond (1919b) early reported increased susceptibility to infections of the respiratory system.

Steenbock, Sell and Buell (1921) confirmed this and suggested that the failure to develop the characteristic eye disease in some cases may be due to a measure of immunity acquired through the respiratory infection. As symptoms of the respiratory infection, the incidence of which is regarded by these authors "as part of the syndrome induced 
by fat-soluble vitamin deficiency," they mention "a nasal or bronchial catarrh or even pulmonary infection with mucous or purulent exudate, at times even resulting in hemorrhage. Animals thus afflicted in the early stages of the disease sneeze and cough violently but later as the inflammation becomes confined more to the lungs, the cough subsides, and dyspnea becomes very pronounced with the slightest activity."

In agreement with other observers, these authors have also noted evidences of cutaneous malnutrition in animals deprived of vitamin A. Such affections are more usual in rats over four months old and may take the form of scabbiness of the tail or ears, sores or abnormal growths on the nose, thin and bushy hair, and sore feet.

They suggested that rats on diets suspected of being deficient in vitamin A should be observed for all such symptoms as well as for eye disease and failure of growth, and that judgment be based on the sum total of indications (Steenbock, Sell and Buell, 1921).

In the work of Sherman and Munsell (1925), in which rats 28 or 29 days of age were placed upon diets devoid of vitamin A, i.e., the "negative controls" of experiments for the measurement of vitamin A values of foods, it was found that 85 per cent of the cases developed unmistakable eye disease before death and that distinct gatherings of pus in one or more of the glands near the base of the tongue appeared in 76 per cent of the cases. In this series of observations, therefore, pus near the base of the tongue (found by careful search at autopsy) was almost as constant a result of the vitamin A deficiency as was the well-developed eye disease. Further observations in the same laboratory, however, lead to the belief that, with sufficient experience and close observation, a very slight swelling of the eyelids and/or discoloration at their junction may be detected after the depletion of bodily reserves in practically all cases of rats kept from four weeks of age upon vitamin-A-free food. (Personal communication from Dr. F. L. MacLeod, 1927.)

Sherman and Storms (1925) also found abscesses near the base of the tongue in about three-fourths of the rats dying from vitamin A deficiency; pus in the middle ear with about equal frequency; sinusitis somewhat less frequently (in about one-half of the cases examined for this condition). These observers also found that when rats of the same stock and previous nutritional history were placed upon the same vitamin-A-free diet at different ages, the ophthalmia developed in a larger proportion of the younger, and respiratory disease in a larger proportion of the older animals.

For the rats just mentioned, transferred from the same normal diet 
to the same vitamin-A-free diet at different ages, the average survival periods were as follows: four-weeks-old rats, 63 days; two months, 111 days; three months, 122 days; four months, 148 days; six months, 171 days; nine months, 140 days.

Thus, on an accurately known diet, which furnishes vitamin A in fairly liberal but not strikingly large proportion, the bodily store as indicated by survival period on vitamin-A-free diet, was found to increase steadily up to six months (in the rat) or about the age at which full adult size has been attained.

That a moderate difference in the vitamin A content of the food, whether during infancy or later life, may result in the acquisition of very significantly different stores of this vitamin by the body was shown by Sherman and Kramer (1924).

Naturally, regularity of outcome is not to be expected in all cases; for a striking result of vitamin A deficiency is that it increases the susceptibility of the body to bacterial invasion at many points and does not kill in such a specific way as does, for instance, a lack of vitamin C.

Wolbach and Howe (1925a) also found that vitamin A deficiency causes a number of pathological changes in addition to those of the eye. These include the presence of abscess-like cavities filled with a yellow cheesy-like material at the base of the tongue, in the pharynx, and in the submaxillary glands. The abnormalities here noted at the base of the tongue are undoubtedly of the same nature as those referred to above as observed by Sherman and Munsell and by Sherman and Storms. Wolbach and Howe found on microscopical examination that the cavities are cysts lined with a stratified keratinizing epithelium and the cheesy mass a mixture of desquamated keratinized cells and leucocytes. Their study of the lesions in rats killed at progressive stages of vitamin-A deficiency showed that the principal change is "transformation of various epithelia into a stratified squamous keratinizing epithelium. This change is practically constant in the upper respiratory tract including the whole of the nasal passages, larynx, trachea, and bronchi." They also reported that all the salivary glands and accessory salivary glands were found affected, and that similar changes occur late in the pancreas; and that, in the genito-urinary tract, this transformation into keratinized epithelium is found in the renal pelvis, bladder, seminal vesicles, epididymis and prostate gland. In all of the glands noted there was a considerable degree of atrophy before the change in character of the epithelium takes place. The histological change, characterized by transformation of cuboidal into squamous epithelium, they find to be directly due to the nutritional deficiency, infection follow- 
ing the weakening of the tissue. Goldblatt and Benischek (1927) and Tyson and Smith (1929) have confirmed the epthelial metaplasia as resulting directly from the vitamin A deficiency. Infection was universally present, and in the more severe cases dominated the picture.

Following corrective dietary treatment the xerophthalmia healed more quickly than the other lesions. Abnormal conditions of the epithelium and chronic or acute infectiun persisted in the tongue and renal pelvis after the rat outwardly appeared healthy.

Falconer (1926), referred to the report of Cramer, Drew and Mottram (1922) that vitamin-A deficiency diminishes the platelet count; but from his own work concluded that, "Changes in the relative number of platelets, red cells, and white cells in the blood of rats are not striking enough, or constant enough, to constitute specific lesion of vitamin-A deficiency in these animals." Falconer also considered the work of Bedson and Zilva (1923) to be in agreement with his own, the greatest diminution which they found being 21.3 per cent.

Cramer and Kingsbury (1924) tested the blood of vitamin-A-deficient rats for ability to develop agglutinins against various microorganisms and found no loss in agglutinating power. They concluded at this time that a deficiency in vitamin A does not diminish the efficiency of the general humoral defenses of the body, but only of the local tissue defenses. "Under the ordinary conditions of vitamin experiments, the breaking down of local defenses allows access to the comparatively avirulent bacteria which normally inhabit the intestine, the respiratory tract or the conjunctival sac, so that the resulting infections are as a rule avirulent in type. When organisms of a more virulent type are present, the condition of the local defenses rather than the mere presence of these organisms may be the factor which determines the onset of a virulent infection."

Turner and his associates (Turner, 1928, 1929; Turner, Anderson, and Loew, 1930) in studies of the bacterial flora of the various organs of rats in which lesions are most frequent as the result of vitamin A deficiency encountered types of pathogenic organisms in increasing frequency with increasing severity of the symptoms of A deficiency and concluded that "these organisms gain a pathogenic hold during the depressed state of their host resulting from vitamin A deficiency."

Further emphasis on the rôle of vitamin A as an anti-infective agent was given by Green and Mellanby (1928) in tabulated data showing the incidence of lesions and infections in various organs of rats suffering from vitamin A deficiency. Among the entire number of 93 vitamin-A-deficient animals examined, all showed lack of adipose 
tissue and general visceral atrophy and all but two showed evidence of infection in some site. Abscesses at the base of the tongue were found in 72 per cent of the entire number and in 90 per cent of those surviving for longer periods than the average. Infection of the lungs occurred in only 9 per cent, while xerophthalmia was evident in over 38 per cent, infection of the alimentary tract in 21 , infection of the kidney or bladder in 44 , and pus in the nasal sinuses or middle ear in 20 per cent. In none of the 50 control animals receiving vitamin $\mathrm{A}$ in the form of dried cabbage, cod-liver oil, or butter was there any evidence of such infection. In a series of several hundred rats receiving adequate vitamin $\mathrm{A}$ and little or no vitamin $\mathrm{D}$ only two instances of gross infection were found.

Mellanby and Green (1929) reported success in the treatment of human puerperal septicemia with vitamin A concentrates. In the first report it was announced that the five patients thus treated had made complete recoveries, while in the same hospital the records for the previous year (1928) had shown 18 cases with no recovery. Mellanby and Green state in their report "it is clear that, impressive as are the results described, they are too few in number to allow the deduction that this form of treatment is specific in its nature for septicemia. They do, however, warrant the belief that the animal experiments pointed correctly to the hypothesis that vitamin A plays a part as an anti-infective agent to bacterial infection, and has the property of raising the resistance of the body to such infection." In a later report Mellanby (1930) stated that of nine puerperal septicemia patients treated with vitamin A concentrates eight had made complete recoveries. In addition two other septicemic patients were similarly treated. One with an infection caused by Bacillus coli recovered, and the other with a staphylococcic infection died. A recent editorial in the Journal of the American Medical Association questions the clinical results of Mellanby and Green on the ground that the high mortality rate which they reported for the cases of puerperal septicemia not receiving vitamin A treatment indicated that in a large proportion of this group the intense fulminating type of septicemia was involved, while Mellanby admitted that the cases in which vitamin A concentrates were used were not of the fulminating type and consequently might be expected to have a low mortality rate. It is suggested, however, that on account of the promising results obtained by Green and Mellanby in their animal work, vitamin A should be given very extensive clinical tests.

At least one such test has been reported. Burton and Balmain (1930) tested the prophylactic effect of a concentrate of vitamin $\mathrm{A}$ in anti- 
toxic immunity, as demonstrated by the Dick test in a group of pregnant women attending an antenatal clinic and in patients recovering from scarlet fever with variable and inconclusive results. The administration of a vitamin A concentrate to one patient with puerperal fever appeared to have a favorable effect, but this was attributed to mere coincidence. Burton and Balmain concluded that "there is no prophylactic value in administering vitamin $\mathrm{A}$ in pregnancy to prevent the development of puerperal fever; also that it is doubtful whether the effects which have been described as following its administration for the purpose of treatment are not a coincidence. The conclusions based on experimental evidence of other workers have recorded that a deficiency of vitamin $\mathrm{A}$ in the diet predisposes to changes in the epithelial linings of mucous tracts in animals, and, therefore, allows infection to occur. This does not, in our opinion, justify a belief that the adequate supply of such vitamin would, in the presence of a sufficient infective dose and the absence of immunity, prevent the occurrence of infection." Cramer (1930) concurred with these views and protested against the use of "antiinfective" as a descriptive name for vitamin $\mathrm{A}$ alone. Admitting and emphasizing that an adequate supply of vitamin $\mathrm{A}$ is a powerful physiological prophylactic against infections entering by the mucous membranes, he states that "it may possibly be of therapeutic value also in the treatment of some chronic intestinal toxemias and of chronic infections of the respiratory and intestinal tracts, especially where these have been associated with defective nutrition. But that has yet to be demonstrated. There is no evidence, however, that vitamin A can cure infections once the barrier of the mucous membranes has been passed or that it can prevent or cure these infections which enter by the blood stream or by the subcutaneous tissues, as they do, for instance, in puerperal septicemia. To call vitamin A an 'antiinfective' vitamin is as much a misnomer as to call it a 'growthpromoting' vitamin. It seems unwise to exaggerate the extent of its real action and to make claims for it which can not be realized. Disappointment is sure to follow. This will discredit the more limited though really effective action of vitamin A of maintaining the physiological defenses of the mucous membranes, which are the portals of entry of most infections in man."

However, there has come from the University Hygienic Institute and the State Serum Institute, Copenhagen, a preliminary report by Lassen (1930) which appears to furnish evidence hitherto lacking, as Cramer has pointed out, of the cure of infections entering the blood stream by the subcutaneous tissues. Having shown that young rats, 
after development of pronounced xerophthalmia symptoms, were much less resistant than normal controls to infection by ingestion of a strain of paratyphoid bacilli, the experiment was repeated with subcutaneous injections of the bacilli instead of inoculation by mouth with like results. Further investigation along these lines will be awaited with interest.

Morgan and Osburn (1925) found that lack of vitamin A altered the quantitative relations of the nitrogen compounds of the urine, at least as regards allantoin, in explanation of which: "It is suggested that in the absence of vitamin A the animal organism fails to produce purine-containing compounds from the ordinary sources, perhaps arginine and histidine, but continues to utilize over again such portions of discarded purine-ring-containing substances as are ordinarily excreted in the form of allantoin. The portions of these compounds which are oxidized only to uric acid are apparently no longer usable and continue to be excreted in proportion to the amount of destruction of cellular material in vitamin-A deficient as in normal animals."

Cramer's suggestion that vitamin A may play both a structural and a humoral rôle in the body, is in a general sense confirmed by the accumulation of evidence that this vitamin increases resistance to infection (not necessarily through the ordinary immunological reactions) and by the fact that actual feeding experiments with tissues of animals of accurately known nutritional history have shown that the richness of the food in this substance may significantly influence the concentration of vitamin A in lung tissue, as well as the amount of the vitamin stored in the liver (Sherman and Boynton, 1925). This direct evidence from the feeding of the tissues as sole sources of vitamin A by quantitative methods such as have been described earlier in this chapter, leaves no room for doubt that it is chiefly the body's store of vitamin A which determines the length of the survival period upon a diet good in other respects but devoid of this essential factor.

Sherman and Cammack (1926) made further use of the method of measuring the survival period upon vitamin-A-free diet as a means of quantitative study of the storage of vitamin A in the body, particularly with reference to the problem of the times and conditions of feeding which may be expected to induce maximum storage of this vitamin by animals of different ages. All of the experimental animals were albino rats of the same stock and of accurately known age, pedigree, and nutritional history, taken from families which had been fed a mixture of one-third whole milk powder and two-thirds ground whole wheat with table salt in the proportion of 2 per cent of the weight of the wheat, the whole so ground and mixed as to ensure the consumption 
of the constituents of the diet in the exact proportions stated. The food mixture and distilled water were furnished ad libitum. Rats which had been separated from their mothers at the age of 28 days were continued either upon the above-mentioned diet alone, or upon this diet plus 1,2, or 4 per cent of cod-liver oil and then at the age of 3,4 , or 6 months transferred to the vitamin-A-free diet above described and kept under identical conditions for the determination of their survival periods. Taking the survival periods as measures of the relative amounts of vitamin A which had been stored under these different conditions of age and feeding, it is clear that at each age studied the animals which had received the richest intake of vitamin $\mathrm{A}$ had acquired the largest bodily store, and that at any given level of feeding, the bodily store became larger at 4 months of age than at 3 , and larger at 6 months than at 4 . It is not to be inferred, however, that the body stores quantitatively the entire surplus received in the intake, for the increases in survival period found are, while very striking and doubtless extremely important, not arithmetically proportional to the concentration of vita$\min \mathrm{A}$ in the food.

Evidently the limit of the body's capacity for storage of vitamin A is not quickly reached, whether regarded from the standpoint of the level of intake or the length of time during which a liberal intake is continued; and it is possible, by building up a bodily store through previous feeding, to prolong very greatly the length of time that an animal can survive upon a diet devoid of vitamin A.

Experiments of Sherman and Burtis (1928) have shown that the level of intake of vitamin A (and perhaps certain other of the chemical factors in nutrition) during early life, may markedly influence subsequent susceptibility to infection. They found that this influence of the level of intake upon the incidence of infection may be pronounced and long continued even when the differences of diet are no greater than may readily occur within the range of ordinary normal or adequate nutrition.

In young rats taken when 4 weeks of age, from families belonging to the same strain, and continued under conditions identical in all respects except as to diet, 38 animals coming from a diet consisting of a mixture of one-sixth dried whole milk and five-sixths ground whole wheat and 37 animals from a diet composed of the same food materials but with a larger proportion of milk (one-third of the dry weight instead of one-sixth), were alike placed upon vitamin-A-free food for periods of about 1 month, i.e., until in each case the surplus store of this vitamin in the body was depleted, and then received during a 
(further) test period of 8 weeks the same limited allowance of vitamin-A-containing food. Autopsies at the end of this test period showed infections established in 75 per cent of the animals which in early life had received the first diet and in only 25 per cent of those which had during the same early period received the diet with the larger proportion of milk. Taking account of the relative lengths of the rat and the human life cycle, and of the similarity of nutrition in the two species, this may perhaps serve as an indication of the differences of incidence of infection to be expected among children of around 10 and 12 years, resulting from differences in the way they were fed before they were 3 years old.

In terms of the chief chemical factors involved, the better diet here used was richer not only in vitamin A but also in vitamin $G$, in calcium, and in certain of the nutritionally important amino acids. It is hoped that experiments now in progress may more fully differentiate the rôles of these several chemical factors.

While the laboratory experiments referred to in the foregoing discussion of the relation of vitamin A to nutrition and health have been made chiefly upon rats, this is only because of the well-established general usefulness of these experimental animals. Other species, varying as widely as the fowl, the rabbit, the dog, the pig, and the monkey, have shown analogous susceptibilities to vitamin A deficiency (Guerrero and Concepcion, 1920; Nelson and Lamb, 1920; Steenbock, Nelson and Hart, 1921; Lamb and Evvard, 1922; Tilden and Miller, 1930).

The response of monkeys to vitamin $A$ deficiency has recently been studied by Tilden and Miller (1930) who while reporting that "the period of survival of monkeys on a diet deficient in vitamin A was shorter than was expected," nevertheless found evidence that, in this species as in others, storage of vitamin A in the body may be a factor of much nutritional importance. Regarding autopsy findings, they report that "the epithelial metaplasia described first by Wohlbach and Howe and later by Goldblatt and Benischek as characteristic of deficiency of vitamin A was found in one or another tissue of 9 of the 11 animals on the A-deficient diet, and was entirely absent in the controls."

The animals subjected to shortage of vitamin A also showed a high incidence of intestinal disease. The widespread weakening of the body tissues which results from shortage of vitamin $\mathrm{A}$ is thus shown to be true of the monkey as well as of the other species which have been studied, while the importance of taking a broad view of vitamin-Adeficiency instead of concentrating attention upon the eye symptoms is 
illustrated in the fact that these monkeys tended to die of the intestinal diseases largely due to vitamin A deficiency, before the development of ophthalmia.

In this connection it may also be recalled that Wohlbach and Howe did not observe ophthalmia in guinea pigs which showed the characteristic histological changes resulting from vitamin A deficiency. Evidently this deficiency may lead to a breakdown sometimes in one direction and sometimes in another.

As the body is evidently able to store relatively large amounts of vitamin $\mathrm{A}$, and as this store is plainly a very important safeguard and resource, it seems wise for those who can afford it to invest rather liberally at all ages in food rich in vitamin A, knowing that in this case the body will store the surplus to an extent and with an efficiency which is not to be expected in the case of most other nutrients.

Relation of Vitamin A to General Vigor.-As the result of his extended investigation of the dietary properties of the various types of food, McCollum concluded that dietaries deficient in vitamin A and in calcium content are of relatively frequent occurrence; that such deficiencies lower the vigor of the body and its ability to resist disease; and that as the result of such weakening there develops an increased susceptibility, so that the incidence of any of a number of diseases (of which tuberculosis serves as the outstanding example) may be largely influenced by the adequacy of the diet as regards vitamin $\mathrm{A}$.

Experiments upon laboratory animals have quite definitely shown that general vigor, as exhibited not only in growth but also in capacity for reproduction and successful suckling of the young, is dependent in large measure upon the amount of vitamin A in the food. Drummond (1919b) stated that a liberal allowance of vitamin A resulted in better reproduction, and McCollum as well as Steenbock and their associates have repeatedly published implied confirmation of this finding by reporting cases in which on diets which were being tested for vitamin A the amount of this factor appeared adequate for growth but not for reproduction, or for reproduction but not for rearing of the young. Reynolds and Macomber (1921) have also found a decrease in fertility to result from a paucity of vitamin A in the diet. More recently, direct comparisons of dietaries otherwise identical but differing in their content of butterfat (or by the substitution of lard or hardened vegetable oil for butterfat) have shown that such differences in the vitamin $\mathrm{A}$ contents of two diets, even though both are adequate for growth and apparently for general health, at least for a relatively long time, may have a most marked influence upon the capacity to 


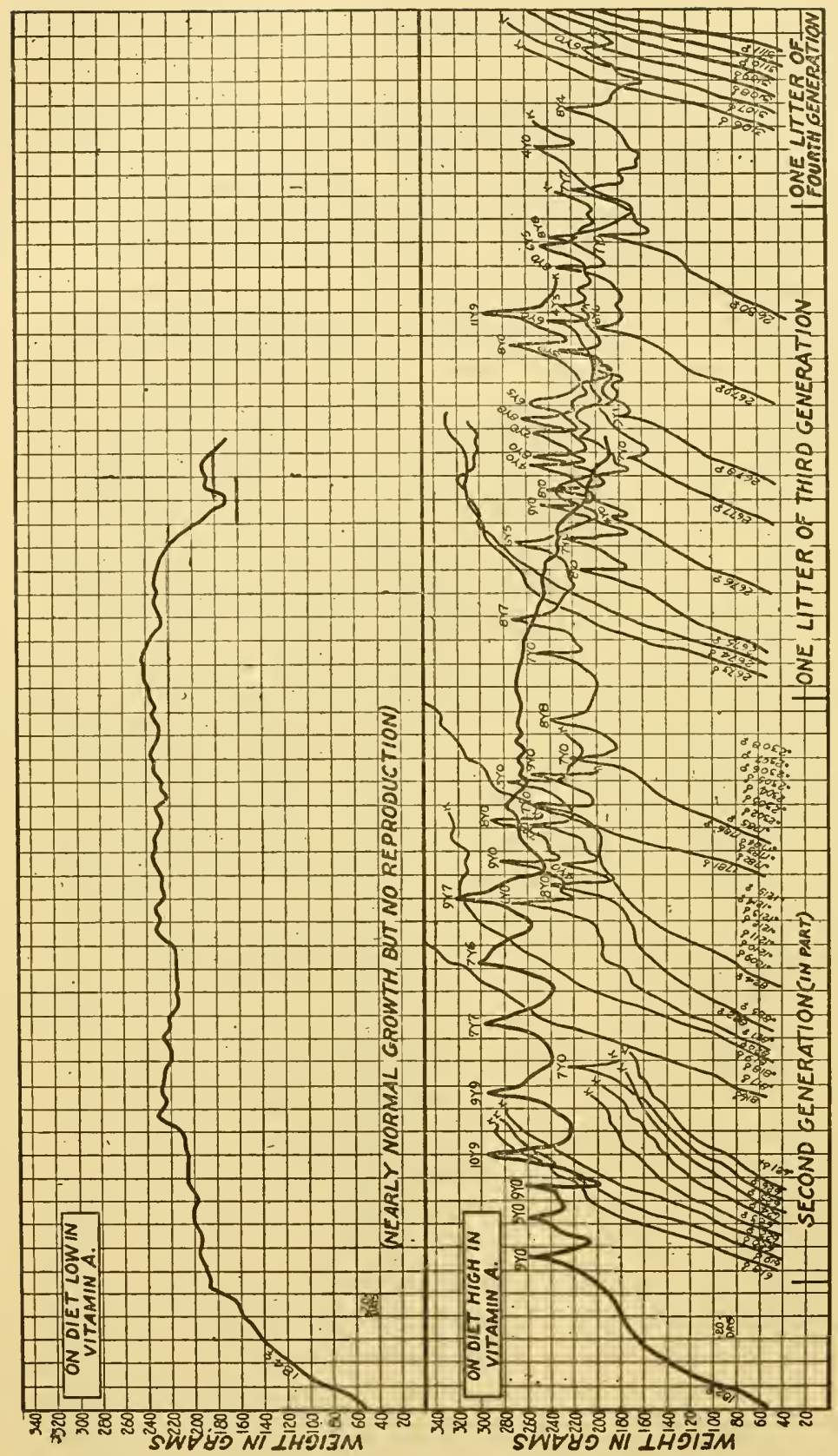

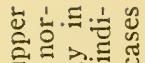

$\exists$

此

+

$\simeq$ 光.

$<$ 递

$\Xi<\%$

ह.

료

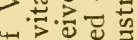

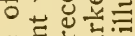

政.

坣焉志

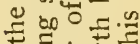

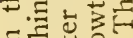

$\Xi w$.

of

政

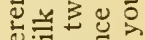

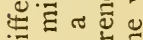

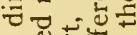

记

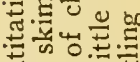

ฮี

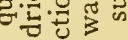

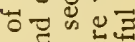

ส

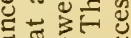

西

त

政

능

政范

+ 당

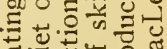

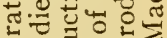

的㦴记

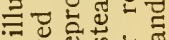

क. 론.

可论。

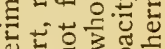

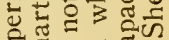

x.

o $5 . \Xi$ อ 1.

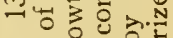

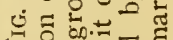

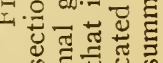


produce and rear young (Sherman and MacLeod, 1925; Batchelder, 1929). See Figs 13 and 14.

By means of the methods developed by Evans and his coworkers in the Department of Anatomy of the University of California, Evans and Bishop (1922) have thrown further light upon the significance of vitamin A in relation to reproduction. As summarized by Evans his studies "confirm the impression that rats may be successfully reared on diets poor in vitamin $\mathrm{A}$ if the diet is not too deficient in this essential. They may for months grow normally and not suffer from the so-called xerophthalmia. It must be admitted that we have not previously had a method for the detection of deficiencies in this vitamin which, nevertheless, permit fair growth and apparent health. Studies on the oestrous cycles of such rats show that they may suffer from an invariable and continuous abnormality or disfunction of the ovaries. It has previously been shown in this laboratory that ovulation in the rat can be detected in the living animal by a series of histological changes in the vaginal smear, changes which are correlated with the growth, maturation and rupture of the Graafian follicles at periodic intervals. When for any reason the follicles are unable to completely mature (as in animals treated with hypophyseal substance) vaginal oestrous changes are absent. A totally different picture is produced if follicles develop but are unable to rupture; under such circumstances, the oestrous changes may be remarkably prolonged, and the dioestrous pause in fact obliterated. As Evans and Long have shown, this occurs as a rare anomaly in large colonies of animals. But this prolongation of oestrous vaginal changes and failure of ovulation occurs in 100 per cent of animals reared on diets which are low in vitamin A but which have nevertheless permitted preliminary normal growth."

Later, Evans (1928) stated that inadequate vitamin A supply characterized by the constant appearance in the female of cornified cells either predominantly or exclusively in the vaginal smear, injures the female reproductive system so that fertilization and implantation usually fail, even though oestrus and ovulation occur fairly frequently.

In the above-mentioned work of Sherman and MacLeod (1925) the comparison of the effects of a low versus moderately liberal intake of vitamin A were not confined to observations upon reproduction but extended throughout the lives of the experimental animals with results which the authors have summarized as follows: "The smaller amount of vitamin A proved sufficient for normal growth up to nearly normal adult size, but not for successful reproduction, and rarely did it support satisfactory longevity. The parallel animals receiving the more 


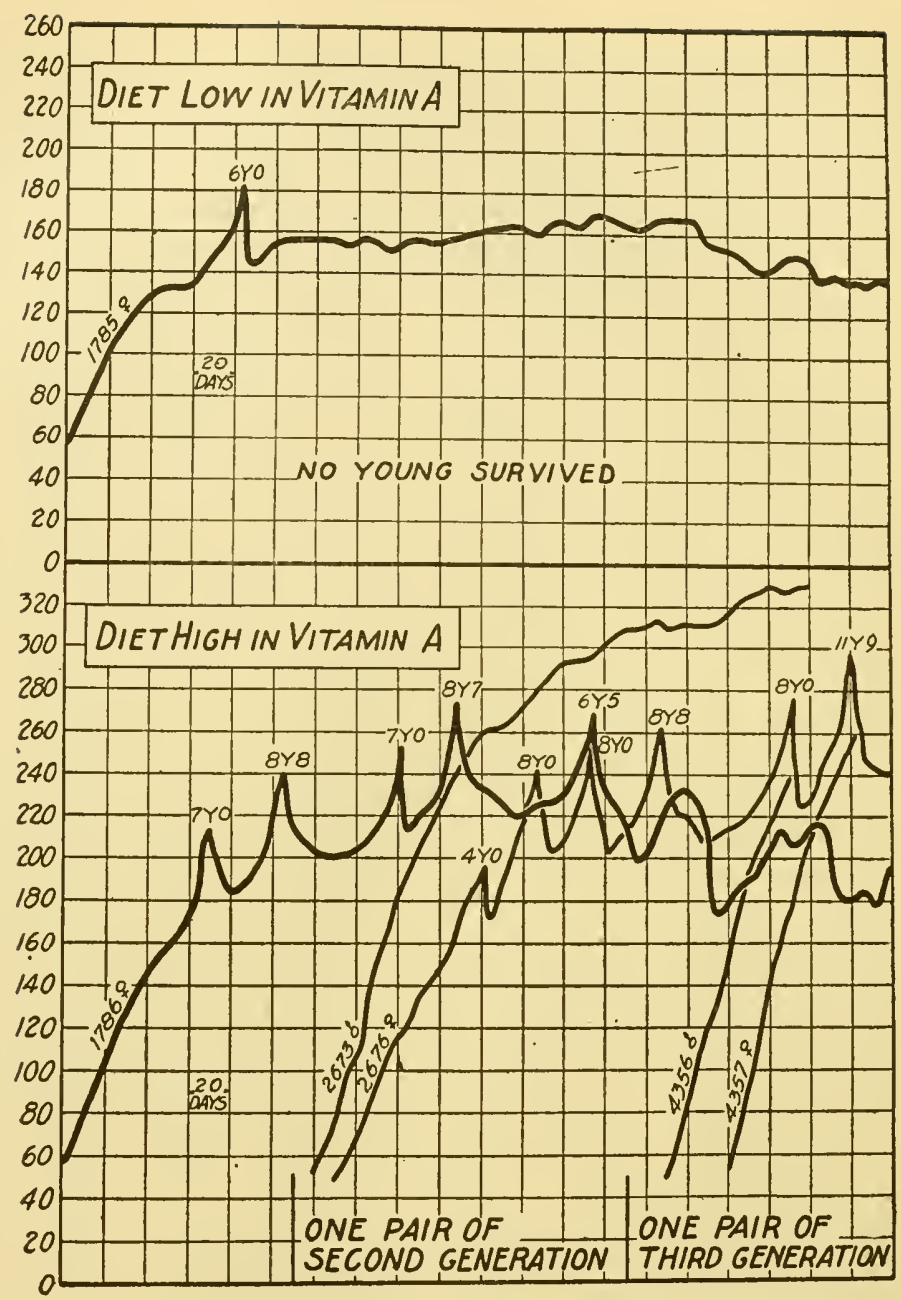

FIG. 14.-Showing comparison of diets containing different amounts of vitamin A similar to the comparison shown in Fig. 13 except that in this case the diets consisted of whole wheat, dried skimmed milk and lard or butter respectively, so as to contain the same proportion of fat and differ only in fat-soluble vitamin contained. Again there was little difference in growth but the diet containing the higher proportion of vitamin A resulted in a much higher degree of vigor as shown by capacity for reproduction and successful suckling of young. In this case only one pair each of the second and third generations is charted. (Sherman and MacLeod, 1925.)

liberal allowance of vitamin A grew to fully average adult size, were successful in reproduction and the rearing of young, and lived on the average a little over twice as long as those on the diet equally good in all other respects but lower in vitamin $\mathrm{A}$. These experiments show 
strikingly that a proportion of vitamin A in the food sufficient to support normal growth and maintain every appearance of good health, for a long time at least, may still be insufficient to meet the added nutritive demands of successful reproduction and lactation.

"Along with the failure to reproduce successfully there usually also appeared in early adult life an increased susceptibility to infection and particularly a tendency to break down with lung disease at an age corresponding to that at which pulmonary tuberculosis so often develops in young men and women. The bacillus involved is different; but the close parallelism of increased susceptibility of the lung to infection at this stage of the life history appears very significant, especially in view of the fact recorded in another paper from this laboratory that the vitamin-A-content of lung tissue varies with that of the food. Especially noteworthy was the repeated observation of young females growing normally and presenting every appearance of good health throughout youth on a diet low in vitamin A, but failing utterly to succeed in the rearing of a second generation, and showing a strong tendency to break down in health at an age at which they should have been in the prime of life."

Batchelder (1929) studied further the effects of successive diminutions of vitamin $\mathrm{A}$ in the food on the nutrition and vitality of all ages in experiments in which rats were fed diets containing 8.22, $4.11,2.06,1.03,0.51$, and 0.00 per cent butterfat. The diets highest and lowest in butterfat were identical with the Sherman and MacLeod diets in vitamin A content. The vitamin A content of the diets decreased practically in proportion to the amount of butterfat present, the vitamin A contents of the other constituents of the diet being shown to be insignificant. Each of the original animals entered the experiment with a bodily store of vitamin.

The only difference here established between rats on 8.22 per cent butterfat and rats on one-half that amount, was that the offspring of the former had larger stores of vitamin $\mathrm{A}$ in their bodies.

A lower weight at all ages was observed for each decrease in butterfat below 4.11 per cent. A significant difference in the weight of young at 28 days was found in rats on diets containing between 4.11 and 2.06 per cent butterfat; in the number of young reared by rats on diets containing below 2.06 per cent; and of young born among rats on diets containing below 1.03 per cent butterfat. Rate of growth, duration of reproductive life, and longevity also were decreased in rats on diets containing significantly below 1.03 per cent of butterfat. 
Tentatively, the less complete data for longevity indicate a distinct tendency toward longer life when the diet is richer in vitamin A.

Urinary calculi were visible to the naked eye in rats on diets very low in vitamin $\mathrm{A}$; but not in rats receiving no vitamin $\mathrm{A}$ at all, probably because of their early deaths. Van Leersum (1928) has reported microscopical examinations showing urinary calculi and calcium deposits present in 88 per cent of rats on a vitamin A-free diet. Evidently these deposits are enabled to grow to macroscopic proportions in rats which, though dying for lack of vitamin $\mathrm{A}$, have had their life prolonged a few weeks beyond that of negative controls.

From these and other observations it is concluded that vitality is affected in various ways by a deficiency of vitamin A, successive diminutions of vitamin $\mathrm{A}$ in the diet first lowering the positive health and then resulting in increasing evidence of nutritive failure.

These results, therefore, confirm and extend the findings previously recorded which had led to the conclusion, "that vitamin A is an even more important factor in the chemistry of food and nutrition than has previously been appreciated, for it must be supplied in liberal proportion not only during growth but in the food of the adult as well, if a good condition of nutrition and a high degree of health and vigor are to be maintained." 


\section{CHAPTER VI}

\section{VITAMIN D}

The medical literature of rickets is authoritatively reviewed both historically and critically by Hess in his recent book Rickets, Osteomalacia and Tetany. In view of this, and of space limits here, the present chapter is confined to the period and literature of the conscious investigation of the vitamin factor.

Mellanby, by his extended study of experimental rickets (1919), showed that cod-liver oil and butterfat were effective in preventing the disorder in puppies subsisting on diets consisting largely of cereal with small allowances of milk. Some of his experiments indicated that still other materials prevented rickets, but these results may have been influenced by the puppies' preexperimental bodily storage of the antirachitic factor, or by the inorganic relations of the food. His results were, for the most part, in keeping with the view that rickets is due primarily to a nutritional lack. He concluded that it is "probable that the cause of rickets is a diminished intake of the antirachitic factor, which is either fat-soluble $A$ or has a somewhat similar distribution to fat-soluble A." This view was adopted, although with some reservation, in the British Committee report of 1919 (Hopkins, Chick, Drummond, Harden and Mellanby, 1919) and was for a time so far accepted as to lead some writers to include prevention of rickets as one of the functions of vitamin A.

Hess and Unger (1919-1920) held that, in their clinical experience, rickets sometimes developed in infants receiving large quantities of milk containing what they believed to be ample fat-soluble vitamin.

In 1921, Sherman and Pappenheimer (1921, 1921a) showed that rickets may be caused or prevented in rats by changes in the mineral elements of the food. Their experimental diets were practically devoid of fat-soluble vitamins; but the animals had not been depleted of their bodily stores of such vitamins.

In the same year Shipley, Park, McCollum and Simmonds (1921) reported that the phosphate ion may be the determining factor in certain cases; whereas previously they had worked on the hypothesis of a joint deficiency of calcium and vitamin $A$. 
McCollum, Simmonds, Shipley and Park (1921, 1922) contrasted cod-liver oil with butterfat in the protection given rats against the effects of insufficient calcium in the diet. They stated that "cod-liver oil contains in abundance some substance which is present in butterfat in but slight amounts, and which exerts a directive influence on the bone development and enables animals to develop with an inadequate supply of calcium much better than they could otherwise do. This substance is apparently distinct from fat-soluble A, which is essential for growth and which is associated definitely with the prevention of ophthalmia (keratomalacia)."

The experimental evidence offered in their paper consisted chiefly of feeding tests with rats upon diets poor in calcium (such as mixtures of seeds with the addition of casein and sodium chloride) from which they found that, "On a diet such as we employed, young rats are much better nourished when supplied with $1 \%$ cod-liver oil than with 10 to 20 per cent of butterfat, as is shown by better growth, fertility, success in rearing young, and in length of life. This is true, notwithstanding that $3 \%$ butterfat is ample for providing the animals with sufficient fat soluble $\mathrm{A}$ and any other organic substance exerting a special effect on the bones when the content of calcium in the diet is raised to approximately one-half the optimal. The provision of nearly seven times this amount does not exert much protection against the specific detrimental effect of lack of calcium when the content of the diet in this element is $1 / 15$ to $1 / 5$ or $1 / 6$ this optimal amount. One per cent of cod-liver oil, on the other hand, seems to increase in a very remarkable manner the effectiveness with which the anatomic elements of the body tissues deal with a very low calcium supply."

In the light of the experiments of Sherman and MacLeod (1925) it would appear that McCollum, Simmonds, Shipley and Park underestimated the importance of an allowance of vitamin A higher than that which 3 per cent of butterfat would usually furnish, and therefore in this paper may have attributed to the antirachitic vitamin some nutritional effects which were really due to the greater amount of vitamin A furnished by the cod-liver oil than by butter. However, a greater calcium-conserving effect of cod-liver oil than of butterfat was clearly postulated, and the attribution of this effect to a distinct "antirachitic" vitamin rather than to vitamin A was supported by experimental evidence.

Their results, they attributed to "a substance which plays an important rôle in influencing the anatomic elements in the osseous tissue and may be designated as a calcium-depositing or phosphorus-mobilizing 
factor. Butter fat is richer in fat-soluble A than in the calcium-depositing factor. Cod-liver oil is exceptionally rich in both substances. It has only been found possible up to the present time to demonstrate the differences between these two fats by using diets poor in calcium, for with the low intake of this element there is a correspondingly high requirement of the substance which we have spoken of as a calciumdepositing factor, but which is more than this. It exerts a distinct influence on the anatomic elements of the growing bone and enables the osteoblasts to form approximately the optimum amount of osteoid tissue. It leads to the deposition of calcium phosphate in a degree which is not possible in its absence when the calcium content of the diet is very low. It improves the general well-being of the animals."

At about the same time Hart, Steenbock and Hoppert (1921) discovered through calcium balance experiments with milking and dry goats, the presence of a calcium-conserving factor in cod-liver oil and in green oats and good oat hay; but not in dry oat straw. This factor in plant tissues, they demonstrated, is distinct from vitamins A, B, or $\mathrm{C}$, and functions in the calcium economy of adults as well as of young.

Later, in July 1922, Hart, Steenbock, Hoppert and Humphrey compared the efficiency of dry and green alfalfa hay in maintaining calcium and phosphorus equilibrium in milking cows. Forbes and his coworkers had shown previously that feeding dry alfalfa hay under conditions of liberal milk production usually results in a considerable loss of calcium. Hart and his coworkers found that this loss can be prevented if the hay has been carefully cured under caps, and can be turned into a decided positive balance if an equivalent amount of fresh alfalfa is fed. They concluded that, "Apparently the question whether positive or negative balances will prevail in liberally milking cows through the use of such an efficient carrier of calcium as alfalfa hay is determined by the quality of the alfalfa hay used. By the term quality, used in this connection, we mean the relative degree of destruction in the curing processes of the unknown factors affecting calcium assimilation."

Bogert and Trail published, in 1922, two papers relating to the inorganic metabolism of women, in which they reported that the addition of yeast or the substitution of an equal weight of purified butterfat for purified fat from nut margarine in a diet very low in vitamins exerted a favorable influence upon the calcium, phosphorus and magnesium balances of the subjects studied.

In August 1922, McCollum and his coworkers furnished explicit 
evidence of the existence of vitamin $\mathrm{D}$, as an entity distinct from vitamin A.

They reported experiments comparing: (1) the potency of different oils to cure xerophthalmia; (2) the values of these same oils in promoting growth of young rats on diets very deficient in calcium; and (3) the values of these oils for inducing deposition of calcium salts in rachitic bones by means of the "line test" (described further on in this chapter). In discussing and summarizing their results, they stated that "cod-liver oil oxidized for 12 to 20 hours does not cure xerophthalmia in rats." It does, however, cause the deposition of calcium in the bones of young rats which are suffering from rickets. This shows that oxidation destroys fat-soluble A without destroying another substance which plays an important rôle in bone growth. They wrote:

"Cocoanut oil is shown to be lacking in fat-soluble A since it will neither prevent nor cure xerophthalmia. This oil, on the other hand, contains a substance which stimulates the deposition of calcium salts in a manner similar to cod-liver oil. It is, like butterfat, far less effective from a quantitative standpoint.

"The evidence set forth in this paper demonstrates that the power of certain fats to initiate the healing of rickets depends on the presence in them of a substance distinct from vitamin A. These experiments clearly demonstrate the existence of a fourth vitamin whose specific property, as far as we can tell at present, is to regulate the metabolism of the bones.

"Animals can undergo apparently normal development when furnished a very small amount of the calcium-depositing vitamin, provided the diet contains somewhere near the optimal content of calcium and phosphorus. The adjustment of the calcium content of these diets at a very low level has enabled us to demonstrate the difference in the quality of fats in respect to their content of calcium-depositing vitamin in a manner that would have been impossible if the diets had contained a more favorable concentration of calcium and phosphorus."

Steenbock and Nelson (1923) confirmed McCollum's finding that the antirachitic and antiophthalmic vitamins are distinct entities in a very extensive and carefully planned series of experiments.

\section{Ultra-violet Light and Vitamin D}

In 1913 Raczynski found sunlight favorable to calcium assimilation in puppies, and definitely suggested a connection between the absence of sunlight and rickets.

Huldschinsky $(1919,1920)$ reported that ultra-violet light cured 
children of rickets. Observations by $\mathrm{X}$-ray showed definite calcium deposition after four weeks, with complete healing after eight. He also found (1920) healing of rickets in 30 children after 22 to 26 treatments covering 2 months of time. Hess and Unger (1921b) confirmed this by showing that exposure to ultra-violet light or sunshine, without any modification of the diet would cure infantile rickets. Even though only one wrist was exposed to light, calcification occurred in both, as judged by $\mathrm{X}$-ray and clinical examination. They reported further, that exposure to sunlight prevented rickets in rats on a ricketsproducing diet.

Howland and Kramer (1921) reported that the serum of infants suffering from rickets contains a diminished amount of inorganic phosphate which, during the process of healing, gradually rises to the normal level; and Hess and Gutman (1922) reported that the cure of rickets by sunlight could be also demonstrated by the rise to the normal level, of the inorganic phosphate of the blood serum. Of this work they said that "it is the first definite evidence of a metabolic change in the animal body brought about by solar rays," and stated it as their belief that the "curative process occasioned by divergent therapeutic agents (cod-liver oil and light) is fundamentally the same."

In studies on 60 young children, Hess and Lundagen (1922) found a seasonal tide of blood phosphate which could be correlated with the seasonal variation of ultra-violet light waves in the sun's spectrum. They say "these meteorological observations confirm our opinion that the dominating factor in the phosphate tide is sunlight, an opinion which is strengthened by the fact that an increase of blood phosphate was brought about during these winter months simply by irradiations of ultra-violet light from an artificial source. In this connection it is also well to bear in mind that rickets and tetany are almost non-existent in the tropics."

This view that it is the short light rays which are curative of rickets was confirmed by the finding by Hess, Pappenheimer and Weinstock (1922) that light waves longer than $334 \mu \mu$ are ineffective while shorter than $310 \mu \mu$ are effective in preventing rickets in rats on a drastically rickets-producing diet.

Hess (1925) reported that from June to August rays from the sun as short as $297 \mu \mu$ penetrate our atmosphere between 11 and 1 o'clock, while from December to February only the longer potent rays, those of about $313 \mu \mu$ are able to reach us. Hess and Anderson (1927) found that with an equal number of ergs of energy per unit surface area, rays shorter than $290 \mu \mu$ were more antirachitic than those between 
290 and $313 \mu \mu$ in wave length. These shorter rays penetrate dark clothing or a heavily pigmented skin less readily than light clothing or a white skin. This indicates one factor in the exceptional susceptibility of negro infants to rickets (Hess, Unger and Pappenheimer, 1922a).

Within a few years (1921-24) evidence was accumulated in many laboratories which showed that irradiation of the body with light rays of short wave length from sunlight, mercury vapor quartz lamps, or from carbon arc lamps promoted growth and improved ossification in children, rats, chicks and pigs, particularly when the diets were decidedly unsatisfactory in the relations of calcium and phosphorus. (As more fully explained elsewhere, this is not to be construed as indicating that irradiation, or vitamin $\mathrm{D}$ in any form, can take the place of the needed calcium.) X-ray studies, analyses of the blood for calcium and inorganic phosphorus and bone analyses were the chief methods employed to study these effects.

Steenbock and Nelson (1923) found 10 minutes of irradiation daily with ultra-violet light to be as effective as 2 per cent aerated cod-liver oil in promoting growth which had failed before the incidence of ophthalmia in their rats on a diet free from fat-soluble vitamins, but otherwise adequate.

Hart, Steenbock and Elvehjem (1924) reported that ultra-violet light can influence the storage of calcium and phosphorus and the equilibrium of these elements in the blood stream of mature animals in a way similar to its effect upon growing animals. Two mature lactating goats and one mature dry goat were brought into distinctly negative calcium balances or calcium equilibrium on a diet deficient in the antirachitic factor; irradiation with a mercury vapor quartz lamp brought them into positive balance. The inorganic phosphorus of the blood was also increased.

Hart, Steenbock, Elvehjem, Scott and Humphrey (1926) found that June sunlight (Wisconsin) could lessen but not obviate the losses of body calcium suffered by cows giving 45 to 60 pounds of milk per day while receiving a ration which contained 70 or 80 grams of calcium oxide. But by increasing the intake of calcium oxide to 200 grams, calcium equilibrium could be established even when the metabolism experiment was conducted indoors.

This work indicates that vitamin D or ultra-violet light can help the organism to utilize advantageously the available calcium or phosphorus, but cannot replace an actual deficiency.

Meanwhile investigators asked if light has also the property of producing, or functioning as an equivalent of, vitamin A. 
Sheets and Funk (1922) concluded that ultra-violet light had no effect upon growth or the incidence of ophthalmia in animals on a diet free from fat-soluble vitamins.

Powers, Park and Simmonds (1923) found that the development of pronounced xerophthalmia was delayed in those of their rats which were exposed to sunlight. They concluded that, "Sunlight possibly exerts no specific antixerophthalmic influence, but acts by raising the level of cellular activity of the organism to a point where the onslaughts of the disease are held in check or allowed to advance very slowly and with relatively little disturbance. It may be true that the nearer the animal to maturity the less vulnerable it is to the effects of a xerophthalmia-producing diet." ... "Previous experiments have shown that the unknown factor in cod liver oil which promotes normal formation of bone (and in that sense may be spoken of as antirachitic) has an equivalent in sunlight. The experiments herein reported indicate that sunlight does not contain at all or only to a very slight degree the equivalent of the antixerophthalmic factor in cod liver oil."

Hume (1922) found that, with rats of matched nutritional history, the irradiated animals developed the typical symptoms of vitamin-A deficiency with greater severity and greater rapidity than did control animals, and that irradiation exerted no curative effect in animals which had succumbed to xerophthalmia, but rather seemed to increase the severity of the symptoms. She states that: "Early cessation of normal growth followed by a long period of maintenance might almost be regarded as a defense mechanism, which enabled the rat to tide over a period of vitamin A famine, such as might easily be a danger in the normal life of a rat. When irradiation is applied it would seem as if, either the low level of vitamin A metabolism is rendered sufficient, i.e., the vitamin is economized-or else the vitamin stores are forced to yield up vitamin $\mathrm{A}$ at a rate sufficient to produce normal growth until the stores are wholly exhausted. This effect can apparently be produced at any time before the end of the period of slow growth, and the amount of normal growth produced at any time would appear to be roughly inversely proportional to the length of time any individual rat has previously been on a deficient diet. Ultimately, when presumably all the stores are exhausted, no normal growth can be evoked by irradiation. Hence it is clear that light can neither create or be substituted for the vitamin; it appears only to act as an economiser or activator when it is already present."

Similar results were reported by Goldblatt and Soames (1922).

Steenbock and Nelson (1923) commented on the paradox of light 
liberating vitamin A for growth but not for curing ophthalmia, and concluded, after a critical examination of Hume's original data, that growth in Hume's animals ceased because of an insufficiency of the antirachitic vitamin as distinguished from vitamin A. When its deficiency was compensated for by radiation, growth was restored and continued until vitamin A was exhausted; then ophthalmia made its appearance and rapid failure supervened. Their theory postulated that the antiophthalmic and antirachitic properties are resident in distinct entities, as proposed by McCollum, Simmonds, Becker and Shipley, and attempted to explain the variable results obtained by different investigators on the basis of variation in the relative amounts of vitamin A and antirachitic vitamin stored in the body of the experimental animal. Regarding their own experiments, they said, "Young rats raised on our stock ration" (poor in the antirachitic factor as compared with its content of vitamin A) "and transferred to a purified ration carrying yeast as its only source of vitamins, will grow for a few weeks then cease growing completely or partially and ultimately will fail due to the incidence of ophthalmia or infections of the respiratory tract. Aerated cod liver oil or light from a quartz mercury-vapor lamp, both well known as antirachitic agents will eliminate the initial failure of growth or when prevalent, will restore it without appreciably postponing the final failure due to ophthalmia or respiratory diseases." They also found that after the incidence of ophthalmia irradiation of animals did not promote growth. Evidently both vitamin A and vitamin D are necessary for growth.

Occasionally experiments may be complicated by the fact that ultraviolet light has a deleterious effect on the eyes. Goldblatt and Moritz (1926) reported that "Direct irradiation induces a conjunctivitis and opacity of the cornea which complicates other eye changes induced by the diet, and also probably affects the general condition of the animal."

Thus while it is now generally accepted that light is not the equivalent of vitamin A, yet in view of the fact that direct irradiation of the animal body by sunlight or ultra-violet light may introduce variables, it is considered good technique to provide vitamin $\mathrm{D}$ in vitamin $\mathrm{A}$ experiments by a method other than direct irradiation of the animal body.

\section{Production of Vitamin D by Irradiation}

Working independently, Hess and Steenbock reported almost simultaneously the possibility of conferring antirachitic potency on food materials by irradiating them with ultra-violet light.*

* According to a recent note in the Journal of the American Medical Association, the first public announcement was by Hess in June 1924, which appeared in print in October 1924; while Steenbock's original announcement appeared in September 1924. 
Steenbock and Black (1924) irradiated diets of purified food materials, thus "making them growth-promoting and bone calcifying to the same degree as when rats are irradiated directly." . . . "The question now presents itself, are we justified in making the further assumption-that the acceptance of the existence of an antirachitic vitamin upon its former premises is no longer justifiable, granted, of course, that we accept a vitamin as a compound of biological origin. If such were justified it would simplify our conceptions materially because it has been difficult though not impossible to conceive how two such apparently different agencies, light and vitamin, should have the same effect. It suggests itself that, in ultimate analysis, both light and the antirachitic vitamin may represent the same antirachitic agent-possibly a form of radiant energy. In this connection it is of interest to mention that the authors have conferred growth-promoting properties upon olive oil and lard by irradiation with ultraviolet light."

Steenbock and Nelson (1924) presented histological evidence to show that a ration which induces rickets in a rat can be made definitely antirachitic by the simple expedient of exposing it to ultra-violet light. In this paper they emphasized their belief "that in the study of the fundamental causes of rickets too much emphasis has been placed heretofore upon the production of the histological picture of rickets characterized primarily by the over production of osteoid and the disorderly occurrence and arrangement of various bone elements in a wide metaphysis. This is produced by a peculiar combination of factors as yet imperfectly understood except that growth is a prerequisite for its production. Physiologically, however, there is operative here, among other factors, the same agency which is responsible for the failure of assimilation of calcium in the mature goat (J. Biol. Chem. 14, 59; 53, $21 ; 58,43$ ) in the growing chicken (J. Biol. Chem. 52, 379; 58, 33) and in the pig (unpublished data) as observed in this laboratory; and that agency is radiant energy either acting directly upon certain compounds in the animal body or else acting indirectly through food materials ingested."

A full account of the experiments upon which Hess had reported in June 1924 was published in December 1924. Hess and his coworkers stated that their first definitely favorable result was obtained when they used irradiated cottonseed oil, 0.1 cubic centimeter daily of which was protective in spite of a severe rickets-producing diet. Other oils were similarly tested. Linseed oil, lettuce and growing wheat plants were successfully activated. The authors concluded that it could not "be definitely stated that it is the same factor which gives codliver 
oil its specificity in rickets. If such prove to be the case, and this factor is regarded as a vitamin, then these results constitute the first demonstration of the production of a vitamin in vitro. All that can be deduced at present is that they furnish evidence of the production of an antirachitic factor outside of a living organism."

They reported success in activating patent flour and the unsaponifiable fraction of linseed oil, and the successful storage of activated material for relatively long periods. Having shown that it is the unsaponifiable fraction of vegetable oils, and not the true fat which is capable of activation, and knowing that the main constituent of the unsaponifiable fraction consists of phytosterol, they next investigated (1924) the possibility of activating such substances by irradiation. They found that 0.25 cubic centimeter of an irradiated 1 per cent suspension of highly purified phytosterol* or 0.1 cubic centimeter of an irradiated 1 per cent cholesterol $*$ (m.p. $148.5^{\circ}$ C.) fed daily to rats on a ricketsproducing diet was protective. "The fact that a well defined chemical substance can be endowed with antirachitic potency by means of irradiation is remarkable and of possible physiologic significance. . . . As is well known, the epidermal portion of the skin contains a large amount of cholesterol situated in its deeper layers in close approximation to the prickle cells. It would seem quite possible that the cholesterol in the skin is normally activated by ultraviolet irradiation and rendered antirachitic - that the solar rays and similar artificial radiations are able to bring about this conversion. This point of view regards the superficial skin as an organ which reacts to particular light waves (the epidermal organ) rather than as a mere protective covering." .. . "It would be premature to more than suggest this hypothesis of the action of cholesterol in rickets."

Similar results were reported by Rosenheim and Webster (1925) and by Steenbock and Daniels (1925).

Hess and Weinstock (1925a) found that 0.1 cubic centimeter of an irradiated 1 per cent suspension of "cholesterol" injected subcutaneously protects against rickets. By the use of light filters they found such cholesterol to be activated by ultra-violet radiations of approximately the same wave lengths as those which had been found to be specific for rickets when animals were subjected to direct irradiation, i.e., rays as short as $289 \mu \mu$ or $280 \mu \mu$ and between 200 and $290 \mu \mu$ with 90 per cent of rays between $254 \mu \mu$ and $265 \mu \mu$.

By means of a spectrograph, Macht, Bell and Elvers (1925) studied

* From later work it appears that such specimens of cholesterol and phytosterol as were accepted as pure at the time of these experiments really contain some ergosterol which, as shown later, is now held to be the substance which yields vitamin $D$ upon irradiation. 
the depth of penetration of ultra-violet rays through animal tissue and found that light waves of $280 \mu \mu$ pass through the skin of an anesthetized rabbit, where the skin is 1 to 2 millimeters thick; and rays of $300 \mu \mu$ pass through the abdominal wall, 3 to 4 millimeters thick. Hume, Lucas and Smith (1927) did not succeed in obtaining any indication of penetration of light waves of $280 \mu \mu$ through skin after 3 hours' exposure to a mercury vapor lamp at 2 feet. Calculating from Hasselbach's (1911) observations, they estimated that 1.56 per cent of the incident light of a wave length of $297 \mu \mu$ penetrates 0.1 millimeter epidermis, and one three-thousandth penetrates 0.2 millimeter epidermis. They found that "vitamin D in an irradiated cholesterol can be absorbed from a small area of undamaged skin in sufficient amounts to supply the needs of the animal" in rats on a diet deficient in fat-soluble vitamins. Further it appeared that rickets could be prevented "in rabbits fed on a rickets-producing diet (McCollum 3143) and almost normal calcification of the bones produced when an area of skin $2.5 \times 3.5$ $\mathrm{cm}$. was irradiated for 10 minutes 3 times a week."

\section{Nature of Vitamin D}

Stability.-The early studies on the nature of vitamin D were conducted on cod-liver oil or fractions thereof, for this oil was the richest known source of the vitamin. As has been pointed out, McCollum and his collaborators (1922) were able to differentiate vitamins A and $\mathrm{D}$ in cod-liver oil on the basis of their differing stability toward oxidation. Cod-liver oil oxidized for 12 to 20 hours (at $100^{\circ} \mathrm{C}$.) did not cure xerophthalmia in rats; it did, however, cause renewed deposition of calcium in bones of young rats which were suffering from rickets. Zucker, Pappenheimer and Barnett (1922) showed the stability of vitamin $\mathrm{D}$ to saponification, by demonstrating that the non-saponifiable fraction of cod-liver oil diluted with 90 parts of cottonseed oil was as potent as the original oil. This was confirmed by Steenbock, Jones and Hart (1923). Southgate (1925) found that the antirachitic vitamin of cod-liver oil was destroyed partially by heating for 2 hours at $200^{\circ} \mathrm{C}$. in absence of air, and to a still greater degree by heating for 4 hours at $200^{\circ} \mathrm{C}$.

Dubin and Funk (1923) have found vitamin D stable to hydrogenation at $55^{\circ} \mathrm{C}$. for 36 hours with colloidal platinum as a catalyst. Bills recorded in 1925 that "it is not destroyed by hydrogen dioxide, hydrogen sulfide, sulfur dioxide or formaldehyde, but is readily destroyed by nitrous fumes and slowly by direct steam or contact with mineral acids." Hart and Steenbock and Lepkovsky (1925) found that 
"cod liver oil mixed with ground grains and stored in cans at room temperature retained its calcifying power for at least 6 months."

Concentration.-Zucker (1922) concentrated the antirachitic potency 1,000 times by extracting cod-liver oil with 95 per cent alcohol, saponifying, precipitating the calcium soaps from an aqueous solution, and finally extracting the vitamin-rich material from these soaps with acetone.

Nelson and Steenbock (1925a) found that the antirachitic substance of cod-liver oil is non-precipitable by digitonin.

By extracting 1,000 grams cod-liver oil with acetic or formic acid and subsequently saponifying the extracted material, Dubin and Funk (1923, 1924) succeeded in securing 0.5 gram of crude concentrate in the form of a brown sirupy mass which on standing crystallized in yellowish brown needle-like crystals radiating from a central point. By eliminating the cholesterol and other inert substances the material was further purified until it represented a concentration of 1 part from 10,000 . The presence of carbon, hydrogen and oxygen was demonstrated, but nitrogen, phosphorus, sulfur and the halogens were reported absent.

Koch, Cahan and Gustavson (1926) secured both from irradiated cholesterol and from cod-liver oil by extraction with liquid ammonia, a brown resinous substance which had great antirachitic potency. Hess, Weinstock and Sherman (1926) likewise found that the ammoniasoluble fraction of irradiated cholesterol was active antirachitically.

Hess and Weinstock (1925a) reported that, "By means of spectral absorption tests a chemical change was demonstrated in cholesterol which had been endowed with antirachitic potency by ultraviolet irradiation. This activated cholesterol absorbs ultraviolet radiations to a less degree than does ordinary cholesterol, an effect which is intensified with increasing degrees of irradiation. If, however, irradiation is prolonged for many hours the activated cholesterol becomes less transparent than even non-irradiated cholesterol. This comparatively opaque product is of a yellowish color and has a lowered melting point." "Neither dihydrocholesterol nor dihydrophytosterol (incapable of activation) undergoes a spectral change as a result of irradiation. When activated cholesterol is kept in a watery suspension or in a dry state its spectral transmission becomes gradually diminished until it reaches a point where it transmits less than it did originally."

In 1925 also, Steenbock and Black pointed out that excessive irradiation (10 to 17 hours) of olive oil as well as cod-liver oil resulted in their inactivation. See also Rosenheim and Webster (1926). 
Hess (1925a) found that activated cholesterol did not lose its potency by prolonged contact ( 24 hours) with acetone, chloroform, or benzene, and Steenbock and Black $(1924,1925)$ reported that liver retained its induced antirachitic potency for at least 2 months after having been dried at $96^{\circ} \mathrm{C}$. for 24 hours, and that activated olive oil kept in a stoppered bottle was found unimpaired in activity after 10 months, while Nelson and Steenbock (1925) reported that a petroleum-ether solution of the unsaponifiable fraction of cod-liver oil maintained its potency for at least one and a half years.

Results not concordant with the theory that cholesterol or phytosterol can always be rendered antirachitic by ultra-violet irradiation were cited by Steenbock and Black in 1925.

\section{Vitamin D from Ergosterol}

Schlutz and Morse (1925) considered the possibility that the "substance in which the absorption spectrum is changed (and antirachitic potency induced by irradiation) may be a small amount of impurity in the cholesterol which is not removed by repeated crystallizations from alcohol, and which is exceedingly absorptive."

Hess, Weinstock and Sherman (1926b) succeeded in separating irradiated cholesterol into an active and inactive portion by precipitating the inactive cholesterol with digitonin. The active fraction constituted 4 or 5 per cent of the original.

Rosenheim and Webster (1927c) found both the unsaturated carbon linkage and the secondary alcohol grouping essential to the precursor of vitamin $\mathrm{D}$. Upon fractionating irradiated cholesterol with digitonin they found the active portion to be about 0.1 per cent when irradiated in nitrogen.

By recrystallizing, from acetone and hot methyl alcohol, some cholesterol which had been irradiated in the air, Shear and Kramer (1926a) obtained from the mother liquor about 5 per cent of crude oil, 40 per cent of which was not precipitable by digitonin. The cholesterolfree oil gave positive reactions with the Shear aniline-hydrochloric acid reagent, Lieberman-Burchard and Salkowski test, and the Lifschütz reaction for oxycholesterol. Both the crude and the cholesterol-free oil obtained by these methods had antirachitic potency. When incorporated in cottonseed oil the cholesterol-free oil retained its potency during two months' storage.

In 1926-1927, Hess and Windaus published evidence that cholesterol or phytosterol recovered after being separated twice as the dibromide, could not be activated. They questioned whether in previous work, 
cholesterol or a contaminating substance had been activated. Soon afterward they reported that ergosterol, a substance having 3 double bonds and an hydroxyl group, could be activated by irradiation. Since cholesterol is usually contaminated by ergosterol they suggested that the latter sterol is the substance which is activated into an antirachitic product by irradiation with ultra-violet light.

Windaus (1927) and Hess (1927) also reported simultaneously upon this work in another form.

Pohl (1927), working with the same materials as Windaus, showed also that the absorption spectra of cholesterol and ergosterol were similar. He found that after irradiation for 25 minutes the ergosterol showed an absorption band only at about $240 \mu \mu$ instead of exhibiting maxima at 293, 280 and $270 \mu \mu$; and attributed this band to vitamin D.

Heilbron, Kamm and Morton (1927) found that cholesterol purified by the usual methods contains another compound in small amount which can be accumulated in a "least-soluble fraction." This substance was found by them to show well-defined absorption bands at $293 \mu \mu, 280 \mu \mu$ and $269 \mu \mu$. "These bands disappear on irradiation with ultraviolet light with concomitant appearance of antirachitic potency. It is obvious that the unknown substance is closely related to the vitamin D precursor."

Rosenheim and Webster (1927c) showed that cholesterol purified by way of the dibromide cannot be activated by ultra-violet light, that it no longer possesses the absorption spectrum in the ultra-violet region which had formerly been considered characteristic for cholesterol (Hess and Weinstock, 1925a; Schlutz and Morse, 1925), and that whereas 0.5 milligram irradiated cholesterol (as formerly prepared) would prevent rickets in rats, doses up to 8 milligrams daily were impotent if the cholesterol used were purified chemically. According to Rosenheim and Webster, "The precursor of vitamin D is not cholesterol itself, but a substance associated with cholesterol as obtained from all natural sources. We have since extended these observations to the phytosterols, and have found that when these are purified by way of the bromides, they also yield no vitamin to irradiation; whereas all vegetable oils and the sterols obtained therefrom and not so purified, readily acquire antirachitic properties under the action of ultraviolet rays." Ordinary cholesterol may be freed from provitamin by purification through treatment with bromine, or with thionyl chloride, by oxidation of the provitamin with potassium permanganate in acetone solution, by overirradiation and subsequent recrystallization, and by treatment with an efficient charcoal. When cholesterol has been thus freed from provitamin, it shows only general absorption of ultra-violet light and cannot 
be rendered antirachitic by irradiation. The sterol nature of the provitamin is shown by the fact that it can be concentrated through precipitation with digitonin and separation from cholesterol-digitonide by fractional extraction with xylene. Since such sensitiveness to oxidation is characteristic of only one known sterol, ergosterol, Rosenheim and Webster studied ergosterol spectroscopically and found absorption bands in the same region as in cholesterol purified by ordinary means, but from 1,500 to 2,000 times more intense. They summarized their findings as follows: "As a result of the irradiation the absorption bands disappear in the critical region of 280 to $310 \mu \mu$ just as in the case of nonpurified cholesterol; at the same time the product loses its property of being precipitated by digitonin. If provitamin is ergosterol, or an unsaturated sterol of similar constitution the amount present in ordinary cholesterol would be of the order of $0.05 \%$." . . "A daily dose of $1 / 10000 \mathrm{mg}$. of irradiated ergosterol cured and prevented rickets in rats on a rachitogenic diet. Tests with still smaller quantities, not yet completed, indicate that the limit of activity will prove to be less than 1/50000 mg." . . " "These results seem to justify the conclusion that the naturally occurring parent substance of vitamin D is ergosterol or a sterol highly unsaturated and of similar constitution possessing the same absorption spectrum and physiological activity." Soon afterward, Rosenheim and Webster (1928) pointed out also that not only the typical ring structure, but also the specific relative positions in which the three double bonds are present in the molecule of ergosterol appear essential for the photochemical conversion of a sterol into vitamin D. They regard the position of ergosterol as parent substance of vitamin $\mathrm{D}$ to be unique.

The finding of Anderson and Shriner (1926) that different fractions of sterols prepared from corn oil show decided differences in optical rotation, stability and solubility, led Hess and Anderson (1927) to study the physiological activity of irradiated sitosterol, a sterol which had exhibited relative instability to oxidation. One kilogram of corn oil yielded 0.5 gram of $\alpha$-sitosterol. After irradiation 2.5 milligrams produced moderate to marked healing of rickets. The authors stated: "Whether traces of ergosterol are present in corn oil is not known, and it cannot, therefore, be definitely stated whether in our experiments ergosterol was the substance extracted and activated. If ergosterol is the sole antirachitic precursor, it is evident that this sterol must be universally present in all fats of animal and of plant origin that are capable of activation by ultraviolet radiations. . . . Considerable further chemical and biologic investigations will be necessary before it can be 
decided whether certain sterols other than ergosterol can contribute to the antirachitic activity of irradiated material."

$\alpha$-sitosterol, like ergosterol, cannot withstand purification by bromination. Therefore the usual method for freeing a sterol from ergosterol is not open.

Bills, Honeywell and MacNair (1928), however, found that their most carefully purified (thrice brominated) cholesterols and also a sample of twice brominated cholesterol prepared by Windaus, when irradiated, appeared slightly active antirachitically when fed to the extent of about thirty times the minimum curative dose of irradiated ordinary cholesterol (mixed apparently, with about 12 parts of ergosterol per 1,000 ). When spectrograms were taken through 20 centimeters of a 15 per cent solution of the thrice brominated cholesterol, absorption bands with maxima at 315, 304, 293.5, 282 and $269 \mu \mu$ were visible. The assumption that the bands characteristic of ergosterol were due to ergosterol itself would account for about one-fifth of the observed antirachitic potency. These authors suggested that the remaining potency (associated with absorption bands at 315 and $304 \mu \mu$ ) was due to the activatability of cholesterol itself or to an undiscovered impurity which persists after 3 purifications with bromine. (See also Schlutz and Morse, 1925.)

Jendrassik and Keményffi (1927) reported that cholesterol could still be activated after purification by way of the dibromide and they believed that ergosterol was not the only substance capable of being activated.

Koch, Koch and Ragins (1929) and Koch, Koch and Lemon (1929) published experiments confirming the results of Bills. They found on purifying cholesterol by the bromine treatment or by a triple treatment with potassium permanganate that the two products upon irradiation induced calcification in dosages 30 to 70 times as large as that required of the original cholesterol. The two products showed no absorption bands corresponding to ergosterol and showed no general absorption in the ultra-violet region. These investigators do not believe this low activatability to be due to a trace of ergosterol. When their purified products were heated slightly above the melting point under conditions that avoided appreciable oxidation, every one of the purified products was about 25 to 70 times as active as the presumably pure cholesterol. With the increase in activatability a strong general absorption was observed in the ultra-violet region but since there were no bands whatever they feel that the potency of the product is not due to ergosterol. They believe that "provitamin D activity" may be a general property in 
varying degrees of various sterols or of certain forms of those sterols.

Using the monochromatic mercury line at $265 \mu \mu$, Fosbinder, Daniels and Steenbock (1928) found a minimum energy input of 234 ergs necessary to activate sufficient "cholesterol" to give a positive test for deposition of calcium in a rachitic rat. According to the quantum theory the total radiation under the conditions of their experiments was $3.2 \times 10^{13}$ quanta. Assuming the Einstein photochemical relation, they calculated that $3.2 \times 10^{13}$ molecules or $2 \times 10^{-8}$ gram of vitamin $D$ (molecular weight of cholesterol, 385 , assumed) is sufficient to give a detectable deposition of calcium in a rachitic rat.

Kon, Daniels and Steenbock (1928) studied the photochemical formation of vitamin $\mathrm{D}$ from ergosterol under the action of monochromatic light for different lines. They found the quantity of radiant energy necessary to form an amount of vitamin D sufficient to cause a demonstrable deposition of calcium in the bones of rachitic rats to be quite constant over a wide range of radiations, 700 to 1000 ergs being necessary for the 256, 265, 280 and $293 \mu \mu$ lines, whether the ergosterol was irradiated in the solid state or in alcoholic solution, and both for ergosterol acetate and for ergosterol. The hydroxyl group apparently plays no part in the activation.

They also irradiated chemically purified cholesterols, purified by the severe methods which Bills believes do not wholly destroy their activatability and found that the highest amount of energy used, 200,000 ergs, was insufficient to activate them. This caused them to conclude "that ergosterol is at present the only substance known to be specifically activated by radiant energy."

In 1927 Rosenheim and Webster (1927e) had noted that exposure of ergosterol to ultra-violet radiation produced a mixture of substances, including vitamin $\mathrm{D}$, and found evidence suggesting that for moderate periods of irradiation, the amount of vitamin D present in the mixture was nearly independent of the period of irradiation, apparently owing to simultaneous formation and destruction of the vitamin.

It had been suggested by Morton, Heilbron and Kamm (1927) and also by Pohl (1927), that as vitamin D seemed to show absorption chiefly between 230 and $260 \mu \mu$ while ergosterol shows it chiefly between 260 and $290 \mu \mu$, the use of a filter cutting off all rays of wave-length shorter than $260 \mu \mu$ should increase the quantity of vitamin D formed, by reducing its rate of destruction. Webster and Bourdillon (1928) were unable to verify this hypothesis. These latter investigators also irradiated ergosterol at temperatures varying from $77.8^{\circ}$ to 
$-195^{\circ} \mathrm{C}$. with so little change in effect on the equilibrium mixture of vitamin $\mathrm{D}$ and ergosterol that it appears that the temperature coefficients for both reactions are very small. The product obtained upon irradiation at very low temperatures was somewhat less potent than the average.

Bills and Brickwedde (1928) had found that if cholesterol were irradiated at the temperature of liquid oxygen $\left(-183^{\circ}\right.$ C.) it was about one-tenth as active as when irradiated at room temperature, and concluded also that the temperature coefficient of the reaction causing the formation of vitamin $\mathrm{D}$ is small.

Webster and Bourdillon (1928), after removing the unchanged ergosterol from their irradiated material by precipitating it with an excess of digitonin, evaporated the filtrate to dryness (to insure precipitation of final traces of ergosterol through concentration) and extracted it with dry ether, in which digitonin and ergosterol digitonide are insoluble. Upon evaporation of the ether extract, they obtained a transparent glassy hard solid, sometimes colorless, sometimes contaminated with yellow pigment. It was soluble in its own weight of alcohol at $30^{\circ} \mathrm{C}$., soluble in ether, chloroform, and light petroleum, but insoluble in water. Its antirachitic activity was high.

Bourdillon, Webster and coworkers (1929) reported that the ultraviolet irradiation of ergosterol produces three substances in succession. The first (antirachitic substance) shows intense absorption for wavelengths of 250 to $310 \mu \mu$ with a maximum absorption at $280 \mu \mu$. The second shows intense absorption at $240 \mu \mu$ and no antirachitic power. The final product shows little or no absorption and no antirachitic power. They give evidence to show that the first substance, formed by irradiating a 0.1 per cent solution of ergosterol in absolute alcohol, removing the unchanged ergosterol by digitonin, evaporating, and extracting with ether, is a single substance and probably vitamin $\mathrm{D}$.

Knudson and Moore $(1928,1929)$ found that ergosterol exposed to cathode rays with a tube operating at 180,000 to 200,000 volts is activated antirachitically, but not to as great a degree as when exposed to ultra-violet light. They found further that irradiation from a CooperHewitt lamp, operating at 5 amperes and 140 volts on a 220 volt D.C. circuit, at a distance of 18 centimeters, is more effective if continued for 15 seconds to 2 minutes than for 30 minutes, and more effective at ordinary temperatures than at the temperature of liquid air. The characteristic absorption spectrum of ergosterol is changed similarly both when exposed to ultra-violet light and to high voltage cathode rays, but the manner in which cathode rays produce their antirachitic action does 
not seem to be due to the production of ultra-violet light. (Compare Bills and Brickwedde, 192\%.)

Using ergosterol $\left([\alpha]_{\mathrm{D}}^{20}=-132^{\circ}\right.$ in $\left.\mathrm{CHCl}_{3}\right)$, Bills and Honeywell (1928) and Bills, Honeywell and Cox (1928) studied the effect upon the antirachitic potency of the product of irradiating for different periods of time. Irradiation was conducted on a 1 per cent solution (in 95 per cent alcohol) in a completely filled cell 2 centimeters deep, placed in contact with the window of a Kromayer lamp, for intervals of $7.5,15,22.5,30,45$ minutes, and $1,2,3,4,5,7.5,10$ and 15 hours.

In the original ergosterol very well-defined absorption bands appeared at 270 and $282 \mu \mu$ with a less defined band at $293.5 \mu \mu$, and no antirachitic potency was evident. During the first 22.5 minutes, they found that the antirachitic potency rapidly increased until it became about 250,000 times as great as that of a good grade of cod-liver oil, with but a slight lowering of the maxima of both of the absorption bands and with a broadening of the $270 \mu \mu$ band. With longer irradiation, the antirachitic potency steadily declined; the band at 282 disappeared, and the band at $270 \mu \mu$ shifted until a new band appeared with a maximum at $248 \mu \mu$, accompanied by almost complete disappearance of antirachitic potency. They conclude, therefore, that the photochemical reaction product which exhibits the absorption bands at $248 \mu \mu$ is not vitamin D, as some investigators had thought, but is a by-product of the vitamin. The wave-lengths which vitamin $\mathrm{D}$ itself absorbs and by which it is destroyed, apparently lie within the same spectral region as the wave-lengths which activate ergosterol. Clearly the spectroscope cannot replace the feeding method of studying the formation of vitamin $\mathrm{D}$.

Oxidation appeared to be involved in the destruction but not in the formation of vitamin $\mathrm{D}$.

These authors suggested that the substance having the absorption band at $248 \mu \mu$ is iso-ergosterol. This same suggestion has been made by Van Wijk and Reerink (1928).

Windaus and coworkers (1929) reported that the effect of the solvent on the antirachitic potency of irradiated ergosterol is small, provided the solvent allows the ultra-violet light to pass through it. Cyclohexane, ether and benzene were used as solvents by these investigators.

Bills, Honeywell, Cox and Wirick (1929) found, however, that the solvent had a very great influence on the potency of the product of irradiation. Alcohol, ether and cyclohexane were used. 
They also reported that a freely transparent solvent is not always required, for extremely high activation can be obtained in arachis oil.

Windaus and Linsert (1928) showed that when ergosterol is dissolved in alcohol, ether, ethyl acetate, benzene, or cyclohexane and irradiated, there is a change in its optical activity from negative to positive. It was later shown by Windaus and coworkers (1929) that when the optical rotation becomes positive, the product of irradiation is no longer active antirachitically.

Windaus and Linsert (1928) also reported a change in the ultraviolet absorption spectra and an increase in solubility with irradiation and showed that the molecular weight of the irradiated ergosterol, which was freed from the unchanged ergosterol by precipitation with digitonin, is the same as that of the unirradiated ergosterol. Their results indicate that the alcohol group in ergosterol does not disappear or the number of double bonds change with irradiation. These investigators point out that on irradiation there may be a shifting of the double bonds inside the ergosterol molecule; or that a stereo-change in the secondary alcohol group may occur, since the irradiated ergosterol is not precipitated by digitonin.

Rosenheim and Adam (1929) found that the monomolecular surface films on water of ergosterol and of the three products formed from it on irradiation, as described by Bourdillon, Webster, et al. (1929), showed a resemblance to those of certain ketonic cholesterol derivatives. The product having an absorption band at $280 \mu \mu$ (probably vitamin D) resembled that of oxycholesterylene. This compound, however, has no antirachitic properties before or after irradiation. They consider it at present impossible to decide which constituent of the mixture containing the active substance represents the vitamin. Alternative possibilities are that the vitamin may be a ketone itself or that the highly absorptive unsaturated ketone may constitute the biologically inactive portion of the mixture, the vitamin itself being in the remaining portion in minute amounts only. It is thought that if the latter explanation proved to be the correct one the vitamin is one of the ergosterol derivatives resulting as by-products in the ketone formation. In either case the lability of the hydrogen of the $\mathrm{CH}(\mathrm{OH})$ group is considered to be the controlling factor of the changes induced by ultra-violet irradiation of ergosterol.

Reerink and Van Wijk (1929, 1929a), by the use of suitably selected light sources and filters, irradiated hexane solutions of ergosterol in quartz tubes and studied the progress of the reaction at different wave- 
lengths by means of absorption spectra and feeding tests and made the apparently important observation that "irradiation of ergosterol with light of wave-length $254 \mu \mu$ ("short wave" irradiation) gives rise to a series of reaction products different from that caused by irradiation with light of wave-length $>275 \mu \mu$ ("long-wave" irradiation). In the case of long-wave irradiation, the reaction proves to be comparatively simple, in so far as only one product is directly formed from the ergosterol. This product itself, which appears to be vitamin D, is on longer irradiation gradually transformed into a new and much more stable product. As the rate of destruction of the first product is rather small, it is possible to convert about 60 per cent of the ergosterol into vitamin $\mathrm{D}$ before the secondary reaction becomes important. The short-wave irradiation causes changes of a more complex character. In this case there are two reaction-products present during the first period of irradiation. It is made probable that one of these products, which is very quickly destroyed on prolonged irradiation, and thus does not accumulate to an appreciable amount is identical with vitamin $\mathrm{D}$. The other product, into which the main portion of the ergosterol is transformed, is much more stable in respect to this irradiation."

The product formed by the long-wave irradiation, about 50 per cent of which is considered to be vitamin $\mathrm{D}$, was capable of curing a rat of rickets in 14 days in daily doses of 0.00001 milligram. Another sample estimated to contain about 30 per cent of vitamin D cured infantile rickets within 14 days by a daily dose of 0.04 milligram, an amount said to be 100 times smaller than the customary dose of irradiated ergosterol. Contrary to previous suggestions that the maximum absorption of vitamin $D$ is at $280 \mu \mu$ the very active substance showed maximum absorption at about $270 \mu \mu$.

Marshall and Knudson (1930), using monochromatic light of various wave-lengths obtained from several intense sources, first determined the effect of varying light intensities on the minimum daily dose of irradiated ergosterol necessary to show the first incidence of healing in rats following the technique of Knudson and Moore (1929) and then varying the wave-lengths (from $302.2 \mu \mu$ to $230 \mu \mu$ ) and the time of exposure calculated the rate of production of vitamin $\mathrm{D}$ with results which they summarize as follows:

"The rate of production of vitamin D from ergosterol is proportional to the first power of the light intensity. The rate of production of vitamin $\mathrm{D}$ is directly proportional to the number of light quanta absorbed by ergosterol and independent of the wave-length of the light used. 
Vitamin D absorbs in the same wave-length region as ergosterol and is destroyed by light of the same wave-length as that which forms it. The highest concentration of vitamin $\mathrm{D}$ which can be produced by direct irradiation of ergosterol is 35 per cent. This is an absolute maximum and the probable value is lower. The quantum efficiency is 0.3 molecules of vitamin D per quantum of light absorbed."

Just as this chapter was being completed (October, 1930) two important papers from Bourdillon, Webster, and their coworkers (Askew, Bourdillon, Bruce, Jenkins, and Webster, 1930, 1930a) appeared which can be quoted only in summary.

In the first paper "a method is described for the partial separation of the irradiation products of ergosterol, by distillation and fractional condensation in a high vacuum. By double distillation, followed by crystallization from aqueous alcohol, a crystalline product has been obtained, showing high antirachitic power, and a melting point of $113^{\circ}$ to $115^{\circ}$. It is not suggested that these crystals are pure vitamin D. They may consist of an inactive substance contaminated with an extremely active vitamin, or of crystalline vitamin forming mixed crystals with an inactive substance. It is possible, on the other hand, that several antirachitic substances are formed by the irradiation of ergosterol, and that the crystals are a relatively pure specimen of one of these substances. Some evidence is given as to the degree of efficiency of the process of fractional condensation in a high vacuum."

The second paper reports a study of the effects of a second irradiation by short waves on the substances formed by a first irradiation of ergosterol by long waves.

"The results show that the product with high absorption at $280 \mu \mu$ is not vitamin D as previously suggested. The substance with high absorption at $280 \mu \mu$ can be formed by the action of short-wave radiations on some product of a previous action of long waves on ergosterol. Reasons are given for supposing that the initial effect of long-wave radiation on ergosterol is the simultaneous production of at least two substances, only one of which is vitamin D."

\section{Effects and Measurement of the Antirachitic Factor}

The Line Test.-In the course of routine autopsies conducted in McCollum's laboratory on all rats dying from any cause in nutritional experiments, evidences of rickets were found in rats which had been fed diverse deficient diets, but particularly in those which had received diets deficient in fat-soluble vitamin(s) or calcium. Some of these faulty diets rendered the cartilage and adjacent portions of the metaphyses of 
the long bones entirely free from visible calcium deposits. Fifteen rats were placed on such a diet until it was evident that they could live but a short time, at which time there was added to the diet of eight of the test animals 1 or 2 per cent of cod-liver oil. After 2 to 8 days all the rats were killed, and the bones examined. Seven of the $S$ animals receiving cod-liver oil showed upon histological examination of the bones, a line of freshly laid down calcium, while none of the control animals showed deposits of calcium. (McCollum, Simmonds, Parsons, Shipley and Park, 1921.) Out of this experience and other work which soon followed was developed a test for calcium-depositing (antirachitic) substances. This "line test" as originally described may be briefly summarized as follows: The rickets-producing diet is fed to rats 55 to 60 grams in weight (weight seemed to be more important than age) for 28 to 40 days. By the time the rats are ready for the test they have a tottering gait and their hind quarters waver in walking. By this time the metaphyses of the bones are usually free from calcium salts. Then the test food is fed for a designated time. During the test, records of food consumption must be kept, because starvation causes a deposition of calcium salts in the epiphyseal cartilage, probably due to release of phosphorus of disintegrating tissue. At the end of the experimental period the proximal end of the tibia is examined for evidences of rickets. The bones are split with a scalpel, immersed in 1 per cent silver nitrate, exposed to light. washed in distilled water, and then studied for signs of deposition of calcium. This treatment stains the newly deposited calcium black. Under the binocular microscope it appears like a cross section of blackened honey-comb (the matrix, not the cartilage cell, is calcified). In a "negative line test" there is no calcification of the epiphyseal cartilage and little if any in the metaphysis. In a "positive line test" there is a broad linear deposit of calcium salts on the metaphyseal side of the epiphyseal cartilage.

For a very detailed description of this technique and for a recent discussion of factors involved in determining vitamin $\mathrm{D}$ quantitatively, by means of it, the reader is referred to an article by Bills, Honeywell and MacNair (1928).

A scale showing line tests of varying degrees of healing has been published by Bills. This is useful in classifying results obtained by this rapid method of diagnosis.

For preparing animals for this line test McCollum, Simmonds, Parsons, Shipley and Park (1921) and McCollum, Simmonds, Shipley and Park (1922) recommended first the following diet, which has been used extensively: 
Diet No. 3143

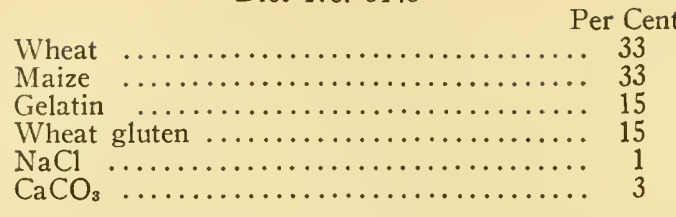

However, because differences in the phosphorus content of hard and soft wheat are great enough to make the production of rickets with diet No. 3143 uncertain unless soft wheat is used McCollum, Simmonds, Becker and Shipley (1925) proposed the use of other diets to prepare animals for the line test, e.g. :

Diet No. 4025

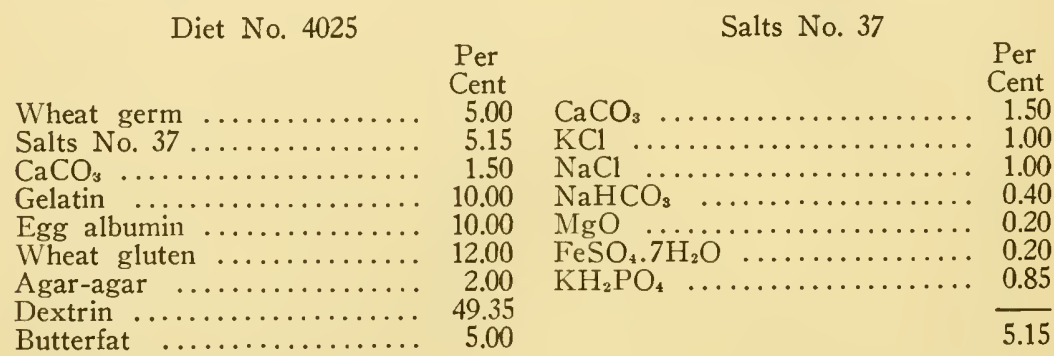

More recently (1926) they have called attention to the fact that even these diets are not altogether reliable for producing rickets, because butterfat introduced at a 5 per cent level to furnish vitamin A, may at the same time furnish enough vitamin D to forestall rickets.

Steenbock and Black (1925) pointed out that the protein content of McCollum's diet No. 3143 is rather high, that the gelatin content is too high, resulting in anorexia or diarrhea, and that the vitamin A content is too low to prevent ophthalmia and infections of the respiratory tract for a period long enough to allow for the depletion of the reserves of the antirachitic vitamin. They recommended the use of their diet No. 2965

Diet No. 2965

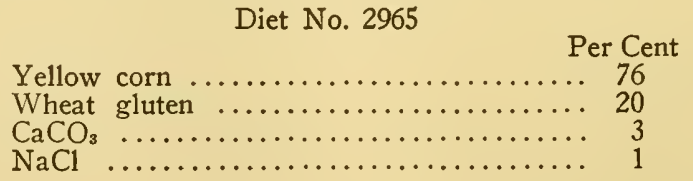

for preparing animals for the line test, because it permits greater growth while shortening the period for the development of rickets, and because it is easier to prepare and is cheaper. Steenbock's diet No. 2965 has practically the same calcium-phosphorus relations as McCollum's 
rickets-producing diets. This diet has been adopted by the Council on Pharmacy and Chemistry of the American Medical Association for standardizing viosterol.

The Steenbock-Black diet and line test technique are also used in the quantitative determination of vitamin D in the Pharmacological Laboratory of the Pharmaceutical Society of Great Britain. Coward (1928), in describing the technique followed in these laboratories, has proposed to overcome the difficulties involved in expressing results obtained in different laboratories by the adoption of a standard preparation of the vitamin in question. The standard recommended is a preparation of irradiated ergosterol of such potency that a daily dose of not more than 0.0001 milligram will under the conditions of the experiment cause complete healing of the induced rickets. By equating by means of the line test, the dosage of the material being tested with the dosage of the standard irradiated ergosterol required for complete healing, it is possible to calculate the amount equivalent to the standard dose of 0.0001 milligram. The amount may then be expressed as the number of units of antirachitic potency contained in any convenient measure of the material being tested.

Using a solution of irradiated ergosterol prepared under conditions calculated to generate its maximum antirachitic activity (although according to Rosenheim and Webster it contained probably less than 10 per cent of vitamin D), Coward (1928c) found that feeding 0.00002 milligram daily resulted in ++ healing, comparable to "positive" rating given by Steenbock, in rats made rachitic by Steenbock's diet No. 2965. Thus for the 10 days' test, $2 \times 10^{-4}$ milligram or $2 \times 10^{-7}$ gram of the activated material was required. The amount of vitamin $\mathrm{D}$ actually fed, then, was approximately $2 \times 10^{-8}$ gram, an amount which confirms the estimation of Fosbinder, Daniels and Steenbock (1928) of the quantity of vitamin D necessary to produce renewed deposition of calcium in the bones of rachitic rats.

Inasmuch as Coward found a certain degree of healing when half or even one-fourth of this amount was fed, she suggested that the line test is suitable for detecting still smaller quantities.

Poulsson and Lovenskiold (1928) recommend taking a skiagram of the left knee joint at the beginning and end of the test period, in experiments conducted similarly to Steenbock's line test, and base estimates of the antirachitic value of supplementary material upon changes in the appearance of the X-ray pictures taken before and after administration of the materials to be tested, rather than upon the "line test."

Knudson and Moore (1929) have used radiographic studies parallel 
with the line test and regard the latter as somewhat more sensitive. However, they recommend the use of radiographic observations to determine when to begin the test period and to follow the progress of the test.

X-ray and Histological Studies of Bone.-Early in 1921 Sherman and Pappenheimer (1921, 1921a) had pointed out that, in their experience, the following diet regularly produced marked rickets in rats:

Diet No. 84

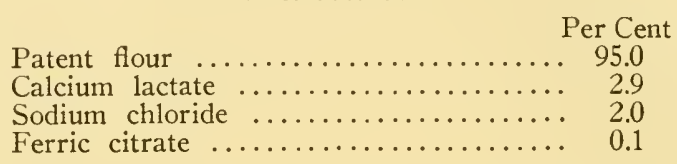

while the introduction of 0.4 per cent secondary potassium phosphate in place of an equal weight, i.e., replacing about one-seventh, of the calcium lactate contained in the rickets-producing diet, completely prevented the development of the rachitic lesions, although it was without influence upon the growth and body weight. Diagnoses of rickets were based upon X-ray studies during life and gross changes found in the thorax and long bones at autopsy, as well as upon microscopic examination of sections of ribs.

The Diet No. 84 of Sherman and Pappenheimer (1921, 1921a) was originally published without further elaboration because it afforded a then-much-needed key to the dietary production of low-phosphorus rickets. The ease, promptness, and regularity with which it permits the experimental induction of the characteristic bone condition has led some investigators to use it as a basal rickets-producing diet in studies of vitamin $D$ values; but it was obviously never offered as in any sense a parallel to the basal diets used, for example, in studies of vitamin A, B and $C$ values of food-diets adequate in all respects except for the vitamin in question. Pappenheimer et al. (1921) suggested modifications in this direction, and Sherman and Stiebeling $(1929,1930)$, as described further on in this chapter, approximated the ideal of a basal diet excellent in all other respects but lacking vitamin $\mathrm{D}$ - a diet which made vitamin $\mathrm{D}$ the sole limiting factor and therefore permitted of its uncomplicated measurement; but which was not in the strictly literal sense a rickets-producing diet. As described later, this and a number of related objectives are embraced in a very far-reaching plan of experimental dietary modification now being developed by Mendel and his coworkers.

When "Diet 84" is used as a basal diet in tests for vitamin D, its drastically rickets-producing deficiencies may result in materials being 
reported as lacking vitamin $D$ when in reality they contain a small, but scientifically significant, amount of it. It is partly for this reason that even some of the leading investigators of vitamin $\mathrm{D}$ do not yet realize that it is widely distributed in nature and that foods not usually looked upon as important sources (even foods upon which rickets sometimes develops) may nevertheless contain significant amounts of vitamin $D$.

Hess and his collaborators determine the vitamin D content of substances sometimes by using them to cure and sometimes to prevent rickets in rats, as judged by $\mathrm{X}$-ray and histological examinations of the bones. They prefer experiments of the protective nature to those of curative nature because, as they say, "in our experience there are numerous complicating factors, difficult of interpretation, which may bring about a greater or less degree of healing in bone."

Bodily Storage of Vitamin D.-Drastically rickets-producing diets may be so severe that rickets is produced in a short time regardless of the stock diet from which the animals are taken. However, Hess, IVeinstock and Tolstoi (1923) have reported instances in which animals were "refractory" to rickets due to the richness of the mothers' diet in vitamin $\mathrm{D}$ and to the small sizes of litters born and reared. Young were rendered susceptible to rickets by feeding the mother and young a less adequate dietary throughout the suckling period. Resistance to rickets in the young was also broken down by means of inadequate lactation during this period as by forcing the mother to suckle young in addition to her own litter. This deprivation of a liberal milk supply resulted in an inability on the part of the young to store a reserve of the antirachitic factor. They said: "Experiences of this kind indicate the necessity of controlling the diet of experimental animals for the entire period preceding the test. They also suggest that the diet of infants during the first weeks of life may be of equal importance to the later development of rickets."

Further research in several laboratories has shown that the diet or irradiation of the mother may have marked effect upon the susceptibility of her offspring to rickets doubtless largely if not mainly through influencing the store of antirachitic vitamin in the body of the human infant or other young animal. Goldblatt stated (1923), "When rats are used which have only a small 'store' of fat-soluble organic factor (rats bred from parents on an ordinary normal diet) grading the content of that factor in their diet results in a corresponding gradation in the percentage of calcium in their bones. Histologically, some of the rats on the diets very deficient in fat-soluble organic factor show slight to severe osteomalacia or rickets. When rats are used which have a large store of 
the fat-soluble organic factor (bred from parents who, in addition to the normal diet, received cod-liver oil breeding paste) grading the content of that factor in their diet results in only the slightest gradation of the percentage of calcium in the bones. Even in some of these wellbred rats, on diets deficient in the organic factor, very slight osteomalacia or rickets may develop."

Further evidence of storage of vitamin D was offered by Goldblatt and Soames (1923a) who found that "if rats are kept on a diet deficient in vitamins $A$ and $D$ until they have ceased gaining weight for about four weeks their livers, fed to other rats, do not possess growthpromoting properties; but if, after complete cessation of growth, rats are irradiated for a few weeks by means of a mercury-vapor quartz lamp, their livers acquire the property of stimulating for a short time the gain in weight of rats that have ceased growing on a diet deficient in the fat-soluble vitamin"-implying, of course, that it was vitamin D and not vitamin A which was here the growth-limiting factor. This was confirmed by Steenbock and Black (1924) who showed that liver from irradiated rats was growth-promoting for those whose growth was inhibited for lack of antirachitic vitamin, while liver from non-irradiated rats was not. The same was found true for lung and muscle tissue. In this work Steenbock and his coworkers used his "synthetic" diet containing an adequate salt mixture with or without the addition of a small amount of dried alfalfa to provide vitamin A. This basal diet consists of purified casein 18 , salts No. 404 , yeast 8 , agar 2, dextrin 68 parts. The addition of the alfalfa ( 0.3 gram per 100 grams ration) postpones for about two weeks the xerophthalmia and infections of the respiratory tract which otherwise occur within 5 to 7 weeks after the animals have been put on the diet at an initial age of 21 to 25 days, and weight of 35 to 60 grams.

The fact that smaller amounts of vitamin $\mathrm{D}$ are detectable by the use of diets having a good salt mixture was indicated by the early work of McCollum, Simmonds, Shipley and Park (1922c) when they found that " 3 per cent butterfat is ample for providing the animals with sufficient fat-soluble $A$ and any other organic substance exerting a special effect on the bones when the content of calcium is raised to approximately half the optimal. The provision of nearly seven times this amount does not exert much protection to the animals against the specific detrimental effect of lack of calcium when the content of the diet in this element is from one-fifteenth to one-fifth or sixth the optimal amount." These findings emphasize the importance of considering the pre-experimental bodily store of vitamin $\mathrm{D}$ in animals used in studies on 
this vitamin made with a basal diet containing a well-balanced salt mixture. Steenbock's stock diet (1923) produces animals which weigh 45 to 68 grams at 21 to 28 days, and which on a diet free from fatsoluble vitamins deplete their stores of vitamin D in about 2 weeks. Dutcher (1925) stated that his 3-week old animals which weigh 30 to 40 grams have "practically no store of vitamin D." Of the Lister Institute stock diet consisting of fresh cow's milk, oats and bran, green cabbage leaves and (occasionally) red meat, Chick wrote "The reserves of vitamin D are usually low compared with those of vitamin A. Great irregularity is caused if materials rich in vitamin $\mathrm{D}$ are at times included in the diet. Substances to be avoided are egg yolk and cod-liver oil. Summer 'pasture-fed' cow's milk should be used sparingly."

Composition of Blood.-Bethke, Steenbock and Nelson (1923) published data on the variation in composition of blood and bone with varying inorganic and vitamin intake. They found that at weaning time in rats of 24 days and 45 to 60 grams weight, the femurs and humeri contained approximately 45 per cent ash. At 66 days, when the body weight had increased to 200 to 275 grams, the percentage of ash had increased to 59. Maximum values were not attained until much later when the animals weighed 375 to 425 grams; the ash content of these bones then totalled 66 per cent. The blood phosphates and calcium were found slightly higher in the very young than in the older animals. They found also, "On a basal ration (synthetic diet) containing $0.105 \%$ calcium and $0.655 \%$ phosphorus marked increase of growth was obtained on the one hand with calcium additions and on the other hand also with the addition of fat-soluble vitamins as found in cod-liver oil. . . . Radiations with ultraviolet light for 10 minutes daily in the absence of fat-soluble vitamins in the diet brought up both the calcium and phosphorus of the blood."

Howland and Kramer (1921) had pointed out that during active rickets in children the inorganic phosphorus of the blood serum is reduced. Since then investigators of the disease have been interested in the use of this criterion for diagnosing rickets. Park (1923) in his review of the etiology of rickets went so far as to say that, "The first detectable signs of rickets are probably a diminution of the inorganic phosphorus or calcium of the blood." Hess and Lundagen (1921), however, reported that in some cases of rickets no blood changes are demonstrable, and Hess and Unger (1922a) stated definitely that while the lowering of inorganic phosphorus generally follows, it is not specific for the disease.

Steenbock and his coworkers confirmed Hess' observations in their 
work with dogs. They believe "that while a reduction of inorganic phosphate need not necessarily precede the incidence of severe rickets, sooner or later in the course of the disease, such a reduction results. When this reduction has taken place, with amelioration of the symptoms and cure of the disease, the composition of the blood is restored to normal." . . . "Just what the normal is for the dog is difficult to say, apparently it varies." ... "It is certain that comparisons can only be made on the same animal."

Cavins (1924) reported that the normal inorganic phosphorus content of the blood serum of rats is "7 to $8.5 \mathrm{mg}$. per $100 \mathrm{cc}$. In animals rendered rachitic by a diet low in phosphorus (such as diet No. 3143 of McCollum) the phosphorus is found to be about $3 \mathrm{mg}$. per $100 \mathrm{cc}$. and may run as low as 2 mg."

Dutcher, Creighton and Rothrock (1925) found the inorganic phosphorus of the blood serum to be 10 milligrams per 100 cubic centimeters in 3-week-old normal rats, and 8 milligrams per 100 cubic centimeters in 11-week-old rats on a normal diet. Rats put on a rachitic diet (No. 2965 Steenbock) at 3 weeks of age, had by the time they were 6 weeks old (21 days on the experimental diet) 1.6 milligrams inorganic phosphorus per 100 cubic centimeters blood serum.

Hess, Weinstock, Rivkin and Gross (1930) found that when inadequate amounts of antirachitic agents were given, such as poorly irradiated milk or irradiated ergosterol, and mild rickets resulted, concentrations of 6 milligrams or more of inorganic phosphorus and of 10 milligrams or more of calcium per 100 cubic centimeters of blood serum were found. In the case of inadequate cod-liver oil, positive line tests were secured, but the blood phosphorus remained low. When extreme rickets was induced by feeding a diet with a very high ratio of calcium to phosphorus and large amounts of cod-liver oil and irradiated ergosterol were fed, the rachitic lesions in the epiphyses were unaffected or gave evidence of slight healing after a 9-day test period, whereas the blood had a normal or an excessive concentration of calcium and phosphorus. These authors state that, "the established fact that the inorganic phosphorus of the blood is generally diminished in rickets has led to the inference that the disturbance associated with this disorder is of a systemic nature and that local factors play no part in its pathogenesis. ... It seems to us that these observations, considered in conjunction with our experiments, indicate that there is probably a local as well as a systemic factor which prevents the calcification of the epiphyses in rickets-something which is lacking in order to bring about a binding of the calcium and phosphorus." 
Growth.-At one time Steenbock, Nelson and Black (1924) suggested that the vitamin $\mathrm{D}$ content of food be measured by finding how much will furnish such a sufficiency of vitamin D that irradiation has no further effect upon growth. They stated, "This, however, has its limitations because vitamin A must be present in sufficient amounts to allow normal growth and also because with the introduction of large amounts of such a material as alfalfa the inorganic balance is changed so that ultimately the amount of vitamin required may actually be much less, and with other materials may be greater."

Composition of Bone.-In other studies involving a quantitative measure for the antirachitic factor, Bethke, Steenbock, and Nelson (1923) used the ash content of bones. They considered the ash content calculated on the basis of dry ether-alcohol extracted bone (femur and humerus) to be more significant than on the "green-bone" basis, "as the bones of animals vary so much in lipoidal and fat content even when taken from animals on the same ration that the accuracy of percentage values is seriously interfered with if omitted." (Cf. Sherman and Stiebeling (1929, 1930) cited briefly below.)

Dutcher, Creighton and Rothrock (1925) established standards for different ages in their animals on normal, rachitic and irradiated rachitic diets, using the level of inorganic phosphorus in the blood and the per cent of bone ash calculated on the basis of dry extracted femurs. They stated their belief, "that these chemical methods should be very useful in making accurate estimations, in a relatively short time, of the antirachitic potency of our common foods. It offers a quantitative method of unusual interest for we should be able to find, by feeding experiments, the minimum amount of any food which will prevent an appreciable fall in inorganic blood phosphorus or bone ash below the normal level of a normal 40 -gram rat or the minimum amount of any food which will bring about normal deposition of mineral matter in bones."

Goldblatt and Soames (1923b), Chick and Roscoe (1926), and other English workers have used percentage of calcium in dry bone or dry extracted bone as a measure of the influence of the antirachitic factor. Goldblatt (1923) found 20.6 per cent calcium in the bones (dry weight) of animals on a diet containing a satisfactory salt mixture, and furnishing ample fat-soluble vitamins, and 14.6 per cent in those receiving no fat-soluble vitamins, after 7 to 8 weeks on the experimental diet.

Chick, Korenchevsky and Roscoe (1926) suggested the use of "A/R ratio" (ash to organic residue) to express degree of calcification. In their work 17 per cent calcium is equivalent to $A / R$ of 0.90 and 20 per 
cent calcium to $A / R$ of 1.20 . This expression serves to magnify differences.

For several years Osborne, Mendel and Park (1923; Osborne and Mendel, 1927) have been engaged in formulating a "synthetic" diet that will permit the study of changes in the development of the bony structure as a result of alterations in a single factor in the food. Obviously such a diet is of importance in vitamin $D$ studies as well as in other connections. They use purified dietary ingredients and a salt mixture that can be maintained comparable, except for a single element, and which can be altered to make the diet vary in its potential acidity or alkalinity. One of their food formulas furnishes the following ingredients: edestin 20 , starch 75 , salt mixture XXX 2.6 parts. Vitamin B is furnished daily in the form of 0.2 gram of dried yeast to which sufficient calcium chloride has been added to maintain the ratio $\mathrm{Ca}: \mathrm{P}:: 1: 0.5$. Vitamin $\mathrm{D}$ is furnished daily by means of cod-liver oil; in experiments that exclude the antirachitic factor the same dose of olive oil is used. Their salt mixture XXX is devoid of calcium and phosphorus and has the following composition:

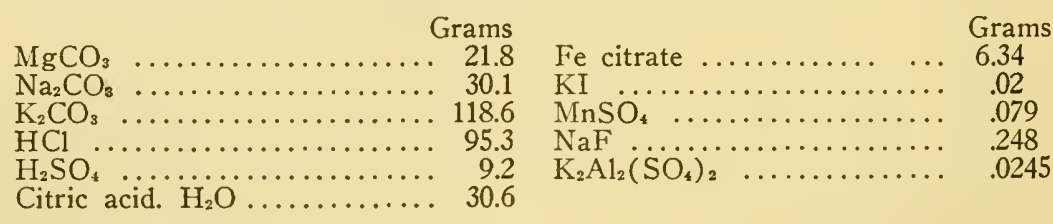

The mixture is neutral. Calcium or phosphorus or both can be added in the form of various salts so as to produce any ratio of $\mathrm{Ca}: \mathrm{P}$ that is desired. The potential reaction can be varied from alkalinity to acidity by the appropriate selection of the compounds of calcium and phosphorus added.

This diet should be useful in the study of many nutritional problems. The vitamin supply, the protein level, the modifying effect of fats and carbohydrates respectively, the potential reaction, the ratio of the boneforming elements to each other, and the relative "levels" at which they occur in the intake can be independently or conjointly modified.

They report that numerous experimental obstacles have arisen in connection with what involves primarily the metabolism of calcium and phosphorus in the growing animal. The presence or absence of fats seems to effect a modifying influence.

By modifying a diet adequate in other respects but free from fatsoluble vitamins to include 20 per cent wheat embryo, 50 per cent hog millet, or 1 per cent alfalfa meal, Soames and Leigh-Clare (1928) found 
that either 20 per cent wheat embryo, or 50 per cent hog millet may be used to supply vitamin A without an appreciable amount of vitamin D, as shown both by the effects upon growth and the degree of calcification, when none or an ample supply of vitamin $\mathrm{D}$ was added to the basal diet.

McCann and Barnett (1922) studied the distribution of phosphorus and calcium between the skeleton and soft parts of rats on rachitic and non-rachitic diets, and Chick, Korenchevsky and Roscoe (1926) compared the chemical composition of the skeletons of young rats fed (1) on diets deprived of fat-soluble vitamins, and (2) on low-phosphorus rachitic diets with those of normally nourished rats.

Sherman and Hessler (1927) used the percentage of calcium in the total body as a means of judging the influence of the antirachitic factor upon calcification.

Sherman and Stiebeling (1929) studied quantitatively the responses in growth and degree of calcification made by young rats to graded allowances of vitamin $D$ at different periods during the first several months of life. They found, in young rats reared by mothers on a diet consisting essentially of two-thirds ground whole wheat and one-third whole milk powder and transferred at the twenty-first or twenty-eighth days of age to a basal diet decidedly deficient in vitamin $\mathrm{D}$ but otherwise adequate (calcium, 0.74 per cent; phosphorus, 0.58 per cent), that practically normal calcification resulted at the fifty-sixth day of age in cases in which the basal diet was supplemented by whole (summer) milk powder to the extent of about 5 per cent of the calories; at the eightieth day of age in cases in which the basal diet was supplemented during the preceding 4 weeks by whole milk powder to the extent of 8 to 9 per cent of the calories; and at 150 and 180 days of age in cases in which the basal diet was supplemented during the preceding 8 weeks by whole milk to 4 to 5 per cent of the calories. Feeding smaller graded portions of the milk as source of vitamin D resulted in corresponding improvements in calcification over their respective negative control. In all these cases, the vitamin $\mathrm{D}$ was the sole significant variable, the calcium and phosphorus content of the food as a whole being kept constant. They found it possible to make satisfactory comparisons only when the groups of test animals are controlled by two other groups, containing representatives from the same litters and matched in sex and weight, the one receiving no added vitamin $\mathrm{D}$, the other an ample supply. They suggested that, at whatever age the studies on rats are terminated, quantitative comparisons of the vitamin $\mathrm{D}$ content of materials under investigation be made at that level of intake which will induce a degree of calcification midway between the minimum and 
maximum values obtainable in rats of the same age and on the same basal diet with (1) no added vitamin D, and (2) with an ample supply, respectively. Their basal diet consisted of: Casein, fat-soluble vitaminfree, 18 per cent; salt mixture (Osborne and Mendel), 4 per cent; dry brewers' yeast, 10 per cent; sodium chloride, 1 per cent ; powdered dry spinach, 1 per cent; cornstarch, 66 per cent. This diet contains enough vitamin $\mathrm{A}$ to permit rats reared under their conditions to make very good growth when vitamin $\mathrm{D}$ is liberally supplied.

In the course of these experiments data were accumulated by the use of which the degree of calcification (ossification) was expressed in each of the following several terms: percentage of ash, or of calcium, in the fresh bone; percentage of ash, or of calcium, in the dried, alcoholether-extracted bone; and/or the ratio of the ash to the organic residue of the dried extracted bone.

From their data it appears that all of these suggested criteria are of about the same order of accuracy, and they recommend the use of the percentage of ash, or of calcium, in the fresh bone as the criterion for the degree of calcification.

They found both growth and calcification improved by supplementary graded portions of vitamin $\mathrm{D}$, but the calcification appeared to be more regularly affected.

Mineral Balance.-Boas (1926) studied the antirachitic value of spinach by carrying out direct metabolic experiments on rats, comparing the infuence upon retention of calcium and phosphorus of daily additions to a diet deficient in fat-soluble vitamins, of cod-liver oil and fresh green winter spinach leaves.

Hart, Steenbock and coworkers (1923) have repeatedly found that balance experiments on goats could measure the effect of the antirachitic factor and Sjollema (1923) has made use of such experiments with rabbits, but the great labor involved in such studies, and the high percentage errors likely to be encountered have deterred most workers from using balance studies with small animals to measure the vitamin $\mathrm{D}$ content of foods.

Reaction of Intestinal Contents.-Jephcott and Bacharach reported (1926) that they had developed a rapid and reliable test for vitamin D based on the observation of Zucker and Matzner to the effect that rats kept on a typical high-calcium low-phosphorus rachitogenic diet develop marked fecal alkalinity, and that the administration of an antirachitic to rats causes the feces again to become acid. This method, they say, is satisfactory only when the test material contains a high concentration of vitamin D, as cod-liver oil, or (in some cases) if the test food can 
be incorporated into the basal diet. It does not seem to have proved generally satisfactory.

Grayzell and Miller (1928) found that normally the reaction of the intestinal contents of dogs is acid practically throughout the entire length of the tract; on a rickets-producing diet, the reaction tends to become alkaline, but administration of cod-liver oil or ultra-violet irradiation under such conditions changes the reaction back to normal values.

The alteration of the reaction of the intestinal tract may be but one mode of action of the antirachitic vitamin. It explains the discovery of Zucker, Johnson and Barnett (1922) that rickets could be prevented by substituting calcium chloride for calcium lactate in a rachitic ration and the ability of Jones (1924) to cure rickets in children by the addition of hydrochloric acid to their diet.

Spectroscopic Tests. - The recently established facts that the provitamin shows well-defined absorption bands with maxima at $293 \mu \mu$, $280 \mu \mu$ and $269 \mu \mu$, enabled Rosenheim and Webster (1927c) to rely to a large extent on the spectroscopic test in place of animal experimentation in their investigations on the parent-substance of vitamin D. They say, "Although such a test had already been tentatively suggested by Schlutz and Morse (1925), a firm basis for its application was only established when we showed by animal experiments that the absence of absorption bands in the ultra-violet coincided with the loss by cholesterol of its property of becoming antirachitic on irradiation. It is evident that the destruction or absence of provitamin can be rapidly proved by a negative spectroscopic test, whilst the animal experiment remains indispensable in confirmation of positive spectroscopic evidence."

Under the auspices of the Committee on Accessory Food Factors of the Medical Research Council, Great Britain, a standard solution of irradiated ergosterol similar to that in use at the Pharmaceutical Societies laboratories (cf. Coward, 1928) has been prepared and tested for stability after prolonged storage. The Medical Research Council in a recent announcement in Lancet has described the preparation of this standard as follows:

"A quantity of ergosterol from yeast, purified and dried by methods carefully recorded, was subjected, in an accurately made alcoholic solution, to irradiation with the rays from a mercury arc, the physical details of the procedure being measured and recorded with the greatest practicable accuracy. All conditions known to influence the amount of vitamin D formed should, therefore, be reproducible at any future date, or in any other laboratory, leaving only the final adjustment of a new standard to equivalence with this original one to be made on the basis of comparative biological assay. The irradiation products were carefully 
freed from alcohol, dissolved in pure, unadulterated olive oil, and the volume adjusted to produce a known concentration, in terms of ergosterol used."

Tests of the stability of the preparation have shown that it retains its activity unchanged for two years if maintained at or below $0^{\circ} \mathrm{C}$., but may suffer some loss in activity at higher temperatures. The recommendations of the committee, which are intended to lead to uniform procedure in Great Britain, are as follows:

“(a) That the standard solution of irradiated ergosterol prepared and maintained by the National Institute for Medical Research be recommended for adoption as a standard for the comparative estimation of vitamin $D$.

"(b) That the unit of vitamin D be defined as the antirachitic potency of a quantity of this preparation corresponding to $0.0001 \mathrm{mg}$. of the ergosterol used in its production.

"(c) That either the X-ray method, the 'Line test,' or chemical analysis of the bones of the experimental animals, be recommended for use in the estimation of vitamin $\mathrm{D}$.

"(d) That supplies of the standard material be made available for general distribution from the National Institute for Medical Research, Hampstead. As the supply is limited, it is hoped that users will themselves prepare standard solutions of irradiated ergosterol for use in individual tests, retaining the National Institute's material for reference. It is important that all such standard materials be kept at temperatures not exceeding $0^{\circ} \mathrm{C}$."

\section{Food Sources of Vitamin D}

That a dietary factor affecting calcium assimilation may appear in plant tissue was shown by Hart, Steenbock and Hoppert (1922) in their study of the comparative efficiency of fresh over dried leaf food in relation to calcium balance of cows during lactation. But that the amounts may be small was indicated by Hess and Unger (1920) who reported that 30 grams spinach daily did not cure rickets in children.

Shipley, Kinney and McCollum (1924) found that "ether, alcohol and acetone extract from alfalfa leaves a substance which exerts an antirachitic effect when fed in amounts corresponding to 250 grams per kilogram of food" (no other levels were reported).... "Ether extracts the antirachitic vitamin from clover blossoms." . . "Ether extracts of dry spinach, brussels sprouts, cabbage, celery, tomato and sweet potato were given in amounts equivalent to feeding 250 grams per kilogram of ration to animals with rickets without any effect on the rachitic process in the bones. These vegetables, therefore, in the dry state are free from, or contain negligible amounts of the antirachitic vitamin." In an accompanying paper (1924a) the same authors confirmed the presence of vitamin D in liver oils and butter and reported that "alcoholic extracts of butterfat contain appreciable amounts of the antirachitic substance, and that the same substance is present in oil of cloves." 
Chick and Roscoe (1926) observed that additions of fresh summergrown spinach to a diet adequate in calcium and phosphorus produced a slight improvement in calcification of bones of young rats. No differences due to size of dose fed could be detected. No improvement in calcification was obtained with spinach grown in the winter. Boas (1926) found in balance studies of rats that the addition of winter spinach up to 3 to 4 grams daily did not increase the storage of calcium in animals beyond that of the controls. Later, working with guinea pigs rendered rachitic by Diet No. 3143, Roscoe (1927) showed that fresh summer spinach had considerable antirachitic potency, when fed in doses of 40 grams daily. (No feedings at other levels were reported.) Mellanby in his work with puppies has also demonstrated antirachitic potency in green vegetables.

Hess (1923) reported that egg yolk has considerable antirachitic potency. He has since found that 0.05 gram fed daily by pipette protects rats on a low phosphorus diet from rickets but is less effective when rats are on low calcium diets. Boiling the egg yolk for 20 minutes did not appreciably lower the vitamin $\mathrm{D}$ content, but keeping it in a dried state caused deterioration. The non-saponifiable fraction obtained from egg yolk was thought to have at least 90 times the antirachitic potency of the original egg.

Tso (1926) found that the Chinese method of preserving ducks' eggs did not seem to destroy the antirachitic value of the yolk, when incorporated in the diet to the extent of 5 per cent.

Zucker and Barnett (1923) using the same method by which they had successfully concentrated 1,000 times the antirachitic potency of cod-liver oil, attempted to concentrate the vitamin D of butter, coconut oil, spinach, carrot, pig's liver, and sheep adrenals, but the amounts present in these substances did not seem large enough to yield satisfactory results.

Bethke, Steenbock and Nelson (1923) reported that "On a synthetic ration of purified food constituents deficient in fat-soluble vitamins poor calcification of bone occurred in rats even with the addition of 15 cubic centimeters of skimmed milk or 0.5 cubic centimeters of whole milk per rat per day. 20 cubic centimeters of skimmed milk or 1 cubic centimeter of whole milk on the other hand, allowed normal calcification."

Later, Steenbock, Hart, Hoppert and Black (1925) reported that from 8 to 12 cubic centimeters of fresh cow's milk daily were required to produce healing in rachitic rats (diet No. 2965); of fresh cow's milk irradiated for 30 minutes 1 cubic centimeter was effective. 
Supplee and Dow (1927) found that upon feeding reconstituted dried milk to rats on the Sherman-Pappenheimer diet No. 84, progressive increases in the ash content of the humeri could be obtained. Fed at 15 cubic centimeter levels, little difference could be seen between non-irradiated summer milk, irradiated summer milk and irradiated winter milk. At lower levels of feeding, higher antirachitic potency could be observed in summer milk than in winter milk, and in irradiated milks than in non-irradiated milks.

Sherman and Stiebeling (1929) added further to the evidence that milk as ordinarily produced (at least under conditions prevailing in this country) contains significant amounts of vitamin $\mathrm{D}$. This evidence remains valid even though rickets occasionally occurs in children receiving fairly liberal amounts of milk; among children less adequately fed the incidence of severe rickets is much greater.

Bills summarized in 1927 an extensive study of the distribution of vitamin $\mathrm{D}$ in various fats and oils. Of all the oils which he examined only the fish oils and the oil of the adult seal were active. He found a great difference in activity between the oils of different species of fish, and even within one species. Puffer fish liver oil (2 samples) was about 1,500 times as potent as cod-liver oil (average of 300 samples): goose fish liver (1 sample), herring (4 samples), sardine oil (4 samples), and cod-liver oil had about the same potency and the oils of several other species ranged in potency from 3 to 40 per cent of the value of the cod-liver oil. Body oil as well as liver oil may contain vitamin $\mathrm{D}$.

Schmidt-Nielsen (1930) reports that the liver oils of cartilaginous fish contain very little vitamin $\mathrm{D}$ in comparison with those of bony fish and suggests selective absorption in the latter to meet the needs of bone formation.

As previously noted in the discussion of rickets-producing diets, some of these are of such drastic character that when employed as a means of testing for vitamin $D$ they have resulted in reports that this vitamin is absent from various natural foods in which it more probably occurs in small or moderate amounts.

Origin of Vitamin $D$ in Nature.-Green leaves have been reported by many investigators to be poor sources of vitamin $\mathrm{D}$. On the other hand, experiments by Hess and Weinstock and others have demonstrated that irradiation with ultra-violet light can confer marked antirachitic potency upon leafy foods, thus showing that they are at least potentially antirachitic to an important degree. Chick and Roscoe (1926) suggested that the explanation of this apparent discrepancy might lie 
either in a difference in the degree of intensity of ultra-violet rays from the sun and from an artificial source, or to the rapid removal or decomposition of any antirachitic substances that might be produced in the growing leaf. The opinion of the botanist that ultra-violet light is possibly harmful and certainly unnecessary to the thriftiness of the plant lends support to the latter theory. No one seems to have yet studied the permanence of activation by ultra-violet irradiation of the vitamin precursor in an uncut and growing plant.

Leigh-Clare (1927a) was unable to demonstrate the presence of vitamin $\mathrm{D}$ in the diatom, Nitzschia clostcrium. It is possible that the amounts fed were not large enough to demonstrate the presence of the vitamin-the largest, 0.46 gram daily (fresh weight), may be too small to prevent, or effect a cure of, rickets in rats on the McCollum diet No. 3143.

It is, of course, possible that the plankton and small fish might receive sufficient solar irradiation to activate the vitamin precursor, and thus become a food source of vitamin $\mathrm{D}$ to the larger fish, including the cod. But from his knowledge of their dietary habits and the vitamin D content of their food, Bills (1927) calculated that codfish would have to eat 26 times their weight in caplin in 4 weeks during the fattening period, if food is the sole source of antirachitic vitamin. $\mathrm{He}$ doubts if the cod eat to that extent. He also cited experiments in which he fattened catfish on raw veal muscle (the fat of which had no apparent antirachitic value when fed at a level of 4 per cent to rachitic rats). The oil extracted from these fish was of normal or enhanced potency. Of this work he says, "In evaluating this additional evidence of the endogenous origin, one should, of course, consider the remote possibility that the veal muscle contained undetected traces of vitamin D which the fish with great economy might have salvaged." Furthermore he found that 5 minutes of irradiation every other day applied to fish under 20 centimeters of water not only did not increase the antirachitic potency of the oil, but exerted a very deleterious effect upon the health of the fish. Bills inclines to the theory that at least some vitamin $\mathrm{D}$ is synthesized by fish.

It is entirely possible that there are enzymes which can activate the vitamin precursor, just as Bills (1926) has shown that antirachitic substances can be formed by the action of a catalyst, floridin, on cholesterol or tricholesterol in carbon tetrachloride. There is also the possibility that for fish the longer light rays are antirachitic (productive of vitamin D). Van Leersum (1924) has shown that the injection of hematoporphyrin in rachitic rats had a therapeutic effect on rickets. 
He explained his results tentatively, on the theory that the hematoporphyrin increased remarkably the sensitivity of the organism to rays of light that lie within the visible part of the spectrum.

Belloc, Fabre, and Simonnet (1930) compared two samples of plankton collected from the same water, one in midsummer and the other in the spring, for their content of ergosterol and vitamin D. The sterols were separated from the material by saponifying the chloroform extract with potassium hydroxide and crystallizing the unsaponifiable fraction from ethyl alcohol, the whole process being conducted rapidly with protection from air and light. The crystallized products were subjected to physical (ultra-violet absorption), chemical (the Rosenheim and Meesemaeker color tests), and biological tests (feeding experiments with rats). As judged by these tests, the "summer" plankton contained both ergosterol and vitamin $\mathrm{D}$, while the "spring" plankton gave tests for the provitamin but not the vitamin. Belloc, Fabre, and Simonnet conclude that the biological activity of plankton is a function of a number of factors, prominent among which is light, but that the presence of the antirachitic factor in the food of fish does not exclude the hypothesis of an activating property of certain of their organs or the intervention of light rays.

Production of Antirachitic Potency in Foods.-Hess and Weinstock $(1924,1925)$ reported success in conferring antirachitic potency by ultra-violet irradiation upon cottonseed oil, linseed oil, green or etiolated lettuce and young growing wheat plant, and showed that irradiated green wheat plant fed at a 10 gram level (no other level tried) to rats on Diet No. 84 was effective in protection from rickets even after being stored from 3 days to 2 weeks and that activated linseed oil in daily doses as low as 0.1 cubic centimeter was potent after 6 months' storage. Irradiated patent flour fed as 95 per cent of the diet was potent (no other level tried). Also they reported that dry milk (5 per cent of diet), flour and spinach (10 gram level) can be rendered antirachitic by radiations from the quartz-mercury vapor lamp. This potency was maintained by the spinach after it had been boiled one-half hour (10 grams daily fed).

Steenbock and Black (1925) found that lard, olive oil, fresh corn oil, and coconut oil could be activated by irradiation (fed at 2 per cent levels) but old corn, peanut, oleo and coconut oils (kept from 6 to 10 years in the laboratory) could not be activated (2 per cent levels fed). They were able to increase the antirachitic potency of whole milk and egg yolk from 15- to 20-fold by irradiation.

Later Steenbock, Hart, Hoppert and Black (1925) recorded that 
"By exposure to the radiations of a quartz-mercury vapor lamp, the antirachitic properties of cow's milk were increased eight or more times. Under the same conditions a sample of goat's milk increased (in potency) about 24 times. The increase in activity can be induced rather promptly though to a lesser degree by direct irradiation of the animal."

Steenbock and Daniels (1925) conferred antirachitic potency by irradiation upon millet, lard, olive oil, wheat, Indian corn, yeast, cream of wheat, crude casein, hominy, rolled oats, shredded wheat biscuit, milk, egg yolk, butter, liver, the unsaponifiable fraction of olive oil, cholesterol, and phytosterol (except when prepared from old oil).

Dutcher and Kruger (1926) found that their irradiated corn oil had at least 38 times the antirachitic potency of irradiated dextrin.

Hart, Steenbock and their collaborators (1925) found that the antirachitic potency of egg yolks from irradiated hens was approximately ten times that of egg yolks from non-irradiated hens.

As yet the experimental evidence is not clear on the relative effect of diet and ultra-violet irradiation of the cow upon the antirachitic value of her milk.

Boas and Chick (1924) reported that rats fed milk from a cow on dry or fresh food, but kept in the dark, showed less calcium retention than rats fed milk from a cow after 2 months on pasture.

Luce (1924) later concluded that "the antirachitic value depends on the diet of the cow and possibly also on the degree of illumination to which she is exposed. Milk from a pasture-fed cow has a definite and high antirachitic value; the same animal when stall-fed in the dark yielded a milk much inferior in antirachitic properties." The part of her study on the effect of light alone was made in November when the average amount of sunshine daily was 3.4 hours and in December when it was but 1.4 hours. This work was later repeated after the cow had been kept for 3 months on a very drastic diet free from fatsoluble vitamins. Under such conditions, exposure to summer sunlight (6.4 hours daily) for 77 days appeared to raise the antirachitic value of her milk slightly but not materially.

In commenting on this work, Steenbock and his collaborators (1925) said that they had come to realize that food may or may not be more important than sunlight-depending upon whether or not the feeds had been exposed to ultra-violet light. "It does, however, appear to be true that sunlight acting upon the animal (cow) itself is not such an extremely active agent as one might be led to assume."

Chick and Roscoe (1926a) in continuing the work of Luce obtained 
results showing that the antirachitic value of the milk (vitamin D content) was found to depend principally on the effect of sunlight on the cow. At the same time there was some indication that the diet of the cow, if consisting of fresh green food, was a contributing factor. Their experiments confirmed those of Luce in showing that "pasturefed" milk possessed the maximum antirachitic value while that yielded by the cow when receiving a diet of cereals and roots appeared, on the whole, inferior in this respect, in spite of exposure to maximum illumination out of doors in summer.

Hart, Steenbock, Scott and Humphrey (1927) reported that ultraviolet light had little if any, direct influence upon the calcium and phosphorus metabolism of dairy cows. It was suggested that this should not be interpreted as indicating that exposure of the dairy cow to sunlight is not beneficial.

Hart, Steenbock, Teut and Humphrey (1929) reported that the feeding of cod-liver oil to dairy cows for more than a year had little influence on calcium assimilation. By feeding the ether extracts of the feces of these animals, they found that there was poor assimilation of vitamin $\mathrm{D}$ from the intestinal tract. Hart, Steenbock, Kline and Humphrey (1930) next fed irradiated yeast at the level of 200 grams per cow per day and found that the absorption of calcium was not increased and that there was vitamin $D$ in the feces. Some vitamin D was absorbed, however, for the amount in the milk was increased. Since calcium retention was secured with an intake of green plant tissue and high calcium, they conclude that something in green plant tissue other than vitamin $D$ is effective in calcium retention.

Golding, Soames and Zilva (1926) have shown that the antirachitic potency of milk fat can be considerably increased by feeding the cows cod-liver oil up to 8 ounces daily in addition to straw, roots and meal mixture; on the other hand, the addition of kale (40 pounds daily) to the cow's diet (straw, mangolds and meal mixture) did not measurably improve the antirachitic potency of milk fat in these experiments.

Supplee and Dow (1927) presented data which "appear to confirm the existence of a significant interdependence between antirachitic properties and the degree of ultra-violet light to which the product itself, or its synthesizing agent the body of the cow has been exposed." ... "Summer-produced milk possessed greater antirachitic and calcifying properties than winter-produced milk. Irradiation of the summerand winter-produced dry milk imparted measurably greater antirachitic and calcifying properties to both products. A greater increment of in- 
crease in calcifying properties was imparted to the winter-produced milk than there was to the summer-produced milk.

"These results indicate that both winter- and summer-produced milk may have the same potential capacity for endowment with antirachitic and calcifying properties by ultraviolet light. The realization of this potential capacity is not attained in milk produced either in the summer or winter months. However, when the cows are subjected to the greater solar irradiation prevailing in the summer months the embodiment of the antirachitic and calcifying properties in the milk is greater than in the winter months when the animals are exposed to the activating rays to a much less degree. Because of the inherent conditions in the quality of the food consumed by cows in the temperate zones during the winter and summer months, the effect of such conditions on the antirachitic properties of the milk is not precluded by the data recorded herein."

Thus it appears that while both fresh green feed and sunshine improve the antirachitic value of the milk, the relative importance of each factor separately depends on the quality of the cow's ration, both from the standpoint of its vitamin and mineral content.

Apparently vitamin D is not abundantly furnished in most food materials. Fish oils, egg yolk, butterfat and whole milk are the best natural sources; green leafy foods contain it in small quantities. Most unrefined foods contain to a greater or less degree the vitamin precursor which can be rendered antirachitic by exposure to light rays of less than $310 \mu \mu$ in wave length.

Several of the topics briefly summarized in this chapter are fully and excellently treated by Blunt and Cowan in their Ultraviolet Light and Vitamin $D$ in Nutrition. 


\section{CHAPTER VII}

\section{VITAMIN E}

Evans and Bishop $(1922,1923 a)$ found that rats kept for relatively long periods of time upon mixtures of purified foodstuffs and with ample provision for all previously known vitamin requirements finally showed evidence of needing, for successful reproduction, an hitherto unknown fat-soluble substance which they tentatively called "X," but which later, in accordance with the chronological alphabetical sequence first suggested by McCollum, became known as vitamin E. Descriptively, this vitamin is often referred to as the "antisterility" vitamin or the "vitamin of reproduction." These terms are unfortunate, for while vitamin $E$ is undoubtedly essential to reproduction, it is certainly no more so than vitamin A. A diet devoid of vitamin A stops reproduction quite as certainly and may stop it quite as quickly as one devoid of vitamin $\mathrm{E}$. Vitamin $\mathrm{E}$, therefore, is no more directly or essentially concerned with reproduction than is vitamin $\mathrm{A}$, and because of its more widespread occurrence is probably of less practical importance than vitamin A. The greater emphasis upon the sterility-preventing properties of vitamin $\mathrm{E}$ than of vitamin $\mathrm{A}$ is probably due to the fact that this is its chief function, while vitamin $A$, as has been shown in Chapter $\mathrm{V}$, has many other important functions in addition to its essential part in the nutritional processes of reproduction.

Lack of vitamin A causes failure of reproduction through interfering with ovulation, whereas lack of vitamin $\mathrm{E}$ interferes with placental function. These two disturbances of the reproductive process are clearly distinguished by the histological technique developed and used by Evans and his collaborators who feel that if this technique is followed carefully there is no danger of shortage of either one of these vitamins being mistaken for a shortage of the other. In their opinion the sterility caused by lack of vitamin $\mathrm{E}$ can be recognized in the living animal only by following each step in the reproductive process. Evans and Burr (1927d) have emphasized in their memoir on vitamin $E$ that in all studies of reproduction "almost no knowledge is gained by merely placing animals of the opposite sex together and ascertaining that infertility results." 


\section{Proof of the Existence of Vitamin E}

Considerable hesitancy was at first shown by various investigators (Hogan and Harshaw, 1924; Anderegg, 1924; Anderegg and Nelson, 1925, 1926; Nelson et al., 1926) in regard to the existence of a new fat-soluble vitamin essential to reproduction. One reason for this was undoubtedly the failure to carry out in every detail the technique which Evans and his collaborators emphasized as essential in differentiating sterility due to lack of vitamin $\mathrm{E}$ from that induced by other causes. Another ground for hesitancy in accepting the vitamin hypothesis for this type of sterility was the failure to understand the significance of the so-called "initial fertility" on diets supposedly lacking in vitamin E. As noted by Evans and Bishop in their early reports, there may be a high percentage of fertility in the first gestation of such a group of rats, but second and subsequent gestations show increasing sterility. Younger animals show greater fertility than older ones on the same deficient diet and there may even be seasonal variations in fertility. Two factors were considered to be chiefly responsible for initial fertility on diets supposed to be free from vitamin E-the transmission of a part of the mother's store of vitamin $\mathrm{E}$ to her offspring during gestation and the inclusion unwittingly of vitamin $\mathrm{E}$ in the basal diet in traces which may vary seasonally.

Evidence of the existence of a vitamin having the properties described by Evans and Bishop was soon forthcoming in two other laboratories where investigations which had been proceeding for some time with quite different objectives from those of Evans led to the same conclusion. Sure, at the Arkansas Agricultural Experiment Station, during the course of an investigation begun in 1919 on the amino acid requirements for reproduction, encountered a dietary sterility in his experimental rats which could not be prevented or cured by the addition of any of the known vitamins or amino acids or by improving the salt mixture. The addition of a plant material, even from a single source such as the Georgia velvet bean pod meal, rice, corn, or oats, to a sterility-producing diet, resulted in fertility. With the announcement by Evans and Bishop of the existence of a hitherto unrecognized dietary factor essential for reproduction, it became evident to Sure (1924, 1924a) that the materials which he had found effective in preventing sterility owed this property to the presence of this same vitamin.

Meantime Mattill and his associates at the University of Rochester, during the course of an extensive investigation (also initiated in 1919) of the nutritive properties of milk, had reported as early as 1920 (Mat- 
till and Conklin, 1920) failures in reproduction in rats on diets consisting chiefly of milk. In the second paper from this laboratory, Mattill and Stone (1923) described degenerative changes in the reproductive organs of male rats on diets in which all of the proteins and vitamins were furnished by milk. They noted also that the infertility of female rats on these rations could not be explained by decline in ovarian functions, since ovulation seemed to be taking place frequently and the ovaries examined, though of small size, appeared to be normal.

Mattill and Carman (1923) reported that the sterility in male rats on milk diets could not be prevented by the inclusion in the ration of rich sources of vitamin B and concluded that "the lack of some other substance than vitamin $B$, and as yet unrecognized, may be solely or jointly concerned in the disappearance of the reproductive function." Mattill, Carman, and Clayton (1924) definitely accepted vitamin E, or $X$ as it was still designated at that time, as the missing factor in the milk diets of their previous studies and reported success in the prevention of this sterility by the various substances considered by Evans to be rich in this vitamin.

The statement by Sure (1926) that cottonseed oil contains vitamin E and by Evans and Burr (1925a), confirmed by Kennedy and Palmer (1926), that this is also true of hydrogenated cottonseed oil led Hogan and Harshaw (1926a) to replace this component of their basal diet with lard, following which change they were able to confirm Evans' findings in every respect. The failure of Anderegg and Nelson to confirm the conclusions of Evans and of Sure was finally accounted for by Nelson, Ohrbeck, Jones, and Taylor (1928) in the discorery that the brand of cod-liver oil which they were using contained appreciable amounts of vitamin $E$. In view of this accumulated evidence there seems to be no longer any skepticism concerning the existence of vita$\min \mathrm{E}$.

\section{Determination of Vitamin $\mathrm{E}$ and Its Distribution in Food Materials of Animal and Vegetable Origin}

The highly special technique required for ascertaining with certainty that the dietary sterility which is used as the criterion of vitamin E deficiency is not due to other causes has served to retard such widespread studies of the occurrence of this vitamin as have been made of the other vitamins. Moreover, too much reliance cannot be placed upon the quantitative aspects of such studies as have been reported, owing to the discovery noted elsewhere that vitamin $\mathrm{E}$ in any foodstuff 
may be destroyed by the oxidizing action of other constituents. As Mattill (1927) has stated:

"Depending on the presence of catalysts of various kinds, or of antioxidizers, the amount of vitamin $\mathrm{E}$ present in an individual foodstuff or in ration mixtures remains unimpaired for shorter or longer periods. Whether the oxidative changes that condition its destruction may take place in the alimentary tract after the ration has been consumed, as well as during its preparation or in containers before its ingestion, remains to be determined. Obviously the assay of foodstuffs for their content of vitamin $E$ has been a resultant of (1) the actual amount of E present and (2) the kind and amount of antioxidizers associated with it."

The most extensive studies of vitamin $E$ distribution are those of Evans and his associates as summarized in the University of California Memoir by Evans and Burr (192/d).

In their earlier studies Evans and his collaborators used as their basal ration a mixture consisting of casein 18 , cornstarch (cooked and subsequently dried) 54 , lard 19 , milk fat 5 , and salts 4 per cent, with dried whole yeast from 0.4 to 0.6 gram daily. Later this diet was changed to one consisting of casein 32, cornstarch 40 , lard 22, cod-liver oil 2, and salts 4 per cent, with the same amount of yeast. The salt mixture in both cases was McCollum mixture No. 1Sj, consisting of sodium chloride 51 , crystals of magnesium sulfate 159.6, monobasic sodium phosphate 104.1, monobasic calcium phosphate 162, dibasic potassium phosphate 286.2, ferric citrate $3 \mathbf{3 . 4}$, and calcium lactate 390 parts.

In Evans' opinion temale rats to be used in detecting the presence of vitamin $E$ should not only show the typical signs of vitamin $E$ deficiency on breeding-implantation of the ovum, but eventual resorption of the fetus-but should give evidence of normal fertility when tested with a material of proven potency in vitamin E. Curative tests are considered much more reliable than prophylactic. The test, therefore, consists in establishing resorption gestation on the basal ration alone and subsequent fertility on the basal ration supplemented by the material in question, the amount required in comparison with a standardized source of vitamin $E$ such as wheat germ oil being a measure of the richness of the material in vitamin $\mathrm{E}$.

With the use of the method as briefly sketched, Evans and his collaborators (see Evans and Burr, 1927d) have shown that vitamin E is present, but never in high concentration, in a great variety of animal tissues. In contrast with vitamin $A$, it is more abundant in the general body musculature and fat than in the viscera. Vitamin $E$ is present in egg yolk and in milk, the amount in the latter being higher in pasturefed than stall-fed cattle. In contrast with Mattill's results with milk, 
whole milk powder as the sole food furnished adequate amounts of vitamin $\mathrm{E}$ in Evans' experiments. Of butterfat, 9 per cent of the ration is usually insufficient to secure fertility, but with rations containing much larger amounts fertility results. Conflicting evidence on the presence of vitamin $\mathrm{E}$ in butterfat can probably be explained by the destruction of the vitamin to a greater or less extent by other constituents of the diet. As is to be expected from the relatively low content of vitamin $\mathrm{E}$ in the viscera, cod-liver oil is extremely low though not entirely lacking in vitamin $\mathrm{E}$.

In contrast with the low content of vitamin $\mathrm{E}$ in animal tissues is its high concentration in the organs of certain plants, especially in green leaves and seeds. The richest known sources of vitamin $\mathrm{E}$ are lettuce leaves and wheat embryo, both of which remain active when desiccated. Successful curative tests have been made with amounts as small as 250 milligrams daily of dried lettuce powder and smaller amounts have been partially successful. Alfalfa leaves and even tea leaves have been found to be fairly rich in vitamin $\mathrm{E}$, although not so rich as lettuce. Daily doses of 1 gram of peanuts or 5 grams of corn embryo, alfalfa seed, and lettuce seed were curative in the experiments reported by Evans and Burr.

Most vegetable and seed oils contain vitamin E, but in not very high concentration. In curative tests (which always require considerably more of the vitamin than prophylactic tests) 100 milligrams of cottonseed oil, olive oil, walnut oil, peanut oil, and flaxseed oil were without effect, although some of these oils were effective in smaller dosage in prophylactic tests. Evans and Burr are of the opinion that all of these oils contain enough vitamin $\mathrm{E}$ to confer fertility if they could be fed in amounts representing the total fat content of the ration. In one trial, cures resulted from the feeding of walnut oil to the extent of 22 per cent of the ration. It is of interest that lettuce-seed oil was effective in dosage of 160 milligrams daily, representing one-half gram of seed.

The only fruits tested by Evans and Burr were bananas and oranges, both of which contained the vitamin but in very low concentration. About 27 grams daily of banana and from 8 to 16 cubic centimeters of orange juice were employed in curative experiments which, in the case of the orange at least, were only partially successful.

Sure has reported from time to time on the occurrence of vitamin $\mathrm{E}$ in various food materials, his results (although obtained with different basal rations and technique) corroborating in a general way those of Evans. Sure (1924b) reported the presence of vitamin E in the 
ether extracts of yellow corn, wheat embryo, and wheat seed, and in commercial cottonseed and olive oils, but not in commercial coconut, linseed, or sesame oils. Evans and Burr had also noted the relatively low content of $\mathrm{E}$ in coconut oil. Sure (1927) also reported the presence of vitamin $\mathrm{E}$ in appreciable amounts in butterfat, but stated that when butterfat was fed to the extent of 10 per cent of the ration the results were not as good as with 1 per cent of wheat oil as the source of vitamin $\mathrm{E}$.

Shortly after the publication of the Memoir in which their work on vitamin $E$ up to that time had been summarized, Evans and Burr (1927a) announced a new basal vitamin-E-free diet dispensing with lard and using sucrose in place of starch. The diet consists of casein prepared by the Van Slyke method 50, sucrose crystallized from 80 per cent alcolol 150, and a salt mixture (185) \& parts, with distilled water ad libitum containing a trace of potassium iodide, cod-liver oil from 2 to 3 drops daily, and yeast from 700 to 1,000 milligrams daily.

More recently Waddell and Steenbock (1928) have reported that a ration composed of natural and varied food materials such as the Steenbock (1923) stock ration can be completely freed from vitamin $\mathrm{E}$ without affecting the vitamin A content of the ration by simple treatment with ferric chloride in the proportion of 1 part to 99 of the ration. The treatment consists in dissolving the correct proportion of the ferric chloride in ether with a small amount of water, pouring the solution over the ration and allowing the ether to evaporate. It is stated that complete sterility is secured in the first gestation on this ration.

\section{Relation to Metabolic Processes}

Although it is beyond the scope of this monograph to discuss in detail the characteristic histopathology of animals suffering from vitamin E deficiency, for excellent discussion of which the reader is referred to Evans and Burr (1927d) and to Mason (1925), attention is called to the different effects produced by lack of vitamin $\mathrm{E}$ upon the male and female reproductive organs. As stated early in the chapter, in the female there is no failure of ovarial but only of placental function. In the male, however, the testes, while at first not adversely affected, ultimately rather completely degenerate. Female sterility can always be cured, since only temporary tissues are damaged, while male sterility tends to go beyond recall through the breaking down of the permanent testicular tissues. In attempting to explain these differences, Evans suggests that both ovary and testis are originally equally favored with the substance, but that the immensely greater cell production occurring 
in the testis leads to earlier exhaustion of the store of vitamin $\mathrm{E}$ in the male than in the female.

Mason (1930) has recently shown that both the A and E vitamins are essential for the maintenance of a normal germinal epithelium of the testis, since neither one of these factors was able to maintain testis normality in the absence of the other. "In other words, they appear to supplement each other in some peculiar way. On the basis of the similarity in distribution, solubilities, and other characteristics of these two factors, it is suggested that they may be concerned in certain phases of fat absorption, assimilation, or synthesis essential for the complete metabolic activities of the developing and maturing germ cells."

As has been shown to be the case with the other fat-soluble vitamins, the animal body is capable of storing a certain amount of vitamin $E$ in its tissues, although curiously enough the chief storage centers are the muscles and fat rather than the active organs as in the case of vitamin A. Evans and Burr found that the tissues of normal rats when fed to female rats which had been made sterile by vitamin $E$ deficiency rendered them fertile, while the tissues of such sterile rats were ineffective. Another proof of the storage of vitamin $\mathrm{E}$ in the animal body is that fertile rats retain their fertility for three or four months after being shifted to a vitamin $\mathrm{E}$ deficient ration and that when sterile rats (female) are cured by the administration of vitamin E, the fertility may last through two or three subsequent gestations, depending upon the amount of vitamin $\mathrm{E}$ administered.

The ability of the body to store and hold in reserve a surplus of vitamin $\mathrm{E}$ has been shown still further by the restoration of fertility in female rats by a single massive dose of vitamin $E$ on, or within five days after, the beginning of gestation. In the case of wheat germ oil, one of the richest sources of vitamin $\mathrm{E}$, the daily administration of one drop (weighing between 23 and 30 milligrams) during the entire gestation period of 22 days, or a single feeding of the total amount, always resulted in the birth of living young. Administration of the oil by parenteral injection, either in a single massive dose or in small daily doses, was likewise effective.

The administration of foods or extracts of foods known to be from twice to twenty times as rich in vitamin $\mathrm{E}$ as is required for normal reproduction did not result, however, in any increase in the number or size of the young nor did it in any other way improve the performance of the reproductive mechanism beyond usual limits.

With this highly specialized function of vitamin $E$ in maintaining fertility, the question naturally arises as to whether this is its sole 
function or, as in the case of the other vitamins, it takes part in some way in the usual metabolic processes of the body. Vitamin $\mathrm{E}$ is always present, though not in large amounts, in the tissues of newborn rats. This in itself would suggest the necessity of the vitamin for the normal functioning of the body. That there is some normal use or wastage of vitamin $\mathrm{E}$ in the usual metabolic processes of the body was inferred by Evans and Burr from observations which indicated that shielding female rats from the nutritional demands of reproduction did not result in any marked prolongation of the period of retention of fertility on vitamin-E-free food.

In an attempt to discover a possible relationship between vitamin $\mathrm{E}$ and the metabolism of other food constituents, Anderson (1926) studied the composition of the blood and the metabolism of nitrogen and fat in rats on rations with and without vitamin $\mathrm{E}$, but discovered no differences which might account for the function of vitamin $\mathrm{E}$.

The possibility that vitamin $\mathrm{E}$ might be a factor concerned in hematin building and consequently with iron assimilation had been suggested by Hart, Steenbock, Elvehjem, and Waddell, and this had led Hogan and Harshaw (1926a) and later Sure, Kik, and Walker (1929a) to examine the blood of rats rendered sterile by lack of vitamin E. Hogan and Harshaw reported no differences in the erythrocyte counts of female rats which had resorbed their fetuses because of vita$\min \mathrm{E}$ deficiency from those of controls which had carried their litters to term on adequate diets. They also found no reduction in the concentration of hemoglobin in either male or female on vitamin-E-deficient rations. Sure, Kik, and Walker likewise reported no reduction in hemoglobin and erythrocytes of female rats during the period of resorption of the fetuses and no reduction in the total leucocyte count as the result of vitamin $\mathrm{E}$ deficiency. Lack of association between iron or other mineral constituents and vitamin $E$ was shown by the failure of ferric citrate or ash of lettuce leaves to prevent female sterility produced by vitamin $\mathrm{E}$ deficiency.

Evans and Burr (1928) described a peculiar form of paralysis developing in suckling rats of mothers on diets low, but not completely lacking, in vitamin E, but abundantly supplied with vitamin B so essential for successful lactation. The paralysis, which develops a day or two before the weaning day (twenty-first day), begins with difficulty in regaining the use of the limbs when the rats are placed on their backs and increases until part of the musculature of the body and the limbs is paralyzed, although the animal appears normal in other ways. 
It was noted that while some of the animals showed an initial flaccid paralysis-a dragging of the legs-this was always succeeded by a spastic condition. Evans and Burr stated that "a more accurate symptomatic and full pathologic-anatomical study of the singular and obscure condition of these animals is under way," but no report of this study had come to our notice at time of writing.

Meantime, Goettsch (1930) announced in a preliminary report that in guinea pigs and rabbits on a diet free from vitamin $\mathrm{E}$ but adequate in other respects (Waddell and Steenbock) changes apparently specific for vitamin $\mathrm{E}$ deficiency take place in the voluntary muscles. "The muscles of the thigh and abdomen were particularly involved. They appeared atrophied and pale and had a yellowish color, quite different from those of the normal controls. Sometimes they were gritty looking and streaked as though calcified or infiltrated with fat. In two cases the thigh muscles were markedly hemorrhagic. The muscles seemed to have lost their irritability."

This report was accompanied by a further description by Pappenheimer (1930) of the pathological changes observed in the affected muscles. The primary alteration was found to be a waxy or hyaline necrosis of the fibers, followed by a great proliferation of the muscle nuclei and in later stages by active regeneration of the muscle cells, accompanied by a variable aniount of interstitial fibrosis. Histological studies of other tissues than the skeletal muscle showed no significant change. The pathological condition is summarized as a universal dystrophy of the entire voluntary muscular system. The significance of these findings awaits further study.

It has been emphasized in the previous chapters that it is ambiguous to apply the term growth-promoting to any one vitamin, since the absence of any of them interferes sooner or later with growth processes. According to Evans (1928c) the time at which vitamin $\mathrm{E}$ affects the growth of rats is after sexual maturity. During the first 90 days of life significant differences may not occur in the growth of the rat on diets rich in or free from vitamin E, but the improvement is evident after the eighth month and marked after one year. The favorable effect on the later growth of male rats was not altered by removal of the testes, thus eliminating the possibility that the favorable effect upon growth may be brought about indirectly through the action of vitamin $\mathrm{E}$ upon the sex glands.

While vitamin $E$ thus appears to be one of the essential food constituents, it is probably not of as great importance from a practical point of view as the other vitamins on account of its wide distribution 
in foods and the extreme smallness of the amounts needed for its special functions.

\section{Physical and Chemical Properties}

In a report to the National Academy of Sciences on April 25, 1925, Evans and Burr (1925a) summarized their findings concerning the physical and chemical characteristics of vitamin $\mathrm{E}$ as follows:

"The vitamin may be called fat soluble, though its range of solubility is far greater than that of ordinary fats. While this range of solubility may really be due to the solubilities of impurities as yet associated with the vitamin, it is a fact that the most concentrated fractions yet obtained have been almost completely miscible with solvents representing such a range as methyl alcohol, ethyl alcohol, ether, pentane, benzene, acetone, etlyyl acetate, carbon disulfide and so forth. The vitamin is almost insoluble in water, yet we have repeatedly encountered its presence in water solutions. There is enough left in the water after precipitation of calcium soaps, for instance, to be extracted with ether and effect cures. The distribution ratio between water and ether is very large, for a few extractions with an equal volume of ether effect quantitative removal. This has been established by a large number of feedings of the non-saponifiable fraction, the residual soap always failing to produce fertility. The solubility of $\mathrm{E}$ in such substances as alcohol and pentane shows a large temperature coefficient and is so much greater than some of the contaminating substances, the sterols, for example, as to permit separation of the vitamin from them.

"Vitamin E is remarkably stable to heat, light, air, and many of the ordinary chemical reactions. As regards temperature, while the ashing of wheat germ completely destroyed the vitamin, yet heating of the germ to $170^{\circ} \mathrm{C}$. so that it was greatly charred left the $E$ unimpaired. Distillation of wheat germ oil, or a fraction out of it, in superheated steam at $180^{\circ} \mathrm{C}$. for several hours has not destroyed it. Distillation in vacuo up to $233^{\circ} \mathrm{C}$. has not, in fact, caused any lowering of the potency of the fractions so treated, nor have any physical changes like changes in solubility been detected. We have not encountered evidence that daylight affects $E$ in wheat germ oil, but there would appear to be partial destruction by exposure in thin layers to a powerful quartz mercury lamp for one hour. As regards oxidation, exposure of wheat germ oil for as many as twelve hours to a stream of air washed with acid and alkali, and at $97^{\circ} \mathrm{C}$., has not destroyed E. At normal temperatures the vitamin is remarkably stable to both acid and alkali and many chemical treatments. It dissolves unchanged, for instance, in saturated alcoholic hydrogen chlorid. We have hydrogenized wheat germ oil in the presence of palladium at $75^{\circ} \mathrm{C}$., and no injury to the vitamin resulted. Further, alcoholic extracts of Crisco, a hydrogenization product of cottonseed oil, are always fairly rich in the vitamin. We have treated the germ oil with both 20 per cent hydrochloric acid and one-tenth normal acid for twenty hours at room temperatures without destruction of the vitamin. It is not destroyed by concentrated sulfuric acid. It resists the action of boiling 20 per cent alcoholic potassium hydrate, though partial destruction would appear to occur on very prolonged hot saponification. The saponification with 20 per cent alcoholic potassium hydrate can be carried out at $30^{\circ} \mathrm{C}$. without great loss of the vitamin which goes into the nonsaponifiable quota, 5 per cent of the oil, so that by this step alone a notable concentration of $\mathrm{E}$ is always attained. The non-saponifiable quota is, in turn, chiefly (73 per cent) sitosterol, which is largely insoluble in pentane in the cold, an excellent solvent for vitamin $E$, which, together with pigments and other materials, can thus be washed out of the sterols, leaving them white. The sterols are inactive. If the orange-red viscous oil obtained from the pentane is treated with methyl alcohol, more extraneous material is removed, and the vitamin goes into the alcohol portions which now can be mixed with petroleum ether or diluted to 90 per cent methyl alcohol, allowing an immediate separation into two layers, the petroleum ether invariably securing more of the vitamin, in fact, all of it, 
if the distribution be done with successive fresh portions of the petroleum ether. Further purifications can now be carried out both with digitonin, boiling methyl alcohol, and finally, distillations in vacuo."

In spite of its great stability toward various reagents, as thus summarized, vitamin $\mathrm{E}$ has been found to be quite unstable in the presence of certain fats and salts; and the failure to recognize this led to some of the discrepancies in results in earlier vitamin $\mathrm{E}$ studies, and it was through efforts to explain these discrepancies that this instability was finally recognized.

Anderegg (1924) reported failure of reproduction in rats on a diet consisting of whole milk powder 50 , starch 38 , lard 10 , and salt mixture 2 per cent and success when part of the lard was replaced by an equivalent amount of dextrin, and concluded that for success in reproduction on synthetic diets the ratio of fat to protein should be kept within certain limits.

Mattill, Carman, and Clayton (1924) reported similar success or failure, depending upon the proportion of lard in the diet, but did not agree with Anderegg in the interpretation of these findings, stating, "this fact is considered not as evidence against the existence of $\mathrm{X}[\mathrm{E}]$, as some have held, but as an indication that the amount of $\mathrm{X}$ required for normal reproductive functions depends upon the nature of the diet."

Clayton (1927), working in Mattill's laboratory, conducted a systematic comparative study of a series of rations containing lard for the fat and cod-liver oil for vitamins $\mathrm{A}$ and $\mathrm{D}$ with a parallel series containing no lard and with separate daily administration of cod-liver oil or with radiation of the animals to furnish vitamin $D$, and reported that "from observations on several hundred animals, many of them in the third generation, it is clear that the presence of these and perhaps other unsaturated animal fats in the ration seriously interfered with reproduction. Reproduction but not lactation was improved when these fats were omitted. ... The presence of wheat germ oil in the fatcontaining rations favored reproduction and also lactation, especially in skim milk powder rations. Depending on as yet unknown factors various rations differ considerably in their ability to protect vitamin $\mathrm{E}$ from the destructive action of unsaturated animal fats."

The same year Evans and Burr (1927) reported that lard at a level of 22 per cent of the ration destroys or renders ineffective the vitamin $\mathrm{E}$ in wheat germ, but not at a lower level ( 7.7 per cent of the ration). Among other materials tested, hydrogenated cottonseed oil and oleic acid rendered vitamin $\mathrm{E}$ ineffective, while butter, cod-liver oil at low concentrations, and stearic acid did not. In this paper they were inclined 
to explain this phenomenon by suggesting that in high fat diets the vitamin is taken up by the fat and excreted with the unabsorbed fraction.

Mattill (1927), attempting to correlate the destruction of vitamin $E$ with that of vitamin $\mathrm{A}$ in butterfat or cod-liver oil through oxidation processes accompanied by the development of rancidity, tested several composite rations used in vitamin $\mathrm{E}$ studies and also mixtures of simple fats for susceptibility to oxidation by the method of Greenbank and Holm (1925) and found that the presence of wheat germ oil in place of lard in the sterility ration rendered it much less susceptible to oxidation, that the rapid oxidation of cod-liver oil was hastened by the presence of traces of ferrous sulfate and retarded by traces of wheat germ oil, that butterfat was less rapidly oxidized than cod-liver oil and that its oxidation was likewise checked by the presence of wheat germ oil. On the theory that the protective action might be due to hydroxyl groups, the acetyl value of wheat germ oil was determined and found to correspond closely to the values for other vegetable oils and to be higher than the values for animal fats and oils, particularly lard and cod-liver oil. The removal of the hydroxyl groups from wheat germ oil by acetylation rendered the oil less effective as an antioxidizer.

Shortly afterward Evans and Burr (1927c), abandoning their theory of the mechanical removal of vitamin $E$ by excess fat in the ration, reported studies along the same general lines as those of Mattill, suggesting the presence in certain fats of a destructive factor for vitamin $\mathrm{E}$ associated with the development of rancidity. They found that rather definite amounts of lard, "oleic acid," or any substance capable of neutralizing the effect of vitamin $\mathrm{E}$ were required for definite amounts of wheat germ oil as the source of vitamin $\mathrm{E}$. While the destruction of vitamin $\mathrm{E}$ was associated with the development of rancidity, even such a good source of vitamin $\mathrm{E}$ as wheat germ oil having a definite destructive effect when rancid, the increase in destructive action did not parallel the acid numbers of the fat. In attempts to remove the destructive substance from the fats, it was found that recrystallized lard is less destructive than market lard, and that on neutralizing the free acids in "oleic acid" with potassium hydroxide and extracting the alkaline aqueous solution of the soaps with ether, most of the strong odor and activity are to be found in the ether extract representing the unsaponifiable fraction.

\section{Concentration and Attempted Isolation}

The method employed by Evans and Burr in their attempts to isolate vitamin $\mathrm{E}$ from wheat germ consists essentially in the following steps: 
Extraction with ether; saponification with alcoholic potassium hydroxide; separation of active material from the non-saponifiable fraction by solution in cold pentane; further concentration by extraction of pentane solution with hot methyl alcohol, and of methyl alcohol solution with petroleum ether; separation of inactive sterols by precipitation with digitonin; saponification with hot 20 per cent alcoholic potassium hydroxide and second precipitation with digitonin; further concentration by solution in boiling methyl alcohol, followed by fractional distillation in vacuo.

The general plan of procedure, approximate yields, and the activity of the fractions thus obtained have been summarized by Evans and Burr in the accompanying outline.

\section{OUTLINE OF FRACTIONATION OF 6 KILOS OF WHEAT GERM}

6 kilos wheat germ.

Extracted with U.S.P. eth:r in Soxh-

let.

Ether extract. Active. Yield: $600 \quad$ Ether-insoluble residue. Inactive. grams $10 \%$.

Saponified in the cold with $20 \%$ alcoholic $\mathrm{KOH}$.

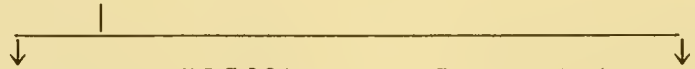

Nonsaponifiable matter (N.S.M.).

Yield: $5 \%$. Contains all the active

Soaps and glycerol. Inactive.

material.

Crystallized from cold pentane.

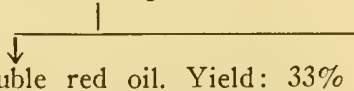

Pentane-soluble red oil. Yield: $33 \%$ of the N.S.M. Contains all the active material.
Pentane insoluble solids. Sitosterol. Yields: $66 \%$ of N.S.M. Inactive.

Extracted with hot $\mathrm{MeOH}$.

Hot methyl alcohol solution. Active.

$\downarrow$

Crystallized from cold $\mathrm{MeOH}$.

Cold $\mathrm{MeOH} \downarrow$

Distributed between dilute $\mathrm{MeOH}$ and

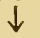
petroleum ether.

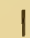

Methyl alcohol insoluble residue. Inactive. Yield: $3 \%$ of N.S.M. 
Petroleum ether soluble. Active.

Yield: $13 \%$ of N.S.M.

Sterols precipitated by digitonin.

$$
\downarrow
$$

Sterol-free orange oil. Yield: $2-3$ grams. Active.

Refluxed in hot $20 \%$ alcoholic $\mathrm{KOH}$.
Dilute $\mathrm{MeOH}$ soluble. Inactive. Yield: $4 \%$ of N.S.M.

N.S.M. Contains all of the active ma- Fatty acids. Inactive. terial.

Sterols again precipitated by digitonin.

Sterols from digitonide. Inactive.

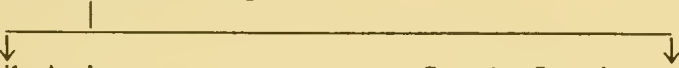

Sterol-free oil. Active.

Treated with boiling $\mathrm{MeOH}$.

Sterols. Inactive.

$$
\downarrow
$$
Orange solution in $\mathrm{MeOH}$. Active.
Yield: $700-1,000$ mgs. (Proven active in single doses of 5-10 mgs.)

Distilled in vacuo.

\section{Fraction I.}

Fraction II.

Residue insoluble in hot $\mathrm{MeOH}$. Inactive. Yield: 50-100 mgs.

Up to $200^{\circ}$ C. at $0.8 \mathrm{~mm}$. Low activity. $38 \%$ of the total.

$200^{\circ}-233^{\circ}$ C. at $0.5 \mathrm{~mm}$.

Highly active. $27 \%$ of the total.

\section{Fraction III.}

Residue above $233^{\circ} \mathrm{C}$.

Highly active. $35 \%$ of the total.

The final fraction is described by them as follows: "The final yellow viscous oil does not develop crystals on long standing. It contains only a trace of ash and no nitrogen, sulfur, phosphorus, or halogen. It is remarkably potent. When $5 \mathrm{mg}$. are fed or injected under the skin of a female of proved sterility at the inception of a new gestation, normal litters of vigorous young are born and have been reared to adolescence. Sister control rats invariably continue sterile. Furthermore, the daily administration of only $0.3 \mathrm{mg}$. of this substance throughout the life of the male results in the retention of complete normality when animals are reared and held on pure foods, a normality proved by the weight and histologic condition of the testis and by weekly functional tests throughout the year, and controlled by the invariable development of sterility at the end of three months in litter-mate brothers held on the identical ration save for omission of the trace of vitamin."

Evans and Burr consider that the best fractions are still not suffciently pure for trustworthy speculation as to the exact chemical nature of the vitamin. 



\section{BIBLIOGRAPHY}

\section{Abderhalden, E.}

1919. Studien über den Einfluss der Art der Nahrung auf das Wohlbefinden des einzelnen Individuums, seine Lebensdaur, seine Fortpflanzungsfähigkeit und das Schicksal der Nachkommenschaft. Arch. ges. Physiol. (Pflüger's) 175, 187-326.

1920. Weitere Beiträge zur Kenntnis von organischen Nahrungsstoffen mit spezifischer Wirkung. Arch. ges. Physiol. (PAïger's) 178, 260-308.

a. Weitere Beiträge zur Kenntnis von organischen Nahrungsstoffen mit spezifischer Wirkung. II. Arch. ges. Physiol. (Pflüger's) 182, 133-156.

1921. Weitere Beiträge zur Kenntnis von organischen Nahrungsstoffen mit spezifischer Wirkung. IV. Gaswechseluntersuchungen an mit geschliffenem Reis mit und ohne Hefezusatz ernährten Tauben. Arch. ges. Physiol. (Pfliiger's) 187, 80-89.

a. Weitere Beitrag zur Kenntnis von organischen Nahrungsstoffen mit spezifischer Wirkung. V. Arch. ges. Physiol. (Pflïger's) 188, 60-66.

b. Weitere Beiträge zur Kenntnis von organischen Nahrungsstoffen mit spezifischer Wirkung. VIII. Versuche an Meerschweinchen. Arch. ges. Physiol. (Pfiüger's) 191, 278-301.

c. Weitere Beiträge zur Kenntnis von organischen Nahrungsstoffen mit spezifischer Wirkung. IX. Arch. ges. Physiol. (Pflïger's) 192, 163-173.

1922. Weitere Beiträge zur Kenntnis von organischen Nahrungsstoffen mit spezifischer Wirkung. XI. Versuche an Tauben. XII. Vergleichende Untersuchungen über das Verhalten des Gewichtes und des Wassergehaltes von einzelnen Organen bei Tauben die normal ernährt wurden, bzw. ausschliesslich geschliffenen Reis mit und ohne Hefezusatz erhielten, bzw. vollstandig hungerten. Arch. ges. Physiol. (Pflügcr's) 193, 329-354, 355-358.

a. Weitere Beiträge zut Kenntnis von organischen Nahrungsstoffen mit spezifischer Wirkung. XV. Ernährungsversuche mit künstlich dargestellten organischen Nahrungsstoffen und ferner mit aus zusammengesetzten organischen Nahrungsstoffen gewonnen Bausteinen mit und ohne Zusatz von Nutraminen. Arch. ges. Physiol. (Pfï̈ger's) 195, 199-226.

b. Weitere Beiträge zur Kenntnis von organischen Nahrungsstoffen mit spezifischer Wirkung. XVI. Vergleichende Untersuchungen über die Wirkung von erwärmter und nichterwärmter Kleie und Hefe und ferner von Organen von normal ernährten und von mit geschiffenem Reis ernährten Tauben. Arch. ges. Physiol. (Pflüger's) 195, 432-459.

c. Weitere Beiträge zur Kenntnis von organischen Nahrungsstoffen mit spezifischer Wirkung. XVIII. Versuche mit reinen Nahrungsstoffen. Arch. ges. Physiol. (Pflüger's) 195, 480-486.

d. Weitere Beiträge zur Kenntnis von organischen Nahrungsstoffen mit spezifischer Wirkung. XIX. Vergleichende Fütterungsversuche mit Fleisch von normal und von ausschliesslich mit geschliffenem Reis ernährten Tauben. XX. Vergleichende Fütterungsversuche mit verschiedenen reinen Nahrungsstoffen. 
XXI. Versuche mit reinen Nahrungsstoffen mit Uberwiegen der Kohlenhydrate bzw. eines Fettsäuren-Glyzeringemisches. XXII. Fütterung von Tauben mit Fleisch ohne und mit Zusätzen. Arch. ges. Physiol. (Pfiüger's) 197, 89-96, 97-104, 105-120, 121-130.

1923. Weitere Beiträge zur Kenntnis von organischen Nahrungsstoffen mit spezifischer Wirkung. XXIII. Vergleichende Versuche ïber das Verhalten von schilddrüsenlosen Meerschweinchen und Solchen, die Schilddrüsen besitzen, gegenüber einer Nahrung die zum Skorbut führt. Arch. ges. Physiol. (Pflïger's) 198, 164-168.

a. Weitere Beiträge zur Kenntnis von organischen Nahrungsstoffen mit spezifischer Wirkung. XXV. Über den Einfluss von Brombenzol auf normal ernährte und mit Reis gefütterte Tauben. Arch. ges. Physiol. (Pflüger's) 198, 179-190.

b. Weitere Beiträge zur Kenntnis von organischen Nahrungsstoffen mit spezifischer Wirkung. XXVI. Arch. ges. Physiol. (Pflüger's) 198, 571-582.

c. Weitere Beiträge zur Kenntnis von organischen Nahrungsstoffen mit spezifischer Wirkung. XXIX. Arch. ges. Physiol. (Pfiïger's) 201, 416-431.

1927. Weitere Beiträge zur Kenntnis der alimentären Dystrophie. Arch. ges. Physiol. (Pflïger's) 217, 88-104.

Abderhalden, E. and Gellhorn, E.

1922. XIV. Weitere Beiträge zur Kenntnis von organischen Nahrungsstoffen mit spezifischer Wirkung. Arch. ges. Physiol. (Pflüger's) 195, 1-21.

Abderhalden, E. and Schaumann, $\mathrm{H}$.

1918. Beitrag zur Kenntnis von organischen Nahrungsstoffen mit spezifischer Wirkung. Arch. ges. Physiol. (Pfï̈ger's) 172, 1-274.

Abderhalden, E. and Schmidt, L.

1920. Weitere Beiträge zur Kenntnis von organischen Nahrungsstoffen mit spezifischer Wirkung. III. Arch. ges. Physiol. (Pfiïger's) 185, 141-146.

Abderhalden, E. and Wertheimer, E.

1921. Weitere Beiträge zur Kenntnis von organischen Nahrungsstoffen mit spezifischer Wirkung. VII. Arch. ges. Physiol. (Pfiüger's) 191, 258-277.

a. Weitere Beiträge zur Kenntnis von organischen Nahrungsstoffen mit spezifischer Wirkung. X. Arch. ges. Physiol. (Pfiüger's) 192, 174-182.

1922. Weitere Beiträge zur Kenntnis von organischen Nahrungsstoffen mit spezifischer Wirkung. XIII. Die mangelhafte Sauerstoffversorgung der Zellen als Ursache der Erscheinungen der alimentären Dystrophie bei Tauben. Arch. ges. Physiol. (Pfiïger's) 194, 647-673.

a. Weitere Beiträge zur Kenntnis von organischen Nahrungsstoffen mit spezifischer Wirkung. XVIII. Arch. ges. Physiol. (Pffïger's) 195, 460-479.

1923. Weitere Beiträge zur Kenntnis von organischen Nahrungsstoffen mit spezifischer Wirkung. XXIV. Weitere Studien über das Wesen der im Stadium der alimentären Dystrophie bei Tauben nach ausschliesslicher Fütterung mit geschliffenem Reis auftretenden Störung der Zellatmung. Arch. ges. Physiol. (Pfïiger's) 198, 169-178.

a. Weitere Beiträge zur Kenntnis von organischen Nahrungsstoffen mit spezifischer Wirkung. XXVII. Versuche an Gänsen. Prüfung des Verhaltens der Zellfermente. Arch. ges. Physiol. (Pflüger's) 198, 583-589.

b. Weitere Beiträge zur Kenntnis von organischen Nahrungsstoffen mit spezifischer Wirkung. XXVIII. Arch. ges. Physiol. (Pfï̈ger's) 199, 352-364. 
1924. Weitere Beiträge zur Kenntnis von organischen Nahrungsstoffen mit spezifischer Wirkung. XXX, XXXI. Arch. ges. Physiol. (Pfiuger's) 202, 395404, 405-409.

Abel, E.

1922. Remarques à propos de quelques expériences d'avitaminose. Compt. rend. soc. biol. $87,1213-1215$.

Abels, $\mathrm{H}$.

1919. Über die Rolle der Infekte bein Skorbut der Kinder und Säuglinge (Möller-Barlowsche Krankheit). Med. Klin. 15, 1084-1086.

1920. Ueber das Verhalten des skorbutischen Organismus gegen Infekte (Skorbutische Dysergie). Wiener klin. Wochschr. 33, 899-901.

1922. Über die Wichtigkeit der Vitamine fur die Entwicklung des menschlichen fötalen und mutterlichen Organismus. Klin. Wochschr. 1, 1785-1787.

1925. Neuer versuch zur Wachstumwirkung des B-Vitamins und zu dessen quantitativen Nachweis. Klin. Wochschr. 4, 1314, 1315.

1927. Zur Physiologie des fettlöschen Vitamins. (Geschlechts- und- Altersunterschiede.) Wicner klin. Wochschr. 40, 1105-1107.

Abraham, A.

1928. Zur Biochemie des Skorbuts. Klin. Wochschr. 7, 353-355.

Abrahamson, E. M. and Miller, E. G. Jr.

1925. Hydrogen-ion concentration in the gastro-intestinal tract of the albino rat. Proc. Soc. Exptl. Biol. Med. 22, 438, 439.

Ackerson, C. W., Blish, M. J. and Mussehl, F. E.

1925. A study of the phosphorus, calcium and alkaline reserve of the blood sera of normal and rachitic chicks. J. Biol. Chem. 63, 75-84.

Ackert, J. E. and Spindler, L. A.

1929. Vitamin D and resistance of chickens to parasitism. Am. J. Hyg. 9, 292-307.

Acuña, E. M.

1923. The vitamin B content of some Philippine fruits and vegetables. Philippine Agr. 12, 293-302.

Adachi, A.

1923. Über den Harnsäure- und Allantoinstoffwechsel bei Avitaminose. Biochem. Z. 143, 408-422.

Adam, A.

1926. Inaktivierung des antirachitischen Faktors im Lebertran durch Bestrahlen mit Ultraviolettlicht. Klin. Wochschr. 5, 1648-1650.

1927. Ist das antirachitische Vitamin des Lebertrans ein bestrahltes Ergosterin? Klin. Wochschr. 6, 1289.

Adam, N. K. and Rosenheim, O.

1929. The structure of surface films. Part XIII. Sterols and their derivatives. Proc. Roy. Soc. (London) A. 126, 25-34.

Adams, G. and McCollum, E. V.

1928. A method for the biological assay of cod liver oil. J. Biol. Chem. 78, 495-524.

Aengenendt, J.

1928. Rachitisbekämpfung im Grossen durch Vigantol. Münch. med. Wochschr. $75,1112,1113$. 
Ahmad, B. and Drummond, J. C.

1930. The relative vitamin A value of the body and liver oils of certain fish. Biochem. J. 24, 27-36.

Aidin, $\mathbf{R}$.

1928. Irradiated ergosterol in the treatment of rickets. Lancet, 1928, I, 229, 230.

Albert, J.

1915. The treatment of infantile beriberi with the extract of tiqui-tiqui. Philippine J. Sci. 10, 80-85.

Allen, E.

1919. Degeneration in the albino rat testis due to a diet deficient in the watersoluble vitamin, with a comparison of similar degeneration in rats differently treated, and a consideration of the Sertoli tissues. Anat. Record 16, 93-117.

Allen, R. S. and Burget, G. E.

1928. The influence of yeast on gastric secretion (man, dog). Am. J. Physiol. 84, 98-102.

Alpern, D.

1923. Untersuchungen über den Rest-Stickstoffgehalt des Blutes bei avitaminösen und hungernden Tauben. Biochem. Z. 138, 142-149.

Alquier, J.

1930. Contribution à l'étude sur le rat du rachitisme expérimental et des régimes ou substances antirachitiques. Bul. soc. hyg. alin1ent. 18, 51-85.

Alquier, J., Asselin, L., Kogane, M. and Silvestre de Sacy, G.

1930. Variations de la composition minérale du tissu osseux chez le rat normal, rachitique, et guéri du rachitisme expérimental. Compt. rend. 190, 334-336.

Anderegg, L. T.

1924. Diet in relation to reproduction and rearing of young. J. Biol. Chem. 59, 587-599.

Anderegg, L. T. and Nelson, V. E.

1925. Whole and skimmed milk powders as food. Observations on a new vitamin for reproduction. Ind. Eng. Chem. 17, 451-455.

1926. Milk powders as food. II. Observations on the existence of vitamin E. Ind. Eng. Chenr. 18, 620-622.

Andersen, A. and Nightingale, E.

1929. Test for vitamin-A in margarine, butter, and other fatty foods. J. Soc. Chem. Ind. 48, $139 \mathrm{~T}, 140 \mathrm{~T}$.

Anderson, E. M.

1926. Preliminary analyses of the tissues and of the metabolism of animals suffering from dietary sterility. Anat. Record 32, 227.

Anderson, E. V., Dutcher, R. A., Eckles, C. H. and Wilbur, J. W.

1921. The influence of heat and oxidation upon the nutritive and antiscorbutic properties of cow's milk. Science 53, 446.

Anderson, R. E.

1929. A quantitative study of the question as to the storage of the antiscorbutic vitamin in the body. Dissertation, Columbia University, New York.

Anderson, R. J. and Kulp, W. L.

1922. A study of the metabolism and respiratory exchange in poultry during vitamin starvation and polyneuritis. J. Biol. Chem. 52, 69-89; also in Nere York State Agr. Expt. Sta. Tech. Bull. 88, 3-22. 
Anderson, R. J. and Shriner, R. L.

1926. The phytosterols of corn oil. J. Am. Chem. Soc. 48, 2976-2986.

Anderson, W. E. and Smith, A. H.

1924. The effect of acute scurvy on the subsequent nutrition and growth of guinea pigs. J. Biol. Chem. 61, 181-191.

Anderson, W. T. and Macht, D. I.

1928. The penetration of ultra-violet rays into live animal tissue. Am. J. Physiol. 86, 320-330.

a. The penetration of ultra-violet rays into live animal tissue. Brit. J. Actinotherapy 3, 123-128.

Andrews, V. L.

1912. Infantile beriberi. Philippine J. Sci., Sect. B. 7, 67-88.

Anrep, G. V. and Drummond, J. C.

1921. Note on the supposed identity of the water-soluble vitamin B and secretin. J. Physiol. 54, 349-352.

Anthony, E. L.

1928. Butter versus oleomargarine in rickets control in pigs. J. Dairy Sci. 11, 66-68.

Appleman, C. O.

1918. Special growth-promoting substances and correlation. Science 48, 319, 320.

Appleton, V. B.

1921. Observations on deficiency diseases in Labrador. Am. J. Pub. Health 11, 617-621.

Arloing, F. and Dufourt, A.

1923. Effets de l'atropinisation sur les phénomènes de carence chez les pigeons soumis au régime du riz décortiqué. Compt. rend. soc. biol. 88, 774, 775.

a. Action de la pilocarpine sur la carence expérimentale du pigeon. Compt. rend. soc. biol. $88,775,776$.

b. Intoxication et carence. Effets de l'adrénaline sur la carence expérimentale du pigeon. Compt. rend. soc. biol. 88, 1037, 1038.

Arneth.

1918. Ueber Skorbut im Felde. Deut. med. Wochschr. 44, 509-513.

Aron, $\mathrm{H}$.

1910. Phosphorus starvation with special reference to beriberi. I. Philippine J. Sci. Sect. B, 5, 81-97.

1911. Nutrition and growth. Philippine J. Sci. Sect. B. 6, 1-51.

1920. Über den "Nährwert" und die Bedeutung der Nahrungsfette. Biochem. Z. $103,172-177$.

Aron, H. and Gralka, R.

1921. Die akzessorischen Nährstoff-Faktoren. I. Zum Sondernährwert verschiedener Nahrungsfette. Biochem. Z. 115, 188-203.

Aron, $\mathrm{H}$. and Hocson, F.

1910. Phosphorus starvation with special reference to beriberi. II. Philippine J. Sci. Sect. B. 5, 98-122.

Artom, C.

1922. Sulle attività enzimatiche dell'apparato digèrente nell'avitaminosi. Arch. farmacol. sper. 33, 127-132, 145-165. 


\section{Arvay, A. von}

1926. Die Wirkung von Überfluss an A- und B- Vitamin in der Nahrung auf den Grundumsatz und die spezifisch- dynamische Wirkung der Nahrungsmittel. Arch. ges. Physiol. (Pflïger's) 214, 421-438.

Asada, K.

1923. Über die Wirkung einseitiger Ernährung bei Avitaminose und das Verhalten der Korpertemperatur bei dieser Krankheit. Biochem. Z. 139, 234-252.

a. Der Fettstoffwechsel bei der Avitaminose I. Der Gesamtfettgehalt und Cholesteringehalt des Körpers bei normaler und avitaminöser Ernährung. Biochem. Z. 141, 166-186.

b. Der Fettstoffwechsel bei der Avitaminose. II. Der Gehalt des Blutes bei normalen, hungernden, avitaminösen und phosphorvergifteten Ratten an Gesamtfett, Neutralfett, Cholesterin und Cholesterinester. Biochem. Z. 142, 44-52.

c. Der Fettstoffwechsel bei der Avitaminose. III. Der Fett und Cholesteringehalt der Leber nach der Phosphorvergiftung bei normalen, hungernden und avitaminösen Ratten. Biochem. Z. 142, 165-180.

d. Der Fettstoffwechsel bei der Avitaminose. IV. Über den Gaswechsel avitaminöser Ratten im nüchteren Zustande, während der Verdaung und nach Adrenalininjekionen. Biochem. Z. 143, 387-398.

1924. Der Fettstoff wechsel bei der Avitaminose. V. Úber die Verteilung der Fette und Lipoide in der Leber nach Phosphorvergiftung bei normalen, hungernden und avitaminösen Tieren. Biochem Z. 144, 203-211.

Aschoff, L. and Koch, W.

1919. "Der Skorbut." Gustav Fischer, Jena.

Ashford, B. K.

1922. Beriberi in the garrison at San Juan, Porto Rico. Am. J. Trop. Med. 2, 305-340.

Ashford, C. A.

1930. The phosphorus distribution in blood and the calcium and phosphorus excretion during hypervitaminosis D. Biochem. J. 24, 661-668.

Askew, F. A., Bourdillon, R. B., Bruce, H. M., Jenkins, R. G. and Webster, T. A.

1930. Distillation of vitamin D. Proc. Roy. Soc. (London) 107 B, 76-90.

a. Effects of further irradiation of the irradiation products of ergosterol. Proc. Roy. Soc. (London) 107B, 91-100.

\section{Avellar de Loureiro J.}

1927. Présence d'un ozonide dans un concentré vitaminique d'huile de foie de morue. Compt. rend soc. biol. 97, 878, 879.

a. L'ozone est-il un component normal de l'huile de foie de morue. Compt. rend. soc. biol. $97,879,880$.

\section{Axtmayer, J. $\mathrm{H}$.}

1930. A study of the vitamin B complex of red kidney beans and polished rice. J. Nutrition 2, 353-357.

Aykroyd, W. R.

1930. The etiology of pellagra. Brit. Med. J. No. 3613, 647, 648.

Aykroyd, W. R. and Roscoe, M. H.

1929. The distribution of vitamin $\mathrm{B}_{2}$ in certain foods. Biochern. J. 23, 483-497. 
Babb, M. F.

1928. The effect of ethylene upon the vitamin B content of celery. Science 68, 231.

Bacharach, A. L.

1925. A note on the basal vitamin B-free diet of Drummond and Watson. Biochem. J. 19, 638-640.

1928. The growth-promoting properties of Vitamin D. Quart. J. Pharm. 1, 49-50.

Bacharach, A. L. and Allchorne, E.

1928. The Vitamin B content of malt extract. Biochcm. J. 22, 313-316.

Bacharach, A. L. and Hartwell, G. A.

1927. A note on technique in testing for vitamin B. Analyst 52, 145-149.

Bacharach, A. L. and Jephcott, H.

1929. Vitamin D and fecal reaction. J. Biol. Chem. 82, 751-758.

Bacharach, A. L. and Smith, E. L.

1928. Some notes on the chemistry of the fat-soluble vitamins in cod-liver oil. Quart. J. Pharm. 1, 539-545.

Bachem, A.

1929. The ultraviolet transparency of the various layers of human skin. Am. J. Physiol. 91, 58-64.

Bachem, A. and Kung, J.

1928. The penetration of ultraviolet light into the human skin. Proc. Soc. Exptl. Biol. Med. 25, 456.

Bailey, E. M.

1922. The potency of some commercial vitamin preparations as compared with that of dry brewers' yeast. Connecticut State Agr. Expt. Sta. Bull. 240, 3-51.

Bakwin, H. and Bakwin, R. M.

1927. Seasonal variation in the calcium content of infants' serum. Am. J. Diseases Children 34, 994-999.

Baldwin, F. M., Cook, H. B. and Nelson, V. E.

1924. Blood pressure in rats under deficient diets. Am. J. Physiol. 68, 379-384.

Balietti, L.

1927. Influenza della vitamina "fattore B" sulla curoa della fatica muscolare. Boll. soc. med. chir. Modena 28, 29-39.

Baly, E. C. C.

1925. High energy chemistry and vitamins. J. State Med. 33, 368-376.

Banks, H. S.

1926. Incidence and treatment of rickets in a Scottish industrial area. Med. Officer 35, 99-101.

Barenberg, L. H. and Lewis, J. M.

1928. The effect of carbon arc irradiation on the health of a group of infants. J. Am. Med. Assocn. 90, 504-506.

Barenberg, L. H., Friedman, I., and Green, D.

1926. The effect of ultraviolet irradiation on the health of a group of infants. J. Am. Med. Assocn. 87, 1114-1117.

Barger, $\mathrm{G}$.

1914. "The Simpler Natural Bases," London and New York, Longmans, Green and Co. 
Barlow, O. W.

1926. Fasting and rice disease in pigeons; the parallelism of loss of body weight, temperature, and respiration rate; the absence of $B$ vitamins from the cortex of the beef suprarenal. Am. J. Physiol. 78, 322-324.

1927. Studies on the anemia of rice disease. The effects produced by the addition of betaine hydrochloride, lactose, vitamins $\mathrm{A}$ and $\mathrm{C}$, magnesium sulfate or mineral oil to the polished rice diet of pigeons. Am. J. Physiol. 83, 237244.

1928. The influence of the daily oral administration of magnesium sulphate or lactose on the blood volumes of normal, beriberi and fasting pigeons. $\mathrm{Am}$. J. Physiol. 86, 594-598.

1930. The influence of inorganic iron on the anemia of rice disease in pigeons. Am. J. Physiol. 93, 156-160.

a. The influence of vitamin $\mathrm{B}$ on the inanition, anemia, and bacteriemia of rice disease in pigeons. Am. J. Physiol. 93, 161-169.

Barlow, O. W. and Whitehead, R. W.

1929. A comparison of the body weights, erythrocyte counts, and total blood volumes of normal, beriberi, and fasting rats. The influence of lactose, mineral oil, and magnesium carbonate on the anemia of rice disease. Am. J. Physiol. 89, 548-554.

Barlow, R. A.

1927. Does vitamin-deficient diet cause deafness? Results of animal experimentation. Laryngoscope 37, 640-648.

Barlow, T.

1883. On cases described as acute rickets. Med. Chir. Trans. 46, 159.

1894. Infantile scurvy and its relation to rickets. Lancet, 1894, II, 1075-1080.

Barnes, D. J., Brady, M. J., and James, E. M.

1930. The comparative value of irradiated ergosterol and cod liver oil as a prophylactic antirachitic agent when given in equivalent dosage according to rat units of vitamin D. Am. J. Diseases Children 39, 45-58.

Barnes, R. E. and Hume, E. M.

1919. A comparison between the antiscorbutic properties of fresh, heated and dried cow's milk. Lancet, 1919, II, 323-324.

Barsickow, M.

1913. Experimentelle Untersuchungen über die therapeutische Wirkung der Hefe bei der alimentären, multiplen Polyneuritis der Meerschweinchen und Tauben. Biochem. Z. 48, 418-424.

Bartenstein, L.

1905. Beiträge zur Frage des künstlichen Morbus Barlow bei Tieren. Jahrb. Kinderheilk. 61, 6-35.

Bassett-Smith, P. W.

1920. Scurvy with special reference to prophylaxis in the Royal Navy. Lancet, 1920, I, 1102-1105.

a. Further experiments for preservation of lemon juice. Lancet, 1920, II, 997, 998.

1921. Preservation of lemon juice and prevention of scurvy. Lancet, 1921, II, $321,322$.

a. Beriberi: A critical review. Trop. Diseases Bull. 18, 307-312. 


\section{Batchelder, E. L.}

1929. The effect of successive diminutions of vitamin $A$ in the food on the nutrition and vitality of albino rats. Dissertation, Columbia University, New York.

Bauer, W., Aub, J. C. and Albright, F.

1929. Studies of calcium and phosphorus metabolism. V. A study of the bone trabeculae as a readily available reserve supply of calcium. J. Exptl. Med. 49, 145-161.

Baumann, L. and Howard, C. P.

1912. Metabolism of scurvy in an adult. Arch. Internal Med. 9, 665-679.

1917. Mineral metabolism of experimental scurvy of guinea-pig. Am. J. Med. Sci. 153, 650-665.

Beach, J. R.

1923. "Vitamin A" deficiency in poultry. Science 58, 542.

1924. Studies on a nutritional disease of poultry caused by vitamin A deficiency. California Agr. Expt. Sta. Bull. 378, 3-22.

Beard, H. H.

1926. Studies in the nutrition of the white mouse. I. The normal growth and nutritive requirements. II. The effect of feeding diets rich in protein and diets containing unbalanced salt mixtures. The rôle of certain sulfur components. III. Vitamin factors in the nutrition of mice. IV. The relation between diet and reproduction. Am. J. Physiol. 75, 645-657, 658-667, 668-681, 682-695.

Beard, H. H. and Pomerene, E.

1929. Studies in the nutrition of the white mouse. V. The experimental production of rickets in mice. Am. J. Physiol. 89, 54-57.

Bechdel, S. I.

1925. Vitamin B requirement of dairy calves. Pennsylvania Agr. Expt. Sta. Bull. 196, 19, 20.

1926. The vitamin B requirement of the calf. Pennsylvania Agr. Expt. Sta. Bull. 204, 18.

Bechdel, S. I. and Honeywell, H. E.

1926. Is the vitamin B potency of milk dependent on the presence of vitamin $\mathrm{B}$ in the ration of the cow? Pennsylvania Agr. Expt. Sta. Bull. 204, 20.

1927. The relation between the vitamin $B$ content of the feed eaten and of the milk produced. J. Agr. Research 35, 283-288.

Bechdel, S. I., Honeywell, H. E., Dutcher, R. A. and Knutsen, M. H.

1928. Synthesis of vitamin B in the rumen of the cow. J. Biol. Chem. 80, 231-238.

Bedson, S. P.

1921. The blood picture in scurvy, with particular reference to the platelet. Brit. Med. J. No. 3176, 792-793.

Bedson, S. P. and Zilva, S. S.

1923. The influence of vitamin A on the blood platelets of the rat. Brit. J. Exptl. Path. 4, 5-12.

a. The platelet count in rats suffering from vitamin A deficiency. Brit. J. Exptl. Path. 4, 305-310.

Bell, M. and Mendel, L. B.

1922. The distribution of vitamin B in the wheat kernel. Am. J. Physiol. 62, 145-161. 
Belloc, G., Fabre, R. and Simonnet, H.

1930. Contribution à l'étude de l'activité biologique des stérols. Etudes des stérols de plankton. Compt. rend. 191, 160-162.

Berczeller, L.

1922. Über die biologisches Wertung der Nahrungsmittel. Biochem. Z. 129, 217-238.

Berczeller, L. and Billig, A.

1923. Über die biologisches Wirkung der einzelnen Nahrungstoffe. II. Biochem. Z. 139, 470-475.

Berg, R.

1927. “Die Vitamine." 2nd Ed. Leipzig, S. Hirzel.

Bergeim, O.

1928. Digestive function in avitaminoses. Proc. Soc. Exptl. Biol. Mcd. 25, $457,458$.

Berglung, H., Keefer, C. S. and Yang, C. S.

1929. Deficiency anemia in Chinese responding to cod liver oil. Proc. Soc. Exptl. Biol. Med. 26, 418-421.

Bertram, S. H.

1929. Some analytical remarks on the vitamin A. Proc. K. Akad. Wetensch. Amsterdam 32, 644-668.

Bertrand, G. and Seidell, A.

1923. Sur la séparation de la vitamine antinévritique de la levure à l'état de picrate. Bul. soc. chim. biol. 5, 794-796.

Bessesen, D. H.

1923. Changes in organ weights of the guinea pig during experimental scurvy. Am. J. Physiol. 63, 245-256.

Best, C. H.

1930. The vitamins. Can. Pub. Health J. 21, 119-131.

Bethke, R. M. and Kennard, D. C.

1927. The growth of chicks as affected by sunlight through glass substitutes. Poultry Sci. 6, 290-301.

1928. Does a mixed grain ration meet the vitamin B requirements of chicks brooded on wire? Poultry Sci. 7, 287-292.

Bethke, R. M., Kennard, D. C. and Kik, M. C.

1925. Nutritional studies of the growing chick. I. The relation of sunlight and green clover to leg weakness in chicks. J. Biol. Chem. 63, 377-390.

Bethke, R. M., Kennard, D. C. and Sassaman, H. L.

1927. The fat-soluble vitamin content of hen's egg yolk as affected by the ration and management of the layers. J. Biol. Chem. 72, 695-706.

Bethke, R. M., Steenbock, H. and Nelson, M. T.

1923. Fat-soluble vitamins. XV. Calcium and phosphorus relations to growth and composition of the blood and bone with varying vitamin intake. J. Biol. Chem. 58, 71-103.

Bethke, R. M., Zinzalian, G., Kennard, D. C. and Sassaman, H. L. 1928. The antirachitic properties of cod-liver meals. J. Agr. Research 36, 747-753.

Beumer, $\mathrm{H}$.

1927. Uber den Versuch einer Ergosterinbilanz. Klin. Wochschr. 6, 941, 942. 
Beumer, H. and Falkenheim, C.

1927. Uber das Ergosterin und eine antirachitischen Wirksamkeit bei Saüglingen. Klin. Wochschr. 6, 798, 799.

Beznák, A. von

1923. Die Rolle der Nebennieren bei Mangel an Vitamin B. Biochem. Z. 141, $1-12$.

1926. Vergleichende Untersuchungen über die Hämolyseresistenz von roten Blutkörperchern von normalen und beriberi-kranken Tauben gegenüber Saponin und Kobragift. Arch. ges. Physiol. (Pfï̈ger's) 212, 246-252.

\section{Bezssonoff, N.}

1921. Influence des oxydases sur la destruction rapide du principe antiscorbutique. Bul. soc. hyg. aliment. 9, 537-546.

a. Du principe antiscorbutique dans le jus de pomme de terre extrait en présence d'acides. Compt. rend. 173, 417-419.

b. Sur une réaction colorée commune aux extraits antiscorbutique et à l'hydroquinone. Compt. rend. 173, 466-468.

1922. Effets sur les cobayes d'une préparation antiscorbutique. Compt. rend. $175,846-848$.

1923. Sur une préparation antiscorbutique et sur le rôle de la vitamine A dans le scorbut expérimental. Bul. soc. sci. hy'g. aliment. 11, 14-38.

a. A simplified method of preparation of the Bezssonoff reagent for vitamin C and some polyphenols. Biochem. J. 17, 420, 421.

1924. Sur la réaction proposée par Jendrassik (1923) pour caractériser la vitamine $\mathrm{B}$ et sur ses rapports avec la fonction phénol. Bul. soc. chim. biol. 6, 35-39.

a. Une condition complémentaire de l'épreuve au réactif de la vitamine C. Bul. soc. chim. biol. 6, 220-225.

b. Necessary conditions for testing with the reagent for vitamin C. Biochem. J. 18, 384-386.

c. Sur les réactions colorées des facteurs liposolubles. Compt. rend. 179, 572-574.

1925. Quelques données sur la nature du principe antiscorbutique dit vitamine C. Compt. rend. 180, 970-972.

1926. Préparation rapide de l'acide monomolybdophosphotungstique réactif des polyphénols et des vitamines. Compt. rend. 182, 1223, 1224.

a. Sur la véritable régime spécifique du scorbut expérimental. Compt. rend. 183, 1309, 1310.

b. L'effet antiscorbutique est-il dù à deux substances différentes? Compt. rend. $183,1309,1310$.

1927. Sur un nouveau régime scorbutigène nettement spécifique. Bull. soc. chim. biol. 9, 555-567.

a. L'effet antiscorbutique est-il dù à deux substances différentes? Bull. soc. chim. biol. 9, 568-579.

b. Du rôle des vitamines chez les végétaux verts. Rev. path. végétale d'entom. agricole 14, 142-155.

1928. Une méthode nouvelle pour caractériser l'action physiologique immédiate des vitamines hydrosolubles. Bull. soc. chim. biol. 10, 1179-1198.

a. Les effets physiologiques immédiate de l'avitaminose C. Bul. soc. chim. biol. 10, 1199-1212. 
b. L'action physiologique immédiate d'une vitamine. Compt. rend. 186, 914-916.

1929. Deux remarques au sujet des réactions colorées des vitamines. Bul. soc. chim. biol. 11, 294-307.

a. Sur les Cobayes nourris à l'avoine irradiée avec et sans apport de vitamines A et C. Bul. soc. chim. biol. 11, 1146-1163.

1930. Vitamine A et carotene. Compt. rend. 190, 529-532.

Bickel, A.

1922. Experimentelle Untersuchungen über den Einfluss der Vitamine auf Verdaung und Stoffwechsel und die Theorie der Vitaminwirkung. Klin. Wochschr. 1, 110-112.

a. Zur pathologischen Physiologie der Avitaminosen. Deut. med. Wochschr. 48, $965,966$.

1924. Das Wesen der Avitaminose nach experimentellen Untersuchungen

über die Abmagerungsform dieser Krankheit. Biochem. Z. 146, 493-521.

1925. Weitere Untersuchungen über den Stoffwechsel bei der Avitaminose.

Biochem. Z. 166, 251-294.

Bickel, A. and Collazo, J. A.

1923. Über den Mechanismus der Insulinwirkung (Beobachtungen an der Kohlenhydratstoff wechsel-störung bei Avitaminose). Deut. med. Wochschr. 49, $1408-1410$.

Bierich, $\mathbf{R}$.

1919. Über Skorbut. Deut. Arch. klin. Med. 130, 151-171.

Bierry, $\mathbf{H}$.

1919. Inanition, temperature, et glycémie. Compt. rend. 169, 1112-1114.

Bierry, H. and Kollmann, M.

1928. Sur le mode d'action de la vitamine B. Compt. rend. 186, 1062-1064.

Bills, C. E.

1925. The resistance of the antirachitic substance in cod liver oil to reagents.

J. Biol. Chem. 64, 1-9.

a. Antiricketic substances. II. The action of $n$-butyl nitrate on activated cholesterol and the antiricketic vitamin. J. Biol. Chem. 66, 451-457.

1926. Antiricketic substances. III. The catalytic formation of an antiricketic cholesterol derivative. J. Biol. Chem. 67, 753-758.

1927. Antiricketic substances. VI. The distribution of vitamin D, with some notes on its possible origin. J. Biol. Chem. 72, 751-758.

Bills, C. E. and Brickwedde, F. G.

1928. The activation of cholesterol at liquid oxygen temperature. Nature 121, 452.

Bills, C. E. and Cox, W. M., Jr.

1929. Studies in the isomerization of ergosterol. J. Biol. Chem. 84, 455-462.

Bills, C. E., Cox, W. M., Jr. and Steel, G. E.

1929. The heat of combustion of ergosterol, isoergosterol, and cholesterol. J. Biol. Chem. 84, 655, 656.

Bills, C. E. and Honeywell, E. M.

1928. Antiricketic substances. VIII. Studies on highly purified ergosterol and its esters. J. Biol. Chem. 80, 15-23.

Bills, C. E., Honeywell, E. M. and Cox, W. M., Jr.

1928. Antiricketic substances. IX. Quantitative biophysical studies on the activation of ergosterol. J. Biol. Chem. 80, 557-563. 
Bills, C. E., Honeywell, E. M., Cox, W. M., Jr. and Wirick, A. M.

1929. Studies on the activation of ergosterol. Abstracted in Am. J. Physiol. $90,286$.

Bills, C. E., Honeywell, E. M. and MacNair, W. A.

1928. Antiricketic substances. VII. Biochemical and spectroscopic studies on purified cholesterol. J. Biol. Chem. 76, 256-261.

Bills, C. E. and McDonald, F. G.

1926. Antiricketic substances. IV. The polymerization of cholesterol. $J$. Biol. Chem. 68, 821-831.

1927. Antiricketic substances. V. The action of ultra-violet rays on the ethers and esters of cholesterol. J. Biol. Chem. 72, 13-19.

1930. Further studies on the isomerization of ergosterol. J. Biol. Chem. 88, 337-346.

Bing, F. C. and Mendel, L. B.

1929. The vitamin $B$ and the vitamin $C$ requirements of the albino mouse. J. Nutrition 2, 49-58.

Birnbacher, $\mathrm{T}$.

1928. Zur Physiologie des fettlöslichen Vitamins A. Münch. med. Wochschr. $75,1114-1115$.

Bisbey, B.

1930. Further experiments upon vitamins $B\left(B_{1}\right)$ and $G\left(B_{2}\right)$. Dissertation, Columbia University, New York.

Bishop, K. S. and Morgan, A. F.

1928. Occurrence of deciduomata in rats low in vitamins $\mathrm{A}$ and $\mathrm{E}$. Proc. Soc. Exptl. Biol. Med. 25, 438.

1929. Reproduction in successive generations of rats on purified rachitogenic diets. Abstracted in Anat. Record 42, 44, 45.

\section{Blackberg, S. N.}

1928. Vitamin A and B content of canned sweet potatoes. Proc. Soc. Exptl. Biol. Med. 26, 254-256.

1929. Effect of carbon arc radiation on resistance of rachitic rats to tetanus toxin. Proc. Soc. Exptl. Biol. Med. 26, 757, 758.

Blacklock, D. B.

1924. Lack of exercise as a determining factor in the epidemiology of beriberi. Brit. Med. J. No. 3311, 1046, 1047.

Blank, V. M. and Graves, G. W.

1929. The unusual association of postinfantile rickets with osteomalacia. $J$. Am. Diseases Children 38, 84-94.

Blegvad, $\mathrm{O}$.

1923. The parenteral vitamin treatment of xerophthalmia. Ugeskr. Laeger 85, 942, 943; Abstracted in Brit. Med. J. No. 3290, 122, 125.

Bleile, A. M. and Seymour, R. J.

1923. The effect of formaldehyde upon the vitamin content of milk. Am. J. Physiol. 63, 421, 422.

Bloch, C. E.

1917. Eye disease and other disturbances in infants from deficiency in fat in the food. (Trans. title.) Ugeskr. Laeger 79, 309-325, 349-370. J. Am. Med. Assoc. 68, 1516. Chem. Abstracts 11, 2222.

1918. Xerophthalmia and dystrophy in infants. (Trans. title.) Ugeskr. Laeger 80, 775-787, 815-827, 868-874. J. Am. Med. Assocn. 71, 322, 416. 
1919. Klinische Untersuchungen über Dystrophie und Xerophthalmie bei jungen Kindern. Jahrb. Kinderheilk. 89, 405-441.

1921. Clinical investigation of xerophthalmia and dystrophy in infants and young children. J. Hyg. 19, 283-304.

1924. Blindness and other diseases in children arising from deficient nutrition (lack of fat-soluble A factor). Am. J. Diseases Children 27, 139-148; also in J. Dairy Sci. 7, 1-10.

a. Further clinical investigations into the diseases arising in consequence of a deficiency in the fat-soluble A factor. Am. J. Diseases Children 28, 659-667.

1925. Light and vitamin A. (Trans. title.) Ugeskr. Laeger 87, 1183-1185.

1926. Light and the A-body. Am. J. Diseases Children 31, 315-322.

Bloch, C. E. and Faber, F.

1925. Light and the antirachitic factor. Am. J. Diseases Children 30, 504512.

Blohm, G. J., Santesson, C. G. and Euler, H. v.

1921. Physiologische Untersuchungen an Vitaminen B und wasserlöslichen Biokatalysatoren. Arkiv. Kemi, Mineral Geol. 8, No. 13, pp. 27.

Bloxsom, A. P.

1929. Vitamin B requirements in infancy. Am. J. Diseases Children 37, 1161-1168.

Blum, J. and Mellion, J.

1926. The rôle of dentition in the diagnosis of rickets. J. Am. Med. Assocn. 86, 677-679.

Blumenfeld, C. M.

1929. Effects of vitamin deficiency on the weight and cortex-medulla ratio of the suprarenal gland in rats. Anat. Record 42, 45 .

Blunt, K., and Cowan, R.

1930. "Ultraviolet Light and Vitamin D in Nutrition," Chicago, University Chicago Press, pp. 229.

Blunt, K. and Wang, C. C.

1921. The present status of vitamins. J. Home Econ. 13, 97-119.

Boas, M. A.

1926. The antirachitic value of winter spinach. Biochem. J. 20, 153-165.

Boas, M. A. and Chick, $H$.

1924. The influence of diet and management of the cow upon the deposition of calcium in rats receiving a daily ration of the milk in their diet. Biochem. J. 18, 433-447.

Bogert, L. J. and Trail, R. K.

1922. Studies in organic metabolism. III. The influence of yeast and butter fat upon calcium assimilation. J. Biol. Chem. 54, 387-397.

a. Studies in inorganic metabolism. IV. The influence of yeast and butter fat upon magnesium and phosphorus assimilation. J. Biol. Chem. 54, 753-761.

Bohstedt, G. et al.

1923. The vitamin content of fish meal, tankage and blood meal. Ohio Agr. Expt. Sta. Mo. Bull. 8, 167-169.

Bohstedt, G., Bethke, R. M., Edgington, B. H. and Robison, W. L.

1924. Rickets and paralysis in swine as affected by nutrition. Ohio Agr. Expt. Sta. Mo. Bull. 9, 139-144. 
Bohstedt, G., Robison, W. L., Bethke, R. M. and Edgington, B. H.

1926. Mineral and vitamin requirements of pigs, with special reference to the effect of diet on bone development. Ohio Agr. Expt. Sta. Bull. 395, 59-229.

Bond, C. J.

1927. A colour test for radio-sensitive substances. Brit. Med. J. No. 3483, 637.

1928. On irradiated ergosterol as a dressing for wounds, with suggestions as to its mode of action. Brit. Med. J. No. 3504, 339, 340.

Bond, $\mathrm{M}$.

1922. A modification of basal diet for rat feeding experiments. Biochem. J. 16, 479-481.

Boock, E. and Trevan, J.

1922. The food value of mangels and the effects of deficiency of vitamin A on guinea pigs. Biochem. J. 16, 780-791.

Borghi, B.

1929. Ricerche sperimentali sugli effetti dell'ergosterina irradiata somministrata ad alte dosi. Biochem. terap. sper. 16, 113-119.

Bosanyi, A. de.

1925. Experimental studies on rickets. III. Protein substances as a factor in normal osteogenesis and in the healing of rickets. Am. J. Discases Children 30, 780-798.

1926. Studies of certain hitherto undescribed antirachitic substances. Bull. Johns Hopkins Hosp. 38, 72-74.

Bourdillon, R. B., Fischmann, C., Jenkins, R. G. C. and Webster, T. A.

1929. The absorption spectrum of vitamin D. Proc. Roy. Soc. (London)

B. $104,561-583$.

Bourquin, A.

1929. Experiments on the quantitative determination of vitamin G. Dissertation, Columbia University, New York.

Bowman, H. H. M. and Yee, M. A.

1925. Crystals of vitamin B from the mung bean. Proc. Soc. Exptl. Biol. Med. 22, 228-231.

Boyenval, L.

1922. Les phénomènes d'avitaminose sont-ils modifiés par l'administration d'histamine chez le rat blanc? Arch. intern. pharmacodyanamie 26, 359-365.

Bracewell, M. F., Hoyle, E. and Zilva, S. S.

1930. The antiscorbutic potency of apples. Biochem. J. 24, 82-90.

Braddon, W. L.

1907. "The Cause and Prevention of Beriberi," London and New York, Rebman Co.

Braddon, W. L. and Cooper, E. A.

1914. The influence of the total fuel value of a dietary upon the quantity of vitamin required to prevent beriberi. Brit. Med. J. No. 2790, 1348, 1349.

a. The influence of metabolic factors in beriberi. I. The effect of increasing the carbohydrate ration on the development of polyneuritis in birds fed on polished rice. J. Hyg. 14, 331-353.

Bradford, W. L.

1928. Mucosus organism from suppurative lesions of rat on diet deficient in vitamin A. J. Infec. Diseases 43, 407-414. 
Bray, G. W.

1928. Vitamin B deficiency in infants. Its possibility, prevalence, and prophylaxis. Trans. Roy. Soc. Trop. Med. Hyg. 22, 9-42.

British Medical Research Committee.

See Hopkins, F. G. et al, 1924.

Brocklesby, H. N.

1929. Vitamin D content of the liver oil of the dogfish. Can. Chem. Met. 13, 74-77.

1930. The nutritive value of marine products. III. Vitamin D content of commercial pilchard oil. Can. Chem. Met. 14, 29-31.

Brougher, J. C.

1928. The value of acterol (irradiated ergosterol) in the treatment of thyroparathyroidectomized dogs. Am. J. Physiol. 86, 538-541.

1930. Viosterol (irradiated ergosterol) in treatment of parathyroid tetany. J. Am. Med. Assocn. 94, 471-473.

\section{Brouwer, E.}

1925. Over het vitamine $\mathrm{C}$ in gras. (Voorloopige mededeeling). Jaarversl. over 1924 Verein tot exploit. proef. te Hoorn, 57-60.

1927. Über das Vitamin C inı frischen Grase (Lolium perenne, englische Raigras) und über das Gewicht verschiedener Organe beim Skorbut. Biochem. Z. 187, 183-193.

a. Über die chemische Zusammensetzung der skorbutischen Meerschweinchenknochen. Biochem. Z. 190, 402-410.

1928. Over het vitamine $\mathrm{C}$ in ingekuild gras. Verslag Land. Onderzock. Rijkslandbouzuproefsta. 33, 95-106.

\section{Brown, A.}

1928. Sunlight. Its effect on the growth of children and resistance to disease. Public Health J. 19, 401-409.

Brown, A. and Tisdall, F. F.

1927. The seasonal variation of the antirachitic effect of sunshine and its effect on resistance to disease. Can. Med. Assocn. J. 17, 1425-1429.

Brown, H. M.

1924. Teeth of children in Cape Province. S. African Med. Record 22, 416. Abstracted in J. Am. Med. Assocn. 83, 1879.

Browne, C. A.

1928. A possible correlation of eye soreness with vitamin A deficiency among the Nez Percés Indians. Science 67, 510, 511.

\section{Bucek.}

1929. Teneur en vitamines des graines de céréales et de Légumineuses. Compt. rend. soc. biol. $100,427,428$.

Buckner, G. D. and Peter, A. M.

1922. The mineral content of the normal white rat during growth. J. Biol. Chem. 54, 5-9.

Budd, G.

1841. "Tweedies' System of Practice of Medicine." Philadelphia, Lea and Blanchard. (Through Hess, 1920.)

Bull, L. B.

1918. Rickets in foxhound puppies: a clinical and experimental investigation. J. Compar. Path. Therap. 31, 193-214. 


\section{Bulley, E. C.}

1919. Note on xerophthalmia in rats. Biochem. J. 13, 103-106.

Bundesen, H. N., Lemon, H. B., Falk, I. S. and Coade, E. N.

1927. Ultra-violet radiation from sunlight and incandescent lamps: Its transmission through window glass and substitutes. J. Am. Med. Assocn. 89, $187-189$.

Bunting, R. W.

1925. The experimental production of dental caries in animals. Dental Cosmos 67, 771-778.

Burnett, F. L. and Howe, P. R.

1927. Malabsorption in deficiency diseases. J. Am. Med. Assocn. 88, 17051709.

Burns, C. M.

1929. Factors influencing bone formation in the albino rat. I. The effect of guanidine intoxication produced by the successive injection of sublethal doses of guanidine salts. II. The effect of the injection of parathyroid extract. Biochem. J. 23, 840-852, 853-859.

Burr, G. O. and Burr, M. M.

1929. A new deficiency disease produced by the rigid exclusion of fat from the diet. J. Biol. Chem. 82, 345-367.

Burrows, M. T.

1929. Further note on the relation of nutrition to the development of cancer. Proc. Soc. Exptl. Biol. Med. 26, 574-577.

Burrows, M. T. and Farr, W. K.

1927. The action of mineral oil per os on the organism. Proc. Soc. Exptl. Biol. Med. 24, 719-723.

Burrows, M. T. and Jorstad, L. H.

1926. On the source of vitamin B in nature. Am. J. Physiol. 77, 24-37.

a. On the source of vitamin A in nature. Am. J. Physiol. 77, 38-49.

Burtis, M. P.

1928. Factors affecting the accuracy of the quantitative determination of vitamin A. Dissertation, Columbia University, New York.

Burton, G. W.

1928. Quantitative determination of vitamins $\mathrm{A}, \mathrm{B}$ and $\mathrm{C}$ in collards and turnip greens. J. Home Econ. 20, 35-42.

Burton, H. G. and Balmain, A. R.

1930. Vitamin A and streptococcal antitoxic immunity. Lancet, 1930, I, 1063. Buschmann, $\mathrm{H}$.

1928. Zur Therapie der Rachitis und Spasmophilie mit durch ultravioletten Strahlen atktivierten Substanzen und besonderer Berücksichtigung des Vigantols. Med. Klin. 24, 302-304.

Busk, G.

1877. "Report of Committee on Scurvy," London. (Through Hess, 1920.)

Byfield, A. H. and Daniels, A. L.

1923. The rôle of parental nutrition in the causation of rickets. J. Am. Med. Assocn. 81, 360-362.

Byfield, A. H., Daniels, A. L. and Loughlin, R.

1920. The antineuritic and growth stimulating properties of orange juice. Am. J. Diseases Children 19, 349-358. 
Cady, O. H. and Luck, J. M.

1930. Studies in the chemistry of vitamin A. J. Biol. Chem. 86, 743-754.

Cajori, F. A.

1920. Some nutritive properties of nuts; their proteins and content of watersoluble vitamin. J. Biol. Chem. 43, 583-606.

Caldwell, G. W. and Dennett, R. H.

1929. The clinical value of sunlight through ultraviolet transmitting glass. J. Am. Med. Assocn. 92, 2088-2090.

Cameron, H. C.

1929. High temperature deaths among experimental rats. Science 69, 576.

a. Changes in the suprarenal gland in vitamin-A deficient rats. Proc. West Virginia Acad. Sci. 3, 83-84.

\section{Campbell, H. L.}

1928. Growth, reproduction and longevity of experimental animals as research criteria in the chemistry of nutrition. Dissertation, Columbia University, New York.

\section{Campbell, J. A.}

1926. Ultra-violet radiation and metabolism with a new method for estimating metabolism. Proc. Roy. Soc. (London) A, 451-461.

Campbell, M. E. D. and Chick, H.

1919. The antiscorbutic and growth-promoting value of canned vegetables. Lancet, 1919, II, 320-332.

Canada Council Sci. Indus. Research Rept.

1922. 34. Report of the associate food research committee for the year 1921-22.

\section{Cannon, A.}

1929. Some observations on beri-beri. Brit. Med. J. No. 3592, 852-854.

Cannon, H. C.

1926. Formules de différents types de régime et méthodes de préparation des aliments, employés pour les expériences de nutrition sur le rat. $B u l$. soc. hyg. aliment. 14, 339-365.

Capper, N. S.

1930. The alleged contamination of carotene by vitamin A. Biochem. J. 24, 453-455.

Card, L. E.

1929. Vitamin E requirements of poultry. Poultry Sci. 8, 328-334.

Caridroit, F.

1922. Contribution a l'étude du métabolisme des pigeons privés du facteur $B$. J. physiol. path. gén. 20, 189-192.

1924. Variation de l'excrétion azotée (azote total urinaire) du scorbut expérimental. Compt. rend. soc. biol. 90, 1379, 1380.

Carlsson, E. V.

1929. Quantitative experiments upon the occurrence of vitamin $G$ in the body, including the influence of the food. Dissertation, Columbia University, New York.

Carr, F. H. and Price, E. A.

1926. Colour reactions attributed to vitamin A. Biochem. J. 20, 497-501.

Carrel, A.

1922. Growth-promoting function of leucocytes. J. Exptl. Med. 36, 385-391. 
Carrick, C. W.

1925. Irradiation of chicks with cod liver oil. Am. J. Physiol. 74, 534-538.

Carrick, C. W. and Carr, R. H.

1925. The antineuritic properties of corn germs. Poultry Sci. 5, 26-34.

Carrick, C. W. and Hauge, S. M.

1925. Presence of the antiscorbutic substances in the livers of chickens fed on scorbutic diets. J. Biol. Chem. 63, 115-122.

Carrick, C. W., Prange, R. W. and Hauge, S. M.

1927. Fat-soluble A requirements for growing chicks. Poultry Sci. 6, 135-140.

a. A milkless, greenless, gritless, all-mash ration for growing chicks. Poultry Sci. 6, 162-166.

Carson, W. C., Irwin, G. F. and Phillips, R. A.

1930. The shortening of the coagulation time of the blood by irradiated ergosterol. Proc. Soc. Exptl. Biol. Med. 27, 488-490.

Carter, C. W. and Drury, A. N.

1929. Heart block in rice-fed pigeons. J. Physiol. 68, I, II.

Cartland, G. F., Speer, J. H. and Heyl, F. W.

1929. Effects of excessive doses of irradiated ergosterol in growing rats and dogs. J. Pharmacol. 36, 619-628.

Casparis, H., Shipley, P. G. and Kramer, B.

1923. The antirachitic influence of egg yolk. J. Am. Med. Assocn. 81, 818, 819.

Cassie, E. and Cox, U.

1930. The effect of ultraviolet light treatment on young children. Lancet, 1930, I, 878-880.

Cavanaugh, G. W., Dutcher, R. A. and Hall, J. S.

1923. The effect of the spray process of drying on the vitamin $C$ content of milk. A preliminary report. Am. J. Discases Children 25, 498-502.

1924. Antiscorbutic potency of whole-milk powder. Ind. Eng. Chem. 16, 1070-1073.

Cavins, A. W.

1924. The effect of fasting (and refeeding) on the calcium and inorganic phosphorus in blood serums of normal and rachitic rats. J. Biol. Chem. 59, 237-242.

Chahovitch, $\mathbf{X}$.

1925. Action de l'insuline sur le béribéri expérimental du Pigeon. Compt. rend. soc. biol. 93, 1333-1335.

Chamberlain, W. P.

1911. Eradication of beriberi from the Philippine (native) scouts by means of a simple change in their dietary. Philippine J. Sci. Sect. B, 6, 133-146.

1915. Prevention of beriberi among Philippine scouts by means of modifications in the diet. J. Am. Med. Assocn. 64, 1215-1220.

Chamberlain, W. P., Bloombergh, H. D. and Kilbourne, E. D.

1911. A study of the influence of rice diets and of inanition on the production of multiple neuritis in fowls and the bearing thereof on the etiology of beriberi. Philippine J. Sci. Sect. B, 6, 177-209.

Chamberlain, W. P. and Vedder, E. B.

1911. A contribution to the etiology of beriberi. Philippine J. Sci. Sect. B, 6, 251-258. 
a. A second contribution to the etiology of beriberi. Philippine J. Sci. Sect. B, 6, 395-404.

1912. The cure of infantile beriberi by the administration to the infant of an extract of rice polishings, and the bearing thereof on the etiology of beriberi. Bull. Manila Med. Soc. 4, 26-29.

Chamberlain, W. P., Vedder, E. B. and Williams, R. R.

1912. A third contribution to the etiology of beriberi. Philippine J. Sci.

Sect. B, 7, 39-52.

Chase, E. F.

1928. A quantitative study of the determination of the antineuritic vitamin.

$\left(\mathrm{F}\right.$ of $\left.\mathrm{B}_{1}\right)$. Dissertation, Columbia University, New York.

Chick, $\mathrm{H}$.

1926. Sources of error in the technique employed for the biological assay of fat-soluble vitamins. Biochem. J. 20, 119-130.

1929. The effect on vitamin $B_{2}$ of treatment with nitrous acid. Biochem. J. 23, 514-516.

Chick, H. et al.

1923. Studies of rickets in Vienna 1919-22. Medical Research Council (Gt. Brit.) Special Rept. No. 77, pp. 230.

Chick, H. and Dalyell, E. J.

1920. Eine Skorbutepidemie unter Kindern im Alter von 6 bis 14 Jahren. Z. Kinderheilk. 26, 257-269.

a. The influence of overcooking vegetables in causing scurvy among children. Brit. Med. J. No. 3119, 546-548.

1921. Observations on the influence of foods rich in accessory factors in stimulating development in backward children. Brit. Med. J. No. 3182, 1061-1066.

Chick, H., Dalyell, E. J., Hume, M., Mackay, H. M. M. and Smith, H. H.

1922. The etiology of rickets in infants. Prophylactic and curative observations at the Vienna University Kinderklinik. Lancet, 1922, II, 7-11.

Chick, H. and Delf, E. M.

1919. The antiscorbutic value of dry and germinated seeds. Biochem. J. 13, 199-218.

Chick, H. and Hume, E. M.

1916-17. (Paper on deficiency diseases and discussion.) Trans. Soc. Trop. Med. Hyg. 10, 179-186.

1917. The distribution among foodstuffs (especially those suitable for the rationing of armies) of the substances required for the prevention of (a) beriberi and (b) scurvy. J. Roy. Army Med. Corps 29, 121-159.

a. The distribution in wheat, rice, and maize grains of the substance, the deficiency of which in a diet causes polyneuritis in birds and beriberi in man. Proc. Roy. Soc. (London) B. 90, 44-60.

b. The effect of exposure to temperatures at or above $100^{\circ} \mathrm{C}$. upon the substance (vitamin) whose deficiency in a diet causes polyneuritis in birds and beriberi in man. Proc. Roy. Soc. (London) B. 90, 60-68.

1919. Note on the importance of accurate and quantitative measurements in experimental work on nutrition and accessory food factors. J. Biol. Chem. 39, 203-207. 
1920. The production in monkeys of symptoms closely resembling those of pellagra, by prolonged feeding on a diet of low protein content. Biochem. J. 14, 135-146.

Chick, H., Hume, E. M. and Skelton, R. F.

1918. An estimate of the antiscorbutic value of milk in infant feeding. Lancet, 1918, I, 1, 2.

a. The antiscorbutic value of cow's milk. Biochem. J. 12, 131-153.

b. The relative content of antiscorbutic principle in limes and lemons. A. Experimental inquiry. Lancet, 1918, II, 735.

1919. The antiscorbutic value of some Indian dried fruits. Lancet, 1919, II, $322,323$.

Chick, H., Korenchevsky V. and Roscoe, M. H.

1926. The difference in chemical composition of the skeletons of young rats fed (1) on diets deprived of fat-soluble vitamins and (2) on a low phosphorus rachitic diet, compared with those of normally nourished animals of the same age. Biochem. J. 20, 622-631.

Chick, H. and Rhodes, M.

1918. An investigation of the antiscorbutic value of raw juices of root vegetables, with a view to their adoption as an adjunct to the dietary of infants. Lancet, 1918, II, 774-775.

Chick, H. and Roscoe, M. H.

1926. The antirachitic value of fresh spinach. Biochem. J. 20, 137-152.

a. Influence of diet and sunlight upon the amount of vitamin $\mathrm{A}$ and vitamin $\mathrm{D}$ in the milk afforded by a cow. Biochem. J. 20,632-649.

1927. On the composite nature of the water-soluble B vitamin. Biochem. J. 21, 698-711.

1928. The dual nature of water-soluble vitamin B. II. The effect upon young rats of vitamin $B_{2}$ deficiency and a method for the biological assay of vitamin B. Biochem. J. 22, 790-799.

1929. A method for the assay in the antineuritic vitamin $B_{1}$, in which the growth of young rats is used as a criterion. Biochem. J. 23, 498-503.

a. An attempt to separate vitamin $B_{2}$ from vitamin $B_{1}$ in yeast and a comparison of its properties with those of the antineuritic vitamin $\mathrm{B}_{1}$. Biochem. J. 23, 504-513.

1930. Heat-stability of the (anti-dermatitis, "anti-pellagra") water-soluble vitamin $\mathrm{B}_{2}$. Biochem. J. 24, 105-112.

Chick, H. and Tazelaar, M.

1924. Note upon the effect on the growth of rats receiving a diet deficient in fat-soluble vitamins, of exposing their environment to the emanation from radium bromide. Biochem. J. 18, 1346-1348.

Chisholm, C. and McKillop, M.

1927. Notes on treatment of nursing mothers with ultra-violet rays. Lancet, 1927, II, 227.

Chittenden, R. H. and Underhill, F. P.

1917. The production in dogs of a pathological condition which closely resembles human pellagra. Am. J. Physiol. 44, 13-66.

Clark, J. H.

1922. The physiological action of light. Physiol. Rev. 2, 277-309.

1923. The effect of ultraviolet light on the condition of calcium in the blood. Am. J. Hyg. 3, 481, 482. 
1924. A simple method of measuring the intensity of ultraviolet light with comparative results on a number of physiological reactions. Am. J. Physiol. $69,200-209$.

1925. Ultraviolet light and scurvy. Science 61, 45-47.

1928. The probable amount of ultra-violet radiation obtained indoors through ultra-violet transmitting glasses. Science 68, 165, 166.

1929. The zinc sulphide method of measuring ultra-violet radiation and the results of a year's observations on Baltimore sunshine. Am. J. Hyg. 9, 646-662.

Clausen, E. M. L.

1928. Effect of infra-red radiation on growth of rachitic rat. Proc. Soc. Exptl. Biol. Med. 26, 77, 78.

1929. The use of isolated radiations in experiments with the rat. I. The effect of infra-red radiation on the growth of the rachitic rat. J. Nutrition 2, 125-153.

Clayton, M. M.

1927. The influence of diets containing unsaturated animal fats on reproduction and lactation in the rat. J. Biol. Chem. 74, LXXIV.

Clenshaw, E. and Smedley-Maclean, I.

1929. The nature of the unsaponifiable fraction of the lipoid matter extracted from green leaves. Biochem. J. 23, 107-109.

Clow, B. and Marlatt, A. L.

1929. The antirachitic factor in burbot-liver oil. Ind. Eng. Chem. 21, 281, 282.

1930. Studies of vitamin C in fresh and canned tomatoes. J. Agr. Research 40, 767-775.

Clow, B., Marlatt, A. L., Peterson, W. H. and Martin, E. A.

1929. The vitamin $\mathrm{C}$ content of fresh sauerkraut and sauerkraut juice. J. Agr. Research 39, 963-971.

Clow, B., Parsons, H. T. and Stevenson, I.

1930. The vitamin-C content of commercially canned sauerkraut, together with some observations on its vitamin-A content. J. Agr. Research 41, 51-64.

Cluver, E. H.

1929. Pellagra among the maize-eating natives of the Union of South Africa. Brit. Med. J. No. 3590, 751-754.

\section{Coblentz, W. W.}

1928. Spectral characteristics of light sources and window materials used in therapy. Trans. Illum. Eng. Soc. (N.Y.) 23, 247-301.

1929. Sources of ultra-violet radiation and their physical characteristics. J. Am. Med. Assocn. 92, 1834-1837.

Cocking, T. T. and Price, E. A.

1926. The search for colour reactions of vitamin "A". Pharm. J. 63, 175-178.

Cohen, B. and Mendel, L. B.

1918. Experimental scurvy of the guinea pig in relation to the diet. J. Biol. Chem. 35, 425-453.

Collazo, J. A.

1922. Untersuchungen über den Kohlehydratstoffwechsel bei der Avitaminose.

I. Über den Blutzucker. Biochem. Z. 134, 194-214. 
1923. Der Kohlehydratstoffwechsel bei Avitaminose. II. Glykogen und Avitaminose. III. Uber den Einfluss von Traubenzuckerzufuhr in kleinen und grossen Mengen auf den Blutzucker beim normalen, hungernden und avitaminösen Körper. IV. Uber die toxische Wirkung intermediärer Stoffwechselprodukte nach der Zuführung verschiedener Zuckerarten bei der Avitaminose. Biochem. Z. 136, 20-25, 26-37, 278-290.

a. Über die Alkalireserve des Blutplasmas bei Avitaminose. Biochem. $Z$. $140,254-257$.

1924. Versuche über den N-Stoffwechsel bei der Avitaminose. Biochem. Z. $145,436-441$.

Collazo, J. A. and Bosch, G.

1923. Über den Fettgehalt des Blutes bei der Avitaminose. Biochem. Z. 141, 370-378.

Collazo, J. A. and Funk, C.

1924. The requirements of vitamine $B$ in the metabolism of foods containing proteins and carbohydrates in varying proportions. J. Metabolic Research 5 , 187-193.

a. The metabolism of vitamin $B$ as one of the food constituents: Its excretion in the pigeon. J. Metabolic Research 5, 195-204.

Collazo, J. A., Rubino, P. and Varela, B.

1929. Experimentelle Hypervitaminose der Ratten durch grosse Dosen bestrahlten Ergosterins. Biochem. Z. 204, 347-353.

\section{Collier, J.}

1924. Prevention of weak legs in experimental chickens. Science 60, 42.

Collison, D. L., Hume, E. M., Smedley-Maclean, I. and Smith, H. H.

1929. The nature of the vitamin A constituent of green leaves. Biochem. J. 23, 634-647.

Comrie, J. D.

1920. Scurvy in North Russia. Edinburgh Med. J. 24, 207-215.

Connell, S. J. B. and Zilva, S. S.

1924. The reducing properties of antiscorbutic preparations. Biochem. J. 18, 638-640.

a. The differential dialysis of the antiscorbutic factor. II. Biochem. J. 18, 641-646.

Cook, D. H.

1927. Vitamin studies in Porto Rico. Porto Rico Health Rev. 2, 22, 23.

Cook, D. H. and Quinn, E. J.

1928. The vitamin B content of white yautia, yellow yautia, and plantain. Am. J. Trop. Med. 8, 73-77.

Cook, D. H. and Rivera, T.

1928. A brief discussion of vitamins A, B, C and D. Porto Rico Rev. Public Health Trop. Med. 3, 267-278.

Cooper, E.

1921. The presence of vitamin A in the peel of common citrus fruits. Proc. Soc. Exptl. Biol. Med. 18, 343, 344.

1924. The distribution of vitamin A in urine and some of the digestive secretions. Am. J. Physiol. 67, 454-463.

Cooper, E. A.

1912. On the protective and curative properties of certain foods against polyneuritis induced in birds by a diet of polished rice. J. Hyg. 12, 436-462. 
1913. The nutritional importance of the presence in dietaries of minute amounts of certain accessory substances. Brit. Med. J. No. 2727, 722-724.

a. The preparation from animal tissues of a substance which cures polyneuritis in birds induced by diets of polished rice. Biochem. J. 7, 268-274.

1914. The curative action of autolyzed yeast against avian polyneuritis. Biochem. J. 8, 250-252.

a. The relation of vitamins to lipoids. Biochem. J. 8, 347-354.

b. On the protective and curative properties of certain foodstuffs against polyneuritis induced in birds by a diet of polished rice. II. J. Hyg. 14, 12-22.

Cooper, E. A. and Funk, C.

1911. Experiments on the causation of beri-beri. Lancet, 1911, II, 1266-1267. Corda, $\mathbf{L}$.

1923. Über die Bedeutung des Vitamins B bei natürlicher Immunität der Tauben gegen Milzbrand. Z. Hyg. Infektionskrankh. 100, 129-139.

Corson, W. C., Irwin, G. F. and Phillips, R. A.

1930. The shortening of the coagulation time of the blood by irradiated ergosterol. Proc. Soc. Exptl. Biol. Med. 27, 488-490.

Council Pharm. Chem. Am. Med. Assocn.

1929. Viosterol. J. Am. Med. Assocn. 93, 693.

Coutts, F. J. H.

1918. Upon an inquiry as to dried milks, with special reference to their use in infant feeding. Rept. Local Govt. Board Public Health (Gt. Brit.) n. ser. No. 116, 1-138.

\section{Coward, K. H.}

1923. The formation of vitamin A in plant tissues. II. Biochem. J. 17, 134-144.

a. The association of vitamin $A$ with the lipochromes of plant tissues. Biochem. J. 17, 145-156.

1925. Synthesis of vitamin A by a freshwater alga, Chlorella. Biochem. J. 19, 240, 241.

a. The persistence of vitamin A in plant tissues. Biochem. J. 19, 500-506.

1927. The influence of light and heat on the formation of vitamin A in plant tissues. J. Biol. Chem. 72, 781-799.

1928. A method of assay of the antirachitic vitamin D. Quart. J. Pharm. I, 27-38.

a. The variations in the amounts of the antirachitic vitamin in different samples of cod liver oil, milk, and butter. Quart. J. Pharm. 1, 534-538.

b. The vitamin content of margarine. Lancet, 1928, II, 726, 727.

c. The minimum amount of vitamin $\mathrm{D}$ required for a positive antirachitic effect in the "line" test. Biochcm. J. 22, 1221, 1222.

1929. The influence of vitamin A deficiency on the oestrous cycle of the rat. J. Physiol. 67, 26-32.

a. The value of irradiated milk compared with cod-liver oil as a source of vitamin D. Lancet, 1929, II, 1090.

Coward, K. H. and Cambden, M. R.

1929. The influence of changes in body weight of the test rats on the accuracy of the assay of vitamin D by means of the line test. Quart. J. Pharm. 2, 44-47.

Coward, K. H. and Clark, A. J.

1923. The vitamin content of certain proprietary preparations. Brit. Med. J. I, 13-15. 
Coward, K. H. and Drummond, J. C.

1920. Researches on the fat-soluble accessory substance. IV. Nuts as a source of vitamin A. Biochem. J. 14, 665-667.

1921. The formation of vitamin $\mathrm{A}$ in living plant tissues. Biochem. J. 15, 530-539.

1922. On the significance of vitamin A in the nutrition of fish. Biochem. J. 16, 631-636.

Coward, K. H. and Eggleton, P.

1928. The content of vitamins A and C in watercress. Lancet, 1928, I, 97, 98.

Coward, K. H. and Key, K. M.

1928. The assay of vitamin A. Biochem. J. 22, 1019-1025.

Coward, K. H., Key, K. M. and Morgan, B. G. E.

1929. Some evidence of the existence of a further factor necessary for growth of the rat. Biochem. J. 23, 695-709.

Coward, K. H., Key, K. M., Morgan B. G. E. and Cambden, M.

1929. The influence of different samples of "casein" on vitamin tests. Biochem. J. 23, 913-920.

Coward, K. H., Lush, J. L. and Palmer, M. G.

1923. A note on the storage of vitamin A in the liver of the rat. Lancet, 1923, I, 124, 125.

Coward, K. H., Morgan, B. G. E. and Dyer, F. J.

1930. The influence of vitamin A deficiency on the oestrus cycle of the rat. II. J. Physiol. 69, 349-352.

\section{Cowell, S. J.}

1925. Irradiation of milk and the healing of rickets. Brit. Med. J. No. 3352, $594,595$.

1928. The antirachitic action of irradiated ergosterol in children and adolescents. Brit. $\bar{M} e d$. J. No. 3522, 5-7.

\section{Cowell et al.}

1926. Discussion on food deficiency conditions in relation to preventable illness. Brit. Med. J. No. 3421, 185-194.

\section{Cowgill, G. R.}

1921. Studies in the physiology of vitamins. Is water-soluble vitamin identical with secretin? Proc. Soc. Exptl. Biol. Med. 18, 148, 149.

a. Studies in the physiology of vitamins. II. Does vitamin B stimulate glands in a manner similar to the alkaloid pilocarpine? Proc. Soc. Exptl. Biol. Med. 18, 290.

b. A contribution to the study of the relation between vitamin $B$ and the nutrition of the dog. Am. J. Physiol. 57, 420-436.

1922. Studies in the physiology of vitamins. III. A comparison of the effects of feeding extracts of muscle and yeast respectively. IV. Parenteral administration of products containing vitamin $\mathrm{B}-$ mammalian experiments. Proc. Soc. Exptl. Biol. Med. 19, 282, 283-284.

1923. An improved procedure for metabolism experiments. J. Biol. Chem. 56, 725-737.

a. Studies in the physiology of vitamins. II. Parenteral administration of vitamin B-mammalian experiments. Am. J. Physiol. 66, 164-175.

1927. Vitamin B in commercial meat residue and commercial egg albumen. Am. J. Physiol. 79, 341-345. 
Cowgill, G. R., Deuel, H. J. Jr., Plummer, N. and Messer, F. C.

1926. Studies on the physiology of vitamins. IV. Vitamin B in relation to gastric motility. Am. J. Physiol. 77, 389-401.

Cowgill, G. R., Deuel, H. J. Jr. and Smith, A. H.

1925. Studies on the physiology of the vitamins. III. Quantitative aspects of the relation between vitamin $\mathrm{B}$ and appetite in the dog. Am. J. Physiol. 73, 106-126.

Cowgill, G. R. and Klotz, B. H.

1927. Determination of the vitamin $B$ requirement of the pigeon and its bearing on the theory of vitamin B function. Am. J. Physiol. 81, 470, 471.

Cowgill, G. R. and Mendel, L. B.

1921. Studies in the physiology of vitamins. I. Vitamin B and the secretory function of glands. Am. J. Physiol. 58, 131-151.

Cowgill, G. R., Smith, A. H. and Beard, H. H.

1925. Quantitative aspects of the function of vitamin $B$ in several species. J. Biol. Chem. 63, XXIII, XXIV.

Cowgill, G. R., Stucky, C. J. and Rose, W. B.

1929. The physiology of vitamins. V. Cutaneous manifestations related to a deficiency of the vitamin B complex. Arch. Path. 7, 197-203.

Cramer, W.

1920. Vitamins and lipoid metabolism. J. Physiol. 54, ii-iv.

a. On glandular adipose tissue, and its relation to other endocrine organs and to the vitamin problem. Brit. J. Exptl. Path. 1, 184-196.

1922. On vitamin underfeeding. Brit. J. Exptl. Path. 3, 298-306.

1923. On the mode of action of vitamins. Lancet, 1923, I, 1046-1050.

1924. Vitamins and the borderland between health and disease. Lancet, 1924, I, 633-640.

1927. Requirements of the population in milk fat and the available supply. Lancet, 1927, II, 774-777.

1930. Vitamin A and infections. Lancet, 1930, I, 1153, 1154.

Cramer, W. and Drew, A. H.

1923. The effect of light on the organism. Brit. J. Exptl. Path. 4, 271-282.

Cramer, W., Drew, A. H. and Mottram, J. C.

1921. Similarity of effects produced by absence of vitamins and by exposure to $\mathrm{x}$-rays and radium. Lancet, 1921, I, 963, 964.

a. On the function of the lymphocyte and of lymphoid tissue in nutrition, with special reference to the vitamin problem. Lancet, 1921, II, 1202-1208.

1922. On blood platelets. Their behavior in vitamin A deficiency and after radiation, and their relation to bacterial infections. Proc. Roy. Soc. (London) B. $93,449-467$.

1923. On the behavior of platelets in vitamin A deficiency and on the technique of counting them. Brit. J. Exptl. Path. 4, 37-44.

Cramer, W. and Kingsbury, A. N.

1924. Local and general defenses against infections, and the effect on them of vitamin deficiency. Brit. J. Exptl. Path. 5, 300-304.

Cramer, W. and Mottram, J. C.

1927. On the nutritive value of bread with special reference to its content in vitamin B. Lancet, 1927, II, 1090-1094. 
Craven, V. and Kramer, M. M.

1927. The vitamin C content of fresh and canned pear. J. Agr. Research 34, 385-392.

Crawford, M. E. F., Golding, J., Perry, E. O. V. and Zilva, S. S. 1930. The fat-soluble vitamins of milk. Biochem. J. 24, 682-691.

Creekmur, F.

1922. The intestinal bacterial flora of rats on a diet deficient in fat-soluble vitamin A. J. Infect. Diseases 31, 461-467.

Crist, J. W. and Dye, M.

1929. The association of vitamin A with greenness in plant tissue. II. The vitamin A content of asparagus. J. Biol. Chem. 81, 525-532.

\section{Crofts, E.}

1928. Studies on basal and resting metabolism after radiation with ultra-violet light. I. The effect of ultra-violet radiation on the resting metabolism of birds. II. The effect of ultra-violet radiation on the basal metabolism of human subjects. Am. J. Hyg. 8, 1014-1019, 1020-1023.

Croll, H. M. and Mendel, L. B.

1925. The distribution of vitamin B in the maize kernel. Am. J. Physiol. 74, 674-694.

Cruickshank, E. M., Hart, E. B. and Halpin, J. G.

1927. The vitamin A and vitamin D content of cod liver meal. Poultry Sci. 7, 9-16.

Csik, L. and Bencsik, J.

1927. Versuche, die Wirkung von B-Vitamin auf die Arbeitsleistung des Menschen festzustellen. Klin. Wochschr. 6, 2275-2278.

Cugnac, A. de.

1929. Essais de purification de la vitamine antinévritique (facteur hydrosoluble B) par précipitations fractionées. Bul. soc. chim. biol. 11, 443-465.

Cunningham, R. L.

1924. An attempt to determine the presence of vitamins $\mathrm{A}$ and $\mathrm{B}$ in tubercle bacilli. Am. Rev. Tuberculosis 9, 487-490.

Curran, J. O.

1847. Observations on Scurvy, etc. Dublin Quart. J. Med. Sciences 4, 107.

Dalldorf, G.

1929. The lesions in the skeletal muscles in experimental scorbutus. J. Exptl. Med. 50, 293-298.

Dalldorf, G. and Zall, C.

1930. Tooth growth in experimental scurvy. J. Exptl. Med. 52, 57-63.

Damianovich, $\mathrm{H}$.

1921. Quelques recherches sur la vitamine B. Compt. rend. soc. biol. 85, 591, 592.

Damianovich, H., Bianchi, A. and Savazzini, L. A.

1923. Le sang des rats avitaminés. Compt. rend. soc. biol. 88, 377, 378.

Damon, S. R.

1921. Bacteria as a source of the water-soluble B vitamin. J. Biol. Chem. 48, 379-384.

1922. Observations on the distribution of vitamin B. J. Am. Med. Assocn. 79, $128,129$. 
1923. Some observations in regard to growth-promoting substances of bacterial origin. J. Biol. Chem. 56, 895-902.

1924. Acid-fast bacteria as a source of vitamin B. J. Path. Bact. 27, 163-169. Daniels, A. I.

1922. Can yeast be used as a source of the antineuritic vitamin in infant feeding? Am. J. Diseases Children 23, 41-50.

1926. Vitamin A content of fecal excretion of a breast-fed and artificially fed infant. Preliminary report. Proc. Soc. Exptl. Biol. Med. 23, 824-826.

Daniels, A. L., Armstrong, M. E. and Hutton, M. K.

1923. Nasal sinusitis produced by diets deficient in fat-soluable A vitamin. J. Am. Med. Assocn. 81, 828, 829.

Daniels, A. L. and Brooks, L. M.

1927. Growth-promoting value of cod liver oil irradiated by sunlight and the mercury vapor lamp. Proc. Soc. Exptl. Biol. Med. 24, 971, 972.

a. Influence of feeding mixture on the antirachitic potency of cod liver oil concentrate. Proc. Soc. Exptl. Biol. Med. 24, 972-974.

b. Further evidence of destruction of vitamin $\mathrm{B}$ in evaporated milk. Proc. Soc. Exptl. Biol. Med. 25, 161-163.

Daniels, A. L., Byfield, A. H. and Loughlin, R.

1919. The role of antineuritic vitamin in artificial feeding of infants. Am.J. Diseases Children 18, 546-554.

Daniels, A. L., Giddings, M. L. and Jordan, D.

1929. The effect of heat on the antineuritic vitamin of milk. J. Nutrition 1, 455-466.

Daniels, A. L. and Hutton, M. K.

1925. Fertility of the white rat on purified rations. Proc. Soc. Exptl. Biol. Med. 23, 225-227.

a. Mineral deficiencies of milk as shown by growth and fertility of white rats. J. Biol. Chem. 63, 143-156.

1928. Relation of bone development in infants to calcium and phosphorus retention ratios. Proc. Soc. Exptl. Biol. Med. 25, 794-797.

Daniels, A. L., Hutton, M. K., Stearns, G. and Hejinian, L. M.

1929. The relation of rate of growth in infants to diet. Am. J. Diseases Children 37, 1177-1186.

Daniels, A. L. and Jordan, D. P.

1928. Influence of wheat germ oil on fertility of rats fed fat free rations. Proc. Soc. Exptl. Biol. Med. 26, 185-188.

1929. Influence of superheating on antirachitic properties of irradiated foods. Proc. Soc. Exptl. Biol. Med. 26, 453-455.

Daniels, A. L., Jordan, D. and Hutton, M. K.

1929. The development of the suckling young of milk fed rats. J. Nutrition 2, 19-29.

Daniels, A. L. and Loughlin, $\mathbf{R}$.

1918. Feeding experiments with peanuts. J. Biol. Chem. 33, 295-301.

1920. Note on the fat-soluble growth-promoting substance in lard and cottonseed oil. J. Biol. Chem., 42, 359-362.

a. A deficiency in heat-treated milks. J. Biol. Chem. 44, 381-397.

Daniels, A. L. and McClurg, N.

1919. Influence of high temperatures and dilute alkalies on the antineuritic properties of foods. J. Biol. Chem. 37, 201-213. 
Daniels, A. L. and Nichols, N. B.

1917. The nutritive value of the soy bean. J. Biol. Chem. 32, 91-102.

Daniels, A. L., Pyle, S. I. and Brooks, L.

1926. Effect of irradiated winter milk and cod liver oil on growth of young of milk-fed rats. Proc. Soc. Exptl. Biol. Med. 23, 821-824.

Daniels, A. L., Stearns, G. and Hutton, M. K.

1929. Calcium and phosphorus metabolism in artificially fed infants. I. Influence of cod liver oil and irradiated milk. Am. J. Diseases Children 37, 296-310.

\section{Danmeyer,}

1927. Kurze Bemerkung über die Ultraviolettstrahlung auf Island, 1926. Arch. ges. Physiol. (Pflïger's) 217, 509, 510.

Danysz-Michel and Koskowski, W.

1922. Etude de quelques fonctions digestives chez les pigeons normaux, nourris au riz poli et inanition. Compt. rend. 175, 54-56.

Darling, S. T.

1914. The pathologic affinities of beriberi and scurvy. J. Am. Med. Assocn. 63, $1290-1294$.

Daubney, C. G. and Zilva, S. S.

1926. The action of reducing agents on the antiscorbutic factor inactivated by aeration. Biochcm. J. 20, 519-523.

a. The antiscorbutic fraction of lemon juice. IV. Biochem. J. 20, 10551059.

Davey, A. J.

1921. Determination of the minimum doses of some citrus fruit juices which will protect a guinea-pig from scurvy, together with some observations on the preservation of such juices. Biochem. J. 15, 83-103.

Davidson, P. B.

1928. The development of deficiency disease during therapeutic tests. J. Am. Med. Assocn. 90, 1014, 1015.

Davis, D. E. and Beach, J. R.

1925. A study of the relative values of certain succulent feeds and alfalfa meal as sources of vitamin A for poultry. California Agr. Expt. Sta. Bull. 384, 3-14.

1926. A study of the relative value of certain root crops and salmon oil as sources of vitamin A for poultry. California Agr. Expt. Sta. Bull. 412, 3-15.

1928. The anti-rachitic value of salmon oil. Poultry Sci. 7, 216-218.

\section{Davis, $M$.}

1923. Effect of rations containing whole and skimmed milk on young growing puppies. J. Metabolic Research 3, 711-723.

Davis, M. and Outhouse, J.

1921. Effect of a ration low in fat-soluble $\mathrm{A}$ on the tissues of rats. Am. J. Diseases Children 21, 307-311.

Davison, W. C.

1922. The failure of yeast therapy. Am. J. Diseases Children 24, 339-345.

Dawbarn, M. C.

1928. The growth and maintenance of white mice fed upon synthetic diets. Aust. J. Exptl. Biol. Med. 5, 149-169. 
Dawkins, V. and Pattison, C. L.

1926. Irradiated milk: Its effect on the blood. Lancet, 1926, $I I, 1314,1315$.

Deas, J.

1924. Yeast growth-promoting vitamin tested for its effects on animals. $J$. Biol. Chem. 61, 5-8.

DeBuys, L. R.

1924. Rickets in breast-fed infants. Am. J. Diseases Childrcn 27, 149-162.

DeBuys, L. R. and Meysenbug, L. von

1924. Correlation of clinical, roentgenologic, and serologic evidences of rickets

in breast-fed. J. Am. Med. Assocn. 83, 1563, 1564.

Delf, E. M.

1918. The antiscorbutic value of cabbage. I. The antiscorbutic and growthpromoting properties of raw and heated cabbage. Biochem. J. 12, 416-447.

1920. Effect of heat on the antiscorbutic factor of vegetable and fruit juices. Biochem. J. 14, 211-228.

a. The distribution of accessory food factors (vitamins) in plants. $S$. African J. Sci. 17, 121-125.

1921. Studies in experimental scurvy with special reference to the antiscorbutic properties of some South African foodstuffs. S. African Inst. Med. Research Pub. No. 14, pp. 105.

1922. Studies in experimental scurvy, with special reference to the antiscorbutic properties of some South African foodstuffs. Lancet, 1922, I, 576-579.

1924. On the properties of certain South African oils with respect to their content of vitamin A. Biochem. J. 18, 93-100.

a. The antiscorbutic value of fresh and canned English tomatoes. Biochem. J. 18, 674-678.

1925. The influence of storage on the anti-scurvy value of fruits and vegetable juices. Biochem. J. 19, 141-152.

Delf, E. M. and Skelton, R. F.

1918. The antiscorbutic value of cabbage. II. The effect of drying on the antiscorbutic and growth-promoting properties of cabbage. Biochcm. J. 12, 448-463.

Delore, $\mathbf{P}$.

1929. Sur la capacité d'oxydation de l'huile de foie de morue et sa teneur en oxygène peroxydique. Influence des rayons ultra-violets. Bul. soc. chim. biol. 11, 74-91.

Denis, W. and Corley, R. C.

1925. Calcium metabolism in tissues affected by calcium salts and ultraviolet light. Proc. Soc. Exptl. Biol. Med. 23, 159.

a. A study of the effect of excessive calcium ingestion on the calcium content of tissues with and without the application of ultra-violet light. J. Biol. Chem. 66, 609-617.

Dennett, R. H.

1929. Routine use of the vitamin B factor in infant feeding. J. Am. Med. Assocn. 92, 769-772.

\section{Denton, J.}

1928. A study of the tissue changes in experimental black tongue of dogs compared with similar charges in pellagra. Am. J. Path. 4, 341-352. 
Denton, M. C. and Kohman, E.

1918. Feeding experiments with raw and boiled carrots. J. Biol. Chem. 36, 249-263.

De Sanctis, A. G., Ashton, L. O., and Stringfield, O. L.

1929. A study of the antirachitic value of irradiated powdered whole milk. Arch. Pediatrics 46, 297-311.

De Sanctis, A. G. and Craig, J. D.

1930. Comparative value of viosterol and cod-liver oil as prophylactic and antirachitic agents. A clinical study. J. Am. Med. Assocn. 94, 1285, 1286.

Desgrez, A., Bierry, H. and Rathery, F.

1923. Utilité de la vitamine $B$ et du lévulose dans la cure par l'insuline. Compt. rend. 177, 795-797.

Deuel, H. J. Jr. and Weiss, R.

1924. The basal metabolism in vitamin B deficiency. Proc. Soc. Exptl. Biol. Med. 21, 456-458.

Dickey, L. B.

1927. The avocado and vitamins. Science $65,15$.

Dixon, W. E. and Hoyle, J. C.

1928. The effects of irradiated ergosteral in large doses. Brit. Med. J. No. $3540,832-835$.

\section{Dodd, W. J.}

1913. Experimental scorbutus and the Roentgen ray diagnosis of scorbutus.

III. Experimental scorbutus in the monkey. Boston Med. Surg. J. 169, 237.

\section{Donaldson, $\mathrm{H}$. $\mathrm{H}$.}

1919. Quantitative studies on the growth of the skeleton of the albino rat. Am. J. Anat. 26, 237-314.

1924. "The Rat," Philadelphia, Wistar Institute, 2nd Ed.

\section{Donaldson, J. C.}

1919. The relative volumes of the cortex and medulla of the adrenal gland in the albino rat. Am. J. Anat. 25, 291-298.

\section{Donath, W. F.}

1924. Changes in the percentage of A-vitamine and in the nature of albumen during the germination of katjang-idjoe (Phaseolus radiatus L). Mededeel. Burgerlijk. Geneeskund. Dienst Nederland-Indië No. 4, 344-362.

1929. The nutritive value of tinmilk and milk powder. Mededeel. Dienst. Volksgezondheid Nederland-Indië 18, 247-303.

a. Synopsis of the anti-xerophthalmic, anti-neuritic and antiscorbutic vitamin percentages, besides the protein, fat, carbohydrate, and water amounts of different Indian foodstuffs. Mededeel. Dienst Volksgezondheid NederlandIndië 18, 334-339.

Dorcas, M. J.

1929. Ultra-violet irradiation of food on a tonnage basis. Food Indus. 1, 504, 505.

Dougherty, J. E.

1926. Egg yolk as a source of the antiscorbutic vitamin. Am. J. Physiol. 76, 265-267.

Downs, A. W. and Eddy, N. B.

1921. Secretin. V. Its effects in anemia, with a note on the supposed similarity between secretin and vitamin B. Am. J. Physiol. 58, 296-300. 
Doyle, L. P.

1925. Enlarged parathyroids in rachitic chickens. Science 61, 118.

a. Rickets in mature chickens. Poultry Sci. 4, 146-150.

Dragstedt, L. R. and Cooper, E. F.

1923. Parabiosis in the study of deficiency diseases. Am. J. Physiol. 67, 48-56.

Drummond, J. C.

1916. The growth of rats upon artificial diets containing lactose. Biochem. $J$. $10,89-102$.

1917. A study of the water-soluble accessory growth-promoting substance of yeast. I. Biochem. J. 11, 255-272.

1918. A study of the water-soluble accessory growth-promoting substance. II. Its influence upon the nutrition and nitrogen metabolism of the rat. Biochem. J. 12, 25-41.

a. The nutritive value of certain fish. J. Physiol. 52, 95-109.

b. Some aspects of infant feeding. Lancet, 1918, II, 482-484.

1919. Note on the rôle of the antiscorbutic factor in nutrition. Biochem. J. 13, 77-80.

a. Researches on the fat-soluble accessory substance. I. Observations upon its nature and properties. Biochem. J. 13, 81-94.

b. Researches on the fat-soluble accessory substance. II. Observations on its rôle in nutrition and influence on fat metabolism. Biochem. J. 13, 95-102. 1920. The nomenclature of the so-called accessory food factors (vitamins). Biochem. J. 14, 660.

1921. Factors influencing the nutritive value of lard and lard substitutes. $J$. Soc. Chem. Ind. 40, 81T-86T.

1923. Recent researches on the causation of rickets. J. Soc. Chem. Ind. 42, 811,812 .

1924. Some modern tendencies of vitamin research. J. Soc. Chcm. Ind. 43, 908-911.

a. Cod liver oil. J. Soc. Chem. Ind. 43, 928-932.

1925. Selection of cod-liver oils for medicinal use. Lancet, 1925, II, 679, 680.

1926. New aspects of the chemistry of the oils and fats. J. Soc. Chem. Ind. 45, 994-999.

a. Modern views on vitamins. J. Roy. Soc. Arts 74, 369-382.

b. New facts concerning the fat-soluble vitamins. Lancet, 1926, I, 272, 273. 1930. Recent progress in the chemical study of the vitamins. J. Soc. Chem. Ind. 49, 1T-10T.

Drummond, J. C., Ahmad, B. and Morton, R. A.

1930. Further observations on the relation of carotene to vitamin-A. J. Soc. Chem. Ind. 49, 291T-296T.

Drummond, J. C. and Baker, L. C.

1929. The composition of wool fat. J. Soc. Chem. Ind. 48, 232T-238T.

a. Further studies of the chemical nature of vitamin A. Biochem. J. 23, 274-291.

Drummond, J. C., Channon, H. J. and Coward, K. H.

1925. Studies on the chemical nature of vitamin A. Biochem. J. 19, 1047-1067.

Drummond, J. C., Channon, H. J., Coward, K. H., Golding, J., Mackintosh, J. and Zilva, S. S.

1924. The influence of the administration of certain oils on the nutritive value of the butter fat of cows on winter rations. J. Agr. Sci. 14, 531-547. 
Drummond, J. C. and Coward, K. H.

1920. Researches on the fat-soluble accessory substance. III. Technique for carrying out feeding tests for vitamin A (fat-soluble A). Biochem. J. 14, 661-664.

a. Researches on the fat-soluble accessory substance. V. The nutritive value of animal and vegetable oils and fats considered in relation to their color. Biochem. J. 14, 668-677.

b. Researches on the fat-soluble accessory factor (vitamin A). VI. Effect of heat and oxygen on the nutritive value of butter. Biochem. J. 14, 734-739. 1921. Nutrition and growth on diets devoid of true fats. Lancet, 1921, II, 698-700.

Drummond, J. C., Coward, K. H., Golding, J., Mackintosh, J. and Zilva, S. S. 1923. Cod liver oil in the winter feeding of milch cows. J. Agr. Sci. 13, 144-152.

Drummond, J. C., Coward, K. H. and Handy, J.

1925. On the technique of testing for the presence of vitamin A. Biochem. J. 19, 1068-1074.

Drummond, J. C., Coward, K. H. and Watson, A. F.

1921. Researches on vitamin A. VII. Notes on the factors influencing the value of milk and butter as sources of vitamin A. Biochem. J. 15, 540-552.

Drummond, J. C., Crowder, G. P. and Hill, E. L. G.

1922. Nutrition on high protein dietaries. J. Phy'siol. 56, 413-420.

Drummond, J. C. and Funk, C.

1914. The chemical investigation of the phosphotungstate precipitate from rice polishings. Biochem. J. 8, 598-615.

Drummond, J. C., Golding, J., Zilva, S. S. and Coward, K. H.

1920. The nutritive value of lard. Biochem. J. 14, 742-753.

Drummond, J. C. and Marrian, G. F.

1926. The physiological rôle of vitamin B. I. The relation of vitamin $B$ to tissue oxidations. Biochem. J. 20, 1229-1255.

Drummond, J. C. and Morton, R. A.

1929. Observations on the assay of vitamin A. Biochem. J. 23, 785-802.

Drummond, J. C., Rosenheim, O. and Coward, K. H.

1925. The relation of sterols to vitamin A. J. Soc. Chem. Ind. 44, 123T, $124 \mathrm{~T}$.

Drummond, J. C. and Watson, A. F.

1922. The testing of foodstuffs for vitamins. Analyst 47, 235-244.

a. The sulphuric acid reaction for liver oils. Analyst 47, 341-349.

Drummond, J. C. and Zilva, S. S.

1922. Studies of the nutritive value of the edible oils and fats. I. The oilbearing seeds and crude vegetable oils and fats. J. Soc. Chem. Ind. 41, 125T-127T.

a. The preparation of cod liver oil and the effect of the processes on the vitamin value of the oils. J. Soc. Chem. Ind. 41, 280T-284T.

Drummond, J. C., Zilva, S. S. and Coward, K. H.

1922. The origin of the vitamin A in fish oils and fish liver oils. Biochem. $J$. $16,518-522$.

Drummond, J. C., Zilva, S. S. and Golding, J.

1923. The use of cod-liver oil in the feeding of farm animals. J. Agr. Sci. 13, 153-162. 
Dubin, H. E.

1925. Antirachitic action of cod liver oil. Comparison of fresh oil with concentrate prepared from oil. Am. J. Diseases Children 30, 72-75.

1927. Nutritional value and standardization of cod liver oil and of its nonsaponifiable fat-soluble vitamine concentrate (Oscodal). J. Am. Pharm. Assocn. 16, 41-45.

Dubin, H. E. and Funk, C.

1923. Studies on the chemistry of cod liver oil. I. The effect of hydrogenation upon the vitamine content. II. A cod liver oil concentrate manifesting both antirachitic and antiophthalmic properties. J. Metabolic Research 4, 461-479.

1924. Studies on the chemistry of cod liver oil. II. A cod liver oil concentrate manifesting both antirachitic and antiophthalmic properties. Proc. Soc. Exptl. Biol. Med. 21, 458-460.

Dulière, W., Morton, R. A. and Drummond, J. C.

1929. The alleged relation of carotin to vitamin A. J. Soc. Chem. Ind. 48, $316 \mathrm{~T}-321 \mathrm{~T}$.

Dunham, G. C.

1921. The water-soluble B vitamin content of certain vegetables. Military Surg. 48, 223-234.

Dunn, L. C.

1924. The effect of codliver oil in various amounts and forms on the growth of young chickens. J. Biol. Chem. 61, 129-136.

Dutcher, R. A.

1918. Vitamin studies. I. Observations on the catalase activity of tissues in avian polyneuritis. J. Biol. Chem. 36, 63-72.

a. Vitamin studies. III. Observations on the curative properties of honey, nectar, and corn pollen in avian polyneuritis. J. Biol. Chem. 36, 551-555.

1919. Vitamin Studies. IV. Antineuritic properties of certain physiological stimulants. J. Biol. Chem. 39, 63-68.

1920. The nature and functions of the antineuritic vitamin. Proc. Natl. Acad. Sci. 6, 10-14.

1921. Factors influencing the vitamin content of foods. J. Ind. Eng. Chem. 13, 1102.

1925. Inorganic blood phosphorus and bone ash in rats fed on normal, rachitic, and irradiated-rachitic rations. Penn. Agr. Expt. Sta. Bull. 196, 4.

1928. Vitamin B terminology. Science 68, 206-209.

Dutcher, R. A. et al.

1929. Vitamin B terminology. Science 69, 276.

Dutcher, R. A. and Collatz, F. A.

1918. Vitamin studies. II. Does water-soluble vitamin function as a catalase activator? J. Biol. Chem. 36, 547-550.

Dutcher, R. A., Creighton, M. and Rothrock, H. A.

1925. Vitamin studies. XI. Inorganic blood phosphorus and bone ash in rats fed on normal, rachitic, and irradiated rachitic diets. J. Biol. Chem. 66, 401-407.

Dutcher, R. A., Eckles, C. H., Dahle, C. D., Mead, S. W. and Schaefer, O. G.

1920. Vitamin studies. VI. The influence of the diet of the cow upon the nutritive and antiscorbutic properties of cow's milk. J. Biol. Chem. 45, 119132. 
Dutcher, R. A., Ely, J. O. and Honeywell, H. E.

1927. Vitamin studies. XV. Assimilation of vitamins $A$ and $D$ in presence of mineral oil. Proc. Soc. Exptl. Biol. Med. 24, 953-955.

Dutcher, R. A. and Francis, E.

1924. Vitamin studies. X. Feeding technique in vitamin studies. Proc. Soc. Exptl. Biol. Med. 21, 189-193.

1926. Vitamin studies. XIII. Vitamin B in evaporated milks made by vacuum and aeration processes. J. Dairy Sci. 9, 379-387.

Dutcher, R. A., Harshaw, H. M. and Hall, J. S.

1921. Vitamin studies. VIII. The effect of heat and oxidation upon the antiscorbutic vitamin. J. Biol. Chem. 47, 483-488.

Dutcher, R. A. and Honeywell, H. E.

1929. Is ossification influenced solely by ultra-violet light? Science 70, 173, 174.

Dutcher, R. A., Honeywell, H. E. and Dahle, C. D.

1927. Vitamin studies. XVI. Vitamin A in evaporated milks made by vacuum and aeration methods. J. Biol. Chem. 75, 85-94.

Dutcher, R. A., Kennedy, C. and Eckles, C. H.

1920. The influence of the diet of the cow upon the fat-soluble and watersoluble vitamin of cow's milk. Science 52, 588, 589.

Dutcher, R. A. and Kruger, J. H.

1926. Vitamin studies. XIV. The influence of ultra-violet light on the antirachitic properties of purified rations used in the study of vitamin A. J. Biol. Chem. 69, 277-282.

Dutcher, R. A. and Outhouse, J.

1923. The vitamin content of raisins, dried raisin seeds and raisin seed oil. Proc. Soc. Exptl. Biol. Med. 20, 450.

Dutcher, R. A., Pierson, E. M. and Biester, A.

1920. Vitamin studies. V. The antiscorbutic properties of raw beef. J. Biol. Chem. 42, 301-310.

Dutcher, R. A. and Wilkins, S. D.

1921. Vitamin studies. VII. The influence of fresh alfalfa upon the weight of testes in Single Comb White Leghorn cockerels. Am. J. Physiol. 57, 437-443.

Dye, J. A. and Waggener, R. A.

1927. The indophenol oxidase content of normal and scorbutic guinea pig's tissues. Proc. Soc. Exptl. Biol. Med. 24, 643.

Dye, M. and Crist, J. W.

1929. Relation of soil fertility to vitamin A content of leaf lettuce. J. Nutrition 1, 335-338.

Dye, M., Medlock, O. C. and Crist, J. W.

1927. The association of vitamin A with greenness in plant tissue. I. The relative vitamin A content of head and leaf lettuce. J. Biol. Chem. 74, 95-106.

Dyke, H. W.

1918. A report on an outbreak of scurvy in the South African native labour corps. Lancet, 1918, II, 513-515.

Earp, J. R.

1929. Note on observation of ultra-violet radiation of sun and sky measured by the zinc sulfide method in Boulder, Colorado. Am. J. Hyg. 9, 663-665. 
Eckman, P. E.

1922. The antiscorbutic value of dehydrated fruits. J. Am. Med. Assocn. 78, $635,636$.

\section{Eckstein, A.}

1923. Einfluss qualitativer Unternährung auf die Funktion der Keimdrüsen. Arch. ges. Physiol. (Pflïger's) 201, 16-24.

Eckstein, A. and Rominger, E.

1924. Ueber den Vitamingehalt der Kuhmilch bei Verfütterung von elektrisch konservierten Grünfutter. Münch. med. Wochschr. 71, 396, 397.

Eddy, W. H.

1916. The isolation of a growth-producing substance from sheep pancreas. $J$. Biol. Chem. 27, 113-126.

1917. The use of pancreatic vitamin in cases of malnutrition. J. Biol. Chem. 29, XVI, XVII.

1920. The use of water-soluble $B$ in the treatment of infant malnutrition. J. Biol. Chem. 41, XXXIV.

1921. "The Vitamin Manual," Baitimore, Williams and Wilkins Co. pp. 121.

1926. The vitamin content of foodstuffs. Am. J. Pub. Health 16, 109-115.

1927. Irradiated milk powder as an antirachitic. Arch. Pediatrics 44, 320, 321.

a. Vitamin B testing revised. Proc. Soc. Exptl. Biol. Med. 25, 125-127.

1928. The use of ultra-violet light transmitting windows. Am. J. Pub. Health $18,1470-1479$.

a. Hunting the vitamin. Am. J. Pub. Health 18, 313-325.

1929. An improvement in the quantitative assay of the antiscurvy vitamin

(C). Am. J. Pub. Health 19, 1309-1320.

Eddy, W. H., Gurin, S. and Keresztesy, J.

1930. The Williams-Waterman vitamin $\mathrm{B}_{3}$ J. Biol. Chem. 87, 729-740.

Eddy, W. H., Heft, H. L., Stevenson, H. C. and Johnson, R.

1921. Studies in the vitamin content. II. The yeast test as a measure of vitamin B. J. Biol. Chem. 47, 249-275.

Eddy, W. H. and Kellogg, M.

1927. The place of the banana in the diet. Am. J. Pub. Health 17, 27-35.

Eddy, W. H., Kerr, R. W. and Williams, R. R.

1924. The isolation of a crystalline substance (M.P. $223^{\circ}$ C.) having the properties of bios. Proc. Soc. Exptl. Biol. Med. 21, 307, 308.

a. The isolation from autolyzed yeast of a crystalline substance melting at $223^{\circ} \mathrm{C}$., having the properties of a bios. J. Am. Chem. Soc. 46, 28462855 .

Eddy, W. H. and Kohman, E. F.

1924. Vitamin $\mathrm{C}$ in canned foods. Ind. Eng. Chem. 16, 52, 53.

Eddy, W. H., Kohman, E. F. and Carlsson, V.

1925. Vitamins in canned foods. III. Canned spinach. Ind. Eng. Chem. 17, 69-74.

1926. Vitamins in canned foods. IV. Green peas. Ind. Eng. Chem. 18, 85-89.

Eddy, W. H., Kohman, E. F. and Halliday, N.

1929. Vitamins in canned foods. VII. Effect of storage on vitamin value of canned spinach. Ind. Eng. Chem. 21, 347. 
Eddy, W. H., Muller, H. R. and Heft, H. L.

1922. Phytin as a source of phosphorus in the prevention of rickets. J. Biol.

Chern. 50, XIX, XX.

Eddy, W. H. and Roper, J. C.

1917. The use of pancreatic vitamin in cases of marasmus. Am. J. Diseascs Children 14, 189-201.

Eddy, W. H., Shelow, E., Pease, R., Richer, M. and Watkins, L.

1923. The effect of cooking on the vitamin content of cabbage. J. Home Econ. 15, 15-30.

Eddy, W. H. and Stevenson, H. C.

1919. The suitability of the Bachmann test for water-soluble B. Proc. Soc. Exptl. Biol. Med. 17, 52-56.

1920. Studies in the vitamin content. J. Biol. Chem. 43, 295-309.

Éderer, S. and Kramár, E.

1923. Die inaktivierende Wirkung der Reduktion auf die Vitamine. Ein Versuch zur Erklärung des Milchnährschadens als eine "endogene Avitaminose." Klin. Wochschr. 2, 2277, 2278.

\section{Edgeworth, H. I.}

1922. Experiments with two methods for the study of vitamin B. Dissertation, Columbia University, New York.

Edie, C. S., Evans, W. H., Moore, B., Simpson, G. and Webster, A.

1912. Antineuritic bases of vegetable origin in relationship to beriberi, with a method of isolation of torulin, the antineuritic base of yeast. Biochem. J. 6, 234-242.

\section{Editorial.}

1921. Nutritional rehabilitation. J. Am. Med. Assocn. 77, 289.

1922. The therapeutic use of yeast and vitamin preparations. J. Am. Med. Assocn. 78, 1127.

1923. The storage of vitamin A in the body. J. Am. Med. Assocn. 80, 851.

1924. Why xerophthalmia deserves attention. J. Am. Med. Assocn. 82, 1048.

a. Adequate versus optimal nutrition. J. Am. Med. Assocn. 83, 768.

1925. The vitamin content of milk fat. J. Am. Med. Assocn. 84, 819.

a. Vitamin A and disease. J. Am. Med. Assocn. 84, 894.

b. The pathologic conditions induced by a deficiency of vitamin A. J. Am. Med. Assocn. 84, 1827.

c. Fresh fruits and vegetables. Vitamins and bacteria. J. Am. Med. Assocn. 85, 1488.

1926. The effect of ultravioiet rays on milk and eggs. J. Am. Med. Assocn. 86,870 .

1927. Pellagra. J. Am. Mcd. Assocn. 89, 1154.

a. The influence of manurial conditions on the vitamin content of grain. Brit. Med. J. No. 3454, 530.

1928. Sunshine, skyshine and rickets. J. Am. Med Assocn. 90, 118, 119.

a. Nutrition and longevity. J. Am. Med. Assocn. 92, 57, 58.

1929. Ergosterol and cathode rays. J. Am. Med. Assocn. 92, 810.

a. Vitamin content of treated tomatoes and asparagus. J. Am. Med. Assocn. 92, 1183, 1184.

b. Viosterol: Irradiated ergosterol. J. Am. Med. Assocn. 93, 694, 695.

c. Vitamin D and milk. J. Am. Med. Assocn. 93, 1386. 
1930. Irradiated ergosterol. Brit. Med. J. No. 3603, 158.

a. Vitamin D in tuberculosis. J. Am. Med. Assocn. 94, 414.

b. The action of vitamin D. J. Am. Med. Assocn. 94, 1505.

c. Carotene and vitamin A. Brit. Med. J. No. 3621, 1013, 1014.

Eggleton, P. and Gross, L.

1925. A note on the blood-sugar levels of rats fed with complete diets and diets deficient in vitamin B. Biochem. J. 19, 633-637.

Eggleton, P. and Harris, L. J.

1925. U1traviolet light and the antiscorbutic vitamin. Brit. Med. J. No. 3387, 987-991.

Eichholtz, F.

1924. Úber die Anwesenheit von Vitamin A in Handels-lecithin. Biochem. $Z$. $144,70,71$.

Eichholz, W. and Kreitmair, H.

1928. Resistenzverminderung infolge vitamin-D-Mangel. Mïnch. medizin. Wochschr. 75, 79-81.

\section{Eidinow, A.}

1929. A new daylight lamp for actinotherapy and general illumination. Brit. Med. J. No. 3562, 680-682.

Eijkman, C.

1890. Polyneuritis bij hoenderen. Geneeskund. Tijdschr. Nederland.-Indië 30, 295-334.

1896. Polyneuritis bij Hoenders. Nieuwe Bijdrage tot de Aetiologie der Ziekte. Geneeskund. Tijdschr. Nederland. Indië 36, 214-269.

1897. Note sur la prophylaxie du beriberi. Janus 2, 23-89.

a. Ein Versuch zur Bekämpfung der Beri-Beri. Arch. path. Anat. (Virchow's) 149, 187-194.

1898. Beriberi en voeding. Geneeskund Tijdschr. Nederland.-Indië 38, 275-284.

1906. Úber Ernahrungspolyneuritis. Arch. Hyg. 58, 150-170.

1911. Polyneuritis gallinarum und Beriberi. Arch. Schiffs-Tropen-Hyg. 15, 698-712.

1927. Experiments with Jansen and Donath's antiberiberi vitamin. Proc. $K$. Akad. Wetensch. Amsterdam 30, 376-382.

Eijkman, C. and van Hoogenhuyze, C. J. C.

1916. The influence of feeding and inanition on the development of polyneuritis gallinarum. Proc. K. Akad. Wetensch. Amsterdam 18, 1467-1480.

Eliot, M. M.

1926. New Haven demonstration of community control of rickets. Pub. Health J. (Toronto) 17, 114-116.

Ellinger, $\mathbf{P}$.

1927. Die Beziehungen zwischen antirachitischer Wirksamkeit und Dielektrizitätskonstante von ultra-violett bestrahlten Cholesterinlösungen. Arch. Exptl. Path. Pharmakol. 122, 238-246.

Elliott, W. E., Crichton, A. and Orr, J. B.

1922. The importance of the inorganic constituents of the food in nutritional disorders. I. Rickets in pigs. Brit. J. Exptl. Pathol. 3, 10-19.

Ellis, C. and MacLeod, H. L.

1922. "Vital Factors of Foods. Vitamins and Nutrition," New York, D. Van Nostrand Co. 
Ellis, N. R., Steenbock, H. and Hart, E. B.

1921. Some observations on the stability of the antiscorbutic vitamin and its behavior to various treatments. J. Biol. Chem. 46, 367-380.

Ely, J. O., Honeywell, H. E. and Dutcher, R. A.

1927. The calcifying potency of codliver oil. Pennsylvania Agr. Expt. Sta. Bull. 213, 4.

1929. Assimilation of vitamin A in the presence of mineral oil. Pennsylvania Agr. Expt. Sta. Bull. 243, 5.

Embrey, H. C.

1921. The investigation of some Chinese foods. China Med. J. 35, 420-427.

1923. The antiscorbutic vitamin in some oriental fruits and vegetables. Philippine J. Sci. 22, 77-82.

Emmett, A. D.

1920. The fat-soluble vitamins and xerophthalmia. Science 52, 157, 158.

1921. The physiological value of the vitamins. J. Am. Pharm. Assocn. 10, 176-182.

a. Standardized methods for the study of vitamins. J. Ind. Eng. Chem. 13, 1104.

1921-22. The vitamins and their relationship to health and disease. Therap.

Gaz. (1921) 45, 768-772, 847-853; 46, (1922) 17-24.

Emmett, A. D. and Allen, F. P.

1919. Nutritional studies on the growth of frog larvae (Rana pipiense). J.

Biol. Chem. 38, 325-344.

Emmett, A. D. and Luros, G. O.

1919. Is lactalibumin a complete protein for growth? J. Biol. Chem. 38, 147159

a. Stability of lactalbumin toward heat. J. Biol. Chem. 38, 257-265.

b. The absence of fat-soluble A vitamin in certain ductless glands. $J$. Biol. Chem. 38, 441-447.

1920. Water-soluble vitamins, I. Are the antineuritic and the growth-promoting water-soluble vitamins the same? J. Biol. Chem. 43, 265-286.

Emmett, A. D. and McKim, L. H.

1917. The value of the yeast vitamin fraction as a supplement to a rice diet.

J. Biol. Chem. 32, 409-419.

Emmett, A. D. and Peacock, G. E.

1922. The chick as an experimental animal in vitamin studies. A preliminary report. J. Biol. Chem. 50, XL.

1923. Does the chick require the fat-soluble vitamins? J. Biol. Chem. 56, 679-693.

1925. Adequacy of pigeons and rats for vitamin studies. J. Biol. Chem. 63, XXIII.

Emmett, A. D. and Stockholm, M.

1920. Water-soluble vitamins. II. The relation of the antineuritic and watersoluble B vitamins to the yeast growth-promoting stimulus. J. Biol. Chem. 43, 287-294.

Emslie, M.

1928. Rheumatic symptomatology and vitamin B. J. Roy. Sanit. Inst. 49, 221-228.

Enright, J. I.

1920. War edema in Turkish prisoners of war. Lancet, 1920, I, 314-316. 
a. The pellagra outbreak in Egypt. II. Pellagra among German prisoners of war; Observations upon the food factor in the disease. Lancet, 1920, I, 998-1004.

Epstein, A.

1918. Über eine auffällige Häufung der Barlowschen Krankheit in den Kriegsjahren 1917-18. Jahrb. Kinderheilk. 88, 237-267.

Estill, H. W. and McCollum, E. V.

1927. The separation of a substance from oils which inhibits the destruction of vitamin A by ferrous sulfate. J. Biol. Chem. 75, 157-162.

Euler, B. v. and Euler, H. v.

1929. A-Vitamin im Tierkörper. Arkiv. Kemi Mineral. Geol. 10B, No. 3, pp. 6.

1930. Neue Ergebnisse über A-Vitamine (Fettlösliche Wachstumfaktoren). Klin. Wochschr. 9, 916-918.

Euler, B. v., Euler, H. v. and Hellström, H.

1928. A-Vitaminwirkung der Lipochrome. Biochem. Z. 203, 370-384.

Euler, B. v., Euler, H. v. and Karrer, P.

1929. Zur Biochemie der Carotinoide. Helv. Chim. Acta. 12, 278-285.

Euler, H. v.

1921. Versuche mit A-Vitaminen aus Möhren. Arkiv. Kemi Mineral. Geol. 8, No. 18 , pp. 7.

1922. Utber Wirkungsbedingungen von A-Vitaminen. Arkiv. Kemi Mineral. Geol. 8, No. 19, pp. 10.

1923. Über Wasser- und Fett-lösliche Wachstumskatalysatoren (D-Vitamine). Arkiv. Kemi Mineral. Geol. 8, No. 31, pp. 10.

Euler, H. v. and Bernton, A.

1922. Chemical studies on vitamines. Arkiv. Kemi Mineral. Geol. 8, No. 21, pp. 9.

Euler, H. v. and Erikson, E.

1925. Zur Kenntnis der Wachstumsfaktoren. V. Z. physiol. Chem. 146, 241-46.

Euler, H. v., Lövgren, T. and Rydbom, M.

1927. Einfluss fettlöslicher Vitamine und Factoren auf den Gehalt des Rattenblutes an gesamter und anorganischer Phosphorsäure. Z. physiol. Chem. 170, 79-90.

Euler, H. v. and Myrbäck, K.

1921. Vitamine (Biokatalysatoren) B und Co-Enzyme. II. $Z$ physiol. Chem. 115, 155-169.

1925. Phosphat- und Calciumgehalt im Blut von Meerschweinchen und Ratten bei wechselnder Zufuhr von C- und A-Vitamin. Z. physiol. Chem. 148, 180-196.

Euler, H. v. Myrbäck, K., Fink, H. and Hellström, H. 1927. Wachstumsfaktoren. X. Z. physiol. Chem. 168, 11-26.

Euler, H. v. and Pettersson, A.

1921. Vitamine B (Biokatalysatoren) und Co-Enzyme. I. Z. physiol. Chem. 114, 4-16.

Euler, H. v., Rydbom, M. and Hellström, H.

1929. Zur Kenntnis der $\mathrm{SbCl}_{3}$-Reaktion von Tranen. Biochem. Z. 208, 73-78.

Euler, H. v. and Steffenburg, S.

1925. Zur Kenntnis der Wachstumsfaktoren. VI. Z. physiol. Chem. 149, 195-202. 
Euler, H. v. and Swartz, O.

1924. Uber den Zusammenhang der wasserlöslichen Wachstumsfaktoren mit Aktivatoren des Zuckerabbaues und über einen thermostabilen Biokatalysator in der Hefe. I. Z. physiol. Chem. 140, 146-163.

Euler, H. v. and Widell, $H$.

1925. Zur Kenntnis der Wachstumsfactoren. IV. Der Einfluss von I $D$-Überschüssen auf das Wachstum von Ratten. Z. physiol. Chem. 144, 132-137.

Euler, H. v., Widell, H. and Erikson, E.

1925. Zur Kenntnis der Wachstumsfaktoren. III. Z. physiol. Chem. 144, 123-131.

Eustis, A. C. and Scott, L. C.

1914. Isolation of vitamin from rice polishings. Biochem. Bull. 3, 466, 467. Evans, H. M.

1923. On the existence of a hitherto unknown dietary factor essential for reproduction. U. S. Dept. Agr. Dairy Div. IVorld's Dairy Cong. Proc. 2, 1027.

1924. Unique dietary needs for lactation. Science 60, 20-22.

1925. Invariable occurrence of male sterility with dietaries lacking fat-soluble vitamine E. Proc. Natl. Acad. Sci. 11, 373-377.

a. The Relations between Fertility and Nutrition, in "Lectures on Nutrition, Mayo Foundation Lectures," W. B. Saunders Co., Philadelphia, pp. 209-234.

1928. The effects of inadequate vitamin $A$ on the sexual physiology of the female. J. Biol. Chem. 77, 651-654.

a. Spontaneous deciduomata in pseudo-pregnancy with low vitamin E. Am. J. Physiol. 85, 149-153.

b. The effect of inadequate vitamin B upon sexual physiology in the male. J. Nutrition 1, 1-21.

c. Relation of vitamin $\mathrm{E}$ to growth and vigor. J. Nutrition 1, 23-28.

Evans, H. M. and Bishop, K. S.

1922. On the existence of a hitherto unrecognized dietary factor essential for reproduction. Science 56, 650, 651; also in Am. J. Physiol. 63, 396, 397.

a. On an invariable and characteristic disturbance of reproductive function in animals reared on a diet poor in fat-soluble vitamin A. Abstracted in Anat. Record 23, 17, 18.

b. On the relations between fertility and nutrition. I. The ovulation rhythm in the rat on a standard nutritional regime. II. The ovulation rhythm in the rat on inadequate nutritional regime. J. Metabolic Research 1, 319-333; 335-356.

1923. The cause of reproductive upset in dietary deficiencies due to lack of vitamin A. Abstracted in Anat. Record 25, 129.

a. Existence of a hitherto unknown dietary factor essential for reproduction. J. Am. Med. Assocn. 81, 889-892.

b. On the relations between fertility and nutrition. III. The normal reproductive performance of the rat. J. Metabolic Research 3, 201-231.

c. The production of sterility with nutritional regimes adequate for growth and its cure with other foodstuffs. J. Metabolic Research 3, 233-316.

1924. Stability and solubilities of the food substance or vitamin X required for reproduction. Proof of the power of the body to store the substance X required for reproduction. Abstracted in Anat. Record 27, 203, 204. 
Evans, H. M. and Burr, G. O.

1924. Preliminary steps in the isolation and concentration of vitamin X. Abstracted in Anat. Record 27, 203.

1925. Distribution of vitamin X in natural foods. Abstracted in Anat. Record $29,356$.

a. The antisterility vitamin fat-soluble E. Proc. Natl. Acad. Sci. 11, 334-341.

1927. Vitamin E: The ineffectiveness of curative dosage when mixed with diets containing high proportions of certain fats. J. Am. Med. Assocn. 88, $1462-1465$.

a. A new dietary deficiency with highly purified diets. Proc. Soc. Exptl. Biol. Med. 24, 740-743.

b. New dietary deficiency with highly purified diets. II. Supplementary requirement of diet of pure casein, sucrose and salt. Proc. Soc. Exptl. Biol. Med. 25, 41-48.

c. Vitamin E. II. The destructive effect of certain fats and fractions thereof on the antisterility vitamin in wheat germ and in wheat germ oil. J. Am. Med. Assocn. 89, 1587-1590.

d. The antisterility vitamine fat soluble E. California Univ. Mem. 8, 176. 1928. Development of paralysis in the suckling young of mothers deprived of vitamin E. J. Biol. Chem. 76, 273-297.

a. On the amount of vitamin B required during lactation. J. Biol. Chem. 76, 263-272.

b. A new dietary deficiency with highly purified diets. III. The beneficial effect of fat in the diet. Proc. Soc. Exptl. Biol. Med. 25, 390-397.

c. A new differentiation between the antineuritic vitamin $B$ and the purely growth-promoting vitamin B. J. Biol. Chem. 77, 231-240.

Evans, H. M. and Hoagland, D. R.

1927. The synthesis of vitamin $\mathrm{E}$ by plants grown in culture solutions. Am. J. Physiol. 80, 702-704.

Evans, H. M. and Lepkovsky, S.

1928. Sparing action of fat on the antineuritic vitamin. Science 68, 298.

1929. Sparing action of fat on the antineuritic vitamin B. J. Biol. Chem. 83, 269-287.

a. Technique for determination of the antineuritic vitamin B. J. Nutrition $2,1-5$.

Evers, N.

1929. Some observations on the antimony trichloride colour test for vitamin A. Quart. J. Pharm. 2, 227-237.

a. The permanence of vitamin A in cod-liver oil as shown by the colour test. Quart. J. Pharm. 2, 556-565.

b. Variations in the results obtained by different observers with the antimony trichloride colour test for cod-liver oil. Quart. J. Pharm. 2, 566-569.

Evers, N. and Foster, H. J.

1923. Note on the sulphuric acid test for fish liver oils. Analyst 48, 58-60.

Faber, H. K.

1920. A study of the antiscorbutic value of honey. J. Biol. Chem. 43, 113-116.

1921. Infantile scurvy following the use of sodium citrate as a modifier of milk. Am. J. Diseases Children 21, 401-405. 
Fabre, R. and Simonnet, $\mathrm{H}$.

1928. Contribution a l'étude des stérols irradiés. Compt. rend. soc. biol. 99, 193-195.

a. L'irradiation des stérols, les rapports des stérols irradiés avec la vitamine antirachitique. J. pharm. chim. 8, 489-506.

1929. Etude comparative de la valeur de l'essai biologique et de l'essai physique de l'ergostérol irradié. Compt. rend. 188, 424-426.

a. Etude physique et biologique du stérol dextrogyre isolé de la levure de bière. Compt. rend. 188, 1312-1315.

Faerber, E. and Telleria, T.

1923. Die Atmung des kindlichen Blutes und ihre Beeinflussung durch Bvitamin (Hefeautolysat). Klin. Wochschr. 2, 2161-2163.

Fairhall, L. T.

1928. Calcium and ultra violet irradiation. A. The effect of ultra violet radiation upon serum calcium. B. Calcium utilization on a calcium-poor diet with ultra violet radiation. Am. J. Physiol. 84, 378-385.

Falconer, E. H. and Peachey, G.

1926. Blood counts in vitamin-A deficiency disease with especial reference to the platelets. Am. J. Physiol. 76, 145-150.

Falkenheim, C.

1927. Zur Therapie der Säuglingsrachitis mit aktiven Ergosterin-präparaten (Vigantol). Deut. med. Wochschr. 53, 1550-1552.

Farmer, C. J. and Redenbaugh, H. E.

1925. A study of heat production in pigeons on diets deficient in vitamin $B$. Am. J. Physiol. 75, 27-44.

a. The decrease in digestive efficiency in Polyneuritis columbarum. Am. J. Physiol. 75, 45-51.

\section{Farnum, M. B.}

1926. Gastric secretion in experimental beriberi in the dog. Arch. Internal Med. 37, 212-216.

Fearon, W. R.

1925. A study of some biochemical colour tests. III. Colour reactions associated with vitamin A. Biochem. J. 19, 888-895.

\section{Fernando, A. S.}

1924. Laryngeal symptoms in beriberi. Philippine J. Sci. 24, 41-43.

Ferry, E. L.

1920. Nutrition experiments with rats-A description of methods and technique. J. Lab. Clin. Med. 5, 735-745.

Findlay, G. M.

1920. A study of the leucocyte changes in pellagra compared with those occurring in beriberi. J. Path. Bact. 23, 490-498.

1921. Glyoxalase in avian beriberi. Biochem. J. 15, 104-106.

a. An experimental study of avian beriberi. J. Path. Bact. 24, 175-191.

b. The effects of an unbalanced diet in the production of guinea-pig scurvy. Biochem. J. 15, 355-357.

c. The blood and blood vessels in guinea pig scurvy. J. Path. Bact. 24, 446-453.

d. Experimental scurvy in the rabbit; effects of antenatal nutrition. $J$. Path. Bact. 24, 454, 455; J. Am. Med. Assacn. 77, 1604, 1605.

1922. B vitamin and pneumococcal infection. Lancet, 1922, I, 714, 715. 
1923. The relation of vitamin C to bacterial infection. J. Path. Bact. 26, 1-18. a. The relation of deprivation of vitamin $B$ to body temperature and bacterial infection. J. Path. Bact. 26, 485-495.

b. The antiscorbutic vitamin and its dual rôle in nutrition. Nation's Health 5, 589, 590.

c. A preliminary note on the destruction of vitamin B by age. Biochem. J. $17,887-890$.

1925. A contribution to the etiology of experimental keratomalacia. Brit. J. Exptl. Path. 6, 16-21.

1928. Pellagra-like lesions associated with deficiency of vitamin $B_{2}$ in the rat. J. Path. Bact. 31, 353-364.

Findlay, G. M. and Mackenzie, R.

1922. Opsonins and diets deficient in vitamins. Biochem. J. 16, 574-577.

a. The bone marrow in deficiency diseases. J. Path. Bact. 25, 402, 403.

Findlay, G. M. and Maclean, I.

1925. The bactericidal action of the blood in certain dietary deficiencies. Biochem. J. 19, 63-70.

Findlay, L.

1924. The underlying cause in the pathogenesis of rickets. J. Am. Med. Assocn. 83, 1473-1479.

Findlay, L., Paton, D. N. and Sharpe, J. S.

1921. Studies in the metabolism of rickets. I. Is rickets due to a deficient supply of calcium in the bones? II. The calcium balance in normal and rachitic children. III. Quart. J. Med. 14, 352-361; 362-397.

Finks, A. J. and Johns, C. O.

1921. Studies in nutrition. VIII. The nutritive value of the protein of tomato-seed press cake. Am. J. Physiol. 56, 404-407.

Finks, A. J. and Jones, D. B.

1923. 'Growth-promoting value of the proteins of the palm kernel and the vitamin content of palm kernel meal. J. Agr. Research 25, 165-169.

Firth, J. B. and Russell, W. K.

1927. The influence of ultraviolet radiation on calcium and phosphorus metabolism in various diseases. Brit. J. Actinotherapy 2, 42.

\section{Fischer, L.}

1923. A study of clinical rickets. Comparison of results obtained on exposure to sunlight and on treatment with cod liver oil or an active concentrate prepared from cod liver oil. J. Metabolic Research 4, 481-489.

1924. Clinical results in cases of rickets treated with an active concentrate prepared from cod liver oil. Proc. Soc. Exptl. Biol. Med. 21, 461, 462.

Fleming, W. D.

1923. Metabolic mechanism in beriberi. Philippine J. Sci. 23, 407-411.

1928. The antirachitic efficiency of winter sunlight of Washington, D. C. Military Surg. 62, 592-609.

a. The anti-rachitic efficiency of skyshine in Washington, D. C. Military Surg. 62, 658-672.

Flesch, M.

1928. Kraft und Stoff. Erkenntnis theoretisches zur Vitaminforschung. Fortschritte Med. 46, 1037-1038. 
Flury, F.

1928. Zur Frage der Standardisierung des Vitamins D in Butter und vitaminhaltiger Margarine. Biochem. Z. 203, 14-21.

\section{Foote, J. A.}

1927. Evidence of rickets prior to 1650 . Am. J. Diseases Children 34, 443452.

Fosbinder, R. J., Daniels, F. and Steenbock, H.

1928. A quantitative study of the photochemical activation of sterols in the cure of rickets. J. Am. Chem. Soc. 50, 923-927.

Fränkel, S. and Hager, J.

1922. Über Vitamine. II. Über die Gärbeschleunigung durch Extrakte tierische Organe. Biochem. Z. 126, 189-226.

Fränkel, S. and Scharf, A.

1922. Über Vitamine. III. Über gärungsbeschleunigende Extrakte aus Pflanzen und über die Wirkung von Cholin und Aminoäthylalkohol auf die Gärung. Biochem. Z. 126, 227-264.

a. Über Vitamine. IV. Versuche über die Adsorption der Vitamine. Biochem. Z. 126, 265-268.

b. Über Vitamine. V. Weitere Versuche über die Chemie der Vitamine. Biochem. Z. 126, 269-280.

Fränkel, S. and Schwartz, E.

1920. Über wasserlösliche Vitamine und gärungsbeschleunigende Substanz aus Hefe und Reiskleie. Biochem. Z. 112, 203-235.

Francis, E. and Dutcher, R. A.

1924. The storage of vitamin B in the albino rat. Science 59, 20.

Francis, L. D., Smith, A. H. and Moise, T. S.

1929. Relation of vitamin $B\left(B_{1}, F\right)$ and $G\left(B_{2}\right)$ to renal enlargement in rats fed high concentrations of protein. Proc. Soc. Exptl. Biol. Med. 26, $725,726$.

Frank, A.

1925. Über den Gehalt der Milch an skorbutverhütenden Stoffen. Klin. Wochschr. 4, 1204-1208.

\section{Frank, M.}

1920. Beitrag zur Klinik und zum Stoffwechsel der Möller-Barlowschen Krankheit. Jahrb. Kinderheilk. 91, 21-42.

Fraser, A. C.

1929. Influence de l'ergostérol irradié sur les globules blancs du sang chez le lapin. Compt. rend. soc. biol. 103, 461, 462.

Fraser, H. and Stanton, A. T.

1909. An inquiry concerning the etiology of beriberi. Studies Inst. Med. Research, Fed. Malay States, No. 10; see also Lancet, 1909, I, 451-455.

1910. The etiology of beriberi. Trans. Soc. Trop. Med. Hyg. 1910, III; Lancet, 1910, II, 1755-1757.

a. The etiology of beriberi. Philippine J. Sci. Sect. B, 5, 55-61.

1911. The etiology of beriberi. Studies Inst. Med. Research Fed. Malay States, No. 12 ; J. Trop. Med. Hyg. 14, 333-341; 349-358; 365-372.

1912. The prevention and cure of beriberi Lancet, 1912, II, 1005-1007.

1914. Unpolished rice and the prevention of beri-beri. Lancet, 1914, I, 96-98.

1915. The chemistry of rice polishings. Lancet, 1915, I, 1021, 1022. 
1924. "Collected Papers on Beriberi." John Bale Sons \& Danielson, Ltd., London.

Freedman, L. and Funk, C.

1922. Nutritional factors in the growth of yeasts and bacteria. I. Vitamins.

II. Protein hydrolysates. J. Metabolic Research 1, 457-468; 469-480.

Freise, E.

1920. Experimentelle Untersuchungen über die Ernahrungs-bedingunen des

Wachstums. Jahrb. Kinderheilk. 91, 79-127.

Freise, E., Goldschmidt, M. and Frank, A.

1915. Experimentelle Beiträge zur Aetiologie der Keratomalazie. Monatschr.

Kinderheilk. 13, 424, 430.

Freudenberg, E. and György, P.

1920. Beitrag zu den biologischen Wirkungen der akzessorischen Nährstoffe. Münch. med. Wochschr. 67, 1061, 1062.

Freudenthal, $\mathrm{P}$.

1927. Rachitisme expérimental. Etiologie du rachitisme. Comp. rend. soc. biol. 97, 1255-1258.

a. Rachitisme expérimental. Importance d'un contenu adéquat en sels, dans un régime alimentaire dont la teneur en vitamine A est variable. Comp. rend. soc. biol. 97, 1259-1262.

b. Rachitisme expérimental. Altérations anatomopathologiques causées par l'avitaminose A. Compt. rend. soc. biol. 97, 1263-1266.

Fridericia, L. S.

1924. Inactivating action of some fats on vitamin A in other fats. J. Biol. Chem. 62, 471-485.

Fridericia, L. S., Freudenthal, P., Gudjonnsson, S., Johansen, G. and Schoubye, N.

1927. Refection, a transmissible change in the intestinal content, enabling rats to grow and thrive without vitamin B in the food. J. Hyg. 27, 70-102.

Fridericia, L. S. and Holm, E.

1925. Experimental contribution to the study of the relation between night blindness and malnutrition. Influence of deficiency of fat-soluble A vitamin in the diet on the visual purple in the eyes of rats. Am. J. Physiol. 73, 63-78.

Fries, M. E.

1927. Effect of therapeutic doses of ultraviolet radiation on basal metabolism in children. I. Am. J. Diseases Children 34, 159-165.

Fries, M. E. and Topper, A.

1927. Effect of therapeutic doses of ultraviolet radiation on basal metabolism in children. II. Am. J. Diseases Children 34, 166-175.

Frölich, T.

1912. Experimentelle Untersuchungen über den infantilen Skorbut. Z. Hyg. Infektionskrankh. 72, 155-182.

Frost, A. W.

1925. The relation of exercise to rickets in white rats. Science 61, 447, 448.

Fürst, V.

1910. Cause of scurvy. Nord. Med. Arch. Abt. II. 1910 Supplement, 349; Chem. Abstracts 5, 1308.

1912. Weitere Beiträge zur Ätiologie des experimentellen Skorbuts des Meerschweinchens. Z. Hyg. Infektionskrankh. 72, 121-154. 
Funk, C.

1911. The chemical nature of the substance which cures polyneuritis in birds induced by a diet of polished rice. J. Physiol. 43, 395-400.

1912. The effect of a diet of polished rice on the nitrogen and phosphorus of the brain. J. Physiol. 44, 50-53.

a. Preparation from yeast and certain foodstuffs of the substance the deficiency of which in the diet causes polyneuritis in birds. J. Physiol. 45, $75-81$.

b. The substance from yeast and certain foodstuffs which prevents polyneuritis (beriberi). Brit. Med. J. No. 2700, 787, 788.

c. The etiology of the deficiency disease. J. State Med. 20, 341-368.

1913. An attempt to estimate the vitamin fraction in milk. Biochem. J. 7, 211-213.

a. Further experimental studies in beriberi. The action of certain purine and pyrimidine derivatives. J. Physiol. 45, 489-492.

b. Studies on beriberi-further facts concerning the chemistry of the vitamin fraction of yeast. Brit. Med. J. No. 2729, 814.

c. Studies on beriberi. VII. Chemistry of the vitamin-fraction from yeast and rice polishings. J. Physiol. 46, 173-179.

d. Studies on pellagra. I. The influence of the milling of maize on the chemical composition and the nutritive value of maize-meal. J. Physiol. 47, 389-392.

e. Studies on growth. I. Growth with food containing vitamin and with vitamin-free food. Z. phy'siol. Chem. 88, 352-356.

1914. "Die Vitamine," Wiesbaden, J. F. Bergmann.

a. Is polished rice plus vitamin a complete food? J. Physiol. 48, 228-232.

b. Studies on growth. The influence of diet on growth, normal and malignant. Lancet, 1914, I, 98-102.

c. Studien über Beriberi XI. Die Rolle der Vitamine beim Kohlenhydratstoffwechsel. Z. physiol. Chem. 89, 378-380.

1915. Results of studies on vitamins and deficiency diseases during the years 1913-1915. Biochem. Bull. 4, 304-364.

1916. The nature of the disease due to the exclusive diet of oats in guinea pigs and rabbits. J. Biol. Chem. 25, 409-416.

a. Fractionation of the phosphotungstic acid precipitate with acetone as a useful method for the preparation of the vitamin fraction from yeast. Biochem. Bull. 5, 1-16.

b. The study of certain dietary conditions bearing on the problem of growth in rats. J. Biol. Chem. 27, 1-14.

1919. Action of substance influencing carbohydrate metabolism in experimental beriberi. J. Physiol. 53, 247-256.

1921. The antiberiberi vitamin. J. Ind. Eng. Chem. 13, 1110, 1111.

1922. "The Vitamines," Baltimore, Williams \& Wilkins Co.

1925. The nomenclature of the vitamines. Science 62, 157, 158.

1927. New series of reagents useful in fractionation of biologically active material. Proc. Soc. Exptl. Biol. Med. 25, 133, 134.

Funk, C. and Collazo, J. A.

1925. Die Zusammensetzung der Nahrung unter der Vitaminbedarf. Chem. Zelle Gewebe. 12, 195-202. 
Funk, C., Collazo, J. A. and Kaczmarek, J.

1925. Composition du régime et vitamine B. Compt. rend. soc. biol. 92, 997-998.

Funk, C. and Douglas, M.

1914. Studies on beriberi. VIII. The relationship of beriberi to glands of internal secretion. J. Physiol. 47, 475-478.

Funk, C. and Dubin, H. E.

1920. A test for antiberiberi vitamin and its practical application. J. Biol. Chem. 44, 487-498.

a. The vitamin requirements of the rat on diets rich in protein, carbohydrate, and fat respectively. Science 52, 447, 448.

1921. Vitamine requirements of certain yeasts and bacteria. J. Biol. Chem. 48, 437-443.

Funk, C. and Freedman, L.

1922. Requirements in the growth of yeasts and bacteria. I. Vitamins. $J$. Metabolic Research 1, 457-468.

1923. Can yeast grow in a chemically pure medium? Proc. Soc. Exptl. Biol. Med. 20, 311-313.

a. The presence of an yeast growth-promoting vitamin in cane sugar. $J$. Biol. Chem. 56, 851-860.

Funk, C., Harrow, B. and Paton, J. B.

1923. Extraction of vitamins from yeast and rice polishings with various water-miscible solvents. J. Biol. Chom. 57, 153-167.

Funk, C. and Lecoq, R.

1927. Note sur différents extraits de levure et sur leur teneur en vitamine $\mathrm{D}$ rapportée à la proportion de levure fraiche initiale utilisée pour leur préparation. Compt. rend. soc. biol. 97, 440-442.

Funk, C. and Levy, A.

1923. Mineral metabolism in rats under the influence of $B$ and $D$ vitamins. J. Metabolic Research 4, 453-459.

Funk, C. and Macallum, A. B.

1914. Die chemischen Determinanten des Wachstums. Z. physiol. Chem. 92, 13-20.

1915. Studies on growth. II. On the probable nature of the substance promoting growth in young animals. J. Biol. Chem. 23, 413-421.

1916. Studies on growth. III. The comparative value of lard and butter fat in growth. J. Biol. Chem. 27, 51-62.

a. Studies on growth. IV. The action of yeast fractions on the growth of rats. J. Biol. Chem. 27, 63-70.

Funk, C. and Paton, J. B.

1922. Studies on vitamins B and D. J. Mctabolic Research 1, 737-775.

Funk, C. and Poklop, J.

1916. The study of certain dietary conditions bearing on the problem of growth. J. Biol. Chem. 27, 1-14.

Funk, C. and Schönborn, E. von

1914. The influence of a vitamin-free diet on the carbohydrate metabolism. J. Physiol. 48, 328-331.

Gargill, S. L., Gilligan, D. R. and Blumgart, H. L.

1930. Metabolism and treatment of osteomalacia: Its relation to rickets. Arch. Internal Med. 45, 879-907. 
Gebhart, J. C.

1924. Preventing rickets in an Italian district in New York City. Am. J.Pub. Health 14, 571.

Georgia Agr. Expt. Sta.

1926. A study of the vitamin content of widely used Georgia foods. Ann. Rept., pp. 152-154.

1927. The vitamin content of Georgia grown foods. Ann. Rept., p. 28.

1928. Vitamin investigations. Ann. Rept., pp. 33, 34.

1929. Vitamin investigations. Ann. Rept., pp. 25-27.

Gerstenberger, H. J.

1921. Malt soup extract as an antiscorbutic. Am. J. Diseases Children 21, 315-326.

1927. Rickets: A brief summary with reflections. J. Am. Med. Assocn. 89, 261-263.

1929. Ultra-violet ray therapy and public health clinics. Am. J. Pub. Health 19, 193-196.

Gerstenberger, H. J., Champion, W. M. and Smith, D. N.

1924. The effect of pregnancy on the course of scurvy in guinea pigs. Am. J. Diseases Children 28, 173-182.

Gerstenberger, H. J. and Hartman, J. I.

1929. Quartz lamp therapy in human rickets and rachitic spasmophilia. J. Am. Med. Assocn. 92, 367, 368.

Gerstenberger, H. J. and Nourse, J. D.

1926. The prevention of rickets in premature infants. J. Am. Med. Assocn. 87, 1108-1114.

Gerstenberger, H. J. and Russell, G. R.

1930. Sunlight type S-1 lamp (G. E.) therapy in human rickets and rachitic spasmophilia. J. Am. Med. Assocn. 94, 1049, 1050.

Ghose, S. N.

1922. The examination of some Indian foodstuffs for their vitamin content. Biochem. J. 16, 35-41.

\section{Giaja, A.}

1927. Etude de la valeur alimentaire des noyaux de pruneaux. Leur teneur en vitamines. Bull. soc. hyg. aliment. 15, 517-523.

Giaume, C.

1929. Azione antirachitica del sangue di animali irradiati con R. U. V. Ricerche sperimentali. Pediatra 37, 119-130.

Gibbons, R. and Barney, C. B.

1930. The vitamin A content of the liver of normal hogs and of hogs developing experimental rickets. J. Home Econ. 22, 491-496.

Gibson, R. B.

1913. The influence of compensated salt mixtures on the development of polyneuritis gallinarum and beri-beri. Philippine $J$. Sci. Sec. B 8, 351-367.

a. The protective power of normal human milk against polyneuritis gallinarum (beri-beri). Philippine J. Sci. Sect. B 8, 469-471.

Gibson, R. B. and Concepcion, I.

1914. Nerve degeneration in fowls fed on unhusked rice (Palay). Philippine J. Sci. Sect. B 9, 119-123.

1916. The influence of fresh and autoclaved cows' milk on the development of neuritis in animals. Philippine J. Sci. Sect. B 11, 119-133. 
Gillam, A. E. and Morton, R. A.

1927. The formation of nitrite from nitrate as a measure of ultra-violet intensity. J. Soc. Chem. Ind. 46, 415T-417T.

a. A comparison of certain methods for determining the ultra-violet intensity of a light source. J. Soc. Chem. Ind. 46, 417T-420T.

Gillern, K., Hussa, V. and Schirmann, M. A.

1928. Ultraviolett bestrahlte Milch als Antirachitikum. Wien. med. Wochschr. 78, 793-802.

Givens, M. H. and Cohen, B.

1918. The antiscorbutic property of desiccated and cooked vegetables. J. Biol. Chem. 36, 127-145.

Givens, M. H. and Macy, I. G.

1921. The antiscorbutic property of some desiccated fruit juices. J. Biol. Chem. 46, XI, XII.

Givens, M. H. and McClugage, H. B.

1919. Antiscorbutic property of vegetables. I. An experimental study of raw and dried tomatoes. J. Biol. Chem. 37, 253-269.

a. The antiscorbutic property of fruits. I. An experimental study of dried orange juice. Am. J. Diseases Children 18, 30-41.

1920. The antiscorbutic property of dehydrated meat. Sciences 51, 273275.

a. Antiscorbutic property of vegetables. II. An experimental study of raw and dried potatoes. J. Biol. Chem. 42, 491-515.

1921. The effect of heat and age upon the antiscorbutic vitamin in tomatoes. Proc. Soc. Exptl. Biol. Med. 18, 164.

Givens, M. H., McClugage, H. B. and Van Horne, E. G.

1921. The antiscorbutic property of raw, dried, and cooked apples and bananas. Proc. Soc. Exptl. Biol. Med. 18, 140, 141.

1922. The antiscorbutic property of fruits. II. An experimental study of apples and bananas. Am. J. Diseases Children 23, 210-225.

Glassmann, B. and Posdeew, A.

1929. Über den chemischen Nachweis des Vitamins C. Z. Untersuch. Lebensm. 57, 191-200.

Glenny, A. T. and Allen, A.

1921. A guinea pig epizootic associated with dietary deficiency. Lancet, 1921, II, 1109.

Godlewski, H.

1921. Carence partielle et préscorbut. Presse méd. 29, 682, 683.

Goebel, F.

1929. Influence de l'ergostérine activée sur le développement des jeunes animaux. Compt. rend. soc. biol. 100, 1155, 1156.

Goettsch, M.

1928. The determination of the antiscorbutic value of foodstuffs by Höjer's method. Quart. J. Pharm. 1, 168-177.

1929. Relationship between vitamin $C$ and oestrus in the guinea pig and the fertilizing power of sperm. Proc. Soc. Exptl. Biol. Med. 27, 71, 72.

1930. The dietary production of dystrophy of the voluntary muscles. Proc. Soc. Exptl. Biol. Med. 27, 564-567. 
Goldberg, B.

1929. An apparatus for mass treatment of children with ultraviolet rays. $J$. Am. Med. Assocn. 93, 1377, 1378.

\section{Goldberger, J.}

1914. The etiology of pellagra. U. S. Pub. Health Repts. 29, 1683-1686.

a. The cause and prevention of pellagra. U. S. Pub. Health Repts. 29, 2354-2357.

1916. Pellagra: Causation and method of prevention. A summary of some of the recent studies of the U. S. Public Health Service. J. Am. Med. Assocn. $66,471-476$.

a. The transmissibility of pellagra. Experimental attempts at transmission to the human subject. U. S. Pub. Health Repts. 31, 3159-3173.

1918. Pellagra.-Its nature and prevention. U. S. Pub. Health Repts. 33, 481-488.

1922. The relation of diet to pellagra. J. Am. Med. Assocn. 78, 1676-1680.

1926. The present status of our knowledge of the etiology of pellagra. Medicine 5, 79-104.

1927. Pellagra. Its nature and prevention. U. S. Pub. Health Repts. 42, 2193-2200.

1929. Pellagra. J. Am. Dietetic Assocn. 4, 221-227.

Goldberger, J. and Lillie, R. D.

1926. A note on an experimental pellagra-like condition in the albino rat. U. S. Pub. Health Repts. 41, 1025-1029.

Goldberger, J. and Sydenstricker, E.

1927. Pellagra in the Mississippi flood area. U. S. Pub. Health Repts. 42, 2706-2725.

Goldberger, J. and Tanner, W. F.

1922. Amino acid deficiency probably the primary etiological factor in pellagra. U. S. Pub. Health Repts. 37, 462-486.

a. An amino acid deficiency as the primary etiologic factor in pellagra. $J$. Am. Med. Assocn. 79, 2132-2135.

1924. A study of the treatment and prevention of pellagra. U. S. Pub. Health Repts. 39, 87-107.

1925. A study of the pellagra-preventive action of dried beans, casein, dried milk and brewers' yeast with a consideration of the essential preventive factors involved. U. S. Pub. Health Repts. 40, 54-80.

Goldberger, J., Waring, C. H. and Tanner, W. F.

1923. Pellagra prevention by diet among institutional inmates. U.S. Pub. Health Repts. 38, 2361-2368.

Goldberger, J., Waring, C. H. and Willets, D. G.

1914. The treatment and prevention of pellagra. U. S. Pub. Health Repts. 29, 2821-2825.

1915. The prevention of pellagra. A test of diet among institutional inmates. U. S. Pub. Health Repts. 30,3117-3131.

Goldberger, J. and Wheeler, G. A.

1915. Experimental pellagra in the human subject brought about by a restricted diet. U. S. Pub. Health Repts. 30, 3336-3339.

1920. Experimental pellagra in white male convicts. Arch. Internal Med. 25, $451-471$. 
a. The experimental production of pellagra in human subjects by means of diet. U. S. Pub. Health Service. Hyg. Lab. Bull. No. 120, 7-116.

1927. A study of the pellagra-preventive action of the tomato, carrot, and rutabaga turnip. U. S. Pub. Health Repts. 42, 1299-1306.

a. A study of the pellagra-preventive action of the cowpea (Vigna sincnsis) and of commercial wheat germ. U. S. Pub. Health Repts. 42, 2383-2391.

1928. Experimental black tongue of dogs and its relation to pellagra. U. S. Pub. Health Repts. 43, 172-217.

1929. A study of the pellagra-preventive action of canned salmon. U. S. Pub. Health Repts. 44, 2769-2771.

Goldberger, J., Wheeler, G. A., Lillie, R. D. and Rogers, L. M.

1926. A further study of butter, fresh beef, and yeast as pellagra-preventives, with consideration of the relation of factor P-P of pellagra (and blacktongue of dogs) to vitamin B. U. S. Pub. Health Repts. 41, 297-318.

1928. A study of the blacktongue preventive action of 16 foodstuffs with special reference to the identity of blacktongue of dogs and pellagra of man. $U . S$. Pub. Health Repts. 43, 1385-1454.

Goldberger, J., Wheeler, G. A., Rogers, L. M. and Sebrell, W. H.

1930. A study of the blacktongue preventive value of leached commercial casein, together with a test of the blacktongue preventive action of a high protein diet. U. S. Pub. Health Repts. 45, 273-282.

a. A study of the blacktongue preventive value of lard, salt pork, dried green peas, and canned haddock. U. S. Pub. Health Repts. 45, 1297-1308.

Goldberger, J., Wheeler, G. A. and Sydenstricker, E.

1918. A study of the diet of non-pellagrous and of pellagrous households in textile mill communities in South Carolina in 1916. J. Am. Med. Assocn. 71, 944-949.

1920. A study of the relation of diet to pellagra incidence in seven textile mill communities of South Carolina in 1916. U. S. Pub. Health Repts. 35, 648-714.

a. Pellagra incidence in relation to sex, age, season, occupation, and "disabling sickness" in seven cotton-mill villages of South Carolina during 1916. U. S. Pub. Health Repts. 35, 1650-1654.

b. A study of the relation of factors of a sanitary character to pellagra incidence in seven cotton-mill villages of South Carolina in 1916. U. S. Pub. Health Repts. 35, 1701-1714.

c. A study of the relation of family income and other economic factors to pellagra incidence in seven cotton-mill villages of South Carolina in 1916. U. S. Pub. Health Repts. 35, 2673-2714.

\section{Goldblatt, $\mathrm{H}$.}

1923. A study of the relation of the quantity of fat-soluble organic factor in the diet to the degree of calcification of the bones and the development of experimental rickets in rats. Biochem. J. 17, 298-326.

1924. Experimental rickets in rats on a purified synthetic diet deficient in phosphorus and fat-soluble organic factor. Biochem. J. 18, 414-418.

Goldblatt, $H$. and Benischek, M.

1927. Vitamin A deficiency and metaplasia. J. Exptl. Med. 46, 699-707.

Goldblatt, H. and Moritz, A. R.

1925. Experimental rickets in rabbits. J. Exptl. Med. 42, 499-506.

1926. On the growth-promoting property of irradiated fat in the diet, of direct irradiation, and of cod-liver oil. J. Biol. Chem. 71, 127-137. 
1927. The effect of heat and oxidation on the nutritive value of a protein.

J. Biol. Chem. 72, 321-326.

Goldblatt, H. and Soames, K. M.

1922. The effect of radiation with the mercury vapor quartz lamp on the growth of rats fed on a diet deficient in the fat-soluble growth-promoting factor. Lancet, 1922, II, 1321-1324.

1923. A study of rats on a normal diet irradiated daily by the mercury vapor quartz lamp or kept in darkness. Biochem. J. 17, 294-297.

a. Studies on the fat-soluble growth-promoting factor. Biochem. J. 17, 446-453.

b. The supplementary value of light rays to a diet graded in its content of fat-soluble organic factor. Biochem. J. 17, 622-629.

Goldblatt, H. and Zilva, S. S.

1923. The relation between the growth-promoting and antirachitic functions of certain substances. Lancet, 1923, II, 647-649.

Golding, J., Soames, K. M., and Zilva, S. S.

1926. The influence of the cow's diet on the fat-soluble vitamins of winter milk. Biochem. J. 20, 1306-1319.

Golding, J. and Zilva, S. S.

1928. The influence of the cow's diet on the fat-soluble vitamins of winter milk.

II. Biochem. J. 22, 173-182.

Golding, J., Zilva, S. S., Drummond, J. C. and Coward, K. H.

1922. The relation of the fat-soluble factor to rickets and growth in pigs. II. Biochem. J. 16, 394-402.

Goodale, H. D.

1926. Early growth rates of chickens with special reference to ultra-violet light. Am. J. Physiol. 79, 44-60.

Goodman, H. and Anderson, W. T., Jr.

1928. Quantitative study of clinical ultraviolet sources. J. Am. Med. Assocn. 90, 671-673.

Gortner, R. A., Palmer, L. S. and Dahl, S. J.

1925. A test of indolinones as agents for prevention and cure of polyneuritis. Proc. Soc. Exptl. Biol. Med. 23, 231-234.

Goss, H.

1925. The antiscorbutic value of commercially concentrated orange juice. Hilgardia [Calif. Sta.] 1, 15-34.

Gotza, H.

1923. Vitamine B et glandes sexuelles. Compt. rend. soc. biol. 88, 373-375.

Gowen, J. W., Murray, J. M., Gooch, M. E. and Ames, F. B.

1926. Rickets, ultra-violet light and milk. Science 63, 97, 98.

Goy, P.

1921. Les végétaux inférieurs et les facteurs accessoires de la croissance. Compt. rend. 172, 242-244.

1922. Physiologie microbienne et facteur accessoire de la croissance. Compt. rend. $174,1579,1580$.

1925. Microbes et vitamines. Ann. Inst. Pasteur 39, 183-195.

Graham, C. E. and Griffith, W. H.

1929. Studies on growth.-Growth factors in liver. Proc. Soc. Exptl. Biol. Med. 26, 715-717. 
Gralka, R. and Aron, $\mathrm{H}$.

1922. Die akzessorischen Nährstoffaktoren. II. Die Bedeutung des Gehaltes an wasserlöslichen Extraktstoffen. Biochem. Z. 126, 147-152.

Grant, A. H.

1926. Effect of the calcium, vitamin C, vitamin D ratio in diet on the permeability of intestinal wall to bacteria. J. Infect. Diseases 39, 502-508.

a. The nutritional requirements of nursing mothers-The effect of lowering both the antirachitic vitamin and calcium in the diet of the mother upon the development of rickets in the young. Am. J. Hyg. 6, 228-237.

1930. The effect of rachitic diets on experimental tuberculosis. III. Resistance to tuberculosis decreased by adding cod-liver oil. IV. Effects of disturbing optimal ratio between calcium, vitamin $\mathrm{C}$ and vitamin D. Am. Rev. Tuberculosis 21, 102-114, 115-126.

Grant, A. H., Bowen, J. A. and Stegeman, D. E.

1928. The effect of rachitic diets on experimental tuberculosis in white rats. II. Vitamine D deficiency as a factor in lowering resistance. Am. Rev. Tuberculosis 16, 642-654.

Grant, A. H. and Goettsch, M.

1926. The nutritional requirements of nursing mothers-The effect of a deficiency of the antirachitic vitamin only, in the diet of the mothers upon the development of rickets in the young. Am. J. Hyg. 6, 211-227.

Grant, A. H., Suyenaga, B., and Stegeman, D. E.

1928. The effect of rachitic diets on experimental tuberculosis in white rats. I. Calcium and vitamine-D deficiencies as factors in lowering resistance. Am. Rev. Tuberculosis 16, 628-641.

Grant, J. H. B. and Gates, F. L.

1924. A preliminary survey of the effects of ultraviolet light on normal rabbits. Proc. Soc. Exptl. Biol. Med. 21, 230-232.

1925. Some factors affecting the levels of the serum calcium and phosphorus of normal rabbits. Proc. Soc. Exptl. Biol. Med. 22, 315-317.

Grayzel, D. M. and Miller, E. G., Jr.

1928. The $\mathrm{pH}$ of the contents of the gastrointestinal tract in dogs, in relation to diet and rickets. J. Biol. Chem. 76, 423-436.

Green, H. H.

1918. Experimental expression of the relationship between the content of a foodstuff in antineuritic hormone and the period of healthy survival of animals upon it. S. African J. Sci. 14, 483, 484.

a. The vitamin content of maize and maize milling products, and the ambiguity of its correlation with the phosphoric oxide content. S. African J. Sci. 14, 519, 520.

b. The deficiency aspect of maize products. Repts. Dir. Vet. Research Union S. Africa 5-6, 753-774.

c. Upon the quantitative relationship between the antineuritic value of a diet and the onset of polyneuritis. Repts. Dir. Vet. Research Union $S$. Africa 5-6, 777-812.

Green, H. N. and Mellanby, E.

1928. A rat technique for demonstrating the interfering effect of cereals on bone calcification. Biochem. J. 22, 108-112.

a. Vitamin $\mathrm{A}$ as an anti-infective agent. Brit. Med. J. No. 3537, 691-696. 
1930. Carotene and vitamin A. The anti-infective action of carotene. Brit. $J$.

Exptl. Path. 11, 81-89.

Greenbank, G. R. and Holm, G. E.

1925. Measurement of susceptibility of fats to oxidation. Ind. Eng. Chem. 17, 624.

Greenbaum, F. R.

1929. Irradiated ergosterol, the modern antirachitic principle. Am. J. Pharm. 101, 417-425.

Greenebaum, J. W., Selkirk, T. K., Otis, F. A. and Mitchell, A. G.

1926. Effects of diet during pregnancy on development of rickets in the offspring. J. Am. Med. Assocn. 87, 1973-1976.

Greenman, M. J. and Duhring, F. L.

1924. "Breeding and Care of the Albino Rat for Research Purposes." Philadelphia, Wistar Institute.

Greenwald, I. and Gross, J.

1929. The prevention of the tetany of parathyroidectomized dogs. I. Cod liver oil, with a note on the effect of cod liver oil on calcium assimilation. II. Lactose-containing diets. J. Biol. Chem. 82, 505-530; 531-544.

Greig, E. D. W.

1917. The sprouting capacity of grains (lentils and peas) issued as rations to troops. Indian J. Med. Research 4, 818-823.

1918. Report on the anti-beri-beri vitamin content of ground-nut meal biscuits. Indian J. Med. Research 6, 143-146.

Greig, E. D. W. and Curjel, D. F.

1918. Report on the anti-beri-beri vitamin content of three kinds of atta biscuits. Indian J. Med. Research 6, 56-67.

Grettie, D. P. and King, C. G.

1929. The preparation and properties of vitamin $C$ concentrates from lemon juice. J. Biol. Chem. 84, 771-776.

Grey, E. C.

1928. The pre-beriberi condition, with special reference to its existence in Japan. J. Hyg. 27, 257-267.

a. Studies in the nutrition of birds. I. Observations on the nutrition of tame and wild Egyptian pigeons. II. Observations on the rate of growth and changes in the organs of wild Egyptian pigeons, fed with various diets. III. A consideration of various factors which protect pigeons against the harmful effects of an exclusively cereal diet, and especially of polished rice. J. Hyg. 27, 268-294.

Grieves, C. J.

1922. The effect of defective diets on teeth. The relation of calcium, phosphorus and organic factors to caries-like and attaching-tissue defects. J. Am. Med. Assocn. 79, 1567-1573.

Griffith, H. D. and Spence, K. C.

1928. The photosynthesis of the antirachitic vitamin by ultra-violet radiation of short wave-lengths. Brit. J. Actinotherapy 3, 69-72.

Grijns, G.

1901. Polyneuritis gallinarum. Genneskund. Tijdschr. Nederland.-Indië 41, 3.

1925. Diet and reproduction. Koninklijke Akad. Wetensch. Amsterdam Proc. 28, 942-952. 
1927. Beiträge zur Geschichte der Erkennung der Beriberi als Avitaminose. Fortschritte Naturw. Forsch. N. Ser. I, pp. 32.

Grijns, G. and Haan, K. de.

1926. Diet and reproduction. II. Koninklijke Akad. Wetensch. Ansterdam Proc. 29, 873-877.

Grijns, G., Haan, K. de and Loeff, J. A. van dy.

1928. Diet and reproduction. III. Koninklijke Akad. Wetensch. Ansterdam Proc. 31, 721-732.

Groebbels, F.

1922. Neue Gesichtspunkte zum Vitaminproblem. Klin. Wochschr. 1, 15481551.

a. Weitere Untersuchungun über das Vitaminproblem. Klin. Wochschr. 1, $2130,2131$.

b. Studien über das Vitaminproblem. I. Untersuchungen über den Gasstoffwechsel avitaminotisch ernährter weisser Mäuse. Z. physiol. Chcm. 122, 104-124.

1923. Studien über das Vitaminproblem. II. Untersuchungen über den Einfluss der Vitaminzufuhr und des Hungerns auf Gasstoffwechsel, Gewicht und Lebensdauer vitaminfrei ernährter weisser Mäuse. Z. physiol. Chem. 131, 214-240.

Grose, M. R.

1923. A quantitative study of the heat destruction of vitamin B. Dissertation, Columbia University, New York.

\section{Gross, L.}

1923. The effects of vitamin-deficient diets on the adrenalin equilibrium in the body. Biochem. J. 17, 569-578.

1924. The effects of vitamin-deficient diets on rats with special reference to the motor functions of the intestinal tract in vivo and in vitro. J. Path. Bact. $27,27-50$.

Grüninger, U.

1928. Über den Vitamin C- und D- Gehalt der Bananen. Arch. Kinderheilk. 84, 284-299.

Gudjónsson, S. V.

1926. Is vitamin A secreted through the skin? Experimental investigations on A-vitamin in wool fat. Am. J. Physiol. 75, 533-541.

1930. Experiments on vitamin A deficiency in rats and the quantitative determination of vitamin A. Acta Path. Microbiol. Scand. Sup. 4, pp. 189.

Guerrero, L. E. and Concepcion, I.

1920. Xerophthalmia in fowls fed on polished rice and its clinical importance. Philippine J. Sci. 17, 99-103.

Guest, A. E., Nelson, V. E., Parks, T. B. and Fulmer, E. I.

1926. Diet in relation to reproduction and rearing of young. II. $A m . J$. Physiol. 76, 339-348.

Gugler, M.

1928. Untersuchungen über in-vitro-Atmung an Beriberi-Tauben. Biochcm. Z. $200,340-350$.

Guha, B. C. and Drummond, J. C.

1929. Observations on the concentration of vitamin $\mathrm{B}_{1}$. Biochem. J. 23, 880897. 
Guha, K. D., Hilditch, T. P. and Lovern, J. A.

1930. The composition of the mixed fatty acids present in the glycerides of cod-liver and certain other fish-liver oils. Biochem. J. 24, 266-290.

Gulick, A.

1922. The influence of a beriberi diet upon the metabolic rate of the white rat. Am. J. Physiol. 59, 483, 484.

1924. The basal metabolism of white rats in relation to the intake of vitamin

B. Am. J. Physiol. 68, 131, 132.

Gulik, P. J. van.

1922. Examen microscopique de la localisation de composés du potassium dans quelques organes du coq dans le cas d'avitaminose. Arch. Nécrland. physiol. $6,328-335$.

Gulland, J. M. and Peters, R. A.

1929. The alleged antineuritic properties of certain quinoline and glyoxaline derivatives. Biochcm. J. 23, 1122-1125.

1930. Observations upon the reducing substances of pigeons' blood. Biochem. J. 24, 91-104.

Gullickson, T. W. and Eckles, C. H.

1927. The relation of sunlight to the growth and development of calves. $J$. Dairy Sci. 10, 87-94.

Gutman, M. B. and Franz, V. K.

1922. Observations on the inorganic phosphate of blood in experimental rickets of rats. Proc. Soc. Exptl. Biol. Med. 19, 171-174.

György, P.

1923. Die Säureausscheidung im Urin bei Rachitis und ihre therapeutische Beeinflussung. Beitrag zur Lebertran- und Strahlen-wirkung. Z. ges. exptl. Med. 38, 9-36.

1925. Therapeutische Versuche mit bestrahlter Milch bei der Rachitis. Klin. Wochschr. 4, 1118.

1927. Therapeutische Versuche mit bestrahltem Ergosterin. Klin. Wochschr. $6,580-584$.

1929. Über Vitamine. Verhandl. naturhistorisch-med. Vereins (Heidelberg) $16,1-27$.

György, P. and Gottlieb, K.

1923. Verstarkung der Bestrahlungstherapie der Rachitis durch orale Eosinverabreichung. Klin. Wochschr. 2, 1302, 1303.

\section{Haag, J. R. and Palmer, L. S.}

1928. The effect of variations in the proportions of calcium, magnesium, and phosphorus contained in the diet. J. Biol. Chem. 76, 367-389.

Haan, J. de.

1910. On the etiology of beriberi. Philippine J. Sci., Sect. B 5, 65-71.

Hahn, F. V. von.

1925. Kolloidbiologische Studien über Oberflächenaktivität und Vitaminwirkung. I. Über den vitaminoiden Zustand. II. Oberflächenaktivität und Vitamingehalt der Nahrungsmittel. Arch. ges. Physiol. (Pflüger's) 208, 732744, 745-760.

a. Zur Kolloidchemie des Vitaminproblemes. Kolloid Z. 36, 271-274.

1930. Vitaminwirkung und Oberfächenaktivität. Bemerkungen zu den Publikationen von N. E. Schepilewska. Biochem. Z. 222, 259-271. 


\section{Hale, W.}

1923. The rôle of bread in nutrition. Ind. Eng. Chem. 15, 1221-1224.

Halliburton, W. D. and Drummond, J. C.

1917. The nutritive value of margarins and butter substitutes with reference to their content of the fat-soluble accessory growth substance. J. Physiol. 51, 235-251.

\section{Halliday, N.}

1929. Quantitative comparative studies of the adsorption of vitamins B and $G$ from protein-free milk by Lloyd's reagent. Dissertation, Columbia University, New York.

Hamano, S.

1925. Photo-activity of vitamin A, cholesterol, fats, and other substances by ultra-violet rays. Japan Med. World 5, 317-319.

a. Photoaktivierung von Vitamin $\mathrm{A}$, Cholesterin von Fetten und anderen Substanzen durch Ultraviolettstrahlen. Biochem. Z. 163, 438-444.

Hamburger, R. and Callazo, J. A.

1923. Fettstoffwechsel und A-Vitamin. Klin. Wochschr. 2, 1756.

Hamilton, B., Kajdi, L. and Meeker, D.

1930. The effect of phosphorus administration, antirachitic treatment, and spontaneous healing on the calcium in the serum of rachitic rabbits. J. Biol. Chem. 88, 331-336.

Hamilton, T. S., Card, L. E. and Kick, C. H.

1927. Do growing chicks require a vitamin B supplement to a mixed grain ration? Poultry Sci. 6, 243-248.

Hanke, M. T.

1929. Relation of diet to caries and other dental disorders. J. Am. Dental Assocn. 16, 2263-2271.

1930. Relation of diet to general health and particularly to inflammation of the oral tissues and dental caries. J. Am. Dental Assocn. 17, 957-967.

Happ, W. M.

1922. Occurrence of anemia in rats on deficient diets. Bull. Johns Hopkins Hosp. 33, 163-172.

Hara, S.

1923. Über den Vitamingehalt verschiedener Speisepilze. Biochem. Z. 142, $79-100$.

1924. Über den Vitamingehalt des Brotes. Biochem. Z. 144, 52-59.

Haramaki, $\mathbf{K}$.

1922. Vitamine und Eisenstoff wechsel beim erwachsenen Individuum. Biochem. Z. 134, 354-359.

Harden, A. and Robison, $\mathbf{R}$.

1919. The antiscorbutic properties of concentrated fruit juices. J. Roy. Army Med. Corps 32, 48-56.

1920. The antiscorbutic properties of concentrated fruit juices. Biochem. J. $14,171-177$.

1921. The antiscorbutic properties of concentrated fruit juices. IV. Biochem. J. $15,521,522$.

1923. Note on the sulphuric test for liver oils. Biochem. J. 17, 115, 116.

Harden, A. and Zilva, S. S.

1917. The alleged antineuritic properties of $\alpha$-hydroxypyridine and adenine. Biochem. J. 11, 172-179. 
1918. The differential behavior of the antineuritic and antiscorbutic factors towards adsorbents. Biochem. J. 12, 93-105.

a. The antiscorbutic factors in lemon juice. Biochem. J. 12, 259-269.

b. Note on the etiology of scurvy in guinea-pigs. Biochem. J. 12, 270-274.

c. Accessory factors in the nutrition of the rat. Biochem. J. 12, 408-415.

d. A note on the susceptibility of the antiscorbutic principle to alkalinity. Lancet, 1918, II, 320.

e. An investigation of beer for antineuritic and antiscorbutic potency. $J$. Institute Brewing 24, 197-208.

1919. Experimental scurvy in monkeys. J. Path. Bact. 22, 246-251.

a. Edema observed in a monkey. Lancet, 1919, II, 780, 781.

1920. The antiscorbutic requirements of the monkey. Biochem. J. 14, 131-134.

a. Dietetic experiments with frogs. Biochem. J. 14, 263-266.

1921. The synthesis of vitamin B by yeasts (preliminary note). Biochem. J. $15,438$.

1924. Investigation of barley, malt and beer for vitamins B and C. Biochem. J. 18, 1129-1132.

Harden, A., Zilva, S. S. and Still, G. F.

1919. Infantile scurvy. The antiscorbutic factor of lemon juice in treatment. Lancet, 1919, I, 17, 18.

Harris, K. D. and Smith, E. A.

1928. Histological study of the thyroid of the guinea pig in experimental scurvy. Am. J. Physiol. 84, 599-602.

Harris, L. J.

1928. Mode of action of vitamin D. Question of regulation of gut acidity. Science Progress 23, 68-74.

1930. Hypervitaminosis D: Calcium-phosphorus intake a determining factor. Lancet, 1930, I, 236.

Harris, L. J. and Moore, T.

1928. "Hypervitaminosis" and "vitamin balance." Lancet, 1928, II, 892, 893.

a. "Hypervitaminosis" and "vitamin balance." Biochem. J. 22, 1461-1477.

1929. Hypervitaminosis and vitamin balance. Part II. The specificity of vitamin $D$ in irradiated ergosterol poisoning. Part III. The pathology of hypervitaminosis D. Biochem. J. 23, 261-273.

a. Hypervitaminosis and vitamin balance. IV. An instance of vitamin balance. Biochem. J. 23, 1114-1121.

Harris, L. J. and Stewart, C. P.

1929. The effect of excessive doses of irradiated ergosterol on the calcium and phosphorus content of the blood. Biochem. J. 23, 206-209.

Harris, S.

1928. Rôle of vitamins in the etiology and cure of gastric and duodenal ulcers. J. Am. Med. Assocn. 91, 1452-1457.

Harrow, B. and Krasnow, F.

1922. Feeding experiments on rats with plants at different stages of development. I. Experiments with corn. J. Metabolic Research 2, 401-415.

1923. Feeding experiments on rats with plants at different stages of development. Part II. J. Metabolic Research 4, 491-497. 


\section{Hart, C.}

1912. Der Skorbut der kleinen Kinder (Moeller-Barlowsche Krankheit) nach experimentellen Untersuchungen. Jahrb. Kinderheilk. 76, 507-541.

Hart, E. B., Halpin, J. G. and McCollum, E. V.

1917. The behavior of chickens fed rations restricted to the cereal grains. $J$. Biol. Chem. 29, 57-67.

Hart, E. B., Halpin, J. G. and Steenbock, H.

1917. The behavior of chickens fed rations restricted to the wheat or maize kernel. J. Biol. Chem. 31, 415-420.

1920. Use of synthetic diets in the growth of baby chicks-a study of leg weakness in chickens. J. Biol. Chem. 43, 421-422.

1922. The nutritional requirements of baby chicks. II. Further study of leg weakness in chickens. J. Biol. Chem. 52, 379-386.

Hart, E. B., and McCollum, E. V.

1914. Influence on growth of rations restricted to the corn or wheat grain. J. Biol. Chem. 19, 373-395.

Hart, E. B., McCollum, E. V., Steenbock, H. and Humphrey, G. C.

1917. Physiological effect on growth and reproduction of rations balanced from restricted sources. J. Agr. Research 10, 175-198.

Hart, E. B., Miller, W. S. and McCollum, E. V.

1916. Further studies on the nutritive deficiencies of wheat and grain mixtures and the pathological conditions produced in swine by their use. J. Biol. Chem. 25, 239-259.

Hart, E. B., Steenbock, H. and Ellis, N. R.

1920. Influence of diet on the antiscorbutic potency of milk. J. Biol. Chem. 42, 383-396.

1921. Antiscorbutic potency of milk powders. J. Biol. Chem. 46, 309-318.

Hart, E. B., Steenbock, H. and Elvehjem, C. A.

1924. Dietary factors influencing calcium assimilation. V. The effect of light upon calcium and phosphorus equilibrium in mature lactating animals. $J$. Biol. Chem. 62, 117-131.

Hart, E. B., Steenbock, H., Elvehjem, C. A., Scott, H. and Humphrey, G. C. 1926. Dietary factors influencing calcium assimilation. VII. The influence of sunlight upon calcium equilibrium in milking cows. J. Biol. Chent. 67, 371-383.

Hart, E. B., Steenbock, H. and Hoppert, C. A.

1920. The influence of dry fresh green plant tissue on calcium assimilation. Science 52, 318.

1921. Dietary factors influencing calcium assimilation. I. The comparative influence of green and dried plant tissue, cabbage, orange juice, and cod liver oil on calcium metabolism. J. Biol. Chem. 48, 33-50.

1922. Dietary factors influencing calcium assimilation. II. The comparative efficiency of dry and green alfalfa in maintaining calcium and phosphorus equilibrium in milking cows. J. Biol. Chem. 53, 21-30.

Hart, E. B., Steenbock, H., Hoppert, C. A., Bethke, R. M. and Humphrey, G. C.

1922. Dietary factors influencing calcium assimilation. III. The comparative efficiency of timothy hay, alfalfa hay and timothy hay plus calcium phosphate (steamed bone meal) in maintaining calcium and phosphorus equilibrium in milking cows. J. Biol. Chem. 54, 75-89. 
Hart, E. B., Steenbock, H., Hoppert, C. A. and Humphrey, G. C.

1923. Dietary factors influencing calcium assimilation. IV. The comparative efficiency of mixed green grasses and this same mixture plus steamed bone meal in maintaining calcium and phosphorus equilibrium in milking cows. J. Biol. Chem. 58, 43-57.

Hart, E. B., Steenbock, H., Humphrey, G. C. and Hulce, R. S.

1924. New observations and a reinterpretation of old observations on the nutritive value of the wheat plant. J. Biol. Chem. 62, 315-322.

Hart, E. B., Steenbock, H., Kletzien, S. W. and Scott, H.

1927. Dietary factors influencing calcium assimilation. IX. Further observation on the influence of cod liver oil on calcium assimilation. J. Biol. Chem. $71,271-280$.

Hart, E. B., Steenbock, H., Kline, O. L. and Humphrey, G. C.

1930. Dietary factors influencing calcium assimilation. XIII. The influence of irradiated yeast on the calcium and phosphorus metabolism of milking cows. J. Biol. Chem. 86, 145-155.

Hart, E. B., Steenbock, H. and Lepkovsky, S.

1922. The antiscorbutic vitamine. I. A study of its solubility from desiccated orange juice. J. Biol. Chem. 52, 241-250.

1923. The nutritional requirements of baby chicks. III. The relation of light to the growth of the chicken. J. Biol. Chem. 58, 33-41.

1925. Is the antirachitic factor of cod liver oil when mixed with ground grains destroyed through storage? J. Biol. Chem. 65, 571-578.

Hart, E. B., Steenbock, H., Lepkovsky, S. and Halpin, J. G.

1924. The nutritional requirements of baby chicks. IV. The chicks' requirement for vitamin A. J. Biol. Chem. 60, 341-354.

1925. The nutritional requirement of the chicken. VI. Does the chicken require vitamin C? J. Biol. Chem. 66, 813-818.

Hart, E. B., Steenbock, H., Lepkovsky, S., Kletzien, S. W. F., Halpin, J. G. and Johnson, O. N.

1925. The nutritional requirement of the chicken. V. The influence of ultraviolet light on the production, hatchability, and fertility of the egg. J. Biol. Chem. 65, 579-595.

Hart, E. B., Steenbock, H., Scott, H. and Humphrey, G. C.

1927. Dietary factors influencing calcium assimilation. VIII. The calcium level and sunlight as affecting calcium equilibrium in milking cows. J. Biol. Chem. 71, 263-269.

Hart, E. B., Steenbock, H. and Smith, D. W.

1919. Studies of experimental scurvy. Effect of heat on the antiscorbutic properties of some milk products. J. Biol. Chem. 38, 305-324.

Hart, E. B., Steenbock, H., Teut, E. C. and Humphrey, G. C.

1929. Dietary factors influencing calcium assimilation. XI. The influence of cod liver oil upon calcium metabolism of milking cows. J. Biol. Chem. 84, 359-365.

a. Dietary factors influencing calcium assimilation. XII. A study of the influence of hays cured with varying exposure to sunlight on the calcium metabolism of milking cows. J. Biol. Chem. 84, 367-376. 


\section{Hart, $\mathrm{K}$.}

1912. Uber die experimentelle Erzeugung der Möller-Barlowschen Krankheit und ihre endgültige Identifizierung mit dem klassischen Skorbut. Arch. Path. Anat. (Virchow's) 208, 367-396.

Hart, M. C., Tourtelotte, D. and Heyl, F. W.

1928. The effect of irradiation and cod liver oil on the calcium balance in the adult human. J. Biol. Chem. 76, 143-148.

\section{Hartwell, G. A.}

1921. The effect of diet on mammary secretion. Biochem. J. 15, 140-162.

a. The evil effect of excess of protein on milk secretion. Lancet, 1921, I, 1240.

b. Excess protein and mammary secretion. Biochem. J. 15, 563-574.

1922. Mammary secretion. III. 1. The quality and quantity of dietary protein. 2. The relation of protein to other dietary constituents. Biochem. J. 16, 78-105.

a. Mammary secretion. IV. The relation of protein to other dietary constituents. Biochem. J. 16, 825-837.

1924. Vitamin B content of white bread. Biochem. J. 18, 120-126.

a. Mammary secretion. V. I. Further research on the threshold and effects of protein "excess." II. The quantitative relation of vitamin B to protein. Biochem. J. 18, 785-794.

b. An experimental study of brown and white bread in the diet of the rat. Biochem. J. 18, 1323-1326.

c. Vitamin B and lactation. Lancet, 1924, II, 956-958.

1925. Sex differences in the requirements of certain food factors. I. During growth. Brit. J. Exptl. Biol. 2, 323-330.

a. A comparison of dried and evaporated milks by a dietetic method. Biochem. J. 19, 226-232.

b. A note on an improved technique for use with synthetic diets. Biochem. J. 19, 729-732.

c.. Mammary secretion. VI. Vitamin B and the lactating rat's diet. 1. The quantitative relation of vitamin B to protein. 2. Vitamin B requirement of the lactating and nonlactating rat (preliminary note). Biochem. J. 19, 10751081.

1926. Growth and reproduction on synthetic diets. Part I. Biochem. J. 20, 1273-1278.

1927. A note on the weight of the rat during gestation. Biochem. J. 21, 572575.

a. Growth and reproduction on synthetic diets. II. Biochem. J. 21, 10761086.

1928. Protein and vitamin B. Biochem. J. 22, 1212-1220.

Hartwell, G. A., Mottram, E. C. and Mottram, V. H.

1923. The technique of breeding rats for feeding experiments. Biochem. J. 17, 208-215.

Hartwell, G. A. and Mottram, V. H.

1929. The brown vs. white bread controversy. Lancet, 1929, II, 892-894.

Harvey, D.

1927. The effects of cod liver oil on the calcium and phosphorus metabolism of the lactating animal. Biochem. J. 21, 1268-1276. 
Hassan, A. and Drummond, J. C.

1927. The physiological rôle of vitamin B. IV. The relation of certain dietary factors in yeast to growth in rats on diets rich in proteins. Biochem. J. 21, 653-661.

Hasselbach, K. A.

1911. Quantitative Untersuchungen über die Absorption der menschlichen Haut von ultravioletten Strahlen. Skand. Arch. Physiol. 25, 55-68.

Hattori, K. and Obata, J.

1923. Unsaponifiable constituents (vitamin A) of cod liver oil. J. Pharm. Soc. Japan No. 497: Chom. Abstracts 18, 410.

Hauge, S. M.

1930. An inheritance study of the distribution of vitamin A in maize. II.

Vitamin A in hybrid red maize. J. Biol. Chem. 86, 161-165.

Hauge, S. M. and Carrick, C. W.

1925. The antiscorbutic properties of eggs. J. Biol. Chem. 64, 111, 112.

1926. A differentiation between the water-soluble growth-promoting and antineuritic substances. J. Biol. Chem. 69, 403-413.

a. The antiscorbutic vitamin in poultry nutrition. Poultry Sci. 5, 166-172.

Hauge, S. M., Carrick, C. W. and Prange, R. W.

1927. Fat soluble A requirements for growing chicks. Poultry Sci. 6, 135-140.

Hauge, S. M., Carrick, C. W. and Prange, R. W.

1928. An inheritance study of the distribution of vitamin A in maize. J. Biol.

Chem. 80, 107-114.

1930. An inheritance study of the distribution of vitamin A in maize. III. Vitamin A content in relation to yellow endosperm. J. Biol. Chem. 86, 167172.

Havard, R. E. and Hoyle, J. C.

1928. Vitamin D in adults: Its effect on the calcium and inorganic phosphate of the blood. Biochem. J. 22, 713-716.

Haverschmidt, J.

1924. Rickets. Nederlandsch. Maadschr. Geneesk. (Leiden) 12, 145. Abstracted in J. Am. Med. Assocn. 82, 2150.

Hawk, P. B.

1929. Cod liver oil and the antimony trichloride reaction for vitamin A. Science 69, 200.

Hawk, P. B., Fishback, H. R. and Bergeim, O.

1919. Compressed yeast as food for the growing organism. Am. J. Physiol. 48, 211-220.

Hawk, P. B., Smith, C. A. and Bergeim, O.

1921. The vitamin content of honey and honey comb. Am. J. Physiol. 55, 339-348.

a. The nutritive value of yeast in bread. Am. J. Physiol. 56, 33-39.

Hawking, F.

1927. The synthesis of the antineuritic factor (Torulin) by yeast. Biochem. J. 21, 728-732.

Heaton, T. B.

1922. On the vitamin D. Biochem. J. 16, 800-808.

Hehir, $\mathbf{P}$.

1919. Beriberi. Indian. J. Med. Research Spec. Indian Sci. Cong. No. 44-59, 1919. 
a. Scurvy. Indian J. Med. Research Spec. Indian Sci. Cong. No. 79-82, 1919.

Heilbron, I. M., Kamm, E. D. and Morton, R. A.

1927. The absorption spectrum of cholesterol and its biological significance with reference to vitamin D. Part I, Preliminary observations. Biochem. J. $21,78-85$.

a. Absorption bands of ergosterol and vitamin D. Nature 120, 617, 618.

b. The absorption spectra of oils and oil constituents with special reference to pro-vitamin D. Biochem. J. 21, 1279-1283.

Heilbron, I. M., Morton, R. A. and Sexton, W. A.

1928. Cholesterol and vitamin D. Nature 121, 452-453.

a. Studies in the sterol group. I. The absorption spectra of some cholesterol derivatives. J. Chem. Soc. Jan. 47-51.

Heilbron, I. M. and Sexton, W. A.

1929. The occurrence of ergosterol in phytosterols. Nature 123, 567.

Heilbron, I. M. and Spring, F. S.

1930. The colour reactions and absorption spectra of sterols in relation to structure. Biochem. J. 24, 133-135.

Heiser, V. G.

1911. Practical experiences with beriberi and unpolished rice in the Philippines. Philippine J. Sci. Sect. B. 6, 229-233.

1912. Beriberi. With a suggestion for governmental aid in its eradication. Med. Record 81, 516, 517.

Hejinian, L. M.

1924. The possible hereditary factors in experimental production of edema and xerophthalmia. Am. J. Physiol. 67, 467-473.

Heller, V. G.

1923. Studies on yeast. V. The vitamin B content of yeast. J. Biol. Chem. 55, 385-398.

1927. Nutritive properties of the mung bean. J. Biol. Chem. 75, 435-442.

1928. Vitamin synthesis in plants as affected by light source. J. Biol. Chem. 76, 499-511.

Heller, V. G. and Caskey, C.

1928. An application of some of the more recent methods of estimating vitamin

D. J. Nutrition 2, 59-65.

Heller, V. G. and Green, R.

1925-26. The chemical and nutritive properties of the grain sorghums. $J$. Metabolic Research 7-8, 205-215.

Heller, V. G., McElroy, C. H. and Garlock, B.

1925. The effect of the bacterial flora on the biological test for vitamin B. J. Biol. Chem. 65, 255-264.

Henderson, J. M.

1925. The effect of irradiation and diet on calcium and phosphorus metabolism. Biochem. J. 19, 52-62.

1926. The influence of ultra-violet light on nutrition. Scottish J. Agr. 9, 33-39.

Henderson, J. McK. and Kelly, F. C.

1930. The influence of certain dietary supplements in relation to the calcium requirements of growing African natives. II. J. Hyg. 29, 429-438. 
a. A note of the influence of the addition of certain supplements to the diets of African natives. III. J. Hyg. 29, 439-442.

Henderson, J. McK. and Magee, H. E.

1926. The effect of ultra-violet light on the calcium and phosphorus metabolism of the lactating animal. Biochem. J. 20, 363-373.

Hentschel, H. and Roszkowski, A.

1929. Über neuere Behandlungsverfahren der englischen Krankheit im Dienste der allgemeinen Rachitisbekämpfung. II. Erfahrungen mit bestrahlter Frischmilch als antirachitikum. Münch. med. Wochschr. 76, 1423-1430.

Hentschel, H. and Zoeller, E.

1927. Über Stoffwechselveränderungen bei Rachitis. I. Der Phosphatstoffwechsel in der Muskulatur bei der experimentellen Rattenrachitis. $Z$ Kinderheilk. 44, 146-162.

\section{Herb, F.}

1928. The functional relationship between complements, vitamins, and hormones. Med. J. Record 128, 268-271; 332-335.

1929. Specificity of the activities of hormones, vitamins, and complements. Med. J. Record 129, 250-254.

\section{Hermano, A. J.}

1930. The vitamin contents of Philippine foods. I. Vitamins A and B in Basella rubra, Capsicum fruitescens and Vigna sinensis. Philippine J. Sci.41, 387-399.

Herter, C. A.

1898. An experimental study of fat starvation with especial reference to the production of serous atrophy of fat. J. Exptl. Med. 3, 293-314.

\section{Hess, A. F.}

1915. Infantile scurvy. II. A new aspect of the symptomatology, pathology and diet. J. Am. Med. Assocn. 65, 1003-1006.

1916. Infantile scurvy. III. Its influence on growth (length and weight). Am. J. Diseases Children 12, 152-165.

1917. Infantile scurvy. IV. The therapeutic value of yeast and of wheat embryo. Am. J. Diseases Children 13, 98-109.

a. Infantile scurvy. V. A study of its pathogenesis. Am. J. Diseases Children 14, 337-353.

b. Subacute and latent infantile scurvy. The cardiorespiratory syndrome (A new sign). J. Am. Med. Assocn. 68, 235-239.

1918. The rôle of antiscorbutics in our dietary. J. Am. Med. Assocn. 71, 941-943.

1920. "Scurvy,-Past and Present," Philadelphia, J. B. Lippincott Co.

a. Scurvy in the world war. Inter. J. Pub. Health I, 302-307.

1921. Newer aspects of some nutritional disorders. J. Am. Med. Assocn. 76, 693-700.

a. The antiscorbutic vitamin. J. Ind. Eng. Chem. 13, 1115, 1116.

1922. Nutritional disorders in the light of recent investigations. Boston Med. Surg. J. 187, 101-108.

a. The prevention and cure of rickets by sunlight. Am. J. Pub. Health 12, 104-107.

b. The influence of light in prevention and cure of rickets. Lancet, 1922, II, 367-369. 
c. Further experiments on the prevention of rickets in rats by exposure to light. J. Biol. Chem. 50, XLIV.

1923. The therapeutic value of egg yolk in rickets. J. Am. Med. Assocn. 81, 15-17.

1925. The ultraviolet rays of the sun. J. Am. Med. Assocn. 84, 1033-1037.

a. The antirachitic activation of foods and of cholesterol by ultraviolet irradiation. J. Am. Med. Assocn. 84, 1910-1913.

1926. Recurrent rickets. Am. J. Diseases Children 31, 380-385.

1927. Antirachitic activity of irradiated cholesterol, ergosterol, and allied substances. J. Am. Mcd. Assocn. 89, 337-339.

1928. The contribution of biology, chemistry and physics to the newer knowledge of rickets. Science 67, 333-335.

1929. "Rickets, Including Osteomalacia and Tetany." Philadelphia, Lea \& Febiger.

Hess, A. F. and Anderson, R. J.

1927. The antirachitic value of irradiated cholesterol and phytosterol. VIII. The activation of sterol fractions by ultra-violet irradiation. J. Biol. Chem. 74, 651-657.

Hess, A. F. and Anderson, W. T., Jr.

1927. The antirachitic activity of monochromatic and regional ultra-violet radiations. J. Am. Med. Assocn. 89, 1222-1225.

Hess, A. F., Bills, C. E. and Honeywell, E. M.

1929. Antirachitic potency in relation to volume of oil in the liver of the cod. J. Am. Med. Assocn. 92, 226-228.

Hess, A. F., Bills, C. E., Weinstock, M., Honeywell, E. and Rivkin, H.

1928. Relation of the antirachitic factor to reproduction in fish. Proc. Soc. Exptl. Biol. Med. 25, 652, 653.

Hess, A. F. and Fish, M.

1914. Infantile scurvy: The blood, the blood vessels, and the diet. Am. J. Diseases Children 8, 385-405.

Hess, A. F. and Gutman, M. G.

1922. The cure of infantile rickets by sunlight accompanied by an increase in the inorganic phosphate of the blood. J. Am. Med. Assocn. 78, 29-31.

Hess, A. F. and Killian, J.

1918. Chemistry of the blood in scurvy. Proc. Soc. Exptl. Biol. Med. 16, 43.

Hess, A. F. and Lewis, J. M.

1928. Clinical experience with irradiated ergosterol. J. Am. Med. Assocn. 91, 783-788.

Hess, A. F., Lewis, J. M. and Rivkin, H.

1929. The status of the therapeutics of irradiated ergosterol. J. Am. Med. Assocn. 93, 661-665.

1930. Irradiated ergosterol. Maintenance of blood phosphate level in the course of development of rickets in infants. Proc. Soc. Exptl. Biol. Med. 27, 611.

a. Newer aspects of the therapeutics of Viosterol (irradiated ergosterol). J. Am. Med. Assocn. 94, 1885-1889.

Hess, A. F. and Lundagen, M. A.

1922. A seasonal tide of blood phosphate in infants. J. Am. Med. Assocn. 79, 2210-2212. 
Hess, A. F. and Matzner, M. J.

1923. Rickets in relation to the inorganic phosphate and calcium in maternal and fetal blood. Am. J. Diseases Children 26, 285-289.

1924. The value of milk acidified with lemon juice. Its combination with egg yolk to add the antirachitic factor. J. Am. Med. Assocn. 82, 1604-1606.

Hess, A. F., McCann, G. F. and Pappenheimer, A. M.

1921. Experimental rickets in rats. II. The failure of rats to develop rickets on a diet deficient in vitamin A. J. Biol. Chem. 47, 395-409.

Hess, A. F., Pappenheimer, A. M. and Weinstock, M.

1922. A study of light waves in relation to their protective action in rickets. Proc. Soc. Exptl. Biol. Med. 20, 14-16.

Hess, A. F., Russell, W. C., Weinstock, M. and Rivkin, H.

1928. Relation of the antirachitic factor to reproduction in birds. Proc. Soc. Exptl. Biol. Med. 25, 651, 652.

Hess, A. F. and Sherman, E.

1925. Comparison of non-irradiated and irradiated cholesterol to inhibit the hemolytic action of digitonin. Proc. Soc. Exphl. Biol. Med. 23, 169, 170.

1927. The antirachitic value of irradiated cholesterol and phytosterol. VI. The effect of irradiated cholesterol on the phosphorus and calcium balance. J. Biol. Chem. 73, 145-151.

Hess, A. F. and Supplee, G. C.

1930. The action of irradiated ergosterol on rats and chickens. Proc. Soc. Exptl. Biol. Med. 27, 609, 610.

Hess, A. F. and Unger, L. J.

1917. Prophylactic therapy for rickets in a negro community. J. Am. Med. Assocn. 69, 1583-1586.

1918. Canned tomatoes as an antiscorbutic. Proc. Soc. Exptl. Biol. Med. 16, $1,2$.

a. The scurvy of guinea-pigs. I. The experimental dietary. II. Experiments on the effect of the addition of fruits and vegetables to the dietary. J. Biol. Chem. 35, 479-496.

b. The diet of the negro mother in New York City. J. Am. Med. Assocn. $70,900-902$.

c. Experiments on antiscorbutics. Report of an antiscorbutic for intravenous use. Proc. Soc. Exptl. Biol. Med. 15, 141, 142.

1919. The scurvy of guinea-pigs. III. The effect of age, heat, and reaction on antiscorbutic foods. J. Biol. Chem. 38, 293-303.

a. Scurvy. VIII. Factors affecting the antiscorbutic value of foods. Am. J. Diseases Children 17, 221-240.

b. The deleterious effect of the alkalinization of infants' food. J. Am. Med. Assocn. 73, 1353-1356.

c. The role of fat-soluble vitamin in the dietary of infants. Proc. Soc. Exptl. Biol. Med. 17, 49, 50.

1920. The clinical role of the fat-soluble vitamin. Its relation to rickets. J. Am. Med. Assocn. 74, 217-223.

a. Scorbutic beading of ribs. Am. J. Diseases Children 19, 331-336.

1921. The destructive effect of oxidation on antiscorbutic vitamin. Proc. Soc. Exptl. Biol. Med. 18, 143.

a. An interpretation of the seasonal variation of rickets. Am.J. Diseases Children 22, 186-192. 
b. The cure of infantile rickets by artificial light and by sunlight. Proc. Soc. Exptl. Biol. Med. 18, 298.

c. The cure of infantile rickets by sunlight (preliminary note). J. Am. Med. Assocn. 77, 39.

d. The destruction of the antiscorbutic vitamin in milk by the catalytic action of minute amounts of copper. Proc. Soc. Exptl. Biol. Mcd. 19, 119, 120.

1922. Use of the carbon arc light in the prevention and cure of rickets. J. Am. Med. Assocn. 78, 1596-1598.

a. Infantile rickets. The significance of clinical, radiographic and chemical examinations in its diagnosis and incidence. Am. J. Diseases Children 24, 327-338.

Hess, A. F., Unger, L. J. and Pappenheimer, A. M.

1922. Experimental rickets in rats. III. The prevention of rickets by exposure to sunlight. J. Biol. Chem. 50, 77-82.

a. A further report on the prevention of rickets in rats by light rays. Proc. Soc. Exptl. Biol. Med. 19, 238, 239.

b. Spontaneous cure of rickets in rats. Proc. Soc. Exptl. Biol. Med. 19, 236-238.

c. Experimental rickets in rats. VII. The prevention of rickets by sunlight, by the rays of the mercury vapor lamp, and by the carbon arc lamp. J. Exptl. Med. 36, 427-446.

Hess, A. F., Unger, L. J. and Steiner, J. M.

1922. Experimental rickets in rats. VIII. The effect of Roentgen rays. $J$. Exptl. Med. 36, 447-452.

Hess, A. F., Unger, L. J. and Supplee, A. C.

1920. Relation of fodder to the antiscorbutic putency and salt content of milk. J. Biol. Chem. 45, 229-235.

Hess, A. F. and Weinstock, M.

1923. A study of light waves in their relation to rickets. J. Am. Med. Assocn. $80,687-690$.

1924. Antirachitic effect of cod liver oil fed during the period of pregnancy or lactation. Am. J. Diseases Children 27, 1-5.

a. The catalytic action of minute amounts of copper in the destruction of antiscorbutic vitamin in milk. J. Am. Med. Assocn. 82, 952-956.

b. Further experiments on the antirachitic action of yolk of egg. Proc. Soc. Exptl. Biol. Med. 21, 441, 442.

c. Rickets as influenced by the diet of the mother during pregnancy and lactation. J. Am. Med. Assocn. 83, 1558-1562.

d. Antirachitic properties imparted to inert fluids by ultra-violet irradiation. J. Am. Med. Assocn. 83, 1845, 1846.

e. Antirachitic properties imparted to inert fluids and to green vegetables by ultra-violet irradiation. J. Biol. Chem. 62, 301-313.

f. Antirachitic properties imparted to lettuce and to growing wheat by ultraviolet irradiation. Proc. Soc. Exptl. Biol. Med. 22, 5, 6.

g. Antirachitic properties imparted to inert fluids by ultraviolet irradiation. Proc. Soc. Exptl. Biol. Med. 22, 6, 7.

1925. A further report on imparting antirachitic properties to inert substances by ultraviolet irradiation. J. Biol. Chem. 63, 297-304. 
a. The antirachitic value of irradiated cholesterol and phytosterol. II. Further evidence of change in biological activity. III. Evidence of chemical change as shown by absorption spectra. J. Biol. Chem. 64, 181-191, 193-201.

b. Some properties of cholesterol and phytosterol activated by irradiation. Proc. Soc. Exptl. Biol. Med. 22, 319.

1926. Ultra-violet irradiation in the activation of cholesterol and foods. Lancet, 1926, I, 12-14.

a. Puffer fish oil: A very potent antirachitic: its elaboration by fish deprived of sunlight. Proc. Soc. Exptl. Biol. Med. 23, 407, 408.

b. The value of elementary phosphorus in rickets-an experimental study. Am. J. Diseases Children 32, 483-496.

1927. The antirachitic activity of monochromatic and regional ultraviolet radiations. Proc. Exptl. Biol. Med. 24, 759, 760.

a. A study of the antirachitic factor in human and in cow's milk. Am. J. Diseases Children 34, 845-853.

1928. An investigation of the prenatal factor in the susceptibility of infants to rickets. Am. J. Diseases Children 38, 966-971.

Hess, A. F., Weinstock, M. and Helman, F. D.

1925. The development of antirachitic potency in phytosterol and cholesterol following irradiation. Proc. Soc. Exptl. Biol. Med. 22, 227.

a. The antirachitic value of irradiated phytosterol and cholesterol. I. J. Biol. Chem. 63, 305-308.

Hess, A. F., Weinstock, M. and Rivkin, H.

1928. Source of increase in serum calcium induced by irradiated ergosterol. Proc. Soc. Exptl. Biol. Med. 26, 199.

1929. Effect of thyroparathyroidectomy on the action of irradiated ergosterol. Proc. Soc. Exptl. Biol. Med. 26, 555, 556.

1930. A further report on the effect of thyroparathyroidectomy on the action of irradiated ergosterol. Proc. Soc. Exptl. Biol. Med. 27, 298, 299.

a. Some differences in action between irradiated ergosterol and cod liver oil. Proc. Soc. Exptl. Biol. Med. 27, 665, 666.

\section{Hess, A. F., Weinstock, M., Rivkin, H. and Gross, J.}

1929. Observations suggesting a local factor in pathogenesis in healing of rickets. Proc. Soc. Exptl. Biol. Med. 27, 140-142.

1930. The lack of relationship between the development and cure of rickets and the inorganic phosphorus concentration of the blood. J. Biol. Chem. 87, $37-46$.

Hess, A. F., Weinstock, M. and Sherman, E.

1925. The antirachitic value of irradiated cholesterol and phytosterol. IV. Factors influencing its biological activity. J. Biol. Chem. 66, 145-160.

1926. The antirachitic value of irradiated cholesterol and phytosterol. V. Chemical and biological changes. J. Biol. Chem. 67, 413-423.

a. The production of antirachitic properties in human milk resulting from irradiation of the mother. Proc. Soc. Exptl. Biol. Med. 23, 636-638.

b. The antirachitic value of irradiated cholesterol. VII. A separation into an active and an inactive fraction. J. Biol. Chem. 70, 123-127.

1927. Antirachitic properties developed in human milk by irradiating the mother. J. Am. Med. Assocn. 88, 24-26. 
Hess, A. F., Weinstock, M. and Tolstoi, E.

1923. The influence of the diet during the preexperimental period on the susceptibility of rats to rickets. J. Biol. Chem. 57, 731-740.

Hess, A. F. and Windaus, A.

1926. Experiments on activation of cholesterol derivatives and allied sterols by ultra-violet irradiation. Proc. Soc. Exptl. Biol. Med. 24, 171, 172.

1927. Contaminating substances as a factor in the activation of cholesterol by irradiation. Proc. Soc. Exptl. Biol. Med. 24, 369, 370.

a. The development of marked activity in ergosterol following ultra-violet irradiation. Proc. Soc. Exptl. Biol. Med. 24, 461, 462.

Hess, J. H., Calvin, J. K., Wang, C. C. and Felcher, A.

1923. Calcium and phosphorus determinations in the blood plasma in rickets and tetany. Am. J. Diseases Children 26, 271-279.

Hess, J. H., Moore, J. J. and Calvin, J. K.

1922. Experimental studies with proprietary vitamin products. J. Am. Med. Assocn. 78, 1441-1445.

Hess, W. R.

1921. Die Rolle der Vitamine im Zellchemismus. Z. physiol. Chem. 117, 284-308.

1922. Die Rolle der Vitamine im Zellchemismus, Z. physiol. Chem. 120, $277-280$.

a. B vitamin and pigeon beriberi. Lancet, 1922, I, 554.

1923. Die Blausäurevergiftung als Methode der Avitaminoseforschung. Arch. ges. Physiol. (Pflüger's) 198, 483-489.

1924. Über die Wirkung der Vitamine. Deut. med. Wochschr. 50, 163-164.

Hess, W. R. and Messerle, N.

1922. Untersuchungen über die Gewebeatmung bei Avitaminose. Z. physiol. Chem. 119, 176-189.

Hess, W. R. and Rohr, K.

1923. Uber den Einfluss thermischer Vorbehandlung von Trockenhefe auf ihre Reduktionsleistung und ihre Vitaminwertigkeit, mit einen Beitrag zur Kenntnis der Vogelberiberi. Z. physiol. Chem. 129, 268-283.

Hess, W. R. and Takahashi, N.

1921. Nachweis eines stofflichen Defizites im Gewebe an Avitaminose erkrankter Tiere. Biochem. Z. 122, 193-203.

Hessler, M. C.

1926. Experiments upon the quantitative differentiation of vitamins $A$ and $D$.

Dissertation, Columbia University, New York.

Hessler, M. C. and Craig, G.

1929. Vitamin C content of spinach and Jonathan apples. Missouri Agr. Expt. Sta. Bull. 272, 67, 68.

Hessler, M. C. and Rogers, E. C.

1929. Influence of the method of preparation on the vitamin B content of spinach and string beans. Missouri Agr. Expt. Sta. Bull. 272, 66, 67.

Hessler, M. C. and Williams, Z.

1929. The antiscorbutic property of apple and rhubarb. Missouri Agr. Expt. Sta. Bull. 272, 68.

Heubner, W. and Holtz, F.

1929. Über die biologische Inaktivität des Ergosterinperoxyds. Klin. Wochschr. 8, 456, 457. 
Heuser, G. F.

1924. Influence of vitamines on growth and resistance to coccidiosis. Rel. Poultry J. 31, 226, 228, 232.

Heuser, G. F. and Norris, L. C.

1926. Rickets in chicks. I. Variations in the antirachitic potency of different brands of cod liver oil. Poultry Sci. 6, 9-17.

1929. Rickets in chicks. IV. The effect of heat and exposure to air on the stability of vitamin D. Am. J. Diseases Children 38, 481-489.

Heyl, F. W., Wise, E. C. and Speer, J. H.

1929. The unsaponifiable fraction from spinach fat. J. Biol. Chem. 82, 111-116. Hift, $R$.

1918. Beobachtungen über Skorbut und Hemeralopie. Wiener klin. Wochschr. $31,939$.

Higgins, G. M. and Sheard, C.

1928. The parathyroid glands as influenced by selective solar radiation. Science $67,536,537$.

a. The effects of selective solar irradiation on the parathyroid glands of chicks. Ain. J. Physiol. 85, 299-310.

Highet, H. C.

1910. Beriberi in Siam. Philippine J. Sci., Sect. B, 5, 73-79.

Hill, C. McD. and Clark, J. H.

1927. The effect of ultraviolet radiation on resistance to infection. Am. J. Hill, L. Hyg. 7, 448-462.

1924. "Sunshine and Open Air. Their Influence on Health, with Special Reference to the Alpine Climate." London, Edward Arnold \& Co.

1927. Measurement of the biologically active ultra-violet rays of sunlight. Proc. Roy. Soc. (London) B. 102, 119-128.

1928. Penetration of the skin by ultra-violet rays. Brit. J. Actinotherapy 3, $147,148$.

Hindhede, $M$.

1920. The effect of food restriction during war on mortality in Copenhagen. J. Am. Med. Assocn. 74, 381, 382.

\section{Hirabayashi, N.}

a. Fettminimum. Skand. Arch. Physiol. 40, 78-131.

1924. Beitrag zum Stickstoff- und Mineralstoffwechsel bei der Avitaminose. Biochem. Z. 145, 18-31.

Hirsch, A.

1885. In "Handbook of Geographical and Historical Pathology," English edition of $1885,2,507$.

Hjort, J.

1922. Observations on the distribution of fat-soluble vitamins in marine animals and plants. Proc. Roy. Soc. (London) B. 93, 440-449.

Hlavaty, J.

1929. Sur la disparition de la vitamine B des graines germant dans un germoir ou dans le sol. Compt. rend. soc. biol. 100, 587-589.

Hoagland, R.

1923. Vitamin B in the edible tissues of the ox, sheep and hog. I. Vitamin B in the voluntary muscle. II. Vitamin $\mathrm{B}$ in the edible viscera. U. S. Dept. Agr. Bull. 1138, pp. 48. 
1924. Antineuritic value of hog muscle. Am. J. Physiol. 67, 300-308.

1929. Antineuritic and water-soluble B vitamins in beef and pork. J. Agr.

Research 38, 431-446.

Hoagland, R. and Lee, A. R.

1924. Antineuritic vitamin in poultry flesh and eggs. J. Agr. Research 28, 461-472.

1926. Vitamin A in poultry flesh and fat. J. Agr. Research 33, 29-40.

Hoagland, R. and Snider, G. G.

1925. Vitamin A in beef, pork and lamb. J. Agr. Research 31, 201-221.

1926. Vitamin A in oleo oil and oleo stearin. J. Agr. Research 32, 397-416.

1930. Beef extract as a source of vitamin G. J. Agr. Research, 40, 977-990.

a. Vitamin G in certain meats and meat by-products. J. Agr. Research 41, 205-220.

Hodgson, A.

1921. Vitamin deficiency and factors in metabolism relative to the development of rickets. Lancet, 1921, II, 945-949.

Höjer, J. A.

1924. Studies in scurvy. Uppsala. (From Acta Paediatrica Vol. III. Supplementum.) pp. 278.

1926. Method for determining the antiscorbutic vaiue of a foodstuff by means of histological examination of the teeth of young guinea pigs. Brit. J. Exptl. Path. 7, 356-360.

Höjer, A. and Westin, G.

1925. Jaws and teeth in scorbutic guinea-pigs: A histopathological study. Dental Cosmos 67, 1-24.

\section{Hoet, J.}

1923. Study of artificial nutrition in the pigeon and of the deficiency in vitamins. Biochem. J. 17, 220-229.

1924. Note concerning the deficiency in vitamin A in the pigeon. Biochem. J. $18,412,413$.

\section{Hoffman, C.}

1925. Production and nutritional value of commercial wheat germ extract. Ind. Eng. Chem. 17, 498-503.

\section{Hofmeister, F.}

1920. Zur Kenntnis der alkaloidischen Bestandteile der Reiskleie. Biochem. Z. $103,218-224$.

1922. Studien über qualitative Unternährung. I. Die Rattenberiberi. Biochem. Z. $128,540-556$.

a. Studien über qualitative Unternährung. II. Der experimentelle Nachweis des Antineuritins. Biochem. Z. 129, 477-486.

1923. Vitaminstudien. III. Die chemische Eigenschaften des Antineuritins. Ergebnisse Physiol. 22, 32-38.

Hogan, A. G.

1917. The effect of high temperatures on the nutritive value of foods. $J$. Biol. Chem. 30, 115-123.

Hogan, A. G., Guerrant, N. B. and Kempster, H. L.

1925. Concerning the adequacy of synthetic diets for the growth of the chick. J. Biol. Chem. 64, 113-124. Missouri Agr. Sta. Research Bull. 81, 1925, 3-51. 
Hogan, A. G. and Harshaw, H. M.

1924. Nutritional requirements for normal reproduction. J. Metabolic Research 5, 111-128.

1926. The relation of diet to bodily activity and the capacity to withstand unfavorable circumstances. Missouri Agr. Expt. Sta. Bull. 236, 35, 36.

a. Some relations between fertility and the composition of the diet. Missouri Agr. Expt. Sta. Research Bull. 94, pp. 23.

b. The relation of diet to bodily activity and to capacity to withstand unfavorable circumstances. Missouri Agr. Expt. Sta. Bull. 244, 29.

Hogan, A. G. and Hunter, J. E.

1928. The action of ultraviolet rays on vitamin B. J. Biol. Chem. 78, XVII.

a. The plural nature of vitamin B. J. Biol. Chem. 78, 433-444.

Hogan, A. G., Hunter, J. E. and Shrewsbury, C. L.

1927. The relation of diet to bodily activity and to capacity to withstand unfavorable circumstances. Missouri Agr. Expt. Sta. Bull. 256, 50, 51.

Hogan, A. G. and Shrewsbury, C. L.

1926. Nutritional requirements of poultry. Missouri Agr. Expt. Sta. Bull. 244, 19.

Holm, E.

1925. Demonstration of hemeralopia in rats nourished on food devoid of fatsoluble-A-vitamin. Am. J. Physiol. 73, 79-84.

Holm, G. E.

1928. Vitamins A, D, and $\mathrm{E}$ and the oxidation of fats and oils. Science 67, 424, 425.

Holmes, A. D.

1922. Studies of the vitamine of cod liver oils. I. The potency of crude cod liver oil, pressed cod liver oil, and cod liver stearin. J. Metabolic Research 2, 113-122.

a. Studies of the vitamine potency of cod liver oils. III. The potency of pollack liver oil-early summer oils. J. Metabolic Research 2, 361-365.

1923. Studies of the vitamin potency of cod liver oils. II. The vitamin potency of "spring" cod liver oil. J. Metabolic Research 3, 393-398.

a. Studies of the vitamin potency of cod liver oils. IV. To what extent is quantitative estimation of vitamin A possible? J. Metabolic Rescarch 3, 583-587.

1924. Preserving the high vitamin potency of cod liver oils. Am. Food J. 19, $55,56,85$.

a. Studies of the vitamin potency of cod-liver oil. VI. The effect of storage of livers on the vitamin A potency of cod-liver oil. Ind. Eng. Chem. 16, 295297.

b. Studies of the vitamin potency of cod-liver oil. VII. The vitamin A potency of hake-liver oil. Ind. Eng. Chem. 16, 379, 380.

c. Vitamin potency of cod-liver oils. IX. Oil produced by the rotting process. Ind. Eng. Chem. 16, 964, 965.

d. Vitamin potency of cod-liver oils. X. Medicinal cod-liver oils. Ind. Eng. Chem. 16, 1181-1184.

e. Studies of the vitamin potency of cod liver oils. XII. The color reactions of cod liver oil. J. Am. Pharm. Assocn. 13, 532-535.

f. Studies of the vitamin potency of cod liver oils. VIII. Potency of haddock liver oil. J. Metabolic Research 5, 259-262. 
1925. Vitamin potency of cod-liver oils. XI. Butter fats produced on summer feeds. Ind. Eng. Chem. 17, 75-78.

a. Studies of the vitamin potency of cod liver oils. XVI. The vitamin potency of shad body oil. Boston Med. Surg. J. 192, 300-302.

1926. Modern cod liver oil as a source of fat-soluble vitamins. Boston Med. Surg. J. 194, 714-716.

a. Studies of the vitamin potency of cod liver oils. XIX. Influence of cod liver oil on reproduction. Poultry Sci. 5, 110-116.

Holmes, A. D., Dolittle, A. W. and Moore, W. B.

1927. Studies of the vitamin potency of cod-liver oils. XXI. The stimulation of reproduction by fat-soluble vitamins. J. Am. Pharm. Assocn. 16, 518-521. Holmes, A. D. and Pigott, M. G.

1925. Vitamin potency of cod-liver oils. XIII. Vitamin A potency of dogfish liver oil. Ind. Eng. Chem. 17, 310, 311.

a. Studies of the vitamin potency of cod liver oils. XVII. The vitamin potency of salmon body oil. Boston Med. Surg. J. 193, 726-728.

1926. Studies of the vitamin potency of cod liver oils. XIV. The variation in daily food consumption of experimental animals. Boston Med. Surg. J. 194, 537, 538.

a. Vitamin potency of cod-liver oils. XVIII. Effect on vitamin potency of cold-pressing cod-liver oils. Ind. Eng. Chem. 18, 188, 189.

b. Studies of the vitamin potency of cod liver oils. XX. Effect of light on the vitamin A content of cod liver oil. Boston Med. Surg. J. 195, 263-265.

Holmes, A. D., Wyman, E. T., Smith, L. W. and Pigott, M. G.

1928. A comparison of cod liver oil and ultra-violet light for use in the prevention of rickets. Am. J. Diseases Children 36, 952-965.

Holst, A.

1907. Experimental studies relating to "ship beri-beri" and scurvy; I. Introduction. J. Hyg. 7, 619-633.

1911. The etiology of beriberi. Trans. Soc. Trop. Med. Hyg. London 5, 76-80.

1912. Diet in relation to disease. Trans. 15 Inter. Cong. Hyg. Demog. Washington 2, 583-590.

1918. Uber die Beriberi-Krankheit und ihre Ursachen auf Norwegischen Schiffen. Centr. Bakt. Parasitenk. Orig. 81, 56-72.

Holst, A. and Frölich, T.

1907. Experimental studies relating to "ship-beri-beri" and scurvy. II. On the etiology of scurvy. J. Hyg. 7, 634-671.

1912. Über experimentellen Skorbut. Ein Beitrag zur Lehre von dem Einfluss einer einseitigen Nahrung. Z. Hyg. Infektionskrankh. 72, 1-120.

1913. Über experimentellen Skorbut. II. Weitere Untersuchungen über das Konservieren und Extrahieren der spezifischen Bestandteile der antiskorbutischen Nahrungsmittel. Z. Hyg. Infektionskrankh. 75, 334-344.

1916. Further investigations on scurvy with special reference to the preservation of white cabbage in such a way as to retain its antiscorbutic properties.

(Translated title.) Norsk. Mag. Laegevidensk. 77, 1008-1039.

1920. On the preservation of the antiscorbutic properties of cabbage by drying.

J. Trop. Med. Hyg. 23, 261-263.

Holst, P. M.

1927. Experimental rickets. J. Hyg. 26, 437-440.

Holt, L. E., Jr. and Shipley, P. G. 
1928. The presence of an unknown factor in serum which influences calcification. Proc. Soc. Exptl. Biol. Med. 25, 572-573.

Holz, F.

1927. Das antirachitische Vitamin. Klin. Wochschr. 6, 535, 536.

Honeywell, E. M. and Steenbock, H.

1924. The synthesis of vitamin C by germination. Am. J. Physiol. 70, 322-332.

Honeywell, H. E.

1921. Studies of the sugar of the blood of pigeons. Am. J. Physiol. 58, 152-167.

Honeywell, H. E. and Dutcher, R. A.

1927. Bone calcification in rats as influenced by ultra-violet light from various sources. Pennsylvania Agr. Expt. Sta. Bull. 213, 5.

Honeywell, H. E., Dutcher, R. A. and Dahle, C. D.

1930. Vitamin studies. XVII. Ossifying potency of raw and evaporated milks. J. Nutrition 2, 251-256.

Honeywell, H. E., Dutcher, R. A. and Ely, J. O.

1928. Vitamin A in yeast. Pennsylvania Agr. Expt. Sta. Bull. 230, 8.

1929. Vitamin A technique as influenced by yeast from various sources. Pennsylvania Agr. Expt. Sta. Bull. 243, 5.

Hoobler, R. R.

1928. Symptomatology of vitamin B deficiency in infants. J. Am. Med. Assocn. 91, 307-310.

Hoobler, R. R., Outhouse, J. and Macy, I. G.

1926. Certain biological properties of human milk. Am. J. Diseases Children $32,456,457$.

\section{Hopkins, F. G.}

1906. The analyst and the medical man. Analyst 31, 385-397.

1912. Feeding experiments illustrating the importance of accessory factors in normal dietaries. J. Physiol. 49, 425-460.

1913. Diseases due to deficiencies in diet. Lancet, 1913, II, 1309, 1310.

1919. A lecture on the practical importance of vitamins. Brit. Med. J. No. $3043,507-510$.

1920. Note on the vitamin content of milk. Biochem. J. 14, 721-724.

a. The effects of heat and aeration upon the fat-soluble vitamin. Biochem. J. 14, 725-733.

1921. Recent advances in science in their relation to practical medicine and the nutritional requirements of the body. Lancet, 1921, I, 1-7.

a. On an autoxidisable constituent of the cell. Biochem. J. 15, 286-305.

1923. The present position of the vitamin problem. I, II. Brit. Med. J. Nos. 3277, 3278, 691-693; 748-750.

Hopkins, F. G. et al.

1919. Report on the present state of knowledge concerning accessory food factors (Vitamins). Natl. Health Inst. Med. Research Comm. (Gt. Brit.) Spec. Rept. No. 38, pp. 107.

1919. Discussion on the treatment and management of diseases due to dietetic deficiencies. Proc. Roy. Soc. Med. Sec. Ther. Pharm. 13, 1.

1920. Discussion on the present position of vitamins in clinical medicine. Brit. Med. J. II, 147-160. 
1924. Report on the present state of knowledge of accessory food factors

(Vitamins). Med. Research Council (Gt. Brit.) Spec. Rept. No. 38 (revised), pp. 171.

Hopkins, F. G. and Chick, $\mathbf{H}$.

1919. Accessory factors in food. Lancet, 1919, II, 28, 29.

Hopkins, F. G. and Neville, A.

1913. A note concerning the influence of diets upon growth. Biochem. J. 7, 97-99.

Hornemann, C.

1925. Über den Vitamingehalt der Sojabohne. Z. Untersuch. Lebensm. 49, 114-120.

Horvath, A. A.

1927. The effect of turnip on avian polyneuritis. Japan Med. World 7, 71-75.

Hotta, K.

1923. Über das Verhalten des Cholesterins bei Tauben-Beriberi. II. Z. physiol. Chem. 128, 85-98.

Hottinger, A.

1926. Bestrahltes Cholesterin in der Therapie der Rachitis. Klin. Wochschr. 5, 2061-2065.

1927. Über den Einfluss des Ultravioletten Lichtes auf den C-Vitamingehalt der Milch. Klin. Wochschr. 6, 1793-1797.

a. Untersuchungen über bestrahltes Ergosterin. Beiträge zur direkten und indirekten Lichttherapie der Rachitis beim Tier, beim Kind und am Erwachsenen. Deut. med. Wochschr. 53, 1549-1550.

b. Experimentelle Untersuchungen über das antirachitische Prinzip bestrahlter Nahrung. Z. Kinderheilk. 43, 8-23.

c. Untersuchungen über bestrahltes Ergosterin. IV. Beiträge zur indirekten Lichttherapie der rachitischen Knochenkrankheiten am Tier beim Kind und am Erwachsenen. Z. Kinderheilk. 44, 282-321.

Hou, H. C.

1928. Studies on the Glandula uropygialis of birds. Chinese J. Physiol. 2, 345-378.

1929. Relation of the preen gland (Glandula uropygialis) of birds to rickets. Chinese J. Physiol. 3, 171-181.

Hou, H. C. and Tso, E.

1930. The rôle of the skin in the cure of rickets by irradiation. Chinese $J$. Physiol. 4, 93-116.

House, M. C., Nelson, P. M. and Haber, E. S.

1929. The vitamin $\mathrm{A}, \mathrm{B}$, and $\mathrm{C}$ content of artificially versus naturally ripened tomatoes. J. Biol. Chem. 81, 495-504.

1930. The vitamin B content of vegetables. I. The distribution of vitamin B in the carrot. II. The effect of sunlight on the vitamin B content of lettuce, kohlrabi, and tomatoes. III. The effect of storage on the vitamin B content of carrots. Iowa Agr. Expt. Sta. Research Bull. 120, 333-344.

Howard, C. P. and Ingvaldsen, $T$.

1917. The mineral metabolism of experimental scurvy of the monkey. Bull. Johns Hopkins Hosp. 28, 222-225. 
Howe, P. R.

1920. Effects of scorbutic diets upon the teeth. Dental Cosmos 62, 586-590.

a. Dental caries. Dental Cosmos 62, 921-929.

1922. Decalcification of teeth and hones, and regeneration of bone through diet. J. Am. Med. Assocn. 79, 1565-1567.

1927. Investigations of dental caries. J. Am. Dental Assocn. 14, 1864-1866.

Howland, J. and Kramer, B.

1921. Calcium and phosphorus in the serum in relation to rickets. Am. J. Diseases Children 22, 105-119.

Hoyle, E.

1929. The vitamin content of honey. Biochem J. 23, 54-60.

Hoyle, E. and Zilva, S. S.

1927. The antiscorbutic fraction of lemon juice. VI. Biochem. J. 21, 11211127.

Hoyle, J. C.

1929. The alleged toxic action of accessory food factors. Lancet, 1929, I, 734736.

1930. The toxic effects of irradiated ergosterol. J. Pharm. 38, 271-289.

Hoyle, J. C. and Buckland, $\mathbf{H}$.

1929. Further observations on the effects of large doses of irradiated ergosterol. Biochem. J. 23, 558-565.

Hryniewicz, M. and Lawrynowicz, A.

1927. Le sang des cobayes au cours du scorbut expérimental. J. physiol. path. gén. 25, 674-677.

Hsu, K. L.

1928. The antiscorbutic vitamin value of some Peking fruits. Chinese J. Physiol. 2, 41-44.

Hughes, J. S., Aubel, C. E. and Lienhardt, H. F.

1928. The importance of vitamin $A$ and vitanin $C$ in the ration of swine, concerning especially their effect on growth and reproduction. Kansas Agr. Expt. Sta. Bull. 23, pp. 48.

Hughes, J. S., Fitch, J. B., Cave, H. W. and Riddell, W. H.

1927. Relation between the vitamin C content of a cow's ration and the vitamin C content of its milk. J. Biol. Chem. 71, 309-316.

Hughes, J. S., Lienhardt, H. F. and Aubel, C. E.

1929. Nerve degeneration resulting from avitaminosis A. J. Nutrition 2, 183-186.

Hughes, J. S., Nitcher, C. and Titus, R. W.

1925. The relative value of ultraviolet light and irradiated air in preventing rickets in chickens. J. Biol. Chem. 63, 205-209.

Hughes, J. S. and Payne, L. F.

1924. Influence of ultraviolet light on young laying hens. Scicnce 60, 549, 550 .

Hughes, J. S., Payne, L. F. and Latshaw, W. L.

1925. The influence of ultraviolet light on leg weakness in growing chicks and on egg production. Poulry Sci. 4, 151-156.

Hughes, J. S., Payne, L. F., Titus, R. W. and Moore, J. M.

1925. The relation between the amount of ultraviolet light received by hens and the amount of antirachitic vitamin in the eggs produced. J. Biol. Chem. $66,595-600$. 
Hughes, J. S. and Pycha, R. L.

1928. A preliminary report of the measurement of variation of energy in the "vita spectrum" of the sunshine in Kansas. Trans. Illum. Eng. Soc. N. Y. 23, 233-246.

Hughes, J. S. and Titus, R. W.

1926. Should leg weakness in growing chicks be called rickets? J. Biol. Chem. 69, 289-294.

Hughes, J. S., Titus, R. W. and Moore, J. M.

1925. Ultraviolet light and antirachitic vitamin in the hen's eggs. Science 62, 492, 493.

Hughes, J. S., Titus, R. W. and Witham, L.

1925-6. The relative values of green oat-sprouts, green alfalfa, and cod liver oil in the prevention of rickets in growing chicks. Poultry Sci. 5, 59-66.

Hughes, T. A., Shrivastava, D. L. and Sahai, P. N.

1929. Observations on the serum calcium and inorganic phosphorus in health and disease. Ind. J. Med. Research 17, 461-469.

a. Observations on the blood chemistry in osteomalacia. Ind. J. Med. Research 17, 470-476.

Hugounenq, L. and Couture, E.

1929. Sur le stérol de l'huile de foie de morue et l'action photochimique de quelques stérols. Bull. soc. chim. biol. 11, 956-964.

a. Action exercée sur la plaque photographique par le cholestérol extrait de l'huile de foie de morue. Compt. rend. 188, 349, 350.

b. Sur l'action photochimique de stérols de diverses origines. Compt. rend. $188,742,743$.

c. Sur l'activité photochimique de divers stérols et sur la nature de leur action. Compt. rend. 189, 47-49.

Huldschinsky, $\mathrm{K}$.

1919. Heilung von Rachitis durch künstliche Höhensonne. Deut. med. Wochschr. 45, 712, 713.

1919-20. Die Behandlung der Rachitis durch Ultraviolettbestrahlung dargestellt an 24 Fällen. $Z$. orthop. Chir. 39, 426-451.

1926. Die antirachitische Zone des Ultraviolett. Klin. Wochschr. 5, 1972-1973.

1928. Preventive irradiation of children against rickets. Brit. J. Actinother. 3, 103-105.

Hulshoff-Pol, D. J.

1902. Katjang Idjo, un nouveau médicament contre le béri-béri. Janus 7, 524-534.

1906. Beriberi and katjang-idjo. Geneeskund. Tijdschr. Nederland.-Indië 46, 477.

1907. X-zuur het tegen beriberi werkzame bestanddeel uit de Katjanghidjoe. Geneeskund Tijdschr. Nederland.-Indï 47, 688-702.

1909. Polyneuritis gallinarum en beri-beri. Geneeskund. Tijdschr. Nederland.Indië 49, 116-129.

1910. Beriberi-Forschungen in den Niederländisch-Ostindischen Kolonien, besonders in bezug auf Prophylaxis und Heilung. Arch. Schiffs Trop. Hyg. Beihefte 3, XIV, p. 38.

1917. X-acid as a remedy in polyneuritis and beriberi. J. Physiol. 51, 432-439. 


\section{Hume, E. M.}

1921. Comparison of the growth-promoting properties for guinea-pigs of certain diets, consisting of natural foodstuffs. Biochem. J. 15, 30-48.

a. Investigation of the antiscorbutic value of full cream sweetened condensed milk by experiments with monkeys. Biochem. J. 15, 163-166.

1922. The effect of radiation with the mercury vapor quartz lamp on the growth of rats fed on a diet deficient in vitamin A. Lancet, 1922, II, 13181321.

1925. The action of ultra-violet light upon the growth of rats. Brit. Med. J. No. 3373, 341, 342 .

Hume, E. M., Lucas, N. S. and Smith, H. H.

1927. On the absorption of vitamin D from the skin. Biochem. J. 21, $362-367$.

Hume, E. M. and Smedley-Maclean, I.

1930. Some further notes on the relation of carotene to vitamin A. Lancet, 1930, I, 290-292.

Hume, E. M. and Smith, H. H.

1923. The effect of air which has been exposed to the radiations of the mercury vapor quartz lamp in promoting the growth of rats fed on a diet deficient in fat-soluble vitamins. Biochem. J. 17, 364-372.

1924. The effect of irradiation of the environment with ultra-violet light upon the growth and calcification of rats fed on a diet deficient in fat-soluble vitamins-The part played by irradiated sawdust. Biochem. J. 18, 1334-1335.

1926. The effect of irradiation of the environment with ultra-violet light upon the growth and calcification of rats fed on a diet deficient in fat-soluble vitamins. Biochem. J. 20, 335-339.

a. A note on the production by irradiation with ultra-violet light of antirachitic properties in sterols derived from the small Siak Illipe nut, Palaquium burckii. Biochem. J. 20, 340-342.

1928. A critical examination of methods of evaluating vitamin A by means of the growth of rats. Biochem. J. 22, 504-521.

Hume, E. M., Smith, H. H. and Smedley-MacLean, I.

1928. The examination of yeast-fat for the presence of vitamins $A$ and D before irradiation and of vitamin D after irradiation. Biochem. J. 22, 27-33.

a. The examination of irradiated zymosterol for the presence of vitamin $D$. Biochem. J. 22, 980-986.

Humphris, F. A.

1925. Light in the prevention of rickets. Lancet, 1925, I, 912, 913.

Hunt, C. $\mathrm{H}$.

1928. Vitamin B. Science 67, 556.

a. The complex nature of vitamin $\mathrm{B}$ as found in wheat and corn. J. Biol. Chem. 78, 83-90.

b. Further evidence of the complex nature of vitamin B. I. Evidence that a third factor exists. J. Biol. Chem. 79, 723-731.

Hunt, C. H. and Hosack, W.

1930. Influence of fertilizers on the vitamin B content of wheat. Ohio Agr. Expt. Sta. Bull. 446, 149.

Hunt, C. H. and Krauss, W. E.

1928. The relative antineuritic and antipellagric potency of cow's milk. J. Biol. Chem. 79, 733-738. 
Hunt, C. H. and Winter, A. R.

1922. Cow's milk versus goat's milk as a source of the antiscorbutic vitamin. Science 56, 114.

1923. A factor causing the assimilation of calcium. Science $57,717,718$.

Hunt, C. H., Winter, A. R. and Miller, R. C.

1923. A possible factor influencing the assimilation of calcium. J. Bial. Chem. 55, 739-742.

Hunter, A., Givens, M. H. and Lewis, R. C.

1916. Preliminary observations of metabolism in pellagra. U. S. Pub. Health Service Hyg. Lab. Bull. 102, 39-67.

Hunter, D.

1930. The significance to clinical medicine of studies in calcium and phosphorus metabolism. Lancet, 1930, I, 897-904.

Hunwicke, R. F.

1928. Recent advances in our knowledge of vitamins. Quart. J. Pharm. I, 581-589.

Husband, A. D., Godden, W. and Richards, M. B.

1923. The influence of cod-liver oil, linseed oil, and olive oil on the assimilation of calcium and phosphorus in the growing pig. Biochem. J. 17, 707-719.

Huston, R. C. and Lightbody, H. D.

1928. Some biochemical relations of phenols. I. Hydroquinone. J. Biol. Chem. 76, 547-558.

Hutchison, H. S.

1920. Is fat starvation a causal factor in the production of rickets? Glasgow Med. J. 93, 8-12.

\section{Idaho Agr. Expt. Sta.}

1929. Vitamin C in Idaho potatoes. Bull. 164, 32.

Ide, $M$.

1907. Über Wildier's Bios. Centr. Bakt. Parasitenk. II. Abt., 18, 193-199.

1921. The "bios" of Wildiers and the cultivation of yeast. J. Biol. Chem. 46, 521-523.

Igarashi, E. and Tagaya, T.

1929. Studies in experimental scurvy. VI. On the amounts of unknown unsaponifiable substance and cholesterol in organ tissues of guinea pigs fed on a vitamin C free diet. J. Biochem. 11, 239-250.

Illinois Agr. Expt. Sta.

1925. Vitamin deficiencies in poultry rations. Ann. Rept. 1924-25, pp. 80-82.

1927. The distribution of vitamin B in the rice kernel and in corn products. Ann. Rept., pp. 263, 264.

1928. Diet rich in vitamin B needed during lactation. Ann. Rept., pp. 302, 303.

a. Vitamin A of corn concentrated in endosperm. Ann. Rept., p. 303.

b. Vitamin B found in embryo and endosperm of oats. Ann Rept., pp. 303, 304.

1929. Further studies show little vitamin A in oats. Ann. Rept., p. 249.

Indiana Agr. Expt. Sta.

1924. The vitamin requirement of animals. Ann. Rept., pp. 35, 36. 
Ingier, $\mathbf{A}$.

1915. A study of Barlow's disease experimentally produced in fetal and newborn guinea pigs. J. Exptl. Med. 21, 525-538.

Iowa Agr. Expt. Sta.

1923. The relation of vitamins to disease resistance. Ann. Rept., p. 35.

Irving, L. and Ferguson, J.

1925. Influence of acidity in the intestine upon the absorption of calcium salts in the blood. Proc. Soc. Exptl. Biol. Med. 22, 527.

Ishido.

1923. Über den kompensatorischen Einfluss des ultravioletten Lichtes auf die avitaminösen Störungen am Knochenmarke. Biochem. Z. 137, 184-192.

Iwabuchi, $\mathbf{T}$.

1922. Über Organanalysen bei experimentellen Skorbut der Meerschweinchen nebst einigen Angaben über den Blutbefund. Z. ges. exptl. Med. 30, 65-79.

Iwasaki, Y.

1927. On the content of vitamin C in Japan sand pear (Cyrus serotina Rehder), kaki (Diospyrus kaki L.), and Satsuma orange (Citrus unshin). J. Okitsu Hort. Soc. 22, 1-10.

Iwata, M.

1928. On polyneuritis of pigeon and albino rat due to the deficiency of oryzanin (vitamin B). Bull. Agr. Chem. Soc. Japan. 4, 63, 64.

Izume, S. and Komatsubara, I.

1928. Studies on experimental rickets. I. On the antirachitic property of crude "biosterin," the cholesterin-free, unsaponifiable fraction of cod-liver oil. $J$. Biochem. 9, 233-241.

Izume, S., Yoshimaru, Y. and Komatsubara, I.

1928. Studies on experimental rickets. II. The influence of ultra-violet irradiation on the antirachitic value of soybean oil. J. Biochem. 10, 177-182.

Jackson, C. M.

1919. The postnatal development of the suprarenal gland and the effects of inanition upon its growth and structure in the albino rat. Am. J. Anat. 25, 221-289.

1925. "The Effects of Inanition and Malnutrition upon Growth and Structure," Philadelphia, P. Blakiston's Son \& Co.

1929. Recent work on the effects of inanition and malnutrition on growth and structure. Arch. Path. 7, 1042-1078; 8, 81-122, 273-315.

Jackson, C. M. and Carleton, R.

1923. The effect of experimental rickets upon the weights of the various organs in albino rats. Am. J. Physiol. 65, 1-14.

Jackson, C. M. and Stewart, C. A.

1919. Recovery of normal weight in the various organs of albino rats on refeeding after underfeeding from birth for various periods. Am. J. Diseases Children 17, 329-352.

1920. The effects of inanition in the young upon the ultimate size of the body and of the various organs in the albino rat. J. Exptl. Zool. 30, 97-128.

Jackson, F. G. and Harley, V.

1900. An experimental inquiry into scurvy. Lancet, 1900, I, 1184-1188. 
Jackson, L.

1918. Demonstration of micrococci in the bones in rickets and scurvy. J. Infect. Diseases 22, 457-461.

Jackson, L. and Moody, A. M.

1916. Bacteriologic studies on experimental scurvy in guinea pigs. J. Infect. Diseases 19, 511-514.

Jackson, L. and Moore, J. J.

1916. Studies on experimental scurvy in guinea pigs. J. Infect. Diseases 19, 478-510.

Jameson, H. L., Drummond, J. C. and Coward, K. H. 1922. Synthesis of vitamin A by a marine diatom (Nitzschia closterium) growing in pure culture. Biochem. J. 16, 482-485.

Janke, A. and Lacroix, $\mathrm{H}$.

1927. Über das Vorkommen von Vitamin D im Gärungsessig. Biochem. $Z$. $190,67-74$.

Jansen, B. C. P.

1917-18. The quantity of fat-soluble vitamins in cocoanut oil. Mededeel. Geneeskund. Lab. Weltevreden 3 Ser. A, 78-94.

a. Is vitamine identical with secretine? Mededeel. Geneeskund. Lab. Weltevreden, 3 Ser. A, 99-104.

1920. Coconut press cake as a protein-containing food for human consumption. Mededeel. Geneeskund. Lab. Welterreden 3 Ser. A, 1-21.

a. On the vitamine-amount of extract of rice bran, and methods of estimating it. Mededeel. Geneeskund. Lab. Weltevreden, 3 Ser. A, 22-49.

1923. On the need of antiberiberi vitamin of the animal organism and on the amount of this vitamin in different foodstuffs. Mededeel. Burgerlijk. Geneeskund Dienst Nederland.-Indie, 1, 1-122.

a. On the nutrient value and the ferment content of 100 years old rice. Mededeel. Burgerlijk. Geneeskund. Dienst Nederland.-Indië 1, 123-135.

1929. Improvements in the method of isolating the anti-beri-beri vitamin. Rec. trav. chim. 48, 984, 985.

Jansen, B. C. P. and Donath, W. F.

1924. The A-vitamin content of different Indian foodstuffs and the value of the proteins of these latter as a supplement to the proteins of rice. Mededeel. Burgerlijk. Geneeskund. Dienst Nederland.-Indië 1, 46-98.

1925. On the quantity of antiscorbutic vitamin in some citrus species and in bananas. Mededeel. Dienst Volksgezondheid Nederland.-Indï Part III, 225239.

1926. Antineuritisch Vitamine. Chem. Weekbl. 23, 201-203.

a. On the isolation of the anti-beriberi vitamin. Proc. Koninklijke Akad. Wetensch. Amsterdam 29, 1390-1400.

1927. Isolation of anti-beriberi vitamin. Mededecl. Dienst Volksgezondheid Nederland.-Indië 16, 186-189.

1928. The nutritive value of maize and its various preparations on Java. Mededeel. Dienst Volksgezondheid Nederland.-Indië 17, 92-119.

a. The amount of vitamin-A in different varieties of batata. Mededeel. Dienst Volksgezondheid Nederland.-Indië 17, 120-125.

b. The amount of vitamin-A in Indian fruits. Mededeel. Dienst Volksgezondheid Nederland.-Indië 17, 126-137. 
Jansen, B. C. P. and Mangkoewinoto, R. M. M.

1920. The respiratory quotient of birds fed on polished rice. Mededecl.

Geneeskund. Lab. Weltureden, 3 Ser. A, 51-65.

Jarussowa, $\mathbf{N}$.

1926. Gas- und Stickstoffwechsel bei dem experimentellen Skorbut der Meerschweinchen. Biochem. Z. 179, 104-111.

1928. Stickstoffbilanz und $\mathrm{C} / \mathrm{N}$ Koefficient des Harns bei dem experimentellen, durch den Hunger nicht komplizierten Skorbut. Biochem. Z. 198, 128-137.

Javillier, M.

1930. Le carotène et la croissance des animaux. Bul. soc. chim. biol. 12, 554578.

Javillier, M., Allaire, H. and Rousseau, S.

1928. Phosphore nucléique et bilans phosphorés chez des souris carencées en facteurs liposolubles. Bul. soc. chim. biol. 10, 294-300.

Javillier, M., Baude, P. and Levy-Lageunesse, S.

1924. Essais d'identification du facteur A. Le facteur A et le phytol. Compt. rend. 179 , 998-1000.

1925. Sur le dosage physiologique du facteur A. Bul. soc. chim. biol. 7, 831-841.

Javillier, M. and Emerique, L.

1929. Le facteur accessoire de l'alimentation dit "Facteur A" dans les huiles d'olive brutes et raffinées. Bul. soc. hyg. aliment. 17, 420-422.

1930. Sur l'activité vitaminique du carotène. Compt. rend. 190, 655-657.

Javillier, M., Rousseau, S. and Emerique, L.

1929. La composition chimique des tissus dans l'avitaminose A : phosphore, extrait lipoidique, cholestérol. Compt. rend. 188, 580-582.

Jendrassik, A.

1923. A color test for water-soluble B. J. Biol. Chem. 57, 129-138.

Jendrassik, A. and Keményffi, A. G.

1927. Zur Kenntnis des D-Vitasterins. I. Uber die Aktivierbarkeit des Cholesterins. Biochem. Z. 189, 180-190.

1928. Zur Kenntnis des D-Vitasterins. Biochem. Z. 201, 269-280.

Jephcott, $\mathrm{H}$.

1923. The stability of vitamins. J. State $\mathrm{Med} .31,471-476$.

Jephcott, H. and Bacharach, A. L.

1921. The antiscorbutic value of dried milk. Biochen. J. 15, 129-139.

1926. A rapid and reliable test for vitamin D. Biochem. J. 20, 1351-1355.

1928. The quantitative estimation of vitamin D. Biochem. J. 22, 60-62.

Jesus, F. de.

1927. The vitamin B content of some Philippine fruits and vegetables. III. Philippine Agr. 15, 533-546.

Jewesbury, R. C. and Ling, T. M.

1929. The relationship between feeding and the development of rickets. Lancet, 1929, I, 872, 873.

Jobling, J. W. and Arnold, L.

1923. Observations and reflections on the etiology of pellagra. J. Am. Med. Assocn. 80, 365-368.

Jobling, J. W. and Peterson, W.

1916-17. Epidemiology of pellagra in Nashville, Tennessee. J. Infect. Diseases $18,501-567$ (1916) ; 21, 109-131 (1917). 
Johns, C. O. and Finks, A. J.

1920. Studies in nutrition. IV. The nutritive value of peanut flour as a supplement to wheat flour. J. Biol. Chem. 42, 569-579.

1921. Studies in nutrition. V. The nutritive value of soy bean flour as a supplement to wheat flour. Am. J. Physiol. 55, 455-461.

Johns, C. O., Finks, A. J. and Paul, M. S.

1919. Studies in nutrition. I. The nutritive value of coconut globulin and coconut press cake. J. Biol. Chem. 37, 497-502.

1920. Studies in nutrition. III. The nutritive value of commercial corn gluten meal. J. Biol. Chem. 41, 391-399.

Johnson, J. M.

1921. The growth-promoting properties of milk and dried milk preparations. U. S. Pub. Health Repts. 36, 2044-2057.

Johnson, J. M. and Hooper, C. W.

1921. Antineuritic vitamin in skim milk powder. U. S. Pub. Health Repts. 36, 2037-2041.

1922. The comparative antiscorbutic values of milk. U. S. Pub. Health Repts. 37, 989-1020.

Jones, D. B.

1926. The nutritional value of oysters and other sea food. Am. J. Pub. Health $16,1177-1182$.

Jones, D. B. and Murphy, J. C.

1924. Cystine deficiency and vitamin content of the lentil. Lens esculenta. Moench. J. Biol. Chem. 59, 243-253.

1926. The vitamin content of oysters. Proc. Soc. Exptl. Biol. Med. 23, 519, 520.

Jones, D. B., Murphy, J. C. and Moeller, O.

1925. The effect of long continued storage at low temperature on the vitamin A content of eggs. Am. J. Physiol. 71, 265-273.

Jones, D. B., Murphy, J. C. and Nelson, E. M.

1928. Biological values of certain types of sea food. II. Vitamins in oysters (Ostrea virginica). Ind. Eng. Chem. 20, 205-210.

Jones, D. B. and Nelson, E. M.

1930. Vitamin content of ethylene-treated and untreated tomatoes. Am. J. Pub. Health 20, 387-394.

Jones, D. B., Nelson, E. M. and Murphy, J. C.

1928. Biological values of certain types of sea foods. III. Vitamins in clams. Ind. Eng. Chem. 20, 648-652.

Jones, I. R., Eckles, C. H. and Palmer, L. S.

1926. The rôle of vitamin A in the nutrition of calves. J. Dairy Sci. 9, 119139.

Jones, J. H.

1927. The relation of the inorganic constituents of a ration to the production of ophthalmia in rats. J. Biol. Chem. 75, 139-146.

Jones, J. H., Rapoport, M. and Hodes, H.

1930. The effect of irradiated ergosterol on thyroparathyroidectomized dogs. J. Biol. Chem. 86, 267-283. 
Jones, J. H., Steenbock H. and Nelson, M. T.

1924. Fat soluble vitamins.-XXII. The comparative amounts of vitamin A and antirachitic factor in butter fat and cod liver oil. J. Metabolic Research 6, 169-187.

Jones, J. M.

1928. Malt extracts and oil emulsions. II. The vitamin A content of commercial malt extract and cod-liver oil emulsions. Quart. J. Pharm. 1, 401, 402. Jones, M. R.

1924. Hydrochloric acid therapy in rickets. J. Am. Med. Assocn. 82, 439-440. Jones, R. L.

1928. Nomenclature of the accessory food factors. Science 68, $480,481$.

a. Avitaminosis in relation to disease resistance. Am. Med. 23, 961-968.

Jones, W. S., Briod, A. E., Arzoomanian, S. and Christensen, W. G.

1929. Limitations of the antimony trichloride test for quantitative estimation of vitamine A. J. Am. Pharm. Assocn. 18, 253-256.

Jonge, G. W. K. de.

1909. Investigations on beriberi. Geneeskund. Tijdschr. Nederland.-Indië 49, 165.

Jorstad, L. H. and Johnston, C. G.

1927. Local and systemic changes induced by lipoid solvents in animals fed on diets varying in vitamin content. Am. J. Path. 3, 489-499.

Jundell, I.

1921. Pathogenesis and treatment of rickets. Hygeia 83, 753. Abstracted in J. Am. Med. Assocn. 78, 772.

\section{Kansas Agr. Expt. Sta.}

1928. The vitamin content of some common fruits and vegetables. Bien. Rept. 123, 124 (1927-28).

Kapsinow, R. and Jackson, D.

1924. The prevention and cure of rickets by means of bile. Proc. Soc. Exptl. Biol. Med. 21, 472, 473.

\section{Karelitz, S.}

1928. Treatment of rickets with irradiated ergosterol (Vigantol). Proc. Soc. Exptl. Biol. Med. 25, 576-578.

a. Activated ergosterol in the treatment of rickets. Am. J. Diseases Children 36, 1108-1120.

Karelitz, S. and Shohl, A. T.

1927. Rickets in rats. I. Metabolism studies on high calcium-low phosphorus diets. II. The effect of phosphate added to the diet of rachitic rats. J. Biol. Chem. 73, 655-664; 665-677.

Karr, W. G.

1920. Some effects of water-soluble vitamin upon nutrition. J. Biol. Chem. 44, 255-276.

a. Metabolism studies with diets deficient in water-soluble B vitamin. J. Biol. Chem. 44, 277-282.

Karrer, P., Euler, B. v. and Euler, H. v.

1929. Zur Kenntnis der zur A-vitamin-Prüfung vorgeschlagenen Antimonchlorid-reaktion. Arkiv. Kemi Mineral. Geol. 10B, No. 2, pp. 6. 
Karshan, M.

1929. Calcification of teeth and bones on rachitic and non-rachitic diets. Proc. Soc. Exptl. Biol. Med. 27, 200-202.

Karshan, N., Krasnow, F. and Harrow, B.

1927. Feeding experiments with plants at different stages of development. III. Synthesis of vitamin in plants. Proc. Soc. Exptl. Biol. Med. 24, 765, 766.

1928. Feeding experiments with plants at different stages of development. III. Synthesis of vitamin in corn. Am. J. Physiol. 84, 314-316.

Kartascheffsky, E.

1926. Zur Frage der Vitamine. I. Über den Einfluss des Vitaminhungers auf den Gaswechsel bei Tauben. Arch. ges. Physiol. (Pfiïger's) 214, 499-515.

a. Zur Frage der Vitamine. II. Über die Auffüterung von Tauben mit vitaminfreier Nahrung nach vorhergehender Hungerperiode. Arch. ges. Physiol. (Pfiüger's) 215, 197-211.

Kato, G.

1924. Further studies on the nervous paralysis of the polished rice disease. Japan Med. World 4, 233-237.

Kato, G. and Shizume, S.

1921. The nature of the paralysis of birds suffering from polished rice disease (preliminary report). Keio Igaku (Keio Med. J.) 1. Abstracted in Japan Med. World 1, 22, See also Japan Med. World 1, 14-19.

Kato, G., Shizume, S. and Maki, K.

1921. The nature of the paralysis in beriberi-like disease or polished rice disease of the bird. II. Keio Igaku (Keio Med. J.) 1. Abstracted in Japan Med. World $1,16,17$.

Kauffman, A. B., Cheekmur, F. and Schultz, O. T.

1923. Changes in the temporal bones in experimental rickets. Their relation to otosclerosis. J. Am. Med. Assocn. 80, 681-685.

Kawakami, K. and Kimm, R.

1929. On the physiological action of carotin. Proc. Imp. Acad. Japan 5, 213215.

1930. On the physiological rôle of carotin and allied substances. Sci. Papers Inst. Phys. Chem. Research (Tokyo) 13, 231-243.

Kawamura, $\mathrm{K}$.

1929. The influence of vitamin B on the intestinal secretion. The method of the quantitative estimation of the proteolytic enzyme of the intestinal juice. Japan J. Exptl. Med. 7, 157-175.

Kay, H. D. and Zilva, S. S.

1923. The alleged specific color reaction for the antiscorbutic factor. Biochem. J. 17, 872-879.

Keefer, C. S.

1930. The beriberi heart. Arch. Internal Med. 45, 3-22.

Keith, M. H. and Mitchell, H. H.

1923. The effect of exercise on vitamin requirements. Am. J. Physiol. 65, 128-138.

Kelleway, C. H.

1921. The effect of certain dietary deficiencies on the suprarenal glands. Proc. Roy. Soc. (London) B 92, No. B 642, 6-27. 
Kelly, F. C. and Henderson, J. McK.

1930. The influence of certain dietary supplements on the nutrition of the African native. I. J. Hyg. 29, 418-428.

Kennedy, C.

1924. The nutritive properties of wild rice. J. Agr. Research 27, 219-224.

Kennedy, C. and Dutcher, R. A.

1922. Vitamin studies. IX. The influence of the diet of the cow upon the quantity of vitamins $\mathrm{A}$ and $\mathrm{B}$ in the milk. J. Biol. Chem. 50, 339-359.

Kennedy, C. and Palmer, L. S.

1922. Yeast as a source of vitamin B for the growth of rats. J. Biol. Chem. 54, 217-232.

1925. The antirachitic properties of breast milk. Proc. Soc. Exptl. Biol. Med. $23,230,231$.

1926. Hydrogenated vegetable oil as a source of vitamin E. Am. J. Physiol. 76, 316-319.

1928. The fundamental food requirements for the growth of the rat. III. Yeast and yeast fractions as a supplement to synthetic rations. IV. Coprophagy as a factor in the nutrition of the rat. J. Biol. Chem. 76, 591-606, 607622.

1929. Heat and ultra-violet irradiation as means of differentiating vitamins $B$ and $\mathrm{G}$ in yeast. J. Biol. Chem. 83, 493-496.

Kennedy, W. P.

1926. Reproduction and diet in the rat. Quart. J. Exptl. Physiol. 16, 281289.

1927. The newest vitamin. Science Progress ( London) 21, 659-664.

Kenny, C. L.

1926. A study of the thermostability of vitamin C. Dissertation, Columbia University, New York.

Kentucky Agr. Expt. Sta.

1928. Vitamin content of kale and mustard greens. Kentucky Agr. Expt. Sta. Rept. pp. 23, 24.

Kerr, R. W.

1928. The isolation of a Beta bios. Proc. Soc. Exptl. Biol. Med. 25, 344, 345.

Kerr, R. W., Eddy, W. H. and Williams, R. R.

1926. Further proof of the "bios" character of crystalline bios 223. Proc. Soc. Exptl. Biol. Med. 23, 416-419.

Kestner, O. and Borchardt, W.

1927. Weitere Strahlenmessungen nördlich des Polarkreises. Arch. ges. Physiol. (Pfïger's) 218, 469-474.

Kierferle, F. and Zeiler, $\mathrm{K}$.

1926. Die antiskorbutische Fähigkeit der Silagemilch, Ihre biologische Wertung im Vergleich mit Trockenfutter- und Schempe- Trebermilch und ihre Eignung als Kindermilch. Fortschritte Landw. 1, 83-90.

Kifer, H. B. and Munsell, H. E.

1929. Vitamin content of honey and honeycomb. J. Agr. Research 39, 355366.

Kik, M. C. and McCollum, E. V.

1928. The nutritive value of haddock and herring (Clupea harengus). Am.J. Hyg. 8, 671-693. 
King, H., Rosenheim, O. and Webster, T. A.

1929. Vitamin D from sterols of mummified Egyptian brain. Biochem. J. 23, $166,167$.

Kingery, H. M. and Kingery, J. R.

1925. A study of the nervous tissues of albino rats fed on a vitamin C-free diet. Anat. Record 29, 364.

Kinnersley, H. W. and Peters, R. A.

1925. Antineuritic concentrates from yeast. I. Biochem. J. 19, 820-826.

1927. Antineuritic yeast concentrates II. The use of norite charcoal in the concentration of torulin. Biochem. J. 21, 777-790.

1928. Antineuritic yeast concentrates. IV. The further purification of yeast vitamin $\mathrm{B}_{1}$ (curative). Biochem. J. 22, 419-433.

1929. Observations upon carbohydrate metabolism in birds. I. The relation between the lactic acid content of the brain and the symptoms of opisthotonus in rice-fed pigeons. Biochem. J. 23, 1126-1136.

1930. A localized lactic acidosis in the brains of pigeons suffering from $B_{1}$ deficiency. J. Physiol. 69, XI, XII.

a. Carbohydrate metabolism in birds. II. Brain localization of lactic acidosis in avitaminosis $\mathrm{B}$ and its relation to the origin of symptoms. Biochem. J. 24, 711-722.

Kinnersley, H. W., Peters, R. A. and Reader, V.

1928. Antineuritic concentrates. III. The curative pigeon test. A critique. Biochem. J. 22, 276-291.

Kinnersley, H. W., Peters, R. A. and Squires, B. T.

1925. Animal quinoidine. Preliminary paper. Biochem. J. 19, 404-413.

Kirsch, W.

1928. Die Aktivierung des antirachitischen Faktors in der Trockenhefe. Bio. chem. Z. 196, 294-300.

Kittelson, J. A.

1920. Effects of inanition and refeeding upon the growth of the kidney of the albino rat. Anat. Record 17, 281-297.

Klein, A., Harrow, B., Pine, L. and Funk, C.

1926. The nutritive value of various layers of the wheat and corn kernels. Am. J. Physiol. 76, 237-246.

Klein, G. T.

1927. A study of various methods of preventing rickets in chicks. Poultry Sci. 7, 31-40.

Klein, I. J.

1929. Effects of massive doses of irradiated ergosterol. J. Am. Med. Assocn. 92, 621, 622.

Knox, C. W. and Lamb, A. R.

1924. The effect of certain vitamin-carrying additions to a normal ration for baby chicks. Poultry Sci. 3, 101-108.

Knudson, A.

1927. Antirachitic activation of substances by cathode rays. Science 66, 176178.

Knudson, A. and Coolidge, W. D.

1927. Effect of high voltage cathode rays on rickets and on the activation of cholesterol. Proc. Soc. Exptl. Biol. Med. 24, 366-369. 
Knudson, A. and Moore, C. N.

1928. Comparison of the antirachitic potency of ergosterol irradiated by ultraviolet light and by exposure to cathode rays. J. Biol. Chem. 78, XIX-XXI. 1929. Comparison of the antirachitic potency of ergosterol irradiated by ultraviolet light and by exposure to cathode rays. J. Biol. Chem. 81, 49-64.

Koch, E. M. and Cahan, M. H.

1926. Inorganic blood phosphate of rats on rachitic and non-rachitic diets.

Proc. Soc. Exptl. Biol. Med. 24, 153, 154.

1927. Inorganic blood phosphate. Studies of rats on rachitic and nonrachitic diets. Am. J. Diseases Children 34, 187-197.

Koch, E. M., Cahan, M. H. and Gustavson, R. C.

1926. The antirachitic properties of certain lipoids. J. Biol. Chem. 67, LII, LIII.

Koch, E. M., Koch, F. C. and Lemon, H. B.

1929. Absorption spectra studies in cholesterol and ergosterol. J. Biol. Chem.

85, 159-167.

Koch, F. C., Koch, E. M. and Ragins, I. K.

1929. Fractionation studies on provitamin D. J. Biol. Chem. 85, 141-158.

Koch, J.

1919. Utber experimentelle Rachitis. Arch. wiss. Prakt. Tierheilk. 45, 263326.

Koch, M. L. and Smith, A. A.

1924. The variation of complement of guinea pigs during scurvy. Proc. Soc.

Exptl. Biol. Med. 21, 366-368.

Koch, M. L. and Voegtlin, C.

1916. Chemical changes in the central nervous system as a result of restricted vegetable diet. U. S. Pub. Health Service Hyg. Lab. Bull. 103, 5-49.

a. Chemical changes in the central nervous system in pellagra. U.S.Pub. Health Service Hyg. Lab. Bull. 103, 51-129.

Koehne, M. and Mendel, L. B.

1929. The utilization of fatty oils given parenterally. J. Nutrition 1, 399-443.

Koessler, K. K. and Maurer, S.

1927. Treatment of pernicious anemia with a high caloric diet, rich in vitamins. J. Am. Med. Assocn. 89, 768-774.

Koessler, K. K., Maurer, S. and Loughlin, R.

1926. The relation of anemia primary and secondary to vitamin A deficiency.

J. Am. Med. Assocn. 87, 476-482.

Koga, Y.

1929. Studies in experimental scurvy. VIII. Contribution to the study on the carbohydrate metabolism of guinea pigs fed on a vitamin C-free diet. $J$. Biochem. 11, 461-477.

Kohls, C. L. and Evans, H. M.

1928. Vitamine E and anemia. Anat. Record 38, 52.

Kohman, E. F.

1923. The protection of vitamin $C$ in foods. Ind. Eng. Chem. 15, 273-275.

1924. Canned cabbage rich in vitamins. Canner 58, 23, 24.

1928. Research findings on corrosion and vitamin destruction. Canning Age 9, 227-231.

1929. Vitamins in canned foods. Natl. Canners Assocn. Bull. 19-L (Revised). 
Kohman, E. F., Eddy, W. H. and Carlsson, V.

1924. Vitamins in canned foods. II. The vitamin $C$ destructive factor in apples. Ind. Eng. Chem. 16, 1261-1263.

Kohman, E. F., Eddy, W. H., Carlsson, V. and Halliday, N.

1926. Vitamins in canned foods. V. Peaches. Ind. Eng. Chem. 18, 302, 303.

Kohman, E. F., Eddy, W. H. and Halliday, N.

1928. Vitamins in canned foods. VI. Strawberries. Ind. Eng. Chem. 20, 202204.

Komm, E.

1926. Über den Gehalt einiger Vitamin-Nährpräparate an Anti-Beriberi-Vitamin. Z. Untersuch. Lebensm. 52, 303-307.

Komm, E. and Dietsch, W.

1927. Über den Gehalt einiger Nährpräparate an Vitasterin A und Vitamin C. Z. Untersuch. Lebensm. 53, 307-310.

Kon, S. K.

1927. A note on the temporary spontaneous disappearance of typical "beriberi" symptoms in pigeons fed on diets deficient in vitamin B. Biochem. J. 21, 834-836.

a. The effect of the administration of glycine to pigeons on a diet deficient in vitamin B. Biochem. J. 21, 837-839.

1928. The photochemistry of ergosterol. Nature 122, 276, 277.

1929. On the carbon: nitrogen $(\mathrm{C} / \mathrm{N})$ ratio in the urine of rats deprived of one or both factors of the vitamin B complex. J. Nutrition 1, 467-473.

Kon, S. K., Daniels, F. and Steenbock, H.

1928. The quantitative study of the photochemical activation of sterols in the cure of rickets. II. J. Am. Chem. Soc. 50, 2573-2581.

Kon, S. K. and Drummond, J. C.

1927. The physiological rôle of vitamin B. Part III. Study of vitamin B deficiency in pigeons. Biochem. J. 21, 632-652.

Kon, S. K. and Mayzner, M.

1930. A study of the antirachitic value of irradiated yeast. Lancet, 1930, I, 794-796.

Kon, S. K. and Moore, T.

1927. Note on the attempted activation of tyrosine by ultra-violet irradiation. Biochem. J. 21, 1368, 1369.

Kon, S. K. and Watchorn, E.

1928. Relation between the nature of the carbohydrate in the diet and refection in rats. J. Hyg. 27, 321-327.

Kondô, M., Matsushima, S., and Okamura, T.

1929. Keimkraft, Nährstoffe und B-Vitamin im Kohlensäuren- und luftdichten Verschlusse 4 Jahre lang aufbewahrten Reiskörner. Proc. Imp. Acad. Tokyo $5,159,160$.

Korenchevsky, V.

1921. Experimental rickets in rats. Brit. Med. J. No. 3171, 547-550.

1922. Experimental rickets in rats. N. Y. Med. J. and Med. Record 115, 612-614.

a. The influence of parathyroidectomy on the skeleton of animals normally nourished, and on rickets and osteomalacia produced by deficient diet. $J$. Path. Bact. 25, 366-392. 
b. The etiology and pathology of rickets from an experimental point of view. Med. Rescarch Council (Gt. Brit.) Spec. Rept. Ser. No. 71, pp. 172.

1923. The influence of removal of sexual glands on the skeleton of animals kept on normal or rickets-producing diets. J. Path. Bact. 26, 207-221.

a. Spontaneous rickets in rats. J. Path. Bact. 26, 222, 223.

b. Glands of internal secretion in experimental avian beriberi. J. Path. Bact. 26, 382-388.

c. Effects of excess of calcium on the skeleton. Brit. Med. J. No. 3254, 802-804.

Korenchevsky, V. and Carr, M.

1923. The influence of the mothers' diet during pregnancy and lactation upon the growth, general nourishment and skeleton of young rats. J. Path. Bact. 26, 389-398.

a. Influence of a milk diet on the skeleton. Biochem. J. 17, 187-203.

b. The influence of the antenatal feeding of parent rats upon the number, weight and composition of the young at birth. Biochem. J. 17, 597-599.

1924. Further experiments on the influence of the parents' diet upon the young. I. The influence of the father's diet. II. The influence upon the young of an excessive amount of fat-soluble factor and calcium in the mother's diet during pregnancy. Biochem. J. 18, 1308-1312; 1313-1318.

1925. The effects of calcium glycerophosphate, sodium glycerophosphate, and sodium dihydrogen phosphate upon the skeleton of rats kept on a diet deficient only in fat-soluble factor. Biochem. J. 19, 101-111.

a. Further experiments on the influence of the parents' diet upon the young. III. The influence upon the young of an excessive amount of calcium in the mother's diet during pregnancy. Biochcm. J. 19, 112-116.

Koskowski, W.

1922. L'action antinévritique de l'histamine chez les pigeons nourris au riz poli. Arch. intern. pharmacodynamie 26, 367-373.

Kozawa, S., Kusunoki, M. and Hosoda, N.

1925. On the contents of nonprotein nitrogen in blood of human beriberi and avitaminosis of rabbits. Japan Med. World 5, 83-86.

\section{Kramár, E.}

1924. Vitaminstudium über das Verhalten des B-Vitamins gegenüber Reduktionsprozessen. Biochem. Z. 154, 343-348.

Kramer, B.

1925. Rickets in infants. Treatment with irradiated milk. Am. J. Diseases Children 30, 195-198.

Kramer, B. and Boone, F. H.

1922. The effect of sunlight upon the concentration of calcium and of inorganic phosphorus of the serum of rachitic children. Proc. Soc. Exptl. Biol. Med. 20, 87-89.

Kramer, B., Casparis, H. and Howland, J.

1922. Ultraviolet radiation in rickets.-Effect on the calcium and inorganic phosphorus concentration of the serum. Am. J. Diseases Children 24, 2026.

Kramer, B., Grayzel, H. G. and Shear, M. J.

1922. Vitamin D in tuberculosis. Proc. Soc. Exptl. Biol. Med. 27, 144-146. 
Kramer, B. and Howland, J.

1922. Factors which determine the concentration of calcium and of inorganic phosphorus in the blood serum of rats. Bull. Johns Hopkins Hosp. 33, 313317.

a. The influence of diet upon the concentration of phosphorus and calcium in the serum of rats. J. Biol. Chem. 50, XXI.

Kramer, B., Kramer, S. D., Shelling, D. H. and Shear, M. J.

1926. Antirachitic properties of cod liver oil concentrates. Proc. Soc. Exptl. Biol. Med. 24, 51.

1926. Cod liver oil concentrate: Its value as an antirachitic agent when injected subcutaneously. J. Biol. Chem. 71, 699-706.

Kramer, B. and Shear, M. J.

1927. Composition of bone. II. Analytical results. Proc. Soc. Exptl. Biol. Med. 25, 141, 142.

1928. Composition of bone. IV. Primary calcification. J. Biol. Chem. 79, 147160.

Kramer, B., Shear, M. J. and McKenzie, M. R.

1929. Composition of bone. VI. Effect of massive doses of irradiated ergosterol. J. Biol. Chem. 82, 555-557.

Kramer, B., Shear, M. J. and Shelling, D. H.

Fractionation of irradiated cholesterol. Antirachitic potency of the fractions. Proc. Soc. Exptl. Biol. Med. 24, 50, 51.

a. Fractionation of irradiated cholesterol. II. Antirachitic potency of the fractions. J. Biol. Chem. 71, 221-233.

Kramer, B., Shelling, D. H. and Orent, E. R.

1927. Studies upon calcification in vitro. I. Effect of reaction in calcification. Proc. Soc. Exptl. Biol. Med. 24, 240, 241.

a. Studies upon calcification in vitro. II. On the inhibitory effect of the magnesium ion. Bull. Johns Hopkins Hosp. 41, 426-431.

Kramer, M. M., Boehm, G. and Williams, R. E.

1929. Vitamin A content of the green and white leaves of market head lettuce. J. Home Econ. 21, 679, 680.

Kramer, M. M., Eddy, W. H. and Kohman, E. F.

1929. Vitamins in canned foods. VIII. Home canning and commercial canning contrasted in their effect on vitamin values of pears. Ind. Eng. Chem. 21, 859-861.

Krause, D. J.

1922. The water content of the tissues in experimental beriberi. Am. J. Physiol. 60, 234-243.

Krauss, W. E.

1929. The effect of the cow's ration on the vitamin-D content of milk. Ohio Agr. Expt. Sta. Bimo. Bull. 14, 57-59.

Kreitmair, $H$. and Moll, $T$.

1928. Hypervitaminose durch grosse Dosen Vitamin D. Münch. med. Wochschr. 75, 637-639.

Křiženecký, J.

1926. Untersuchungen über die Beeinflussung der Avitaminosen durch ultraviolette Strahlen. II. Weitere Versuche bei Taubenberiberi: Versuche mit Tauben, welche direkt an entfiederter Haut bestrahlt wurden. Arch. ges. Physiol. (Pfiüger's) 211, 663-665. 
a. Untersuchungen über die Beeinflussung der Avitaminosen durch ultraviolette Strahlen. III. Versuche bei Meerschweinchenskorbut. Arch. ges. Physiol. (Pflüger's) 212, 45-53.

Křiženecký, J. and Petrov, I.

1926. Über die Bedeutung des antineuritischen (B-) Vitamins für die Neubildung des Gefieders. Arch. ges. Physiol. (Pfiüger's) 213, 5-18.

Kronacher, C., Kliesch, J. and Schäper, W.

1929. Untersuchungen über die Ziegenmilchanämie an wachsenden Schweinen unter besonderer Berücksichtigung der Vitaminfrage. Z. Tierzücht. Zuchtungsbiol. Tierernähr. 14, 231-294.

Kruger, J. H. and Bechdel, S. I.

1928. Studies in the normal depositions of mineral in the bones of dairy calves. J. Dairy Sci. 11, 24-34.

Kruse, H. D. and McCollum, E. V.

1929. Biochemical investigation of vitamin B. Physiol. Rev. 9, 126-239.

Kučera, C.

1928. L'influence de l'alimentation de base dans la détermination de la teneur en vitamines de diverses sources. Compt. rend. soc. biol. 99, 988-990.

a. Uber die Inhaltsschwankung des Vitamins $\mathrm{B}$ und $\mathrm{C}$ im keimenden Getreidekorn und über die Lokalisation des Vitamins $B$ und $C$ in der jungen Pflanze. Z. Tierzücht. Züchtungsbiol. Tierermähr. 12, 239-247.

1929. Nouvelles recherches sur la diminution de la teneur en vitamine B dans la graine des céréales et des légumineuses au cours de la germination. Compt. rend. soc. biol. $100,429,430$.

a. Weitere Versuchsergebnisse über Abnahme des Vitamin-B-Gehaltes beim Keimen der Getreidekörner und Hülsenfrüchte. Z. Tierzïcht. Züchtungsbiol. Tierernähr. 13, 387-390.

b. Praktische Erfahrungen über den Einfluss der Nahrung bei experimenteller Erforschung des Vitamingehalts verschiedener Stoffe. Z. Tierzücht. Ziichtungsbiol. Tierernähr. 13, 391-394.

Kudrjawzewa, A.

1924. Uber den Einfluss der Polyneuritis auf den Kreatingehalt in den Muskeln.

Z. physiol. Chem. 141, 105-109.

Kugelmass, I. N. and McQuarrie, I.

1924. The photoactivity of substances curative of rickets and the photolysis of the oxy-products by ultraviolet radiation. Science $60,272-274$.

1925. "Russell effect," not ultraviolet light, responsible for changes produced in the photographic plate by antirachitic substances. Science $62,87,88$.

Kühl, G.

1929. Zur therapeutsichen Dosierung von Vigantol dilutum. Münch. med. Wochschr. 76, 1419, 1420.

Kunde, M. M. and Williams, L. A.

1927. The influence of the thyroid gland on experimental rickets. Proc. Soc. Exptl. Biol. Med. 24, 631, 632.

a. Experimental cretinism. II. The influence of the thyroid gland on the production and control of experimental rickets. Am. J. Physiol. 83, 245249. 
Kuroya, M. and Hosoya, S.

1923. The synthesis of the water-soluble vitamin by coli bacillus grown on synthetic medium. Sci. Repts. Tokyo Imp. Univ. Govt. Inst. Infect. Diseases 2 , 287-304.

Laird, C. N.

1926. A comparison of the pigeon and rat as test subjects for vitamin B. $A \mathrm{~m}$. J. Hyg. 6, 201-210.

Laird, D. A.

1929. A small animal cage with sanitary features. Science 70, 241.

Lamb, A. R. and Evvard, J. M.

1922. Vitamins on the farm. Their practical relation to live-stock feeding. Iowa Agr. Expt. Sta. Circ. 73, pp. 8.

Lambert, R. A. and Yudkin, A. M.

1923. Changes in the paraocular glands accompanying the ocular lesions which result from a deficiency of vitamin A. J. Exptl. Med. 38, 25-32.

La Mer, V. K.

1921. Vitamins from the standpoint of physical chemistry. J. Ind. Eng. Chem. $13,1108-1110$.

a. The effect of temperature and hydrogen ion concentration upon the rate of destruction of the antiscorbutic vitamin. Dissertation, Columbia University, New York.

La Mer, V. K. and Campbell, H. L.

1921. Changes in organ weight produced by diets deficient in antiscorbutic vitamin. Proc. Soc. Exptl. Biol. Med. 18, 32.

La Mer, V. K., Campbell, H. L. and Sherman, H. C.

1921. The effect of temperature and of $\mathrm{H}$-ion concentration upon the rate of destruction of antiscorbutic vitamin. Proc. Soc. Exptl. Biol. Med. 18, 122, 123.

1922. The effect of temperature and concentration of hydrogen ions upon the rate of destruction of the antiscorbutic vitamin (vitamin C). J. Am. Chem. Soc. 44, 172-181.

Lane-Claypon, J. E.

1909. Observations on the influence of heating upon the nutrient value of milk as an exclusive diet for young animals. J. Hyg. 9, 233-238.

Lange, L. B.

1925. Experimental tuberculosis in rats on varied diets. Fat and vitamin factors. Am. Rev. Tuberculosis 11, 241-246.

Langen, C. D. de.

1929. Stone formation and diet. Mededeel. Dienst. Volksgezondheid. Nederland.-Indië 18, 315-333.

Langstein, L. and Edelstein, F.

1917. Die Rolle der Ergänzungstoffe bei der Ernährung wachsender Tiere. Ernährungsversuche an jungen wachsenden Ratten. Z. Kinderheilk. 16, 305402.

Laqueur, E., Wolff, L. K. and Dingemanse, E.

1928. Uber den Gehalt der Leber an Vitamin A (im besonderen beim Menschen). Deut. med. Wochshr. 54, 1495-1497. 
Larimore, J. W.

1928. Chronic ulcerative colitis. Observations of treatment by diet. J. Am. Med. Assocn. 90, 841-844.

Larson, J. H.

1920. Butter fat and the child's weight. Arch. Pediatrics 37, 610-614.

Lasch, $\mathbf{F}$.

1928. Über den Einfluss von bestrahltem Ergosterin (Vigantol) auf den Cho-

lesterin- und Calciumgehalt des Blutserums beim Erwachsenen. Klin. Wochschr. 7, 2148-2150.

Lasch, W.

1921. Über die Wirkung der künstlichen Höhensonne auf den Stoffwechsel. Deut. med. Wochschr. 47, 1063.

Lasch, W. and Behrens, A.

1927. Über die Wirkung des Ergosterins auf die Rachitis. Deut. med. Wochschr. 53, 1552, 1553.

Latzke, A.

1929. Penetration of uitra-violet rays through fabrics. Am. J. Hyg. 9, 629645.

Laufberger, W.

1923. Über die Avitaminose bei Fischen. Arch. Physiol. 198, 31-36.

Laurens, $\mathrm{H}$.

1928. The physiological effects of radiation. Physiol. Rev. 8, 1-91.

Laurens, H. and Sovy, J. W.

1924. Effect of light and darkness on rats. Proc. Soc. Exptl. Biol. Med. 22, $112-114$.

Lavialle, $\mathbf{P}$.

1923. Contribution à l'étude des vitamines (specialement du lait de vache). Compt. rend. soc. biol. 89, 1031-1033.

1927. Le facteur $C$ dans le lait de vache. Ses rapports avec la concentration, l'homogénéisation et la stérilisation. Bull. soc. chim. biol. 9, 208-221. Also in Lait 7, 621-624.

1928. Le facteur antiscorbutique. Ses rapports avec la dessiccation. Bull soc. chim. biol. 10, 1293-1297.

Lavinder, C. H., Francis, E., Grimm, R. M. and Lorenz, W. F.

1914. Attempts to transmit pellagra to monkeys. J. Am. Med. Assocn. 63, 1093, 1094.

Lawrow, B. A. and Matzko, S. N.

1926. Über den Gaswechsel im Anfangsstadium der B-Avitaminose bei Vögeln. Biochem. Z. 179, 332-347.

1928. Stickstoffumsatz bei einseitiger Ernährung. II. Der Stickstoffwechsel bei Hühnern wahrend der B-Avitaminose. Biochen. Z. 198, 138-148.

Lax, $H$.

1921. Untersunchungen über die Ergänzungstoffe des Lebertrans. Biochem. $Z$. $125,265-271$.

Lecoq, $\mathbf{R}$.

1927. Quelques considérations sur les vitamines des levures. J. pharm. chim. 3, 289-295.

Leersum, E. C. van

1924. On the effect of hematoporphyrin on the deposition of calcium in the bones of rachitic rats. J. Biol. Chem. 58, 835-844. 
1926. Sur la teneur en vitamine C du lait cru ou pasteurisé. Bull. soc. hyg. aliment. 14, 391-405.

a. The discovery of vitamins. Science 64, 357, 358 .

1927. Vitamin A deficiency and urolithiasis. Brit. Med. J. No. 3488, 873, 874.

1928. Vitamin A deficiency and urolithiasis. J. Biol. Chem. 76, 137-142.

a. Vitamin A deficiency and calcification of the epithelium of the kidney. J. Biol. Chem. 79, 461-468.

1929. Terminology of vitamin B. Science 69, 166, 167.

Leggate, A. R.

1920. Observations on beriberi among the Chinese in France. Edinburgh Med. J. 24, 32-36.

Leichtentritt, B. and Zielaskowski, M.

1922. Untersuchungen über den wachstumfördernden Faktor des Citronensaftes. I. Auf welche Weisse lässt sich der bakterienwachstum-fördernde Faktor in dem Citronensaft durch physikalische, chemische, kolloid-chemische Methoden beeinflussen. II. Vergleiche zwischen dem Meerschweinchen- und Bakterienplattenversuch. Biochem. Z. 131, 499-512, 513-524.

Leigh-Clare, J. L.

1927. The effect of excessive radiation with ultra-violet light upon the growth of rats. Biochem. J. 21, 208-210.

a. A search for vitamin $\mathrm{D}$ in the diatom Nitzschia closterium. Biochem. J. $21,368-372$.

b. A note on the vitamin $\mathrm{D}$ content of the stomach oil of the Australasian petrel. (Aestralata lessoni). Biochem. J. 21, 725-727.

Leigh-Clare, J. L. and Soames, K. M.

1928. The relative content of the fat-soluble vitamins $A$ and $D$ in a series of cod-liver oils. Lancet, 1928, I, 150-152.

Lepkovsky, S., Hart, E. B., Hastings, E. G. and Frazier, W. C.

1925. The effect of fermentation with specific microörganisms on the vitamin C content of orange and tomato juice. J. Biol. Chem. 66, 49-56.

Lepkovsky, S. and Nelson, M. T.

1924. Observations on the persistence of vitamin $C$ in the livers of rats on a scorbutic ration. J. Biol. Chem. 59, 91-96.

Lepkovsky, S., Wood, C. and Evans, H. M.

1930. Glucose tolerance in avitaminosis due to low antineuritic vitamin B. J. Biol. Chem. 87, 239-250.

Lepper, E. H. and Zilva, S. S.

1925. The bicarbonate of the plasma and the hydrogen-ion concentration of the blood of guinea pigs suffering from scurvy. Biochem. J. 19, 581-588.

Lesné, E., Christou and Vaglianos.

1923. Passage dans le lait des vitamines $C$ introduites par voie parenterale. Compt. rend. 176, 1006-1008.

Lesné, E. and Clément, $\mathbf{R}$.

1929. Activité antirachitique spontanée de certaines algues. Compt. rend. soc. biol. 101, 1021, 1022.

a. Innocuité des fortes doses d'ergostérol irradié. Compt. rend. soc. biol. 101, 1023.

Lesné, E., Geunes, L. de and Guillaumin.

1923. L'action de la lumière sur les variations de la calcémie chez les rachitiques. Compt. rend. 177, 291-294. 
Lesné, E. and Vagliano, M.

1923. De l'utilisation par l'organisme des vitamines C introduites par voie parentérale. Compt. rend. 176, 614-615.

a. Différenciation de la vitamine $\mathrm{A}$ et du facteur antirachitique. Compt. rend. $177,711,712$.

1924. Le pouvoir antiscorbutique du lait condensé sucré de vieille préparation. Compt. rend. soc. biol. 90, 393, 394.

a. Production d'un lait de vache doué de propriétés antirachitiques. Compt. rend. 179, 539-541.

Lesné, E., Vagliano, M. and Christou.

1923. Contribution a l'étude du rachitisme expérimental chez le rat. Rev. path. compar. 23, 561-567.

Levaditi, C. and Po, L. Y.

1930. Etude expérimentale de l'ergostérol irradié. Presse méd. 38, 168-172.

Levene, P. A.

1928. The concentration of vitamin B. IV. On the concentration and the separation of the two components of vitamin B. J. Biol. Chem. 79, 465-470. 1930. Vitamin $B_{2}$. Science $71,668$.

Levene, P. A. and Muhlfeld, M.

1923. On the identity or nonidentity of antineuritic and water-soluble B vitamins. J. Biol. Chem. 57, 341-349.

Levene, P. A. and van der Hoeven, B. J. C.

1924. Concentration of vitamin B. Science 59, 276.

a. The concentration of vitamin B. J. Biol. Chem. 61, 429-443.

1925. Concentration of the growth-promoting principle obtained from yeast (Vitamin B). Science 62, 594.

a. The concentration of vitamin B, II. J. Biol. Chem. 65, 483-489.

1926. The concentration of vitamin B. III. J. Pharmacol. 29, 227-231.

Levine, V. E。

1924. A critical study of the Jendrassik reaction for water-soluble B. J. Biol. Chem. 62, 157-161.

Levine, V. E., McCollum, E. V. and Simmonds, N.

1922. Glacial acetic acid as a solvent for the antineuritic substance, watersoluble B. J. Biol. Chem. 53, 7-11.

Levinsohn, S. H.

1927. Rickets in the negro. Effect of treatment with ultra-violet rays. $A m . J$. Diseases Children 34, 955-961.

Lewis, H. B.

1919. The antiscorbutic value of the banana. J. Biol. Chem. 40, 91-101.

Lewis, R. C.

1920. The chemical composition of the blood of pellagrins. U.S. Pub. Health Service Hyg. Lab. Bull. 116, 37-44.

Liang, B. and Wacker, L.

1925. Studien über den Fett-, Cholesterin- und Steroid-Stoffwechsel im Organismus wachsender Ratten bei An- und Abwesenheit von Vitamin A. Biochem. Z. 164, 371-393.

Liégeois, F. and Lefèvre, A.

1929. L'équilibre acide-base et la calcémie dans le rachitisme et l'ostéomalacie spontanés chez le chien. Compt. rend. soc. biol. 102, 957, 958. 
a. Le calcium du sang total au cours du traitement du rachitisme par les stérols irradiés. Compt. rend. soc. biol. 102, 960-962.

Light, R. F., Miller, G. and Frey, C. N.

1929. Studies on the effects of overdosage of vitamin D. J. Biol. Chem. 84, $487-494$.

Lind, J.

1757. "A Treatise on Scurvy," London. Second edition.

Lindsay, B. and Medes, G.

1926. Histological changes in the testis of the guinea pig during scurvy and inanition. Am. J. Anat. 37, 213-235.

1926. Histological changes in the adrenal glands of guinea pigs subjected to scurvy and severe inanition. Proc. Soc. Exptl. Biol. Med. 23, 293, 294.

\section{Linossier, G.}

1919. Les vitamines et les champignons. Compt. rend. soc. biol. 82, 381-384. Liotta, D.

1923. Sulla scorbuto sperimentale. 1. Le moderne vedute sulla etiologia dello scorbuto. 2. L'influenza della costipazione intestinale nello sviluppo dello scorbuto. 3. Le modificazioni emoleucocitarie. Arch. farmacol. sper. 35, 184$191 ; 36,1-5,76-80$.

Lippmann, A. and Völker, $H$.

1928. Beiträge zur Frage der Stoffwechselbeeinflussung durch Ultraviolettbestrahlung. Klin. Wochschr. 7, 213-214.

Lipschitz, W.

1923. Die Wirkung von Tyramin auf die Tauben-Beriberi. Z. physiol. Chem. 124, 194-201.

\section{Little, J. M.}

1912. Beriberi caused by fine white flour. J. Am. Med. Assocn. 58, 2029, 2030. 1914. Beriberi. J. Am. Med. Assocn. 63, 1287-1290.

Ljubarskaja, T.

1928. Der Kreatinstoffwechsel im Gehirne von Tauben im Hunger und bei Polyneuritis. Arch. ges. Physiol. (Pfïger's) 218, 627-634.

Löwy, E.

1923. Histologische Untersuchung einiger Drüsen mit innerer Sekretion bei Skorbut-kranken Meerschweinchen. Z. ges. exptl. Med. 38, 407-409.

Looser, E.

1905. Über die Knochenveränderungen beim Skorbut und bei der Barlowschen Krankheit (Säuglings-Skorbut). Jahrb. Kinderheilk. 62, 743-768.

Lopez-Lomba, J.

1923. Modifications pondérales des organes chez le pigeon au cours de l'avitaminose B. Compt. rend. 176, 1417-1419.

a. Modifications pondérales des organes chez le cobaye au cours de l'avitaminose C. Compt. rend. 176, 1752-1755.

\section{Lopez-Lomba and Randoin.}

1923. Production du scorbut chez le cobaye et le lapin jeune au moyen d'un nouveau régime complet et biochimiquement équilibré, uniquement dépourvu de facteur C. Compt. rend. 176, 1003-1008.

a. Contribution à l'étude de l'avitaminose $\mathrm{B}$ chez le pigeon. Compt. rend. $176,1249-1251$.

b. Etude du scorbut produit par un régime complet et biochimiquement équilibré, uniquement dépourvu de facteur C. Compt. rend. 176, 1573-1576. 
Lucas, G. H. W.

1924. The fractionation of bios, and comparison of bios with vitamins $B$ and $C$. J. Phys. Chem. 28, 1180-1200.

Lucas, N. S.

1926. The nature of the action on a photographic plate of sawdust and cholesterol irradiated by a inercury vapour quartz lamp. Biochem. J. 20, 23-25.

Luce, E. M.

1923. The size of the parathyroids of rats and the effect of a diet deficiency of calcium. J. Path. Bact. 26, 200-206.

1924. The influence of diet and sunlight upon the growth-promoting and antirachitic properties of the milk afforded by a cow. Biochem. J. 18, 716739.

a. Further observations on the influence of sunlight upon the growthpromoting and antirachitic properties of cow's milk. Biochem. J. 18, 12791288.

1926. Glass screens for the transmission of the light radiations curative of rickets. J. Biol. Chem. 71, 187-190.

Luce, E. M. and Maclean, I. S.

1925. The presence of vitamin A in yeast fat. Biochem J. 19, 47-51.

Luckiesh, M. and Pacini, A. J.

1926. "Light and Health," Baltimore, Williams \& Wilkins.

\section{Luettmerding, A.}

1929. Influence de l'acidité du milieu sur la formation de la vitamine C. Compt. rend. soc. biol. 100, 589-591.

\section{Lumière, $\mathbf{A}$.}

1920. Sur les accidents polynévritiques et cérébelleux chez le pigeon soumis au régime du riz décortiqué. Bull. acad. méd. (Paris) 3. ser. 83, 96-101.

a. Sur l'anorexie chez le pigeon nourri au riz décortiqué et le rôle des vitamines dans la nutrition. Bull. acad. méd. (Paris) 3 ser. 83, 310-313.

b. Avitaminose et inanition. Bull. acad. méd. (Paris) 3 ser. 84, 274-276.

c. $\AA$ propos des réserves de vitamines. Paris méd. 10, 474-477.

d. Les vitamines sont-elles nécessaires au développement des végétaux? Compt. rend. 171, 271-273.

1921. Influence des vitamines et des auximones sur le croissance des végétaux. Ann. Inst. Pasteur. 35, 102-123.

\section{Lunin, N.}

1881. Über die Bedeutung der anorganischen Salze für die Ernährung des Thieres. Z. Physiol. Chem. 5, 31-39.

Lust, F. and Klocman, L.

1912. Stoff wechsel-versuche bei Barlowscher Krankheit. Jahrb. Kinderheilk. 75, 663-685.

Lyman, F. J. and Blystone, I.

1926. The white potato as a source of vitamin B. J. Home Econ. 18, 199-204.

McAmis, A. J., Anderson, W. E. and Mendel, L. B.

1929. Growth of rats on fat-free diets. J. Biol. Chem. 82, 247-262.

\section{McCandlish, A. C.}

1924. Growth and nutrition of dairy calves. IX. The addition of tomatoes to a milk ration. J. Dairy Sci. 7, 94-106. 
McCann, G. F. and Barnett, M.

1922. Experimental rickets in rats. IX. The distribution of phosphorus and calcium between the skeleton and soft parts of rats on rachitic and nonrachitic diets. J. Biol. Chem. 54, 203-212.

McCarrison, $\mathrm{R}$.

1919. The influence of deficiency of accessory food factors on the intestine. Brit. Med. J. No. 3053, 36-39.

a. The pathogenesis of deficiency disease. Indian J. Med. Research 6, 275-355.

b. The pathogenesis of deficiency disease. II. The effects of deprivation of 'B' accessory food factors. Indian J. Med. Research 6, 550-556.

c. Involution of the thymus in birds. Indian J. Med. Research 6, 557-559.

d. The pathogenesis of deficiency disease. III. The influence of dietaries deficient in accessory food factors on the intestine. IV. The influence of a scorbutic diet on the adrenal glands. V. Histopathology. VI. The influence of a scorbutic diet on the bladder. VII. The effects of autoclaved rice dietaries on the gastro-intestinal tract of monkeys. VIII. The general effects of deficient dietaries on monkeys. IX. On the occurrence of recently developed cancer of the stomach in a monkey fed on food deficient in vitamins. Indian J. Med. Rescarch 7, 167-187, 188-194, 269-278, 279-282, 283-307, 308$341,342-345$.

1920. The effects of deficient dietaries on monkeys. Brit. Med. J. No. 3806, 249.

a. Deficiency disease with special reference to gastro-intestinal disorders. Brit. Med. J. No. 3103, 822-826.

b. Dietetic deficiency and endocrine activity with special reference to deficiency edemas. Brit. Med. J. No. 3111, 236-239.

c. The genesis of edema in beriberi. Proc. Roy. Soc. (London) B. 91, 103-110.

d. The pathogenesis of deficiency disease. X. The effects of some food deficiencies and excesses on the thyroid gland. Indian J. Med. Research 7, 633-647.

1921. "Studies in Deficiency Disease," London, Henry Frowde and Hodder and Stoughton.

1922. Faulty food in relation to gastro-intestinal disorder. J. Am. Med. Assocn. 78, 1-8; also in Lancet, 1922, I, 207-212. 314.

a. Effects of faulty foods on endocrine glands. N. Y. Med. J. 115, 309-

1923. The relation of faulty nutrition to the development of the Epithelioma contagiosium of fow1s. Brit. Med. J. No. 3266, 172-174.

a. The function of the adrenal gland and its relation to concentration of hydrogen ions. Brit. Med. J. No. 3238, 101, 102.

b. Pathogenesis of deficiency disease. XII. Concerning the function of the adrenal gland and its relation to concentration of hydrogen ions. XIII. Effects of heat, cold, serum and sunlight on the action of epinephrin and adrenalin hydrochloride. Indian J. Med. Research 10, 861-899; 900-907.

c. Pathogenesis of deficiency disease. XIV. On the occurrence of ophthalmia in pigeons fed exclusively on parboiled rice and its prevention by the addition of soil to the food. Indian J. Med. Research 11, 323-335.

1924. The relation of manure to the nutritive and vitamin value of certain grains. Brit. Med. J. No. 3300, 567-569. 
a. Rice in relation to beriberi in India. Brit. Med. J. No. 3297, 414-420.

b. Pathogenesis of deficiency disease. XVII. The relation of faulty nutrition to the development of Epithelioma contagiosum. Indian J. Med. Research 11, 1119-1130.

1926. The effect of manurial conditions on the nutritive and vitamin values of millet and wheat. Indian J. Med. Research 14, 351-378.

1927. The experimental production of stone in the bladder. Brit. Med. J. No. $3458,717,718$.

a. The experimental prevention of stone in the bladder in rats. Brit. Med. J. No. $3473,159-160$.

b. The nutritive value of wheat, paddy, and certain food-grains. Ind. J. Med. Rescarch 14, 631-639.

c. A good diet and a bad one. An experimental contrast. Ind. J. Med. Research 14, 649-654.

1928. The influence of irrigation on the nutritive value of rice. Ind. J. Med. Research 15, 915-920.

a. Beri-beri columbarum. Indian Med. Research Mem. 10, pp. 146.

1930. The effect on the tracheal mucous membrane and on the thyroid gland of faulty food containing an insufficiency of vitamin A. Ind. J. Med. Research 17, 693-699.

McCay, C. M. and Nelson, V. E.

1925-1926. Metabolism and vitamin A. J. Metabolic Research 7 \& 8, 199-204. McClendon, G. C.

1924. The probable occurrence of xerophthalmia in turkeys. Proc. Soc. Exptl. Biol. Med. 22, 184.

McClendon, J. F.

1921. Methods of extracting and concentrating vitamins $A, B$ and $C$ together with an apparatus for reducing milk, fruit juices, and other fluids to a powder without destruction of vitamins. J. Biol. Chem. 47, 411-420.

1922. Metabolism of calcium and phosphoric acid on isorachitic diets. $J$. Biol. Chem. 50, XI, XII.

a. The diagnostic value of phosphate metabolism in experimental rickets. Proc. Soc. Exptl. Biol. Med. 19, 412, 413.

McClendon, J. F., Bowers, W. S. and Sedgwick, J. P.

1921. The antiscorbutic properties of commercially dried orange juice. J. Biol. Chem. 46, IX, X.

McClendon, J. F. and Cole, W. C. C.

1919. The antiscorbutic properties of green malt. Am. J. Physiol. 49, 145, 146. McClendon, J. F., Cole, W. C. C., Engstrand, O. and Middlekauff, J. E.

1919. The effects of malt and malt extracts on scurvy and the alkaline reserve of the blood. J. Biol. Chem. 40, 243-258.

McClendon, J. F. and Dick, S. M.

1921. The commercial drying of orange juice retaining its antiscorbutic properties. J. Biol. Chem. 46, X, XI.

McClendon, J. F. and Shuck, C.

1923. The presence of antiophthalmic vitamin and the absence of antirachitic vitamin in dried spinach. Proc. Soc. Exptl. Biol. Med. 20, 288.

McCallum, E. V.

1917. Supplementary dietary relationships among our natural foodstuffs. $J$. Am. Med. Assocn. 68, 1379-1386. 
1918. "The newer knowledge of nutrition." New York, The Macmillan Co. 1918a. Influence of heat on growth-promoting properties of food. Am. J. Pub. Health 8, 191-194.

1919. Relation of the diet to pellagra. Proc. Am. Phil. Soc. 58, 41-54.

1920. Nutrition and physical efficiency. J. Franklin Inst. 189, 421-440.

1927. Water soluble vitamins. In "Contemporary Developments in Chemistry,"

New York, Columbia University Press, pp. 10.

McCollum, E. V. and Davis, M.

1913. The necessity of certain lipins in the diet during growth. J. Biol. Chem. $15,167-175$.

1914. Observations on the isolation of the substance in butter fat which exerts a stimulating influence on growth. J. Biol. Chem. 19, 245-259.

a. Further observations on the physiological properties of the lipins of the egg yolk. Proc. Soc. Exptl. Biol. Med. 11, 101, 102.

1915. Nutrition with purified food substances. J. Biol. Chem. 20, 641-658.

a. The influence of certain vegetable fats upon growth. J. Biol. Chem. 21, 179-182.

b. The nature of the dietary deficiencies of rice. J. Biol. Chem. 23, 181-230.

c. The essential factors in the diet during growth. J. Biol. Chem. 23, 231-246.

d. The cause of the loss of nutritive efficiency of heated milk. J. Biol. Chem. 23, 247-254.

McCollum, E. V. and Kennedy, C.

1916. The dietary factors operating in the production of polyneuritis. J. Biol. Chem. 24, 491-502.

McCollum, E. V. and Kruse, H. D.

1926. Some observations on the extraction of the vitamin B from wheat germ. Am. J. Hyg. 6, 197-200.

McCollum, E. V. and Parsons, H. T.

1920. The antiscorbutic requirement of the prairie dog. J. Biol. Chem. 44, 603-607.

McCollum, E. V. and Pitz, W.

1917. The "vitamin" hypothesis and deficiency diseases. A study of experimental scurvy. J. Biol. Chem. 31, 229-253.

McCollum, E. V. and Simmonds, N.

1917. A biological analysis of pellagra-producing diets. I. The dietary properties of mixtures of maize kernel and bean. J. Biol. Chem. 32, 29-61.

a. A biological analysis of pellagra-producing diets. II. The minimum requirements of the two unidentified dietary factors for maintenance as contrasted with growth. J. Biol. Chem. 32, 181-193.

b. A biological analysis of pellagra-producing diets. III. The value of some seed proteins for maintenance. J. Biol. Chem. 32, 347-368.

1918. A study of the dietary essential, water-soluble $B$, in relation to its solubility and stability toward reagents. J. Biol. Chem. 33, 55-89.

a. A biological analysis of pellagra-producing diets. IV. The cause of failure of mixtures of seeds to promote growth in young animals. J. Biol. Chem. 33, 303-311.

b. The nursing mother as a factor of safety in the nutrition of the young. Am. J. Physiol. 46, 275-301. 
1922. The potency of commercial vitamin preparations. J. Am. Med. Assocn. 78, 1953-1957.

McCollum, E. V., Simmonds, N. and Becker, J. E.

1922. On a type of ophthalmia caused by unsatisfactory relations in the inorganic portion of the diet. An ophthalmia not due to starvation for fatsoluble $\mathrm{A}$ and not curable by its administration. J. Biol. Chem. 53, 313-321.

1925. Technique in the use of the rat for vitamin B studies. J. Biol. Chem. 63, 547-551.

a. Further studies on the cause of ophthalmia in rats produced with diets containing vitamin A. J. Biol. Chem. 64, 161-179.

1927. Studies on "salt ophthalmia." III. Proc. Soc. Exptl. Biol. Med. 24, 952. McCollum, E. V., Simmonds, N., Becker, J. E. and Shipley, P. G.

1922. Studies on experimental rickets. XXI. An experimental demonstration of the existence of a vitamin which promotes calcium deposition. Bull. Johns Hopkins Hosp. 33, 229; J. Biol. Chem. 53, 293-312.

a. Studies on experimental rickets. XXIII. The production of rickets in the rat by diets consisting essentially of purified food substances. J. Biol. Chem. 54, 249-252.

1925. Studies on experimental rickets. XXVI. A diet composed principally of purified foodstuffs for use with the "line test" for vitamin D studies. J. Biol. Chem. 65, 97-100.

1926. Studies on experimental rickets. XXVII. Variation of vitamin D content of butter fat as a factor in the development of rickets induced by diets suitable for preparing rats for the line test. J. Biol. Chem. 70, 437, 438.

1927. Studies on experimental rickets. XXVIII. Does Vitamin D pass into the milk? Am. J. Diseases Children 33, 230-243.

McCollum, E. V., Simmonds, N., Kinney, E. M. and Grieves, C. J.

1922. The relation of nutrition to tooth development and tooth preservation.

I. A preliminary study of gross maxillary and dental defects in two hundred and twenty rats on defective and deficient diets. Bull. Johns Hopkins Hosp. 33, 202-215.

McCollum, E. V., Simmonds, N., Kinney, M., Shipley, P. G. and Park, E. A. 1922. Studies on experimental rickets. XVII. The effects of diets deficient in calcium and in fat-soluble $A$ in modifying the histological structure of the bones. Am. J. Hyg. 2, 97-106.

McCollum, E. V., Simmonds, N. and Parsons, H. T.

1918. A biological analysis of pellagra-producing diets. V. The nature of the dietary deficiencies of a diet derived from peas, wheat flour, and cottonseed oil. J. Biol. Chem. 33, 411-423.

a. The dietary properties of the potato. J. Biol. Chem. 36, 197-210.

1919. Supplementary relationships between the proteins of certain seeds. $J$. Biol. Chem. 37, 155-178.

a. The dietary properties of the pea. (Vicia sativa). J. Biol. Chem. 37, 287-301.

b. A biological analysis of pellagra-producing diets. VI. Observations on the faults of certain diets comparable to those employed by man in pellagrous districts. J. Biol. Chem. 38, 113-146.

1921. Supplementary protein values in foods. I. The nutritive properties of animal tissues. II. Supplementary dietary relations between animal tissues and cereal and legume seeds. III. The supplementary dietary relations be- 
tween the proteins of the cereal grains and the potato. IV. The supplementary relations of cereal grain with cereal grain; legume seed with legume seed; and cereal seed with legume seed with respect to improvement in the quality of their proteins. V. Supplementary relations of the proteins of milk for those of cereals and of milk for those of legume seeds. J. Biol. Chem. 47, 111-137, 139-173, 175-206, 207-234, 235-247.

McCollum, E. V., Simmonds, N., Parsons, H. T., Shipley, P. G. and Park, E. A.

1921. Studies on experimental rickets. I. The production of rachitis and similar diseases in the rat by deficient diets. J. Biol. Chem. 45, 333-341.

McCollum, E. V., Simmonds, N. and Pitz, W.

1916. The nature of the dietary deficiencies of the wheat embryo. J. Biol. Chem. 25, 105-131.

a. The vegetarian diet in the light of our present knowledge of nutrition. Am. J. Physiol. 41, 333-360.

b. The distribution in plants of the fat-soluble $A$, the dietary essential of butterfat. Am. J. Physiol. 41, 361-375.

c. The relation of the unidentified dietary factors, the fat-soluble $\mathrm{A}$ and water-soluble $\mathrm{B}$, of the diet to the growth-promoting properties of milk. $J$. Biol. Chem. 27, 33-43.

d. Dietary deficiency of the maize kernel. J. Biol. Chem. 28, 153-165.

e. The effects of feeding the proteins of the wheat kernel at different planes of intake. J. Biol. Chem. 28, $211-229$.

1917. The nature of the dietary deficiencies of the oat kernel. J. Biol. Chem. 29, 341-354.

a. The dietary deficiencies of the white bean, Phaseolus vulgaris. J. Biol. Chem. 29, 521-536.

b. The supplementary dietary relationship between the leaf and seed as contrasted with combinations of seed with seed. J. Biol. Chem. 30, 13-32.

McCollum, E. V., Simmonds, N., Shipley, P. G. and Park, E. A.

1921. Studies on experimental rickets. IV. Cod liver oil as contrasted with butter fat in the protection against the effects of insufficient calcium in the diet. Proc. Soc. Exptl. Biol. Med. 18, 275-280.

a. Studies on experimental rickets. VI. The effects on growing rats of diets deficient in calcium. Am. J. Hyg. 1, 492-511.

b. Studies on experimental rickets. VIII. The production of rickets by diets low in phosphorus and fat-soluble A. J. Biol. Chem. 47, 507-527.

1922. Studies on experimental rickets. XII. Is there a substance other than fat-soluble A associated with certain fats which plays an important rôle in bone development. J. Biol. Chem. 50, 5-30.

a. Studies on experimental rickets. XV. The effect of starvation on the healing of rickets. Bull. Johns Hopkins Hosp. 33, 31-33.

b. Studies on experimental rickets. XVI. A delicate biological test for calcium-depositing substances. J. Biol. Chem. 51, 41-49.

c. Studies on experimental rickets. XXII. Conditions which must be fulfilled in preparing animals for testing the antirachitic effect of individual foodstuffs. Bull. Johns Hopkins Hosp. 33, 296-302.

McCollum, E. V., Simmonds, N. and Steenbock, H.

1917. A method for the separation of the dietary essential "fat-soluble A" from butterfat. J. Biol. Chem. 29, XXVI. 
McCordock, H. A. and Congdon, C. C.

1924. Suppurative otitis of the albino rat. Proc. Soc. Exptl. Biol. Med. 22, 150-154.

McGowan, J. P.

1924. The modification of the bone lesions in rickets due to mobility of the part. J. Path. Bact. 27, 409-415.

McHargue, J. S.

1924. The association of manganese with vitamins. J. Agr. Research 27, 417424.

1925. The association of copper with substances containing the fat-soluble A vitamin. Am. J. Physiol. 72, 583-594.

McHargue, J. S. and Roy, W. R.

1930. Effect of ultraviolet irradiation on the magnesium content of rats receiving reflected sunlight and a uniform stock ration. Am. J. Physiol. 92, 651-655.

McKay, H. M. M.

1928. Treatment of rickets. I. Prophylactic measures. II. Curative measures. Lancet, 1928, I, 923, $924 ; 979,980$.

McKinnis, R. B. and King, C. G.

1930. The nature of vitamin C-A study of its electrical transference. J. Biol. Chem. 87, 615-623.

McLaughlin, L.

1929. The relation of vitamin A content to size of leaves. J. Biol. Chem. 84, 249-256.

a. The nutritive value of New Zealand spinach. J. Nutrition 2, 197-202.

McLean, S. and McIntosh, R.

1928. Healing in infantile scurvy as shown by $\mathrm{x}$-ray. Am. J. Diseases Children $36,875-930$.

McRobert, G. R.

1928. Observations on the hydrogen-ion concentration of the alimentary canal of the albino rat. Indian J. Med. Research 16, 545-552.

Macallum, A. B.

1920. The relation of vitamins to the growth of young animals. Trans. Roy. Can. Inst. 12, 175-237.

1925. Some features in the chemistry and physiology of the vitamins. Can. Chem. Met. 9, 175-177.

MacArthur, C. G. and Luckett, C. L.

1915. Lipins in nutrition. J. Biol. Chem. 20, 161-174.

MacArthur, E. H.

1926. A quantitative study of the determination of vitamin B. Dissertation, Columbia University, New York.

MacDonald, M. B.

1922. The synthesis of water-soluble B by yeast grown in solutions of purified nutrients. J. Biol. Chem. 54, 243-248.

1923. The synthesis of "bios" by yeast grown in a solution of purified nutrients. J. Biol. Chem. 56, 489-499.

1925. Notes on the multiplication of yeast in solutions of purified nutrients. Am. J. Hyg. 5, 622-634.

1926. A plea for the retention of the term "bios." Science 63, 187. 
MacDonald, M. B. and McCollum, E. V.

1921. The cultivation of yeast in solutions of purified nutrients. J. Biol. Chem.

45, 307-311.

Macht, D. I., Anderson, W. T. and Bell, F. K.

1928. The penetration of ultraviolet rays into live animal tissues. J. Am. Med.

Assocn. 90, 161-165.

Macht, D. I., Bell, F. K. and Elvers, C. F.

1925. Penetration of ultraviolet rays through animal tissues. Am. J. Physiol. 76, 210, 211.

Mackay, H. M. M.

1920. Observations on cases of rickets in an out-patient department. Brit. Mcd. J. No. 3129, 929-932.

Mackay, H. M. M. and Shaw, H. F.

1925. Foodstuffs irradiated with ultra-violet light. Their effect on the bone lesions of rachitic children. Brit. Med. J. No. 3373, 344, 345.

1926. Irradiation of foodstuffs with ultra-violet light. A clinical investigation of the curative value of irradiated food in rickets. Lancet, 1926, I, 8-11.

Mackenzie, J.

1927. Beriberi in Aden. J. Roy. Army Med. Corps 49, 256-260.

Mackie, F. P. and Chitre, G. D.

1928. The association of bowel disease with vitamin C deficiency. Ind. J. Med. Research 16, 77-94.

Macklin, A. H. and Hussey, L. D. A.

1921. Scurvy. A system of prevention for a polar expedition, based on presentday knowledge. Lancct, 1921, II, 322-326.

MacLeod, F. L.

1927. Antiscorbutic vitamin content of milk of stall-fed cows throughout a year. Probability that ensilage is an important source of this vitamin. J. Am. Mcd. Assocn. 88, 1947-1949.

1930. The chemistry of vitamin D. J. Nutrition 2, 517-530.

MacLeod, G. and Booher, L.

1930. The antiscorbutic vitamin content of some preserved foods. J. Homc Econ. 22, 588-593.

MacNeal, W. J.

1916. The alleged production of pellagra by an unbalanced diet. J. Am. Med. Assocn. 66, 975-977.

1921. Pellagra. Am. J. Med. Sci. 161, 469-501.

Macomber, D.

1923. Defective diet as a cause of sterility. J. Am. Med. Assocn. 80, 978-980.

1927. Effect of a diet low in calcium on fertility, pregnancy and lactation in the rat. J. Am. Med. Assocn. 88, 6-13.

a. The effect of diet upon reproduction. J. Am. Dietet. Assocn. 3, 57-67.

Macy, I. G. and Outhouse, J.

1929. The vitamin content of milk used in infant feeding. Am. J. Discases Children 37, 379-400.

Macy, I. G., Outhouse, J., Graham, A. and Long, M. L.

1927. Human milk studies. II. The quantitative estimation of vitamin A. J. Biol. Chem. 73, 175-187.

a. Human milk studies. III. The quantitative estimation of vitamin B. J. Biol. Chem. 73, 189-201. 
Macy, I. G., Outhouse, J., Long, M. L. and Graham, A.

1927. Human milk studies. I. Technique employed in vitamin studies. J. Biol. Chem. 73, 153-174.

Magne, $H$. and Simonnet, $H$.

1922. Sur les variations du quotient respiratoire chez le pigeon carencé. Influence des injections intraveineuses de glucose. Bull. soc. chim. biol. 4, 419425.

Malcolm, J.

1926. Food values of New Zealand fish. Part 6. The vitamin A content of mutton-bird oil and of some fish oils. Trans. Proc. New Zealand Inst. 56, 650-658.

1927. The food values of New Zealand fish. Part 7. The vitamin content of the Tarakihi. (Chilodactylus macropterus.) Trans. Proc. New Zealond Inst. $57,879,880$.

a. Food values of New Zealand fish. Part 8. Stewart Island oysters. Trans Proc. New Zealand Inst. 58, 167-173.

1928. Food values of New Zealand fish. Part 9. Tinned toheroa and toheroa soup. Trans. Proc. New Zealand Inst. 59, 85-90.

a. Food values of New Zealand fish. Part 10. Seasonal variations in Stewart Island oysters. Trans. Proc. Nezv Zealand Inst. 59, 668-677.

Mallon, M. G. and Clark, M.

1922. Vitamin A content of lard obtained from hogs on a control ration. J. Biol. Chem. 54, 763-766.

\section{Manning, J. R.}

1929. Bibliography on cod-liver oil in animal feeding, with noncritical comments and abstracts. U. S. Dept. Com. Bur. Fisheries Doc. 1065, 333-365.

\section{Manville, I. A.}

1925. Pathologic changes occurring in white rats raised on diets deficient in vitamin A. Arch. Internal Med. 35, 549-556.

1926. A vitamin B deficiency manifesting itself for the first time in the second generation. Science 64, 256-257.

a. Variations in the fat-soluble vitamin content of eggs, resulting from different methods of preservation. Am. J. Hyg. 6, 238-253.

1929. The ultraviolet component of the sunlight of Portland, Ore., measured by the acetone-methylene blue method. Am. J. Diseases Children 37, 972-996.

Marchlewski, L. and Wierzchowski, $Z$.

1922. Vitamin studies. Pam. Panst. Inst. Nauk. Gosp. Wiejsk. Pularvach. 2, A $160-187$.

1924. Recherches sur les vitamines. I. Bull. soc. chim. biol. 6, 40-43.

Marcus, J. K.

1928. A new process for the separation of the vitamin fraction from cod liver oil. J. Biol. Chem. 80, 9-14.

Marrian, G. F.

1928. The effect of inanition and vitamin B deficiency on the adrenal glands of the pigeon. Biochem. J. 22, 836-844.

Marrian, G. F., Baker, L. C., Drummond, J. C. and Woollard, H.

1927. The physiological role of vitamin B. Part V. The relation of inanition to vitamin B deficiency in the pigeon. Biochem. J. 21, 1336-1348. 
Marrian, G. F. and Parkes, A. S.

1928. The effects of inanition and vitamin B deficiency on the testis of the pigeon. J. Roy. Micros. Soc. 48, 259-270.

Marshall, A. L. and Knudson, A.

1930. The formation of vitamin D by monochromatic light. J. Am. Chem. Soc. 52, 2304-2314.

Marshall, J. A.

1927. Dental caries and pulp sequelae resulting from experimental diets. $J$. Am. Dental Assocn. 14, 3-37.

Masaki, $\mathrm{T}$.

1927. Studies on vitamin deficiency diseases. J. Am. Dental Assocn. 14, 16541656.

Maslow, H. L., Shelling, D. H. and Kramer, B.

1926. Irradiated orange juice: Its value as an antirachitic agent. Bull. Johns Hopkins Hosp. 39, 56-61.

Mason, K. E.

1925. A histological study of sterility in the albino rat due to a dietary deficiency. Proc. Natl. Acad. Sci. 11, 377-382.

1929. The effect of purified diets, and their modifications, on growth and testicular degeneration in male rats. J. Nutrition 1, 311-334.

1930. The specificity of vitamin $E$ for the testis.- $I$. Relation between vitamins A and E. J. Exptl. Zoöl. 55, 101-122.

Matheu, C. P.

1921. Recherches cliniques sur la vitamine B. Compt. rend. soc. biol. 85 , 593.

Matsumura, S. et al.

1929. The etiology of beriberi. J. Am. Med. Assocn. 92, 1325-1327.

Matsuoka, T.

1930. Studies on vitamin C. I. On the occurrence of vitamin C in celery. II. The production of vitamin $\mathrm{C}$ by germination. Part I. III. The production of vitamin C by germination. Part II. Mem. Coll. Agr., Kyoto Imp. Univ. No. 9, Art. 1-3, 1-13, 15-21, 23-27.

Mattei, P. di.

1923. L'azione vitaminica di sostanze termostabili. Arch. farmacol. sper. 35, 5-30.

Mattill, H. A.

1921. Nutritive properties of milk with special reference to growth and reproduction in the white mouse. Proc. Soc. Exptl. Biol. Med. 18, 242, 243.

a. The influence of fasting and of vitamin $B$ deprivation on the nonprotein nitrogen of rats' blood. Abstracted in Science 54, 176.

1923. The utilization of carbohydrate by rats deprived of vitamin B. J. Biol. Chem. 55, 717-727.

a. The effect of fasting and of vitamin B deprivation on the chemical composition of rats' blood. Proc. Soc. Exptl. Biol. Med. 20, 537, 538.

1927. The relation of vitamins $\mathrm{B}$ and $\mathrm{E}$ to fertility in the male rat. $A m . J$. Physiol. 79, 305-315.

a. The oxidative destruction of vitamins $\mathrm{A}$ and $\mathrm{E}$ and the protective action of certain vegetable oils. J. Am. Med. Assocn. 89, 1505. 
Mattill, H. A. and Carman, J. S.

1923. The degeneration of the testis of rats on a milk diet. Proc. Soc. Exptl. Biol. Med. 20, 420.

Mattill, H. A., Carman, J. S. and Clayton, M. M.

1924. The nutritive properties of milk. III. The effectiveness of the $X$ substance in preventing sterility in rats on milk rations high in fat. J. Biol. Chem. 61, 729-740.

Mattill, H. A. and Claytor, M. M.

1925. The influence of milk rations high and low in fats on the sex glands of male albino rats with special reference to substance X. J. Biol. Chem. 63, XXVII, XXVIII.

1926. Vitamin $\mathrm{E}$ and reproduction on synthetic and milk diets. J. Biol. Chem. 68, 665-685.

Mattill, H. A. and Congdon, C. C.

1924. The influence of yeast product additions to milk rations on the infertility of rats. J. Biol. Chem. 59, XII, XIII.

Mattill, H. A. and Conklin, R. E.

1920. The nutritive properties of milk, with special reference to reproduction in the albino rat. J. Biol. Chem. 44, 137-158.

Mattill, H. A. and Pratt, A. D.

1928. Note on tea as a source of vitamin C. Proc. Soc. Exptl. Biol. Med. 26, $82-85$.

Mattill, H. A. and Stone, N. C.

1923. The nutritive properties of milk with special reference to reproduction in the albino rat. II. J. Biol. Chem. 55, 443-455.

Matzko, S. N.

1929. Gehalt an Vitamin " $D$ " in den Fetten der Meerssäugetiere. Biochem. $Z$. 213, 391-398.

Maughan, G. H.

1928. Ultra-violet wave lengths valuable in the cure of rickets in chickens. Am. J. Physiol. 87, 381-398.

Maughan, G. H. and Dye, J. A.

1929. Amount of ultra-violet radiation necessary to cure rickets. Proc. Soc. Exptl. Biol. Med. 27, 158, 159.

1930. The amount of ultra-violet radiation necessary to cure rickets. J. Optical Soc. America 20, 279-292.

Maurer, S. and Tsai, L. S.

1929. Vitamin B deficiency in nursing young rats and learning ability. Science 70, 456-468.

Maver, M. B.

1930. Nutritional edema and "war dropsy." J. Am. Med. Assocn. 74, 934941.

Mayerson, H. S., Gunther, L. and Laurens, $H$.

1925. The effects of radiation on calcium and phosphorus. Proc. Soc. Exptl. Biol. Med. 22, 469, 470.

Maynard, L. A., Goldberg, S. A. and Miller, R. C.

1925. The influence of sunlight on the mineral nutrition of swine. Proc. Soc. Exptl. Biol. Med. 22, 494, 495.

a. The influence of sunlight on bone development in swine. J. Biol. Chem. $65,643-655$. 
Medes, G.

1926. Germinal epithelium of guinea pigs during early stages of scurvy. Proc. Soc. Exptl. Biol. Med. 23, 294.

a. Rats on diets high in phosphorus and low in calcium. Proc. Soc. Exptl. Biol. Med. 23, 679.

b. The antiscorbutic vitamine in fresh beef. J. Lab. Clin. Med. 11, 871873.

Medical and Surgical History of the War of the Rebellion

1888. Washington, I, Part 3, Chap. 8.

\section{Meesemaecker, $\mathbf{R}$.}

1930. Nouvelle réaction colorée de l'ergostérol. Differenciation de l'ergostérol et de l'ergostérol irradié. J. pharm. chim. 11, 380-384.

\section{Mellanby, E.}

1919. An experimental investigation of rickets. Lancet, 1919, I, 407-412.

a. A further demonstration of the part played by accessory food factors in the etiology of rickets. J. Physiol. 52, LIII, LIV.

1920. Accessory food factors (vitamins) in the feeding of infants. Lancet, 1920, I, 856-862.

1921. Experimental rickets. Med. Research Council (Gt. Brit.) Spec. Rept. Ser. No. 61, pp. 28.

1922. The rickets-producing effect of dried thyroid. J. Physiol. 57, II, III.

1924. Deficiency diseases, with special reference to rickets. Brit. Med. J. No. $3308,895-900$.

1925. Experimental rickets. The effect of cereals and their interaction with other factors of the diet and environment in producing rickets. Med. Research Council (Gt. Brit.) Spec. Rept. Ser. No. 93, pp. 66.

1926. Diet and disease, with special reference to the teeth, lungs, and prenatal feeding. Brit. Med. J. No. 3403, 515-519.

a. The presence in foodstuffs of substances having specific harmful effects under certain conditions. J. Physiol. 61, XXIV.

1930. The relation of diet to health and disease. Brit. Med. J. No. 3614, 677-681.

Mellanby, E. and Green, H. N.

1929. Vitamin A as an anti-infective agent. Its use in the treatment of puerperal septicemia. Brit. Med. J. No. 3569, 984-986.

Mellanby, E., Surie, E. M. and Harrison, D. C.

1928. The antirachitic effect of ergot. J. Physiol. 65, XXIX.

1929. Vitamin D in ergot of rye. Biochem. J. 23, 710-717.

Mellanby, M.

1918. Influence of diet on teeth formation. Lancet, 1918, II, 767-770.

1924. The influence of light in relation to diet on the formation of teeth. Brit. Dental J. 45, 545-552.

1928. The influence of diet on the structure of teeth. Physiol. Rev. 8, 545-577. 1929. Diet and the teeth: An experimental study. Part I. Dental structure in dogs. Med. Research Council (Gt. Brit.) Spec. Rept. Ser. No. 140, pp. 308.

Mellanby, M. and Killick, E. M.

1926. A preliminary study of factors influencing calcification processes in the rabbit. Biochem. J. 20, 902-926. 
Mellanby, M. and Pattison, C. L.

1928. The action of vitamin D in preventing the spread and promoting the

arrest of caries in children. Brit. Med. J. No. 3545, 1079-1082.

Mellanby, M., Pattison, C. L. and Proud, J. W.

1924. The effect of diet on the development and extension of caries in the teeth of children. Brit. Med. J. No. 3324, 354.

Mendel, L. B.

1915. Nutrition and growth. J. Am. Med. Assocn. 64, 1539-1547.

1917. Abnormalities of growth. Am. J. Med. Sci. 153, 1-20.

1923. "Nutrition: The Chemistry of Life," New Haven, Yale University Press.

Mendel, L. B. and Cannon, H. C.

1927. The relation of the rate of growth to diet. II. J. Biol. Chem. 75, 779787.

Mendel, L. B. and Vickery, H. B.

1928-29. Continuation and extension of work on vegetable proteins. Carnegie Inst. Wash. Yearbook 28, 367-377.

1929. An attempt to secure "refection" in rats. Proc. Soc. Exptl. Biol. Med. 26, 552-555.

Menville, L. J., Blackberg, S. N. and Ané, J. N.

1929. Radiological study of the motility of gastro-intestinal tract of rachitic rats. Proc. Soc. Exptl. Biol. Med. 26, 758-760.

Mercer, E. W. and Tozer, F. H. W. P.

1927. The effect of irradiation and cod-liver oil upon poultry. J. Ministry Agr. 34, 624-626.

Merjanian, A.

1926. Über den Vitamingehalt von Trauben und Traubenweinen. Z. Untersuch. Lebensmittel. 52, 307-311.

Meyer, A. W. and McCormick, L. M.

1928. Experimental scurvy in the guinea pig. Proc. Soc. Exptl. Biol. Med. 25, 494-496.

a. Studies on scurvy. Stanford Univ. Pubs. Univ. Ser. Med. Sci. 2, pp. 107.

Meyer, C. R. and Hetler, R. A.

1929. The distribution of vitamin A in some corn-milling products. J. Agr. Research 39, 769-780.

Meyer, L. F.

1926. Die Altersdisposition zu Avitaminosen. Deut. med. Wochschr. 52, 2070-2072.

Meyer, L. F. and Nassau, E.

1924. Experimentelle Untersuchungen über den Vitamingehalt der Milch. Klin. Wochschr. 3, 2132-2135.

1925. Über den Vitamingehalt der Frauenmilch. Klin. Wochschr. 4, 23802383.

Meyerstem, A.

1922. Anatomische Untersuchungen zur Frage der akzessorischen Nährstoffe. Arch. path. Anat. (Virchow's) 239, 350-362.

Meysenbug, L. von.

1922. The inorganic phosphate content of breast milk of mothers with normal and with rachitic infants. Am. J. Diseases Children 24, 200-203. 
Mignon, H. L.

1923. Biological food tests. IV. The protein and the vitamin A content of the English walnut. Am. J. Physiol. 66, 215-231.

Miles, L. M. and Feng, C. T.

1925. Calcium and phosphorus metabolism in osteomalacia. J. Exptl. Med. 41, 137-157.

Miller, C. D.

1924. Vitamins $\mathrm{A}$ and $\mathrm{B}$ in fresh and canned pineapple. J. Home Econ. 16, 18-26, 74-79.

1925. Vitamin $C$ in fresh and canned pineapple. J. Home Econ. 17, 377-382.

1926. The vitamins (A, B, and C) of papaya. Biochem. J. 20, 515-518.

a. The vitamin and mineral content of pineapple bran. Hazeaii Pineapple Canner's Sta. Bull. 6, pp. 11.

1927. Food values of poi, taro and limu. Bernice P. Bishop Museum Bull. 37, pp. 25.

1928. The vitamin A and B content of the pigeon pea. J. Agr. Research 18, 569-573.

Miller, C. D. and Hair, D. B.

1928. The vitamin content of mung bean sprouts. J. Home Econ. 20, 263-271.

Miller, H. G.

1926. Vitamin B requirements for successful reproduction and rearing of the young. Am. J. Physiol. 79, 255-259.

Miller, H. G. and Yates, W. W.

1924. The relation of natural foodstuffs and their treatment on growth and reproduction. J. Biol. Chem. 62, 259-268.

Miller, R. C. and Maynard, L. A.

1927. Calcification studies with rats fed menhaden oil and various menhaden fish meals. Am. J. Physiol. 79, 626-632.

Miller, R. C., Wohlwend, I. and Maynard, L. A.

1926. Vitamin studies with menhaden fish meal and menhaden oil. Proc. Soc. Exptl. Biol. Med. 23, 283, 284.

Miller, R. J., Dutcher, R. A. and Knandel, H. C.

1929. Nutritional leg-weakness in poultry. Poultry Sci. 8, 113-123.

Miller, S. P.

1922. Effects of various types of inanition upon the mitochondria in the gastrointestinal epithelium and in the pancreas of the albino rat. Anat. Record 22, 205.

\section{Miller, W. L.}

1924. Wildiers' Bios. Science 59, 197-199.

1930. Bios. J. Chem. Ed. 7, 257-267.

Millicer-Szymanska, R. and Funk, C.

1926. Die Wirkung von einigen Puridinderivaten auf reisgefütterte Tauben. Chem. Zelle Gewebe 13, 44-45.

Mills, C. A.

1928. Treatment of diabetes with an acid-alcoholic extract of plants rich in vitamin B. Am. J. Med. Sci. 175, 376-383.

a. Effects on carbohydrate metabolism of an acid-alcoholic extract of plants rich in vitamin B. Am. J. Med. Sci. 175, 384-386. 


\section{Mirvish, L.}

1929. Nature of the rickets-producing factor in cereals. Nature 124, 410, 411.

1930. The effect of cereal extracts on blood-calcium. Biochem. J. 24, 233-238. Mitchell, H. H.

1919. On the identity of the water-soluble growth-promoting vitamin and the antineuritic vitamin. J. Biol. Chem. 40, 399-413.

1922. The necessity of balancing dietaries with respect to vitamines. Science $56,34-37$.

Mitchell, H. H. and Carman, G. C.

1926. Effect of excessive amounts of vitamin B on the basal metabolism of rats of different ages. Am. J. Physiol. 76, 385-397.

Mitchell, H. H., Kendall, F. E. and Card, L. E.

1923. The vitamin requirements of growing chickens. Poultry Sci. 2, 117-124.

\section{Mitchell, H. S.}

1922. An improved method of caging and feeding mice. J. Lab. Clin. Med. 7, $299,300$.

a. Reproduction on synthetic diets when purified agar is added to the mixture. Am. J. Physiol. 62, 557, 558.

1928. Nutritive value of the garbanza pea. West. Hosp. Nurses' Rev. II, $26,27,52,53$.

1929. Vitamin C content of Japan green tea. J. Am. Dietetic Assocn. 5, 28-31. Mitchell, H. S., Bradshaw, P. J. and Carlson, E. R.

1924. Hair ball formation in rats in relation to food consistency. Am. J. Physiol. 68, 203-206.

Mitchell, H. S. and Johnson, F.

1925. Ultraviolet radiations in conditions of extreme calcium and phosphorus deficiency. Am. J. Physiol. 72, 143-150.

Mitchell, H. S. and Mendel, L. B.

1921. Studies in nutrition. The choice between adequate and inadequate diet as made by rats and mice. Am. J. Physiol. 58, 211-225.

Miyadera, $\mathbf{K}$.

1922. Über die Beziehungen der Vitaminfunktion zum Kalkstoffwechsel. Biochem. Z. 130, 199-208.

\section{Monasterio, G.}

1929. Über die sogenannten Reaktionen des Vitamine A. Biochem. Z. 212, 66-70.

Moore, C. U.

1924. Newer clinical signs of early rickets. J. Am. Med. Assocn. 83, 14691473.

Moore, C. U. and Brodie, J. L.

1927. The relation of maternal diet to hemorrhage in the new-born. Am. J. Diseases Children 34, 53-60.

Moore, C. U., Brodie, J. L. and Hope, R. B.

1927. Some effects upon the young of inadequate maternal diets. I. Polyneuritis and hemorrhages. Am. J. Physiol. 82, 350-357.

Moore, J. J. and Jackson, L.

1916. Experimental scurvy produced in guinea-pigs by milk and milk products. J. Am. Med. Assocn. 67, 1931-1935. 
Moore, T.

1927. Vitamin A formation in the etiolated wheat shoot. Biochem. J. 21,870874.

1928. Vitamin A formation: The feeding of etiolated wheat shoots to rats kept in darkness. Biochem. J. 22, 1097-1101.

1929. A note on carotin and vitamin A. Lancet, 1929, I, 499, 500.

a. The colorimetric estimation of vitamin A. Lancet, 1929, II, 219, 220.

b. The relation of carotin to vitamin A. Lancet, 1929, II, 380, 381.

c. Vitamin A and carotene. I. The association of vitamin A activity with carotene in the carrot root. Biochem. J. 23, 803-811.

d. Vitamin A and carotene. II. The vitamin A activity of red palm oil carotene. III. The absence of vitamin D from carotene. IV. The effect of various dietary modifications upon the vitamin A activity of carotene. Biochem. J. 23, 1267-1269, 1270, 1270-1272.

1930. Vitamin A and carotene. V. The absence of the liver oil vitamin A from carotene. VI. The conversion of carotene to vitamin A in vivo. Biochem. J. 24, 692-702.

Moore, T. and Willimott, S. G.

1927. On the development of chromogenic properties in cholesterol by the action of heat. Biochem. J. 21, 585-588.

Morel, A., Mouriquand, G., Michel, P. and Thévenon, L.

1921. Sur l'absence de troubles électifs du métabolisme du calcium osseux. dans le scorbut expérimental. Compt. rend. soc. biol. 85, 469, 470.

Morgan, A. F.

1923. Biological food tests. I. Vitamin A in some citrus fruit products. II. Vitamin A in skim milk. Am. J. Physiol. 64, 522-537; 538-546.

1924. Biological food tests. VIII. Vitamins $\mathrm{A}$ and $\mathrm{B}$ in radish. Am. J. Physiol. 69, 634-637.

1926. Biological food tests. IX. Vitamin A in three varieties of cheese. Am. J. Physiol. 78, 11-16.

Morgan, A. F. and Barry, M. M.

1930. Underweight children: Increased growth secured through the use of wheat germ. Am. J. Diseases Children 39, 935-947.

Morgan, A. F. and Chaney, M. S.

1924. Biological food tests. VI. Further experiments upon the vitamin A and $\mathrm{B}$ content of citrus fruit products. Am. J. Physiol. 68, 397-406.

Morgan, A. F. and Field, A.

1929. The effect of drying and of sulfur dioxide upon the antiscorbutic property of fruits. J. Biol. Chem. 82, 579-586.

1930. Vitamins in dried fruits. II. The effect of drying and of sulfur dioxide upon the vitamin A content of fruits. J. Biol. Chem. 88, 9-25.

Morgan, A. F. and Francis, L. D.

1924. Biological food tests. VII. The vitamin A and B content of fresh and dehydrated pumpkin. Am. J. Physiol. 69, 67-77.

Morgan, A. F. and Garrison, E. A.

1930. The effect of vitamin $D$ and of reaction of diet upon response to parathyroid extract. J. Biol. Chem. 85, 687-711.

Morgan, A. F., Newbecker, B. M. and Bridge, E.

1923. Biological food tests. V. The biological value of almond proteins and of almond oil. Am. J. Physiol. 67, 173-192. 
Morgan, A. F. and Osburn, D. F.

1925. The effect of vitamin A deficiency upon the character of nitrogen metabolism. J. Biol. Chem. 66, 573-594.

Morgan, A. F. and Smith, L. L. W.

1928. Development of vitamin A during ripening of tomatoes. Proc. Soc. Exptl. Biol. Med. 26, 44-47.

Morgan, A. F. and Stephenson, H. D.

1923. Biological food tests. 1II. Changes in vitamins $\mathrm{A}$ and $\mathrm{B}$ of the globe artichoke due to various canning and drying processes. Am. J. Physiol. 65, 491-502.

Morgan, R. S. and MacLennan, K.

1928. The fluorescence of some vitamin A-containing fats. Biochem. J. 22, 1514-1522.

Mori, M.

1904. Über den sog. Hikan (Xerosis conjunctivae infantum ev. Keratomalacie).

II. Jahrb. Kinderheilk. 59, 175-195.

Mori, S.

1922. Primary changes in eyes of rats which result from deficiency of fatsoluble $\mathrm{A}$ in diet. J. Am. Med. Assocn. 79, 197-200.

a. The changes in the para-ocular glands which follow the administration of diets low in fat-soluble A with notes of the effect of the same diets on the salivary glands and the mucosa of the larynx and trachea. Bull. Johns Hopkins Hosp. 33, 357-359.

1923. The pathological anatomy of ophthalmia produced by diets containing fat-soluble A, but unfavorable contents of certain inorganic elements. Am. J. Hyg. 3, 99-102.

Morinaka, $\mathrm{K}$.

1922. Über die anorganischen Bestandteile des Körpers bei Avitaminose. Biochem. Z. 133, 63-66.

1923. Zum Phosphorstoffwechsel bei Avitaminose. Biochem. Z. 142, 381-384. Moritz, A. R.

1925. The effect of ultraviolet irradiation on the state of the serum calcium. J. Biol. Chem. 64, 81-89.

Moritz, A. R. and Goldblatt, H.

1925. Studies on the state of the serum calcium. Proc. Soc. Exptl. Biol. Med. 23, 111-113.

Moritz, A. R. and Krenz, C.

1930. The relation of the fat-soluble vitamins (A and D) to the development of experimental rickets in rabbits. J. Nutrition, 2, 257-264.

Morrison, R. R., Peacock, P. R. and Wright, S.

1928. The action of $\mathrm{X}$-radiation upon vitamin $\mathrm{D}$ in activated ergosterol. Biochem. J. 22, 1138-1141.

Morton, R. A. and Heilbron, I. M.

1928. The absorption spectrum of vitamin A. Nature 122, 10.

a. The absorption spectrum of vitamin A. Biochem. J. 22, 987-996.

Morton, R. A., Heilbron, I. M. and Kamm, E. D.

1927. The absorption spectrum of ergosterol in relation to the photosynthesis and formation of vitamin D. J. Chem. Soc. 2000-2005.

Morton, R. A., Heilbron, I. M. and Spring, F. S.

1930. Absorption spectra in relation to vitamin A. Biochem. J. 24, 136-140. 
Mottram, J. C., Cramer, W. and Drew, A. H.

1922. Vitamins, exposure to radium and intestinal fat absorption. Brit. J. Exptl. Path. 3, 179-181.

Mouriquand, G. and Bernheim, M.

1925. Nouvelles recherches sur les relations entre l'âge et l'apparition des trou-

bles d'avitaminose C. Compt. rend. 181, 1103, 1104.

Mouriquand, G. and Leulier, A.

1927. Recherches expérimentales sur la biochemie du scorbut. J. physiol. path. gén. 25, 308-318.

1929. Sur l'action antirachitique de certains lipides cholesteriques de l'escargot de Bourgogne (Helix Pomatia). Compt. rend. 188, 1701-1702.

Mouriquand, G., Leulier, A., Bernheim, M. and Schoen, J.

1928. Recherches sur les fixateurs du calcium. Presse méd. 36, 209-213.

Mouriquand, G., Leulier, A. and Michel, P.

1925. Avitaminose C et glycémie. Compt. rend. soc. biol. 92, 271-273.

a. Fluctuations du fer sanguin au cours du scorbut expérimental. Compt. rend. $180,86-88$.

b. Dosage du phosphore et de la chaux du tissu osseux et des dents des animaux soumis à l'avitaminose C. Compt. rend. soc. biol. 92, 269-271.

Mouriquand, G., Leulier, A. and Sédallian, P.

1927. Le $\mathrm{pH}$ et la réserve alcaline dans l'avitaminose C. Compt. rend. 185, 551-553.

Mouriquand, G. and Michel, P.

1921. Parallélisme entre le degré de dessiccation et la perte du pouvoir antiscorbutique des végétaux frais. Compt. rend. soc. biol. 84, 41, 42.

a. Accidents du type scorbutique chez des animaux à une alimentation normale, non carencée, soumis à l'action de l'extrait thyroïdien. Compt. rend. soc. biol. $84,43-45$.

b. Le jus de citron stérilisé est-il antiscorbutique? Compt, rend. soc. biol. $85,470-472$.

1922. De l'action de certains aliments gras sur le métabolisme osseux. Adjuvants et antagonistes de la substance antiscorbutique. Compt. rend. soc. biol. 86, $1170-1172$.

a. Sur la valeur antiscorbutique du jus de citron stérilisé et sur la question des doses d'antiscorbutique necessaires au métabolisme. Compt. rend. soc. biol. 87, 1403, 1404.

1923. Sur les conditions expérimentales d'action de l'huile de foie de morue. Son pouvoir ostéodystrophique en présence d'un régime deficient. Compt. rend. 176, 338-340.

a. Sur quelques facteurs ostéodystrophiques et leur action suivant les espèces animales. Compt. rend. 176, 1655-1657.

1924. Relations entre l'âge, l'apparition et l'évolution des troubles d'avitaminose

C. Compt. rend. 178, 652-654.

Mouriquand, G., Michel, P. and Barre, L.

1922. Croissance et substance antiscorbutique. Compt. rend. soc. biol. 86, 1167-1169.

Mouriquand, G., Michel, P. and Bernheim.

1924. La sensibilisation de l'organisme vis-à-vis des régimes carencés. Compt. rend. 178, 1098-1099. 
a. Nouvelles recherches sur la sensibilisation de l'organisme du cobaye à l'avitaminose C. Compt. rend. 179, 541-543.

Mouriquand, G., Michel, P. and Sanyas, R.

1923. Extrait thyroïdien et lesions de carence expérimentale. Compt. rend. soc. biol. 88, 214, 215.

a. Les régimes adjuvants et antagonistes de l'action dystrophique de l'extrait thyroidien. Compt. rend. soc. biol. 88, 216-218.

Mouriquand, G., Michel, P. and Uicodiévitch.

1922. Polynévrite expérimentale par le riz decortiqué et inanition. Compt. rend. soc. biol. 87, 168, 169.

Muckenfuss, A. M.

1918. The presence of food accessories in urine, bile, and saliva. J. Am. Chem. Soc. 40, 1606-1611.

1919. The excretion of vitamins. Arch. Pediatrics 36, 80-81.

Munsell, H. E.

1924. The quantitative determination of relative amounts of vitamin A. Dissertation, Columbia University, New York.

1929. Rice polishings as a source of vitamin B. J. Home. Econ. 21, 124-129. 1930. The vitamin A, B, C, and $\mathrm{G}$ content of watermelon (Citrullus vulgaris). J. Home Econ. 22, 680-685.

Munsell, H. E. and Black, $H$.

1928. "The assay of a so-called cod-liver oil extract" for vitamin-A content and calcifying properties compared to cod-liver oil. J. Am. Pharm. Assocn. 17, 139-144.

Munsell, H. E. and Kifer, H. B.

1929. Green tea as a source of vitamin C. J. Home Econ. 21, 514-518.

Murphy, J. C. and Jones, D. B.

1924. Vitamin A content of fresh eggs. J. Agr. Research 29, 253-257.

Murray, I.

1923. The thyroid gland in experimental rickets. Brit. J. Exptl. Path. 4, 335-339.

Murray, J. M. and Little, C. C.

1924. The influence of ultra-violet light on nutrition in poultry. Maine Agr. Exptl. Sta. Bull. 320, 141-164.

Mussehl, F. E., Hill, R. and Ackerson, C. W.

1928. The antirachitic value of cod liver meal. Poultry Sci. 7, 239-242.

Myers, C. N. and Voegtlin, C.

1920. The chemical isolation of vitamins. J. Biol. Chem. 42, 199-205.

Nabarro, D. and Hickman, J. O.

1930. The irradiation of milk for the increasing of its antirachitic potency. Lancet, 1930, I, 127-129.

Nagayama, T. and Munelusa, T.

1929. Studies in experimental scurvy. V. The calcium and phosphorus metabolism of guinea pigs fed on a vitamin C-free diet. J. Biachem. 11, 191-201.

Nagayama, T. and Tagaya, T.

1929. Studies in experimental scurvy. V. The lipoid metabolism of the guinea pigs fed on a vitamin C-free diet. J. Biochem. 11, 225-237. 
1930. Studies in experimental scurvy. VII. The effect of a vitamin C given parenterally to guinea pigs fed on a vitamin free diet. J. Biochem. 11, 445460.

Nagayo, M.

1923. Beriberi and rice neuritis. J. Am. Med. Assocn. 81, 1435-1437.

Naito, $\mathrm{H}$.

1923. Uber den Kephalin- und Lecithingehalt des Gehirns bei Avitaminose. Biochem. Z. 142, 385-392.

a. Über den Lecithingehalt des Gehirns und der Leber normaler und avitaminöser Tauben nach farcierter Lecithinfütterung. Biochem. Z. 142, 393397.

Nakahara, W.

1926. Influence of vitamin A on the absorption of a foreign fat. Sci. Papers Inst. Phys. Chem. Research (Tokya) 4, 161-166.

Nakahara, W. and Somekawa, E.

1928. Vitamins and tumor growth. I. The deficient vitamin B content of chicken sarcoma tissue. Proc. Imperial Acad. Tokyo 4, 440-443. Also in Japan. Med. World 8, 231-233.

1929. Vitamins and tumor growth. II. Preliminary note on the non-consumption of vitamin $\mathrm{B}$ by growing chicken sarcoma. Proc. Imperial Acad. Tokyo 5, 55, 56.

a. Non-consumption of vitamin B by growing chicken sarcoma. Sci. Papers Inst. Phy. Chem. Research (Tokyo) 10, 211-220.

1930. Vitamins and tumor growth. III. Vitamin B content of rat tumors. Proc. Imp. Acad. Tokyo 6, 116-118.

Nakahara, W. and Yokoyama, Y.

1926. Effect of combined fat and vitamin A deficiency on growth and organ weight of albino rats. Sci. Papers Inst. Phys. Chem. Research (Tokyo) 5, 63-78.

Nakamiya, Z. and Kawakami, K.

1927. On the hydrogenation of "Biosterin." Sci. Papers Inst. Phys. Chem. Research 7, 123-142.

Narayanan, B. T.

1930. The chemical investigation of "bios." Part I. Biochem. J. 24, 6-18.

Narayanan, B. T. and Drummond, J. C.

1930. The concentration of vitamin B. Biochem. J. 24, 19-26.

Nassau, E.

1928. Praktische Anwendung der Vitamine. Deut. med. Wochschr. 54, 15901592.

Nebraska Agr. Expt. Sta.

1925. A study of fat soluble A as present in the milk of the four dairy breeds. Ann. Rept., p. 12.

Nelson, E. M. and Jones, D. B.

1928. Observations bearing on the determination of vitamin A. J. Biol. Chem. 80, 215-226.

Nelson, E. M. and Steenbock, $\mathrm{H}$.

1925. Fat-soluble vitamins. XXI. Observations bearing upon the alleged induction of growth-promoting properties in air by irradiation with ultra-violet light. J. Biol. Chem. 62, 575-593. 
a. Fat-soluble vitamins. XXIV. The non-precipitability of the antiophthalmic and antirachitic properties from cod-liver oil by digitonin. J. Biol. Chem. 64, 299-312.

b. Fat-soluble vitamins. XXV. Further observations on the antirachitic action of irradiated animals on the non-irradiated when placed in the same cage. Am. J. Physiol. 73, 341-345.

Nelson, P. M.

1926. Maternal diet and lactation. J. Home Econ. 18, 383-338.

Nelson, V. E., Baldwin, F. M., Riggs, A. G. and Cunningham, N.

1925. The relation of vitamin deficiency to muscle fatigue. Am. J. Physiol. 72, 69-75.

Nelson, V. E., Fulmer, E. I. and Cessna, R.

1921. The nutritional requirements of yeast. III. The synthesis of watersoluble B by yeast. J. Biol. Chem. 46, 77-81.

Nelson, V. E., Heller, V. G. and Fulmer, E. I.

1923. Studies on yeast. VII. The dietary properties of yeast. J. Biol. Chem. 57, 415-424.

1925. Molasses as a source of vitamin B. Ind. Eng. Chem. 17, 199-201; also in Planter Sugar Mfr. 74, 128, 129.

Nelson, V. E., Jones, R. L., Heller, V. G., Parks, T. B. and Fulmer, E. I.

1926. Diet in relation to reproduction and rearing of young. I. Observations on the existence of vitamin E. Am. J. Physiol. 76, 325-328.

Nelson, V. E. and Lamb, A. R.

1920. The effect of vitamin deficiency on various species of animals. I. The production of xerophthalmia in the rabbit. Am. J. Physiol. 51, 530-535.

Nelson, V. E., Lamb, A. R. and Heller, V. G.

1922. The effect of vitamin deficiency on various species of animals. II. Observations on the comparative vitamin A requirement of rabbits, rats, swine and chickens. Am. J. Physiol. 59, 335-345.

Nelson, V. E., Ohrbeck, E., Jones, R. L. and Taylor, M. W.

1928. Codliver oil for reproduction. Am. J. Physiol. 85, 476-481.

Never, H. E.

1928. Avitaminose und Verdaungssorgane. Arch. ges. Physiol. (Pflüger's) 219, 554-563.

Newcomb, C.

1930. The water content of the heart muscle in beri-beri columbarum. Indian J. Med. Research 17, 721-734.

Nitzescu, I-I.

1923. L'action de la chaleur humide (autoclave) et de la chaleur sèche (étuve) sur le facteur B. Compt. rend. soc. biol. 89, 1244, 1245.

Nitzescu, I-I., and Cadarin, I.

1923. Le sang chez les pigeons dans l'avitaminose. Compt. rend. soc. biol. 89, 1245-1247.

Nitzescu, I-I., Popoviciu, G. and Denes-Goetz, J.

1927. Contribution a l'isolement de la fraction antirachitique du cholestérol irradié par les rayons ultra-violets. Bull. soc. chim. biol. 9, 126-136.

Nobel, E.

1921. Zur Barlow-Frage. Bedeutung der Hitzewirkung auf die VitamineBeitrag zur Frage der Nahrungskonzentration. Z. Kinderheilk. 28, 348-370. 
1923. Uber die Beeinflussung des experimentellen Meerschweinchenskorbuts durch die Gravidität. Z. ges. exptl. Med. 38, 528-536.

Nobel, E. and Wagner, $\mathbf{R}$.

1923. Beeinflussung von experimentellen Skorbut durch Schilddrüsenfütterung. Z. ges. exptl. Med. 38, 181-190.

Nonidez, J. F.

1927. Studies on the bones in avian rickets. I. Bone lesions in chickens deprived of the antirachitic factor after five weeks of normal growth. Am. J. Path. 4, 463-480.

Nonidez, J. F. and Goodale, H. D.

1927. Histological studies on the endocrines of chickens deprived of ultraviolet light. Parathyroids. Am. J. Anat. 38, 319-347.

Normak, P.

1924. Beiträge zur Biochemie der Avitaminosen. 3. Blutfermente bei experimentellen Skorbut. Biochem. Z. 152, 420-425.

Norris, E. R. and Church, A. E.

1930. A study of the antimony trichloride color reaction of vitamin A. J. Biol. Chem. 85, 477-489.

a. A study of the antimony trichloride reaction for vitamin A. II. The dilution curve of cod liver oil with antimony trichloride reagent. J. Biol. Chem. 87, 139-146.

Norris, E. R. and Danielson, L. S.

1929. Ratfish-liver oil as a source of vitamin A. Ind. Eng. Chem. 21, 1078.

a. Comparison of biological and colorimetric assays for vitamin A as applied to fish oils. J. Biol. Chem. 83, 469-475.

Norris, L. C., Heuser, G. F. and Wilgus, H. S., Jr.

1929. Effect of storage in finely divided feeds upon the stability of the D vitamin of cod-liver oil. N. Y. Cornell Agr. Expt. Sta. Mem. 126, pp. 15.

1930. Is the chief value of milk for feeding poultry due to the presence of a new vitamin? Poultry Sci. 9, 133-140.

Norris, L. C. and Ringrose, A. T.

1930. The occurrence of a pellagrous-like syndrome in chicks. Science 71, 643.

Novaro, $\mathbf{P}$.

1920. Ricerche calorimetriche comparative sul Digiuno e sull'Avitaminosi. I. Del Digiuno. II. Dell'Avitaminosi. Pathologica 12, 87-100; 133-156.

Ogata, C., Kawakita, S., Oka, C. and Kagoshima, S.

1921. Polished rice disease of birds. Nisshin Igaku (Progress in Med) 10, No. 6. Abstracted in Japan Med. World 1, 23.

Ogata, D.

1022. Kann die avitaminöse Wachstumsstörung durch chemisch definierte Substanzen beeinflusst werden? Biochem. Z. 132, 89-94.

Ohdake, $\mathrm{S}$.

1927. On the chemical constituents of yeast extract. J. Agr. Chem. Soc. Japan 3, 98-122.

Ohio Agr. Expt. Sta.

1924. Influence of fertilizers on nutritive value of wheat. Bull 382, pp. 56, 57. 
1927. Growth of chicks as affected by sunight. Bull. $402,88,89$.

a. Calcium requirements of the growing chick. Bull. 402,90 .

b. The effect of cod liver oil and ultra-violet light on egg production and hatchability. Bull. 402, 91, 92.

c. Fat-soluble vitamin content of hen's eggs. Bull. 402, 92, 93.

Ohler, W. R.

1914. Experimental polyneuritis. Effects of exclusive diet of wheat flour in the form of ordinary bread on fowls. J. Med. Research 31, 239-246.

Ohomori, K., Ohhashi, Y., Nakanichi, H., Hara, M. and Ota, I. 1922. Studies on the etiology of beriberi. Japan Med. World 2, 128-133.

Okada, S. and Sakurai, E.

1926. The basal metabolism in beriberi. Arch. Internal Med. 38, 770-778.

Okada, S., Sakurai, E., Ibuki, T. and Kabeshima, $\mathrm{H}$.

1923. On the basal metabolism in vitamin B starvation and in beriberi. Japan Med. World 3, 102-104.

1927. Basal metabolism in vitamin B starvation. Arch. Internal Med. 40, 292-313.

Okamato, T.

1925. Studies on experimental xerophthaimia. Japan Med. World 5, 180-182.

Okuda, M.

1922. On the action of rice embryo against avian polished rice disease. Kenyokwai Zasshi. J. Kenyo Med. Soc. No. 158, May, 1922. Abstracted in Japan Med. World 2, 305.

Olson, T. M. and Copeland, L.

1924. The influence of pasteurization and the diet of the cow on the antiscorbutic potency of the milk. J. Dairy Sci. 7, 370-380.

Onohara, $\mathrm{K}$.

1925. Über den Einfluss der Insulinbehandlung auf den Fettgehalt des Körpers bei avitaminösen Ratten und verschiedenen Ernährungsbedingungen. Biochem. Z. 163, 51-60.

a. Untersuchungen über den Einfluss des Insulins auf den Blutfettgehalt bei der Avitaminose des Hundes. Biochem. Z. 163, 67-74.

Orgler, A.

1911. Uber den Kalkstoffwechsel bei Rachitis. Monatsschr. Kinderheilk. 10, 373-387.

1912. Der Kalkstoff wechsel des gesunden und des rachitischen Kinder. Ergeb. inn. Med. Kinderheilk. 8, 142-182.

Orr, J. B. and Crichton, A.

1924. The requirements of the pig for vitamin A and vitamin C. J. Agr. Sci. 14, 114-126.

Orr, J. B., Crichton, A. et al.

1924. The effects of adding vitamin-rich substances to normal rations for poultry. I. The fat-soluble vitamin or vitamin A. Scottish J. Agr. 7, 266277.

Orr, J. B., Henderson, J. M. and Crichton, A.

1926. The influence on nutrition of sunlight and artificially produced ultraviolet rays. Trans. Highland Agr. Soc. Scotland 38, 88-105.

Orr, J. B., Magee, H. E. and Henderson J. McA.

1925. The effect of ultra-violet light on the mineral metabolism of the lactating animal. Biochem. J. 19, 569-572. 


\section{Orr, J. B., Moir, M., Newbigin, H., Robertson, G. S. and Murphy, M.}

1925. The nutritive requirements of poultry. I. The effect of adding vitaminrich substances to normal rations. II. Vitamin B or the water-soluble vitamin of yeast. Scottish J. Agr. 8, 62-69.

Orr, W. J., Holt, L. E., Jr., Wilkins, L. and Boone, F. H.

1923. The calcium and phosphorus metabolism in rickets, with special reference to ultra-violet ray therapy. Am. J. Diseases Children 26, 362-372.

1924. Relation of calcium and phosphorus in the diet to the absorption of these elements from the intestine. Am. J. Diseases Children 28, 574-581.

Orr-Ewing, J. and Reader, V.

1928. Strcptothrix corallinus in the estimation of vitamin $\mathrm{B}_{1}$. Biochem. J. 22, 440-442.

a. Note on the Meningococcus as a source of the growth factor for Streptothrix corallinus. Biochem. J. 22, 443-444.

Orton, C. R., McCollum, E. V. and Simmonds, N.

1922. Observations on the presence of the antineuritic substance water-soluble B in chlorophyll-free plants. J. Biol. Chem. 53, 1-6.

Osborne, T. B. and Leavenworth, C. S.

1921. The effect of alkali on the efficiency of the water-soluble vitamin B. J. Biol. Chem. 45, 423-426.

Osborne, T. B. and Mende1, L. B.

1911. Feeding experiments with isolated food-substances. Carnegie Inst. Wash. Pub. 156, pt. 2.

1912. The rôle of gliadin in nutrition. J. Biol. Chcm. 12, 473-510.

a. Maintenance experiments with isolated proteins. J. Biol. Chem. 13, 233-276.

1913. The relation of growth to the chemical constituents of the diet. J. Biol. Chem. 15, 311-326.

a. The influence of butter fat on growth. J. Biol. Chem. 16, 423-437.

1914. The influence of cod liver oil and some other fats on growth. J. Biol. Chem. 17, 401-408.

a. The suppression of growth and the capacity to grow. J. Biol. Chem. 18, 95-106.

1915. The comparative nutritive value of certain proteins in growth, and the problem of the protein minimum. J. Biol. Chem. 20, 351-378.

a. Further observations of the influence of natural fats upon growth. $J$. Biol. Chem. 20, 379-389.

b. Protein minima for maintenance. J. Biol. Chem. 22, 241-257.

c. The resumption of growth after long-continued failure to grow. J. Biol. Chem. 23, 439-454.

1916. The stability of the growth-promoting substance in butter fat. J. Biol. Chem. 24, 37-39.

a. The amino acid minimum for maintenance and growth, as exemplified by further experiments with lysine and tryptophane. J. Biol. Chem. 25, 1-12.

b. A quantitative comparison of casein, lactalbumin and edestin for growth and maintenance. J. Biol. Chem. 26, 1-23.

c. Acceleration of growth after retardation. Am. J. Physiol. 40, 16-20.

d. The growth of rats upon diets of isolated food substances. Biochem. J. 10, 534-538. 
1917. The use of cottonseed as food. J. Biol. Chem. 29, 289-317.

a. The rôle of vitamins in the diet. J. Biol. Chem. 31, 149-163.

b. Nutritive factors in animal tissues. I. J. Biol. Chem. 32, 309-323.

c. The use of soy bean as food. J. Biol. Chem. 32, 369-387.

d. The incidence of phosphatic urinary calculi in rats fed on experimental

rations. J. Am. Med. Assocn. 69, 32, 33.

1918. The growth of chickens in confinement. J. Biol. Chem. 33, 433-438.

a. Nutritive factors in animal tissues. II. J. Biol. Chem. 34, 17-27.

b. Nutritive factors in plant tissues. I. The protein factor in the seeds of cereals. J. Biol. Chem. 34, 521-535.

c. Milk as a source of water-soluble vitamin. J. Biol. Chem. 34, 537-551.

d. The choice between adequate and inadequate diets, as made by rats. $J$.

Biol. Chem. 35, 19-27.

1919. The vitamins in green foods. J. Biol. Chem. 37, 187-200.

a. The nutritive value of the wheat kernel and its milling products. J. Biol. Chem. 38, 557-601.

b. The nutritive value of yeast protein. J. Biol. Chem. 38, 223-227.

c. Nutritive factors in plant tissues. II. The distribution of water-soluble vitamin. J. Biol. Chem. 39, 29-34.

d. The extraction of fat-soluble vitamin from green foods. Proc. Soc. Exptl. Biol. Med. 16, 98, 99.

1920. Nutritive value of the proteins of the barley, oat, rye and wheat kernels. J. Biol. Chem. 41, 275-303.

a. Nutritive factors in plant tissues. III. Further observations on the distribution of water-soluble vitamin. J. Biol. Chem. 41, 451-468. 523.

b. Milk as a source of water-soluble vitamin. II. Biol. Chem. 41, 515-

c. Nutritive factors in plant tissues. IV. Fat-soluble vitamin. J. Biol. Chem. 41, 549-565.

d. The occurrence of water-soluble vitamin in some common fruits. $J$. Biol. Chem. 42, 465-489. $1-4$.

e. Skimmed milk as a supplement to corn in feeding. J. Biol. Chem. 44,

f. Growth on diets poor in true fats. J. Biol. Chem. 45, 145-152.

1921. A critique of experiments with diets free from fat-soluble vitamin. $J$. Biol. Chem. 45, 277-288.

a. Ophthalmia and diet. J. Am. Med. Assocn. 76, 905-908.

b. Does growth require preformed carbohydrate in the diet? Proc. Soc. Exptl. Biol. Med. 18, 136, 137.

c. Growth on diets containing more than 90 per cent of protein. Proc. Soc. Exptl. Biol. Med. 18, 167, 168.

d. Continuation and extension of work on vegetable proteins. Carnegie Inst. Wash. Yearbook 20, 432-441.

1922. Further observations on the distribution of vitamin B in some vegetable foods. J. Am. Med. Assocn. 78, 1121, 1122. 367.

a. Milk as a source of water-soluble vitamin. III. Biochem. J. 16, 363-

b. Quantitative aspects of the rôle of vitamin B in nutrition. J. Biol. Chem. 54, 739-752. 
1923. Eggs as a source of vitamin B. J. Am. Med. Assocn. 80, 302, 303.

a. The effect of diet on the content of vitamin B in the liver. J. Biol. Chem. 58, 363-367.

1924. Nutrition and growth on diets highly deficient or entirely lacking in preformed carbohydrates. J. Biol. Chem. 59, 13-32.

a. Ophthalmia as a symptom of dietary deficiency. Am. J. Physiol. 69, 543-547.

b. (Annual report to Carnegie Institution) Carnegie Inst. Wash. Yearbook 22, 340-346.

1925. The rôle of vitamin $\mathrm{B}$ in relation to the size of growing rats. J. Biol. Chem. 63, 233-238.

1926. The relation of the rate of growth to diet. J. Biol. Chem. 69, 661-673. 1928. Continuation and extension of work on vegetable proteins. Carnegie Inst. Wash. Yearbook 27, 369-375 (1927-28).

Osborne, T. B., Mendel, L. B. and Ferry, E. L.

1919. A method of expressing numerically the growth-promoting value of protein. J. Biol. Chem. 37, 223-229.

Osborne, T. B., Mendel, L. B. and Park, E. A.

1923. Experimental production of rickets with diets of purified food substances. Proc. Soc. Exptl. Biol. Med. 21, 87-90.

Osborne, T. B. and Wakeman, A. J.

1915. Some new constituents of milk. I. The phosphatids of milk. J. Biol. Chem. 20, 539-550.

a. Does butter fat contain nitrogen and phosphorus? J. Biol. Chem. 21, 91-94.

1916. Some new constituents of milk. II. The distribution of phosphatids in milk. J. Biol. Chem. 28, 1-9.

1919. Extraction and concentration of the water-soluble vitamin from brewer's yeast. J. Biol. Chem. 40, 383-394.

Osborne, T. B., Wakeman, A. J. and Ferry, E. L.

1919. Preparation of proteins free from water-soluble vitamin. J. Biol. Chem. $39,35-46$.

Oser, B. L.

1928. The intestinal $\mathrm{pH}$ in experimental rickets. J. Biol. Chem. 80, 487-497.

Outhouse, J., Macy, I. G. and Brekke, V.

1928. Human milk studies. V. A quantitative comparison of the antiricketic factor in human milk and cow's milk. J. Biol. Chem. 78, 129-144.

Outhouse, J., Macy, I. G., Brekke, V. and Graham, A.

1927. Human milk studies. IV. A note on the vitamin A and $B$ content of cow's milk. J. Biol. Chem. 73, 203-208.

Padua, R. G.

1919. Cystolithiasis among Filipinos in association with dietetic deficiency. Philippine J. Sci. 14, 481-498.

Page, I. H.

1930. The distribution of ergosterol administered to rabbits. Proc. Soc. Exptl. Biol. Med. 27, 314, 315 .

Palladin, A.

1923. Uber den Gehalt des Leinöls an "fettlöslichen Faktor A." Biochem $Z$. 136, 339-345. 
a. Enthält der Buchweizen alle dem wachsenden Organismus notwendigen Nahrungsfaktoren? Biochem. Z. 136, 346-352.

1924. Beiträge zur Biochemie der Avitaminosen. I. Kohlehydratstoffwechsel bei experimentellen Skorbut. Biochem. Z. 152, 228-245.

Palladin, A. and Epelbaum, S.

1929. Beitrag zur Biochemie der Avitaminosen. 12. Úber den Kreatinphosphorsäuregehalt in den weissen und roten Muskeln bei experimentellen Skorbut und bei Polyneuritis. Biochem. 2. 204, 140-149.

Palladin, A. and Kratinowa, $\mathbf{K}$.

1925. Beiträge zur Biochemie der Avitaminosen. 6. Über den Einfluss der Maisnahrung auf die Stickstoff-, Kreatinin-, und Kreatinausscheidung bei Kaninchen und auf die Gewichtskurve der Meerschweinchen. Biochem. $Z$. 159, 179-191.

Palladin, A. and Kudrjawzewa, A.

1924. Beiträge zur Biochemie der Avitaminosen. 2. Stickstoffwechsel (insbesondere Kreatinstoff wechsel) bei experimentellen Skorbut. Biochem. Z. 152, 373-387.

a. Beiträge zur Biochemie der Avitaminosen. 5. Untersuchungen über den Stoffwechsel bei avitaminös ernährten Kaninchen. Biochem. Z. 154, 104-124.

Palladin, A. and Ssawron, E.

1924. Beiträge zur Biochemie der Avitaminosen. 4. Kalkausscheidung und Blutkalk beim experimentellen Skorbut. Biochem. Z. 153, 86-96.

1928. Beiträge zur Biochemie der Avitaminosen. II. Über den Einfluss des Skorbuts und des Hungerns auf die chemische Zusammensetzung, insbesondere auf den Kreatingehalt des Gehirns. Biochem. Z. 200, 244-249.

Palladin, A. and Utewski, A.

1928. Beiträge zur Biochemie der Avitaminosen. 10. Acetaldehydbildung im Muskelgewebe von normalen, avitaminösen und hungernden Tauben. Biochem. Z. 200, 108-114.

Palladin, A., Utewski, A. and Ferdmann, D.

1928. Beiträge zur Biochemie der Avitaminosen. 2. Über den Einfluss der Avitaminose normaler und thyreoidektomierter Kaninchen auf die Stickstoff-, Kreatinin- und Kreatinausscheidung und auf den Blutzucker. Biochem. $Z$. 198, 402-419.

Palladin, A. and Zuwerkalow, D.

1928. Beiträge zur Biochemie der Avitaminosen. 7. Über den Einfluss des experimentellen Skorbuts auf die Hippursäuresynthese. Biochem. Z. 195, 8-13.

Palmer, L. S.

1915. Xanthophyll, the principal natural yellow pigment of the egg yolk, body fat, and blood serum of the hen. The physiological relation of the pigment to the xanthophyll of plants. J. Biol. Chem. 23, 261-279.

1916. The physiological relation of plant carotinoids to the carotinoids of the cow, horse, sheep, goat, pig and hen. J. Biol. Chem. 27, 27-32.

1919. Carotinoids as fat-soluble vitamin. Science 50, 501, 502.

Palmer, L. S. and Eckles, C. H.

1914. Carotin, the principal natural yellow pigment of milk fat. Its relations to plant carotin and the carotin of the body fat, corpus luteum, and blood serum. J. Biol. Chem. 17, 191-249. 
Palmer, L. S. and Kempster, H. L.

1919. The relation of plant carotinoids to growth, fecundity, and reproduction of fowls. J. Biol. Chem. 39, 299-312.

a. The physiological relation between fecundity and the natural yellow pigmentation of certain breeds of fowls. J. Biol. Chem. 39, 313-330.

b. The influence of specific feeds and certain pigments on the color of the egg yolk and body fat of fowls. J. Biol. Chem. 39, 331-337.

Palmer, L. S. and Kennedy C.

1922. Yeast as a source of vitamin B for the growth of rats. J. Biol. Chem. 54, 217-232.

1923. Growth and reproduction of rats on whole milk as the sole diet. Proc. Soc. Exptl. Biol. Med. 20, 506-508.

1927. The fundamental food requirements for the growth of the rat. I. Growth on a simple diet of purified nutrients. J. Biol. Chem. 74, 591-611.

a. The fundamental food requirements for the growth of the rat. II. The effect of variation in the proportion and quality of recognized nutrients. $J$. Biol. Chem. 75, 619-659.

1929. Fundamental food requirements for growth of rat. V. Influence of fat in diet. Proc. Soc. Exptl. Biol. Med. 26, 427-430.

Palmer, L. S., Kennedy, C. and Kempster, H. L.

1921. The relation of plant carotinoids to growth and reproduction of albino rats. J. Biol. Chem. 46, 559-577.

Pappenheimer, A. M.

1922. Experimental rickets in rats. VI. The anatomical changes which accompany healing of experimental rat rickets under the influence of cod liver oil or its active derivatives. J. Exptl. Med. 36, 335-355.

1924. A note on the prevention of experimental low-phosphorus rickets in rats by the subcutaneous administration of potassium phosphate. Proc. Soc. Exptl. Biol. Med. 21, 504-506.

1930. Pathological changes in the skeletal muscles produced by dietary means. Proc. Soc. Exptl. Biol. Med. 27, 567, 568.

Pappenheimer, A. M. and Dunn, L. C.

1925. The relation of leg weakness in growing chicks to mammalian rickets. J. Biol. Chem. 66, 717-729.

Pappenheimer, A. M. and Larimore, L. D.

1923. The occurrence of gastric lesions in rats, and their possible relation to dietary deficiency. Prac. Soc. Exptl. Biol. Med. 21, 141, 142.

1924. The occurrence of gastric lesions in rats. Their relation to dietary deficiency and hair ingestion. J. Exptl. Med. 40, 719-732.

Pappenheimer, A. M., McCann, G. F., Zucker, T. F. and Hess, A. F.

1921. The effect of various modifications of a diet producing rickets in rats. Proc. Soc. Exptl. Biol. Med. 18, 267-270.

1922. Experimental rickets in rats. IV. The effect of varying the inorganic constituents of a rickets-producing diet. $V$. The effect of varying the organic constituents of a rickets-producing diet. J. Exptl. Med. 35, 421-446; 447-466.

Pappenheimer, A. M. and Minor, J.

1921. Hyperplasia of the parathyroids in human rickets. J. Med. Research 42, 391-403. 
Park, E. A.

1923. The etiology of rickets. Physiol. Rev. 3, 106-163.

a. Certain factors causing the deposition of lime salts in bone. Dental Cosmos 65, 176-185.

Park, E. A., Guy, R. A. and Powers, G. F.

1923. A proof of the regulatory influence of cod liver oil on calcium and phosphorus metabolism. Am. J. Diseases Children 26, 103-111.

Park, E. A. and Howland, J.

1921. The radiographic evidence of the influence of cod liver oil in rickets. Bull. Johns Hopkins Hosp. 32, 341-344.

Park, E. A., Shipley, P. G., McCollum, E. V. and Simmonds, N.

1922. The effect of diets very high in phosphorus and very low in calcium on the development of the bones in young rats. J. Biol. Chem. 50, VII, VIII.

a. Is there more than one kind of rickets? Proc. Soc. Exptl. Biol. Med. 19, 149-154.

Parkes, A. S.

1928. The nature of the anoestrous condition resulting from vitamin B deficiency. Quart. J. Exptl. Physiol. 18, 397-401.

Parkes, A. S. and Drummond, J. C.

1925. The effect of vitamin B deficiency on reproduction. Proc. Roy. Soc. (London) B. 98, 147-171.

1926. The effect of fat-soluble vitamin A deficiency on reproduction in the rat. Brit. J. Exptl. Biol. 3, 251-273.

Parkhurst, R. T. and Neidig, R. E.

1924. The effect of accessory food factors on egg production. Poultry Sci. 3, 149-152.

Parsons, H. T.

1920. The antiscorbutic content of certain body tissues of the rat. The persistence of the antiscorbutic substance in the liver of the rat after long intervals on a scorbutic diet. J. Biol. Chem. 44, 587-602.

Parsons, H. T. and Alexander, N.

1924. A new feeding device for use with the experimental rat. J. Lab. Clin. Med. 9, 576-580.

Parsons, H. T. and Hutton, M. K.

1924. Some further observations concerning the antiscorbutic requirement of the rat. J. Biol. Chem. 59, 97-105.

Parsons, H. T. and Reynolds, M. S.

1924. The depletion of vitamin $C$ in the liver of the guinea pig on a scorbutic ration. J. Biol. Chem. 59, 731-736.

\section{Parsons, L. G.}

1926. The value of irradiated cholesterol in the treatment of rickets. Brit. Med. J. No. 3403, 519-521.

Paton, D. N.

1922. Rickets: A theory of the metabolic disturbances and of its association with tetany. Brit. Med. J. No. 3193, 379-381.

Paton, D. N., Findlay, L. and Watson, A.

1918. Observations on the cause of rickets. Brit. Med. J. No. 3023, 625-626.

Paton, D. N. and Watson, A.

1921. The etiology of rickets. An experimental investigation. Brit. J. Exptl. Path. 2, 75-94. 
1923. The etiology of rickets. An experimental investigation. II. Brit. J. Exptl. Path. 4, 177-195.

Pattison, C. I.

1926. Dietetic conditions which influence the calcium content of saliva. The possible significance of these facts in tuberculosis. Brit. Med. J. No. 3417, 6-8.

1929. Vitamin D and the calcification of bone in tuberculosis. Brit. Med. J. No. 3556, 419, 420.

Peacock, P. R.

1925. Quantitative data in tissue reactions to ultra-violet radiations. Lancet, 1925, II, 369-372.

1926. The action of light on cod-liver oil. Lancet, 1926, II, 328-330.

Pekelharing, C. A.

1905. Over onze kennis van de waarde der voedingsmiddelen uit chemische fabrieken. Nederland. Tijdschr. Geneeskunde 70, 111-124.

Pénau, $H$. and Hardy, $Z$.

1929. Etude du complexe digitonoside-ergostérol. J. pharm. chem. 9, 145-151.

Pénau, $H$. and Simonnet, $H$.

1921. Les extraits alcooliques de levure de bière dans la polynévrite aviare. Compt. rend. soc. biol. 85, 198-200.

Pénau, H. and Tanret, G.

1929. Sur un stérol dextrogyre de la levure, le zymosterol. Compt. rend. 188, 1317-1319.

Perlmann, S. and Weber, W.

1928. Experimentelle Erzeugung von Blasensteinen durch Avitaminose. Deut. med. Wochschr. 54, 1045, 1046. See also Münch. med. Wochschr. 75, 2167, 2168.

Peskett, G. L.

1927. The synthesis of antineuritic vitamin by yeast. Biochem. J. 21, 11021103.

1928. Critical concentrations of bioses. Proc. Soc. Exptl. Biol. Med. 25, 340343.

Peters, R. A.

1924. The action of nitrous acid upon the antineuritic substances in yeast. Biochcm. J. 18, 858-865.

1929. Vitamin B. Nature 124, 411.

1929-1930. Co-ordinative bio-chemistry of the cell and tissues. I. Cell surfaces. II. The ministers of metabolic change. III. Tissue anarchy. J. State Med. 37, 683-709; 38, 3-30, 63-87.

Peters, R. A., Kinnersley, H. W., Orr-Ewing, J. and Reader, V.

1928. The relation of vitamin $B_{1}$ to the growth-promoting factor for a Streptothrix. Biochem. J. 22, 445-450.

Petersen, H. A.

1924. An experimental study of ununited fractures with especial reference to the inorganic bone-forming elements in the blood serum. Bull. Johns Hopkins Hosp. 35, 378-381.

Pfannensteil, W.

1928. A summary of recent work on Vigantol (irradiated ergosterol). Lancet, 1928, II, 845-847. 
a. Weitere Beobachtungen über Wirkung bestrahlten Ergosterins im Tierversuch. Münch. med. Wochschr. 75, 1113-1114.

Pfannensteil, W. and Scharlau, B.

1930. Die Wirkung gesteigerter Zufuhr von Vitaminen bzw. Vitaminkombinationen auf experimentelle Infektionen (Lungentuberkulose, Staphylomykose der Haut). Münch. med. Wochsch. 77, 619-622.

Pfaundler, M.

1929. Ueber neuere Behandlungsverfahren der englischen Krankheit im Dienste der allgemeinen Rachitisbekämpfung. V. Vorschläge zur Rachitisabwehr in der Fürsorge. Miinch. med. Wochschr. 76, 1437-1441.

Pfund, A. H.

1927. Windows and actionometers for heliotherapy. Bull. Johns Hopkins Hosp. 40, 228-237.

1928. A practical window for transmitting ultraviolet rays. J. Am. Med. Assocn. 91, 18, 19.

Pharmaceutical Society of Great Britain, Pharmalogical Laboratories Vitamin Testing Department.

1927. Pharm. J. 64, 49.

Phemister, D. B., Miller, E. M. and Bonar, B. E.

1921. The effect of phosphorus in rickets. J. Am. Med. Assocn. 76, 850-854.

Pico Estrada, O.

1927. Sensibilité des rats privés de surrenales à l'avitaminose B. Compt. rend. soc. biol. 97, 1031, 1032.

Pierce, H. B., Osgood, H. S. and Polansky, J. B.

1929. Absorption of glucose from alimentary tract of rats deprived of vitamin B complex. Proc. Soc. Exptl. Biol. Med. 26, 347.

Pierson, E. M. and Dutcher, R. A.

1920. Rhubarb as an antiscorbutic. Science $51,70$.

Pilcher, J. D. and Sollmann, T.

1925. Deficient and surplus consumption of vitamin B. Their quantitative relation to weight changes and to vitamine storage in adult pigeons. J. Pharmacol. 26, 203-213.

Pilley, V. E.

1929. An X-ray study of osteomalacia. Ind. J. Med. Research 17, 348-350.

Pincussen, $\mathrm{L}$.

1925. Über Veranderungen des Stoffwechsels unter Bestrahlung. II. Zur Beeinflussung des Mineralstoffwechsels durch Sonnenlicht. Biochem. Z. 161, $67-70$.

Pincussen, L. and Naarkrineos, I.

1925. Über Veranderungen des Stoff wechsels unter Bestrahlung. I. Über Veranderungen des Kalium- und Calciumgehaltes des Blutes unter Bestrahlung. Biochem. Z. 161, 61-66.

Pitz, W.

1918. Studies of experimental scurvy. II. The influence of grains other than oats, and specific carbohydrates on the development of scurvy. J. Biol. Chem. $33,471-482$.

a. Studies of experimental scurvy. III. The influence of meat and various salts upon the development of scurvy. J. Biol. Chem. 36, 439-466. 
Platon, J. B.

1925. Über die Oxydation der A-Vitamine in Milchfett beim Buttern. Biochem. Z. 155, 228-234.

1927. Der A-Vitamingehalt der Magermilch. Biochcm. Z. 185, 238-241.

Plimmer, R. H. A.

1920. Note on scurvy in pigs. Biochem. J. 14, 570, 571.

Plimmer, R. H. A. and Plimmer, V. G.

1928. "Food, Health, and Vitamins," 3rd Ed., London and New York, Longmans, Green \& Co.

Plimmer, R. H. A., Raymond, W. H. and Lowndes, J.

1929. Experiments on nutrition. IX. Comparative vitamin B values of foodstuffs, pulses, and nuts. Biochem. J. 23, 546-557.

Plimmer, R. H. A. and Rosedale, J. L.

1926. Observations on vitamin B and metabolism. J. State Med. 34, 117-121.

Plimmer, R. H. A., Rosedale, J. L., Crichton, A. and Topping, R. B.

1922. The rearing of chickens on the intensive system. I. The vitamin requirements. II. The effect of good protein. Biochem. J. 16, 11-18; 19-22.

Plimmer, R. H. A., Rosedale, J. L. and Raymond, W. H.

1923. The rearing of chickens on the intensive system. III. B-vitamin requirements. Comparison of yeast extracts and dried yeast. IV. C-vitamin requirements of chickens and other birds. V. Comparative B-vitamin requirements of pigeons, chickens and other birds. Biochem. J. 17, 772-786, 787-793, 79े4-799.

1927. Experiments on nutrition. VI. Balance of food by vitamin B. Biochem. J. 21, 913-939.

a. Experiments on nutrition. VII. Fat-soluble vitamin requirement of chickens. Biochem. J. 21, 940-944.

Plimmer, R. H. A., Rosedale, J. L., Raymond, W. H. and Lowndes, J.

1927. Experiments on nutrition. VIII. Comparative vitamin $B$ value of foodstuffs: Cereals I. Biochem. J. 21, 1141-1161.

Plummer, B. A.

1927. The motility of the intestinal tract in experimental beriberi (rats) and scurvy (guinea pigs). Am. J. Physiol. 80, 278-287.

Pohl, R.

1927. Zum optischen Nachweis eines Vitamines. Naturvissenschaften 15, 433-438.

a. Über das Absorptionsspektrum des antirachitischen Provitamins und Vitamins. Nach. Ges. Wiss. Göttingen Math-Physik. Klasse 2, 185-191.

Polansky, J. B.

1928. The response of the isolated segment of small intestine (rabbit) to extracts of yeast and other substances during their passage through the lumen. Am. J. Physiol. 83, 488-498.

Pomerene, E. and Beard, H. H.

1930. Studies in the nutrition of the white mouse. VI. The experimental production of xerophthalmia in mice. Am. J. Physiol. 92, 282-286.

Portier, P.

1920. Modifications du testicule des oiseaux sous l'influence de la carence. Compt. rend. 170, 755-757.

a. Régénération du testicule chez le pigeon carencé. Compt. rend. 170, 1339-1341. 
Portier, P. and Randoin, L.

1919. Sur la technique des expériences d'avitaminose par sterilisation. Compt. rend. soc. biol. 82, 990-992.

1920. Création de vitamines dans l'intestin des lapins recevant une nourriture sterilisée à haute temperature. Compt. rend. 170, 478-480.

Portman, K.

1927. Investigations on the histological changes in the skin of rats on various diets. Acta. Path. Microbiol. Scand. 4, 341-348.

\section{Poulsson, E.}

1924. A note on the durability of vitamin A of cod-liver oil. Biochem. J. 18, 919, 920.

1926. Das fettlösliche Vitamin. Deut. med. Wochschr. 52, 6-8.

Poulsson, E. and Lovenskiold, $\mathrm{H}$.

1928. The quantitative determination of vitamin D. Biochem. J. 22, 135-141.

Powers, G. F., Park, E. A., Shipley, P. G., McCollum, E. V. and Simmonds, N.

1922. The prevention of the development of rickets in rats by sunlight. XIV. Studies on experimental rickets. J. Am. Med. Assocn. 78, 159-165.

a. Studies on experimental rickets. XIX. The prevention of rickets in the rat by means of radiation with the mercury vapor quartz lamp. Bull. Johns Hopkins Hosp. 33, 125-127.

Powers, G. F., Park, E. A. and Simmonds, N.

1923. The influence of radiant energy upon the development of xerophthalmia in rats. A remarkable demonstration of the beneficial influence of sunlight and out-of-door air upon the organism. J. Biol. Chem. 55, 575-597.

Powick, W. C.

1925. Inactivation of vitamin A by rancid fat. J. Agr. Research 31, 1017-1026.

Pringle, E. F.

1928. A note on the treatment of rickets with irradiated ergosterol and ultraviolet light. Lancet, 1928, II, 1237.

Pringle, $\mathrm{H}$.

1925. The identity of vitamin A. The comparative effects of human and cow's milk. Proc. Roy. Dublin Soc. n. ser. 18, 93-97.

\section{Prinke, G.}

1927. Klinische Erfahrungen mit dem neuen Rachitisheilmittel von WindausHess. Klin. Wochschr. 6, 1644-1647.

Priston, J. L.

1926. The prevention of scurvy in the navy. J. Roy. Naval Med. Service 12, $1-20$.

Pritchard, E.

1919. Cause and treatment of rickets. Brit. Med. J. No. 3072, 627-629.

1923. The pathogenesis of rickets. Brit. Med. J. No. 3256, 887-889.

Puckner, W. A.

1922. Yeast preparations and vitamin B concentrates. J. Am. Med. Assocn. $78,1146$.

Putzig, $\mathrm{H}$.

1920. Die Behandlung der Rachitis mit künstlicher Höhensonne. Therap. Halbmonatsh. 34, 234-236. 
Quinn, E. J.

1929. What is involved in testing a food for vitamin D. Food Industries 1, 249-251.

Quinn, E. J. and Brabec, L. B.

1930. The vitamin A, B, and $\mathrm{G}$ content of malted milk. J. Home Econ. 22, 123-128.

Quinn, E. J., Burtis, M. P. and Milner, E. W.

1927. Quantitative studies of vitamins A, B, and C in green plant tissues other than leaves. J. Biol. Chem. 72, 557-563.

Quinn, E. J. and Cook, D. H.

1928. The vitamin A content of white yautia, yellow yautia, and plantain with further evidence of a possible relationship between the color of a natural food and its richness in vitamin A. Am. J. Trop. Med. 8, 503-506.

Quinn, E. J., King, C. G. and Dimit, B. H.

1929. A study of the effects of certain diets upon the growth and form of albino rats. J. Nutrition 2, 7-18.

Quinn, E. J., Whalen, F. B. and Hartley, J. G.

1930. The vitamin B and $\mathrm{G}$ contents of certain yeast samples. J. Nutrition 3, 257-263.

Rabl, C. R. H.

1929. Organverkalkungen unter dem Einfluss von vitamin D. Deut. med. Wochschr. 55, 63, 64.

Raczynski, J.

1913. Recherches expérimentales sur le manque d'action du soleil comme cause du rachitisme. Compt. rend. assocn. intern. pédiatrie, 308, 309.

Randoin, L.

1923. Etude des vitamines chez les mollusques. Sur la presence du facteur antiscorbutique dans l'huitre. Compt. rend. 177, 498-501.

a. La question des vitamines. II. Le facteur antiscorbutique. Bull. soc. chim. biol. 5, 806-864.

b. Constitution de deux régimes définis pour l'étude du scorbut et de la polynévrite aviare. Bull. soc. hyg. aliment. 11, 453-467.

1925. La question des vitamines en matière d'expertise. Ann. fals. 18, 325-331.

1928. Recherches sur la valeur alimentaire des jus de raisins frais et des vins, au point de vue de leur teneur en vitamines. Bull. soc. hyg. aliment. 16, 464-486.

1929. Les aliments irradiés. Bull. soc. hyg. aliment. 17, 405-419.

Randoin, L., Alquier, J., Asselin and Charles.

1926. Reproduction, croissance et équilibre alimentaîre. Compt. rend. 182, 94-96.

Randoin, L., André, E. and Lecoq, R.

1928. Valeur antirachitique comparée de quelques huiles d'animaux marins et de l'huile de foie de morue. J. pharm. chim. 7, 529-539.

Randoin, L. and Fabre, R.

1927. Recherches comparatives sur la teneur en glutathion de quelques tissues et du sang, chez le pigeon normal, chez le pigeon sous-alimenté, et chez le pigeon privé de vitamines B. Compt. rend. 185, 151-153. 
Randoin, L. and Lecoq, R.

1926. Résistance des vitamines liposolubles à l'hydrogénation. Ann. fals. 19, 518-523.

a. Å propos des variétés commerciales de dextrine et de leur emploi dans la constitution de régimes artificiels destinés à l'analyse biologique des aliments. J. pharm. chim. 8, 289-294.

b. Inégalité de la teneur en vitamines hydrosolubles (B) d'extraits de levure d'origine différente. Compt. rend. 182, 1408-1410.

c. Les vitamines hydrosolubles $\mathrm{B}$ contenus dans la levure de bière existentelles préalablement dans le milieu de culture? Compt. rend. 182, 1564-1566.

1927. Å propos de l'existence probable de deux vitamines antiscorbutiques. Compt. rend. soc. biol. 96, 671-673.

a. Constitution d'un nouveau régime artificiel pour l'étude du rachitisme expérimental. Compt. rend. soc. biol. 97, 1277-1279.

b. L'évolution de l'avitaminose B dans ses rapports avec la constitution des glucides du régime. Compt. rend. 184, 1347-1349.

c. Influence de la nature des glucides alimentaires sur la production d'états polynévritiques aigus, récidivants ou chroniques, obtenu malgré la presence de levures ou d'extraits de levure. Compt. rend. 185, 1068-1070.

d. Recherches expérimentales sur la sensibilité des vitamines hydrosolubles $\mathrm{B}$ à la dessiccation. Bull. sci. pharmacol. 34, 129-138.

e. Valeur biologique d'un extrait de levure "standard" utilisé comme source de vitamines hydrosolubles B. J. pharm. chim. 5, 147-154.

f. Recherches qualitatives et quantitatives sur les vitamines hydro-solubles B contenues dans les extraits de levure, dans les levures et dans les milieux de culture de ces levures. J. pharm. chim. 5, 193-208.

g. Influence des ferments amylolytiques sur l'évolution de l'avitaminose B provoquée au moyen de régimes riches en amidon. J. pharm. chim. 5, 340346.

h. Le malt et l'extrait de malt envisagés comme sources de vitamines hydrosolubles B et C. Bull. soc. chim. biol. 9, 49-58.

i. Polynévrite et scorbut chroniques. Bull. soc. chim. biol. 9, 513-526.

1928. Existe-t-il dans la levure de bière, un facteur hydrosoluble thermostable et alcalinostable nécessaire à la vie distinct des vitamines $B$, d'utilisation nutritive et antinévritique? Compt. rend. soc. biol. 99, 586-588.

a. Essai biologique de la valeur antirachitique des lipides effectué sur le jeune rat au moyen d'un nouveau régime rachitigène simple composé de substances définies. Ann. fals. 21, 68-74.

1929. Production de polynévrite chronique typique au moyen d'extraits de levure de bière privés de la vitamine $B$ antinévritique par traitement préalable à la terre à foulon. Compt. rend. soc. biol. 101, 11-14.

a. Production du scorbut chez le cobaye, jeune ou adulte, au moyen d'un nouveau régime artificiel bien défini. Compt. rend. soc. biol. 101, 877-879.

b. Constitution d'un nouveau régime artificiel défini, producteur d'avitaminose $\mathrm{C}$, permettant l'étude de scorbut et la recherche de la vitamine antiscorbutique. J. pharm. chim. 10, 337-344.

c. Action curative du lait de vache entier desseché et du lait concentré sucré sur le rachitisme expérimental du rat. J. pharm. chim. 10, 496-500.

d. Les vitamines du groupe B. Etat actuel de la question. Rev. path. comparće 29, 747-762. 
Randoin, L. and Lelesz, E.

1925. Variations comparatives de la glycémie arterielle (effective et protéidique) et de la teneur du foie en glycogène chez le pigeon normal, et chez le pigeon soumis a un régime déséquilibré par manque de facteur hydrosoluble B. Compt. rend. 180, 1366-1368.

Randoin, L. and Michaux, A.

1927. Variations de la teneur en fer du foie, de la rate et du sang, sous l'influence d'un régime déséquilibré par absence complète de vitamine antiscorbutique. Compt. rend. 185, 365-368.

1928. Variations comparatives de la teneur du foie et de la rate en eau, acides gras et cholestérol, chez le cobaye normal et chez le cobaye soumis à un régime privé de vitamine antiscorbutique. Compt. rend. 187, 146-149.

a. Sur la teneur des reins en eau, acides gras et cholestérol chez le cobaye normal et chez le cobaye soumis a un régime scorbutigène. Compt. rend. soc. biol. 99, 584-586.

1929. Variations comparatives de la teneur du sang en eau et de la résistance globulaire, chez le cobaye normal et chez le cobaye soumis à un régime privé de vitamine antiscorbutique. Compt. rend. 188, 729-731.

Randoin, L. and Simonnet, $\mathrm{H}$.

1923. Influence de la nature et de la quantité des glucides présents dans une ration privée de facteur $B$ sur la précocité de l'apparition des accidents de la polynévrite aviare. Compt. rend. 177, 903-906.

1924. Sur l'équilibre alimentaire. Entretien du pigeon au moyen d'un régime totalement privé de facteur hydrosoluble B. Compt. rend. 179, 700-703.

a. Croissance et entretien du rat soumis à un régime artificiel privé à la fois de facteur B et de glucides. Compt. rend. 179, 1219-1222.

b. Recherches expérimentales relatives au mécanisme de la production des troubles caractérisant une maladie par déséquilibre alimentaire: la polynévrite aviare. Bull. soc. hyg. aliment. 12, 86-112.

c. Equilibre alimentaire, isodynamie et substances elémentaires fondamentales. Bull. soc. chim. biol. 6, 601-624.

1925. Essai de définition des vitamines. Bull. soc. chim. biol. 7, 1020-1023.

1926. Régime alimentaire, lumière et valeur biologique du lait. Bull. soc. hyg. 14, 217-243.

1928. Hormones et vitamines. Ā propos d'une nouvelle dénomination des vitamines. Bull. soc. chim. biol. 10, 745-756.

Read, J. W.

1924. The relative vitamin and mineral values of cow pea, soy bean, alfalfa, lespedeza, and prairie hays as ordinarily cured. Abstracted in Science 59, 20.

Read, J. W. and Sure, B.

1923. Nutritive value of the Georgia velvet bean (Stizolobium deeringianum). J. Agr. Research 24, 433-440.

Reader, V.

1929. The relation of the growth of certain micro-organisms to the composition of the medium. IV. The addition of mannitol. Biochem. J. 23, 61-67.

a. A second thermolabile water-soluble accessory factor necessary for the nutrition of the rat. Biochem. J. 23, 689-694.

1930. Further evidence for a third accessory "B" factor. Biochem. J. 24, $77-80$. 
Reader, V. and Drummond, J. C.

1925. Further observations on nutrition with diets rich in protein. $J$. Physiol. 59, 472-478.

1926. Relation between vitamin B and protein in the diet of growing rats.

Physiological rôle of vitamin B. Part II. Biochem. J. 20, 1256-1263.

Redman, T.

1928. The hydrogen-ion concentration of the faeces of rachitic children. Biochem. J. 22, 14-21.

1929. The hydrogen-ion concentration and the calcium and phosphorus content of the faeces of rachitic children. Biochem. J. 23, 256-260.

Redman, T., Willimott, S. G. and Wokes, F.

1927. The $\mathrm{pH}$ of the gastro-intestinal tract of certain rodents used in feeding experiments, and its possible significance in rickets. Biochem. J. 21, 589-605.

Reed, C. I. and Thacker, E. A.

1929. The intravenous administration of irradiated ergosterol. Proc. Soc. Exptl. Biol. Med. 28, 187, 188.

Reed, H. S.

1920. The nature of the growth rate. J. Gen. Physiol. 2, 545-561.

1921. The rate of growth following an initial period of suppression. $A m$. Naturalist 55, 539-555.

Reerink, E. H. and Wijk, A. Van.

1929. On the photochemical reactions of ergosterol. Proc. K. Akad. Wetensch. Amsterdam. 32, 845-848.

1929. The vitamin D problem. I. The photochemical reactions of ergosterol. Biochem. J. 23, 1294-1307.

Remy, E.

1929. Vergleichende Untersuchungen über fleisch und vitamin-haltige Hefeextrakte unter besonderer Berücksichtigung der chemisch- physiologischen sowie bakteriologisch- serologischen Verhältnisse. Arch. Hyg. 101, 27-38.

Rendenbaugh, H. E.

1927. Blood sugar changes in avian polyneuritis. Proc. Soc. Exptl. Biol. Med. $24,842,843$.

Report of a committee of inquiry regarding the prevalence of pellagra among Turkish prisoners of war.

1919-20. J. Roy. Army Med. Corps. 33, 426-447, 508-527; 34, 70-79, 173-184, 272-292.

Reyher, P.

1928. Úber den Gehalt der Kuhmilch an antineuritischen B-Vitamin. Arch. Kinderheilk. 84, 55-67.

Reyher, P. and Walkhoff, E.

1928. Uber die toxische Wirkung ultraviolettbestrahlten Milch und anderer Substanzen. Münch. med. Wochschr. 75, 1071-1073.

Reyher, P., Walkhoff, E. and Walkhoff, $\mathrm{O}$.

1928. Studien über die Wirkung C-hypo-vitaminotischer Nahrung auf

Schwangere, Feten und Neugeborene. Mïnch. med. Wochschr. 75, 2087-2090.

Reynolds, E. and Macomber, D.

1921. Defective diet as a cause of sterility. A study based on feeding experiments with rats. J. Am. Med. Assocn. 77, 169-175. 
Richardson, A. E. and Green, H. S.

1916. Nutrition investigations upon cottonseed meal. I. J. Biol. Chem. 25, $307-318$.

1917. Nutrition investigations upon cottonseed meal. II. J. Biol. Chem. 30, 243-258.

a. Nutrition investigations upon cottonseed meal. III. Cottonseed flour. Nature of its growth-promoting substance and a study in protein minimum. J. Biol. Chem. 31, 379-388.

Richardson, J. E. and Jacobs, E.

1927. Effect of storage on vitamin C in potatoes. Montana Agr. Expt. Sta. Rept., pp. 78, 79.

Richardson, J. E., Palmer, L. S. and Kennedy, C.

1928. The interrelationship of the carriers of vitamins $A$ and $B$ as affecting the growth and development of the tissues and organs of young animals. Am. J. Physiol. 83, 712-728.

Richmond, H. D. and England, E. H.

1922. Note on the sulphuric acid reaction for liver oil. Analyst 47, 431.

Riddle, A. R.

1930. The ultraviolet limit in sunlight. Am. Rev. Tuberculosis 21, 278-289.

Riesenfeld, E. A., Handelman, I. and Rose, A. R.

1925. Inorganic phosphorus in the blood of the new-born; Its seasonal variation and its relation to rickets. Am. J. Diseases Children 30, 646-658.

Rigobello, G.

1928. Sull azione vitaminica dell 'olio di oliva irradiato cor raggi ultravioletti. Rev. Sud-Americana Endocrinol. Immunol. Quimioterap. II, 456-466.

Robb, E. F.

1920. The influence of dry versus fresh green plant tissue on calcium metabolism. Science 52, 510.

Robb, E. F., Medes, G. et al.

1921. A study of scurvy and its bearing on the preservation of the teeth. J. Dental Research 3, 39-61.

Roberts, L. R.

1920. Types and treatment of pellagra. J. Am. Med. Assocn. 75, 21-25.

Robertson, E. C.

1927. The effect of exposure to the sun on the susceptibility of rachitic rats to infection. Preliminary note. Can. Med. Assocn. J. 17, 1033-1035.

1929. A study of the effect of various agents, chiefly sunlight, upon the susceptibility of rachitic rats to infection. Am. J. Hyg. 9, 75-96.

Robertson, T. B. and Ray, L. A.

1920. Experimental studies on growth. XV. On the growth of relatively long-lived as compared with that of relatively short-lived animals. J. Biol. Chem. 42, 71-107.

a. Experimental studies on growth. XVI. The influence of brain tissue freed from cholesterol upon the growth of the white mouse. J. Biol. Chem. 44, 439-453.

\section{Robison, $\mathbf{R}$.}

1919. The antiscorbutic properties of concentrated fruit juices. J. Roy. Army Med. Corps 32, 48-56. 
Robison, R. and Soames, K. M.

1925. A chemical study of defective ossification in rachitic animals. Biochem.

J. 19, 153-161.

Roche, J.

1925. La respiration des tissus dans l'avitaminose et l'inanition. Compt. rend. 180, 467-469.

1928. Essai d'isolement physiologique de la vitamine provoquant la dyscarbonurie et l'élimination urinaire de produits intermédiares du métabolisme des glucides. Compt. rend. soc. biol. 99, 589-590.

a. Des variations du rapport $\mathrm{C} / \mathrm{N}$ urinaire du rat au cours de l'inanition et de la carence en facteur B. Différenciation de la mort par inanition et de la mort par avitaminose B. Compt. rend. soc. biol. 99, 671-673.

1929. Du rôle de l'inanition dans la mort par carence en facteur B. Remarques sur les variations du rapport $\mathrm{C} / \mathrm{N}$ urinaire au cours de l'avitaminose $\mathrm{B}$ chez le rat. Compt. rend. soc. biol. 100, 849-852.

1930. Recherches sur le syndrome urinaire des troubles métaboliques provoqués par la carence en facteurs B chez le rat. Bull. soc. chim. biol. 12, 342-356.

Röhmann, F.

1916. UUber Künstliche Ernährung und Vitamine," Berlin, Borntraeger Bros.

Roelli, P.

1923. Die Aktivierung der Invitroatmung durch Muskelkochsaft, untersucht an verschiedenen Gewebearten von gesunden Tauben, Beriberitauben und Hungertauben. Z. physiol. Chem. 129, 284-303.

\section{Röslo, B.}

1929. Beitrag zur Kenntnis des Vorkommens des A-Vitamines in Blut und Blutserum von Haustieren, in Kuhmilch, Milchprodukten und in einigen Futtermitteln. Z. physiol. Chem. 182, 289-304.

Roger, H., Binet, L. and Vagliano, M.

1924. Action des graisses du poumon sur la croissance. Compt. rend. soc. biol. 90, 1310, 1311.

a. Action des graisses du poumon sur la fixation du calcium. Compt. rend. soc. biol. 91, 357-360.

Rogers, E. C.

1928. Quantitative determination of vitamin B in spinach and string beans, with a preliminary report on the vitamin A content of the same foods. Dissertation, University of Missouri.

Rohr, F. and Schultz, O.

1927. Ultraviolettbestrahltes enteiweisstes Milchfett, ein wirksames, wohlschmechendes Antirachiticum. Klin. Wochschr. 6, 848-853.

Rohr, K.

1923. Vergleichende Untersuchungen über die Atmungsgrösse verschiedener Gewebearten und ihren Gehalt an Vitaminfaktor B. Z. physiol. Chem. 129, 248-267.

Rondoni, P.

1919. Remarks on the pathogenesis of deficiency diseases and on pellagra. Brit. Med. J. No. 3044, 542-544.

Roscoe, M. H.

1927. A further note on the antirachitic value of fresh spinach. Biochem. J. 21, 211-215. 
a. Spontaneous cures in rats reared upon a diet devoid of vitamin B and antineuritic vitamin. J. Hyg. 27, 103-107.

Rose, M. S. and McCollum, E. L.

1928. Supplementary values among foods. I. Adding egg to an adequate diet compared with increasing vegetables. Proc. Soc. Exptl. Biol. Med. 25, 697, 698.

a. Studies in nutrition. I. Growth, reproduction, and lactation on diets with different proportions of cereals and vegetables. II. The effect of adding egg to a diet already adequate. J. Biol. Chem. 78, 535-547; 549-555.

Rose, M. S. and MacLeod, G.

1922. The almond as a source of the A vitamin. Proc. Soc. Exptl. Biol. Med. 19, 391-393.

1928. Supplementary values among foods. II. Growth and reproduction on white bread with various supplements. J. Nutrition I, 29-38.

Rose, S. G.

1921. Nutritional keratomalacia in infants. Am. J. Diseases Children 22, 232-243.

Rose, W. B.

1928. Relation of vitamin " $B$ " to infection and immunity with special reference to Bacillus welchii. Proc. Soc. Exptl. Biol. Med. 25, 657, 658.

Rose, W. B. and Stucky, C. J.

1928. Anhydremia in rats suffering from lack of what has been called vitamin B. Proc. Soc. Exptl. Biol. Med. 25, 687, 688.

a. Gastric motility in relation to anhydremia. Proc. Soc. Exptl. Biol. Med. 25, 688.

1930. Studies in the physiology of vitamins. VIII. The effect of parathormone on normal and vitamin B-deficient rats. Am. J. Physiol. 91, 513-562.

Rose, W. B., Stucky, C. J. and Cowgill, G. R.

1930. Studies in the physiology of vitamins. X. Further contributions to the study of gastric motility in vitamin B deficiency. Am. J. Physiol. 91, 531-546.

a. Studies in the physiology of vitamins. XI. The effect of insulin on gastric atony in vitamin B deficiency. Am. J. Physiol. 91, 547-553.

b. Studies in the physiology of vitamins. XII. The effect of parathormone on gastric motility in vitamin B-deficient and normal dogs. Am. J. Physiol. 91, 554-562.

c. Studies in the physiology of vitamins. XIII. The relation of gastric motility to anhydremia in vitamin B-deficient dogs. Am. J. Physiol. 92, 83-91.

Rose, W. B., Stucky, C. J. and Mendel, L. B.

1930. Studies in the physiology of vitamins. IX. Hemoglobin, sugar, and chloride changes in the blood of vitamin B-deficient rats. Am. J. Physiol. 91, 520-530.

Rosedale, J. L.

1927. Studies on the antineuritic vitamin. I. Preliminary note on a possible second factor. Biochem. J. 21, 1266, 1267.

1929. Studies on the antineuritic vitamin. Part III. Further evidence of the composite nature of B vitamin. Indian J. Med. Research 17, 216-221. 
Rosedale, J. L. and Oliveiro, C. J.

1928. Studies on the antineuritic vitamin. II. The properties of the "curative" substance. Biochem. J. 22, 1362-1367.

Rosenau, M. J.

1921. Vitamins in milk. Boston Med. Surg. J. 184, 455-458.

Rosenheim, O.

1927. Note on some sterol colour reactions in their relation to vitamin $\mathrm{A}$. Biochem. J. 21, 386-388.

a. A new colorimeter based on the Lovibond colour system and its application to the testing of cod-liver oil and other purposes (with Addendum by E. Schuster). Biochem. J. 21, 1329-1334.

b. Note on the induced fluorescence of ergosterol. Biochem. J. 21, 1335.

1929. A specific colour reaction for ergosterol. Biochem. J. 23, 47-53.

Rosenheim, O. and Adam, N. K.

1929. Monomolecular films of irradiated ergosterol in relation to the production of vitamin D. Proc. Roy. Soc. (London) B. 105, 422-428.

Rosenheim, O. and Drummond, J. C.

1920. On the relation of the lipochrome pigments to the fat-soluble accessory food factor. Lancet, 1920, I, 862-864.

1925. A delicate colour reaction for the presence of vitamin A. Biochem. J. 19, 752-756.

Rosenheim, O. and Webster, T. A.

1925. Rickets and cholesterol. Lancet, 1925, I, 1025, 1026.

1926. The antirachitic properties of irradiated sterols. Biochem. J. 20, 537544.

a. Note on the antirachitic action of irradiated sawdust. Biochem. J. 20, 1340, 1341.

b. The nature of Fearon's colour reaction and its nonspecificity for vitamin A. Biochem. J. 20, 1342-1345.

c. A critical study of colour tests suggested for vitamin A. Lancet, 1926, II, $806,807$.

1927. The stomach oil of the Fulmar petrel. (Fulmarus glacialis). Biochem. J. 21, 111-118.

a. The relation of cholesterol to vitamin D. Biochem. J. 21, 127-129.

b. On the nature of the parent substance of vitamin D. Lancet, 1927, I, $306,307$.

c. The parent substance of vitamin D. Biochem. J. 21, 389-397.

d. The sources of supply of vitamins A and D. Nature 120, 440.

e. The photochemical production of vitamin D from ergosterol. Lancet, 1927, II, 622-625.

1928. The specificity of ergosterol as parent substance of vitamin D. Biochem. J. 22, 762-766.

1929. Note on the absorption spectrum of vitamin A. Biochem. J. 23, 633.

Rothlin, E.

1922. Untersuchungen über den Gehalt an diastatischem Ferment des Pancreas bei Beri-Beritauben. Z. physiol. Chem. 121, 300-306.

Rousseau, E.

1928. Action photochemique oxydante du cholestérol et de l'ergostérol après leur irradiation par un arc au mercure. Compt. rend. soc. biol. 99, 1844, 1845. 
a. Dosages du pouvoir oxydant des solutions de cholésterol et d'ergostérol après leur irradiations. Compt. rend. soc. biol. 99, 1845, 1846.

1929. Recherches complémentaires et critiques au sujet du cholestérol dissous et irradié. Rôle d'un antioxydant. Compt. rend. soc. biol. 100, 82, 83.

a. Deplacement de l'iode d'un iodure par une solution huileuse de cholestérol ou d'ergostérol irradiée par la lumière solaire. Compt. rend. 189, 37-39.

Roussel, G. and Guillion, G.

1928. Recherches sur l'activation de l'ergostérol par l'irradiation ultraviolette. Compt. rend. soc. biol. 99, 592-594.

Rowlands, M. I.

1929. Vitamin content of grass seeds from treated plots. Nature 124, 760.

Rowlands, M. J. and Browning, E.

1928. The relation of deficiency of vitamin B to atony of the stomach. Lancet, 1928, I, 180.

Rowles, E. K.

1925. Quantitative study of the hypophysis cerebri of the guinea pig in experimental scurvy. Anat. Record 29, 394, 395.

Rubino, P. and Collazo, J. A.

1923. Untersuchungen über den intermediären Kohlenhydratstoffwechsel bei Avitaminose. I. Glykogenbildung und Umsatz bei der Avitaminose. Biochem. Z. 140, 258-267.

Russell, W. C.

1930. The vitamin A content of yellow and white-capped yellow dent corn. $J$. Nutrition 2, 265-269.

Russell, W. C. and Massengale, O. N.

1928. The effect on bone formation of winter sunlight transmitted by a glass substitute. Poultry Sci. 7, 85-91.

Russell, W. C., Massengale, O. N. and Howard, C. H.

1928. The duration of the effect of ultra-violet radiation of chickens. J. Biol. Chem. 78, XXI, XXII.

a. The duration of the effect of ultra-violet radiation on chickens. J. Biol. Chem. 60, 155-162.

\section{Rutherford, W. J.}

1921. Eye disease resulting from malnutrition. Brit. J. Ophthalm. 5, 60-64.

Sahashi, Y.

1925. Über das Vorkommen von Di-hydroxy-chinolin-carbonsäure ( $\beta$-Säure von U. Suzuki) in der Reiskleie. Biochem. Z. 159, 221-234.

1926. The constitution of $\beta$-acid (dioxyquinolinecarboxylic acid) obtained from crude oryzanine by hydrolysis. Sci. Papers Institute Phys. Chem. Research (Tokyo) 4, 207-234.

a. Über die Konstitution der durch Hydrolyse von Rohoryzanin entstehenden $\beta$-Säure (Dioxychinolincarbonsäure). II. Biochem. Z. 168, 69-72.

1927. On the behavior of 2, 6-dioxyquinoline obtained from $\beta$-acid of crude oryzanine upon the polyneuritis of pigeons. Sci. Papers Inst. Phys. Chem. Research (Tokyo) 5, 191-198.

Saleck, W.

1928. Über den C-Vitamingehalt von frischer und von gefrorener roher Winterkuhmilch. Milchwirtschaft Forsch. 6, 464-486. 
Saleeby, N. M.

1919. Treatment of human beriberi with autolyzed yeast extract. Philippine J. Sci. 14, 11, 12.

Salmon, W. D.

1922. The effect of feeding velvet beans to pigeons. Science 56, 368 .

1925. Vitamin B in the excreta of rats on a diet low in this factor. J. Biol. Chem. 65, 457-462.

1927. On the existence of two active factors in the vitamin B complex. $J$. Biol. Chem. 73, 483-497.

Salmon, W. D., Guerrant, N. B. and Hays, I. M.

1928. On the existence of two active factors in the vitamin B complex. II. J. Biol. Chem. 76, 487-497.

a. The effect of hydrogen-ion concentration upon adsorption of the active factors of vitamin B complex by fuller's earth. J. Biol. Chem. 80, 91-101.

Salmon, W. D., Hays, I. M. and Guerrant, N. B.

1928. Etiology of dermatitis of experimental pellagra in rats. J. Infect. Diseases 43, 426-441.

Salmon, W. D. and Livingston, C. W.

1925. Vitamins A and B in the pecan nut. J. Home Econ. 17, 129-135.

Salmon, W. D. and Miller, E. R.

1925. The water-soluble vitamin content of the velvet bean. J. Agr. Research 31, 793-799.

Sammartino, U.

1921. Uber Vitamine. VI. Biochem. Z. 125, 25-42.

Samson, K.

1926. Die Bedeutung des Serumphosphors für Pathogenese, Diagnose und

Therapie der Rachitis. Deut. med. Wochschr. 52, 579-580.

Sandels, M. R.

1928. Relative solubilities of the antineuritic and antipellagric vitamins in alcohol and a study of certain properties of these substances. Dissertation, Columbia University, New York.

1930. Experimental nutritional polyneuritis in the rat. J. Nutrition 2, 409-413.

Sandwith, F. M.

1914. Is pellagra a disease due to a deficiency of nutrition? Trans. Soc. Trop. Med. Hyg. 6, 143-148. See also Trans. Natl. Assocn. Study Pellagra 2, 97-100 (1912).

Santos, F. O.

1922. Some plant sources of vitamins B and C. Am. J. Physiol. 59, 310-334.

Santos, F. O. and Collado, E. G.

1925. Vitamin B in tikitiki extract prepared by the Philippine Bureau of Science. Philippine Agr. 14, 243-245.

1926. The nutritive value of Philippine cereals. I. The vitamin B content of glutinous rice, dead rice, and adlay. Philippine Agr. 14, 473-477.

1928. The anti-beriberi vitamin content of sweet potato leaves and shoots. Philippine Agr. 16, 513-520.

Santos, F. O. and Santos, S.

1926. The vitamin B content of some Philippine fruits and vegetables. II. Philippine J. Sci. 30, 307-323. 
Saunders, E. B.

1914. The coexistence of pellagra and beriberi. Trans. Natl. Assocn. Study Pellagra 2, 325-331 (1912).

Schabad, J. A.

1910. Zur Bedeutung des Kalkes in der Pathologie der Rachitis. I. Der Mineralgehalt gesunder und rachitischer Knochen. II. Der physiologische Kalkbedarf und Rachitis infolge von unbefriedigtem Kalkbedarf. III. Der Kalkstoffwechsel bei Rachitis. IV. Der Phosphorstoffwechsel bei Rachitis. Arch. Kinderheilk. 52, 46-47, 68-106; 53, 380-414; 54, 83-110.

Schaumann, $\mathrm{H}$.

1908. Beriberi und Nuclein-phosphorsäure in der Nahrung. Arch. SchiffsTropen-Hyg. Beihefte 12, [5] 37-57.

1909. Weitere Beiträge zur Ätiologie der Beriberi. Arch. Schiffs-Tropen-Hyg. Beihefte 13, [6] 82-90.

1910. Die Ätiologie der Beriberi unter Berucksichtigung des gesamten Phosphorstoffwechsels. Arch. Schiffs-Tropen-Hyg. Beihefte 14, [8] 5-397.

1911. Further contributions to the etiology of beriberi. Trans. Soc. Trop. Med. Hyg. 5, 59-75.

1912. Weitere Beiträge zur Ätiologie der Beriberi. Arch. Schiffs-TropenHyg. Beihefte 16, [1] 137-170.

a. Über die Darstellung und Wirkungsweise einer der in der Reiskleie enthaltenen, gegen experimentelle Polyneuritis wirksamen Substanzen. Arch. Schiffs-Tropen-Hyg. 16, 349.

b. Le béribéri, maladie de nutrition. Bull. soc. path. cxot. 5, 125-134.

c. $\mathrm{Zu}$ dem Problem der Beriberiätiologie. Arch. Schiffs-Tropen-Hyg. 16, 825-838.

\section{Scheer, K.}

1925. Über die Einwirkung der Thymusdrüse und des Wachstumvitamins auf das Wachstum. Klin. Wochschr. 4, 702, 703.

1928. Die Behandlung der Rachitis mit in Kohlensäureatmosphäre bestrahlter Milch. Münch. med. Wochschr. 75, 642-644.

Schepilewskaja, N. E.

1929. Vitaminwirkung und Oberffächenaktivität. I. Die Anwendung oberflächenaktiver vitaminfreier Stoffe bei der Avitaminose der Meerschweinchen. Biochem. Z. 204, 371-388.

a. Vitaminwirkung und Oberflächenaktivität. II. Die antiscorbutische Wirkung einiger Pflanzensäfte und ihre Oberflächenaktivität. Biochem. Z. 208, 334-351.

b. Vitaminwirkung und Oberflächenaktivität. III. Zur Frage nach dem Parallelismus zwischen den Veränderungen der antiskorbutischen Wirkung und der Oberflächenaktivität des Kohlsaftes. Biochem. Z. 210, 334-347.

Schepilewskaja, N. and Jarussova, N.

1926. Zur Frage nach dem experimentellen Skorbut der Meerschweinchen. Biochem. Z. 167, 245-264.

\section{Scheunert, A.}

1926. Beitrag zum Gehalt der Butter an Vitamin A unter dem Einfluss der üblichen Fütterung. Milchwirtschaft. Forsch. 3, 117-121.

1927. Über den Vitamingehalt der bei der Margarinefabrikation verwendsten technischen Sojaphosphatidpräparate. Z. Untersuch. Lebensm. 54, 302-307. 
a. Über den Vitamingehalt der für die Futterung wichtigen Rübenarten. Zuchtungskunde 2, 264-278.

b. Über den Gehalt von Weizen und Roggenkeimen an Vitaminen. Biochem. Z. 183, 113-121.

c. Über den Vitamingehalt der Silagefutter. Z Tier-ïcht. Züchtungsbiol. Tierernähr. 8, 349-377.

1929. Der Vitamingehalt der Deutschen Nahrungsmittel. I. Teil. "Obst und Gemüse," Berlin, Julius Springer, pp. 37.

a. Beitrag zum Vitamingehalt der Wiesen- und Weidegräser. Biochem. Z. 207, 447-457.

Scheunert, A. and Hermersdörfer, C.

1925. Zur Kenntnis der Vitamine. IV. Über den Gehalt des Pferdefleisches an Vitamin A und B. Biochem. Z. 156, 58-62.

Scheunert, A., Reschke, J. and Schaker, B.

1929. Über den Vitamin-D-Gehalt handelsüblicher Fischmele. Vorläufige Mitteilung. Z. Tiersücht. Zïchtungsbiol. Tierernähr. 15, 273-276.

Scheunert, A. and Schieblich, M.

1922. Studien über die Magendarmflora polyneuritischen Tauben und die Bildung antineuritischen Vitamins durch Darmbakterien. Centbl. Bakt. Parasitenk. I. Abt. Orig. 88, 290-298.

1923. Zur Kenntnis der Vitamine. II. Über die Bildung von Vitamin B durch obligate Darmbakterien. Biochem. Z. 139, 56-65.

1924. Zur Kenntnis der Vitamine. III. Über den Vitamingehalt des Bieres. Chem. Zelle Gewebe. 12, 45-46.

1926. Vergleich des Gehaltes von Frischhefe und der daraus hergestellten Trockenhefe an Vitamin B. Chem. Zelle Gewebe. 13, 79-86.

1927. Über das verschiedene Verhalten von Tauben und Hühnern bei Deckung ihres Vitamin-B Bedarfs durch frisches grünes Pflanzenmaterial. Biochem. $Z$. 186, 222-228. 231.

a. Weiterer Beitrag zum Vitamingehalt des Bieres. Biochem. Z. 186, 229-

b. Ist der Taubenversuch zum Nachweis von Vitamin B in frischen und konserviertem Grünfutter geeignet? Z. Tiersïcht. Züchtungsbiol. Tierernähr. 8, 315-320.

1928. Über den Vitamin-B-gehalt von unter Zusatz verschieden grosser Hefemengen gebackenen Weizenbroten. Biochem. Z. 202, 380-386.

1929. Zur Wertbestimmung von Vitamin D-Präparaten. I. Biochem. Z. 209, 290-303.

a. Über den Vitamin-B-Gehalt verschiedener Hefen und damit hergestellter Weizenbrote. Biochem. Z. 212, 80-86.

b. Ueber eine auf dem Schutzversuch beruhende Methode zur Wertbestimmung von Vitamin D-Präparaten. Klin. Wochschr. 8, 699.

Scheunert, A., Schieblich, M. and Schwanebeck, E.

1923. Zur Kenntnis der Vitamine. I. Über den Vitamingehalt des Honigs. Biochem. Z. 139, 47-56.

Schieblich, M.

1929. Nachweis der Bildung von Vitamin B durch Bacillus vulgatus (Flügge) Migula mittels einer neuen einwandfreien Methode. Biochem. Z. 207, 458461. 
Schimizu, K.

1924. Experimentelle Untersuchungen über die Kohlenstoffausscheidung durch den Harn in der Norm, bei der Avitaminose, der Unternährung und dem Hunger. Biochem. Z. 153, 424-455.

Schioss, E.

1913. Zur Therapie der Rachitis. I. Die Wirkung von Phosphorlebertran und Calcium aceticum auf den Stoffwechsel des natürlich ernahrten rachitischen Kind. Jahrb. Kinderheilk. 78, 694-722.

Schlutz, F. W.

1927-1927. Heliotherapy and actinotherapy in relation to pediatrics. Am. J. Diseases Children 32, 900-921; 33, 122-135.

Schlutz, F. W. and Morse, M.

1925. Some spectroscopic observations on cod-liver oil. Am. J. Diseases Children 30, 199-209.

Schlutz, F. W. and Ziegler, M. R.

1929. Is irradiated farina as bought in the market enriched with vitamin D? J. Am. Med. Assocn. 93, 1466, 1467.

Schlutz, F. W., Ziegler, M. R. and Morse, M.

1927. The influence of irradiation upon oxidation products of cholesterol. $J$. Biol. Chem. 73, 209-213.

Schmidt-Nielsen, S. and Schmidt-Nielsen, S.

1929. A preliminary note on the liver-oil of the basking shark. Kongelige Norske Videnskabers Selskab Forhandl. 1, 5-7 (1926-1928).

a. Some liver oils yielding a strong color reaction with antimony trichloride. Biochem. J. 23, 1153-1157.

b. On the vitamin content of kippered herrings. Kongelige Norske Videnskabers Selskab Forhandl. 1, 45-47 (1926-1928).

c. On the value of the antimony trichloride test for $\AA$-vitamin. Kongelige Norske Videnskabers Selskab Forhandl. 1, 77-80 (1926-1928).

b. Pasteuriseringens indflytelse po melkens A-vitamin. Kongelige Norske Videnskabers Selskab Forhandl. 1, 126-128 (1926-1928).

e. On a difference between the vitamin content of the flesh-oil and the liver-oil of the salmon. Kongelige Norske Videnskabers Selskab Forhandl. 1, 189-191 (1926-1928).

1930. On the value of the antimony trichloride test for vitamin A. II. Kongelige Norske V'idenskabers Selskab Forhandl. 2, $44-47$ (1929).

a. A lack of D-vitamin in the liver-oil of the rabbit-fish (Chimaera monstrosa). Kongelige Norske Videnskabers Selskab Forhandl. 2, 48-50 (1929).

b. Puberteten som feilkilde ved A-vitaminforsk. (With an English summary.) Kongelige Norske Videnskabers Selskab Forhandl. 2, 51-54 (1929).

c. A lack of vitamin D in the liver-oil of the rabbit-fish. II. Kongelige Norske Videnskabers Selskab Forhandl. 2, 128-130 (1929).

d. Overdosering some feilkilde ved kvantitativ bestemmelse av Vitamin A. (With an English summary.) Kongelige Norske Videnskabers Selskab Forhandl. 2, 125-127 (1929).

e. Der Gehalt der Knorpelfische an antirachitische Vitamin. Z. physiol. Chem. 189, 229-238.

Schmidt-Weyland, P. and Koltzsch, W.

1927. Experimentelle Untersuchungen über den Einfluss des Skorbuts auf die Disposition zu Infektionen. Z. Hyg. Infektionskrankh. 108, 199-219. 
Schmitz, E. and George, E.

1929. Beitrag zur Frage der Einheitlichkeit des B-Vitamins. Biochem. Z. 204, 165-178.

Schmitz, E. and Hiraoka, T.

1928. Uber den Phosphatidstoffwechsel bei der B-Avitaminose der Tauben. Biochem. Z. 193, 1-17.

Schmitz, E. and Pollack, H. J.

1928. B-Avitaminose und Nebenniere. II. Úber das Verhalten von B-avitaminösen Tauben gegen Adrenalin und Cholin. Biochem. Z. 195, 428-441.

Schmitz, E. and Reiss, M.

1927. B-Avitaminose und Nebenniere. Biochem. Z. 183, 328-340.

Schoedel, J.

1928. Die augenblickliche Bewertung bestrahlter Tiermilch als Prophylaktikum und Therapeutikum gegen die Rachitis. Münch. med. Wochschr. 75, 644645.

Schoubye, B. N.

1928. Studies on the hydrogen-ion concentration in the different regions of the intestinal canal in animals on a normal diet and on a diet containing no vitamin B. Acta Med. Scand. Sup. 26, 537-546.

Schulz, O.

1929. Die Vitamin-A-Mangelkrankheiten (Keratomalacie und Xerophthalmie) unserer Haustiere. Z. Tierziicht. Züchtungsbiol. Tierernähr. 15, 123-134.

Schulz, O. and Maurmann, G.

1928. Indirekte Strahlentherapie der Rachitis. Sammelreferat. Arch. wissensch. prakt. Tierheilk. 57, 148-163.

\section{Schultzer, P.}

1925. Le métabolisme du phosphore et du calcium chez de jeunes rats soumis au régime rachitigène riche en calcium, sous l'influence des rayons ultraviolets de l'huile de foie de morue et des phosphates. Compt. rend. soc. biol. 93, 1005-1007.

a. Le calcium et le phosphore minéral du sérum des rats rachitiques sous l'influence de différents traitements. Compt. rend. soc. biol. 93, 1008-1010.

1927. Studien über Phosphor- und Calciumstoffwechsel bei mangelhaften Kostformen. I. Die Einwirkung ultravioletten Lichtes. II. Die Einwirkung von Lebertran. III. Veränderung des Phosphor oder Calciumgehalts der Kost. Biochem. Z. 188, 409-426, 427-434, 435-447.

1928. Studies on phosphorus and calcium metabolism. Acta Med. Scand Sup. 26, $560-565$.

Schwartze, E. W., Murphy, F. J. and Hann, R. M.

1929. Rolled oats and bran in a scurvy producing diet and the negative control test. J. Nutrition 2, 171-181.

Scott, L. C. and Hermann, G. R.

1928. Beriberi ("maladie des jambes") in Louisiana. J. Am. Med. Assocn. 90, 2083-2090.

Scotti-Foglieni, L.

1925. Sur la vitamine B. Arch. ital. biol. 75, 183-185.

1926. Sulla vitamina C. Boll. soc. biol. sper. 1, 627-628.

1927. Ulteriori richerche sulla vitamina C. Boll. soc. biol. sper. 2, 150, 151.

a. Sul reattivo di Bezssonoff per la vitamina C. Boll. soc. biol. sper. 2, 152. 
Seaman, E. C.

1920. The influence of an alcoholic extract of the thyroid gland upon polyneuritic pigeons and the metamorphosis of tadpoles. Am. J. Physiol. 53, 101108.

Sebrell, W. H.

1929. Fatty degeneration of the liver and kidneys in the dog apparently associated with diet. U.S. Pub. Health Repts. 44, 2697-2701.

Seel, $\mathrm{H}$.

1929. Wirkungen und Nebenwirkungen des bestrahlten Ergosterins. Münch. med. Wochschr. 76, 1413-1417.

\section{Segawa, W.}

1914. Über das Wesen der experimentellen Polyneuritis der Hühner und ihre Beziehung sur Beriberi des Menschen. Arch. path. Anat. (Virchow's) 215, 404-443.

Seidell, A.

1916. Vitamins and nutritional diseases. A stable form of vitamin, efficient in the prevention and cure of certain nutritional deficiency diseases. $U . S$. Pub. Health Repts. 31, 364-370.

1917. The vitamin content of brewers' yeast. J. Biol. Chem. 29, 145-154.

1921. The chemistry of vitamins. J. Ind. Eng. Chem. 13, 72-75.

a. Preliminary note on a stable silver vitamin compound obtained from brewers' yeast. U. S. Pub. Health Repts. 36, 665-670.

b. Experiments on the isolation of the antineuritic vitamin. J. Ind. Eng. Chem. 13, 1111-1115.

1922. Improved method for the preparation of vitamin-activated fuller's earth. U. S. Pub. Health Repts. 37, 801-803.

a. A physiological test for the activity of vitamin preparations. U. S. Pub. Health Repts. 37, 1519-1523.

b. Further experiment on the isolation of the antineuritic vitamin. J. Am. Chem. Soc. 44, 2042-2051.

1924. The preparation of a crystalline picrate having the antineuritic properties of vitamin B. U. S. Pub. Health Repts. 39, 294-299.

a. The chemistry of vitamines. Science 60, 439-447.

1926. Concentrated antineuritic vitamin prepared from brewer's yeast. J. Biol. Chem. 67, 593-600.

a. Comparaison entre la méthode du pigeon et celle du rat pour l'essai de la vitamine antinévritique. Bull. soc. chim. biol. 8, 746-750.

1929. Further progress towards the isolation of the antineuritic vitamin (vitamin B) from brewers' yeast. J. Biol. Chem. 82, 633-640.

a. The activity and nitrogen content of fractions obtained in the concentration of the antineuritic vitamin of brewers' yeast. Rec. trav. chim. 48, 855-859.

Sekine, $\mathrm{H}$.

1930. Fat-soluble A in fish oils. J. Tokyo Chem. Soc. 41, 426.

Sequeira, J. H. and O'Donovan, W. J.

1925. The practice of artificial light-bath treatment. Lancet, 1925, I, 909-912. Selter, H. and Nehring.

1921. Einfluss der Ernährung auf die Tuberkulosesterblichkeit. Z. Tuberk. 34, $1-7$. 
Sexton, W. A.

1928. The colour reactions of substances containing vitamin D. Biochem. J. 22, 1133, 1134.

Sharpe, J. S.

1922. The phospholipin of the blood and liver in experimental rickets in dogs. Biochem. J. 16, 486-488.

Shattuck, G. C.

1928. Scurvy with reference especially to adults. J. Am. Med. Assocn. 90, 1861-1864.

a. The relation of beriberi to polyneuritis from other causes. Am. J. Trop. Med. 8, 539-543.

Shear, M. J.

1926. A color reaction associated with vitamin D. Proc. Soc. Exptl. Biol. Med. 23, 546-549.

Shear, M. J. and Kramer, B.

1926. Fractionation of irradiated cholesterol. Chemical observations, Proc. Soc. Exptl. Biol. Med. 24, 48, 49.

a. Fractionation of irradiated cholesterol. I. Chemical observations. J. Biol. Chem. 71, 213-220.

Shear, M. J., Washburn, M. and Kramer, B.

1929. Composition of bone. VIII. Equilibration of serum solutions with CaHPO4. Science 69, 335, 336.

Sheard, C. and Higgins, G. M.

1928. The effects of selective solar irradiation on the growth and development of chicks. Am. J. Physiol. 85, 290-298.

1929. Influence of the ultra-violet light, solar irradiation and cod-liver oil on production, fertility, and incubation of eggs. Proc. Soc. Exptl. Biol. Med. $26,615-618$.

Sheard, C., Higgins, G. M. and Foster, W. L.

1930. The growth and development of chicks as influenced by solar irradiation of long visible and ultraviolet wave lengths respectively, with and without supplementary irradiation of various types. Am. J. Physiol. 94, 84-90.

Sheehy, E. J.

1924. Experiments on the possible effect of vitamins on quantity of milk and butterfat. Sci. Proc. Roy. Dublin Soc. n. ser. 17, 333-336.

1927. The relative food values of brown (from "entire" wheat grain) and white (from endosperm of grain) wheaten flour and their comparative potency for the prevention of xerophthalmia in guinea pigs. Proc. Roy. Irish Acad. B. $37,415-425$.

Sheehy, E. J. and Senior, B. J.

1929. The effect of cod-liver oil feeding on the calcium and phosphorus content of cow's milk. Biochem. J. 23, 898-901.

\section{Sheer, $\mathrm{K}$.}

1925. Úber die Einwirkung der Thymusdrüse und des Wachstumvitamins auf das Wachstum. Klin. Wochschr. 4, 702, 703.

Sheets, O. and Funk, C.

1922. The effect of ultraviolet rays on rats deprived of vitamin $A$ in their diet. Proc. Soc. Exptl. Biol. Med. 20, 80, 81. 
Shelling, D. H., Kramer, B. and Orent, E. R.

1928. Studies upon calcification in vitro. III. Inorganic factors determining calcification. J. Biol. Chem. 77, 156-170.

Sherman, H. C.

1926. "Chemistry of food and nutrition." 3d ed., New York, The Macmillan Co.

Sherman, H. C. and Axtmayer, J. H.

1927. A quantitative study of the problem of the multiple nature of vitamin $B$. J. Biol. Chem. 75, 207-212.

Sherman, H. C. and Boynton, L. C.

1925. Quantitative experiments upon the occurrence and distribution of vitamin $\mathrm{A}$ in the body and the influence of the food. J. Am. Chem. Soc. 47, 1646-1653.

Sherman, H. C. and Burtis, M. P.

1928. Vitamin A in relation to growth and to subsequent susceptibility to infection. Proc. Soc. Exptl. Biol. Med. 25, 649, 650.

a. Factors affecting the accuracy of the quantitative determination of vitamin A. J. Biol. Chem. 78, 671-680.

Sherman, H. C. and Burton, G. W.

1926. Effect of hydrogen-ion concentration upon the rate of destruction of vitamin B. J. Biol. Chem. 70, 639-645.

Sherman, H. C. and Cammack, M. L.

1926. A quantitative study of the storage of vitamin A. J. Biol. Chem. 68, 69-74.

Sherman, H. C. and Campbell, H. L.

1924. Growth and reproduction upon simplified food supply. IV. Improvement in nutrition resulting from an increased proportion of milk in the diet. J. Biol. Chem. 50, 5-15.

1928. The influence of food upon longevity. Proc. Natl. Acad. Sci. 14, 852855.

1930. Further experiments on the influence of food upon longevity. J. Nutrition 2, 415-417.

Sherman, H. C. and Edgeworth, H.

1923. Experiments with two methods for the study of vitamin B. J. Am. Chem Soc. 45, 2712-2718.

Sherman, H. C. and Gloy, O. H. M.

1927. Vitamin B determination and requirement with special reference to protein intake. J. Biol. Chem. 74, 117-122.

Sherman, H. C. and Grose, M. R.

1923. A quantitative study of the destruction of vitamin B by heat. J. Am. Chem. Soc. 45, 2728-2738.

Sherman, H. C. and Hessler, M. C.

1927. Quantitative differentiation of vitamins A and D. J. Biol. Chem. 73, 113-120.

Sherman, H. C. and Kramer, M. M.

1924. Experiments upon vitamin A. J. Am. Chem. Soc. 46, 1055-1063.

Sherman, H. C., La Mer, V. K. and Campbell, H. L.

1921. The effect of temperature and of the concentration of hydrogen ions upon the rate of destruction of antiscorbutic vitamin (vitamin C). Proc. Natl. Acad. Sci. 7, 279-281. 
1922. The quantitative determination of the antiscorbutic vitamin (vitamin C).

J. Am. Chem. Soc. 44, 165-172.

Sherman, H. C. and MacArthur, E. H.

1927. A quantitative study of the determination of vitamin B. J. Biol. Chem. 74, 107-115.

Sherman, H. C. and MacLeod, F. L.

1925. The relation of vitamin A to growth, reproduction, and longevity. $J$. Am. Chom. Soc. 47, 1658-1662.

a. The calcium content of the body in relation to age, growth, and food.

J. Biol. Chem. 64, 429-459.

Sherman, H. C. and Munsell, H. E.

1925. The quantitative determination of vitamin A. J. Am. Chem. Soc. 47, 1639-1646.

Sherman, H. C. and Pappenheimer, A. M.

1921. A dietetic production of rickets in rats and its prevention by an inorganic salt. Proc. Soc. Exptl. Biol. Med. 18, 193-197.

a. Experimental rickets in rats. I. A diet producing rickets in white rats, and its prevention by the addition of an inorganic salt. J. Exptl. Med. 34, 189-198.

Sherman, H. C., Quinn, E. J., Day, P. L. and Miller, E. H.

1928. The relative stability of vitamin A from plant sources. J. Biol. Chem. 78, 293-298.

Sherman, H. C. and Sandels, M. R.

1929. Experiments with reference to the more heat-stable factor of the vitamin $\mathrm{B}$ group (factor P-P, vitamin $\mathrm{B}_{2}$ or G). Proc. Soc. Exptl. Biol. Med. 26, 536-540.

1931. Further experimental differentiation of vitamins B and G. J. Nutrition 3, 395-409.

Sherman, H. C. and Spohn, A.

1923. A critical investigation and an application of the rat-growth method for the study of vitamin B. J. Am. Chem. Soc. 45, 2719-2728.

Sherman, H. C. and Stiebeling, H. K.

1929. Quantitative studies of responses to different intakes of vitamin D. J. Biol. Chem. 83, 497-504.

1930. The relation of vitamin D to deposition of calcium in bone. Proc. Soc. Exptl. Biol. Med. 27, 663-665.

Sherman, H. C. and Storms, L. B.

1925. The bodily store of vitamin A as influenced by age and other conditions. J. Am. Chem. Soc. 47, 1653-1657.

Sherman, H. C. and Whitsitt, M. L.

1931. A study of the effect of nitrous acid upon components of the Vitamin B complex. J. Biol. Chem. 90, 153-160.

Sherman, H. E.

1929. Relative vitamin A content of four Oriental foods. Philippine J. Sci. 38, 1-7.

a. Relative content of water-soluble vitamin B in thirty Oriental foods. Philippine J. Sci. 38, 9-36.

b. Relative water-soluble vitamin $\mathrm{C}$ content of nine Oriental fruits and vegetables. Philippine J. Sci. 38, 37-46. 


\section{Shibayama, G.}

1910. Some observations concerning beriberi. Philippine J. Sci. Sect. B, 5, 123-125.

Shimizu, T. and Hatakeyama, T.

1929. UUber das Wachstumsvitamin A. I. Vitamin A-Choleinsäure. Z. physiol. Chem. 182, 57-71.

Shinoda, Fujimaki and Saiki, S.

1927. Teneur en vitamines des produits alimentaires Japonais. Bull. soc. hyg. aliment. 15, 524-530.

Shinoda, G.

1924. Stoffwechseluntersuchungen bei Avitaminose. Arch. ges. Physiol. (Pflïger's) 203, 365-393.

Shipley, P. G.

1924. Healing of rickety bones in vitro. Bull. Johns Hopkins Hosp. 35, 304.

Shipley, P. G. and Holt, L. E., Jr.

1927. Effect of starvation on healing of rickets in rats. Proc. Soc. Exptl. Biol. Med. 25, 32, 33.

a. Studies on calcification in vitro. IV. The effect of inorganic salts. Bull. Johns Hopkins Hosp. 41, 437-446.

Shipley, P. G., Kinney, E. M. and McCollum, E. V.

1924. Studies on experimental rickets. XXIV. The effect of certain extracts of plant tissues on florid rickets. J. Biol. Chem. 59, 165-175.

a. Studies on experimental rickets. XXV. A study of the antirachitic effect of certain oils. J. Biol. Chem. 59, 177-182.

Shipley, P. G., Kramer, B. and Howland, J.

1925. Calcification of rachitic bones in vitro. Am. J. Diseases Children 30, 37-39.

1926. Studies upon calcification in vitro. Biochem. J. 20, 379-387.

Shipley, P. G., McCollum, E. V. and Simmonds, N.

1921. Studies in experimental rickets. IX. Lesions in the bones of rats suffering from uncomplicated beriberi. J. Biol. Chem. 49, 399-410.

Shipley, P. G., Park, E. A., McCollum, E. V. and Simmonds, N.

1921. Studies on experimental rickets. III. A pathological condition bearing fundamental resemblance to rickets of the human being resulting from diets low in phosphorus and fat-soluble $A$. The phosphate ion in its prevention. Bull. Johns Hopkins Hosp. 32, 160-166.

a. Studies on experimental rickets. V. The production of rickets by means of a diet faulty in only two respects. Proc. Soc. Exptl. Biol. Med. 18, 277280.

b. Studies on experimental rickets. VII. The relative effectiveness of cod liver oil as contrasted with butter fat for protecting the body against insufficient calcium in the presence of the normal phosphorus supply. Am. J. Hyg. 1, 512-525.

1922. Studies on experimental rickets. X. Rickets and rickets-like disease produced in rats on deficient diets. Dental Cosmos 64, 265-273.

a. Studies on experimental rickets. XVIII. Is there more than one kind of rickets? Am. J. Diseases Children 23, 91-106. 
Shipley, P. G., Park, E. A., McCollum, E. V., Simmonds, N. and Kinney, E. M.

1922. Studies on experimental rickets. XX. The effect of strontium administration on the histological structure of the growing bones. Bull. Johns Hopkins Hosp. 33, 216-220.

Shipley, P. G., Park, E. A., McCollum, E. V., Simmonds, N. and Parsons, H. 'T.

1921. Studies on experimental rickets. II. The effect of cod liver oil administered to rats with experimental rickets. J. Biol. Chem. 45, 343-348.

Shipp, H. L. and Zilva, S. S.

1928. Metabolism in scurvy. I. The lactic acid excretion of scorbutic guinea pigs. Biochem. J. 22, 408-415.

a. Metabolism in scurvy. II. The nitrogen absorption and retention of guinea pigs. Biochem. J. 22, 1449-1460.

Shohl, A. T. and Bennett, H. B.

1927. Rickets in rats. III. Metabolism of calcium and phosphorus of rats on restricted food intake. J. Biol. Chem. 74, 247-256.

1928. Rickets in dogs. Metabolism of calcium and phosphorus. J. Biol. Chem. 76, 633-642.

Shohl, A. T., Bennett, H. B. and Weed, K. L.

1928. Rickets in rats. IV. The effect of varying the acid-base content of the diet. J. Biol. Chem. 78, 181-190.

a. Rickets in rats. V. Comparison of effects of irradiated ergosterol and cod liver oil. Proc. Soc. Exptl. Biol. Med. 25, 551-554.

b. Rickets in rats. VI. Effect of phosphate added to the diet of non-ricketic rats. Proc. Soc. Exptl. Biol. Med. 25, 669-671.

c. Rickets in rats. VII. Metabolism of calcium and phosphorus of rats fed upon non-ricketogenic diets. J. Biol. Chcm. 79, 257-267.

Shohl, A. T. and Bing, F. C.

1928. Neuro-muscular response of rats to galvanic stimuli. Am. J. Physiol. 86, 628-632.

a. Rickets in rats. VIII. Rickets and tetany. Am. J. Physiol. 86, 633638.

b. Rickets in rats. IX. $\mathrm{pH}$ of the feces. J. Biol. Chem. 79, 269-274.

Shohl, A. T. and Brown, H. B.

1929. Rickets in rats. X. Fasting tetany and phosphate tetany. J. Biol. Chem. 84, 501-509.

Shorten, J. A.

1922. The rôle of vitamins in tropical diseases. Indian Med. Gazette 57, 164169.

Shorten, J. A. and Roy, C. B.

1919. Report on the antiberiberi vitamin content and antiscorbutic property of sun-dried vegetables. Indian J. Med. Rescarch Spec. Indian Sci. Congress No. $1919,60-78$.

1921. The antiscorbutic and antiberiberi property of certain sun-dried vegetables. Biochem. J. 15, 274-285.

Shrader, J. H., Coblentz, M. H. and Korff, F. A.

1929. Effect of atmospheric pollution upon incidence of solar ultra-violet light. Am. J. Pub. Hcalth 19, 717-724. 
Shulman, D. N. and Mendel, L. B.

1924. The blood platelets in rats on adequate and inadequate diets. Proc. Soc. Exptl. Biol. Med. 21, 435, 436.

Shurley, B. R. and Turner, R. G.

1930. Infection of accessory sinuses and upper respiratory tract in vitamin A deficiency. J. Am. Med. Assocn. 94, 539-543.

Siler, J. F., Garrison, P. E. and MacNeal, W. J.

1914. Introduction to the second progress report of the Thompson-McFadden pellagra commission. Arch. Internal Med. 14, 289-292.

a. A statistical study of the relation of pellagra to use of certain foods and to location of domicile in six selected industrial communities. Arch. Internal Med. 14, 293-373.

b. The relation of methods of disposal of sewage to the spread of pellagra. Arch. Internal Med. 14, 453.

c. Further studies of the Thompson-McFadden pellagra commission. A summary of the second progress report. J. Am. Med. Assocn. 63, 1090-1093.

\section{Simmonds, $\mathbf{N}$.}

1924. Observations on reproduction and rearing of young by the rat as influenced by the diet. Am. J. Hyg. 4, Sept. Sup., pp. 108.

Simmonds, N., Becker, J. E. and McCollum, E. V.

1927. The relation of vitamin $\mathrm{E}$ to iron assimilation. J. Am. Med. Assocn. 88, 1047-1050.

1928. The distribution of vitamin E. J. Nutrition I, 39-47.

Simonik, F.

1929. Teneur en vitamine $\mathrm{C}$ des graines de légumineuses pendant la germination. Compt. rend. soc. biol. 100, 431, 432.

\section{Simonnet, $\mathrm{H}$.}

1920. Obtention chez le pigeon des accidents de polynévrite par l'emploi d'une alimentation synthétique.

1921. Régime artificiel chez le pigeon. Effets de la carence en levure de bière. Bull. soc. hyg. aliment. 9, 69-85.

1922. L'analyse biologique du lait (au point de vue des vitamines). Bull. soc. hyg. aliment. 10, 125-178.

a. Quelques remarques sur la carence en facteur $\mathrm{A}$ et les carences associées. Bull. soc. hyg. aliment. 10, 356-364.

1923. La question des vitamines. I. Le facteur lipo-soluble. Bull. soc. chim. biol. 5, 539-689.

a. Régimes simples carencés en facteur lipo-soluble A. Bull. soc. chim. biol. 5, 739-747.

1924. Les besoins nutritifs sont-ils les mêmes pour les deux sexes durant la croissance? Compt. rend. 178, 235-237.

1925. Etude comparative des effets de la carence en facteur lipo-soluble A et des effets de la sous-alimentation sur le développement de l'organisme. Bull. soc. hyg. aliment. 13, 419-443.

Simonnet, $H$. and Randoin, L.

1925. La question des vitamines. III. Le facteur hydro-soluble B. Bull. soc. chim. biol. 7, 678-749.

Simonnet, H. and Tanret, G.

1929. Au sujet de la toxicité de l'ergostérol irradié. Presse méd. 37, 468, 469. 
a. Au sujet de la toxicité de l'ergostérol irradié. Compt. rend. soc. biol. $100,548-550$.

1930. Sur la toxicité pour les animaux de laboratoire de hautes doses d'ergostérol irradié. Compt. rend. 190, 400-402.

Sipple, H. L. and King, C. G.

1930. A study of the preparation and properties of vitamin $C$ fractions from lemon juice. J. Am. Chem. Soc. 52, 420-423.

Sjörslev, N.

1924. On the sulfuric acid reaction of butter fat and the disappearance of the reaction from vitamin $\mathrm{A}$-containing butter fat through the action of oxidized fat. J. Biol. Chem. 62, 487-493.

Sjollema, B.

1923. Studies in inorganic metabolism. I. The influence of cod liver oil upon calcium and phosphorus metabolism. J. Biol. Chem. 57, 255-270.

Skarzynska-Gutowska, M.

1928. Action physiologique de la vitamine $B$. Phénomènes de sécretion de quelques glandes. Compt. rend. soc. biol. 99, 1168, 1169.

Slanetz, E. J.

1923. Effect of autoclaving on vitamin content in milk. J. Dairy Sci. 6, 237242.

Slonaker, J. R. and Card, T. A.

1923. The effect of a restricted diet. I. On growth. II. On pubescence and the menopause. III. On the number of litters and young born. Am. J. Physiol. 63, 503-512; 64, 35-43, 167-180.

a. The effect of a restricted diet. IV. On the age of greatest reproductivity. V. On mortality, cannibalism and the sex ratio. Am. J. Physiol. 64, 203-209, 297-310.

Smith, A. H. and Anderson, W. E.

1929. Lactation vs. improved growth in stock albino rats. Science 70, 98, 99.

Smith, A. H. and Bing, F. C.

1928. Improved rate of growth of stock albino rats. J. Nutrition 1, 179-189.

Smith, A. H. and Bogin, M.

1927. Experimental gangrene produced by dietary means. Am. J. Path. 3, 67-74.

Smith, A. H. and Carey, E.

1923. Growth on diets high in carbohydrate and high in fat. J. Biol. Chem. 58, 425-433.

Smith, A. H., Cowgill, G. R. and Croll, H. M.

1925. A note on the technique for studying vitamin B. J. Biol. Chem. 66, 15-21.

Smith, A. H. and Sah, P. P. T.

1927. Vitamins A and B in the Chinese litchi nut. Proc. Soc. Exptl. Biol. Med. 25, 63, 64 .

Smith, A. Henderson.

1918. Beer and scurvy. Lancet, 1918, II, 813-815.

1919. An historical inquiry into the efficacy of lime juice for the prevention and cure of scurvy. J. Roy. Army Med. Corps 32, 93-116, 188-208.

Smith, D. T. and Webster, L. T.

1925. Epidemiologic studies on respiratory infections of rabbits. VI. Etiology of otitis media. J. Exptl. Med. 41, 275-283. 
Smith, E. A.

1927. A study of the alimentary tract in experimental scurvy (guinea pig). Am. J. Physiol. 80, 288-300.

a. Gastric motility in vitamin B deficiency in the dog. Am. J. Physiol. 80, 485-487.

Smith, E. A. and Medes, G.

1921. Effect of heating the antiscorbutic vitamin in the presence of invertase. J. Biol. Chem. 48, 323-327.

Smith, E. A. and Plummer, B. A.

1928. The influence of yeast in the diet of the rat upon the activity of isolated segments of duodenum and colon. Am. J. Physiol. 84, 200-201.

Smith, G. H. and Wason, I. M.

1923. Serological factors of natural resistance in animals on a deficient diet. J. Immunol. 8, 195-200.

Smith, H. H. and Chick, H.

1926. Maintenance of a standardized breed of young rats for work upon fatsoluble vitamins, with particular reference to the endowment of the offspring. Biochem. J. 20, 131-136.

Smith, L. M. and Lewis, H. B.

1917. A study of the normal metabolism of the guinea pig. J. Am. Chem. Soc. 39, 2231-2239.

Smith, M. I.

1923. Studies on nutrition in experimental tuberculosis. I. The effect of the fat-soluble A vitamin on tuberculosis of the guinea pig with especial reference to the value of cod liver oil in experimental tuberculosis. Am. Rev. Tuberculosis $7,33-48$.

1926. Studies on nutrition in tuberculosis. II. Experimental tuberculosis infection in the albino rat and the influence of vitamin deficient diets thereon. J. Lab. Clin. Med. 11, 712-732.

1930. A new method of evaluating the potency of antineuritic concentrates. U. S. Pub. Health Repts. 45, 116-129.

Smith, M. I. and Elvove, E.

1929. The action of the irradiated ergosterol in the rabbit. U. S. Pub. Health Repts. 44, 1245-1256.

Smith, M. I. and Hendrick, E. G.

1926. Some nutrition experiments with brewers' yeast with especial reference to its value in supplementing certain deficiencies in experimental rations. $U . S$. Pub. Health Repts. 41, 201-207.

Smith, M. I., McClosky, W. T. and Hendrick, E. G.

1926. The influence of vitamin deficiencies on susceptibility to certain poisons. U. S. Pub. Health Repts. 41, 767-779.

Smith, S. L.

1928. Vitamin B. A question of nomenclature. Science 67, 494-496.

1929. Vitamins in food materials. U. S. Dept. Agr. Circ. 84, pp. 55.

Smith, Th.

1896. Bacilli in swine disease. U. S. Dept. Agr. Bureau Animal Industry Ann. Rept. 1895-6, 172.

Soames, K. M.

1924. A preliminary note on the growth-promoting and antirachitic value of cod liver oil when injected intraperitoneally. Biochem. J. 18, 1349-1353. 
Soames, K. M. and Leigh-Clare, J. C.

1928. The assay of the antirachitic vitamin D. Biochem. J. 22, 522-527.

Sobel, J. and Claman, I.

1929. Observations on the use of irradiated ergosterol in active rickets. Arch. Pediatrics 46, 1-16.

Sopp, E.

1924. Bananens vitaminindhold. Norsk. Mag. Lacgevidensk 85, 732-735.

Souba, A. J.

1923. Influence of the antineuritic vitamin upon the internal organs of Single

Comb White Leghorn cockerels. Am. J. Physiol. 64, 181-201.

Souba, A. J., Knandel, H. C. and Dutcher, R. A.

1923. The effect of the antineuritic vitamin (accessory food factor B) on the egg production and condition of laying hens. Pennsylvania Agr. Expt. Sta. Bull. 181, 26.

South Dakota Agr. Expt. Sta.

1929. Vitamin C content of canned spinach and Swiss chard. Ann. Rept. pp. $15,16$.

\section{Southgate, H. W.}

1924. The dietetic value of barley, malt, and malted liquors as determined by their vitamin content. Biochem. J. 18, 769-776.

a. The effect of fermentation on the water-soluble vitamin content of wort. Biochem. J. 18, 1248-1251.

1925. Note on the effect of high temperatures on the accessory food factor content of cod liver oil. Biochcm. J. 19, 733-736.

Souza, G. deP. and McCollum, E. V.

1920. A study of the factors which interfere with the use of yeast as a test organism for the antineuritic substance. J. Biol. Chem. 44, 113-129.

Speer, J. H., Wise, E. C. and Hart, M. C.

1929. The composition of spinach fat. J. Biol. Chem. 82, 105-110.

Spinka, J.

1923. The action of ultra-violet rays on accessory substances. Chem. Listy 17, 217, 257, 309. Chem. Abstracts 18, 1321.

Sprawson, C. A.

1920. Beriberi in the Mesopotamian Force. Quart. J. Med. 52, 337-355.

Spruyt, J. P.

1930. A research after a colorimetric method for estimating the silverskin (anti-beriberi-vitamin) percentage of rice. Mededeel. Dienst. Volksgezondheid Nederland.-Indië 19, 46-65.

a. Een onderzoek naar een colorimetrische methode voor de keuring van rijst op zilvervlies (anti-beri-beri-vitamine) gehalte. Chem. Weekblad 27, 298-304.

Stammers, A. D.

1921. Feeding experiments in connection with vitamins A and B. I. The value of steam-distilled palm kernel oil as a control fat. II. Wheat bran as a source of vitamins A and B. Biochem. J. 15, 489-493.

1922. Feeding experiments in connection with vitamins A and B. III. Milk and the growth-promoting vitamin. IV. The vitamin A content of refined cod liver oil. Biochem. J. 16, 659-667.

a. On the incidence of keratomalacia among rats suffering from avitaminosis. S. African J. Sci. 19, 241-243. 
1924. Feeding experiments in connection with vitamins A and B. V. Orange juice as a source of vitamin B. VI. Ophthalmia in rats affected with avitaminosis. VII. The vitamin content of cod liver oil and malt extract. Biochem. J. 18, 9-15.

a. Vitamin A content of vegetable oils. Lancet, 1924, II, 598-599.

1925. On the effect of deficiency of vitamin A on the blood-platelet count in rats. Brit. J. Exptl. Path. 6, 312, 313.

Steenbock, $\mathrm{H}$.

1917. Antineuritic substances from egg yolk. J. Biol. Chem. 29, XXVII.

1919. White corn vs. yellow corn and a probable relation between the fatsoluble vitamin and yellow plant pigments. Science 50, 352, 353.

1923. A satisfactory ration for stock rats. Science 58, 449, 450.

Steenbock, H. and Black, A.

1924. Fat-soluble vitamins. XVII. The induction of growth-promoting and calcifying properties in a ration by exposure to ultra-violet light. J. Biol. Chem. 61, 405-422.

1925. Fat-soluble vitamins. XXIII. The induction of growth-promoting and calcifying properties in fats and their unsaponifiable constituents by exposure to light. J. Biol. Chem. 64, 263-298.

Steenbock, H., Black, A. and Thomas, B. H.

1927. Cereals and rickets. Ind. Eng. Chem. 19, 906, 907.

1930. Cereals and rickets. III. The comparative rickets-producing properties of corn, wheat, and oats, and the effect of irradiation and mineral supplements. J. Biol. Chem. 85, 585-606.

Steenbock, H. and Boutwell, P. W.

1920. Fat-soluble vitamin. III. The comparative nutritive value of white and yellow maizes. J. Biol. Chem. 41, 81-96.

a. Fat-soluble vitamin. V. Thermostability of the fat-soluble vitamin in plant materials. J. Biol. Chem. 41, 163-171.

b. Fat-soluble vitamin. VI. The extractability of the fat-soluble vitamin from carrots, alfalfa, and yellow corn by fat solvents. J. Biol. Chem. 42, 131152.

Steenbock, H., Boutwell, P. W. and Kent, H. E.

1918. Fat-soluble vitamin. I. J. Biol. Chem. 35, 517-526.

Steenbock, H. and Coward, K. H.

1927. Fat-soluble vitamins. XXVII. The quantitative determination of vitamin A. J. Biol. Chem. 72, 765-779.

Steenbock, H. and Daniels, A. L.

1925. Irradiated foods and irradiated organic compounds: Therapeutic possibilities. J. Am. Med. Assocn. 84, 1093, 1097.

Steenbock, H. and Gross, E. G.

1919. Fat-soluble vitamin. II. The fat-soluble vitamin content of roots, together with some observations on their water-soluble vitamin content. J. Biol. Chem. 40, 501-532.

1920. Fat-soluble vitamin. IV. The fat-soluble vitamin content of green plant tissues together with some observations on their water-soluble vitamin content. J. Biol. Chem. 41, 149-162.

Steenbock, H. and Hart, E. B.

1913. The influence of function on the lime requirements of animals. J. Biol. Chem. 14, 59-73. 
Steenbock, H., Hart, E. B., Elvehjem, C. A. and Kleitzien, S. W. F.

1925. Dietary factors influencing calcium assimilation. VI. The antirachitic properties of hays as related to climatic conditions with some observations on the effect of irradiation with ultra-violet light. J. Biol. Chem. 66, 425440.

Steenbock, H., Hart, E. B., Hanning, F. and Humphrey, G. C.

1930. Fat-soluble vitamins. XXX. The antirachitic value of cow's milk as modified by the feeding of irradiated yeast. J. Biol. Chem. 88, 197-214.

Steenbock, H., Hart, E. B., Hoppert, C. A. and Black, A.

1925. Fat-soluble vitamins. XXVI. The antirachitic property of milk and its increase by direct irradiation and by irradiation of the animal. J. Biol. Chem. 66, 441-449.

Steenbock, H., Hart, E. B. and Jones, J. H.

1924. Fat-soluble vitamins. XVIII. Sunlight in its relation to pork production on certain restricted rations. J. Biol. Chem. 61, 775-794.

Steenbock, H., Hart, E. B., Jones, J. H. and Black, A.

1923. Fat-soluble vitamins. XIV. The inorganic phosphorus and calcium of the blood used as criteria in the demonstration of the existence of a specific antirachitic vitamin. J. Biol. Chem. 58, 59-70.

Steenbock, H., Hart, E. B., Riising, B. M., Hoppert, C. A., Basherov, S. and Humphrey, G. C.

1930. Fat-soluble vitamins. XXVIII. The antirachitic value of cow's milk as modified by exposure of the cow to sunlight and to radiations from a quartz mercury vapor lamp. J. Biol. Chem. 87, 103-126.

Steenbock, H., Hart, E. B., Riising, B. M., Kleitzien, S. W. F. and Scott, H. T.

1930. Fat-soluble vitamins. XXIX. Is antirachitic activation induced by ultra-violet radiations a panacea for negative calcium balances? J. Biol. Chem. 87, 127-137.

Steenbock, H., Jones, J. H. and Hart, E. B.

1923. Fat-soluble vitamins. XVI. Stability of the antirachitic vitamin to saponification. J. Biol. Chem. 58, 383-393.

Steenbock, H., Kent, H. E. and Gross, E. G.

1918. The dietary qualities of barley. J. Biol. Chem. 35, 61-74.

Steenbock, H. and Nelson, E. M.

1923. Fat-soluble vitamin. XIII. Light in its relation to ophthalmia and growth. J. Biol. Chem. 56, 355-373.

Steenbock, H., Nelson, E. M. and Hart, E. B.

1921. Fat-soluble vitamin. IX. The incidence of an ophthalmic reaction in dogs fed a fat-soluble vitamin deficient diet. Am. J. Physiol. 58, 14-19.

Steenbock, H. and Nelson, M. T.

1924. Fat-soluble vitamins. XIX. The induction of calcifying properties in a rickets-producing ration by radiant energy. J. Biol. Chem. 62, 209-216.

Steenbock, H., Nelson, M. T., and Black, A.

1924. Fat-soluble vitamins. XX. A modified technique for the determination of vitamin A. J. Biol. Chem. 62, 275-286.

Steenbock, H., Riising, B., Black, A. and Thomas, B.

1929. Cereals and rickets. II. Antirachitic activation of cereals. J. Am. Med. Assocn. 93, 1868-1874. 
Steenbock, H. and Sell, M. T.

1922. Fat-soluble vitamin. X. Further observations on the occurrence of the fat-soluble vitamin with yellow plant pigments. J. Biol. Chem. 51, 63-76.

Steenbock, H., Sell, M. T. and Boutwell, P. W.

1921. The fat-soluble vitamin. VIII. The fat-soluble vitamin content of peas in relation to their pigmentation. J. Biol. Chem. 47, 303-308.

Steenbock, H., Sell, M. T. and Buell, M. V.

1921. Fat-soluble vitamin. VII. The fat-soluble vitamin and yellow pigmentation in animal fats with some observations on its stability to saponification. J. Biol. Chem. 47, 89-109.

Steenbock, H., Sell, M. T. and Jones, J. H.

1923. Vitamin B. II. Storage of vitamin B by the rat. J. Biol. Chem. 55, 411-419.

a. Fat-soluble vitamin. XII. The fat-soluble vitamin content of millets. J. Biol. Chem. 56, 345-354.

Steenbock, H., Sell, M. T. and Nelson, E. M.

1923. Vitamin B. I. A modified technique in the use of the rat for determination of vitamin B. J. Biol. Chem. 55, 399-410.

a. Fat-soluble vitamin. XI. Storage of the fat-soluble vitamin. J. Biol. Chem. 56, 327-343.

Stefánsson, V.

1918. Original observations on scurvy. Med. Rev. Rcv. 24, 257-264.

a. Observations on three cases of scurvy. J. Am. Med. Assocn. 71, 1715.

Steidle, $H$.

1924. Besitzen essbare Pilze antiskorbutische Wirkung? Biochem. Z. 151, 181-186.

Stephenson, M.

1920. A note on the differentiation of the yellow plant pigments from the fatsoluble vitamin. Biochem. J. 14, 715 .

Stephenson, M. and Clark, A. B.

1920. A contribution to the study of keratomalacia among rats. Biochem. $J$. 14, 502-521.

Stepp, W.

1909. Versuche über Fütterung mit lipoidfreier Nahrung. Biochem. Z. 22, $452-460$.

1911. Experimentelle Untersuchungen über die Bedeutung der Lipoide für die Ernährung. Z. Biol. 57, 135-170.

1912. Weitere Untersuchungen über die Unentbehrlichkeit der Lipoide für das Leben. Über die Hitzezerstörbarkeit lebenswichtiger Lipoide der Nahrung. Z. Biol. 59, 366-395.

1913. Fortgesetzte Untersuchungen über die Unentbehrlichkeit der Lipoide für das Leben. Über das Verhalten der lebenswichtigen Stoffe zu den Lipoidextraktionsmitteln. Z. Biol. 62, 405-417.

1914. Über lipoidfreie Ernährung und ihre Beziehungen zu Beriberi und Skorbut. Deut. med. Wochschr. 40, 892-895.

1916. Ist die durch Lipoidhunger bedingte Ernährungskrankheit identisch mit Beriberi? Z. Biol. 66, 339-349.

a. Zur Frage der synthetischen Fähigkeiten des Tierkörpers. Z. Biol. 66, 350-358. 
b. Die Lipoide als unentbehrliche Bestandteile der Nahrung. Z. Biol. 66, 365-386.

1919. Über Versuche mit lipoidfreier Ernährung an Ratten und Hunden ( $\mathrm{Zu}$ gleich ein Beitrag zur Wirkung des Beriberischutzstoffes). Z. Biol. 69, 495513.

1921. Zum Frage der Verwertung der Trockenmilch vom Standpunkt der Vitaminlehre aus. Med. Klin. 17, 287-288.

1922. Über den derzeitigen Stand der Vitaminlehre mit besonderer Berücksichtigung ihrer Bedeutung für die klinische Medizin. Klin. Wochschr. 1, 881-885, 931-935.

1923. Über die Bedeutung gewisser fettlöslicher Nahrungstoffe für Wachstum und Erhaltung des tierischen Organismus. Naturwissenchaften 11, 33-37.

1925. Zur Frage der Bildung des Vitamins A bei der Keimung des Samens. Z. Biol. 83, 94-98.

a. Über die Wirksamkeit parenteraler Zufuhr der Vitamine A und D bei experimentellen Avitaminosen. Z. Biol. 83, 102-106.

b. Über gleichzeitige experimentelle Erzeugung schwerer Xerophthalmie und Rachitis bei jungen Ratten. Ergebnisse Physiol. 24, 67-70.

Stepp, W. and Friedenwald, J. S.

1924. Zur Frage der experimentellen Erzeugung von Schichtstar bei jungen Ratten durch Vitaminmangel der Nahrung. Klin. Wochschr. 3, 2325-2327.

Stepp, W. and György, P.

1927. "Avitaminosen und Verwandte Krankheitszustände," Berlin, Julius Springer.

Stern, R. O. and Findlay, G. M.

1929. The nervous system in rats fed on diets deficient in vitamins $B_{1}$ and $B_{2}$. J. Path. Bact. 32, 63-69.

Steudel, $\mathrm{H}$.

1927. Über das Verhalten des Vitamins A. Z. physiol. Chem. 170, 13-22.

1929. Uber die antirachitische Wirkung eines eiweissfreien Dotterextraktes (Heliocitin). Klin. Wochschr. 8, 830-833.

a. Über den Nachweis des Vitamins A. Biochem. Z. 207, 437-440.

Steudel, H. and Peiser, E.

1928. Über den Nachweis des Vitamin A. Z. physiol. Chem. 174, 191-195.

Stevenson, A. G.

1920. Notes on the etiology of an outbreak of scurvy in North Russia, with an experiment in test dieting. J. Roy. Army Med. Corps 35, 218-223.

Stewart, C. A.

1918. Changes in the relative weights of the various parts, systems and organs of young albino rats underfed for various periods. J. Exptl. Zo.öl. 25, 301353.

1919. Changes in the weights of the various parts, systems and organs in the albino rats kept at birth weight by underfeeding for various periods. Am. J. Physiol. 48, 67-78.

Stiebeling, H. K.

1928. Studies of the relation of vitamin D to the deposition of calcium in the bones of experimental animals with special reference to the quantitative determination of vitamin D. Dissertation, Columbia University, New York. 
Stockard, C. R.

1928. Rickets in dogs as probably related to sex. Am. J. Diseases Children 36, 310-314.

Stoeltzner, W.

1921. Die Rachitis als Avitaminose. Münch. med. Wochschr. 68, 1481, 1482. 1928. Eine chemische Reaktion auf antirachitisches Vitamin. Münch. med. Wochschr. 75, 1584.

1929. Hie bestrahlte Milch hie Vigantol. Münch. med. Wochschr. 76, 920.

Stolk, D. van, Dureuil, E. and Heudebert.

1929. Conditions de formation et de destruction de la vitamine $\mathrm{D}$ au cours de l'irradiation de l'ergostérol. Compt. rend. 187, 854-856.

Storms, L. B.

1927. The vitamin "A" (anti-ophthalmic) content of butter and spinach. Trans. Proc. New Zealand Inst. 58, 264-276.

Storm von Leeuwen, W. and Verzár, F.

1921. The sensitiveness to poisons in avitaminous animals. J. Pharmacol. 18, 293-311.

Strandberg, 0.

1928. Heliotherapy and artificial light. Treatment of tuberculous conditions and particularly laryngeal tuberculosis. J. Am. Med. Assocn. 90, 1595-1602.

Strong, R. P. and Crowell, B. C.

1912. The etiology of beriberi. Philippine J. Sci., Sect. B, 7, 271-413.

Strote, $\mathrm{H}$.

1928. Klinische Erfahrungen mit dem bestrahlten Ergosterin Windaus. Klin. Wochschr. 7, 114-116.

Stuart, H. O.

1928. Antirachitic values of cod liver oil, cod liver meal, and fish meal. New Hampshire Agr. Expt. Sta. Circ. 28, pp. 4.

Stucky, C. J.

1928. Does commercial insulin contain what has hitherto been called vitamin B? Arch. Internal Med. 42, 780-783.

Stucky, C. J. and Rose, W. B.

1929. Studies in the physiology of vitamins. VII. Hemoglobin, solids, sugar, and chloride changes in the blood of vitamin B-deficient dogs. $A m$. J. Physiol. 89, 1-17.

Stucky, C. J., Rose, W. B. and Cowgill, G. R.

1928. Studies in the physiology of vitamins. VI. The effect of insulin on gastric motility in vitamin B deficiency. Am. J. Physiol. 87, 85-92.

Suda, G.

1923. Kann die avitaminöse Wachstumshemmung durch anorganische Substanzen kompensiert werden? Biochem. Z. 138, 269-273.

Sudholt, H.

1929. Die Ziegenmilchanämie als C-Avitaminose (Auf Grund von Versuchen an Meerschweinchen). Z. Tierzïcht. Zïchtungsbiol. Tierernähr. 14, 175-230.

Sugiura, K.

1918. A preliminary report on the preparation of antipolyneuritic substances from carrots and yeast. J. Biol. Chem. 36, 191-196.

Sugiura, K. and Benedict, S. R.

1918. The nutritive value of the banana. J. Biol. Chem. 36, 171-189. 
1919. The action of radium emanation on the vitamins of yeast. J. Biol. Chem. $39,421-433$.

a. The nutritive value of the banana. II. J. Biol. Chem. 40, 449-468.

1923. A study of the adequacy of certain synthetic diets for the nutrition of pigeons. J. Biol. Chem. 55, 33-44.

Sullivan, M. X.

1920. A biological study of a diet resembling the Rankin Farm diet. U. S. Pub. Health Service, Hyg. Lab. Bull. No. 120, 127-140.

a. Feeding experiments with the Rankin Farm pellagra-producing experimental diet. U. S. Pub. Health Service, Hyg. Lab. Bull. No. 120, 141-156.

Sullivan, M. X. and Jones, K. K.

1920. The chemical composition of the Rankin Farm pellagra-producing experimental diet. U. S. Pub. Health Service, Hyg. Lab. Bull. No. 120, $117-$ 126.

Sullivan, M. X., Stanton, R. E. and Dawson, P. R.

1921. Metabolism in pellagra. A study of the urine. Arch. Internal Med. 27, 387-405.

Sullivan, M. X. and Voegtlin, C.

1916. The distribution in foods of the so-called vitamins and their isolation. J. Biol. Chem. 24, XVI.

\section{Sumi, M.}

a. The relation of the lipoids to the vitamins. J. Biol. Chem. 24, XVII.

1928. On ergosterin, isolated from a Japanese edible mushroom. Cortinellus shiitake. Proc. Imp. Acad. (Japan) 4, 116-119.

Sunderlin, G. and Werkman, C. H.

1927. The synthesis of vitamins by microörganisms. Proc. Iowa Acad. Sci. 34, 93, 94.

1928. Synthesis of vitamin B by microörganisms. J. Bact. 16, 17-33.

Supplee, G. C. and Dow, O. D.

1925. Reproductive potency of dry milk as affected by oxidation. J. Biol. Chem. 63, 103-114.

1926. Variations in the antiscorbutic properties of dry milk. Am. J. Diseases Children 31, 41-50.

1927. The antirachitic and calcifying properties of summer- and winter-produced dry milk, irradiated and non-irradiated. J. Biol. Chem. 73, 617-622.

a. Vitamin A potency of irradiated milk. J. Biol. Chem. 75, 227-239.

b. Antirachitic properties of irradiated dry milk. Am. J. Diseases Children 34, 364-371.

Supplee, G. C., Dow, O. D. and Flanigan, G. E.

1928. The concentrated water-soluble fraction of milk as a source of vitamin B. J. Dairy Sci. 11, 420-425.

Sure, $B$.

1924. Suggestive evidence for the existence of a specific vitamin for reproduction. Science 59, 19.

a. Dietary requirements for reproduction. I. The nutritive value of milk proteins from the standpoint of reproduction. II. The existence of a specific vitamin for reproduction. J. Biol. Chem. 58, 661-692; 693-709.

b. Dietary requirements for reproduction. III. The existence of the reproductive dietary complex (vitamin E) in the ethereal extracts of yellow corn, wheat embryo and hemp-seed. J. Biol. Chem. 62, 371-396. 
1925. Dietary requirements for reproduction. IV. Solubility of the reproductive dietary complex (vitamin E) in various organic solvents. J. Biol. Chem. 63, 211-223.

1926. Dietary requirements for reproduction. V. The rôle of various vegetable and fruit oils in fertility and lactation. VI. Types of sterility produced on a skimmed milk powder reproduction-deficient diet. VII. The existence of a lactation-producing factor in the unsaponifiable matter from wheat oil. J. Biol. Chem. 69, 29-40, 41-51, 53-74.

1927. Dietary requirements for reproduction. VIII. Further studies of a skimmed milk powder reproduction-deficient diet. IX. Cod liver oil versus wheat oil as sources of vitamin E. X. Vitamin B requirements for normal lactation. XI. The potency of butter fat in vitamin E. J. Biol. Chem. 74, $37-44,45-53,55-69,71-84$.

a. Vitamin requirements of nursing young. I. The unusual response of nursing young (Mus norvegicus albinus) to vitamin B administrations. Preliminary report. J. Am. Med. Assocn. 89, 675, 676.

b. Dietary requirements for reproduction. XII. The inefficiency of the lactating mother (Mus norvegicus albinus) to secrete vitamin B in the milk and the relation of such phenomenon to infant mortality. Science 66, 265-266.

1928. Dietary requirements for fertility and lactation. Arkansas Agr. Expt. Sta. Bull. 231, 5-8.

a. A detailed study of the rôle of vitamin $\mathrm{B}$ in anorexia in the albino rat. J. Nutrition I, 49-56.

b. Vitamin requirements of nursing young. III. A quantitative biological method for the study of vitamin $B$ requirements of nursing young of the albino rat. IV. Vitamin $B$ r's. vitamin $A$ and $D$ requirement for growth of nursing young of the albino rat. J. Nutrition 1, 139-153, 155164.

c. Dietary requirements for fertility and lactation. XIII. Storage of fatsoluble vitamins for lactation, with some observations on the cod liver oil requirements of nursing young. XIV. A quantitative biological method for the study of vitamin $B$ requirements for lactation. XV. The inefficiency of the lactating mother (Mus norvegicus albinus) in secreting vitamin B in the milk, and the relation of this phenomenon to infant mortality (detailed report). J. Biol. Chem. 76, 659-671, 673-683, 685-700.

d. Dietary requirements for fertility and lactation. XVI. Potency of "Vitavose" versus dehydrated yeast in vitamin B. Proc. Soc. Exptl. Biol. Med. 25, 603-605.

e. Dietary requirements for fertility and lactation. XVII. A dietary sterility associated with vitamin A deficiency. XVIII. The vitamin A content of wheat oil. J. Agr. Research 37, 87-92, 93-99.

f. Dietary requirements for fertility and lactation. XIX. Does copper supplement vitamin $B$ for lactation? XX. A differentiation of the vitamin $B$ complex in rice polishings as evidenced in studies of lactation. J. Biol. Chem. 80, 289-295, 297-307.

1929. Cow's milk as a source of vitamin B for lactation. Science 70, 583, 584.

1930. Dietary requirements for fertility and lactation. I. The rôle of fat soluble vitamins in fertility and lactation. Arkansas Agr. Expt. Sta. Bull. 250 , pp. 67. 
a. Dietary requirements for fertility and lactation. II. The rôle of vitamin $\mathrm{B}$ in lactation and vitamin requirements of nursing young. Arkansas Agr. Expt. Sta. Bull. 251, pp. 62.

b. Dietary requirements for fertility and lactation. III. Pathological changes in nursing young of the albino rat suffering from vitamin B deficiency, and the rôle of vitamin B in infant nutrition. Arkansas Agr. Expt. Sta. Bull. 252, pp. 50.

c. Dietary requirements for fertility and lactation. XXII. Further studies of the rôle of milk fat in fertility and lactation. J. Nutrition 2, 485-489.

Sure, B., Kik, M. C. and Walker, D. J.

1928. Vitamin requirements of nursing young. IV. (a) A quantitative biological method for the study of vitamin B requirements of nursing young. (b) Marked anhydremia associated with marked disturbance in hematopoietic function of nursing young suffering from vitamin B deficiency. J. Biol. Chem. 78, XVIII, XIX.

1929. Vitamin requirements of nursing young. VI. Anhydremia associated with disturbance in hematopoietic function in nursing young of the albino rat suffering from a deficiency of the vitamin B complex. J. Biol. Chem. 82, 287-306.

a. The effect of avitaminosis on hematopoietic function. I. Vitamin A deficiency. II. Vitamin B deficiency. III. Vitamin E deficiency. J. Biol. Chem. 83, 375-385, 387-400, 401-408.

Sure, B. and Read, J. W.

1921. Biological analysis of the seed of the Georgia velvet bean, Stizolobium deeringiamum. J. Agr. Research 22, 5-15.

Sure, B. and Schilling, S. J.

1927. Vitamin requirements of the nursing mother. I. The production of beriberi in the nursing young of the albino rat on diets entirely satisfactory for growth. J. Biol. Chem. 74, LXXIV-LXXVI.

1928. Vitamin requirements of nursing young. II. The production of beriberi in the nursing young (Mus norvegicus albinus) associated with hemorrhages. Am. J. Diseases Children 35, 811-822.

Sure, B. and Smith, M. E.

1928. Vitamin requirements of nursing young (Mus norvegicus albinus). V. Hypoglycemia in nursing young suffering from vitamin B deficiency. Proc. Soc. Exptl. Biol. Med. 26, 72, 73.

1929. Effect of vitamin deficiencies on carbohydrate metabolism. I. Hypoglycemia associated with anhydremia and disturbance in hematopoietic function in nursing young of the albino rat suffering from uncomplicated vitamin-B deficiency. J. Biol. Chem. 82, 307-315.

a. The effect of vitamin deficiencies on carbohydrate metabolism. II. The influence of uncomplicated vitamin-B deficiency on concentration of true sugar, reducing non-sugar, and alkaline reserve in the blood of the albino rat. $J$. Biol. Chem. 84, 727-740.

b. Vitamin requirements of nursing young. VII. The production of uncomplicated vitamin-B deficiency in nursing young of the albino rat. $J$. Nutrition 1, 537-540.

Suski, P. M.

1923. Kann ultraviolettes Licht die Ausbildung der avitaminösen hypochromen Anämie verhindern? Biochem. Z. 139, 258-260. 
1927. Uber den Einfluss der Verfütterung verschiedener aktiver Eisenverbindungen auf den Verlauf der Avitaminose bei Reistauben. Biochem. Z. 188, 459-464.

Suzuki, K. and Hatano, T.

1927. The influence of ultraviolet light upon the growth of animals. Proc. Imp. Acad. (Japan) 3, 94-96.

Suzuki, T.

1927. Studies on the vitamin content (the yeast growth-promoting stimulus) of mother's milk whose infants are suffering from beri-beri. Oriental $J$. Diseases Infants 2, 113-117.

Suzuki, U.

1926. Chemical studies of vitamin B in Japan. Sci. Papers Inst. Phys. Chem. Research (Tokyo) 4, 295-302.

Suzuki, U., Nakahara, W. and Hashimoto, N.

1927. The sterility of white rats maintained on certain synthetic diets. Proc. Imp. Acad. (Japan) 3, 619-621. Also in Japan Med. World 8, 31, 32 (1928).

a. On the reproductive failure of white rats on synthetic diets. Sci. Papers Inst. Phys. Chem. Research (Tokyo) 7, 143-151.

Suzuki, U., Odake, S. and Mori, T.

1924. Über einen schwefelhaltigen Bestandteil der Hefe. Biochem. Z. 154, 278-289.

Suzuki, U., Shimamura, T. and Odake, S.

1912. Über Oryzanin, ein Bestandteil der Reiskleie und seine physiologische Bedeutung. Biochem. Z. 43, 89-153.

Sweetman, M. D. and Palmer, L. S.

1928. Insects as test animals in vitamin research. I. Vitamin requirements of the flour beetle, Triboliun confusum Duval. J. Biol. Chem. 77, 33-52.

Swingle, W: W. and Rhinhold, J. G.

1925. The effect of ultra-violet radiation upon experimental tetany. Am. J. Physiol. 75, 59-69.

Swoboda, F. K.

1920. A quantitative method for the determination of vitamin in connection with determinations of vitamin in glandular and other tissues. J. Biol. Chem. 44, 531-551.

Sydenstricker, E.

1915. The prevalence of pellagra. Its possible relation to the rise in the cost of food. U. S. Pub. Health Repts. 30, 3132-3148.

Szarka, A.

1929. Die Wirkung von "E" Vitamin auf die Ovarialfunktion. XI. Inkretion und Avitaminose. Arch. Physiol. (Pflüger's) 223, 657-662.

Taguchi, K., Hiraishi, S. and Kiva, F.

1922. Experimental polished rice diseases in humans. Japan Med. World 2, 133-135.

Takahashi, $\mathbf{K}$. and Kawakami, $\mathbf{K}$.

1923. Chemistry of vitamin A. I. Separation of the effective constituent of cod liver oil and its properties. J. Chem. Soc. Japan (Nippon Kwagku Kwai Shi) 44, 590-605. Abstracted in J. Soc. Chem. Ind. 42, 904A. 
Takahashi, K. and Nakamiya, J.

1925. Physiological significance of biosterin (so-called vitamin A). Japan Med. World 5, 2-9.

Takahashi, K., Nakamiya, Z., Kawakami, K. and Kitasato, T.

1925. On the physical and chemical properties of biosterin (a name given to fat-soluble A) and on its physiological significance. Inst. Phys. Chem. Research (Tokyo) Sci. Papers 3, 81-146.

Takaki, $\mathbf{K}$.

1885. Prevention of Kakke in Japanese Navy. Sei-i-K-wai, August 1885 and April 1886, v, 41.

1887. Special report of the kakke patients in the Imperial Japanese Navy from 1878-86. Sci-i-K-zcai 6, 73. Abstracted in Lancet, 1887, II, 189.

1888. On the prophylactic influence upon other diseases of preventive measures against kakke. Sei-i-K-wai, 7, 187.

1906. Three lectures on the preservation of health amongst the personnel of the Japanese Navy and Army. Lancet, 1906, I, 1369, 1451, 1520. See also N. Y. Med. J. 83, 1161-1166.

a. Military hygiene of the Japanese Army. N. Y. Med. J. 83, 1161-1166.

Talbot, F. B., Dodd, W. J. and Peterson, H. O.

1913. Experimental scurvy and the Roentgen-ray diagnosis of scorbutus. Trans. Am. Pediat. Soc. 25, 195-212. See also Boston Med. Surg. J. 169, 232-239.

Tange, U. and McCollum, E. V.

1928. The allophanates of certain sterols. J. Biol. Chem. 76, 445-456.

Tanner, F. W.

1925. The "bios" question. Chem. Revier's 1, 397-472.

Tanner, W. F. and Echols, G. L.

1921. The occurrence of pellagra in patients apparently receiving an ample diet. J. Am. Med. Assocn. 76, 1337, 1338.

Tasawa, $\mathbf{R}$.

1915. Experimentelle Polyneuritis, besonders bei Vögeln, im Vergleich zur Beriberi des Menschen. Z. exptl. Path. Therap. 17, 27-46.

Taylor, J. and Thant, U.

1929. Observations on vitamin B deficiency in pigeons (including the occurrence of refection). Indian J. Med. Research 16, 747-765.

Taylor, M. W. and Nelson, V. E.

1929. Molasses, sorghum and honey as sources of vitamin E. Proc. Soc. Exptl. Biol. Med. 26, 521.

Taylor, N. B., Branion, H. D. and Kay, H. D.

1930. Some effects of the administration of excessive doses of irradiated ergosterol to normal and parathyroidectomized dogs. J. Physiol. 49, XXXVXXXVII.

\section{Tazawa, $R$.}

1923. Weitere Untersuchungen zur Erforschung der sogenannten Avitaminose. Biochem. Z. 137, 105-116.

Teding van Berkhout, P. J.

1926. Contribution to knowledge of the influence of B-vitamine on metabolism. Mededeel. Dienst Volksgezondheid Nederland.-Indië Part 4, 410-418. 
Telfer, S. V.

1921. The influence of codliver oil and butterfat on the retention of calcium and phosphorus. J. Physiol. 54, CV, CVI.

1922. Studies on calcium and phosphorus metabolism. Part I. The excretion of calcium and phosphorus. Part II. The metabolism of calcium and phosphorus in rickets. Quarterly J. Med. 16, 45-62, 63-72.

1924. The mineral content of human milk in normal and rachitic families. Biochem. J. 18, 809-813.

a. Studies on calcium and phosphorus metabolism. III. The absorption of calcium and phosphorus and their fixation in the skeleton. Quart. J. Med. 17, 245-259.

Tern-uchi, Y., Oyama, T., Nakamura, K. and Wada, C.

1929. On the mode of action of vitamin B (Aetiology of Beri-beri). Japan Med. World 9, 309-312.

Tholin, $T$.

1921. Über die Thermostabilität des Co-Enzyms und seine Abscheidung von Hefevitamin B. Z. physiol. Chem. 115, 235-256.

Thomas, D. R.

1927. A note on vegetable ghee (vanaspali) with a short experiment on its food value. Ind. J. Med. Research 14, 659-666.

Thomas, T. H.

1928. The treatment of progressive muscular atrophy by parathyroid, calcium and vitamin D. Brit. Med. J. No. 3518, 978, 979.

Thompson, H. B. and Mendel, L. B.

1918. Alternating growth and suppression of growth in the albino mouse, with special reference to the economy of food consumption. Am. J. Physiol. 45, 431-460.

Thompson, T. J. and Carr, I. L.

1923. The relation of certain blood constituents to a deficient diet. Biochem. J. 17, 373-375.

Thorup, D. W. and Carlson, A. J.

1928. The influence of yeast on the alimentary rate. Am. J. Physiol. 84, 90-97.

Thunberg, $\mathrm{T}$.

1918. Zur Kenntnis der Einwirkung tierischer Gewebe auf Methylenblau. Skand. Arch. Physiol. 35, 163-195.

Thurman, B. H. and Vahlteich, H. W.

1929. The vitamin $\mathrm{C}$ content of cucumbers and cucumber pickles. J. Home Econ. 21, 510-513.

Thurston, L. M., Eckles, C. H. and Palmer, L. S.

1926. The rôle of the antiscorbutic vitamin in the nutrition of calves. J. Dairy Science 9, 37-49.

Thurston, L. M., Palmer, L. S. and Eckles, C. H.

1929. Further studies of the rôle of vitamin $\mathrm{C}$ in the nutrition of calves. $J$. Dairy Sci. 12, 394-404.

Tilden, E. B. and Miller, E. G., Jr.

1929. The response of the monkey (Macacus rhesus) to withdrawal of vitamin A from the diet. Dissertation, Columbia University, New York. 
1930. The response of the monkey (Macacus rhesus) to withdrawal of vitamin

A from the diet. J. Nutrition 3, 121-140.

Tisdall, F. F.

1922. The calcium and phosphorus concentration in the serum of infants with

mild rickets or a condition simulating mild rickets. Am. J. Diseases Children 24, 382-386.

a. The effects of ultraviolet rays on the calcium and inorganic phosphate content of the blood serum of rachitic infants. Can. Med. Assocn. J. 12, 536-538.

Tisdall, F. F. and Brown, A.

1927. Seasonal variation of the antirachitic effect of sunshine. Am. J. Diseases Children 34, 721-736.

a. Antirachitic effect of skyshine. Am. J. Diseases Children 34, 737-741.

b. Antirachitic value of the sun's rays through various special window glasses. Am. J. Diseases Children 34, 742-752.

1928. Seasonal variation of the antirachitic effect of sunshine. Am. J. Diseases Children 36, 734-739.

1929. Relation of the altitude of the sun to its antirachitic effect. J. Am. Med. Assocn. 92, 860-864.

1930. The antirachitic value of irradiated wheat. J. Am. Med. Assocn. 94, 854-856.

Tisdall, F. F., Drake, T. G. H., Summerfeldt, P. and Brown, A.

1930. A new wheat irradiated biscuit containing vitamins and mineral elements. Can. Pub. Health J. 21, 68-75.

Tisdall, F. F. and Price, H. W.

1927. The effect of sunshine on the acidity of the intestinal tract of rachitic rats. Bull. Johns Hopkins Hosp. 41, 432-436.

Titus, R. W., Hughes, J. S., Hinshaw, W. R. and Fitch, J. B.

1926. Destruction of vitamin $\mathrm{A}$ in milk by ultraviolet light. Ind. Eng. Chem. $18,843$.

Tixier, G.

1929. Vérification spectrographique de l'activation de l'ergostérol sous l'in-

fluence de l'irradiation par les rayons ultraviolets. Compt. rend. 188, 206-208.

Tobler, W.

1918. Der Skorbut im Kindesalter. Z. Kinderheilk. (Originalien) 18, 63-158.

Tonney, F. O., Hoeft, G. L. and Somers, P. P.

1930. Loss in actinic intensity in urban sunshine due to air pollution. J. Preventive Med. 4, 139-148.

Tonney, F. O., Somers, P. P. and Marti, W. C.

1928. Actinic measurement of solar ultra-violet light and some correlations with the erythema dose. J. Preventive Med. 2, 493-511.

Toverud, $\mathrm{G}$.

1923. The influence of diet on teeth and bones. J. Biol. Chem. 58, 583-600.

Towle, E. C. and Merrill, E. C.

1928. Preliminary studies of the Rosenheim-Drummond color tests of vitamin A in cod-liver oil. Am. J. Pharm. 100, 601, 602.

Toyoda, $\mathrm{T}$.

1923. Experimental studies on vitamin with special reference to the vitamin content of the Japanese eggplant and orange. Chugwai Iyi Shimpo (Intern. 
J. Med. (Japan)) June 1923, No. 1036, 1037. Abstracted in Japan Med. World $3,275$.

Tozer, F. M.

1921. The effect of a diet deficient in animal fat on bone tissue (rib junctions) of kittens. Biochem. J. 15, 28, 29.

a. The effect on guinea pigs of deprivation of vitamin $A$ and the antiscorbutic factor with special reference to the condition of the costochondral junctions of the ribs. J. Path. Bact. 24, 306-325.

Tscherkes, L.

1922. Die Bedeutung der Vitamine im Haushalt des tierischen Körpers. I.

Die Rolle der Proteine und Kohlehydrate bei Vitaminhunger. Biochem. $Z$. 133, 75-84.

1923. Die Bedeutung der Vitamine im Haushalt des tierischen Körpers. II.

Der Verlauf der experimentellen Avitaminose bei dem unvollständigen Hungern. Biochem. Z. 137, 121-124.

a. Kalorische und qualitätive Eigenschaften von Ernährungsregimen bei Versuchen mit experimenteller Avitaminose. Biochem. Z. 143, 1-9.

1924. Die Bedeutung der Vitamine im Haushalt des tierischen Körpers. III. Der Verlauf des Eiweiss-, Kohlehydrat- und Fetthungers bei Avitaminose. Biochem. Z. 149, 51-62.

1926. Studien über B-Avitaminose. I. Biochem. Z. 167, 203-220.

Tscherkes, L. A. and Kuperman, T. M.

1926. Upon the localization of the disturbance in the nervous system by the B-avitaminosis. I. The development of the B-avitaminosis in pigeons with destroyed labyrinth. Russian J. Biol. Med. exptl. 1, No. 8, p. 13.

Tscherkes, L. A. and Litwack, I. I.

1927. Über das antirachitische Prinzip des Lebertrans. Z. Kinderheilk. 43, 403-410.

Tso, E.

1924. The value of egg yolk in supplementing diets deficient in calcium. Am. J. Physiol. 77, 192-198.

1925. A method for the preparation of basal dietary free from vitamin A. Proc. Soc. Exptl. Biol. Med. 22, 265, 266.

1926. The effect of chemical preservation of eggs upon the stability of their vitamin contents. Biochem. J. 20, 17-22.

1927. The effects of synthetic diets on fertility and lactation. Proc. Soc. Exptl. Biol. Med. 24, 465-468.

1928. The anti-scorbutic vitamin in cabbage soup, cabbage purée, and turnip juice. Chinese J. Physiol. 2, 403-408.

1929. Growth and reproduction of rats on vitamin C free diet. Proc. Soc. Exptl. Biol. Med. 26, 276, 277.

\section{Tsuji, M.}

1922. Über den Stoffwechsel bei vitaminfreier Ernährung. Biochem. Z. 129, 194-207.

Turner, R. G.

1928. Microbic studies of acute infections in animals (albino rat) deprived of an adequate supply of vitamin A. Proc. Soc. Exptl. Biol. Med. 26, 233-235.

1929. Bacteria isolated from infections of the nasal cavities and middle ear of rats deprived of vitamin A. J. Infect. Diseases 45, 208-213. 
Turner, R. G., Anderson, D. E. and Loew, E. R.

1930. Bacteria of the upper respiratory tract and middle ear of albino rats deprived of vitamin A. J. Infect. Diseases 46, 328-334.

Tyson, M. D. and Smith, A. H.

1929. Tissue changes associated with vitamin A deficiency in the rat. $A m . J$. Path. 5, 57-70.

\section{Uhlmann, F.}

1918. Beitrag zur Pharmakologie der Vitamine. I. Z. Biol. 68, 419-456.

a. Weiterer Beitrag zur Pharmakologie der Vitamine. II. Z. Biol. 68, 457-498.

Underhill, F. P. and Mendel, L. B.

1925. The effective agent in the prevention or alleviation of the ChittendenUnderhill pellagra-like syndrome in dogs. U. S. Pub. Health Repts. 40, 1087-1089.

1928. A dietary deficiency canine disease-further experiments on the diseased condition in dogs described as pellagra-like by Chittenden and Underhill and possibly related to so-called black tongue. Am. J. Physiol. 83, 589-633. Underhill, S. W. F.

1928. "Hypervitaminosis." Lancet, 1928, II, 948.

Ungar, A.

1927. Über den Ca-Gehalt des Blutserums bei Mangel an Vitamin B. Biochem. Z. $180,357,358$.

Urechia, C. I. and Popoviciu, G.

1927. L'ergostérine irradiée dans la tétanie expérimentale. Compt. rend. soc. Uyei, U. biol. 98, 405-407.

1927. Food accessory substances (vitamins) and tubercle bacilli. 1. Do tubercle bacilli contain or produce growth-promoting substances? 2. The effect of the commonly known vitamins on the growth of tubercle bacilli. J. Infect. Diseases 40, 425-432, 433-437.

\section{Van-Leent, J. B.}

1903. Note sur une forme mixte et peu connue de béri-béri et de scorbut, avec quelques remarques sur la thérapeutique alimentaire. Arch. méd. navale $79,275-279$.

Van Wyk, A. and Reerink, E. H.

1928. Vitamin D and isoergosterol. Nature 122, No. 3078, Sup. 648.

Vasarhelyi, B.

1926. Untersuchung über Gewebsatmung bei Mangel an Vitamin B. Arch. ges. Physiol. (Pfiüger's) 212, 239-245.

Vedder, E. B.

1912. A fourth contribution to the etiology of beriberi. Philippine J. Sci. Sect. B, 7, 415-422.

a. The prevention of beriberi. Trans. I5 Intern. Cong. Hyg. Demog. Washington 5, Sect. 2, 671-678.

b. The etiology of beriberi. Brit. Med. J. No. 2712, 1731.

1913. "Beri-beri," New York, Wm. Wood and Co.

1916. Dietary deficiency as the etiological factor in pellagra. Arch. Internal Med. 18, 137-172. 
a. The relation of diet to beriberi and the present status of our knowledge of the vitamins. J. Am. Med. Assocn. 67, 1494-1497.

1918. Is the neuritis-preventing vitamin concerned in carbohydrate metabolism? J. Hyg. 17, 1-9.

1921. The etiology of scurvy. Military Surg. 49, 133-150, 502-512.

1930. Deficiency diseases and the vitamins. 1 \& 2. Porto Rico J. Pub. Health Trop. Med. 5, 283-301, 458-476.

Vedder, E. B. and Clark, E.

1912. A study of Polyneuritis gallinarum. A fifth contribution to the etiology of beriberi. Philippine J. Sci. Sect. B, 7, 423-461.

Vedder, E. B. and Feliciano, R. T.

1928. An investigation to determine a satisfactory standard for beriberi-preventing rices. Philippine J. Sci. 35, 351-389.

Vedder, E. B. and Lawson, W. E.

1927. Solubilities of the antiscorbutic factor present in lemon juice. J. Biol. Chem. 73, 215-218.

Vedder, E. B. and Williams, R. R.

1913. Concerning the beri-beri-preventing substances or vitamins contained in rice polishings-a sixth contribution to the etiology of beri-beri. Philippine J. Sci. Sect. B, 8, 175-195.

Verder, E.

1928. Effect of diets deficient in vitamin A or B on resistance to paratyphoidenteritidis organisms. J. Infect. Diseases 42, 589-604.

1929. Observations following the feeding of Salmonella enteritidis to rats on diets varying in vitamin A content. J. Preventive Med. 3, 489-497.

Verzár, F, and Bogel, J.

1920. Untersuchungen über die Wirkung von akzessorischen Nahrungssubstanzen. Biochem. Z. 108, 185-206.

Viswalingam, A.

1918. Pellagra. J. Trop. Med. Hyg. 21, 153-158.

1920. Some further observations on the etiology of pellagra. J. Trop. Med. Hyg. 23, 46, 47.

Voegtlin, C. and Harris, R. H.

1920. The occurrence of pellagra in nursing infants, with observations on the chemical composition of the human milk from pellagrous mothers. U. S. Pub. Health Service Hyg. Lab. Bull. 116, 73-100.

Voegtlin, C. and Lake, G. C.

1919. Experimental mammalian polyneuritis produced by a deficient diet. Am. J. Physiol. 47, 558-589.

Voegtlin, C., Lake, G. C. and Myers, C. N.

1918. The dietary deficiency of cereal foods with reference to their content in "antineuritic vitamin." U. S. Pub. Health Repts. 33, 647-666.

Voegtlin, C. and Myers, C. N.

1918. The growth-promoting properties of foods derived from corn and wheat. U. S. Pub. Health Repts. 33, 843-868.

a. Phosphorus as an indicator of the vitamin content of corn and wheat products. U. S. Pub. Health Repts. 33, 911-917.

1919. Distribution of the antineuritic vitamin in the wheat and corn kernel. A contribution to the biology of the antineuritic vitamin. Am. J. Physiol. 48, 504-511. 
a. A comparison of the influence of secretin and the antineuritic vitamin on pancreatic secretion and bile flow. J. Pharmacol. 13, 301-315.

Voegtlin, C., Neill, M. H. and Hunter, A.

1920. The influence of vitamins on the course of pellagra. U.S. Pub. Health Service, Hyg. Lab. Bull. 116, 7-35.

Voegtlin, C., Sullivan, M. X. and Myers, C. N.

1916. Bread as a food-Changes in its vitamin content and nutritive value with reference to the occurrence of pellagra. U.S. Pub. Health Rcpts. 31, 935-943, 2205.

Voegtlin, C. and White, G. F.

1916. Can adenine acquire antineuritic properties? J. Pharmacol. 9, 155-166. Völtz, W. and Kirsch, W.

1927. Der Nachweis des antirachitischen Faktors bei im Dunkeln und hinter Fensterglas gewachsenen Gräsern. Biochem. Z. 186, 255-263.

1928. Der Nachweis des antirachitischen Faktors bei Gras das im Dunkeln auf einer künstlichen Nährlösung gewachsen ist. II. Biochem. Z. 193, 281-284.

Völtz, W., Kirsch, W. and Falkenheim, C.

1927. Der Einfluss der Bestrahlung von Kühen mit der künstlichen Höhensonne und mit Sonnenlicht auf die Sekretion von antirachitisch wirkender Milch. Landw. Jahrb. 65, 375-397.

\section{Vogt, E.}

1927. Über die Beziehungen zwischen Hormonen und Vitaminen. Münch. med. Wochschr. 74, 2125-2128.

1928. Erfahrungen mit der Anreicherung der Nahrung an Vitaminen durch Zufuhr von Vigantol während der ersten Lebenszeit. Münch. med. Wochschr. $75,721-723$.

1929. Fetus und Vitamin A. Münch. med. Wochschr. 76, 1748-1750.

a. Über Reformen der Schwangerschaftsdiät mit Rücksicht auf das Vitaminbedürfnis des Fetus. Mïnch. med. Wochschr. 76, 1959-1961.

Vogt, $H$.

1920. Säuglingskorbut. Jahrb. Kinderheilk. 91, 278-299.

Voit, C.

1881. Hermann's "Handbook of Physiology," VI, 19 (through Osborne and Mendel 1911).

\section{Vollmer, $\mathrm{H}$.}

1928. Beitrag zur Ergosterinbehandlung der Rachitis. Deut. med. Wochschr. 53, 1634, 1635.

1929. Zum Problem des planmässigen Rachitisbekämpfung in der Fürsorge. Deut. med. Wochschr. 55, 1882-1884.

\section{Wachtel, M.}

1929. Die Vermehrung und Vitaminreicherung der Muttermilch und Kuhmilch mittels bestrahlter Hefe. Münch. med. Wochschr. 76, 1513, 1514.

Waddell, J. and Steenbock, $\mathrm{H}$.

1928. The destruction of vitamin $E$ in a ration composed of natural and varied foodstuffs. J. Biol. Chem. 80, 431-442.

Wagner, R. and Wimberger, $H$.

1924. Clinical observations upon the value of oxidized cod-liver oil in the therapy of rickets. Lancet, 1924, II, 55-57. 


\section{Walker, S.}

1922. The relationship between xerophthalmia and fat-soluble A. J. Am. Med. Assocn. 78, 273, 274.

Walle, N. van der.

1922. The presence of the antineuritic and antiscorbutic vitamins in urine. Biochem. J. 16, 713-726.

Wallis, R. L. M.

1918. The food value of the groundnut (Arachis). Ind. J. Med. Research 6, 46-55.

Walshe, F. M. R.

1918. The "food deficiency" or "vitamine" theory in its application to infantile beri-beri. Brit. J. Children's Diseases 15, 258-268.

a. On the deficiency theory of the origin of beriberi in the light of clinical and experimental observations. Quart. J. Med. 11, 320-338.

1920. The nervous lesion of beriberi and its bearing on the nature and cause of the disease. Med. Sci. Abs. Rev. 2, 41-46.

1922. Some recent observations on the nervous lesion in experimentally produced avian polyneuritis (experimental beriberi). Med. Sci. Abs. Rev. 5, 314-319.

Waring, J. I.

1929. Beriberi in infants. Am. J. Diseases Children 38, 52-56.

Wason, I. M.

1921. Ophthalmia associated with a dietary deficiency in fat-soluble vitamin A. A study of the pathology. J. Am. Med. Assocn. 76, 908-912.

Watchorn, E.

1930. The absorption and excretion of calcium and phosphorus by rats receiving excessive doses of irradiated ergosterol. Biochem. J. 24, 631-640.

Waters, H. J.

1908. The capacity of animals to grow under adverse conditions. Proc. Soc. Prom. Agr. Sci. 29, 71-96.

1910. The influence of nutrition upon the animal form. Proc. Soc. Prom. Agr. Sci. 30, 70-98.

Watson, C. and Finlay, T. Y.

1929. The therapeutic value of irradiated milk in the treatment of rickets. Lancet, 1929, II, 704-707.

Watson, C. and Hunter, A.

1906. Observations on diet. The influence of diet on growth and nutrition. J. Physiol. 34, 111-130.

Weatherby, L. S. and Waterman, E. W.

1928. Vitamin B content of avocados. Ind. Eng. Chem. 20, 968-970.

Weatherby, L. S., Youtz, J. E. and Watson, R. V.

1929. The vitamin A content of avocados. J. Home Econ. 21, 360-364.

Webster, T. A. and Bourdillon, R. B.

1928. Notes on the irradiation of ergosterol. Biochem. J. 22, 1223-1230.

1929. The absorption spectrum of vitamin D. Nature 123, 244.

Webster, T. A. and Hill, L.

1923. Note on the alleged growth-promoting effect of air irradiated with the quartz mercury vapor lamp. J. Physiol. 57, LXXVIII-LXXX.

1924. The supposed influence of irradiated air on growth. Biochem. J. 18, 340-346. 
1925. Causation and prevention of rickets. Brit. Med. J. No. 3360, 956-960.

Wedgewood, P. E. and Ford, F. L.

1924. Sur la valeur de la réaction de Bezssonoff comme indicateur de la présence de la vitamine $\mathrm{C}$ dans le jus de choucroute. Bull. soc. chim. biol. 6, 217-219.

Weech, A. A.

1927. The influence of the administration of cod-liver oil to the mother on the development of rickets in the infant. Bull. Johns Hopkins Hosp. 40, 244-258.

1930. Association of keratomalacia with other deficiency diseases. Am. J. Diseases Children 39, 1153-1166.

\section{Weidemann, G.}

1926. Studies on the chemical nature of the unsaponifiable fraction of fish oil. Biochem. J. 20, 685-691.

Weill, E., Arloing, F. and Dufourt, A.

1922. Sur l'hématologie du pigeon carencé par alimentation au riz décortiqué. Compt. rend. soc. biol. 86, 1175, 1176.

a. Ả propos du rôle de l'inanition dans la carence des pigeons soumis au régime du riz décortiqué. Compt. rend. soc. biol. 87, 169, 170.

Weill, E. and Mouriquand, G.

1915. Recherches sur les maladies par "carence." Béribéri experimental provoqué par une alimentation exclusive par l'orge cortiqué stérilisé. Compt. rend. soc. biol. 78, 649-651.

1916. Graines de céréales décortiquées, "hypercarencées" par la stérilisation. Compt. rend. soc. biol. 79, 194-199.

a. Inanition et carence. Compt. rend. soc. biol. 79, 382, 383.

b. Troubles de la digestion dans la carence expérimentale. Compt. rend. soc. biol. 79, 384-386.

1917. Recherches expérimentales sur la valeur alimentaire du mais. Mais cru, stérilisé et décortiqué. Compt. rend. soc. biol. 80, 372-375.

1919. Syndrome béribérique expérimental chronique. Compt. rend. soc. biol. 81 , 432-436.

a. Sur le moment d'apparition de la substance antiscorbutique et sur les accidents provoqués chez les cobayes par les grains d'orge aux différents stades de leur germination. Compt. rend. soc. biol. 82, 184-186.

Weill, E., Mouriquand, G. and Michel, P.

1916. L'alimentation exclusive et la carence alimentaire. Compt. rend. soc. biol. 79, 37-39.

a. Recherches sur la carence alimentaire. Effets comparés de la nourriture exclusive des chats par la viande crue, congelée, salée, cuite, et stérilisée. Compt. rend. soc. biol. 79, 189-193.

Wellman, C., Eustis, A. C. and Scott, L. C.

1913. The rapid cure of Polyneuritis gallinarum by intramuscular injection of a substance isolated from rice-Note on the pathology of the disease. Am. J. Trop. Diseases Preventive Med. 1, 295-299.

Wells, A. H.

1921. The preparation of tikitiki extract for the treatment of beriberi. Philippine J. Sci. 19, 67-73.

Wells, C. A. and Ewing, P. V.

1916. Cottonseed meal as an incomplete food. J. Biol. Chem. 27, 15-25. 
Wells, F. M.

1919. Food deficiencies as a factor influencing the calcification and fixation of the teeth. Proc. Roy. Soc. Med. (Sec. Odontology) 12, 23-38.

Wells, H. G.

1921. Eye troubles of Roumanian children. Through Blunt and Wang, 1921, See p. 364.

Wenckebach, K. F.

1928. Heart and circulation in a tropical avitaminosis (beri-beri). Lancet, 1928, II, 265-268.

Wendt, G. von.

1925. Studien über das Vorkommen und die Bedeutung der A-Vitamingruppe. Klin. Wochschr. 4, 2389, 2390.

Werkman, C. H.

1923. Immunologic significance of vitamins. I. Influence of the lack of vitamins on the production of specific agglutinins, precipitins, hemolysins and bacteriolysins in the rat, rabbit and pigeon. II. Influence of lack of vitamins on resistance of rat, rabbit and pigeon to bacterial infection. III. Influence of the lack of vitamins on the leucocytes and on phagocytosis. J. Infect. Diseases 32, $247-254,255-262,263-269$.

1927. Vitamin effects in the physiology of microörganisms. J. Bact. 14, 335-347.

Werkman, C. H., Baldwin, F. M. and Nelson, V. E.

1924. Immunologic significance of vitamins. V. Resistance of the avitaminic albino rat to diphtheria toxin; production of antitoxin and blood pressure effects. J. Infect. Diseases 35, 549-556.

Werkman, C. H., Nelson, V. E. and Fulmer, E. I.

1924. Immunologic significance of vitamins. IV. Influence of lack of vitamin $\mathrm{C}$ on resistance of the guinea pig to bacterial infection, on production of specific agglutinins, and on opsonic activity. J. Infect. Diseases 34, 447-453.

Wesselow, O. L. V. de.

1922. The calcium and inorganic phosphorus content of the maternal blood during pregnancy and lactation. Lancet, 1922, II, 227, 228.

West, J. H.

1929. Supplying the breast-fed baby with vitamin B. Arch. Pediatrics 46, 646-650.

Wheeler, G. A.

1922. Treatment and prevention of pellagra by a daily supplemental meal. J. Am. Med. Assocn. 78, 955-957.

Wheeler, G. A., Goldberger, J. and Blackstock, M. R.

1922. On the probable identity of the Chittenden-Underhill pellagra-like syndrome in dogs and blacktongue. U. S. Pub. Health. Repts. 37, 1063-1069.

Wheeler, $\mathbf{R}$.

1913. Feeding experiments with mice. J. Exptl. Zoöl. 15, 209-223.

1915. A study of the nutritive value of some proprietary infant foods. II. As milk modifiers. Am. J. Diseases Children 9, 300-317.

Wheeler, R. and Biester, A.

1914. A study of the nutritive value of some proprietary infants foods. $A m . J$. Diseases Children 7, 169-183. 
Whitehead, R. W. and Barlow, O. W.

1929. Studies on the anemia of rice disease in rats. The influence of vitamins

A, B, and D, iron, copper, beef muscle, and liver on the course and regeneration from the anemia of rice disease. Am. J. Physiol. 89, 542-547.

Widmark, E.

1924. Vitamin A deficiency in Denmark and its results. Lancet, 1924, I, 12061209.

a. Der Zusammenhang zwischen der Bildung des A-Vitamins und den Farbstoffen der Pflanzen. Skand. Arch. Physiol. 45, 7-11.

Widmark, E. M. P. and Svensson, B.

1928. Studien über den Bedarf und zur Verfügung stehende Mengen an fettlöslichen Vitaminen der Kinder in den Volksschulen zu Malmö. Skand. Arch. Physiol. 54, 127-144.

Wierzchowski, $Z$.

1924. Vitamin studies. II. (Translated title). Pam. Państ. Inst. Nauk. Gosp. Wiejsk Pulawach. (Mém. Inst. Natl. Polon. Econ. Rurale Pulawy) 5, 15-69. 1928. On the contents of vitamin $B$ in ripening and germinating wheat grains. (Translated title). Pam. Państ. Inst. Nauk. Gosp. Wiejsk Pulawach. (Mém. Inst. Natl. Polon. Econ. Rurale Pulawy) 9, 537-554.

Wilder, T. S.

1929. The tetany of fasting in experimental rickets. J. Biol. Chem. 81, 65-72. Wilder, T. S. and Vack, C.

1930. Antirachitic effect of winter sunshine through Celoglass. Results of experimentation on rats. Am. J. Diseases Children 39, 930-934.

Wilkes, E. T., Follett, D. W. and Marples, E.

1929. The treatment of rickets with irradiated ergosterol. Am. J. Diseases Children 37, 483-496.

Wilkins, I. and Kramer, B.

1927. Infantile rickets. Treatment by intramuscular injection of a cod-liver oil concentrate. Bull. Johns Hopkins Hosp. 40, 52-57.

Willcox, W. H.

1916. Beriberi-with special reference to prophylaxis and treatment. J. Roy. Army Med. Corps 27, 191-202.

1917. Rations in relation to disease in Mesopotamia. Lancet, 1916, II, 677.

1920. The treatment and management of diseases due to deficiency of diet :Scurvy and beriberi. Brit. Med. J. No. 3081, 73-77.

Williams, C. T.

1928. The infrequency of severe rickets in New Orleans and vicinity. An attempt to correlate some of the responsible factors. Am. J. Diseases Children $35,590-606$.

Williams, J. and Corran, J. W.

1930. The preservation of the antiscorbutic vitamin in lemon juice. Biochem. $J$. $24,37-50$.

Williams, R. J.

1919. The vitamin requirement of yeast. A simple biological test for vitamin. J. Biol. Chem. 38, 465-486.

1920. A quantitative method for determination of vitamin. J. Biol. Chcm. 42, 259-265.

1921. Vitamins and yeast growth. J. Biol. Chem. 46, 113-118.

1928. "Nutrilites." Science 67, 607, 608. 
Williams, R. J. and Roehm, R. R.

1930. The effect of antineuritic vitamin preparation on the growth of yeasts.

J. Biol. Chem. 87, 581-590.

Williams, R. J., Warner, M. E. and Roehm, R. R.

1929. The effect of various preparations on the growth of bakers' and brewers' yeasts. J. Am. Chem. Soc. 51, 2764-2774.

Williams, R. J., Wilson, J. L. and Von der Ahe, F. H.

1927. The control of "bios" testing and the concentration of a "bios." J. Am.

Chem. Soc. 49, 227-235.

Williams, R. R.

1916. The chemistry of the vitamines. Philippine J. Sci. Sect. A, II, 49-57.

a. The chemical nature of the "vitamins." I. Antineuritic properties of the hydroxypyridines. J. Biol. Chem. 25, 437-445.

1917. The chemical nature of the "vitamins." III. The structure of the curative modifications of the hydroxypyridines. J. Biol. Chem. 29, 495-520.

1921. Vitamins from the standpoint of structural chemistry. J. Ind. Eng. Chem. 13, 1107, 1108.

1924. Ultra-violet light and the antineuritic vitamin. Science 60, 499.

1927. A note on the effects on pigeons of an exclusive diet of rice meal, bran and polish. Biochem. J. 21, 1349-1351.

Williams, R. R. and Crowell, B. C.

1915. Thymus gland in beriberi. Philippine J. Sci. Sect. B, 10, 121-125.

Williams, R. R. and Eddy, W. H.

1928. Physiological functions of vitamins. Carnegie Inst. Wash. Yearbook 27, 375-380 (1927-28).

1929. Physiological functions of vitamins. Carnegie Inst. Wash. Yearbook 28, 377-385 (1928-29).

Williams, R. R. and Johnston, J. A.

1915. Miscellaneous notes and comments on beriberi. Philippine J. Sci. Sect. B, 10, 337-343.

Williams, R. R. and Saleeby, N. M.

1915. Experimental treatment of human beriberi with constituents of rice polishings. Philippine J. Sci., Sect. B, 10, 99-118.

Williams, R. R. and Seidell, A.

1916. The chemical nature of the "vitamins." II. Isomerism in natural antineuritic substances. J. Biol. Chem. 26, 431-456.

Williams, R. R. and Waterman, R. E.

1926. The solubility of vitamin B in benzene. J. Biol. Chem. 68, 499-501.

1927. The composite nature of vitamin B. Proc. Soc. Exptl. Biol. Med. 25, $1-3$.

1928. The tripartite nature of vitamin B. J. Biol. Chem. 78, 311-322.

Williams, R. R., Waterman, R. E. and Gurin, S.

1929. The effect of $\mathrm{pH}$ control in the autoclaving of yeast with respect to the vitamin B factors. J. Biol. Chem. 83, 321-330.

1930. The Jansen and Donath procedure for the isolation of antineuritic vitamin. J. Biol. Chem. 87, 559-579.

Willimott, S. G.

1926. The vitamin B of lemon rind. Biochem. J. 20, 31-36.

1927. Some less appreciated constituents of orange juice. Pharm. J. 64, 773775. 
1928. The vitamins of orange juice Biochem. J. 22, 67-76.

a. The vitamins of commercially concentrated orange juice. Biochem. J. 22, 535-544.

Willimott, S. G. and Moore, T.

1926. Fearon's "pyrogallol" test as a possible basis for the estimation of vitamin A. Biochem. J. 20, 869-872.

1927. The feeding of xanthophyll to rats on a diet deficient in vitamin A. Biochem. J. 21, 86-88.

Willimott, S. G., Moore, T. and Wokes, F.

1926. Effects of various agents on colour tests for vitamin A. Biochem. J. 20, 1292-1298.

\section{Willimott, S. G. and Wokes, F.}

1925. The vitamin content of Cortex Limonis B. P.-Preliminary note. Pharm. J. [4] $61,164-168$.

a. The assay of cod-liver oil for vitamin "A." Pharm. J. [4] 61, 718-720.

1926. The vitamin content of Tinct. Limonis Fort. B. P. C. Pharm. J. [4] $63,184-186$.

a. The antirachitic vitamin D of cod-liver oil. Pharm. J. [4] 63, 473-476, 495-497, 521-524.

b. The vitamin C of lemon rind. Biochem. J. 20, 1013-1015.

c. Vitamins and other constituents of grapefruit rind. Biochem. J. 20, 1299-1305.

1927. The effect of irradiation on vitamin A. Pharm. J. [4] 64, 217, 218.

a. Vitamins A and D of spinach. Biochem. J. 21, 887-894.

b. Colour tests for vitamin A: Their application to naturally occurring products. Lancet, 1927, II, 8-11.

c. Some constituents of citrus fruits. Pharm. J. 64, 770-773.

1928. Some constituents of dried yeast and yeast extracts. Their application in human nutrition. Lancet, 1928, II, 668-673.

\section{Wills, L. and Mehta, M. M.}

1930. Production of pernicious anemia (Bartonella anaemia) in rats by deficient feeding. Brit. Med. J. No. 3625; 1167, 1168.

\section{Wilson, D. C.}

1929. Osteomalacia (late rickets) studies. I. Clinical symptoms in relation to bone changes as shown by X-ray examination. Indian J. Med. Research 17, 339-347.

Wilson, D. C. and Patel, G. P.

1930. Osteomalacia (late rickets) studies. II. The blood picture. Indian J. Med. Riesearch 17, 881-887.

Wilson, D. C. and Surie, E.

1930. Osteomalacia (late rickets) studies. III. Dietary factors in the aetiology of osteomalacia. IV. A preliminary note on the incidence of rickets and dental caries among school children in India. Indian J. Med. Research 30, 889-902, 903-905.

\section{Wilson, J. N.}

1922. The relation of photosynthesis to the production of vitamin A in plants. J. Biol. Chem. 51, 455-459. 
Wilson, M. G.

1926. The prevention of rickets. The influence of the routine administration of cod liver oil in the prevention of rickets in infants. Am. J. Diseases Children $31,603-630$.

Wilson, W. H.

1921. The diet factor in pellagra. J. Hyg. 20, 1-59.

1927. Reaction of fatty extracts of certain organs with the antimony trichloride test for vitamin A (preliminary note). Biochem. J. 21, 1054-1058.

1930. Note on the etiology of pellagra. Brit. Med. J. No. 3602, 101-103.

Wiltshire, H. W.

1918. A note on the value of germinated beans in the treatment of scurvy, and some points in prophylaxis. Lancet, 1918, II, 811-813.

Wimberger, $\mathrm{H}$.

1922. Note on the X-ray diagnosis of rickets. Lancet, 1922, II, 11, 12.

Windaus, $\mathbf{A}$.

1927. Sterine und antirachitisches Vitamin. Chem.-Ztg. 51, 113, 114.

Windaus, A. and Hess, A.

1927. Sterine und antirachitisches Vitamin. Nachr. Ges. Wiss. Göttingen. Math-physik. Klasse. 1926, 2, 175-184.

Windaus, A. and Linsert, $\mathrm{O}$.

1928. Uber die Ultraviolett-Bestrahlung des Dehydro-ergosterol. Liebig's Ann. 465, 148-166.

Windaus, A., Westphal, K., Weider, F. V., and Rygh, O.

1929. Einige Beobachtungen über die Ultraviolettbestrahlung des Ergosterins. Nachr. Ges. Wiss. Göttingen. Math.-physik. Klasse., 1929, 45-59.

Winfield, G.

1918. Some investigations bearing on the nutritive value of dried milk. Rept. Local Govt. Board (Gt. Brit.) Pub. Health, etc. n. Ser. No. 116, 139-156.

Wisconsin Agr. Expt. Sta.

1923. Chemistry of the antiscurvy (antiscorbutic) vitamin. Bull. 352, 13, 14.

a. Vitamin A in whole milk, skimmed milk and filled milk. Bull. 352, 14, 15.

b. Relation of vitamin A to rickets. Bull. 352, 16-18.

1926. Antirachitic content of canned oysters. Bull. 388, 124, 125.

1928. Irradiating food products on commercial scale proves feasible. Bull. $405,37,38$.

1929. Household storage of eggs does not impair antirachitic value. Bull. 405, 47.

Wiskott, A.

1929. Uber neuere Behandlungsverfahren der englischen Krankheit im Dienste der allgemeinen Rachitisbekämpfung. III. Bestrahltes Ergosterin gegen Rachitis. Münch. med. Wochschr. 76, 1430-1433.

Woenckhaus, E.

1927. Blutuntersuchungen an weissen Laboratoriums-ratten bei experimenteller Rachitis. Arch. exptl. Path. Pharmakol. 122, 44-60.

Wokes, F.

1928. Studies on colour tests for sterols and vitamin A. I. Sterol tests. Biochem. J. 22, 830-835.

a. Studies on colour tests for sterols and for vitamin A. II. A spectroscopic study of the colorations attributed to vitamin A. Biochem. J. 22, 995-1006. 
Wokes, F. and Barr, J. R.

1927. A note on antimony trichloride and some factors affecting its sensitivity as a reagent for vitamin A. Pharm. J. [4] 64, 758-760.

Wokes, F. and Willimott, S. G.

1927. A study of the effect of heat and oxidation on cod-liver oil as measured by colour tests. Biochem J. 21, 419-425.

a. A study of antimony trichloride as a possible quantitative reagent for vitamin A. Analyst 52, 515-524.

b. The detection and estimation of vitamin A and of vitamin D in cod-liver oil and various food products. Pharm. J. [4] 64, 752-757.

Wolbach, S. B. and Howe, P. R.

1925. The effect of the scorbutic state upon the production and maintenance of intercellular substances. Proc. Soc. Exptl. Biol. Bed. 22, 400-402.

a. The epithelial tissues in experimental xerophthalmia. Proc. Soc. Exptl. Biol. Med. 22, 402, 403.

b. Tissue changes following deprivation of fat-soluble A vitamin. J. Exptl. Med. 42, 753-777.

1926. Intercellular substances in experimental scorbutus. Arch. Path. Lab. Med. 1, 1-24.

1928. Vitamin A deficiency in the guinea pig. Arch. Path. Lab. Med. 5, 239-253.

Wolff, L. K., Overhoff, J. and van Eckelen, M.

1930. Über Carotin und Vitamin A. Deut. med. Wochschr. 56, 1428, 1429.

Wollman, E.

1921. Sur le rôle des microorganismes dans la production des vitamines.

Compt. rend. soc. biol. 85, 801-803.

Wollman, E. and Vagliano, $\mathbf{M}$.

1922. Influence de l'avitaminose sur la lactation. Compt. rend. 174, 1637-1639.

a. Sur le rôle des microorganismes dans la production des vitamines. Recherches sur la production des vitamines de croissance par le Bacille bulgare et l'Amylomucor B. Compt. rend. soc. biol. 86, 832, 833.

1923. Action sur la croissance de la vitamine B administrée par voie parentérale. Compt. rend. soc. biol. 88, 163, 164.

a. Action de la vitamine de croissance A administrée par voie parentérale. Compt. rend. soc. biol. 88, 336-338.

b. Action de la lumière sur la croissance. Compt. rend. 176, 1653-1655.

Wood, W. F., Jr.

1926-27. A comparison of the growths of chicks behind window glass and a glass substitute. Poultry Sci. 6, 62-70.

Woollard, H. H.

1927. The nature of the structural changes in nerve endings in starvation and in beriberi. J. Anatomy 61, 283-287.

Woringer, $\mathrm{P}$.

1924. La carence solaire dans la première enfance. Rev. méd. (Paris) 41, 356-378.

Wright, A. M.

1923. The presence of vitamin B in frozen flesh food. J. Soc. Chem. Ind. 42, 403T, 404T. Also in New Zealand J. Sci. Technol. 6, 129-133.

a. The presence of vitamin A in frozen pork. J. Soc. Chem. Ind. 42, 509T. Also in New Z.ealand J. Sci. Technol. 6, 133, 134. 
Wright, $\mathbf{O}$. $\mathbf{K}$.

1922. The action of yeast-growth stimulant. Biochem. J. 16, 137-142.

Wright, $\mathrm{S}$.

1921. The effect of B-vitamin on the appetite. Lancet, 1921, II, 1208, 1209.

a. A study of the combined action of raw cow's milk and orange juice as antiscorbutic substances. Biochem. J. 15, 695-702.

1922. B vitamin and pigeon beriberi. Lancet, 1922, I, 297.

$\mathrm{Wu}, \mathrm{H}$. and Chen, T-T.

1929. Growth and reproduction of rats on vegetarian diets. Chinese J. Physiol. $3,157-169$.

$\mathrm{Wu}, \mathrm{H}$. and $\mathrm{Wu}, \mathrm{D} . \mathrm{Y}$.

1928. Growth of rats on vegetarian diets. Chinese J. Physiol. 2, 173-194.

Wurzinger, S.

1928. Erfahrungen mit Vigantol bei der Behandlung der Rachitis. Klin. Wochschr. 7, 1859-1860.

Wyard, S.

1922. The treatment of malignant disease by a diet free from fat-soluble vitamin A. Lancet, 1922, I, 840, 841.

Wyman, E. T.

1927. The prevention and treatment of rickets. Boston Med. Surg. J. 197, 376-388.

Wyman, E. T., Drinker, P. and Mackenzie, K. H.

1930. Antirachitic value of winter sunlight in the latitude of $42^{\circ} 21^{\prime}$ (Boston). Am. J. Diseases Children 39, 969-979.

Wyman, E. T., Holmes, A. D., Smith, L. W., Stockbarger, D. C. and Pigott, M. G.

1926. A comparison of the antirachitic potency of irradiated cod liver oils. Boston Med. Surg. J. 195, 525-535.

1927. Does the irradiation of cod liver oil increase its antirachitic potency? Am. J. Diseases Children 34, 753-764.

1929. The value of different types of glass for transmitting ultra-violet light. Am. J. Diseases Children 37, 473-482.

Wyman, E. T. and Weymuller, C. A.

1924. The organization of a special clinic for the treatment of rickets with the mercury vapor quartz lamp in an outpatient department. J. Am. Med. Assocn. 83, $1479-1483$.

Wyss, W. H. de.

1922. B-vitamin and pigeon beriberi. Lancet, 1922, I, 100, 101.

Yang, F. H.

1924. Beiträge zum Meerschweinskorbut. Z. Hyg. 102, 493-502.

Yaoi, H.

1928. Glutathione and reducing power of muscle in vitamin B deficiency. Proc. Imp. Acad. (Tokyo) 4, 233-235. Also in Japan Med. World 8, 85, 86.

Yoder, L.

1926. The relation between peroxidation and antirachitic vitamin. J. Biol. Chem. 70, 297-307.

1927. Effect of antirachitic vitamin on the phosphorus, calcium and $\mathrm{pH}$ in the intestinal tract. J. Biol. Chem. 74, 321-329. 
Yoshiue, S.

1922. Úber die Bedeutung der verschiedenen Vitamine für die Eisenassimilation beim heranwachsenden Individuum und über die Zusammensetzung der Körperasche bei vitaminfreier und vitaminhaltiger Ernährung. Biochem. Z. 134, 363-374.

a. Uber den Einfluss der Funktion auf den Verlauf der Avitaminosen. Biochem. Z. 134, 375-380.

1924. Úber den Stickstoffwechsel bei der Avitaminose. Biochem. Z. 148, 1-48. Young, C. $\mathrm{H}$.

1929. The transmission of ultra-violet light through tracing cloth. Nature 123, 47.

Yudkin, A. M.

1922. Ocular manifestations of the rat which result from deficiency of vitamin A in the diet. J. Am. Med. Assocn. 79, 2206-2208.

1924. An experimental study of ophthalmia in rats on rations deficient in vitamin A. Arch. Ophthalmol. 53, 416-425.

Yudkin, A. M. and Lambert, R. A.

1922. Location of the earliest changes in experimental xerophthalmia in rats. Proc. Soc. Exptl. Biol. Med. 19, 375.

a. Lesions in the lacrimal glands of rats in experimental xerophthalmia. Proc. Soc. Exptl. Biol. Med. 19, 376, 377.

1923. Pathogenesis of the ocular lesions produced by a deficiency of vitamin A. J. Exptl. Med. 38, 17-24.

\section{Zäch, C.}

1928. Ubber die Methoden zum chemischen Nachweis der Vitamine. Mitt. Lebensm. Hyg. 19, 61-65.

\section{Zajdel, R. and Funk, C.}

1926. The use of colloidal ferric hydroxide for adsorbing vitamins $B$ and D. Biochem. J. 20, 26-30.

Zih, A.

1926. Die Wirkung des Thyreoidea-Inkrets auf den Gaswechsel bei Mangel an B-vitamin. Arch. ges. Physiol. (Pfiügcr's) 214, 449-462.

Zilva, S. S.

1919. The action of ultra-violet rays on the accessory food factors. Biochem. J. 13, 164-171.

a. The influence of deficient nutrition on the production of agglutinins, complement and amboceptor. Biochem. J. 13, 172-194.

1920. The extraction of the fat-soluble factor of cabbage and carrots by solvents. Biochem. J. 14, 494-501.

a. The action of ozone on the fat-soluble factor, preliminary note. Biochem. J. 14, 740, 741 .

1921. The infuence of aeration on the stability of the antiscorbutic factor. Lancet, 1921, I, 478.

1922. Conditions of inactivation of the accessory food factors. Biochem. J. $16,42-48$.

a. A note on the relative influence of cod liver oil and butter on the deposition of calcium in the bone of growing rats. Lancet, 1922, I, 1244.

1923. The influence of reaction on the oxidation of the antiscorbutic factor in lemon juice. Biochem. J. 17, 410-415. 
a. A note on the conservation of the potency of concentrated antiscorbutic preparations. Biochem. J. 17, 416, 417.

1924. The antiscorbutic fraction of lemon juice. I. Biochem. J. 18, 182-185.

a. A note on the conservation of the potency of concentrated antiscorbutic preparations. II. Biochem. J. 18, 186, 187.

b. The antiscorbutic fraction of lemon juice. II. Biochem. J. 18, 632-637.

c. The stability of the vitamin A of cod liver oil towards the hardening process. Biochem. J. 18, 881, 882.

1925. Recent progress in vitamin research. J. Soc. Chem. Ind. 44, 445T$450 \mathrm{~T}$.

a. The antiscorbutic fraction of lemon juice. III. Biochem. J. 19, 589-594.

1925. Untersuchungen über den Einfluss ultravioletter Strahlen auf die akzessorischen Nährstoff faktoren. Biochem. Z. 155, 333.

1927. A note on the precipitation of the antiscorbutic factor from lemon juice. Biochem. J. 21, 354, 355 .

a. The antiscorbutic fraction of lemon juice. V. Biochem. J. 21, 689-697.

1928. The antiscorbutic fraction of lemon juice. VII. Biochem. J. 22, 779-785.

1929. The antiscorbutic fraction of lemon juice. VIII. Biochem. J. 23, 11991205.

Zilva, S. S. and Drummond, J. C.

1921. Vitamin A content of oils prepared from livers of the cod, coal-fish and haddock. Lancet, 1921, II, 753, 754.

1922. Fish-liver oils and other highly potent sources of vitamin A. Lancet, 1922, I, 1243.

1923. The cod-liver oil industry in Newfoundland. J. Soc. Chem. Ind. 42, $185 \mathrm{~T}-188 \mathrm{~T}$.

Zilva, S. S., Drummond, J. C. and Graham, M.

1924. The relation of the vitamin A potency of the liver oil to the sexual condition and age of the cod. Biochem. J. 18, 178-181.

Zilva, S. S., Golding, J., Drummond, J. C. and Coward, K. H.

1921. The relation of the fat-soluble factor to rickets and growth in pigs. Biochem. J. 15, 427-437.

Zilva, S. S., Golding, J., Drummond, J. C. and Korenchevsky, V.

1924. The relation of the fat-soluble factor to rickets in pigs. III. Biochem. J. $18,872-880$.

Zilva, S. S. and Miura, M.

1921. A note on the relative activity of the fat-soluble accessory factor in codliver oil and butter. Lancet, 1921, I, 323.

a. The differential dialysis of the antineuritic and the antiscorbutic factors. Biochem. J. 15, 422-426.

b. The quantitative estimation of the fat-soluble factor. Biochem. J. 15, 654-659.

Zilva, S. S. and Still, G. F.

1920. Orbital hemorrhage with proptosis in experimental scurvy. Lancet, 1920, I, 1008.

Zilva, S. S. and Wells, F. M.

1919. Changes in the teeth of the guinea pig produced by a scorbutic diet. Proc. Roy. Soc. (London) B. 90, 505-512. 
Zoelch, P.

1929. Über neuere Behandlungsverfahren der englischen Krankheit im Dienste der allgemeinen Rachitis-bekämpfung. I. Uber Erfahrungen mit der Quartzlampe bei Rachitis. Münch. med. Wochschr. 76, 1420-1423.

Zucker, T. F.

1922. Further observations on the chemistry of cod-liver oil. Proc. Soc. Exptl. Biol. Med. 20, 136.

Zucker, T. F. and Barnett, M.

1923. Observations on the distribution of antirachitic substances. Proc. Soc. Exptl. Biol. Med. 20, 375-378.

Zucker, T. F. and Gutman, M.

1923. The various forms of phosphoric acid in the blood. Findings in rickets. Proc. Soc. Exptl. Med. Biol. 20, 372-375.

Zucker, T. F., Johnson, W. C. and Barnett, M.

1922. The acid-base ratio of the diet in rickets production. Proc. Soc. Exptl. Biol. Med. 20, 20-22.

Zucker, T. F. and Matzner, M. J.

1924. On the pharmacological action of the antirachitic active principle of cod liver oil. Proc. Soc. Exptl. Biol. Med. 21, 186, 187.

Zucker, T. F., Pappenheimer, A. M. and Barnett, M.

1922. Observations on cod liver oil and rickets. Proc. Soc. Exptl. Biol. Med. 19, $167-169$. 



\section{AUTHOR INDEX}

Abderhalden, E., 77, 351-353

Abel, E., 353

Abels, H., 353

Abraham, A., 353

Abrahamson, E. M., 353

Ackerson, C. W., 353, 467

Ackert, J. E., 353

Acuna, E. M., 353

Adachi, A., 353

Adam, A., 353

Adam, N. K., 312, 489

Adams, G., 353

Aengenendt, J., 353

Ahmad, B., 247, 354

Aidin, R., 354

Albert, J., 354

Albright, F., 359

Alexander, N., 477

Allaire, H., 433

Allchorne, E., 357

Alleman, I., 136

Allen, A., 400

Allen, E., 82, 354

Allen, F. P., 389

Allen, R. S., 354

Alpern, D., 354

Alquier, J., 354, 482

Ames, F. B., 403

Anderegg, L. T., 337, 338, 346, 354

Andersen, A., 242, 354

Anderson, D. E., 519

Anderson, E. M., 343, 354

Anderson, E. V., 354

Anderson, R. E., 160, 354

Anderson, R. J., 307, 354, 355, 416

Anderson, W. E., 355, 449

Anderson, W. T., Jr., 297, 355, 403, 416, 456

André, E., 482

Andrews, V. L., 75, 355

Ané, J. N., 461

Anrep, G. V., 73, 355

Anthony, E. L., 355

Appleton, V. B., 277, 355

Arloing, F., 355, 523

Armstrong, M. E., 378

Arneth, 355

Arnold, L., 433

Aron, H., 355, 404

Artom, C., 355

Árvay, A. von, 356

Arzoomanian, S., 435

Asada, K., 356
Aschoff, L., 356

Ashford, B. K., 356

Ashford, C. A., 356

Ashton, L. O., 381

Askew, F. A., 314, 356

Asselin, L., 354, 482

Aub, J. C., 359

Aubel, C. E., 160, 427

Avellar de Loureiro, J., 356

Axtmayer, J. H., 90, 92, 123, 356, 498

Aykroyd, W. R., 132, 137, 138, 140, 356

Babb, M. F., 357

Bacharach, A. L., 326, 357, 433

Bachem, A., 357

Bailey, E. M., 357

Baker, L. C., 237, 382, 457

Bakwin, H., 357

Baldwin, F. M., 357, 469, 524

Balietti, L., 357

Balmain, A. R., 282, 367

Baly, E. C. C., 357

Banks, H. S., 357

Barenburg, L. H., 357

Barger, G., 27, 357

Barlow, O. W., 358, 525

Barlow, T., 358

Barnes, D. J., 358

Barnes, R. E., 210, 358

Barnett, M., 303, 325, 327, 329, 450, 533

Barney, C. B., 399

Barr, J. R., 529

Barre, L., 466

Barry, M. M., 464

Barsickow, M., 358

Bartenstein, L., 358

Basherov, S., 507

Bassett-Smith, P. W., 182, 358

Batchelder, E. L., 288, 291, 359

Baude, P., 433

Bauer, W., 359

Baumann, L., 359

Beach, J. R., 359, 379

Beard, H. H., 77, 359, 376

Bechdel, S. I., 83, 84, 88, 359, 443

Becker, J. E., 117, 300, 316, 453, 502

Bedson, S. P., 281, 359

Behrens, A., 445

Bell, F. K., 456

Bell, M., 90, 92, 359 
Bencsik, J., 377

Benedict, S. R., 46, 112, 160, 510, 511

Benischek, M., 281, 286, 402

Bennett, H. B., 501

Berczeller, L., 360

Bergeim, O., 360, 413

Berglund, H., 360

Bernheim, M., 164, 466, 467

Bernton, A., 390

Bertram, S. H., 242, 360

Bertrand, G., 360

Bessesen, D. H., 360

Best, C. H., 360

Bethke, R. M., 321, 323, 329, 360, 365, 410

Beumer, H., 360, 361

Beznák, A. von, 361

Bezssonoff, N., 150, 175-177, 215, 361, 362

Bianchi, A., 377

Bickel, A., 362

Bierich, R., 362

Bierry, H., 362, 381

Biester, A., 207, 385, 524

Billig, A., 360

Bills, C. E., 308-312, 315, 330, 331, 362, 363, 416

Binet, L., 487

Bing, F. C., 363, 501, 503

Birnbacher, T., 363

Bisbey, B., 102, 108, 136, 363

Bishop, K. S., 82, 288, 336, 337, 363, 391

Black, A., 255, 301, 304, 305, 316, 317, $320,329,332,506,507$

Black, H., 467

Blackberg, S. N., 363, 461

Blacklock, D. B., 363

Blackstock, M. R., 524

Blank, V. M., 363

Blegvad, O., 363

Blish, M. J., 353

Bloch, C. E., 276-278, 363, 364

Blohm, G. J., 364

Bloombergh, H. D., 369

Bloxsom, A. P., 364

Blum, J., 364

Blumenfeld, C. M. 364

Blumgart, H. L., 398

Blunt, K., 277, 364

Blystone, I., 92, 449

Boas, M. A., 326, 329, 333, 364

Boehm, G., 268, 442

Bogel, J., 520

Bogert, L. J., 295, 364

Bogin, M., 503

Bohstedt, G., 364, 365

Bonar, B. E., 479

Bond, C. J., 365

Bond, M., 365

Boock, E., 365

Booher, L., 206, 456
Boone, F. H., 441, 472

Borchardt, W., 437

Borghi, B., 365

Bosanyi, A. de, 365

Bosch, G., 373

Bourdillon, R. B., 240, 309, 310, 312, $314,365,522$

Bourquin, A., 133, 134, 137, 139, 365

Boutwell, P. W., 227, 229, 231, 245 , 255, 506, 508

Bowen, J. A., 404

Bowers, W. S., 451

Bowman, H. H. M., 365

Boyenval, L., 365

Boynton, L. C., 255, 270, 284, 498

Brabec, L. B., 482

Bracewell, M. F., 190, 365

Braddon, W. L., 365

Bradford, W. L., 365

Bradshaw, P. J., 463

Brady, M. J., 358

Branion, H. D., 515

Bray, G. W., 366

Brekke, V., 89, 474

Brickwedde, F. G., 310, 311, 362

Bridge, E., 464

Briod, A. E., 241, 435

Brocklesby, H. N., 366

Brodie, J. L., 86, 463

Brooks, L. M., 378, 379

Brougher, J. C., 366

Brouwer, E., 366

Brown, A., 366, 517

Brown, H. B., 501

Brown, H. M., 366

Browne, C. A., 366

Browning, E., 490

Bruce, H. M., 314, 356

Bucek, 366

Buckland, H., 427

Buckner, G. D., 366

Budd, G., 14, 366

Buell, M. V., 245, 278, 508

Bull, L. B., 366

Bulley, E. C., 275, 367

Bundesen, H. N., 367

Bunge, G., 18

Bunting, R. W., 367

Burget, G. E., 354

Burnett, F. L., 367

Burns, C. M., 367

Burr, G. O., 131, 145, 338-341, 343-345, 367, 392

Burr, M. M., 367

Burrows, M. T., 367

Burtis, M. P., 93, 206, 245, 256, 258, $259,263,264,285,367,482,498$

Burton, G. W., 93, 106, 107, 367, 498

Burton, H. G., 282, 367

Buschmann, H., 367

Busk, G., 367

Byfield, A. H., 367, 378 
Cadarin, I., 469

Cady, O. H., 234, 368

Cahan, M. H., 304, 439

Cajori, F. A., 94, 368

Caldwell, G. W., 368

Calvin, J. K., 420

Cambden, M. R., 374, 375

Cameron, H. C., 368

Cammack, M. L., 284, 498

Campbell, H. L., 145, 146, 169, 172, 176 , $188,211-213,368,444,498$

Campbell, J. A., 368

Campbell, M. E. D., 199, 206, 368

Cannon, A., 368

Cannon, H. C., 368, 461

Capper, N. S., 368

Card, L. E., 160, 368, 408, 463

Card, T. A., 503

Carey, E., 503

Caridroit, F., 368

Carleton, R., 431

Carlson, A. J., 516

Carlson, E. R., 463

Carlsson, V., 86, 92, 138, 146, 190, 191, $200,206,368,386,440$

Carman, G. C., 463

Carman, J. S., 338, 346, 459

Carr, F. H., 239, 240, 368

Carr, I. L., 516

Carr, M., 441

Carr, R. H., 369

Carrel, A., 368

Carrick, C. W., 92, 116, 120, 160, 369, 413

Carson, W. C., 369

Carter, C. W., 369

Cartland, G. F., 369

Caskey, C., 414

Casparis, H., 369, 441

Cassie, E., 369

Cavanaugh, G. W., 210, 369

Cave, H. W., 427

Cavins, A. W., 369

Cessna, R., 469

Chahovitch, X., 369

Chamberlain, W. P., 16, 26, 112, 369, 370

Champion, W. M., 399

Chaney, M. S., 464

Channon, H. J., 235, 237, 382

Charles, 482

Chase, E. F., 85, 100-102, 370

Cheekmur, F., 436

Chen, T. T., 530

Chick, H., 45, 72, 90, 97, 112, 114, 122, $125,126,128,130-133,136,142-145$, $154,155,169,176,180,187,192,193$, $199,202,203,206-208,259,293,323$, $325,329,330,333,364,368,370,371$, 426,504

Chisholm, C., 371

Chitre, G. D., 456
Chittenden, R. H., 119, 140, 141, 371

Christensen, W. G., 241, 435

Christou, 446, 447

Church, A. E., 242, 243, 470

Claman, I., 505

Clark, A. B., 275, 508

Clark, A. J., 374

Clark, E., 105, 112, 520

Clark, J. H., 371, 372, 421

Clark, M., 457

Clausen, E. M. L., 372

Clayton, M. M., 338, 346, 372, 459

Clément, R., 446

Clenshaw, E., 372

Clover, E. H., 372

Clow, B., 188, 198, 372

Coade, E. N., 367

Coblentz, M. H., 501

Coblentz, W. W., 372

Cocking, T. T., 372

Cohen, B., 355, 361, 372, 400

Cole, W. C. C., 202, 451

Collado, E. G., 491

Collatz, F. A., 384

Collazo, J. A., 79, 362, 372, 373, 397, $398,408,490$

Collier, J., 373

Collison, D. L., 246, 373

Comrie, J. D., 373

Concepcion, I., 112, 286, 399, 406

Congdon, C. C., 455, 459

Conklin, R. E., 338, 459

Connell, S. J. B., 215, 218, 373

Cook, D. H., 246, 373, 482

Cook, H. B., 357

Coolidge, W. D., 438

Cooper, E., 373

Cooper, E. A., 26, 31, 55, 85, 86, 365, 373,374

Cooper, E. F., 382

Copeland, L., 471

Corda, L., 374

Corley, R. C., 380

Corran, J. W., 525

Corson, W. C., 374

Coutts, F. J. H., 374

Couture, E., 428

Cowan, R., 364

Coward, K. H., 130, 147, 201, 228, 230 , $235,237,244,251,252,255,256,259$, $261,263,270,272,273,317,318,327$, $374,375,382,383,403,432,506$

Cowell, S. J., 375

Cowgill, G. R., 30, 73, 74, 77, 99, 375, $376,488,503,510$

Cox, W. M., Jr., 362, 363

Cox, U., 369

Craig, J. D., 381

Cramer, W., 281, 283, 284, 376, 466

Craven, V., 192, 377

Crawford, M. E. F., 272, 377

Creekmur, F., 377 
Creighton, M., 322, 323, 384

Crichton, A., 388, 471, 480

Crist, J. W., 245, 377, 385

Croll, H. M., 91, 99, 377, 503

Crowder, G. P., 383

Crowell, B. C., 510, 526

Cruickshank, E. M., 377

Csik, L., 377

Cugnac, A. de, 47, 48, 377

Curjel, D. F., 405

Curran, J. O., 377

Dahl, S. J., 403

Dahle, C. D., 209, 384, 385, 425

Dalldorf, G., 179, 377

Dalyell, E. J., 370

Damianovich, H., 377

Damon, S. R., 377, 378

Daniels, A. L., 83, 89, 114, 253, 302, $333,367,378,379$

Daniels, F., 309, 317, 333, 395, 440

Danielson, L. S., 240, 242, 470

Danmeyer, 379

Danysz-Michel, 379

Darling, S. T., 151, 379

Daubney, C. G., 216, 379

Davey, A. J., 184, 379

Davidson, P. B., 379

Davis, D. E., 379

Davis, M., 13, 19, 52, 112-114, 223, 225, $228,253,267,379,452$

Davison, W. C., 379

Dawbarn, M. C., 379

Dawkins, V., 380

Dawson, P. R., 511

Day, P. L., 138, 232, 499

Deas, J., 380

DeBuys, L. R., 380

Delf, E. M., 169, 185, 192-194, 196-199, $203,205,213,370,380$

Delore, P., 380

Denes-Goetz, J., 469

Denis, W., 68, 176, 380

Dennett, R. H., 368, 380

Denton, J., 380

Denton, M. C., 381

De Sanctis, A. G., 381

Desgrez, A., 381

Deuel, H. J., Jr., 74, 376, 381

Dick, S. M., 451

Dickey, L. B., 381

Dietsch, W., 440

Dimit, B. H., 85, 482

Dingemanse, E., 444

Dixon, W. E., 381

Dodd, W. J., 381

Donaldson, H. H., 381

Donaldson, J. C., 381

Donath, W. F., 42, 45-47, 49, 67-69, $190,381,432$

Doolittle, A. W., 424
Dorcas, M. J., 381

Dougherty, J.'E., 381

Douglas, M., 82, 86, 398

Dow, O. D., 329, 334, 511

Downs, A. W., 73, 381

Doyle, L. P., 382

Dragstedt, L. R., 382

Drake, T. G. H., 517

Drew, A. H., 281, 376, 466

Drinker, P., 530

Drummond, J. C., 21, 32, 36, 47, 50, 53, $56,62,63,65,67,68,72,73,75-80$, $82,95,96,105,107,109,113,114,122$, $127,136,145,157,159,228-230,235$, $237,238,240,241,244,247,251,253$, $255,256,258,259,263,270,272,273$, $278,287,293,354,355,375,382,383$, $403,406,408,413,432,440,457,468$, $477,485,489,532$

Drury, A. N., 369

Dubin, H. E., 115, 303, 304, 384, 398

Dufourt, A., 355, 523

Duhring, F. L., 405

Dulière, W., 247, 384

Dunham, G. C., 99, 384

Dunn, L. C., 384, 471

Dureuil, E., 510

Dutcher, R. A., 88, 99, 197, 207, 209, $210,264,321-323,333,354,359,369$, $384,385,389,395,425,462,479,505$

Dye, J. A., 385

Dye, M., 245, 377, 385

Dyer, F. J., 375

Dyke, H. W., 385

Earp, J. R., 385

Echols, G. L., 515

Eckelen, M. van, 529

Eckles, C. H., 160, 208, 209, 354, 384, $385,407,475,516$

Eckman, P., 386

Eckstein, A., 386

Eddy, N. B., 381

Eddy, W. H., 47, 49, 53, 65, 69, 73, 86, $92,94,113,131,176,178,189-192$, $200,206,213,386,387,437,440,442$, 526

Edelstein, F., 444

Ederer, S., 387

Edgeworth, H. I., 387, 498

Edginton, B. H., 364, 365

Edie, C. S., 28, 40, 387

Eggleton, P., 79, 201, 375, 388

Eicholtz, F., 388

Eicholz, W., 388

Eidinow, A., 388

Eijkman, C., 15, 24, 25, 42, 68, 113, 388

Eliot, M. M., 388

Ellinger, P., 388

Elliott, W. E., 388

Ellis, C., 388 
Ellis, N. R., 198, 208, 389, 410

Elvehjem, C. A., 298, 343, 507

Elvers, C. F., 302, 456

Elvove, E., 504

Ely, J. O., 385, 389, 425

Embrey, H. C., 193, 205, 389

Emerique, L., 433

Emmett, A. D., 55, 114, 116, 160, 275, 389

Emslie, M., 389

England, E. H., 486

Engstrand, O., 202, 451

Enright, J. I., 389

Epelbaum, S., 475

Epstein, A., 390

Erikson, E., 390, 391

Estill, H. W., 390

Euler, B. v., 246, 248, 390, 391, 435

Euler, H. v., 246, 248, 249, 364, 390, 391,435

Eustis, A. C., 391, 522

Evans, H. M., 82, 131, 145, 289, 336$349,387,391,392$

Evans, W. H., 28, 387

Evers, N., 242, 392

Evvard, J. M., 286, 444

Ewing, P. V., 523

Faber, F., 364

Faber, H. K., 392

Fabre, R., 332, 393, 482

Faerber, E., 393

Fairhall, L. T., 393

Falconer, E. H., 281, 393

Falk, I. S., 367

Falkenheim, C., 361, 393, 521

Farmer, C. J., 79, 393

Farnum, M. B., 393

Farr, W. K., 367

Fearon, W. R., 239, 393

Felcher, A., 420

Feliciano, R. J., 520

Feng, C. T., 462

Ferdmann, D., 475

Ferguson, J., 431

Fernando, A. S., 393

Ferry, E. L., 112, 114, 393, 474

Field, A., 191, 464

Findlay, G. M., 71, 82, 142, 143, 154, $157,163,393,394,509$

Findlay, L., 394, 477

Fink, H., 390

Finks, A. J., 394, 434

Finlay, T. Y., 522

Fischer, L., 394

Fischmann, C., 365

Fish, M., 152, 416

Fishback, H. R., 413

Fitch, J. B., 427, 517

Flanigan, G. E., 511

Fleming, W. D., 394
Flesch, M., 394

Flury, F., 395

Follett, D. W., 525

Folin, O., 68, 176

Foote, J. A., 395

Ford, F. L., 177, 523

Fosbinder, R. J., 309, 317, 395

Foster, W. L., 497

Fränke1, S., 395

Francis, E., 385, 395, 445

Francis, L. D., 395, 464

Frank, A., 395, 396

Frank, M., 395

Franz, V. K., 407

Fraser, A. C., 395

Fraser, H., 26, 27, 113, 395, 396

Frazier, W. C., 446

Freedman, L., 396, 398

Freise, E., 396

Freudenberg, E., 396

Freudenthal, P., 396

Frey, C. N., 448

Fridericia, L. S., 84, 277, 396

Friedenwald, J. S., 509

Friedman, I., 357

Fries, M. E., 396

Frölich, T., 16, 148-151, 155, 168, 173 , $189,197,198,202,203,211,396,424$

Frost, A. W., 396

Fürst, V., 149, 150, 202, 396

Fuj imaki, 194, 500

Fulmer, E. I., 164, 406, 469, 524

Funk, C., 16, 20, 21, 26-28, 30-33, 53, $54,79,82,86,113,115,128,299,303$, $304,373,374,383,384,397,398,438$, $462,497,531$

Gargill, S. L., 398

Garlock, B., 414

Garrison, E. A., 464

Garrison, P. E., 502

Gates, F. L., 404

Gebhart, G. C., 399

Gellhorn, E., 352

George, E., 495

Gerstenberger, H. J., 399

Geunes, L. de, 446

Ghose, S. N., 399

Giaja, A., 399

Giaume, C., 399

Gibbons, R., 399

Gibson, R. B., 112, 399

Giddings, M. L., 378

Gillam, A. E., 400

Gillern, K., 400

Gilligan, D. R., 398

Givens, M. H., 184, 187, 188, 190, 195, $196,198,400,430$

Glassmann, B., 400

Glenny, A. T., 400

Gloy, O. H. M., 81, 103, 498 
Godden, W., 430

Godlewski, H., 400

Goebel, F., 400

Goettsch, M., 178, 344, 400, 404

Goldberg, B., 401

Goldberg, S. A., 459

Goldberger, J., 92, 118-123, 130, 132, $137-141,145,401,402,524$

Goldblatt, H., 281, 286, 299, 300, 319, $320,323,402,403,465$

Golding, J., 253, 272, 334, 377, 382, 383, 403,532

Goldschmidt, M., 396

Gooch, M. E., 403

Goodale, H. D., 403, 470

Goodman, H., 403

Gortner, R. A., 403

Goss, H., 185, 403

Gotta, H., 403

Gottlieb, K., 407

Gowen, J. W., 403

Goy, P., 403

Graham, A., 89, 456, 457, 474

Graham, G. E., 403

Graham, M., 532

Gralka, R., 355, 404

Grant, A. H., 404

Grant, J. H. B., 404

Graves, G. W., 363

Grayzel, D. M., 327, 404

Green, D., 357

Green, H. H., 250, 281, 282, 404, 405

Green, H. N., 404, 460

Green, H. S., 486

Green, R., 414

Greenbank, G. R., 347, 405

Greenbaum, F. R., 405

Greenman, M. J., 405

Greenwald, I., 405

Greig, E. D. W., 405

Grettie, D. P., 219, 220, 405

Grey, E. C., 405

Grieves, C. J., 405, 453

Griffith, H. D., 405

Griffith, W. H., 403

Grijns, G., 24, 25, 405, 406

Grimm, R. M., 445

Groebbels, F., 406

Grose, M. R., 106, 107, 406, 498

Gross, E. G., 93, 255, 506, 507

Gross, J., 322, 405, 419

Gross, L., 74, 79, 388, 406

Grüninger, U., 406

Gudjónsson, S., 396, 406

Guerrant, N. B., 63, 64, 109, 123, 124, 143,422

Guerrero, L. E., 286, 406

Guest, A. E., 406

Gugler, M., 406

Guha, B. C., 47, 50, 62, 63, 65, 67, 68, $95,107,109,406,407$

Guillaumin, 446
Guillion, G., 490

Gulick, A., 98, 407

Gulik, P. J. van, 407

Gulland, J. M., 50, 407

Gullickson, T. W., 407

Gunther, L., 459

Gurin, S., 47, 102, 128, 386, 526

Gustavson, R. C., 304, 439

Gutman, M., 533

Gutman, M. B., 407

Gutman, M. G., 297, 416

Guy, R. A., 477

György, P., 396, 407, 509

Haag, J. R., 407

Haan, J. de, 407

Haan, K. de, 406

Haber, E. S., 189, 253, 426

Hager, J., 395

Hahn, F. V. von, 407

Hair, D. B., 462

Hale, W., 408

Hall, J. S., 210, 369

Halliburton, W. D., 408

Halliday, N., 108, 109, 136, 189, 191, $386,408,440$

Halpin, J. C., 160, 208, 377, 410, 411

Hamano, S., 408

Hamburger, R., 408

Hamilton, B., 408

Hamilton, T. S., 408

Handy, J., 259, 383

Hanke, M. T., 168, 408

Hann, R. M., 495

Hanning, F., 507

Hansen, 177,178

Happ, W. M., 408

Hara, M., 471

Hara, S., 408

Haramaki, K., 408

Harden, A., 35, 49, 157-159, 181-184, $198,207,212,293,408,409$

Hardy, Z., 478

Harley, V., 431

Harris, 129

Harris, K. D., 409

Harris, L., 388

Harris, L. J., 409

Harris, R. H., 520

Harris, S., 409

Harrison, D. C., 460

Harrow, B., 398, 409, 436, 438

Harshaw, H. M., 337, 338, 343, 385, 423

Hart, C., 410

Hart, E. B., 112, 160, 198, 208, 210, 286, $295,298,303,326,328,329,333,334$, $343,377,389,410,411,446,506,507$

Hart, K., 412

Hart, M. C., 412, 505

Hartley, J. G., 137, 139, 141 
Hartman, J. I., 399

Hartwell, G. A., 80, 81, 145, 357, 412 Harvey, D., 412

Hashimoto, N., 514

Hassan, A., 80, 122, 145, 413

Hasselbach, K. A., 345, 413

Hastings, E. G., 446

Hatakeyama, T., 500

Hatano, T., 514

Hattori, K., 413

Hauge, S. M., 92, 116, 120, 160, 246, 369,413

Havard, R. E., 413

Haverschmidt, J., 413

Hawk, P. B., 413

Hawking, F., 413

Hays, I. M., 63, 64, 123, 124, 143, 491

Heaton, T. B., 413

Heft, H. L., 386, 387

Hehir, P., 179, 413

Heilbron, I. M., 240, 248, 306, 309, 414, 465

Heiser, V. G., 414

Hejinian, L. M., 378, 414

Heller, V. G., 414, 469

Hellström, H., 390

Helman, F. D., 419

Henderson, J. M., 414, 471

Henderson, J. McK., 414, 415, 437, 471

Hendrick, E. G., 62, 92, 117, 118, 120 , 130,504

Hentschel, H., 415

Herb, F., 415

Hermann, G. R., 495

Hermano, A. T., 415

Hermersdörfer, C., 493

Herter, C. A., 415

Hess, A. F., 14, 151-155, 157, 158, 161 , $164,168,169,171,172,180,183,187$, $188,194,195,207-210,293,297,298$, $300-302,304-307,319,321,322,328$, $329,332,415-420,528$

Hess, J. H., 420

Hess, W. R., 77, 420

Hessler, M. C., 259, 325, 420, 498

Hetler, R. A., 461

Heubner, W., 420

Heudebert, 510

Heuser, G. F., 421, 470

Hey1, F. W., 369, 412, 421

Hickman, J. O., 467

Hift, R., 421

Higgins, G. M., 421

Highet, $H$. C., 421

Hilditch, T. B., 407

Hill, E. L. G., 383

Hill, C. McD., 421

Hill, L., 421, 522

Hill, R., 467

Hindhede, M., 421

Hinshaw, W. R., 517

Hirabayashi, N., 421
Hiraishi, S., 514

Hiraoka, T., 495

Hirsch, A., 421

Hjort, J., 421

Hiavaty, J., 421

Hoagland, R., 87, 139, 421, 422

Hocson, F., 355

Hodes, H., 434

Hodgson, A., 422

Hoeft, G. L., 517

Höjer, A. A., 164-167, 175, 177-179, 422

Hoet, J., 98, 422

Hoffman, C., 422

Hofmeister, F., 35, 36, 104, 105, 422

Hogan, A. G., 124, 337, 338, 343, 422, 423

Holm, E., 277, 396, 423

Holm, G. E., 405

Holmes, A. D., 423, 424, 530

Holst, A., 16, 148-151, 155, 168, 173, $189,197,198,202,203,211,424$

Holst, P. M., 424

Holt, L. E., Jr., 424, 472, 500

Holtz, F., 420

Holz, F., 425

Honeywell, E. M., 203, 311, 315, 416, 425

Honeywell, H. E., 84, 362, 363, 425

Hoobler, R. R., 425

Hooper, C. W., 434

Hope, R. R., 463

Hopkins, F. G., 13, 18, 20, 25, 51, 52, $222,223,230,293,425,426$

Hoppert, C. A., 295, 328, 329, 333, 410 , 411,507

Hornemann, C., 426

Horvath, A. A., 426

Hosack, W., 429

Hosoda, N., 441

Hosoya, S., 444

Hotta, K., 426

Hottinger, A., 426

Hou, H. C., 426

House, M. C., 189, 253, 426

Howard, C. H., 490

Howard, C. P., 359, 426

Howe, P. R., 162, 163, 167, 280, 287, $367,427,529$

Howland, J., 297, 321, 427, 441, 442, 477,500

Hoyle, E., 365, 427

Hoyle, J. C., 413, 427

Hryniewicz, M., 427

Hsu, K. L., 427

Hughes, J. S., 160, 427, 428, 517

Hughes, T. A., 428

Hugounenq, L., 428

Hulce, R. S., 411

Huldschinsky, K., 296, 428

Hulshoff-Pol, D. J., 15, 16, 428

Hume, E. M., 112, 114, 154, 155, 169 , $176,180,192,202,207,208,210,246$, 
$247,258,266,299,300,303,358,370$, $371,373,429$

Humphrey, G. C., 295, 298, 334, 410, 411,507

Humphris, F. A., 429

Hunt, C. H., 62, 92, 126, 130, 429, 430

Hunter, A., 521, 522

Hunter, D., 430

Hunter, J. E., 124, 423

Hunwicke, R. F., 430

Husband, A. D., 430

Hussa, V., 400

Hussey, L. D. A., 182, 456

Huston, R. C., 430

Hutchison, H. S., 430

Hutton, M. K., 159, 378, 379, 477

Ibuki, J., 471

Ide, M., 430

Igarashi, E., 430

Ingier, A., 153, 431

Ingvaldsen, T., 426

Irving, L., 431

Irwin, G. F., 369, 374

Ishido, 431

Iwabuchi, T., 431

Iwasaki, Y., 193, 431

Iwata, M., 431

Izume, S., 431

Jackson, C. M., 431

Jackson, D., 435

Jackson, F. G., 431

Jackson, L., 154, 432, 463

Jacobs, 53

Jacobs, E., 486

James, E. M., 358

Jameson, H. L., 251, 432

Janke, A., 432

Jansen, B. C. P., 42, 44-47, 49, 67-69, 190, 432, 433

Jarussowa, N., 433, 492

Javillier, M., 250, 264, 433

Jendrassik, A., 308, 433

Jenkins, R. G. C., 314, 356, 365

Jephcott, H., 326, 357, 433

Jesus, F. de, 433

Jewesbury, R. C., 433

Jobling, J. W., 433

Johansen, G., 396

Johns, C. O., 394, 434

Johnson, F., 463

Johnson, J.' M., 434

Johnson, O. N., 411

Johnson, R., 386

Johnson, W. C., 327, 533

Johnston, C. G., 435

Johnston, J. A., 526

Jones, D. B., 92, 189, 253, 394, 434, 467,468
Jones, I. R., 434

Jones, J. H., 303, 434, 435, 507, 508

Jones, J. M., 435

Jones, K. K., 511

Jones, M. R., 327, 435

Jones, R. L., 338, 435, 469

Jones, W. S., 241, 435

Jonge, G. W. K. de, 435

Jordan, D., 378

Jorstad, L. H., 367, 435

Jundell, I., 435

Kabeshima, H., 471

Kaczmarek, J., 398

Kagoshima, S., 470

Kajdi, L., 408

Kamm, E. D., 306, 309, 414, 465

Kapsinow, R., 435

Karelitz, S., 435

Karr, W. G., 72, 73, 435

Karrer, P., 390, 435

Karshan, M., 436

Kartascheffsky, E., 436

Kato, G., 436

Kauffman, A. B., 436

Kawakami, K., 235, 250, 436, 468, 514, 515

Kawakita, S., 470

Kawamura, K., 436

Kay, H. D., 177, 436

Keefer, C. S., 360, 436

Keith, M. H., 436

Kelleway, C. H., 436

Kellogg, M., 94, 190, 386

Kelly, F. C., 414, 437

Keményffii, A. G., 308, 433

Kempster, H. L., 244, 245, 422, 476

Kendall, F. E., 160, 463

Kennard, D. C., 360

Kennedy, C., 53, 112, 113, 124, 129, 147, $244,338,385,437,452,476,486$

Kennedy, W. P., 437

Kenny, C. L., 175, 179, 212, 213, 437

Kent, H. E., 229, 255, 506

Keresztesy, J., 386

Kerr, R. W., 386, 437

Kestner, O., 437

Key, K. M., 178, 375

Kick, E. H., 408

Kierferle, F., 209, 437

Kifer, H. B., 437, 467

Kik, M. C., 343, 360, 437, 513

Killian, J., 416

Killick, E. M., 460

Kimm, R., 250, 436

King, C. G., 85, 219, 220, 405, 455, 482, 503

King, H., 438

Kingery, H. M., 438

Kingery, J. R., 438

Kingsbury, A. N., 281, 376 
Kinnersley, H. W., 40, 44-46, 66, 68, 69, $78,81,96,104,107-109,127,131,438$, 478

Kinney, E. M., 328, 453, 500, 501

Kirsch, W., 438, 521

Kitasato, T., 235, 515

Kittelson, J. A., 438

Kiva, F., 514

Klein, A., 438

Klein, G. T., 438

Klein, I. J., 438

Kletzien, S. W. F., 411, 507

Kliesch, J., 443

Kline, O. L., 334, 411

Klocman, L., 449

Klotz, B. H., 376

Knandel, H. C., 462, 505

Knox, C. W., 438

Knudson, A., 310, 313, 317, 438, 439, 458

Knutsen, M. H., 359

Koch, E. M., 304, 308, 439

Koch, F. C., 308, 439

Koch, J., 439

Koch, M. L., 439

Koch, W., 167, 356

Koehne, M., 439

Koessler, K. K., 439

Koga, Y., 439

Kogane, M., 354

Kohls, C. L., 439

Kohman, E., 381

Kohman, E. F., 92, 188-192, 200, 206, $213,386,439,440,442$

Kollmann, M., 362

Koltzsch, W., 494

Komatsubara, I., 431

Komm, E., 440

Kon, S. K., 78, 79, 81, 96, 105, 145, 309, 440

Kondô, M., 440

Korenchevsky, V., 323, 325, 371, 440, 441

Korff, F. A., 501

Koskowski, W., 379, 441

Kozawa, S., 441

Kramár, E., 387, 441

Kramer, 13

Kramer, B., 297, 305, 321, 369, 427, 441, $442,458,497,498,500,525$

Kramer, M. M., 192, 258, 268, 377, 442, 498

Krasnow, F., 409, 436

Kratinowa, K., 475

Krause, D. J., 442

Krauss, W. E., 442

Kreitmair, 388, 442

Krenz, G., 465

Křriženecký, J., 442, 443

Kronacher, C., 443

Kruger, J. H., 333, 385, 443

Kruse, H. D., 62, 136, 443, 452
Kučera, C., 204, 205, 443

Kudrjawzewa, A., 443, 475

Kühl, G., 443

Kugelmass, I. N., 443

Kulp, W. L., 354

Kunde, M. W., 443

Kuperman, T. M., 518

Kuroya, 444

Kusunoki, M., 441

Lacroix, H., 432

Laird, C. N., 121, 444

Laird, D. A., 444

Lake, G. C., 520

Lamb, A. R., 286, 438, 444, 469

Lambert, R. A., 140, 444, 531

La Mer, V. K., 169, 172, 176, 188, 211 $213,444,498,499$

Lane-Claypon, J. E., 444

Lange, L. B., 444

Langen, C. D. de, 444

Langstein, L., 444

Laqueur, E., 444

Larimore, J. W., 445

Larimore, L. D., 476

Larson, J. H., 445

Lasch, F., 445

Lasch, W., 445

Latshaw, W. L., 427

Latzke, A., 445

Laufberger, W., 445

Laurens, H., 445, 459

Lavialle, P., 445

Lavinder, C. H., 445

Lawrow, B. A., 445

Lawrynowicz, A., 427

Lawson, W. E., 520

Lax, H., 445

Leavenworth, C. S., 58, 472

Lecoq, R., 56, 84, 98, 105, 125, 150, 398, $445,482,483$

Lee, A. R., 88, 422

Leersum, E. C. van, 18, 292, 331, 445, 446

Lefèvre, A., 447, 448

Leggate, A. R., 446

Leichtentritt, B., 446

Leigh-Clare, J. L., 324, 331, 446, 505

Lelesz, E., 79, 484

Lemon, H. B., 308, 367, 439

Lepkovsky, S., 82, 159, 160, 208, 392, 411,446

Lepper, E. H., 446

Lesné, E., 446, 447

Leulier, A., 466

Levaditi, C., 447

Levene, P. A., 41, 59-61, 64, 65, 68, 107, $115,116,128,447$

Levine, V. E., 447

Levinsohn, S. H., 447

Levy, A., 398 
Levy-Lageunesse, S., 433

Lewis, H. B., 190, 447, 504

Lewis, J. M., 357, 416

Lewis, R. C., 430, 447

Liang, B., 447

Liégeois, F., 447, 448

Lienhardt, H. F., 160, 427

Light, R. F., 448

Lightbody, H. D., 430

Lillie, R. D., 118-122, 137, 139-141, 401, 402

Lind, J., 14, 448

Lindsay, B., 448

Ling, T. M., 433

Linossier, G., 448

Linsert, O., 312, 528

Liotta, D., 448

Lippmann, A., 448

Lipschitz, W., 448

Little, C. C., 467

Little, J. M., 448

Litwack, I. I., 518

Livingston, C. W., 94, 491

Ljubarskaja, T., 448

Lloyd, J. U., 59, 108, 109, 114, 136

Loeff, J. A. van dy., 406

Lövgren, T., 390

Loew, E. R., 281, 519

Löwy, E., 448

Long, M. L., 89, 456, 457

Looser, E., 448

Lopez-Lomba, J., 98, 175, 448

Lorenz, W. F., 445

Loughlin, R., 253, 367, 378, 439

Lovenskiold, H., 317, 481

Lovern, J. A., 407

Lowndes, J., 95, 480

Lucas, G. H. W., 449

Lucas, N. S., 303, 429, 449

Luce, E. M., 334, 449

Luck, J. M., 234, 368

Luckett, C. L., 455

Luckiesh, M., 449

Luettmerding, A., 204, 449

Lumière, A., 449

Lundagen, M. A., 297, 321, 416

Lunin, N., 17, 449

Luros, G. O., 114, 116, 389

Lush, J. L., 375

Lust, F., 449

Lyman, J. F., 92, 449

McAmis, A. J., 449

McCandlish, A. C., 449

McCann, G. F., 325, 417, 450, 476

McCarrison, R., 70, 71, 74, 82, 85, 450, 451

McCay, C. M., 451

McClendon, G. G., 451

McClendon, J. F., 163, 202, 451

McClosky, W. T., 504
McClugage, H. B., 184, 188, 190, 196, 400

McClurg, N., 114, 378

McCollum, E. L., 488

McCollum, E. V., 13, 19-21, 52-54, 56, $60,65,75,86,92,99,104,107,111-$ $114,117,121,136,223,225,226,228$, $253,267,276-278,287,293-296,300$ $303,314-316,320,327,328,339,353$, $390,410,437,443,447,451-454,456$, $472,477,481,500-502,505,515$

McCordock, H. A., 455

McCormick, L. M., 168, 461

McDonald, F. G., 363

McElroy, C. H., 414

McGowan, J. P., 455

McHargue, J. S., 455

McIntosh, R., 455

McKay, H. M. M., 455

McKenzie, M. R., 442

McKillop, M., 371

McKim, L. H., 55, 114, 389

McKinnis, R. B., 455

McLaughlin, L., 268, 455

McLean, S., 455

McQuarrie, I., 443

McRobert, G. R., 455

Macallum, A. B., 53, 54, 113, 398, 455

MacArthur, C. G., 455

MacArthur, E. H., 103, 455, 499

MacDonald, M. B., 453, 455, 456

Macht, D. I., 302, 355, 456

Mackay, H. M. M., 370, 456

Mackenzie, J., 456

Mackenzie, K. H., 530

Mackenzie, R., 394

Mackie, F. B., 456

Mackintosh, J., 382, 383

Macklin, A. H., 182, 456

Maclean, I., 394

MacLennan, K., 465

Macleod, F. L., 209, 210, 288, 290, 291, 294, 456, 499

MacLeod, G., 206, 456, 488

MacLeod, H. L., 388

MacNair, W. A., 308, 315, 363

MacNeal, W. J., 456, 502

Macomber, D., 287, 456

Macy, I. G., 89, 187, 400, 425, 456, 457, 474

Magee, H. E., 415, 471

Magne, H., 457

Maki, K., 436

Malcolm, J., 457

Mallon, M. G., 457

Mangkoewinoto, R. M. M., 433

Manning, J. R., 457

Manville, I. A., 457

Marchlewski, L., 457

Marcus, J. K., 457

Marlatt, A. L., 188, 198, 372 
Marples, E., 525

Marrian, G. F., 70, 77, 79, 82, 383, 457, 458

Marshall, A. L., 313, 458

Marti, W. C., 517

Martin, A. L., 372

Masaki, T., 458

Maslow, H. L., 458

Mason, K. E., 341, 342, 458

Massengale, O. N., 490

Matheu, C. P., 458

Matsuoka, T., 458

Matsumura, S., 458

Matsushima, S., 440

Mattei, P. di, 458

Mattill, H. A., 82, 337-339, 346, 347, 458,459

Matzko, S. N., 445, 459

Matzner, M. J., 417, 533

Maughan, G. H., 459

Maurer, S., 439, 459

Maurmann, G., 495

Maver, M. B., 459

Mayerson, H. S., 459

Maynard, L. A., 459, 462

Mayzner, M., 440

Mead, S. W., 209, 384

Medes, G., 163, 448, 460, 486, 504

Medlock, O. C., 245, 385

Meeker, D., 408

Meesemaecker, R., 460

Mehta, M. M., 527

Mellanby, E., 250, 281, 282, 293, 329, $404,405,460$

Mellanby, M., 460, 461

Mellion, J., 364

Mendel, L. B., 17, 19, 25, 50-52, 54, 55, $71-73,76,77,80,82,84,86-93,98-100$, $111,113,133,138,140-142,155-158$, $169,222-229,231,253-257,261,267$, 274-276, 278, 359, 363, 372, 376, 439, $449,461,463,472-474,488,516,519$

Menville, L. J., 461

Mercer, E. W., 461

Merjanian, A., 461

Merrill, E. C., 241, 517

Messer, F. C., 74, 376

Messerle, N., 77, 420

Meyer, A. W., 168, 461

Meyer, C. R., 461

Meyer, L. F., 461

Meyerstem, A., 461

Meysenbug, L. von, 380, 461

Michaux, A., 484

Michel, P., 464, 466, 467, 523

Middlekauff, J. E., 202, 451

Mignon, H. L., 462

Miles, L. M., 462

Miller, C. D., 94, 193, 196, 462

Miller, E. G., Jr., 286, 327, 353, 404, 516

Miller, E. H., 232, 499
Miller, E. M., 479

Miller, E. R., 491

Miller, G., 448

Miller, H. G., 462

Miller, R. C., 430, 459, 462

Miller, R. J., 462

Miller, S. P., 462

Miller, W. L., 462

Miller, W. S., 112, 410

Millicer-Szymanska, R., 462

Mills, C. A., 462

Milner, E. W., 93, 206, 245, 268, 48?

Minor, J., 476

Mirvish, L., 463

Mitchell, A. G., 405

Mitchell, H. H., 111, 113, 114, 160, 436, 463

Mitchell, H. S., 463

Miura, M., 218, 263, 532

Miyadera, K., 463

Moeller, O., 434

Moir, M., 472

Moise, T. S., 395

Moll, T., 442

Monasterio, G., 463

Moody, A. M., 154, 432

Moore, B., 28, 387

Moore, C. N., 310, 313, 317, 439

Moore, C. U., 463

Moore, J. J., 154, 420, 432, 463

Moore, J. M., 427

Moore, T., 239, 241, 246, 248, 249, 409, $440,464,527$

Moore, W. B., 424

Morel, A., 464

Morgan, A. F., 191, 252, 284, 363, 464, 465

Morgan, B. G. E., 375

Morgan, R. S., 465

Mori, M., 465

Mori, S., 276, 465

Mori, T., 514

Morinaka, K., 465

Moritz, A. R., 300, 402, 403, 465

Morrison, R. R., 465

Morse, M., 305, 306, 308, 327, 494

Morton, R. A., 240, 241, 247, 248, 306, $309,383,384,400$

Mottram, E. C., 412

Mottram, J. C., 281, 376

Mottram, V. H., 412

Mouriquand, G., 105, 164, 464, 466, 467, 523

Muckenfuss, A. M., 467

Muhlfeld, M., 115, 116, 447

Muller, H. R., 387

Munelusa, T., 467

Munsell, H. E., 91, 92, 205, 256, 264, $279,280,437,467,499$

Murphy, F. J., 495

Murphy, J. C., 92, 434, 467

Murphy, M., 472 
Murray, I., 467

Murray, J. M., 403, 467

Mussehl, F. E., 353, 467

Myers, C. N., 35, 73, 467, 520, 521

Myrbäck, K., 390

Naakrineos, I., 479

Nabarro, D., 467

Nagayama, T., 467, 468

Nagayo, M., 468

Naito, H., 468

Nakahara, W., 468

Nakamiya, J., 235, 515

Nakamiya, Z., 468

Nakamura, K., 516

Nakanichi, H., 471

Narayanan, B. T., 127, 136, 468

Nassau, E., 461

Nehring, 496

Neidig, R. E., 477

Neill, M. H., 521.

Nelson, E. M., 99, 129, 189, 253, 259, $261,262,286,296,298,299,301,304$, $305,323,434,468,469,507,508$

Nelson, M. T., 159, 255, 321, 323, 329, $360,435,446,507$

Nelson, P. M., 189, 253, 426, 469

Nelson, V. E., 164, 286, 337, 338, 354, $357,406,451,469,515,524$

Never, H. E., 469

Neville, A., 53, 426

Newbecker, B. M., 464

Newbigin, H., 472

Newcomb, C., 469

Nichols, N. B., 379

Nightingale, E., 354

Nitcher, C., 427

Nitzescu, I. I., 469

Nobel, E., 469, 470

Nonidez, J. F., 470

Normak, P., 470

Norris, E. R., 240, 242, 243, 470

Norris, L. C., 421, 470

Nourse, J. D., 399

Novaro, P., 470

Obata, J., 413

Odake, S., 29, 31, 514

O'Donovan, W. J., 496

Ogata, C., 470

Ogata, D., 470

Ohdake, S., 470

Ohhashi, Y., 471

Ohler, W. R., 471

Ohomori, K., 471

Ohrbeck, E., 338, 469

Oka, C., 470

Okada, S., 471

Okamato, T., 471

Okamura, T., 440
Okuda, M., 471

Oliveiro, C. J., 489

Olson, T. M., 471

Onohara, K., 471

Orent, E., 442, 498

Orgler, A., 472

Orr, J. B., 389, 471, 472

Orr, W. J., 472

Orr-Ewing, J., 472, 478

Orton, C. R., 472

Osborne, T. B., 13, 17, 19, 25, 37, 39, $50,52,54,55,57-61,63,64,71,72$, $76,80,82,86-93,98,99,111-114,116$, $129,130,133,158,222-227,229,231$, $253-257,259,261,267,274,275,278$, $324,472-474$

Osburn, D. F., 284, 465

Oser, B. L., 474

Osgood, H. S., 479

Ota, I., 471

Otis, F. A., 405

Outhouse, J., 89, 379, 385, 425, 456, 457

Overhoff, J., 249, 529

Oyama, T., 516

Pacini, A. J., 449

Padua, R. G., 474

Page, I. H., 474

Palladin, A., 474, 475

Palmer, L. S., 124, 129, 147, 160, 208, $243-245,338,403,407,434,437,475$, $476,486,514,516$

Palmer, M. G., 375

Pappenheimer, A. M., 293, 297, 298, 303, $318,330,344,417,418,476,499,533$

Park, E. A., 293, 299, 315, 316, 320 , $321,324,453,454,474,477,481,500$, 501

Parkes, A. S., 82, 458, 477

Parkhurst, R. T., 477

Parks, T. B., 406

Parsons, H. T., 92, 111, 158-160, 198, $203,207,208,277,315,316,372,452-$ $454,477,501$

Patel, G. P., 527

Paton, D. N., 394, 477, 478

Paton, J. B., 398

Pattison, C. L., 380, 461, 478

Paul, M. S., 434

Pauly, 35, 44, 46, 68

Payne, L. F., 427

Peachey, G., 393

Peacock, G. E., 160, 389

Peacock, P. R., 465, 478

Pease, R., 387

Peiser, E., 509

Pekelharing, C. A., 18, 478

Pénau, H., 478

Perlmann, S., 478

Perry, E. O. V., 272, 377 
Peskett, G. L., 478

Peter, A. M., 366

Peters, R. A., 39, 40, 44-46, 50, 66, 69, $78,81,96,104,107-109,123,126$, $127,130,131,143,147,407,438$, 478

Petersen, H. A., 478

Peterson, W., 433

Peterson, W. H., 372

Petrov, I., 443

Pettersson, A., 390

Pfannensteil, W., 478, 479

Pfaundler, M., 479

Pfund, A. H., 479

Phemister, D. B., 479

Phillips, R. A., 369, 374

Pico Estrada, 479

Pierce, H. B., 479

Pierson, E. M., 197, 207, 385, 479

Pigott, M. G., 424, 530

Pilcher, J. D., 77, 479

Pilley, V. E., 479

Pincussen, L., 479

Pine, L., 438

Pitz, W., 75, 114, 225-227, 452, 454, 479

Platon, J. B., 480

Plimmer, R. H. A., 90, 95, 160, 480

Plimmer, V. G., 480

Plummer, B. A., 74, 480, 504

Plummer, N., 376

Po, L. Y., 447

Pohl, R., 306, 480

Poklop, J., 398

Polansky, J. B., 479, 480

Pollack, H. J., 495

Pomerene, E., 359, 480

Popoviciu, G., 469

Portier, P., 480, 481

Portman, K., 481

Posdeew, A., 400

Poulsson, E., 317, 481

Powers, G. F., 299, 477, 481

Powick, W. C., 481

Prange, R. WV., 369, 413

Pratt, A. D., 459

Price, E. A., 239, 240, 368, 372

Price, H. W., 517

Pringle, E. F., 481

Pringle, H., 481

Prinke, G., 481

Priston, J. L., 186, 481

Pritchard, E., 481

Proud, J. IW., 461

Puckner, W. A., 481

Putzig, N., 481

Pycha, R. L., 428

Pyle, S. I., 379

Quinn, E. J., 85, 93, 137, 206, 232, 245, $246,268,373,482,499$
Rabl, C. R. H., 482

Raczynski, J., 296, 482

Ragins, I. K., 308, 439

Randoin, L., 56, 71, 79, 84, 95, 98, 105, $125,150,175,448,481-484,502$

Rapaport, M., 434

Rathery, F., 381

Ray, L. A., 486

Raymond, W. H., 480

Read, J. W., 484, 513

Reader, V., 46, 62, 80, 96, 130, 438, $472,478,484,485$

Redenbaugh, H. E., 79, 393, 485

Redman, T., 485

Reed, C. I., 485

Reed, H. S., 485

Reerink, E. H., 312, 485, 519

Reiss, M., 495

Remy, E., 485

Reschke, J., 493

Reyher, P., 485

Reynolds, E., 287, 485

Reynolds, M. S., 159, 208, 477

Rhinhold, J. G., 514

Rhodes, M., 187, 193, 371

Richards, M. B., 430

Richardson, A. E., 486

Richardson, J. E., 486

Richer, M., 387

Richmond, H. D., 486

Riddell, W. H., 427

Riddle, A. R., 486

Riesenfeld, E. A., 486

Rigobello, G., 486

Riising, B. M., 507

Ringrose, A. J., 470

Rivera, T., 373

Rivkin, H., 322, 416, 417, 419

Robb, E. F., 163, 486

Roberts, L. R., 486

Robertson, E. C., 486

Robertson, G. S., 472

Robertson, T. B., 486

Robison, R., 184, 408, 486, 487

Robison, W. L., 364, 365

Roche, J., 77, 487

Roehm, R. R., 526

Röhmann, F., 487

Roelli, P., 487

Röslo, B., 487

Roger, H., 487

Rogers, E. C., 420, 487

Rogers, L. M., 118, 119, 137, 139, $40 \dot{z}$

Rohr, K., 77, 420, 487

Rominger, E., 386

Rondoni, P., 487

Roper, J. C., 387

Roscoe, M. H., 45, 72, 84, 90, 102-104, $122,125,126,128,130,132,133,136-$ $138,140,142-145,323,325,329,330$, $333,356,371,487,488$

Rose, M. S., 488 
Rose, W. B., $81,376,488,510$

Rosedale, J. L., 160, 480, 488, 489

Rosenau, M. J., 489

Rosenheim, O., 238-240, 244, 302, 304$307,309,312,327,353,383,438$, 489

Roszkowski, A., 415

Rothlin, E., 489

Rothrock, H. A., 322, 323, 384

Rousseau, E., 489, 490

Rousseau, S., 433

Roussel, G., 490

Rowlands, M. I., 490

Rowlands, M. J., 490

Rowles, E. K., 490

Roy, C. B., 197, 501

Roy, W. R., 455

Rubino, P., 373, 490

Russell, G. R., 399

Russell, W. C., 490

Rutherford, W. J., 490

Rydbom, M., 249, 390

Sah, P. P. T., 503

Sahai, P. N., 428

Sahashi, Y., 49, 50, 490

Saiki, S., 194, 500

Sakurai, E., 471

Saleck, W., 490

Saleeby, N. M., 491, 526

Salmon, W. D., 63, 64, 84, 92, 94, 99, $122-124,131,143,491$

Sammartino, U., 491

Samson, K., 491

Sandels, M. R., 72, 85, 105, 108, 132, $135,144,491,499$

Sandwith, F. M., 491

Santesson, C. G., 364

Santos, F. O., 94, 491

Santos, S., 491

Sanyas, R., 467

Sassaman, H. L., 360

Saunders, E. B., 492

Savazzini, L. A., 377

Schabad, J. A., 492

Schaefer, O. G., 209, 384

Schäper, W., 443

Schaker, B., 493

Scharf, A., 395

Scharlau, B., 479

Schaumann, H., 28, 104, 352, 492

Scheer, K., 492

Schepilewskaja, N. E., 492

Scheunert, A., 189, 193, 196, 197, 201, 206, 492, 493

Schieblich, M., 493

Schilling, S. J., 83, 513

Schimizu, K., 494

Schirmann, M. A., 400

Schloss, E., 494

Schlutz, F.' W., 305, 306, 308, 327, 494
Schmidt, L., 77, 352

Schmidt-Nielsen, S., 330, 494

Schmidt-Weyland, P., 494

Schmitz, E., 495

Schoedel, J., 495

Schoen, J., 466

Schönborn, E. von, 79, 398

Schoubye, B. N., 495

Schoubye, N., 396

Schuck, C., 451

Schultz, O., 436, 487

Schultzer, P., 495

Schulz, O., 495

Schwanebeck, E., 493

Schwartz, E., 395

Schwartze, E. W., 495

Scott, H., 298, 334, 410, 411

Scott, H. T., 507

Scott, L. C., 391, 495, 523

Scotti-Foglieni, L., 495

Seaman, E. C., 496

Sebrell, W. H., 402, 496

Sédallian, P., 466

Sedgwick, J. P., 451

Seel, H., 496

Segawa, W., 496

Seidell, A., 32, 34-39, 41, 42, 47, 48, 55$57,59,60,118,119,121,360,496,526$

Sekine, H., 496

Selkirk, T. K., 405

Sell, M. T., 99, 129, 245, 278, 508

Selter, H., 496

Senior, B. J., 497

Sequeira, J. H., 496

Sexton, W. A., 414, 497

Seymour, R. J., 363

Shackleton, 182

Sharpe, J. S., 394, 497

Shattuck, G. C., 497

Shaw, H. F., 456

Shear, M. J., 305, 441, 442, 497

Sheard, C., 421, 497

Sheehy, E. J., 497

Sheer, K., 497

Sheets, O., 299, 497

Shelling, D. H., 442, 458

Shelow, E., 387

Sherman, E., 417, 419

Sherman, H. C., 72, 86, 89-92, 100, 105, $123,125,132,133,135,144,145,169$, $172,176,179,188,211-213,232,255$, $256,258,259,263,264,270,279,280$, $284,285,288-291,293,294,305,318$, $323,325,330,444,498,499$

Sherman, H. E., 499

Shibayama, G., 500

Shimamura, T., 29, 31, 514

Shimizu, T., 500

Shinoda, 77, 194, 500

Shipley, P. G., 293, 294, 300, 315, 316, $320,328,369,424,453,454,477,481$, 500,501 
Shipp, H. L., 501

Shizume, S., 436

Shohl, A. T., 435, 501

Shorten, J. A., 197, 501

Shrader, J. H., 501

Shrewsbury, C. L., 423

Shriner, R. L., 307, 355

Shrivastava, D. L., 428

Shuck, C., 451

Shulman, D. N., 502

Shurley, B. R., 502

Siler, J. F., 502

Silvestre de Sacy, G., 354

Simmonds, N., 56, 62, 75, 92, 104, 107, $111-114,117,225-227,277,293,294$, $298,299,315,316,320,447,452-454$, $477,481,500-502$

Simonik, F., 204, 502

Simonnet, H., 71, 98, 332, 393, 457, 478, $484,502,503$

Simpson, G., 28, 387

Sipple, H. L., 220, 503

Sjollema, B., 326, 503

Skarzynska-Gutowska, M., 503

Skelton, R. F., 371, 380

Slanetz, E. J., 503

Slonaker, J. R., 503

Smedley-MacLean, I., 246, 247, 373, 429

Smith, A. A., 439

Smith, A. H., 99, 281, 355, 376, 395, 503, 519

Smith, A. Henderson, 180, 503

Smith, C. A., 413

Smith, D. T., 503

Smith, D. W., 210, 411

Smith, E. A., 409, 504

Smith, E. L., 357

Smith, H. H., 246, 258, 266, 303, 370, $373,429,504$

Smith, L. L. W., 252, 465

Smith, L. M., 504

Smith, L. W., 424, 530

Smith, M. E., 81, 513

Smith, M. I., 48, 92, 105, 117, 118, 120 , 130,504

Smith, S. L., 504

Smith, Th., 148, 504

Snider, G. S., 139, 422

Soames, K. M., 299, 320, 323, 324, 334, $403,446,487,504,505$

Sobel, J., 505

Sollmann, T., 77, 479

Somekawa, E., 468

Somers, P. P., 517

Sopp, E., 505

Souba, A. J., 505

Southgate, H. W., 303, 505

Souza, G. de P., 505

Sovy, J. W., 445

Speer, J. H., 369, 421, 505

Spence, K. C., 405

Spindler, L. A., 353
Spinka, J., 505

Spohn, A., 72, 89, 94, 100, 125, 133, 499

Sprawson, C. A., 505

Spring, F. S., 240, 414, 465

Spruyt, J. P., 505

Squires, B. T., 438

Ssawron, E., 475

Stammers, A. D., 505

Stanton, A. T., 26, 27, 113, 395, 396

Stanton, R. E., 511

Stearns, G., 378

Steel, G. E., 362

Steenbock, H., 92, 93, 99, 114, 129, 160 , $198,203,208,210,227-229,231,243$, $245,255,259,261,262,278,286,287$, $295,296,298-305,309,316,317,320$, $321,323,328,329,332-334,341,343$, $344,360,389,395,410,411,425,435$, $440,454,468,469,506-508,521$

Stefánsson, V., 208, 509

Steffenburg, S., 390

Stegeman, D. E., 404

Steidle, H., 508

Steiner, J. M., 418

Stephenson, H. D., 465

Stephenson, M., 244, 275, 508

Stepp, W., 221, 222, 508, 509

Stern, R. O., 509

Steudel, H., 241, 509

Stevenson, A. G., 509

Stevenson, H. C., 386

Stevenson, I., 198, 372

Stewart, C. A., 431, 509

Stewart, C. P., 409

Stiebeling, H. K., 139, 147, 259, 318, $323,330,499,509$

Still, G. F., 181, 409, 532

Stockard, C. R., 510

Stockbarger, D. C., 530

Stockholm, M., 389

Stoeltzner, W., 510

Stolk, D. van, 510

Stone, N. C., 459

Storm von Leeuwen, W., 510

Storms, L. B., 279, 280, 499, 510

Strandberg, O., 510

Stringfield, O. L., 381

Strong, R. P., 510

Strote, H., 510

Stuart, H. O., 510

Stucky, C. J., 81, 376, 488, 510

Suda, G., 510

Sudholt, H., 510

Sugiura, K., 111, 160, 510, 511

Sullivan, M. X., 511, 521

Sumi, M., 511

Summerfeldt, P., 517

Sunderlin, G., 511

Supplee, G. C., 209, 330, 334, 417, 418, 511

Sure, B., 81, 83, 337, 338, 340, 341, 343 , $484,511-513$ 
Surie, E., 460, 527

Suski, P. M., 513

Suzuki, K., 514

Suzuki, T., 514

Suzuki, U., 29-32, 39, 49, 514

Suyenaga, B., 404

Svensson, B., 525

Swartz, O., 391

Sweetman, M. D., 514

Swingle, W. W., 514

Swoboda, F. K., 514

Sydenstricker, E., 401, 402, 514

Szarka, A., 514

Tagaya, T., 430, 467, 468

Taguchi, K., 514

Takahashi, W., 235, 237, 248, 420, 514, 515

Takaki, K., 14, 25, 515

Talbot, F. B., 515

Tange, U., 515

Tanner, W. F., 119, 138, 401, 515

Tanret, G., 478, 502, 503

Tasawa, R., 515

Taylor, J., 515

Taylor, M. W., 338, 469, 515

Taylor, N. B., 515

Tazawa, R., 515

Tazelaar, M., 371

Teding van Berkhout, 515

Telfer, S. V., 516

Telleria, T., 393

Tern-uchi, Y., 516

Teut, E. C., 334, 411

Thévenon, L., 464

Tholin, T., 516

Thomas B., 507

Thomas, B. H., 506

Thomas, D. R., 516

Thomas, T. H., 516

Thompson, H. B., 516

Thompson, T. J., 516

Thorup, D. W., 516

Thunberg, T., 77, 516

Thurman, B. H., 205, 516

Thurston, L. M., 160, 208, 516

Tilden, E. B., 286, 516, 517

Tilt, J., 94

Tisdall, F. F., 366, 517

Titus, R. W., 427, 517

Tixier, G., 517

Tobler, W., 517

Tolstoi, E., 319, 420

Tonney, F. O., 517

Topper, A., 396

Tourtelotte, D., 412

Toverud, G., 163, 165, 517

Towle, E. C., 241, 517

Toyoda, T., 517

Tozer, F. H. W. P., 461

Tozer, F. M., 162, 518
Trail, R. K., 295, 364

Trevan, J., 365

Trost, J. F., 246, 413

Tscherkes, L., 518

Tscherkes, L. A., 80, 518

Tso, E., 88, 329, 426, 518

Tsuji, M., 518

Turner, R. G., 281, 502, 518, 519

Tyson, M. D., 281, 519

Uhlmann, F., 519

Uicodiévitch, 467

Underhill, F. P., 119, 138, 140, 141, 371,519

Underhill, S. W. F., 519

Ungar, A., 519

Unger, L. J., 157, 162, 169, 183, 187, $188,194,209,212,293,297,298,321$, $328,417,418$

Urechia, C. I., 519

Utewski, A., 475

Uyei, U., 519

Vack, C., 525

Vagliano, M., 446, 447, 487, 529

Vahlteich, H. W., 205, 516

Van Eckelen, M., 249, 529

Van der Hoeven, B. J. C., 41, 59-61, 67,447

Van' Hoogenhuyze, C. J. C., 388

Van Horne, E. G., 190, 400

Van Leent, J. B., 519

Van Slyke, 341

Van Wijk, A., 311, 312, 485, 519

Varela, B., 373

Vasarhelyi, B., 519

Vedder, E. B., 16, 26, 30, 31, 105, 112, $369,370,519,520$

Verder, E., 520

Verzár, F., 510, 520

Vickery, H. B., 84, 461

Viswalingam, A., 520

Voegtlin, C., 34, 35, 73, 439, 467, 511, 520,521

Vogt, E., 521

Vogt, H., 521

Voit, C., 221, 521

Völker, H., 448

Vollmer, H., 521

Völtz, W., 521

Von der Ähe, F. H., 526

Wachtel, M., 521

Wacker, L., 447

Wada, C., 516

Waddell, J., 341, 343, 344, 521

Waggener, R. A., 385

Wagner, R., 470, 521

Wakeman, A. J., 37, 57-61, 63, 64, 112, $114,116,129,130,474$ 
Walker, D. J., 343, 513

Walker, S., 522

Walkhoff, E., 485

Walkhoff, O., 485

Walle, N. van der, 522

Wallis, R. L. M., 522

Walshe, F. M. R., 522

Wang, C. C., 277, 364, 420

Waring, C. H., 401

Waring, J. I., 118, 522

Warner, M. E., 526

Washburn, M., 497

Wason, I. M., 276, 504, 522

Watchorn, E., 440, 522

Waterman, E. W., 522

Waterman, R. E., 41, 47, 56, 62, 69, $98,102,113,125,128,526$

Waters, H. J., 522

Watkins, L., 387

Watson, A., 477

Watson, A. F., 238, 272, 273, 383

Watson, C., 522

Watson, R. V., 522

Weatherby, L. S., 522

Weber, W., 478

Webster, A., 28, 387

Webster, L. T., 503

Webster, T. A., 239, 302, 304-307, 309, $310,312,327,365,438,489,522,523$

Wedgewood, P. E., 177, 523

Weech, A. A., 523

Weed, K. L., 501

Weideman, G., 523

Weill, E., 105, 523

Weinstock, M., 297, 302, 304, 306, 319, $322, \quad 332, \quad 416-420$

Weiss, R., 381

Wellman, C., 523

Wells, A. H., 523

Wells, C. A., 523

Wells, F. M., 162, 164, 165, 524, 532

Wells, H. G., 524

Wenckebach, K. F., 524

Wendt, G. von, 524

Werkman, C. H., 154, 164, 511, 524

Wertheimer, E., 352

Wesselow, O. L. V. de, 524

West, J. H., 524

Westin, G., 166, 423

Weymuller, C. A., 530

Whalen, F. B., 137, 146, 482

Wheeler, G. A., 118, 119, 137, 139, 401, 402,524

Wheeler, R., 524

White, G. F., 34, 521

Whitehead, R. W., 358, 525

IVhitsitt, M. L., 108, 499

Widell, H., 391

Widmark, E., 525

Wierzchowski, Z., 457, 525

Wijk, A. van, 312,485

Wilbur, J. W., 354
Wilder, J. S., 525

Wilgus, H. S., Jr., 470

Wilkes, E. T., 525

Wilkins, I., 525

Wilkins, L., 472

Wilkins, S. B., 385

Willcox, W. H., 179, 525

Willets, D. G., 118, 401

Williams, C. T., 525

Williams, I., 525

Williams, L. A., 443

Williams, R. E., 442

Williams, R. J., 525, 526

Williams, R. R., 30, 31, 33-35, 37, 41, $47,49,56,62,65,69,98,102,112$, $113,118,125,128,131,370,386,437$, 520,526

Williams, Z., 268, 420

Willimott, S. G., 186, 239, 245, 464, 485, $526,527,529$

Wills, L., 527

Wilson, D. C., 527

Wilson, J. L., 526

Wilson, J. N., 252, 527

Wilson, M. G., 528

Wilson, W. H., 528

Wiltshire, H. W., 202, 528

Wimberger, H., 521, 528

Windaus, A., 305, 306, 312, 420, 528

Winfield, G., 528

Winter, A. R., 430

Wise, E. C., 421, 505

Wiskott, A., 528

Witham, L., 428

Woenckhaus, E., 528

Wohlwend, I., 462

Wokes, F., 186, 239, 242, 485, 527-529

Wolbach, S. B., 167, 280, 287, 529

Wolff, L. K., 249, 444, 529

Wollman, E., 529

Wood, C., 82, 446

Wood, W. F., Jr., 529

Woollard, H. H., 78, 457, 529

Woringer, P., 529

Wright, A. M., 529

Wright, O. K., 530

Wright, S., 87, 465, 530

Wu, D. Y., 530

Wu, H., 530

Wurzinger, S., 530

Wyard, S., 530

Wyman, E. T., 424, 530

Wyss, W. H. de, 530

Yang, C. S., 360

Yang, F. H., 530

Yaoi, H., 530

Yates, W. W., 462

Yee, M. A., 365

Yoder, L., 530

Yokoyama, Y., 468 
Yoshimaru, Y., 431

Yoshiue, S., 531

Young, C. H., 531

Youtz, J. E., 522

Yudkin, A. M., 444, 531

Zäch, C., 531

Zajdel, R., 531

Zall, C., 179, 377

Zeiler, K., 209, 437

Ziegler, M. R., 494

Zielaskowski, M., 446
Zih, A., 531

Zilva, S. S., 35, 49, 157-159, 162-165,

$177,181-183,190,198,207,212-218$,

$227,230,253,258,263,270,272,281$,

$334,359,365,373,377,379,382,383$,

$403,408,409,427,436,446,501,531$, 532

Zinzalian, G., 360

Zoelch, F., 533

Zoeller, E., 415

Zucker, T. F., 303, 304, 326, 327, 329, 476,533

Zuwerkalow, D., 475 


\section{SUBJECT INDEX}

"A" vitamin. See Vitamin A

Abscesses, 279, 280, 282. See also Cavities, Cysts

Absorption band of vitamin A, 240-249, 489

Absorption of alveolar processes of teeth, 162

Absorption spectrum, effect of time of irradiation on, 311,312

of cholesterol, 306, 308, 414, 439

of ergosterol, 306, 307

of vitamin D, 414, 480

use of, in detection of ergosterol in cholesterol, 305

Accessory substances, 25. See also each Vitamin

Acetic acid, 47, 62, 108, 304, 447. See also Vitamin $\mathrm{B}$, concentration of

Acetone, 129, 305, 328, 345. See also Vitamins $B$ and $G$, concentration of

Acetyl chloride, 234

Acetylation, 237

Acid. See name of each

Acid-base balance of diet in production of rickets, 501,533

Acid clay, 42

Acid, effect of, on vitamins. See under each Vitamin, chemical properties, stability

Acidification of alcohol in extraction of vitamins $B$ and $G, 136$

Acids. See name of each; see also Amino acids

Activated fullers' earth, 32, 37, 47. See also Vitamin B, concentration of

Activation, antirachitic, 507. See also Irradiation; Vitamin D

Activity, hydrogen-ion, as affecting stability of vitamins, $59,63,64,66$, $69,106,107,109,124,126,127$, $128,136,148,149,181,183,188$, $211-213,216,233-234,444,483$, $485,491,495,498,501,530$. See also descriptions of Vitamins $\mathrm{B}$, $\mathrm{C}$ and $\mathrm{G}$

Adenine, 32, 34, 35, 37, 128, 408

Adjustment, period of, in measurement of vitamin values, 132,135

Adrenaline, 43, 406, 495

Adrenals, $70,160,163,329,361,381$, $450,457,479$
Adsorbent, relation of amount of, to adsorption of vitamin B, 109, 409 of vitamin G, 136

Adsorbents. See name of each; also Adsorption, and under each Vitamin

Adsorption, 26, 109, 114, 122, 127, 531 of vitamin B, 26, 109, 110

of vitamin G, 127

See also names of adsorbents and Vitamins $\mathrm{B}, \mathrm{C}$ and $\mathrm{G}$

Aeration, 106, 229-238, 531. See also Oxidation

Agar, 54, 56, 225, 316, 320, 463

Age, as influencing vitamin A content of body, 254

of vegetables as affecting vitamin C values, 194, 195, 196

See also each Vitamin, relation to nutrition

Agglutinins, 524, 531

Aging of food in relation to vitamin values. See each Vitamin; also names of individual foods

Air, apparently deleterious in extraction of vitamin by alcohol, 136

See also Aeration, Oxidation

Alcohol, as solvent for ergosterol, 312

extraction of vitamin $A$ value from plant tissues, 227-228

for concentration of vitamin D, 304

in extraction of vitamin D, 328

purification of casein by, 100

solubility of vitamins in, 20 ; and under properties of each Vitamin

See also Vitamin B, concentration of Alcohol-petroleum ether mixture, 227228

Alcohol-water-benzene mixture, 113

Alfalfa, 169, 225, 227, 232, 234, 268, 295, $320,323,324,328,340,379,385$, 506

Algæ, 251, 446

Alimentary tract, 142, 144

Alkali, effects of, on vitamins. See description of each Vitamin

Alkalinized orange juice, 183

Alkaloid, 26, 109

Alkylamines, 68

Allantoin, 284, 353

Alligator pear. See Avocado

Allophanates of certain sterols, 515

Almond oil, 253, 270, 464 
Almonds, 94, 95, 149, 269, 464, 488

Alopecia, 143. See also Dermatitis; and Vitamin $\mathrm{G}$ deficiency

$\alpha$-crocetin, 246

$\alpha$-hydroxypyridine, $33,34,35,49,408$

Amboceptor, 531

Amines, $40,44,57$

Amino acids, 26, 58, 286, 472

nitrogen, 62 , 126-127

in relation to vitamin $G, 126-127$

Ammonia, 234, 304

liquid, for concentration of vitamin D, 304

Amyl alcohol, 53. See also Vitamin B, concentration of

Anemia, 360, 408, 439, 513, 527

Anhydremia in vitamin B deficiency, 81, 513

Anhydrous sodium sulfate, use of, for drying solution of vitamin $A$, 236

Animal fats, 229, 271. See also name of each

Animals, experimental, 258. See also description of each Vitamin, and of methods of determination or measurement

Anorexia, 512. See also Appetite

Antiberiberi factor and torulin, 45. See also Vitamin B

Anti-infective agent or action, 281, 282. See also Infection

Antimony trichloride reagent, 239-249, $392,413,435,470,494,529$

Antineuritic (factor, substance or vitamin), 15, 16, 20, 24-31, 37, 42-44, $47-50,69,104-105,131$

chemical nature of, $26,27,48-50$

concentrate, $27,31,42,43,47,131$

crystallization of, 27

from various sources, $27,28,29,30$, 31

hydrochloride of, $42,43,44,47$

isomeric changes, 49

See also Vitamin B

"Antineuritin," 36, 422

Antiophthalmic, 20. Sec also Vitamin A

Antipellagric factor, 131. See also Vitamin G

Antirachitic, 238, 257. See also Vita$\min \mathrm{D}$

activation of cholesterol, 308

potency, 311, 332-335

Antiscorbutic, 20, 150, 169, 170, 175, 184, 417. See also Vitamin C

"Antisterility" vitamin. Sce Vitamin E

Appetite, 71-73, 85, 142, 143, 278. See also Vitamin $\mathrm{B}$

Apples, 94, 148, 189-191, 200, 269, 365, $400,420,440$

Apricots, 189
$A / R$ ratio in measurement of vitamin D, 323, 324

Arachis (peanut) oil, 270

as solvent for ergosterol, 312

Arctic expeditions, scurvy in, 181

Arginine, 26, 284

Army medical commission, 16, 26

Arsenic trichloride reaction, 238-240

Arthritis, 143

Artichoke, 268, 465

Artificial food mixtures, 221-224

Asparagine, 26

Asparagus, 93, 196, 197, 245-246, 377

Assimilation. See Metabolism

Atony, gastric and intestinal, 74, 75

Atrophy, 70, 282. See also under each Vitamin deficiency

Atta, 180, 405

Autoclaved bakers' yeast, 100, 102 brewers' yeast, 105

milk, 162,163

Autolyzed yeast, 32, 35. Sec also Vita$\mathrm{min} \mathrm{B}$, concentration of

Autopsies, 286

in vitamin $\mathrm{C}$ deficiency, 171-175

score, 174,175

Avitaminosis, 81, 449, 500, 510, 513, 514, $515,518,531$. Sec also under each Vitamin deficiency

Avocado (alligator pear), 94, 269, 381

Aykroyd-Roscoe unit of vitamin G, 137

B avitaminosis, 445. Sec also Vitamin B deficiency

"B" vitamin. Sec Vitamin B

"B," vitamin. See Vitamin B

"B. vitamin. See Vitamin G

Bacillus coli, 282

Bacteria, 154, 281, 377, 378, 398, 414, 519. See also Infection

as source of vitamin $\mathrm{B}, 83,84$

susceptibility to, on diets deficient in vitamins. See individual Vitamins

Bacteriolysins, 524

Balance experiments in measurement of Vitamin D, 326

Banana, 94, 148, 178, 190, 269, 340, 386, $400,406,432,447,505,510,511$

Bands, spectrographic, 237-249

Barcelona nuts, 269

Barium acetate. See Vitamin B, concentration of

Barium hydroxide, 127, 128 . See also Vitamin $B$, concentration of

Barium sulfide, 39

Barley, 55, 90, 92, 155, 156, 203, 204, $269,409,473,505,507$

Barlow's disease, 390, 431. See also Scurvy

Bases. See names of individual bases 
Basal diets. See methods of testing for individual vitamins

Basic lead acetate, 28. See also Vitamin $\mathrm{B}$, concentration of

Beans, 15, 24, 56, 73, 92, 93, 95, 178, $199,202,203,205,206,245-246$, $268,269,337,452,528$

dried, 401

germinated, Vitamin C value of, 202

haricot, 95

mung, 414

red kidney, 356

soy, 92,95

string, 93, 178, 199, 205, 206, 245-246, 268

velvet, 513

white, 454

Beef, 86, 87, 119, 120, 130, 138, 139, 207, $270,385,402,422,460$

extract, 139,422

fat, $17,253,271$

Beer, 158, 409, 493, 503

Beets, 93, 269, 493

Behavior of vitamins upon heating at different hydrogen-ion concentrations. See description of individual Vitamins

Benedict's alkaline copper reagent, 234

Benedict test, 46

Benzene, 31, 345, 526

alcohol-water mixture, 113

as solvent for ergosterol, 312

as solvent for vitamins. See description of individual Vitamins

effect on Vitamin D, 305

Benzoic acid, 62, 108

Benzoyl chloride, 42

substituted, 237

Benzoylation, 237

Beriberi, 14, 15, 16, 20, 24, 25, 81, 85, $162,354,355,356,358,368,369$, $370,379,393,394,395,397,398$, $399,405,406,407,413,414,421$, $424,426,428,435,440,441,442$, $446,448,451,456,458,468,469$, $471,480,491,492,495,496,497$, $500,505,508,510,513,514,516$, $519,520,522,523,526,529$

compared with inanition, 77

dry, 81

eradication from Philippine Scouts, 26

in British troops in Mesopotamia, 179

in Japanese navy, 25

prevention of, 14-17

relationship to other deficiency diswet, 81 eases, 148,149

"Beriberi vitamine," 26. See also Vita$\min B$

Berkfeld candle, 183

Berries, 149, 189 $\beta$-hydroxypyridine, 34,37

$\beta$-methylpyridine, 37

Betaine, 32, 36, 128

ring, 34

Bile, vitamins in, 467

Biological assays. See Feeding methods methods. See Feeding methods; see also under each Vitamin

Bios, 22, 95, 127, 386, 430, 437, 449, 455, $462,468,478,515,526$

Biosterin, 235-238, 431, 468, 515

Biosterol, 234-238

Birds, polished rice disease in, 470

Bismuth iodide, 43

potassium iodide, 35,36 . See also Vitamin B, concentration of

Bisulfite, 234

Black-tongue, 119, 380, 402, 524

Bladder, 163

stones, experimental production of 451

Blood, 81, 86, 142, 143, 145, 207, 271, $281,297,321,322,373,377,394$, $399,416,446,497,502,506,510$, $513,516,517,524$

composition of, in rickets, 321, 322

composition of, in Vitamin B deficiency, 81

phosphate, 416

plasma, 420

platelets, 359, 376, 393

serum of, in rickets, 297

sugar, 388

ureal, 364

Body stores of vitamins, 130-131, 134. See also Vitamin A; Vitamin B; Vitamin C; Vitamin D; Vitamin E; Vitamin G

vitamin $A$ in, 270

"Bondols," 43

Bone, 354, 367, 378, 384, 425, 427, 436, $442,443,477,478,497,499,500$, $501,517,518,527$

ash, 384

calcification, 425,436

changes. See Rickets and Scurvy

composition, in measurement of vita$\min \mathrm{D}, 323$

decalcification, 427

development, $367,378,478$

lesions, need for antiscorbutic vitamin in cure of, 167

marrow, 163, 164, 166, 394, 431

Boron, 216

Bottom yeast, 57

Bourquin method for measurement of vitamin $G$ values, $133-135$

Bowel function, derangement of, in deficiency disease. See Vitamin B, deficiency of; Vitamin C, deficiency of

Brain, $28,70,86,146,475$ 
Bran, 90, 157, 163, 164, 169, 175, 269, 321, 505. Sec also Rice; Wheat

Brazil nuts, 94, 269

Bread, 158, 180, 269, 376, 408, 412, 493 brown, 412

white, 412

yeast, 493

Breadstuffs, 89, 90. See also Bread

Brewer's yeast, 169, 175

British Committee Report, 425, 426

British troops, beriberi in, 179

Bromine, 27, 306

Bromocresol purple, 127

Bronchi, 280. See also Vitamin A deficiency

Brown rice, 55

Brussels sprouts, 201, 268, 328

Buckwheat, 90

nutritive value of, 475

Burbot liver oil, vitamin $D$ in, 372

Butterfat, 17, 21, 52, 54, 56, 100, 119, $130,138,140,169,170,175,223$, $224,226,228,229,234,250,253$, $272,273,274,287,291,293,294$, $295,316,320,328,329,333,335$, $340,341,354,374,382,383,394$, $398,402,424,435,452,453,472$, $474,492,497,510,512,516,531$

antirachitic potency of, on irradiation, 333

as source of vitamin D, 329

vitamin $\mathrm{D}$ in, $328,329,335$

vitamin $\mathrm{D}$ in alcohol extract of, 328

Butternuts, 269

Butyl alcohol, 128, 238

"C" vitamin. Sec Vitamin C

Cabbage, 53, 148, 149, 151, 156, 192, 197 , $198,199,200,201,212,218,225$, $227,238,246-247,250,251,268$, $321,328,380,387,424,439,518$, 531

juice, 212

Cadmium chloride, 43, 44, 62. See also Vitamin B, concentration of

Cage for experimental animals, 444

Calcification, 436, 460, 482, 498, 500, 524. See also Bone, Rickets and Scurvy

Calcium, 286, 293-296, 298, 301, 303, $322,328,334,357,359,364,378$, $380,393-394,407,409,410,412$, $414,417,419,427-428,430-431$, $441-442,450-451,456,459,463$, $466-467,472,477,479,486,492$, $495,499,501,503,506-507,509$, $516-518,522,524,530$

carbonate, 181

lactate, 169

metabolism, 328, 334, 359, 380, 394, $414,417,441,451,463,467,472$,
Calcium (continued)

metabolism (continued)

$477,486,495,501,503,506-507$, 516, 522. See also Rickets, Scurvy, Vitamin C, Vitamin D soaps, 304

Calculi, urinary, 292, 473

100-Calorie portion of food, 103

Calves, $160,359,407,434,449,516$

Canning. See Cooking

of fruits and vegetables. See name of each

Cantaloupe, 269

Capacity of body to store vitamins. See under individual Vitamins

Carbohydrate, $79-80,82,126,372-373$, $397-398,438,462,474-475,483-$ $484,490,494,503$

metabolism, 79-80, 82, 372-373, 397$398,438,462,474-475,483-484$, $490,494,503$

Carbon arc lamps, 298, 418

Carbon dioxide. See Vitamin B, concentration of

Carbon disulfide, 345

Carbylamine, 220

Carnosine, 31

Carotene, 140, 141, 227, 228, 238, 243$250,368,382,384,388,405,429$, $433,436,464,475$. See also Carotinoids; Pigments; Vitamin A

loss of, in oleic acid or ethyl oleate as solvent, 247

Carotin. See Carotene. See also Carotinoids; Pigments; Vitamin A

Carotinoid, 227, 243, 250, 390, 475-476. See also Carotene; Vitamin A

Carrageen moss, 251

Carrots, 93, 139, 148, 156, 192, 194, 227, $228,231,240,249,250,268,329$, $381,390,402,426,464,506,531$. See also Carotene; Vitamin A; Vitamin D

"Cart-wheel turnings," 80, 105

Cartilage in rickets, 314

Casein, 52, 53, 56, 65, 100, 103, 130, 132, $256-257,294,320,326,333,375$, 401-402

purification of, 52

for use in measurements of vitamin $A$ values, $256-257$

of vitamin $B$ values, 100

Castor oil, 270

Cat fish, 331

Catarrh, bronchial, 279

nasal, 278

Cathode rays, 310,438

Cauliflower, 268

Cavities, abscess-like, 280. See also Abscesses

Celery, 268, 328, 357, 458 
Cells, 281, 289, 425

blood, 281

cornified, 289

Cellulose, 169

Centrifugation in purification of butterfat, 224

Cephalin, 468

Cereals, $89,90,94,137,149,153,155-$ $156,162,225,269,334,366,404$, 460, 463, 480, 491, 506, 520. See also Grains, Rice, Wheat, Maize, etc.

Charcoal, 109, 306

Chard, 231, 268, 505

Chase method for measurement of vita$\min B$ values, 100,101

Chayote, 269

Cheese, 464

Cherries, 189

Chestnuts, 94, 95

Chicago Dental Research Club, 168

Chicken liver, 369

Chicks (and chickens), 116, 353-354, $360,373,382,384,389,408,410$, $411,413,417,421-422,428,438$, $463,469-471,473,476,480,490$, 497,529

Chimyl alcohol, 238

Chittenden-Underhill syndrome, 140, $141,519,524$

Chlorine, 234

Chloroform, 31, 42, 305, 310

Chlorophyll, 237. See also Vitamin A Chloroplatinic acid, 46

Cholesterol, 235-238, 302, 304-306, 308, $362-363,414,416-417,419,426$, $433,438,442,464,489,494,497$. See also Ergosterol

irradiated, 238, 308, 362, 416-417, 419, $426,433,494$. See also Vitamin D

Choline, 26-27, 32, 36, 495

Chondrus crispus, 251

Chromogenic factor of cod-liver oil, 238-249

Cider, 14

Cinchomeric acid, 33

Citrazinic acid, 33

Citric acid, 62, 151, 181

Citrus fruits, 94, 180-186, 192, 373, 379, 432,464 . See also under name of each

Cladophora, 251

Cloth, tracing, transmission of ultraviolet light, 531

Cloudberries, 148, 149, 189

Clover, 226, 232, 268, 328

Cloves, 328

Co-adsorbent, 109

Cobalt, 216

Coccus reported by Salmon as characteristic of "pellagra-like" condition, 144
Coconut, 95

globulin, 434

oil, 270, 296, 329, 332, 341, 432

press cake, 432,434

Cocum, 192

Cod, 252, 270, 331, 360, 377, 467, 510

Cod-liver oil, 17, 65, 100, 103, 119, 138, $176,225-226,234-238,240-249$, $252-253,271,274,293-301,303-$ $305,311,315,320-322,324,326-$ $327,329-330,334,338,340,353$, $357,374,378-380,382-384,390$, $392,394,402,411-413,416,418$, $421,423-424,435,442,445-446$, $457,467,469-472,477-478,481$, $494,495,497,501,503-505,510$, $514,516,521,527-528,531-533$

oil concentrate, 442,525

Coffee, 95

Colitis, 445

Collagen, 177

Collards, 93, 201, 367

Color reactions, $361,362,365,368,393$, $423,433,436,460-464,470,489$, 494,503 . See also under the individual Vitamins

Colostrum, 272

Commercial fats, 253

Committee on Accessory Food Factors of the Medical Research Council, Great Britain, 327

Concentrates, cod-liver oil, 442, 525

Concentration. See under each Vitamin.

Condensing agents, 239. See also under name of each

Congo red, 127

Conjunctivitis, 142, 274. See also Vitamin A deficiency

Convulsions in vitamin $B$ deficiency, $79-80$

Cooking (and canning), 194, 195, 196, 197, 198, 199, 200-201. See also names of foods concerned

Copper, 418, 455, 512

Coprophagy, 129, 437

Corn. See Maize

Corn, embryo, 340

endosperm, 430

germ, 369

gluten meal, 434

meal, 119, 132, 225

oil, $269,307,332-333,355$

sirup, 184

Cornstarch, 326

Cornification, 289

Cortex Limonis B. P., 527

Cottonseed, 269, 473

meal, 486, 523

oil, 103, 138, 225, 253, 269-270 $301,303,305,332,338,340-341$, 378

Cough, 279 
Council on Pharmacy and Chemistry of the American Medical Association, 317

Cow, 383, 410, 411, 507

Coward factor, 130,147

Cowpeas, 119, 137, 268, 402

Cream of wheat, 333

Creatin, 448

Creatinuria, 76

Cress, 197

Crystalline concentrate of vitamin C, 215

Cucumber, 205, 268, 516

Cupric acetate, 43

Currants, 189

Cutaneous symptoms. See Dermatitis

Cycles, œstrous, 289. See also Vitamin $\mathrm{A}$ and Vitamin $\mathrm{E}$

Cyclohexane, 312

Cysts, 280, 474. See also Abscesses and Cavities

"D" vitamin. See Vitamin D

$d l-\alpha$-hydroxy- $\beta$-glyoxaline-4-(or 5 ) propionic acid, 50

Dandelion, 148, 164, 197

Dari, 90

Dasheens, 93, 196, 269

Date, 269

Decalcification, 162, 163, 177

Decitrated lemon juice, 181, 182

Decorticated grain, 151

Defences of body, 281, 283. See also Infection; also each Vitamin as related to health

Deficiency. See under each Vitamin

Deficiency diseases, 14-17. See aiso Beriberi, Ophthalmia, Pellagra, Polyneuritis, Rickets, Scurvy

Dehydration, 151

Dehydroergosterol, 240, 528

Dental caries. See Dentition

Dentition, 162, 168, 177, 178, 364, 367, $427,458,461,527$

Depletion of body stores of test animals, in vitamin A measurements, 260-261 in vitamin B measurements, 100,103

Dermatitis, 121, 141-145. See also Vita$\min \mathrm{G}$

Desquamation, 142, 145

Desiccation, effects of. See dried foods under names of individual foods

Destruction of vitamins. See under each Vitamin

Determination, quantitative, of vitamin A values, $253-267$

of vitamin $B$ values, 95-105

of vitamin C values, $168-179$

of vitamin $\mathrm{D}$ values, $314-319$

of vitamin $G$ values, $129-135$

Dextrin, 56, 316, 320, 333, 483
Diabetes treatment with vitamin B extracts, 462

Diacetyl, 27

Dialysis, 24, 26, 126, 216, 532

"Dialyzed iron," 40

Diarrhea, 103, 278

Diatoms, 251, 331, 432, 446

Diazo, 27

Diet. See Food

diet, basal, for study of vitamin values. See under each Vitamin, determination of measurement

Dietary deficiencies. See Beriberi, Dental caries, Ophthalmia, Pellagra, Rickets, Scurvy

Differentiation of vitamins $B$ and $G$, $111-126,499$

Digestion in avitaminoses, 360

Digitonin, 236, 304, 305, 310, 312, 348, $349,417,469$

Dihydro- $\alpha$-crocetin, 246, 247, 248

Dihydroxychinolin carbonic acid, 490

Dihydrocholesterol, 304

Dihydrophytosterol, 304

Diketopiperazines, 49

2, 6-Dihydroxyquinoline, 50

2, 6 - Dihydroxyquinoline - 4 - carboxylic acid, 50

Dilution curve, 242-243

Dimethyl urea, 40

m-Dinitrobenzene, 77

Diospyros kaki, 193

2, 6-Dioxyquinoline, 49

hydrochloride of, 49

2, 6-Dioxyquinoline-4-carboxylic acid, 49

Discovery of vitamins, 13, 18-22. See also under each vitamin

Diseases, "deficiency," 14-17. See also Beriberi, Ophthalmia, Pellagra, Polyneuritis, Rickets, Scurvy

Distillation, fractional, in concentration of vitamin A, 236-238

Distribution of vitamins. See under each Vitamin

Djave oil, 270

Dog fish liver oil, 366,424

Dogs as affected by lack of vitamins, $77,119,139,141,286,371,375$, $393,404,405,447,501,507,519$

Drying. See names of foods concerned and also description of each Vitamin

Duration of reproductive life, 291. See also Reproduction

Dystrophy of muscles, 344

"E" vitamin. See Vitamin E

Ear, infections of, 279, 518, 519. See also Otitis

Edema, 81, 142, 389, 414, 459 
Edestin, 324

Egg albumin, 316, 375

fat, 21, 226, 253

plant, 268, 517

white, 130,160

yolk, 52, 87, 138, 140, 160, 175, 176 , $250,271,272,321,329,332,333$, $335,339,360,369,381,416,417$, $418,452,475,476,506,518$

Eggs, 87, 88, 92, 94, 138, 160, 223, 250, $253,270,271,329,339,411,413$, $427,428,434,457,467,471,474$, $477,488,497,518,528$

Embryo, 75, 90

Emprosthotonus, 80

Endive, 148, 197

Endosperm, 90, 246

Endothelium, 163

Energy, radiant. See Irradiation

Enzymes, 79, 195, 355

Ensilage, 209, 456

Eosin, 407

Epithelium, 280, 283, 286, 529. See also Vitamin A deficiency; also Infection

Ergosterol, 305-314, 317, 322, 327, 328, $332,356,358,360,362,363,365$, $369,374,375,381,387,393,395$, $400,405,407,409,414,416,417$, $419,420,426,427,434,438,439$, $440,442,446,447,460,465,474$, $478,479,485,489,496,502,503$, $504,505,515,517,522$. See also Vitamin D

absorption spectrum of, $306,309,327$, 414,465

as precursor of vitamin D, 305-308, 489

chemical nature of, $306,460,489$

effect of time of irradiation, 309, 311

estimation of amount in cholesterol, 307

Ergosterol, irradiated, absorption spectrum of, $306,307,309,312$

amount for cure or prevention of rickets, 307,313

effect of solvent on antirachitic potency of, 311,312

effects of excessive doses of, 309, 381, $409,427,438,446$

nature of products of irradiation of, 309-314, 489

optical rotation of, effect of irradiation on, 312

radiant energy required to form vitamin $D$ from, 309

standard solution of, $317,327,328$

Ergot, 460

Ergothioneine hydrochloride, 50

Escarole, 268

Ether, 226-228, 310, 312, 328, 345, 348, 349
Ethyl acetate, 31, 312, 345

Ethyl alcohol, 345

Ethylene, 188, 234, 253, 357

Exercise, effect on vitamin requirements, 436

Experimental period, length of, in measurements of vitamin values, 266, 267. See also under each Vitamin, determination

Extraction of vitamins. See under each Vitamin

Eye disease, 144, 224, 274, 275, 276, 277, 278, 279, 444, 490, 524, 531. See also Vitamin $\mathrm{A}$; Vitamin $\mathrm{G}$; and Ophthalmia

Factors, chemical, in superior nutrition, 286. See also under each Vitamin, relation to health or to nutrition

which may interfere in measurement of vitamin values, $129-135$

Farina, 494

Fasting, 501, 502, 525. See also Starvation

Fat, relation to vitamin $B$ requirement, $82,83,392$

Fat-free diet, 225

Fat-soluble A, 21, 157. See also Vita$\min \mathrm{A}$

factor, 52, 53, 531. See also Vitamins A, D, E

vitamins, 417, 421. See also Vitamin A, Vitamin D, Vitamin E

Fats, 52, 138, 223, 238, 253, 270, 271, $302,339,356,367,372,383,392$, $396,398,405,408,415,421,430$, $452,466,468,472,473,476,503$

commercial, 253

Fatty oils, 439

Fearon reagents, 239

Feces, 84, 130

vitamins in, 84

Feeding, as influencing vitamin A content of body, 254

of breeding-stock for production of rats for vitamin A testing, 259, 260

Fermentation, 505

Ferric chloride, 341

Ferric phosphate, 35. See also Vitamin $\mathrm{B}$, concentration of

Fertility, 378, 423, 458, 512, 513, 518

Fertilizers, 429, 470

Fever, 283. See also Infection

Figs, 269

Fir tops as antiscorbutic, 197

Fish, 149, 251, 252, 271, 330, 331, 364, $375,382,445,493,510$

dried cooked, 149

meal, $364,493,510$

oils, $330,335,354,470,523$ 
Fishermen, 16

Flattening of growth curves, 134, 135

Flavedo of oranges, lemons and grapefruit, 186

Flavor of butter and vitamin $\mathrm{A}$ value, 273

Flaxseed oil, 340. See also Linseed oil

Flesh, horse, 30, 31

Flora, bacterial, 281. See also Infection

Floridin, 331

Flour, 137, 225, 302, 318, 332, 497

Fluorescence, 465

Folin-Denis phenol reagent, 68, 176

Folin-Macallum phosphotungstic sodium carbonate reagent, 34

Follicles, Graafian, 289

Food, as influencing ability of body to withstand subsequent vitamin deficiencies, 280. See also Storage in body, under each vitamin

as influencing vitamin $A$ content of body and of tissues, 270-273

as influencing vitamin value of body fat, 253

consumption and vitamin $B, 72,73,75$ relation of, to longevity, 498

Foods, 271, 328-335, 386, 399, 402, 415, $416,417,421,432,451,452,456$, $473,480,482,488,500,502,504$, 514

individual. See name of each

Indian, 399, 432

Japanese, 500. See also names of individual foods

Philippine, 415

"protective," 271

Foodstuffs, purified mixtures of, for nutrition investigations, 221-224, 225

Form of body as influenced by vitamin deficiency, 85

Formaldehyde, 303

Formation of vitamin A, 250-253, 374, $375,383,464,465,482$

of vitamin B, 84, 359, 409, 455, 469, 493

of vitamin C, 155, 159, 160, 202-205, $425,449,458$

of vitamin $\mathrm{D}, 300-303,305-314,330$ $335,458,465$

of vitamin $\mathrm{E}, 336,338-341,392$

Formic acid, 47, 304. See also Vitamin $\mathrm{B}$, concentration of

Formulas suggested. See Chemical Nature under the vitamin concerned

4-glyoxalinemethylethyl carbinol hydrochloride, 50

4-hydroxy-2, 6-dimethyl pyrimidine, 50

4-(or 5)-[glyoxaline-4 (or 5)-methyl] glyoxaline-5-(or 4)-methyl alcohol, 50
4 - (or 5) - glyoxalinemethylethyl carbinol, 49

4-phenylisocytosine, 37

Fowls, 14, 15, 24, 160, 271, 286, 476, 496,505

Fractures, 478

Frogs, 409

Fruit oils, 512

Fruits, citrus, 269

individual. See names of each

Fruits and fruit juices, 93, 168, 180-193, $269,353,371,380,386,389,400$, $408,427,432,433,435,464,473$, $486,493,499$

Fuller's earth, 32, 109, 114, 122, 123, 127, 128, 496. See also Vitamin $\mathrm{B}$, concentration of

Function, placental, 336

"Funk's compound," 128

"G" vitamin. See Vitamin G

Gallic acid, 62, 108, 136

$\gamma$-hydroxypyridine, 34

Gangrene, 503

Gastric motility, 461, 488, 490, 504, 510

Gastrointestinal disorders, 71, 143, 144, 450,476

Gelatin, 316

Germination in relation to vitamin formation, 155, 202-205, 251, 421, $425,458,502$

Glacial acetic acid, 41. See also Vita$\min \mathrm{B}$, concentration of

Gliadin, 472

Glands, $55,280,450$. See also name of each

Glandular organs, 270

Glass, different types, in relation to transmission of ultra-violet light, 530

Glucose, 29, 47, 64, 82, 182, 446, 479

tolerance, as influenced by vitamin B, 82

Glutathione, 482, 530

Glutazine, 33

Glycemia, 466, 484

Glycogen, 484

Glyoxaline, 50,407

Glyoxaline-4-(or 5)-acetic acid, 50

Glyoxylic acid, 27

Glyoxaline-4-(or 5)-formaldehyde, 50

4-Glyoxalinemethylethyl carbinol hydrochloride, 50

5-Glyoxalinemethylethyl carbinol hydrochloride, 50

4-(or 5) - [Glyoxaline-4 (or 5) -methyl]glyoxaline-5-(or 4)-methyl alcohol, 50

4 - (or 5)-Glyoxalinemethylethyl carbinol, 49

Glycine, 440 
Gold chloride, 36. See also Vitamin B, concentration of

Goose fish liver, 330

Gooseberries, 189

Graafian follicles, 289

Grains, 225, 479. See also under name of each; also Cereals

Grapefruit, 93, 186, 269

Grapefruit peel oil, 270

Grape juice, 187, 482

Grapes, 94, 187, 269, 461

Grass, 130

seeds, 490

Green herbs, 179

Green vegetables. See under each Vitamin; also Leaves; Greens

Greens, 193, 201. See also Leaves

Groundnut, 522. See also Peanut

Growth, 18-21, 25, 54, 76, 77, 88, 89, 94, $115-117,120,123,129-135,137$, $221-225,231,253-255,273,274$, $278,289,323,344,368,382,396$, $398,403,425,452,461,466,472-$ $474,485,486,492,499,503,507$, $516,518,522,530$

Guanidine, 32, 40

Guanine, 32, 128

Guinea pigs. See Vitamin C and Scurvy

Gum tragacanth, 182

Haddock, 270, 402, 437

Hair ball formation in rats, 463

loss of. See Vitamin G deficiency symptoms

Hake liver oil, 423

Halogen, 349

Hay, 169, 295, 411, 493, 507

alfalfa, 295

oat, vitamin D in, 295

Hazel nuts, 95

Head retraction, 40, 104

Health, relation of vitamin A to, 273292. For relation of other vitamins to health, see individual vitamins; also Growth, Reproduction, and $\mathrm{Vigor}$

Heart, 70, 86, 146, 253, 436, 524

block and vitamin $B$ deficiency, 81

"Heat cure," 46, 78

Heat-labile factor. See Vitamin B

Heat-stable factor. See Vitamin G

Heating experiments, 24

influence of, upon stability of vitamins, 503

upon stability of vitamin B, 498

upon stability of vitamin C, 498. See also description of each vitamin

Heliotherapy, 479, 494, 510. See also Irradiation; Sunlight

Hematoporphyrin, effect of, in rickets, 331, 445
Hematoxylin-eosin, 177

Hemeralopia, 277, 421, 423

Hemoglobin, 81, 510

Hemolysins, 524

Hemorrhage, 463, 532

from vitamin $B$ deficiency, 83

Hempseed oil, 270, 511

Hens, 427

Herbs, 179

Herring, 437

kippered, 494

Hexane, 312

Hexylamine, 40

Histamine, $35,40,441$

Histidine, 26, 27, 35, 284

Histological changes, 289. See also under each Vitamin, relation to health or nutrition, or symptoms of deficiency

Histology of teeth in relation to scurvy, 177-178

of vitamin A deficiency, 286, 287. See also Vitamin A

Höjer method, 177-178, 400

Hog muscle, 421, 422

Hominy, 333

Honey, 384, 392, 413, 427, 437, 493, 515

Hormones, 415, 484, 521

Horse flesh, 104, 493

denatured, 104

Humoral róle of vitamin A, 284

Hunt factor, 126, 130, 147

Hydrochloric acid, 108. See also Vitamin B, concentration of

Hydrogen, 216, 229

Hydrogen dioxide, 303

Hydrogen-ion activities (or concentrations), 59, 63, 66, 69, 106, 107, $109,124,126-128,136,148,149$, $181,183,188,211-213,216,233-$ $234,326,444,483,485,491,495$, $498,501,530$

of alimentary tract, 501,530

relation of, to adsorption of vitamin B, 59, 63, 64, 66, 69, 109, 491

of vitamin $G, 124,127,136$

relation of, to precipitation of vita$\min$ C, 216

of vitamin $G, 126$

relation of, to stability of vitamins, 498

relation of, to stability of vitamin $\mathrm{A}$, 233-234; of vitamin B, 106, 107

of vitamin C, 148, 149, 181, 183, $188,211-213,444$

of vitamin $\mathrm{G}, 128$

See also pH

Hydrogen peroxide, 46, 107, 234

Hydrogen sulfide, 62, 127, 131, 234. See also Vitamin B, concentration of, properties of

Hydrogenated oil, 338 
Hydrogenation, 303, 483, 532

effect upon vitamin A values of fats, 229

Hydrous aluminum silicate, 32

4-Hydroxy-2, 6-dimethyl pyrimidine, 50

2-Hydroxy-4, 6-methyl pyrimidine, 50

$\gamma$-Hydroxypyridine, 34

6-Hydroxynicotinic acid, 33

Hydroxyphenyl, 68

Hydroxypyridines, 33

Hyperemia, 148, 163

Hyperemic pulp of teeth, 177

Hyperglycemia and vitamin B deficiency, 79

Hypervitaminosis, 409, 442. See also Vitamin D

Hypogly cemia, 513

Illustrations of relation of vitamin A to nutrition, health, and vitality, 287-292

Imidazole, 44

Immunity, 283, 524. See also Infection; also under each Vitamin as related to health

Inactivation, $107,108,304,306,531$. See also individual Vitamins.

Inanition, 77-80, 431, 438, 457, 523. See also Fasting; Starvation

Incidence of different symptoms. See under each Vitamin deficiency.

of infection. See Infection; also under each Vitamin, relation to health

of ophthalmia in vitamin A deficiency, 274-276

Incisor, 177

Indian foodstuffs, 381

Indian troops, scurvy in, 179, 180

Indole, 68

Infantile scurvy cured by decitrated, concentrated lemon juice, 214

Infants, antiscorbutics for, 211

Infants' foods, $378-380,382,386,417$, $456,460,524$

Infection, $85,143-144,224,250,274-$ $292,320,393,394,404,421,479$, $486,488,494,498,502-504,518-$ 520,524

Infusorial earth, 114

Injection method of vitamin B tests, 25 , 74,105

Infra-red radiation, 372

Inositol, 128

Insects, 225, 514

as test animals, 514

Insulin, 369, 471, 510

Interrelationships of scurvy and other deficiency diseases, 151

Intestine, $70,163,281,326,404,406$. $409,431,450,474,480$

See also Infection
Introduction to vitamin theory, 13

Invertase, 504

Iodine, 216

Iodine-potassium iodide, 43

Iron, $216,466,502$

Irradiation, 238, 257, 296-313, 317, 319$321,328,330-335,357,371,385$, $401,403,414,419,421,429,441-$ $443,445,447-448,463,465,479$, $481,490,495-497,505-507,510$, $513-514,517,522,526,528,530$ 533

effect of excess on inactivation of vitamin D, 304, 306

of body with ultra-violet light, 296$298,319-321,334,335,371,403$. $414,421,429,447-448,463,465$, $479,481,490$

of cholesterol, 238, 302-305, 308-310, 313

of ergosterol, 306, 307, 309-313, 317. 328

of foods, 300-302, 330-334, 385, 448, $517,522,528$

See also Cholesterol; Ergosterol; Light; Ultra-violet; Vitamin D; under each Vitamin

Isoergosterol, 311, 519

Isolation experiments. See under each Vitamin, chemical nature; concentration

Japan sand pear, 431

Japanese navy, conquest of beriberi in, 25

Jaw bone, decalcification of, 177

Jendrassik, 447

Juices. See Fruit juices

Kaki, 193

Kakke, 515. See also Beriberi

Kale, 93, 201, 437

Katjang idjo, 15, 381, 428

Keratinization, 280 . See also Vitamin A deficiency

Keratoconjunctivitis, 274

Keratomalacia, 274, 294, 394, 396, 488, $495,508,523$. See also Vitamin A

Kidney, 56, 70, 86, 139, 146, 160, 163 , $253,270,446,478,496$

stones, 478

Kieselguhr, 114

Kinnersley-Peters concentrate used as source of vitamin B in vitamin G investigation, 127

Kohlrabi, 197, 426

Kromayer lamp, 311 
Labrador, deficiency diseases in, 355

Lactalbumin, 389

Lactation, 82-84, 145-146, 286-292, 391, $392,412,415,430,452,469,471$, $503,512,513,518$

Lactic acid, 62, 81, 438

in tissues in vitamin $B$ deficiency, 81

Lactose, 21, 25, 52, 53, 182

Lamb, 139, 270, 422

Lamps, carbon arc, 298

Kromayer, 311

Mercury vapor quartz, 298, 320

Lard, 51, 54, 253, 271, 274, 287, 301 , $332,333,346,378,382,383,398$, 402,457

Larynx, 280, 465

Latent scurvy, 152, 153, 162-164, 166

Leaching of casein, 132

Lead acetate, 36, 43, 131

Lead, precipitation of vitamin C, 215 precipitation of vitamin $\mathrm{G}, 126-128$

Leaves, 122, 123, 164, 197, 201, 225, $226,232,234,251,268,340,373$, 454,455 . See also Grass

Lecithins, 26, 468

Leg weakness, 373, 427, 428, 476

Legumes, 24, 94, 95, 137, 155, 202, 203, 204,366

Lemon peel, 186

Lemons and lemon juice, 13, 14, 93, 158, $168,176,180-185,213-220,269$, $358,371,379,405,409,427,466$, $520,525,531,532$

concentrated, 185, 213-217

decitrated, 213-217

treated, 181

Length of experimental period in measurements of vitamin values, 266 , 267. See also determination and measurement of each Vitamin

Lentils, 92, 149, 203, 269, 405, 434

Lesions, ocular, 444. See also Eye

Lettuce, 93, 148, 168, 197, 201, 245, 268, $301,332,340,385,426,442$

Lettuce-seed oil, 340

Light, 124, 126, 230-231, 297, 303, 313, $371,372,417,418,426,507,529$

effect upon vitamin A, 230-231

therapy, 426

See also Irradiation; Sunlight

Lime juice, 28, 180, 181, 184, 371, 503

Line test, 296, 314, 315, 453

Linseed oil, 225, 270, 301, 332, 341, 474. See also Flaxseed oil.

Lipins, 223, 455. See also Lipoids

Lipochromes, 242, 271, 390, 489. See also Carotene; Vitamin A

Lipoids, 26, 221, 222, 439, 467, 508, 509, 511

Litchi nut, 503

Live-stock feeding, 444
Liver, $55,70,86,130,138,140,143$, $146,147,159,160,163,207,208$, $250,253,270,276,305,320,329$, $333,399,403,444,446,474,496$, 497,532

oils, 238-245, 247-250, 271, 328, 408, 486,494

Lloyd's reagent, 92, 109, 114 . See also Vitamin B, concentration of

Longevity, 146, 289-292, 368, 498, 499

Loss of body weight and deficiency of vitamin $B, 70$

Lung, 270, 279, 284, 291, 320

disease, 291

fat, 487

Lymphocytes, 376

Mafurreira oil, 270

Magnesium, 295, 364, 407, 455

Maize (corn), 55, 63, 64, 90, 91, 109, $116,137,227,231,240,246,251$, $252,269,316,333,337,341,370$, $377,404,410,413,429,430,432$, $436,438,452,454,461,475,490$, $506,511,520$

See also Corn

Maize oil, 225

Malt, 357, 409, 435, 451, 483, 505

sirup, 399

Malted grains, 202

Manganese, 455

Mangels, 269

Mango, 192

Manure, 450

Margarine, 295, 354, 395, 408

Marmite, 65, 78, 90

Marrow, vegetable, 205

Mastic, 35. See also Vitamin B, concentration of

Measurement of vitamin values. See determination and measurement under each Vitamin

Meat, 24, 86, 87, 119, 138, 179, 180 , $207,208,253,321,375,422,479$

dehydrated, 400

extract, 55

juice, 192

peptone, 175

Medical Research Council, Committee on Accessory Food Factors of, 327

Menhaden oils, 462

Mercuric chloride. See Vitamin B, concentration of

Mercuric sulfate, 109, 131

Mercury vapor quartz lamps, 298, 320

Mesopotamia, beriberi and scurvy in, 179

m-dinitrobenzene, 77 
Metabolism, 72, 73, 75-82, 122, 377, $407,433,436,463,471,475$

relation of vitamin $\mathrm{B}$ to, $72,73,75-82$, 463,471

See also Calcium; Phosphorus; Protein

Metaphyses in rickets, 314

Metaplasia, 281, 286. See also Vitamin A deficiency

Method of measuring vitamin values. See each Vitamin, determination, measurement

Methy1 alcohol, 35, 305, 345, 348, 349

2-Methyl glyoxaline, 50

Methylene blue, 77

Mice, $17,77,359,363,406,458,463$, 480,524

Microörganisms, 524, 529

Middlings, 90

Milk, 17, 18, 19, 28, 51, 87-89, 92, 94, $105,106,119,123,130,134,138$, $150,152,153,156,160,162-164$, $169,170,175,180,208-211,221$, $222,224,270-272,286,293,319$, $321,322,325,330,332-335,339$, $354,358,371,374,375,377-381$, $383,384,386,395,399,400,407$, $410-412,415,418,419,425-427$, $429,430,434,437,441,442,444-$ $447,449,452-454,456,458,459$, $461,463,467,468,471,473-476$, $480-482,484,485,487,489,490$, $492,494,497,498,503,507,509-$ $512,521,522,528,530$

ash, 25,51

autoclaved, 158, 169, 180

boiled with aeration, 175

condensed, 272, 447, 483

dried, $210-211,272,330,369,374,401$, $433,434,483,528$

evaporated, $210,272,378,381,384$, 385

human, 399, 419, 425, 437, 456, 461, $474,481,516$

pasteurization of, 150-153, 471, 494

powder, 51, 52, 105, 108, 109, 340, 381, $386,410,434$

skimmed, 329, 480, 528

Millet, 90, 269, 324, 333, 451, 508

Milling products of wheat, vitamin B values, $90-92$

Millon reagent, 27

Mineral acids, 303

elements, 293, 326, 421, 426, 479

See also Calcium; Phosphorus

"Minimum protective dose," 96, 158, $175,176,178$.

Mixture, ternary, of alcohol, water and benzene, 113

Molasses, 469, 515

Monkeys, 20, 157, 158, 286, 287, 381, $409,426,445,450,516,517$
Moss, 251

Motor functions and vitamin B, 74-75

Mouth symptoms, 140-145

Mung bean, 365, 462

Muscle tissue, $31,55,76,77,86,130$, $138-140,146-147,159,207,253$, $270,320,339,344,400,421,469$, 475, 476. See also Meat

Muscular control, loss of, in vitamin B deficiency, 104.

Mushrooms, 251, 269, 408, 448

Mustard greens, 93, 437

Mustardseed oil, 270

Mutton, 87, 271

fat, 271

Nasal passages, 280, 378, 518. See also Vitamin A deficiency

Navy, 14

beans, 73

Negative controls. See determination and measurement of each Vita$\min$

Nervous system, 70, 71, 74, 78, 79, 112, 427,436

"New" factors, 110, 129-131, 134, 147

Nickel, 216

Nicotinic acid, 16, 29, 32, 33, 34, 35 methyl ester hydrochloride of, 33

Night blindness, 277, 278, 396

Ninhydrin, 35

Nitrogen, 58, 76, 220, 349, 373, 421, $433,441,475$

Nitrogenous base and vitamin B, 26

Nitrous acid, $57,65,107,108,126,127$, $234,303,499$

Nitszchia closterium, 331

Nomenclature, 20-23, 504

Norite, 39, 48, 109, 127, 128, 239

Nuclear metabolism, relation of vitamin B to, 71

Nucleic acid, 26, 58, 128

"Nuclein bases," 34

Nutrition, 13-22, 24, 161-168, 221-225, 273-292, 387, 437, 452, 461, 488, 522. See also under each Vita$\min$

"Nutrilites," 525

Nuts, 94, 95, 269, 368, 375, 480

Oatmeal, 90

Oats, 53, 55, 90, 117, 148, 155-157, 163, $164,169,170,175,204,225,295$, $321,333,337,430,454,473,506$

Ocular lesions in vitamin A deficiency, 531. See also Vitamin A.

Odontoblasts, 163, 165, 167, 177, 178

Oestrus, 289

Oil, extraction of vitamin A of plants by, $227-228$ 
Oils, 302, 328, 383, 392, 407, 414, 482 See also under name of each

Okra, 94

Old vs. young fresh vegetables as sources of vitamin C, 194

Oleo oil, 332, 422

Oleyl alcohol, 237

Olive oil, 225, 253, 270, 301, 304, 305, $324,328,332,333,340,341,433$, 486

Onions, 197, 269

Ophthalmia, 17, 20, 143, 224-225, 261, $274,294,299,300,316,434,450$, $453,465,473,474,507,522$

"salt," 453

See also Xerophthalmia

Opisthotonic, 104

Opisthotonus, 80, 81, 104

Opsonins and vitamin deficiency, 394

Optical activity of ergosterol on irradiation, 312

Orange peel, 153, 186

Orange rind oil, 240

Oranges and orange juice, 13, 14, $93,152,156,158,161,163,167$, $168,176,178,182,184,185,186$, $269,340,367,400,403,411,431$, $446,451,458,506,517,526,527$, 530

canned, 185

dried, 184-186, 400, 411, 451, 458

frozen, 185

sirup, 185,186

Organic phosphorus compounds, 26

Organs, 270, 444, 509. See also name of each

Oridin, 36

hydrochloride, 36

Oryzanine, $29,30,49,431,490,514$

$\alpha$-acid of, 29,49

$\beta$-acid of, 29,49

picrate, 29

"Oscodal," 384

Osteoblast, 165, 167, 295

Osteodentin, 163

Osteomalacia, 319, 320, 398, 428, 440, $447,462,479,527$

Otitis media, 503. See also Ear; Vita$\min \mathrm{A}$

suppurative, 455

Ovaries, 70, 76, 289, 341-344

Ovulation, 289, 336, 338. See also Vitamin E; Reproduction

Ox brains, 28 tissue, 421

Oxidases, 198

Oxidation, 77, 78, 228-238, 303, 311, 347, 511

Oxidation-reduction potential, 210, 212213

Oxidizing agents, 46

Oxycholesterylene, 312
Oxygen, effect of, upon vitamin A, 230238

upon vitamin C, 213

utilization and vitamin $\mathrm{B}, 77-79$

Oxygenase in citrus fruits, 186

Oysters, 434, 457, 482, 528

Ozone, 230-231, 356, 531

effect upon vitamin A, 230-231

Palm kernel meal, 394

kernel oil, 505

oil, 464

Pancreas, 53, 55, 70, 86, 280, 386, 489

Papaya, 94, 192, 193, 269, 462

p-oxy-nicotine acid, 33

Paralysis, 85, 104, 105, 123, 343, 344. See also Vitamin B

Parathyroid, 421, 440, 464, 470, 476

Parsley, 93

Parsnips, 269

Pasteurization of milk, 150-153, 471, 494

Pasture-feeding, 333, 334

Patent flour, 302. See also Flour

Pathology, 152, 153. See also under each Vitamin deficiency, and as related to health

Pauly reaction, $35,44,46,68$

Peaches, 94, 189, 191, 269, 440

Peanut (arachis) oil, 270, 340

antirachitic potency of on irradiation, 332

Peanuts, 95, 158, 269, 340, 378, 405, 434 meal, 405

Pears, 94, 189, 192, 377, 442

Peas, 92,95, 149, 203-206, 245, 251, 268, $402,405,453,463,486$

green, 206, 402, 486

sprouted, 405

Pecans, 94, 269, 491

Pellagra, 118-145, 162, 356, 371, 372, $390,393,394,397,401,402,430$, $433,439,445,447,452,453,456$, $470,485-487,491,492,502,511$, $514,515,519,520,524,528$

Pellagra-like condition, 519. See also Vitamin G

Pellagra-preventive (P-P) factor. See Vitamin G

Pelvis, 281

Pentane, 345, 348

Peppers, green, 93, 205, 206, 245-246, 268

red, 268

Peptides, 58

Perchloric acid, 44

Period, length of experimental, in measurements of vitamin values. 266, 267

Permanganate, 215

Peroxidase, 186, 218

Peroxidation, 530 
Persimmon, 193

Persistence of vitamin A in plant tissues, 252

Peters' antineuritic concentrate, 131

Petroleum, 310

ether, 305, 348, 349

as solvent for vitamin $\mathrm{D}, 305$

$\mathrm{pH}, 59,63,64,66,69,106,107,109$, $124,126-128,136,148,149,181$, $183,188,211-213,216,233-234$, $326,444,446,483,485,491,495$, $498,501,530$

effect on vitamin C, 148, 149, 181, 183,188

influence of, in adsorption of vitamin B, 59, 63, 64, 66, 69, 109, 491

influence of, in adsorption of vitamin G, $124,127,136$

influence of, on precipitation of vitamin C by lead, 216; of vitamin $\mathrm{G}$, by lead, 126

influence of, upon solubility of vita$\min B, 59,63-66$

of alimentary tract, 326, 485, 495, 501, 530

in relation to rickets, 501

of feces of rachitic children, 485

relation of, to stability of vitamin $\mathrm{A}$, 233-234

relation of, to stability of vitamin $B$, 106,107

relation of, to stability of vitamin $\mathrm{C}$, 211-213, 444

relation of, to stability of vitamin G, 128

See also Hydrogen-ion activities

Phagocytosis, 524

Pharmaceutical Society of Great Britain, 317

Pharmaceuticals, 240. See also under name of each

Pharmacology of the vitamins, 519. See also description of each Vita$\min$

Pharyngitis, 140

Phaseolus, 24

radiatus, 15

Phenol indophenol, 216, 218

Phenols, 220, 430

4-Phenylisocytosine, 37

Philippine cereals, fruits and vegetables, 353,491

Scouts, eradication of beriberi from, 26

Phosphate, 415, 435, 439, 491

Phosphatid, 495

Phosphoric acid, 62, 176

Phosphorus, 25, 216, 293, 295, 296, 298, $322,334,349,359,364,379,384$, $390,394,407,409,412,414,415$, $417,419,428,430,433,441,442$, $450,451,459,463,465-467,472$,
Phosphorus (continued)

$477,479,486,492,495,501,503$, $507,516,517,522,524,530,533$ metabolism, 293, 295, 296, 298, 322, $334,359,364,379,384,390,394$, $409,412,414,415,417,419,428$, $430,433,441,451,459,463,465-$ $467,472,477,479,486,492,495$, $501,516,522$

Phosphorus pentachloride, 234

Phosphorus pentoxide, 239

Phosphotungstic acid. See Vitamin B, concentration of

Photoactivity, 443

Phthalic anhydride, 237

Phytol, 237, 433

Phytosterol(s), 305, 306, 414, 419

Pickles, 205, 516

Picric acid, 38. See also Vitamin B, concentration of

Picrolonic acid, 43, 107. See also Vitamin $B$, concentration of

Pidan, 88

Pig, 286, 365, 388, 403, 430, 471, 480 kidney, 56, 70

Pigeons, 77, 114, 115, 118, 125, 379, 389, $405-407,422,425,444,469,480$, $495,496,511,523,526,530$

Pigmentation, 227, 272, 508. See also Carotene.

Pigments, 227, 272, 508. See also Carotene

Pimiento, 206

Pineapple, 94, 192, 193, 269, 462 juice, 192

Piperidine, 36

Pitchard oil, 366

Pituitary gland, 70, 76

Pituitrin, 41

Placental function, 336

Plankton, 252, 331, 360

Plantain, 269, 373, 482

Plants, 251, 375, 454, 473, 482, 491, 493 green, 493

tissues, 375, 473, 482

See also under each Vitamin, occurrence or distribution, in nature or in foods

Platelets, 281

Platinic chloride, 43. See also Vita$\min \mathrm{B}$, concentration of

Platinum, colloidal, 303

Plums, 189

Poi, 196

Polenta, 137

Polished rice, 47, 52, 55, 56, 65, 70, 71, $148,151,436,470$

Polishings, rice, 14,15

Pollack liver oil, 423

Polyneuritis, 15, 16, 24, 25, 27, 31, 39, $72,73,78,85,98,104-105,115-$ $117,121,365,384,388,399,405$, 
Polyneuritis (continued)

$428,443,449,452,463,467,471$, $478,482-485,491,502$

special basal diet for study of, 98

See also Beriberi; Vitamin B

Polysiphonia, 251

Pork, 87, 139, 270, 402, 422, 507, 529

Potassium, 407, 479

bisulfite, 185

chromate, 43

iodide, 43

permanganate, $107,306,308$

sulfate, 38

thiocyanate, 43

Potatoes, 53, 92, 93, 148, 153, 192, 195, $269,328,363,400,430,432,449$, 453,486

sweet, 328,363

Poultry, 270, 271, 359, 368, 379, 413, $422,423,430,461,462,467,471$, 472

fat, 271

P-P factor. See Vitamin G

Prairie dog, 452

Precautions against interfering factors in vitamin work, 129-131. See also determination and measurement under each Vitamin

Precipitins, 524

Precursor, 250, 305, 306. See also Vitamin A; Carotene; Vitamin D

Preen gland, 426

Pregnancy, 75, 405. See also Reproduction; also under each Vita$\min$

Preparation of alcohol extract of wheat as source of vitamin $B$ in vitamin $G$ experiments, 133

"Primary" form or source of vitamin A, 250

Production of antirachitic potency in foods, 332-335

of vitamin D by irradiation, 300303

Prophylaxis, 283. See also Infection; also under each Vitamin as related to health

"Protective foods," 271

Protein-free milk, 19, 25, 50, 51, 54, $108,109,222,224$

Protein metabolism, 80, 81, 83, 122, 383 $398,403,412,453,454,472,473$, 474,485

relation to vitamin need, $25,80,81$, $83,398,474$

Protocols in experimental scurvy, 174

Provitamin D, 307, 439

Prunes, 94, 183, 269, 399 pits, 399

Pseudo base, 34

cures, 50
Puffer fish, liver oil, 330

oil, 419

Pulp, 163

Pulses, 155, 192, 480

Pumpkin, 205, 268, 464

Puppies, 366

Pure compounds, mixtures of, as food, 221-224

Purine, 30, 32, 35, 58, 68, 284, 397

"fraction," 30

Pyorrhea, 162, 168

Pyrimidine, 16, 26, 44, 50, 397

Pyridine, 33, 36

Pyrogallol, 239

Pyrophosphate, 128

Quantitative. See determination, distribution, measurement, occurrence, values, under each Vitamin

Quinine, 64

Quinoline, 50, 407

Quinolinic acid, 33

Rabbits, 157, 250, 286, 393, 404, 460, $465,469,474,475$

Rachitic rosary, 162

Radiations. See Irradiation

Radishes, 194, 269, 464

Raisin seed oil, 385

seeds, 385

Raisins, 269, 385

Rapeseed oil, 270

Raspberry, 149, 189

Ratfish-liver oil, 470

Reactions to vitamin deficiency, 85. See also Symptoms; also under each Vitamin

Reader factor, 130, 147

Rearing of young, 259, 260, 287. See also Reproduction; also under each Vitamin, relation to health or to nutrition

Refection, 84, 103, 396, 440, 461

Relation of vitamin A to nutrition and health, 273-292

of other Vitamins. See under each

of plant pigments to vitamin A, 243250. See also Carotene; Carotinoids; Vitamin A

Reproduction, 75, 82-84, 145-146, 287 $292,336,354,363,368,391,405$, $406,417,423,424,437,456,462$, $469,476,477,482,503,511,512$, $514,518,530$. See also under each Vitamin

Reserves of vitamins in body. See under each Vitamin, storage in body

Resistance, 504, 520. See also Health, Infection, Vigor

Resorption, 339 
Respiratory disease. See Infections and Vitamin $\mathrm{A}$

quotient, 433, 457

Resting stage, relation to vitamin C, 206-207

Rhubarb, 196, 197, 420, 479

Ribs, beading of, $162,163,165$

Rice, $14,15,24,43,55,70,91,112,115$, $149,158,269,337,370,430,432$, $437,440,451,452,492,505,514$, 520

birds, 43

brown, 55

bran, 29,432

embryo, 422, 471

polishings, 26-28, 30-33, 35, 42, 43, $45,47,56,69,73,370,383,391$, $395,397,398,467,512,526$

starch, 65, 103

Rickets, 162, 293-335, 353-355, 357, 359, $363,366,367,370,371,380,382$, $388,393-397,399,402,404,407$, $408,413,415-420,422,424,426-$ $432,435,436,438-441,443,447$, $450,453-456,459-461,463,467$, $470,472,474,476-479,481,482$, $487,489,491,492,494,497,499$, $500,501,505,506,509,510,516$, $517,521,523,525,527,528,532$, 533

calcium and phosphorus of blood and bone in, 293-297, 321-323, 371, $407,420,427,491,492$

diet of mother as factor in development of, $319,320,367,380,404$, 419

diets for production of, $315,316,318$, $453,460,474,476$

line test for determination of, 296, 315

$\mathrm{pH}$ of intestine in, 326, 327, 474

prevention and cure of, 293, 294, 296, $297,299-303,305-319,322,328-$ $335,353,393,394,407,408,415$, $416,418,426,428,429,435,438$, $472,477,481$

seasonal variation in, 297, 417

Rind, grapefruit, 527

lemon, 527

Roots, 92, 93, 112, 334

"Russell effect," 443

Rutabagas, 93, 140,157, 164, 193, 199, $218,269,402$

Rye, 90, 149, 203, 204, 473, 493

Sailors, 14, 16

Saliva, 467, 478

Salivary glands, 280 . See also Vitamin A deficiency

Salmon, 139, 270, 402 oil, 379,424
Salt mixtures for experimental diets, $100,103,175,257$

Sapodilla, 269

Saponification, 225, 303, 304, 507

Sardine oil, 330

Sauerkraut, 198, 201, 372

Scorbutic diets, 153, 161-168, 482

effect on teeth, 161-168

effect on calcium metabolism, 163, 166

Scurvy, 13-17, 148-156, 158, 160-168, 171-178, 181-182, 353, 355, 358$360,362,366,368,370,372,373$, $377,379,381,385,392,393,396$, $399,409,410,412,414-417,421$ $422,424,426,427,430-433,439$, $446,448,449,452,455,456,460$, $461,463,464,466,467,470,475$, $479-484,488,490,492,494,497$, $501,503,504,508,509,515,519-$ $521,528-530$. See also Vitamin C

score, $160,175,176$

Seal oil, 271, 330

Seasonal variation in rickets, 297

Seaweeds, 251

"Secondary" form or source of vitamin A, 250

Secretin, 73-74, 355, 381, 432

Seedlings, 251, 252

Seedpods, 201

Seeds, 92, 149, 150, 207, 225, 340, 370, $452,473,513$. See also Grains

Selachyl alcohol, 237, 238

Septicemia, 282, 283, 460. See also Infection

Sesame seed oil, 270, 341

Shad body oil, 424

Shark-liver oil, 238

Sheep's pancreas, 53, 421

Ship beriberi, 148, 149, 424

Shoots. See Seedlings

Shredded wheat, 333

Siak Illipe nut oil, 270, 429

Silage, 210, 493

Silicotungstic acid, 44

Silver, as a precipitant for vitamin B, 38 nitrate. See Vitamin B, concentration

use of in line test for vitamin $D$, 315

nitrite, 41

sulfate, 41. See also Vitamin B, concentration of

Sitosterol, 307

6-hydroxynicotinic acid, 33

Skatole, 68

Skimmed milk powder, 108, 169, 170

Skin, 302, 413, 421, 426, 481

Skyshine, 394

Smears, vaginal, 289 . See also Vita-

Snails, 466 min A; Vitamin E 
Sodium nitrite, 40 sulfite, 184

Solubilities of vitamins. See under each Vitamin

Sorghum, 515

Sorrel, 148, 197

South African foodstuffs, 380

Soy-bean, 119, 122, 137, 155, 156, 379, 426,473

cracker, 169

flour, $169,184,434$

oil, 225, 270, 431

Sparing action of fat for vitamin B, 82

Spectroscopic tests, 250, 302, 304, 327

Spinacene, 237

Spinach, 54, 93, 197, 200, 226, 232, 234, $250,268,272,326,328,329,332$, $364,371,386,420,421,451,455$, $487,500,510,527$

fat, 505

Spleen, 70, 139, 146, 163

Spontaneous cures in vitamin B deficiency, $79-80$

Squalene, 238

Squash, 231, 268

Stability. See under each Vitamin

Starch, 15, 51, 54, 100, 103, 175, 324, 326

Starvation, 71, 500, 523, 529. See also Inanition

Stems, 93

Steenbock theory of relationship of plant pigments to vitamin A, 243250

Sterility, 336, 338, 341-344, 349, 354, $391,456,458,485,514$. See also Reproduction; Vitamin E

Sterols, 235-238, 348, 349, 383, 393, 395, $414,416,428,440,489,515$

Stomatitis, 143, 144

Storage, of food, effect on vitamins. See under each Vitamin

of vitamins in body. See under each Vitamin

Strawberries, 189,440

String beans, 205, 420, 487

Strontium, 501

Structure. See chemical nature under the Vitamin concerned

Sulfanilic acid, 44

Sulfur, 62, 216, 349 dioxide, 191, 234, 303

Sulfuric acid test for liver oils, 238, 383 , $392,408,486$

Sunflower, 251

Sunflower-seed oil, 225, 270

Sunlight, 251, 252, 296, 297, 333, 366, $368,394,428,441,457,471,479$, $481,482,486,490,497,501,507$, $517,525,530$. See also Irradiation; Light; Rickets; Vitamin D Suprarenal glands, 364, 368, 431, 436
Survival period on Vitamin-A-free diet, 258, 280, 284-286

on vitamin-B-free diet, $100-101$

on vitamin-C-free diet, 171-174

Swedes, 164, 193, 199. See also Rutabagas

Sweet potato, 93, 196, 231, 268

leaves and shoots, 491

Swine, 160, 364, 410, 427, 459, 469, 504

Symbiosis, bacterial, as factor in vitamin B supply, 84

Symptoms, 85, 171-174. See also under each Vitamin

Synthesis of vitamins, 83, 250, 331, 444, 511. See also under each Vita$\min$

Tamarinds, 192

Tangerines, 192

Tankage, 364

Tannic acid, 29, 62

Taro, 196

Tea, 463, 467

Technique. See Methods; also name of each Vitamin

Teeth, 161-163, 165, 167, 168, 177, 178, $179,366,405,408,422,427,436$, $453,460,488,517,524,532$

Temperature in relation to formation and stability of vitamin D, 305, $310,328,329$

in relation to stability of vitamin $\mathrm{A}$, 228-250

in relation to stability of vitamin $\mathrm{C}$, 444

of body in vitamin B deficiency, 77-79

Terminology, 20-23

Testis, 70, 76, 341-344, 458, 459, 480

Tetany, 420, 477, 501, 514, 519, 525

Tetrahydroxypyridine, 33

Thermo-labile factor. See Vitamin B

Thermo-stable factor. See Vitamin G

Thermostability. See Heating and Temperature

2-Thiol-4-amino methyl glyoxaline, 50

2-Thiol-5-amino methyl glyoxaline, 50

Thionyl chloride, 306

Thymine, 26

Thymus, 70, 76, 492, 497, 526

Thyroid, $70,76,409,443,451,460,467$, 496

Thyroxine, 43

Tibia, 315

Tiki-tiki, 131, 354, 491, 523

Timothy, 232

Tinct. Limonis Fort. B.P.C., 527

Tintometer, 239, 249

Tissues, $31,55,75,86,270,420,433,487$

Togi, 94

Tomato and tomato juice, $73,92-94,106$, $119,139,160,172-175,187-189$, 
Tomato and tomato juice (cantinued) $212,218,252,253,269,328,372$, $380,400,402,417,426,434,446$, 449,465

seed press cake, 394

Tongue, abscess-like cavities or cysts at base of, 280. See also Vitamin A

Torulin, 28, 40, 44, 45, 50,81, 387, 413, 438

Toxemia, 283. See also Infection

Toxicity of irradiated ergosterol, question of, 502-503. See also Ergosterol

theory of beriberi, 15

Trachea, $280,465,467$

Tradescantia, 251

Trichloroacetic acid, 177, 239

Trigonelline, 33

2,3,4-Trihydroxypyridine, 33

2,4,6-Trihydroxypyridine, 33

Trimethylamine, 28

Trimethyluracil, 37

Tri-oxyhematin, 177

"Tripartite nature" of vitamin B complex, 125

Tuberculosis, 287, 291, 404, 441, 444, $478,496,504,510$. See also Infection; also Vitamin A, relation to health

Tubers, 112

Tumors, 75,468

Turkeys, 451

Turnips, 192, 193, 251, 269, 426, 518

Turnip greens, 201, 367

Tyrosine, 440

2,6-dihydroxyquinoline, 50

2,6-dihydroxyquinoline-4-carboxylic acid, 50

2,6-dioxyquinoline, 49

hydrochloride of, 49

2,6-dioxyquinoline-4-carboxylic acid, 49 2-hydroxy-4, 6-methyl pyrimidine, 50 2 -methyl glyoxaline, 50

2-thiol-4-amino-methyl glyoxaline, 50

2-thiol-5-amino-methyl glyoxaline, 50

2,4,6-trihydroxypyridine, 33

2,3,4-trihydroxypyridine, 33

Ultra-violet light, 124, 126, 230-231, 239. $252, \quad 296-303,305-314,332-334$, $355,357,367,369,371,372,379$, $381,385,386,394,396,399,400$, $403,404,423,424,426,427-429$, $437,445,446,456,459,471,478$, 479

antirachitic zone in, 297,428

depth of penetration of, in tissues, $303,355,357,456$

discovery of antirachitic value of, 296, 297, 459

effect upon vitamin A, 230-231
Ultra-violet light (continued)

in antirachitic activation of ergosterol, 305-314

in antirachitic activation of foods, 300-303, 332-334, 381

in promotion of growth, 298, 429

relation to vitamin A, $298-300$

See also Irradiation; Lamps; Sunlight; Vitamin D

Ulva, 251

Unit of vitamin A, 265

of vitamin B, 102-103

of vitamin C, 176

of vitamin $\mathrm{D}, 317,328$

of vitamin G, 137

Underhill-Mendel factor, 140-141

United States Army Medical Commission, 26

Uracil, 26

Uric acid, 68, 284, 353

Urine, 373, 407, 467, 468, 522

Urolithiasis, 446

Valine, 36

Values, vitamin. See under each Vitamin

Vanaspali, 516

Veal, 270, 331

Vegetable ghee, 516

marrow, 205

oils, 253, 287, 306, 340, 437, 506, 512

Vegetables, 13, 19, 92, 93, 112, 139, 149, $168,180,202-207,231,250,268-$ $269,328,368,370,371,380,384$, $433,435,449,466,473,493,499$, 501

Velvet beans, 484, 491

Vetch, 204

"Vigantol," 367, 393, 443, 445, 478, 510, 530

Vigor, relation of vitamin A to, 287292. See also under each Vitamin, relation to health

"Viosterol," 317, 366, 374, 381, 416

Viscera, 339, 421. See also names of individual organs

Vitality. See under each Vitamin, relation to health or to nutrition.

Vitamin A, 21-23, 221-292, 293, 336, $354,359,360,362-369,372-383$, $385,387-393,396,402,404,406$, $408,413,417,420,422-427,429-$ $435,437,439,442,444,446,447$, $449,451,454-458,460-471,474$, $477,480-482,486-495,497-500$, $502-506,509,510,513-520,522$, $524,525,529,531,532$

absorption spectrum of, $249,465,489$ acetylation, 237

action of various reagents upon, 234250 
Vitamin A (continued)

and carotene, $249,362,374,382$

and disease, 221-292, 367, 376, 387, 404, 439

and inheritance of yellow color in maize, 246, 413

and light, 230, 231, 298-300, 364, 424

and metabolism of fat, 408, 447, 468

antimony trichloride method, 238249. (See also color reactions)

assimilation in presence of mineral oil, 385,389

attempts to isolate, 235-238. See also concentration

benzoylation, 237

bromination, 237

chemical nature, $228-250,368,382$, $383,390,423,433,458$

color reactions and spectrographic studies, 238-250, 368, 372, 392, $393,413,435,463,464,470,489$, $527-529$

concentration of, 235-238, 454. See also chemical nature

condition in plant tissue, 226, 227, $249,362,374,382$

correlation with green and yellow color, $243-250,362,374,382,413$

deficiency, 162, 256-259, 274-291, 359, $364-366,368,374,375,377,379$, $391,393,396,406,417,422,423$, $427,433,446,451,457,465,468$, 477

determination (measurement), 238$249,253-273,360,367,375,383$, $420,423,425,429,447,456,466-$ $468,498,507$

distillation of, $235-238$

distribution of, in nature, 267-273, $454,455,489$. See also names of foods

effect of aeration on, 229-238

effect of light on, 230-238, 298-300, 364,424

effect of ozone, 230, 231

feeding tests and color reactions compared, 238-250

formation in nature, $250-253,374$, $375,383,464,465,482$

from plant and animal sources compared, 226-250

genetical studies, 246, 413

heating experiments with, 229-234

in foods, 267-273. See also names of foods

in plant and animal tissues and products, $267-273$

irradiation, effects of, 230-238

measurement of values, 253-273, 360, $367,375,383,420,423,425,429$, $447,456,466-468,498,507$
Vitamin A (continued)

method. See determination; measurement

nutritional effect of successive diminution of amount fed, 291, 359

oxidation of, 228-238, 247

properties, 226-250. See also chemical nature

reduction, 237

relation to health, 273-292 (and bibliography)

to nutrition, 273-292 (and bibliography)

to plant pigments, $243-250$

resistant to saponification, 225

solubility and extraction, 226-229

stability, 228-250, 464, 481, 499

supposed solubility in water, 228229

synthesis, 246-253, 374, 432

thermostability, 228-250

values, 253-273. See also bibliography and names of individual foods

Vitamin-A-free diet, 256-259

Vitamin B, 14-23, 24-110, 120-129, 142, $147,176,194,226,353,356,357$, $359-366,368,370,373,375-378$, $380-395,398,403,406-409,412-$ $414,420-423,425,426,429,430$, $433-438,440,442,443,446-449$, $452,455-459,462-465,467-491$, $493,495,496,498,499,502-506$, $510,512,513,515-520,522,525$, $526,529-532$

action of ultra-violet rays on, 423 . See also chemical nature, properties, stability

adsorption of, 45, 59-61, 63-67, 69, $109,110,408$

alcoholic extraction of, 108 . See also concentration

and carbohydrate metabolism, 79, 80 . See also relation to metabolism, and to nutrition

and functional disorder of nervous system, 70-71

and growth, 76-77, 479. See also Growth

and metabolism, 71-73, 76-81, 376, $395,407,412,463,480,485$

as an antineuritic substance, 24-50, 104,362

basal diet for study of, 100 . See also determination

casein in study of, 100

chemical nature, 24-50, 56-69, $105-$ 110,472

coefficient of destruction upon heating, 106

comparative needs for, in avian and mammalian nutrition, 65

"complex," 22-23, 104 
Vitamin B (continued)

concentration of, 26-48, 55-70, 77, 360, $377,406,447,452,468,474,481$, 496

conditions for testing antineuritic action of, 39, 40, 100. See also determination; measurement

crystalline salts of, $38,39,43,44,60$ day-dose of, 67

deficiency, 14-16, 20, 24-26, 50-57, 70 $85,95,96,100-105,366,368,376$, $381,391,393,394,425,440,446$, $448,457-459,471,477,479,483$, $484,488,490$

determination, $37,39,45,48,67,85$, $95-105,353,357,370,371,386$, $387,392,455,456,498$

destruction of, $106-108,394,406,452$, 469

development of knowledge of, 24-70

distribution of, $85-95,356,377,395$, 473

effect of nitrous acid on, 107, 108

of other agents, see stability

extractability of, 108 . See also concentration of

factors of, $22,23,45,56,57,62,63$, $68,69,95,102,110,111-126,129-$ 131,147

feeding method for quantitative determination of, 99-105, 353, 370, $386,387,392,455,456,498,508$

fractionation. See concentration, and factors

gold compound of, 44, 67, 68

growth-promoting function, 50-70. See also factors, and the general description of vitamin $B$

impure lactose as source of, 52, 53

in body, 85-95, 377, 395, 473, 474, 508

in foods, 85-95, 377, 473, 474

in infant feeding, 380,386 . See also relations to nutrition

in insulin treatment, 381

inactivation of, 46, 106-108, 394, 406, $452,469,472$. See also chemical nature, and the general description of vitamin B

injection of, 74

injection method for determination, 105

instability of, as possibly related to isomeric changes, 34-37

Jendrassik reaction for, 447

measurement of activity, potency, or value, $37,39,4563,65-66,85-$ $95,95-105,353,357,370,386$, $387,392,455,456,498,508$

metallic double salts of, $37,38,43$, $44,62,67,68$

nomenclature, $20-23,384,446$
Vitamin B (continued)

Osborne-Wakeman concentrate of, from yeast, $57-58$

oxidation of, 107, 108

parallelism between lack of, and simple starvation, 70-79

paralysis in rats upon diets deficient in, 104,105

partial deficiency, 70. See also deficiency

parenteral administration of, 74,105 , 375

physiological properties and significance of, $70-85,440,485$

picrate. See concentration of

picrolonate of, 43, 66-69

precipitation of, with chloroplatinic acid, 46

with gold chloride, 67-68

preparation of concentrates of, 47,48 , $55-70,77,360,377,447,452,468$, $474,481,496$

of proteins free from, 474

presence in animal organs and tissues, $55,75,76,85-95,377,395,473$, 474

properties of, $24-85,104-110,394,406$, $452,469,472$

protective $v s$. curative tests, 37 . See also determination

quantitative determination, 95-105

rate of destruction upon heating, 106 relation to appetite, $54,71-73,376$

to gastric motility, 376

to infection, 393, 488

to metabolism, $54,75-82,373,382$, $383,384,412,462,485$

to muscular work, 377

to nutrition, $52,70-85,364,373,376$, $380,382,383,386,392,407,412$, $430,437,458,462,463,474,479$, 480

to renal enlargement on high protein diet, 395

to secretion, $73,74,375,376,381$, 436

silver complex of, 37,38

solubility, 56, 57, 69, 108. See also general description of vitamin B

sources of, in experimental diets, 73 , $100,102,131-133$

sparing action of fat, 392

stable silver compound of, 36,37

stability of, $46,58,59,105-108,394$, $406,452,469,472$

standardized animals for determination of, 101, 102, 103, 105

subcutaneous injection of, $56,74,105$, 375

successive adsorptions of, 66

suitability of rats as test animals for, 104 
Vitamin B (continued)

synthesis of, $84,359,409,455,469$, 493

tests for, see determination

"unit" of, 102, 103

Vitamin-B-free basal diet, 100, 103

Vitamin $B_{1}$, see Vitamin B

Vitamin $B_{2}$, see Vitamin $G$

Vitamin $\mathrm{B}_{3}, 125,386,484$

Vitamin C, 13-15, 21-23, 148-220, 354, $361,362,366,370,373,375,377$, $379-382,385,386,388,389,394$, $400,405,406,408,411,417,418$, $420,422,424-427,429-432,434$, $436,437,439,440,443-449,451$, $455-458,460,462-464,466-468$, $471,477,479,482,483,486,490$, $491,495,499,502,503,505,510$, $516,518,522,523,525,531$, 532

adsorption of, 183, 409

advantages of young fresh over old vegetables, 194

basal diets in study of, 162-164, 167, $169,175-177,448,483$

chemical properties, $211-220,373,379$, $385,388,389,409,417,437,445$, 455

color test for, 176, 177, 361, 362, 400, 436

computation of potency, 175

concentration of, 181, 182, 213-220, $379,405,424,427$

conditions influencing stability, 211$220,385,388,389,409,417,437$, 445

crystalline concentrate of, 215

deficiency, 156, 163-168, 171-174, 456, 466, 477. See also general account of vitamin $\mathrm{C}$

influence of, upon bones, 163-167 upon growth, 172

upon infection, 163-166, 456

upon teeth, 161-163, 165-168

determination of, $168-179,361,386$, $400,422,448,483,499$

destruction of, 173-175, 183-188, 194$201,211-220,361,385,388,389$, $409,417,437,445$

development of, in sprouting of seed, $202-205,425,449,458$

dialysis of, $216,218,373$

distribution in nature and in foods, 14 , $148-157,168-211,366,370,375$, $377,380,381,385,386,389,400$, $406,408,409,418,420,424,426$, $427,429-434,439,440,445-447$, $451,456-458,460,462-464,466$, $467,471,479,482,483,486,590$, 491

estimation of, by influence on histology of teeth, 177
Vitamin C (continued)

in nutrition and health, $148-168,354$, $382,394,400,427,446,447,456$, $466,468,471,477$

injury to teeth in deficiency of, 161 168

loss of calcium in deficiency of, 163 , 166

measurement of vitamin $C$ values, 168 $179,361,386,400,422,448,483$, 499

"minimum protective dose," 165,166 , 176

newer view of protective dose, 177 , 178

occurrence, see distribution

partial deprivation of, 161-168

properties of concentrates, $211-220$

protocols of experiments, 172-175

quantitative studies, $168-220$

requirements of different species, 148168

results with graded allowances of, $172-175$

stability (and stabilization) of, 148$157,173-175,179-213,217,385$, $388,389,409,417,437,445$, 446

standard animals for study of, 169 , 173, 176

storage in body, 158-161, 207

suggested duality of, 483

synthesis of, $155,159,160,202-205$, $425,449,458$

"unit" of, 176

Vitamin D, 22, 176, 227, 257, 293-335, $356,357,362,365,366,372,374$, $377,385-388,395,405,406,409$, $413,414,417,420,421,423,425$, $427-429,433,435,437,438,441$, $442,446-448,454,456,458-460$, $462,464-466,470,474,478,480$ $483,487,489,493-495,497,499$, $501,505,507,509,510,518,519$, $522,525,527-531,533$

absorption from skin, 429

absorption spectrum of, $327,328,365$, 480

and ultra-violet light, 296-300

chemical nature of, 303-314

concentrate, chemical properties of, 310,456

concentration of, 304, 310,329, 456, 470

deficiency, see Rickets

determination, 314-319, 323-326, 374, $414,420,433,454,481,482$, 509

diets for determination of, 316-326

differentiation (quantitative) from vitamin A, 293-296, 420

distillation of, 356 
Vitamin D (continued)

distribution of, $328-335,362,366,372$, $374,377,387,406,427,428,435$, $437,438,446,459,460,462,466$, $474,483,487,489,493$

effect of mineral oil on assimilation of, 385

of overdosage, 448

of solvent in formation of, 311

of temperature on stability of, 328

of time of irradiation on stability of, 311,328

of wave-length of light on formation of, 313

effect of, on calcium and phosphorus in body, 323, 325, 326, 413, 478, 482

on response to parathyroid extract, 464

energy required for formation of, 309

extraction from plant tissues, 328 . See also concentration of

food sources of, 328-335, 374, 387, $406,427,428,435,437,466,474$, 483,487

formation of, 300-303, 305-314, 330$335,458,465$

in growth, $300,326,357$

in irradiated foods, 300-303, 332-335, $374,386,429,487,494$

in plankton, 332

in plant tissues, 328,329

in tuberculosis, 388

increase of, in milk by feeding codliver oil, 334

intensity of light in production of, 313

line test for, 314-317, 374

measurement of, $314-328,374$

minimum detectable amount, 317,374

nature of, 303-314. See also general account of vitamin D

oils as source of, 330. See also names of individual oils

origin in nature, 330-332. See also distribution

production of, by cathode rays, 310

by floridin, 331

by irradiation, 300-303, 305-314, $332-335,374,386,429,487,494$

from ergosterol, 305-314

in foods, 332-335, 374, 386, 429, 487,494

photosynthesis of, 300-303, 305-314, $330-335,405,458,465,489$

proof of existence of, 293-296

quantity estimated as detectable, 317 relation to ergosterol (and cholesterol), 305-314, 489

seasonal variations, $329,330,332,334$ specificity of ergosterol as precursor, $305-314,489$

spectroscopic studies of, 327
Vitamin D (continued)

stability of, 303-305, 328-332, 362, 421, $423,438,470$

standard solution for reference in measurements of, 328

standardization in butter and margarine, 395

storage in body, 293, 294, 319-321

temperature coefficient for formation of, 310

theory as to synthesis by cod, 331

"unit" of, 317,328

Vitamin E, 54, 82, 336-349, 368, 391, $392,437,439,458,459,469,502$, $511-513,515,521$

chemical properties, 341, 345-349, 391, 458

concentration of, $347-349,392$

deficiency, 341-344, 391, 392, 439, 458, 459

destruction of, $341,392,458$

determination of, $338-341$

distribution of, 336, 338-341, 392

evidence of existence, 337-338, 469

in foods, 336, 338-341, 437

initial fertility, 337

occurrence, 336, 338-341, 392

physical properties, 345-349

relation to metabolism, 341-344, 368, 391

stability of, 341, 345-349, 391, 458

storage in body, 337, 342, 343

synthesis, 336, 338-341, 392

Vitamin F, 22

Vitamin G, 22, 23, 82, 90, 91, 94, 100 , $102-105,109,111-147,286,363$, $365,368,370,371,394,395,408$, $422,429,447,467,482,499$

activity of Levene concentrate, 129

adsorption of, 63, 64, 123, 124, 408

apparent losses in presence of air and alcohol, 136

concentration of, 126-129, 135

deficiency of, 140-147, 394

determination of, $129-135,365,371$

differentiation from vitamin $B, 111$ 126,135

distribution in body and in foods, 137 $140,368,422,429,467,482$

evidence of existence, 111-126

experiments with ultra-violet light, 124-126

extraction experiments, $135-136$

in foods, $137-140,422,429,467,482$

in normal nutrition, $145-147$

measurement of, $129-135,365,371$

occurrence, $137-140,368,422,429$, 467,482

precipitation of, $126-129$

probable relation to longevity, 146

to renal enlargement on high protein diet, 145,395 
Vitamin G (contimued)

quantitative determination, 129-135, 365,371

relation to lactation, 145-146

to nutrition, $140-147,395$

to protein metabolism, 145,395

to reproduction, $145-146$

solubility in alcohol of different strengths, 135,136

stability of, $124-126,136,370,371$, 482

storage in body, 146, 147

"unit" of, 137

Vitamin theory, 13-25, 157

Vitamins, fat-soluble. See Vitamins A, D, E

water-soluble. See Vitamins B, C, G

"Vitavose," 512

Walnut oil, 340

Walnuts, 94, 269, 462

IVater, shaking butterfat with, 228229

Watercress, 201, 375

Watermelon, 205, 269, 467

Water-soluble B, 21, 25, 52, 53, 157. Sce also Vitamin B

Water-soluble C, 21. See also Vitamin C

Water-soluble vitamins, differentiation of, 111

Wave-lengths of light, in formation of vitamin $\mathrm{D}, 313$

in prevention and cure of rickets, 297

Whale oil, 271

Wheat, 53, 90, 108, 123, 125, 129-130, $132-133,137,203-204,252,201$, $316,325,333,341,359,370,410$ $411,429,438,451,454,470,473$, $497,506,520$

extraction of with alcohol, 53, 133

embryo, 47, 52, 53, 56, 57, 62, 67, 69, $73,109,123,129-130,225,324$, $340-341,454,511$

germ, 56, 62, 90, 117, 158, 316, 402, $422,452,464,493$

germ oil, 347,378

gluten, 316

oil, 512

plant, 332

shoots, 464
White beans, 56

bread, 180

v's. yellow turnips as source of Vitamin C, 193

Williams-Waterman factor, 125

Wool fat, 406

Worms, 225

Wort, 505

"X." See Vitamin E

X-rays, 317-319

Xanthophyll, 227, 250, 527, 475. See also Carotinoids; Pigments

Xanthoproteic, 27

Xerophthalmia, 224-225, 231, 250, 274, $284,299,303,320,363-364,367$, $387,389,406,414,451,469,471$, 480-481, 495, 497, 509, 522. See also Infection; Ophthalmia; Vita$\min \mathrm{A}$

Yautia, 246, 268, 373, 482

Yeast, 28, 31, 33-35, 37, 39-42, 44-48, $53-58,63-65,68,69,73,76-80,90$, $95,100-103,108,109,115-117$, $119,120,122,123,125-131,137-$ $138,140,164,169,176,257,300$, $320,324,326,327,333,334,354$, $371,374,378,380,382,386,387$, $389,391,396-398,401,402,409$, $411,413,414,425,429,437,438$, $440,445,449,456,469,470,472-$ $474,476,478,481,483,493,496$, $504,507,511,514,516,525-527$, 530

autoclaved, 100,102

concentrates of, $37,39,45,65,397$, 438,481

extracts of, $45,64,78,79,398,470$

for use in experimental diet, 257,425

irradiated, 334, 411, 440

protein, 473

fat, 128, 429, 449

Yeast-growth method, 95, 386

Yellow vegetables. See Vitamin A

Yolk. See Egg.

Young, nutrition of, 513. See also Growth

rearing of, 286-292. See also Lactation

Zinc chloride, 43,62

Zymosterol, 429, 478 



\section{American Chemical Society MONOGRA PH SERIES}

\section{PUBLISHED}

No.

1. The Chemistry of Enzyme Actions (Revised Edition)

By K. George Falk.

2. The Chemical Effects of Alpha Particles and Electrons (Revised Edition)

By Samuel C. Lind.

3. Organic Compounds of Mercury

By Frank C. Whitmore.

4. Industrial Hydrogen

By Hugh S. Taylor.

5. Zirconium and Its Compounds

By Francis P. Venable.

6. The Vitamins (Revised Edition)

By H. C. Sherman and S. L. Smith.

7. The Properties of Electrically Conducting Systems By Charles A. Kraus.

8. The Origin of Spectra

By Paul D. Foote and F. L. Mohler.

9. Carotinoids and Related Pigments

By Leroy S. Palmer.

10. The Analysis of Rubber

By John B. Tuttle.

11. Glue and Gelatin

By Jerome Alexander.

12. The Chemistry of Leather Manufacture (Revised Edition) By John A. Wilson. Vol. I. and Vol. II.

13. Wood Distillation By L. F. Hawley.

14. Valence and the Structure of Atoms and Molecules By Gilbert N. Lewis.

15. Organic Arsenical Compounds

By George W. Raiziss and Jos. L. Gavron.

16. Colloid Chemistry (Revised Edition) By The Svedberg.

17. Solubility By Joel H. Hildebrand.

18. Coal Carbonization

By Horace C. Porter.

19. The Structure of Crystals

By Ralph W. G. Wyckoff.

20. The Recovery of Gasoline from Natural Gas By George A. Burrell.

21. The Chemical Aspects of Immunity (Revised Edition) By H. Gideon Wells. 


\section{American Chemical Society MONOGRAPH SER I ES}

\section{PUBLISHED}

No.

22. Molybdenum, Cerium and Related Alloy Steels By H. W. Gillett and E. L. Mack.

23. The Animal as a Converter of Matter and Energy By H. P. Armsby and C. Robert Moulton.

24. Organic Derivatives of Antimony By Walter G. Christiansen.

25. Shale Oil By Ralph H. McKee.

26. The Chemistry of Wheat Flour By C. H. Bailey.

27. Surface Equilibria of Biological and Organic Colloids By P. Lecomte du Noüy.

28. The Chemistry of Wood By L. F. Hawley and Louis E. Wise.

29. Photosynthesis By H. A. Spoehr.

30. Casein and Its Industrial Applications By Edwin Sutermeister.

31. Equilibria in Saturated Salt Solutions By Walter C. Blasdale.

32. Statistical Mechanics as Applied to Physics and Chemistry By Richard C. Tolman.

33. Titanium By William M. Thornton, Jr.

34. Phosphoric Acid, Phosphates and Phosphatic Fertilizers By W. H. Waggaman.

35. Noxious Gases By Yandell Henderson and H. W. Haggard.

36. Hydrochloric Acid and Sodium Sulfate By N. A. Laury.

37. The Properties of Silica By Robert B. Sosman.

38. The Chemistry of Water and Sewage Treatment By Arthur M. Buswell.

39. The Mechanism of Homogeneous Organic Reactions By Francis O. Rice.

40. Protective Metallic Coatings By Henry S. Rawdon.

41. Fundamentals of Dairy Science By Associates of Rogers.

42. The Modern Calorimeter By Walter P. White. 


\section{American Chemical Society \\ MONOGRA PH SER IES}

\section{PUBLISHED}

No.

43. Photochemical Processes

By George B. Kistiakowsky.

44. Glycerol and the Glycols

By James W. Lawrie.

45. Molecular Rearrangements

By C. W. Porter.

46. Soluble Silicates in Industry

By James G. Vail.

47. Thyroxine

By E. C. Kendall.

48. The Biochemistry of the Amino Acids

By H. H. Mitchell and T. S. Hamilton.

49. The Industrial Development of Searles Lake Brines By John E. Teeple.

50. The Pyrolysis of Carbon Compounds By Charles D. Hurd.

51. Tin

By Charles L. Mantell.

52. Diatomaceous Earth By Robert Calvert.

53. Bearing Metals and Bearings

By Willian M. Corse.

54. Development of Physiological Chemistry in the United States

By Russell $\mathrm{H}$. Chittenden.

\section{IN PREPARATION}

Piezo-Chemistry

By L. H. Adams.

The Biochemistry of the Fats and Related Substances

By W. R. Bloor.

Absorptive Carbon

By N. K. Chaney.

The Activated Sludge Process of Sewage Disposal

By Robert Cramer and John Arthur W'ilson.

Fixed Nitrogen

By Harry A. Curtis.

The Manufacture of Sulfuric Acid

By Andrew M. Fairlie.

Liquid Ammonia as a Solvent

By E. C. Franklin. 


\section{American Chemical Society MONOGRA PH SERIES}

\section{IN PREPARATION}

Surface Energy and Colloidal Systems

By W. D. Harkins and T. F. Young.

The Structure of Rubber

By Ernst A. Hauser.

Absorption Spectra

By Victor Henri and Emma P. Carr.

The Rate of Homogeneous Gas Reactions

By Louis S. Kassel.

The Effect of Radiant Energy on Physiological Processes By H. Laurens.

Nucleic Acids

By P. A. Levene.

Vapor Phase Catalytic Oxidation of Organic Compounds and Ammonia

By L. F. Marek and Dorothy A. Hahn.

The Corrosion of Alloys

By Robert J. McKay.

Significance of Manganese, Iron and Aluminum to Soil Acidity and Plant Life

By Forman T. McLean.

Physical and Chemical Properties of Glass

By Geo. W. Morey.

Acetylene

By J. A. Nieuwland.

The Free Energies of Organic Compounds

By G. S. Parks and H. M. Huffman.

Furfural

By F. N. Peters, Jr., and H. J. Brownlee.

Carbon Dioxide

By Elton L. Quinn and Charles L. Jones.

Aliphatic Sulfur Compounds

By E. Emmet Reid.

The Chemistry of Intermediary Metabolism By Wm. C. Rose.

Electrical Precipitation of Suspended Particles from Gases By W. A. Schmidt and Evald Anderson.

Dielectric Constants and Molecular Structure By Charles P. Smyth.

Precise Electric Thermometry

By W. P. White and E. F. Mueller.

Modern Theories of Electrolytes

By John Warren Williams.

Measurement of Particle Size and Its Application By L. T. Work. 



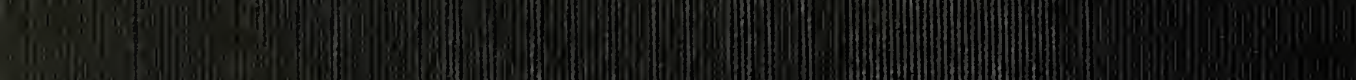

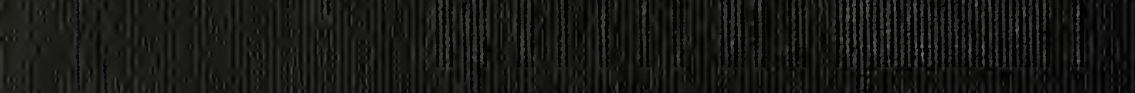

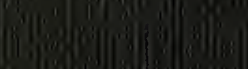
19.1 $\mid$ Not. $1 / 20$ 